\title{
A LABORATORY MANUAL FOR
}

\section{COMPARAIIVE VERTEBRATE ANATOMY}

\section{HYMAN}

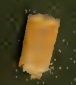




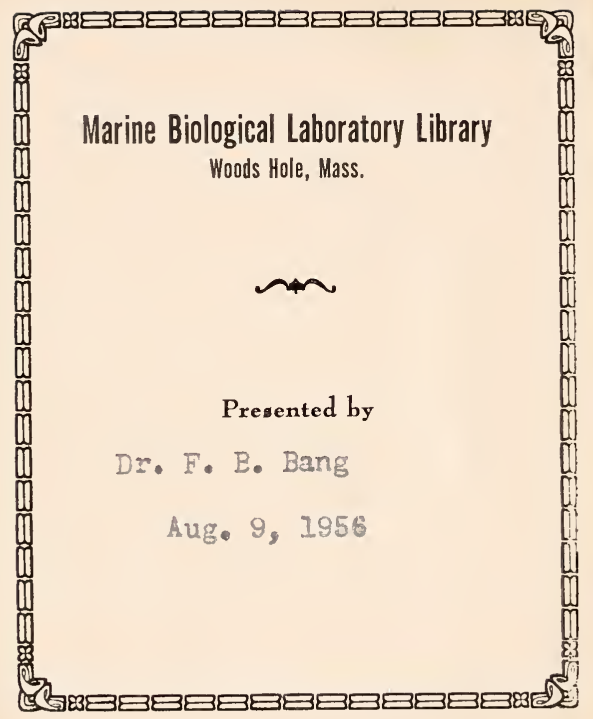


Frederik Karry Bana.

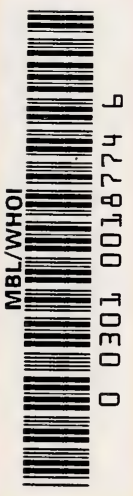



A LABORATORY MANUAL FOR COMPARATIVE VERTEBRATE ANATOMY 


\section{THE UNIVERSITY OF CHICAGO PRESS CHICAGO, ILLINOIS}

THE BAKER \& TAYLOR COMPANY NEW YORK

THE CAMBRIDGE UNIVERSITY PRESS LONDON

THE MARUZEN-KABUSHIKI-KAISHA TOKYO, OSAKA, KYOTO, FUKUOKA, SENDAI

THE COMMERCIAL PRESS, LIMITED SHANGHAI 


\section{A LABORATORY MANUAL FOR}

\section{COMPARATIVE VERTEBRATE ANATOMY}

By

LIBBIE HENRIETTA HYMAN, Pн.D.

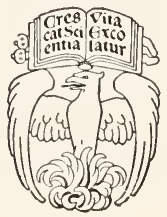

THE UNIVERSITY OF CHICAGO PRESS

CHICAGO, ILLINOIS 
COPYRIGHT I922 BY THE UNIVERSITY OF CHICAGO ALL RIGHTS RESERVED. PUBLISHED FEBRUARY 1922 FIFTEENTH IMPRESSION AUGUST 1934

COMPOSED AND PRINTED BY THE UNIVERSITY OF CHICAGO PRESS CHICAGO, ILLINOIS, U.S.A. 


\section{PREFACE}

Several years ago the method of procedure in the laboratory work in vertebrate zoölogy in this University was changed from the type plan, then in common use, to the comparative plan. No doubt a similar change has been made in many other institutions. A suitable manual for the comparative method has, however, hitherto been lacking; the present publication attempts to supply this need. There can scarcely be any question of the superiority of the comparative method of study of vertebrate anatomy, for by this method the student not only learns all of the anatomical facts brought out by the type method but he also acquires an understanding of vertebrate and human structure which he is quite unable to attain by the older method of study. In view of the fact that the majority of the students taking courses in vertebrate anatomy at the present time are preparing for medicine, it seems obligatory that they be taught the "history of the human body" as revealed by the study of the anatomy of vertebrates. On the other hand, the comparative method is perhaps faulty in that it may not give the student a clear-cut picture of the characteristics of the various classes of vertebrates. Thus, while the student readily learns the history of the aortic arches, for example, he does not readily associate any particular group of vertebrates with a particular condition of the arches, when the comparative method is followed. This defect should probably be remedied in the lecture part of the course. I have attempted to remedy it to a slight extent by introducing the section on the general features of typical chordates.

In this manual $\mathrm{I}$ have attempted not only to give the laboratory directions for the dissection of the various systems, but I have also presented in connection with each system a very brief, generalized, and simplified account of the development and evolution of that system. It has seemed to me essential that such an account precede or accompany the laboratory directions in order to give a significance to the facts revealed by the dissection at the time when the student becomes aware of those facts. As the consultation of other texts during a dissection is inconvenient and time-consuming, I have thought it the most practical plan to include such explanatory matter in the laboratory manual. Simple illustrations have been added to clarify further the explanations. I have not, however, in the least intended that these explanations should take the place of reading in the standard texts of comparative anatomy. The students should understand that additional outside reading is expected of them.

In making such brief and generalized explanations as are given here it is impossible to take into account numerous exceptions and variations. I must therefore ask the indulgence of the expert in vertebrate anatomy for the omission of qualifying clauses in the explanatory accounts of the various systems; in 
some cases, no doubt, I have been unaware of the exceptions; in others I have knowingly omitted them on the grounds that statements of exceptions are more confusing than informative to the beginner.

To avoid confusion the explanatory matter is printed in slightly smaller type than the directions for the dissections.

I have included in the manual such materials as seemed to me to bear most directly on the story of the evolution of the various systems. I have treated the skeleton and the coelom at greater length than is commonly the case. The prevailing neglect of the study of the skeleton in courses in vertebrate anatomy seems to me unjustifiable in view of the importance of this system in the study of phylogenetic and evolutionary questions. It is true that skeletal material is somewhat expensive to purchase and maintain, but a good many of the more important materials can be prepared by the instructor or students. In presenting the vertebral column I have adopted Gadow's conception of the development of the vertebrae from separate arcualia, an idea also adopted by Schauinsland in his account of the development of the vertebrae in Hertwig's Handbuch. The conception would appear to be correct in the main, and at least furnishes a simple explanation for the variety of vertebral columns encountered among vertebrates. The difficulties attending the study of the coelom and mesenteries do not seem to me to justify one in disregarding them. I hope the simplified account I have presented after long study and thought on the matter will aid in the understanding of this complex subject. As to the animals to be dissected, the elasmobranch must naturally form the point of departure in the study of comparative anatomy. I have described the skate in addition to the dogfish, since some teachers prefer it; it is certainly more favorable than the dogfish for the study of the nervous and urogenital systems, but less favorable, in my opinion, for the study of the circulation. The not infrequent scarcity of dogfish in recent years makes it desirable that an alternative form be described. The bony fish is omitted because its specialized structure would confuse rather than aid the student in following out the evolution of the systems. The frog is so often used in general and beginning courses that it seems superfluous to consider it here. Further, the urodeles serve our purpose much better. I should like to have included Cryptobranchus as alternative to Necturus, but the limits of space forbade. The reptile is important for the purposes of a comparative course, and the turtle is perhaps the most readily obtainable form of sufficient size. The bird has been included since it seemed inadvisable to omit altogether an entire vertebrate class. I have described both the cat and rabbit, as the former animal, though perhaps preferable in some respects, is not always readily obtainable.

At the University of Chicago the course consists of a brief glance at the external features of the lower chordates and of representative vertebrates; of all of the work given in the manual on the exoskeleton, endoskeleton, and muscles, with the exception of the muscles of Necturus; and of the complete dissection 
of the dogfish, turtle, and mammal, except the peripheral distribution of the cranial nerves in the mammal. The elasmobranch, urodele, and mammal would make a combination nearly as desirable.

The general statements and explanations given in the manual have been taken from standard works and original papers on comparative anatomy, vertebrate zoölogy and embryology, and human anatomy. I have adopted, for the most part, the usual accounts of the evolution of the various systems and parts, not regarding myself as competent to criticize them. In a few cases I have presented some recent views appearing in the literature. The laboratory directions and descriptions of the anatomy of the several animals have, however, been written entirely from the specimens. Practically the whole of the dissection has been performed twice, some of it more than twice. The dissections have been carried on simultaneously with the writing of the directions. In locating and naming the structures I have been assisted by various texts, manuals, and original papers. In a number of cases I have found it desirable to devise additional names or to change old names. I have employed the terms dorsal, ventral, anterior, and posterior as they are used in vertebrate anatomy, abandoning the usage common in human anatomy. This has involved a change in some familiar names, such as that of the "anterior" abdominal vein.

I have made a particular effort to use all technical words in a very precise and exact manner and to define each such word where it first occurs. One is continually surprised and annoyed in a study of vertebrate structure to note the loose and inexact way in which many terms are employed. It is, in fact, practically impossible to find any precise usage for many terms, such as urethra, bulbus arteriosus, peritoneum, olfactory lobe, perineum, and others. In such cases I have been compelled to adopt such a definition as seems consistent with the majority of the anatomical facts.

I have attempted nothing in this manual but a plain account of the anatomy of the several animals, which account the student follows. This "verification" type of laboratory manual has been recently subjected to much criticism, much of it, in my opinion, undeserved. The critics seem to forget that the student is not in reality engaged in "verifying" the statements in the manual; he is engaged in learning the anatomy of an animal by the shortest and easiest route, a route which the critics themselves would follow if confronted with an animal with which they were not familiar. It is my opinion that human beings in general see chiefly that which is pointed out to them; this has been proved over and over again in the history of biology. The large number and complexity of the anatomical facts to be acquired, the limited time allowed for their acquisition, the large size of the classes, and the limited number of laboratory assistants available seem to me to necessitate that detailed and specific laboratory directions be provided. If the directions are not given in the manual, then the laboratory instructors are compelled to provide them verbally. Personally I am unable 
to see any pedagogical difference between directions and explanations written in the manual and those given out verbally by the instructor, but I do see a great deal of difference as concerns the time, patience, and energy of instructors and students. Our experience with laboratory manuals of the type in which the burden of discovery is left to the student is that the student becomes highly dissatisfied and that the instructors are brought into a state of irritation and fatigue by the continuous demands for assistance with which they are bombarded. Frankly, I believe in the conservation of instructors, and have written this manual with that end in view. In place of inserting questions in the laboratory manual our method is to hold thorough oral quizzes on the laboratory and textbook work at frequent intervals.

Although a number of drawings are called for in the manual, it is probable that each instructor will prefer to decide for himself what drawings are to be made. Drawings might profitably be omitted altogether, their place being taken by rigorous practical individual quizzes on the dissected specimen.

I am indebted to a number of authors and publishers for permission to reproduce figures from their publications. Due acknowledgment is made in the legends to these figures. I have not listed the numerous original papers to which I have referred, since most of them are given in the bibliographies appended to Kingsley's Comparative Anatomy of Vertebrates and Goodrich's account of the fishes in Part IX, first fascicle, of Lankester's A Treatise on Zoölogy. I am indebted to Dr. C. R. Moore, Dr. B. H. Willier, and Dr. J. W. Buchanan for calling my attention to errors and omissions in the first draft of the manual which has been used in the laboratory under their direction during the past two years. My thanks are also due to Mr. Kenji Toda for his patience and skill in drawing the illustrations. Finally, I wish to acknowledge the fact that the previous organization of the laboratory work along comparative lines, chiefly through the efforts of Dr. J. IW. MacArthur and Mr. J. G. Sinclair, has facilitated the task of preparing this manual.

So laborious has been this task and so great is the number of facts to be considered that I can scarcely hope to have avoided errors, omissions, and statements lacking in clarity. I shall be more than grateful to have my attention called to them.

University of Chicago

L. H. HymaN

November, I921 


\section{GENERAL DIRECTIONS}

\section{Supplies}

I. Dissecting instruments necessary for the course are:

Medium-sized scalpel.

Fine scissors.

Coarse scissors.

Stout probe for dissecting.

Long slender probe for probing.

Medium-sized forceps with straight points.

A towel and a laboratory coat or gown to protect the clothes are desirable.

Bone scissors or forceps will be provided in the laboratory.

2. Drawing materials necessary for the course are:

Drawing paper, No. 6. This paper must be stiff and hard and have a smooth surface.

Hard drawing pencil, 6 or $8 \mathrm{H}$.

Eraser.

Ruler.

Red, yellow, and blue pencils.

Pad of emery paper to sharpen the hard pencil.

3. Obtain the supplies named above and present yourself with a complete outfit at the first laboratory period. Do not handicap yourself at the start by neglecting to provide yourself with the necessary materials.

\section{Drawings}

I. All drawings must be made with a hard pencil on good quaiity drawing paper, unless otherwise specified. Colors are to be used only when specified in the directions. Shading, crosshatching, etc., are undesirable and are to be avoided. Drawings made otherwise than as here specified will not be accepted.

2. Drawings are to be line drawings only, that is, only the outlines of the structures are to be drawn. Every line must represent a structure actually present on the specimen. Lines must be smooth and clean. Correct proportions are of the utmost importance and are to be obtained by use of a ruler. In making a drawing it is best to outline the drawing first with very light lines, correcting these until accurate appearance and proportions are obtained. Then erase the light lines until they are barely visible and go over them with a well-sharpened pencil, making the final lines firm and clear.

3. Drawings are not to be diagrammatized unless so directed in the manual. Many students do not seem to understand the difference between a diagram and a drawing. As an illustration, Figure 9, page I 5, in Kingsley's Comparative A natomy of Vertcbrates, is a diagram, and Figure 45, page 5I, is a drawing of the upper half of the same structures represented in the diagram. The latter shows what the object actually looks like; the former is for purposes of explanation only.

4. All drawings must be made directly from the object with the object before the student and completed in the laboratory. The making of rough sketches in the laboratory to be "improved" elsewhere is unscientific, inaccurate, and absolutely not permitted.

5. Remember that the prime requisite of a drawing is accuracy. A drawing is for the instructor a record of what you have actually seen upon your specimen. If you have not 
dissected the structures called for, then it is obvious that you cannot draw them accurately. Poor laboratory work invariably reflects itself in the quality of the drawings.

6. Drawings must contain all of the details mentioned in the manual. If, after honest effort and with the aid of the laboratory assistants, you are unable to identify certain structures, omit them from the drawing and make a note to the effect that you were unable to find them. An unreasonable amount of time should not be spent in locating small or unimportant details.

7. All drawings must be thoroughly labeled. Every drawing must be completely labeled regardless of whether the same structures have already been labeled in some preceding drawing. Labels are to be written or printed in hard pencil parallel to the top and bottom edges of the page and lines drawn with a ruler from the labels to the parts indicated.

8. Draw on the right-hand surface of the page only.

9. Remember that the laboratory instructors are familiar with all of the figures in the various textbooks and that undue resemblance between your drawings and such figures will reflect upon your honesty and raise a suspicion that you have not been exerting yourself in the laboratory.

ı. The drawings will be called in at intervals. The dates on which they are due will be announced in advance by the laboratory instructors.

\section{Notes and Quizzes}

I. No notes are required in this course. The notebooks will consist of drawings only.

2. Oral and written quizzes upon the subject-matter of the laboratory work are to be expected at any time. These quizzes will deal with the anatomy of the animals you are dissecting and with comparative anatomy. You will be expected to know thoroughly the animals and materials which you dissect and study in the laboratory, and to be able to compare them with one another, stating resemblances and differences. You will be required to exhibit your dissections and to be able to identify the structures present on the dissections.

3. An important quiz will follow the completion of each section of the laboratory work and will deal with that section.

4. Reading in the textbooks of comparative anatomy is expected as a part of the laboratory work. Quizzes will include material in such textbooks.

\section{Dissection}

I. Dissection does not consist in cutting an animal to pieces. Dissection consists in separating the parts of an animal so that they are more clearly visible, leaving the parts as intact as practicable.

2. In dissecting an animal very little cutting is required. Cleaning away the connective tissue which binds together and conceals structures is the chief process in dissection. In doing this, use blunt instruments, as the probe, forceps, or fingers. Avoid the use of scalpel and scissors. You will probably cut something you will need later on. In short, do not cut; separate the parts.

3. Have the animal firmly fastened. Smaller animals are generally pinned to waxbottomed dissecting pans. Larger animals, such as are used in the greater part of this course. are tied to screw eyes in the corners of the dissecting pan. Put the particular part you are dissecting on a stretch.

4. Do your own dissecting. Do not watch somebody else do it. Begin at the most easily accessible point of the system you are studying and follow out your structure, cleaning away the tissues that conceal it.

5. Exercise patience and care. Clean the structures by small portions. 
6. Follow the directions precisely. Do not cut anything or remove anything unless specifically directed to do so.

7. Your laboratory grade is partly determined by the kind of dissections you make.

\section{Materials}

I. But one specimen of each animal is allowed to each student. Each student will be given the necessary specimens and will retain them to the end of the course. Do not discard any animal until the manual so directs.

2. The smaller materials which are provided for the class as a whole should be returned to the bottles or jars from which they came as soon as you have finished studying them.

3. The larger specimens will be kept in large cans. Each table will be allotted the necessary number of cans. Students will attach tags bearing their names to their specimens and keep them in the cans when they are not in use.

4. Specimens must always be kept moist and must never be allowed to dry up, as this ruins them for dissection. Do not go away and leave your specimens out on the table. When ready to leave the laboratory, wrap the animals in moistened cheesecloth provided in the laboratory and put them into the cans. See that the cans are always covered.

5. Students who, through their own carelessness, render their specimens unfit for further dissection will have to pay for new specimens.

6. The skeletal material provided in this course is expensive. Handle it with care. Be particularly careful with skeletons preserved in fluid.

\section{References}

Throughout this manual reference is made to a number of texts and manuals which may profitably be consulted by the student. They are obtainable in the library. These references are indicated as follows:

B-Bensley, Practical Anatomy of the Rabbit.

CNH-The Cambridge Natural History: Volume VII, "Fishes, Ascidians," etc.; Volume VIII,

"Amphibia and Reptiles."

D-Davison, Mammalian Anatomy with Special Reference to the Cat.

H-Hertwig, Manual of Zoölogy, translated by Kingsley, 3d ed., r9i 2.

K-Kingsley, Comparative Anatomy of Vertebrates, 3d ed., I926.

L-Lankester, A Treatise on Zoölogy. Part IX, first fascicle, "Cyclostomes and Fishes." $\mathrm{N}$-Newman, Vertebrate Zoölogy.

$\mathrm{P}$ and H-Parker and Haswell, A Textbook of Zoölogy, Volume II, 3d ed., I92 I.

$\mathrm{R}-$ Reynolds, The Vertebrate Skeleton.

$\mathrm{R}$ and J-Reighard and Jennings, Anatomy of the Cat.

W-Wilder, The History of the Human Body.

Wd-Wiedersheim, Comparative Anatomy of Vertebrates. 



\section{TABLE OF CONTENTS}

I. General Considerations on Animal Form .
A. Descriptive Terms
B. Planes and Axes
C. Symmetry .
D. Metamerism or Segmentation
E. Cephalization
F. Homology and Analogy

II. The Phylum Chordata

A. The Characteristics of the Chordates

B. The Characteristics of the Vertebrates

C. The Classification of the Chordates

iII. General Study of Typical Chordates
A. Amphioxus
B. A Tunicate
C. Balanoglossus
D. Anatomy of a Lamprey
E. External Anatomy of the Dogfish
F. External Anatomy of the Skate
G. External Anatomy of a Teleost
H. Some Ganoid Fishes
I. External Anatomy of Necturus
J. External Anatomy of a Lizard
K. External Anatomy of the Turtle
L. External Anatomy of the Pigeon
M. External Anatomy of a Mammal
N. Summary

IV. General Features of Chordate Development • • • • • • 3 I

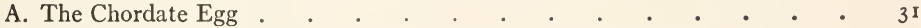

B. The Cleavage of the Egg and the Formation of the Blastula . . . . 32

C. Formation of the Gastrula . . . . . . . . . . 34

D. Formation of the Third Germ Layer, the Neural Tube, and the Notochord 36

E. Further History of the Mesoderm . . . . . . . . . 39

F. The Fate of the Ectoderm . . . . . . . . . . . . $4 \mathrm{I}$

G. The Fate of the Entoderm . . . . . . . . . . . . 4 I

H. The Fate of the Mesoderm and the Formation of Mesenchyme . . . 42

V. The Comparative Anatomy of the Integument and the Exoskeleton . 45

A. General Considerations on the Skeleton . . . . . . . . 45

B. The Structure of the Skin . . . . . . . . . . . 45

C. The Exoskeleton in General . . . . . . • . • • 447

D. Exoskeleton of Fishes . • • • • • • • • • • 447

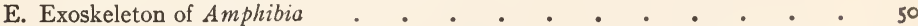

F. Exoskeleton of Reptiles . . . . . . . . . . . . 50 
G. Exoskeleton of Birds

H. Exoskeleton of Mammals

I. Summary

VI. The Endoskeleton: the Comparative Anatomy of the Vertebral Column AND RIBS .
A. General Considerations on the Endoskeleton
B. The Embryonic Origin of the Vertebrae and Ribs
C. Some Primitive Vertebral Columns
D. The Vertebral Column of the Dogfish .
E. Vertebral Column of Teleosts
F. Vertebral Column of Amphibia
G. Vertebral Column of Reptiles
H. Vertebral Column of Birds
I. Vertebral Column of Mammals
J. Summary of the Vertebral Column and Ribs

VII. The Endoskeleton: the Comparative Anatomy of the Girdles, the Sternum, and the Paired Appendages

A. General Considerations 78

B. The Pelvic Girdle and the Posterior Paired Appendages . . . . . . 79

C. The Pectoral Girdle, the Sternum, and the Anterior Paired Appendages - 85

D. General Summary of the Girdles, the Sternum, and the Paired Appendages

VIII. The Endoskeleton: the Comparative Anatomy of the Skull and the Visceral Skeleton
A. The Cartilage Stage of the Skull .
B. The Visceral Skeleton
C. The Formation of the Membrane Bones of the Skull
D. The Formation of the Cartilage Bones of the Skull and the Composition of the Complete Skull .

E. The Skull of Necturus, a Partially Ossified Skull

F. The Skull of the Alligator, a Typical Modern Skull .

G. The Bones of the Mammalian Skull

H. General Summary of the Skull and Visceral Skeleton
A. General Considerations
B. The Muscles of the Dogfish
C. The Muscles of Necturus
D. The Muscles of the Cat and Rabbit
E. Summary

X. The Comparative Anatomy of the Coelom, Digestive, and Respiratory Systems
A. The Origin and Parts of the Coelom and the Mesenteries
B. The Digestive Tract and Its Derivatives
C. The Coelom, Digestive, and Respiratory Systems of Elasmobranchs
D. The Coelom, Digestive, and Respiratory Systems of Necturus
E. The Coelom, Digestive, and Respiratory Systems of the Turtle
F. The Coelom, Digestive, and Respiratory Systems of the Pigeon 
G. The Coelom, Digestive, and Respiratory Systems of a Mammal

H. The Comparative Anatomy of the Coelom and the Mesenteries

I. Summary

Xi. The Comparative Anatomy of the Circulatory System

A. General Considerations

B. The Circulatory System of Elasmobranchs .

C. The Circulatory System of Necturus

D. The Circulatory System of the Turtle

E. The Circulatory System of the Pigeon

F. The Circulatory System of the Mammal

G. Summary of the Circulatory System

XII. The Comparative Anatomy of the Urogenital System

A. Embryonic Origin and Evolution of the Urogenital System

B. The Urogenital System of Elasmobranchs .

C. The Urogenital System of Necturus

D. The Urogenital System of the Turtle

E. The Urogenital System of the Pigeon

F. The Urogenital System of the Mammal

G. The Embryonic Membranes .

H. Summary of the Urogenital System

XiII. The Comparative Anatomy of the Nervous System and the Sense Organs

A. General Considerations . . . . . . . . . . . . 296

B. The Nervous System and Sense Organs of Elasmobranchs . . . . 301

C. The Nervous System and Sense Organs of Necturus . . . . . . 318

D. The Nervous System and Sense Organs of the Turtle . . . . . 321

E. The Nervous System and Sense Organs of the Pigeon . . . . $\quad 328$

F. The Nervous System and Sense Organs of the Mammal . . . . 333

G. Summary of the Nervous System and the Sense Organs . . . . 359

Appendix A. Pronunciation and Derivation of Technical Words . . . 362

Appendix B. Preparation of Materlals . . . . . . . . . 370

INDEX • • • • • • • • • • • • • • • • • • 373 



\section{GENERAL CONSIDERATIONS ON ANIMAL FORM}

\section{A. DESCRIPTIVE TERMS}

The body of vertebrates is carried in the horizontal position and the various surfaces are designated as follows with reference to this position:

Dorsal - the back or upper side (posterior in human anatomy).

Ventral - the under side (anterior in human anatomy).

Lateral - the sides, right and left.

Anterior, cephalic, or cranial - the head end of the animal (superior in human anatomy).

Posterior or caudal-the tail end of the animal (inferior in human anatomy).

Median - the middle.

Adverbs made by substituting $d$ for the terminal letter of these words mean "in the direction of," as craniad, toward the head, caudad, toward the tail, etc.

Other descriptive terms are:

Central-the part of a system nearest the middle of the animal.

Peripheral-the part nearest the surface.

Proximal-near the main mass of the body, as the thigh.

Distal-away from the main mass of the body, as the toes.

Superficial一on or near the surface.

Deep-some distance below the surface.

Superior-above.

Inferior-below.

\section{B. PLANES AND AXES}

The structures of most animals are arranged symmetrically with reference to certain imaginary planes and axes.

I. The median plane is a vertical longitudinal plane passing from head to tail through the center of the body from dorsal to ventral surfaces. It divides the body into two nearly identical halves, right and left.

2. The sagittal plane or section is any vertical longitudinal plane through the bodythat is, the median plane or any plane parallel to it. Sagittal planes other than the median plane are sometimes designated as parasagittal to avoid misunderstanding.

3. The horizontal or frontal plane or section is any horizontal longitudinal section through the body - that is, all planes at right angles to the median plane and parallel to the dorsal and ventral surfaces.

4. The transverse or cross plane or section cuts vertically across the body at right angles to the sagittal and horizontal planes.

5. The longitudinal or anteroposterior axis is a line in the median sagittal plane extending from head to tail; a sagittal or dorsoventral axis is any line in the median sagittal plane extending from dorsal to ventral surfaces; a transverse or mediolateral axis is any line in the transverse plane running from side to side.

\section{SYMMETRY}

The forms of symmetrical animals are dependent upon the arrangement of their parts with regard to the foregoing axes and planes. There are four fundamental types of animal symmetry-spherical, radial, biradial, and bilateral. Since all vertebrates are bilaterally 
symmetrical, the other types of symmetry will not be considered here. For them consult $\mathrm{H}$, pages $123-25$.

Bilateral symmetry.-The parts of a bilaterally symmetrical animal are arranged symmetrically with reference to three axes, the longitudinal, transverse, and sagittal axes; the two ends of the sagittal axis in any given cross-section are unlike. There is but one plane of symmetry in such an animal-that plane which passes through the longitudinal and sagittal axes-namely, the median sagittal plane. It divides the animal into approximately identical right and left halves, which are mirror images of each other. The structures of vertebrates are either cut in half by the median sagittal plane, in which case they are spoken of as unpaired structures, or they are placed symmetrically on each side of this plane, equidistant from it, in which case they are paired structures. The digestive tract is the only system which does not exhibit a symmetrical relation to the median plane in the adult, although it, too, is bilaterally symmetrical in early embryonic stages.

\section{METAMERISM OR SEGMENTATION}

Segmentation or metamerism is that structural condition occurring in certain groups of animals in which all or most of the paired parts or structures are repeated at regular intervals along the anteroposterior axis. The body of such animals consequently is composed of a longitudinal series of divisions or elements, in each of which all or most of the systems of the body are represented, either by entire paired organs or structures or by a portion of the median unpaired structures. Each such division or element of the body is called a metamere, somite, or segment. The anterior and posterior boundaries of each segment may or may not be marked externally by a constriction of the body wall. In the former case the animal is said to exhibit both external and internal metamerism; in the latter case internal metamerism alone is present.

In an ideal segmented animal all of the segments are identical with each other in all of the details of structure. No such animal exists because both the head and the terminal segments must of necessity differ, if only slightly, from the other segments, but the ringed worms, such as Nereis and the earthworm, closely approach the ideal. Such segmented animals in which the various segments are nearly alike are said to possess homonomous segmentation. The majority of segmented animals, however, display heteronomous segmentation, in which the various segments have become unlike each other in many respects.

The segmented groups of animals are the annelids, the arthropods, and the vertebrates and their relatives. In the evolution of segmented animals there has been a continuous progression from the homonomous to the extreme heteronomous condition. Homonomous segmentation represents a primitive and generalized type of structure in which the various segments are more or less independent of each other and each is capable of performing all of the necessary functions. But with the evolution of heteronomy, the segments become unlike and there is a division of labor among them, some portions of the body elaborating certain functions and others other functions. Each segment is then no longer capable of performing all of the functions, but is dependent upon its fellow-segments with a resulting unification and organization which is lacking in the homonomously segmented forms.

The heteronomous condition is derived from the homonomous through a number of different processes, such as loss of segments, fusion of adjacent segments, enlargement or reduction of segments, loss of organs or parts from some segments with their retention in other segments, structural changes among the repeated organs or parts so that those of different segments become unlike, etc.

The vertebrates are heteronomously segmented animals with internal segmentation only. The embryos of vertebrates much more nearly approach the homonomous condition, an 
indication that the vertebrates arose from homonomously segmented forms. In the embryonic development of vertebrates the change from a somewhat homonomous condition to an extreme heteronomy can be directly followed.

\section{E. CEPHALIZATION}

In the evolution of animals there is a pronounced tendency for the anterior end of the body to become more and more distinctly separated and differentiated from the rest of the body as a head. This differentiation of the head consists chiefly of the localization within the head of the main part of the nervous system-i.e., the brain-and of the most important sense organs. Since the brain and the sense organs control, to a very large degree, the activities and responses of the rest of the body, the head thus becomes the dominant part of the organism. This centralization or localization of nervous structures and functions in the head with accompanying dominance of the head is called cephalization. Cephalization is more and more marked the higher one ascends in the animal kingdom, and is particularly prominent as a structural and functional feature of the vertebrates.

In segmented animals the advance in cephalization is correlated with the progression of the heteronomous condition. Heteronomy, in fact, appears first in the head region and gradually progresses posteriorly. The anterior end thus retains the least and the posterior end the most resemblance to the original homonomous condition. This results in an illusion of a retreat of certain systems toward the posterior regions of the body, whereas the situation in reality arises from the fact that these systems have disappeared from the anterior segments and are retained in the posterior segments. In the case of certain vertebrate organs, as the heart, a real posterior descent occurs during the evolution of the vertebrates. In the vertebrates, as in other heteronomously segmented animals, the head is produced through the fusion of a certain number of the most anterior segments with a loss of some segments or of parts of segments and the disappearance from these head segments of nearly all systems except the nervous system. As cephalization progresses the head appropriates more and more of the adjacent segments, incorporating them into its structure, so that in general it may be said that the higher the degree of cephalization, the greater is the number of segments composing the head. In advanced cephalization, such as is possessed by vertebrates, it is very difficult, indeed, almost impossible, to decipher the number and boundaries of the segments which originally went into the composition of the head; in fact, the problem of the segmentation of the vertebrate head has not been completely solved, although it has received the attention of the foremost vertebrate anatomists.

The vertebrates are, then, animals characterized by the possession of bilateral symmetry, internal and markedly heteronomous segmentation, and a high degree of cephalization. The details of their structure are understandable only in the light of these three broad anatomical conditions.

\section{F. HOMOLOGY AND ANALOGY}

Homologous structures are those which, however unlike in function or superficial appearance, have the same origin, as demonstrated by the study of their embryonic origin and development and their paleontological history. Thus the wing of birds, the flipper of the seals, and the fore leg of the cat are homologous structures because they are all modifications of the original fore limb and develop in the same way up to a certain point. The whole aim of comparative anatomy is to discover what structures are homologous and to trace the modifications of such homologous structures in the course of evolution.

Analogous structures are those which resemble each other either as to superficial appearance or in function but which have had different origins. Thus, both fish and snakes are 
covered with scales for protective purposes, but these scales are not the same morphologically because they originate from the skin in different ways. Such functional correspondences are usually the result of environmental conditions, i.e., they are "adaptations." Not only parts of animals but whole animals may come to resemble each other through the action of the same environment, as whales and fishes. Such resemblance, not based on actual relationship, is called convergence. On the other hand, animals closely related to each other may differ greatly in appearance, owing to the different environments in which they live, as seals and cats. This is known as divergence. (See further, N, pp. I5-20; W, pp. r6 ff.) 


\section{THE PHYLUM CHORDATA}

\section{A. THE CHARACTERISTICS OF THE CHORDATES}

While the vertebrates comprise the greater part of the phylum Chordata, three small groups of animals are united with them in this phylum because they possess certain characteristics in common with the vertebrates. These characteristics are:

I. The wall of the pharynx of the embryo or adult is pierced by openings, the gill slits, originally for respiratory purposes.

2. A notochord is present in embryo or adult. The notochord is a rod lying dorsal to the intestine, extending from anterior to posterior end, and serving as a skeletal support. In vertebrates the notochord is partially or wholly replaced by the skull and vertebral column.

3. The central nervous system is nearly always hollow (in the tunicates in the embryo only), containing usually a single continuous cavity but in some cases a number of isolated spaces, and is situated entirely on the dorsal side of the body (except in the Hemichordata where there are both dorsal and ventral portions). In the invertebrates, the central nervous system is always solid and lies mainly ventral in the body.

For further discussion of these characters see $\mathrm{P}$ and $\mathrm{H}$, pages $\mathrm{I}-2 ; \mathrm{N}$, pages $3 \mathrm{I}-32$; $\mathrm{K}$, pages $\mathrm{I}-2$.

\section{B. THE CHARACTERISTICS OF THE VERTEBRATES}

The morphological characters of the vertebrates are the following: animals with bilateral symmetry, internal heteronomous segmentation, and cephalization; with generally two pairs of paired jointed locomotor appendages, in the form of fins or limbs, and sometimes with unpaired appendages in addition; skin separable from the rest of the body wall and commonly producing protective structures, such as scales, feathers, hair, etc., cellular in nature; muscle layer of the body wall decidedly metameric in arrangement; with an internal skeleton, of cartilage or bone, consisting of a skull and gill supports in the head, vertebral column, ribs, and breastbone in the body, and supports for the appendages; vertebral column highly metameric, composed of successive rings around the notochord; central nervous system consisting of a brain, much enlarged, within the skull, and a spinal cord within the vertebral column; nerves highly metameric in arrangement; head with three pairs of sense organs, eyes, ears, and nose; digestive tract giving rise by outgrowth to two digestive glands, the liver and the pancreas; pharynx intimately connected with the respiratory system, either opening to the exterior by openings, the gill slits, in the walls of which the gills are borne, or giving rise by outgrowth to the lungs; heart always ventral in the body; circulatory system closed, always with a median dorsal artery, the aorta, and with one or two portal systems; genital and excretory systems closely related, the excretory ducts generally serving as genital ducts; excretory and genital ducts opening in common with the intestine into a cloaca, or opening separately near the anus; with a well-developed coelom, never segmented, and divided in the adult into two or four compartments; viscera supported by mesenteries. (See further, K, pp. 2-4; $\mathrm{P}$ and $\mathrm{H}$, p. Ir $;$; $\mathrm{H}$ pp. 575-76; Wd, pp. Ir-I3; and study the diagrams in $\mathrm{P}$ and $\mathrm{H}$, p. 69.)

\section{THE CLASSIFICATION OF THE CHORDATES}

Since in this manual reference by their scientific names to groups of chordates is unavoidable, it is essential that the student learn at once the following scheme of classification. Only 
those groups are included which are actually met with or referred to in the manual. For complete classification see the various textbooks, as $\mathrm{N}, \mathrm{P}$ and $\mathrm{H}, \mathrm{CNH}$, etc. Specimens of these different groups will be seen in the laboratory in the next section of the manual.

\section{Phylum Chordata}

Subphylum I. Cephalochordata (Acrania), Amphioxus and its allies.

Subphylum II. Urochordata or Tunicata, the tunicates or sea squirts.

Subphylum III. Hemichordata (Enteropneusta), Balanoglossus and its allies.

Subphylum IV. Vertebrata, all animals with a vertebral column.

Class I. Cyclostomata, the cyclostomes or round-mouthed fishes, fishlike animals without lower jaws or paired fins.

Class 2. Pisces, the true fishes, with jaws and paired fins.

Subclass I. Elasmobranchii, fishes with a cartilaginous skeleton and exposed gill slits, including the dogfish, skates, sharks, etc.

Subclass 2. Teleostomi, fishes with a more or less bony skeleton and with gill slits concealed under an operculum.

Order I. Crossopterygii, paired fins with a basal stalk; Polypterus. (See Fig. I.)
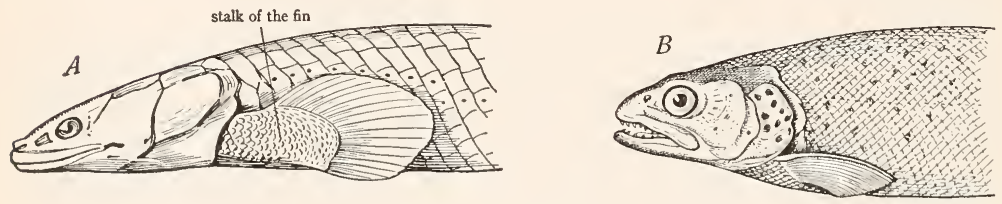

FIG. I. - A, anterior end of a crossopterygian fish (Polypterus) to show the lobe or stalk on which the fin is borne. $B$, anterior end of a common teleost fish (trout) to show the absence of such a stalk. This difference is not, however, an important distinguishing character between groups of fishes. (From Bridge and Boulenger in the Cambridge Natural History, courtesy of the Macmillan Company.)

Order 2. Chondrostei, paired fins without a stalk (Fig. I), skeleton largely cartilaginous, with a spiral valve in the intestine, heart with a conus arteriosus; the sturgeon (Acipenser) and the spoonbill (Polyodon).

Order 3. Holostei, like the preceding, but skeleton well ossified; the gar pike (Lepidosteus) and the river dogfish or bowfin (Amia).

These three orders are commonly referred to as the ganoid fishes owing to the shiny scales (ganoid scales) with which most of them are covered.

Order 4. Teleostei, paired fins without a stalk, skeleton well ossified, without spiral valve or conus arteriosus; all of the common fishes. This order is commonly referred to as the teleosts or bony fishes. The three orders Chondrostei, Holostei, and Teleostei are often grouped together as the Actinopterygii or ray-finned fishes in which the fin rays spring directly from the body in contrast to the Crossopterygii or fringe-finned fishes, in which the fin rays spring from a stalk and form a sort of fringe on the end of the stalk. 
Class 3. Amphibia, amphibians, lowest four-legged vertebrates, skins naked and slimy, or with bony plates (extinct), living in or near water.

Subclass r. Stegocephala, extinct amphibia, with tails, and covered with an armor of bony plates.

Subclass 2. Lissamphibia, present-day amphibia, with naked slimy skins (a few with minute, concealed scales).

Order I. Urodela, with tails; the salamanders and newts, Necturus, Amblystoma, Cryptobranchus, etc.

Order 2. Anura, tailless; frogs, toads.

Class 4. Reptilia, reptiles, air-breathing vertebrates covered with horny scales.

Order I. Cotylosauria. Most primitive group of extinct reptiles, resembling Stegocephala in skeletal characters. Seymouria.

Order 2. Chelonia, the turtles, body inclosed in a hard case.

Order 3. Rhyncocephalia, including but one animal, the Sphenodon (Hatteria) or tuatara of New Zealand, a lizard-like animal with primitive skeletal characters.

Order 4. Squamata, usually of small or moderate size and covered with horny scales.

Suborder I. Lacertilia, the lizards, nearly always with limbs, eyelids movable.

Suborder 2. Ophidia, the snakes, devoid of limbs, eyelids immovable.

Order 5. Crocodilia, the crocodiles, alligators, gavials, and caimans, large reptiles with both horny scales and bony plates in the skin.

Class 5. Aves, birds, vertebrates with feathers.

Class 6. Mammalia, mammals, vertebrates with hair and milk glands.

Subclass r. Prototheria, mammals laying eggs.

Order I. Monotremata, the monotremes, or egg-laying mammals, including only the duckbill (Ornithorhynchus) and the spiny anteaters (Echidna and Proechidna) of the Australian region.

Subclass 2. Eutheria, mammals bearing the young alive.

Division I. Didelphia or Metatheria.

Order I. Marsupialia, the marsupials, mammals bearing the young in a very immature state, and carrying them in a pouch formed by a fold of skin on the abdomen, placenta absent or primitive; kangaroos, opossums, etc.

Division 2. Monodelphia or Placentalia, the placental mammals, without a pouch, young nourished in the uterus by a placenta, which is produced by a fusion between certain parts of the embryo and certain parts of the maternal uterus. This division includes sixteen orders which are described in detail in $\mathrm{N}$, pages $346-404$. In this manual, we meet with three orders:

Order Carnivora, the carnivorous mammals, with claws and sharp, cutting teeth; the bears, raccoons, minks, martens, weasels, otters, dogs, foxes, wolves, cats, lions, tigers, hyaenas, seals, walruses.

Drder Rodentia, the rodents, with chisel-like front teeth, and back teeth with flat, grinding surfaces; the hares, rabbits, squirrels, rats, mice, porcupines, guinea pigs.

Order Edentata, teeth lacking or degenerate; the ant bears, sloths, and armadillos. 
Additional scientific names used for convenience in grouping the classes of vertebrates are:

Ichthyopsida. This term, meaning fishlike animals, includes the two classes, Pisces and Amphibia.

Sauropsida. This term, meaning reptile-like animals, includes the two classes, Reptilia and $A$ ves.

Anamniota or Anamnia. This term includes the classes Pisces and Amphibia and refers to the fact that their embryos are naked.

Amniota. This embraces the three classes, Reptilia, Aves, and Mammalia, and refers to the fact that the embryos of these groups are covered by a membrane, the amnion.

Acraniata or Acrania. This name is synonymous with Cephalochordata and is derived from the absence of the skull in Amphioxus.

Craniata. This term is synonymous with Vertebrata and refers to the fact that all vertebrates possess a skull.

Agnathostomata. This term, meaning without jaws, is synonymous with Cyclostomata.

Gnathostomata. This name, meaning jawed, includes all of the classes of vertebrates, except Cyclostomata.

Tetrapoda. This term, meaning four footed, includes all of the land vertebrates, i.e., the classes Amphibia, Reptilia, Aves, and Mammalia. 


\section{GENERAL STUDY OF TYPICAL CHORDATES}

\section{A. AMPHIOXUS}

I. External anatomy of Amphioxus.-Obtain a specimen and place in a dish of water. The body is slender, fishlike, pointed at each end, and compressed laterally. The more blunt end is anterior, the more pointed end, posterior; the dorsal surface is sharp, the ventral surface, for the greater part of its length, flattened. The anterior end represents a poorly developed, somewhat degenerate head. The ventral and greater part of the head consists of an expanded membrane, the oral hood, which incloses a cavity, the stomodaeum or vestibule, at the bottom of which the mouth is located. The borders of the oral hood are extended into a series of stiff tentacles or cirri.

Turn the animal ventral side up and observe that the flattened portion of the ventral surface is bounded laterally by two membranous folds, the metapleural folds, or lateral fins, extending posteriorly from the oral hood. These folds meet at a point nearly three-fourths of the distance from anterior to posterior end, behind a median opening, the atriopore. From this point a median membranous fold, the fin, passes to the posterior end of the body, around to the dorsal side, and forward along the dorsal side to the anterior end. The slightly wider portion of this fin which surrounds the pointed posterior end is the caudal fin, that along the dorsal side, the dorsal fin. The anal opening will be found on the ventral side very near the posterior end, just behind the point where the fin widens. The anus is on the left side.

The body is covered by a thin epidermis under which is a muscle layer. The greater part of the muscle layer consists of the lateral muscles, forming the side walls of the body, and divided into a large number of $\mathrm{V}$-shaped muscle segments, or myotomes, clearly visible through the transparent epidermis. Each myotome is separated from its neighbor by a connective tissue partition, the myocomma. Note that the myotomes extend nearly to the tip of the anterior end, diminishing in size above the oral hood. The number of myotomes in Amphioxus is definite, about sixty. The myotomes are one expression of the metamerism of the Amphioxus body. The ventral portion of the body is provided with a thin layer of transverse or ventral muscles, whose fibers run circularly; these muscles are not visible externally.

Immediately below the ventral terminations of the myotomes will be seen. in some individuals at least, a row of square white masses, the gonads or reproductive organs. Their arrangement is metameric. The ventral part of the body. extending posteriorly from the oral hood, appears somewhat clear and is occupied by a large cavity, the atrium, which surrounds the digestive tract and opens to 
the exterior through the atriopore. Amphioxus maintains a continuous circulation of water through its digestive tract; water enters the mouth, passes through the walls of the pharynx into the atrium, and out of the atriopore. Make a drawing of the animal from the side.

2. Internal anatomy.-The internal anatomy is most easily studied on small mounted specimens. Examine with the low power of the microscope. Identify: the various parts of the fin, containing rectangular bodies, the fin rays, serving as the skeletal support of the fins; the muscle segments; and the digestive tract, occupying the ventral half of the body. The parts of the digestive tract may be studied in some detail. Note again the oral hood with its cirri. The vestibule is narrowed posteriorly by a membrane, the velum, pierced by a small opening, the mouth. The mouth opens into a wide cavity, the pharynx, extending half the length of the body. Its walls are perforated by numerous oblique slits, the gill slits or pharyngeal clefts. The solid portions of the pharyngeal wall between the gill clefts are called the branchial bars and each is supported by an internal skeletal branchial rod. Surrounding the pharynx is a large cavity, the atrium, the ventral boundary of which is visible as a line below the pharynx. This line may be traced to the atriopore. The posterior end of the pharynx opens into a tubular intestine which extends straight to the anus. The first part of this intestine for a short distance posterior to the pharynx is narrow and dorsally located; the intestine then widens suddenly and from this widened part a blind sac, the liver, extends forward beneath the narrowed portion.

Immediately dorsal to the digestive tract and nearly as wide as the pharynx is a rod, the notochord, extending the length of the body. It will be seen by focusing down through the myotomes and is most distinct in the head, where it runs forward nearly to the extreme tip. Just above the notochord is situated the much smaller neural tube, best recognized by the row of black pigment spots which it bears. These pigment spots have been shown to be sensitive to light. Draw the whole mount, showing its structure.

3. Cross-section through the pharyngeal region.-Examine the crosssection with the low power and identify the following: (a) The epidermis, the outer covering of the body composed of a single layer of columnar epithelial cells. (b) The dorsal median projection, the dorsal fin, containing an oval mass, the fin ray, which supports it. (c) The two ventrolateral projections, the metapleural folds. There are a number of smaller folds in the ventral wall between the two metapleural folds. (d) The myotomes, a series of circular masses filling the dorsal and lateral portions of the body wall, and separated from each other by connective tissue partitions. The myotomes are thick dorsally and thin out ventrally. Transverse muscles are present in the ventral body wall, just above the small folds of the epidermis. (e) The neural tube, a median dorsal mass, oval or trapezoidal in section, lying between the dorsal portions of the myotomes, below the fin ray. Observe that it contains a central canal, the neurocoel. 
Black spots in the neural tube are the pigment cups of simple eyes or lightperceiving organs. These are distributed along the anterior part of the neural tube of Amphioxus and are similar in structure to the eyes of planarians, consisting of a pigment cup filled with nerve cells. Experiment has shown that these optic cups are sensitive to light while the eyespot on the anterior end of the brain is insensitive to light. $(f)$ The notochord, an oval mass, larger than the neural tube and directly ventral to it. (g) The atrium, the large cavity occupying the ventral half of the section. ( $h$ ) The pharynx, the structure occupying the center of the atrium. It is elongated in some regions, heart shaped in others. It consists of separate pieces, the branchial bars, each inclosing in its outer extremity a stiff support, the branchial rod. The spaces between adjacent gill bars are the gill slits or pharyngeal clefts, by means of which the cavity of the pharynx communicates with the atrium. In the median dorsal line of the pharynx is a deep groove, the epibranchial groove; in the median ventral line, a broad slightly depressed structure, the hypobranchial groove, or endostyle. These grooves secrete mucus in which the minute food particles are caught. (i) The liver, an oval hollow structure present in some sections to the right of the pharynx. It is composed of tall epithelial cells, which probably produce a digestive secretion. (j) The gonads. In cross-sections of larger specimens these are found as masses, one on each side of and projecting into the atrium. Draw the crosssection.

For further details of the anatomy of Amphioxus consult $\mathrm{P}$ and $\mathrm{H}$, pages 44-56; CNH, Vol. VII, pages II 2-30; N, pages 30-42.

\section{B. A TUNICATE}

I. External anatomy.-Obtain a specimen and place in a dish of water. It is an oval saclike creature, scarcely recognizable as an animal. The animal is in life permanently attached to rocks or other objects. The end which was attached is recognizable by its rough and irregular form and by the fragments of wood or other materials which adhere to it. The opposite end, which in the living state extends free into the water, bears two openings, the siphons. When the animal is gently squeezed, jets of water are observed to squirt from the siphons; hence the name sea squirt popularly applied to these animals. The upper of the two siphons is the oral or incurrent siphon and is the degenerate anterior end of the animal; the lower siphon is the atrial or excurrent siphon and represents the posterior end. The anteroposterior axis of the tunicates is bent into a $\mathrm{U}$-shape. The region of the body between the two siphons is therefore dorsal, and the remaining and much larger part of the surface is ventral. When the animal is undisturbed, the siphons are widely expanded, and a current of water passes into the oral siphon and out of the atrial siphon. When disturbed and also generally in the preserved state, the siphons are closed and retracted. Closure and reaction is accomplished by circular and longitudinal muscles located 
in the body wall. In closing, the rim of the siphons is thrown into folds, whose number and arrangement varies with different species.

The outer layer of the body wall consists of a thick, tough, sometimes somewhat gelatinous, often warty, covering, the tunic or test, which is secreted by the ectoderm beneath it. In some tunicates (Ciona, Corella) the test is so transparent that practically all of the internal organs are visible through it. The test is attached to the underlying body only at the siphons.

Draw the animal.

2. Dissection of a tunicate.-(A demonstration dissection may be substituted for individual dissection by the student.) As different tunicates vary in the details of their internal structure, the following account is of necessity somewhat generalized. It is based chiefly on the anatomy of Ciona. Make a cut through the base of the tunic, avoiding injury to the underlying wall, and peel off the tunic. Note its attachments at the siphons, and cut through these attachments, discarding the tunic. The soft body wall or mantle is thus revealed, the tunic being, as already explained, a secretion of the outer layer of the mantle. The muscle fibers which operate the siphons may be visible as stripes in the mantle; they are conspicuous in Ciona.

Fasten the animal in a wax-bottomed dissecting pan by pins through the rims of the siphons and through the extreme basal end. Fill the pan with water to cover the animal.

The mantle incloses the viscera which tend to adhere to it, so that its removal without injury to the viscera is difficult. In transparent forms the viscera may be studied through the mantle. In case it is necessary to remove the mantle, make a longitudinal slit in it from the atrial siphon to the basal end and pull it off in small strips, separating each strip gently from the underlying parts. The principal internal parts are the following. The oral siphon leads into a large thin-walled bag, which in some forms, as Ciona, extends the length of the body. This bag is the pharynx. Its wall appears to the naked eye like mosquito netting, because it is pierced by numerous minute openings, the gill slits or visceral clefts. The cavity outside of the pharynx is the atrium; it communicates with the exterior by way of the atrial siphon. The lower end of the pharynx narrows abruptly into a short esophagus which opens into a widened stomach, situated below or to one side of the basal end of the pharynx. The stomach is curved so that its long axis lies at right angles to the long axis of the pharynx. In some cases (Molgula) brown digestive glands can be seen covering the stomach. The stomach leads into an intestine which immediately makes a loop, doubling back so as to lie parallel to the stomach. It then bends and extends straight upward toward the atrial siphon, terminating by an anus situated within the atrial cavity.

The reproductive organs consist of a single or paired mass, each being hermaphroditic, that is, composed in part of an ovary and in part of a testis. This hermaphroditic gonad is located either in the space between the intestinal loop 
and the stomach (Ciona, Corella, Ascidia) in which case it is commonly single, or may be attached to the inside of the mantle (Cynthia, Molgula), in which case a pair of such glands is often present. From the ovarian part arises an oviduct and from the testicular part a vas deferens. The two ducts run in close contact with each other (and in some cases fuse to one duct) and open into the atrial cavity near the atrial siphon. In Ciona the two genital ducts are found closely attached to that side of the intestine which faces the pharynx, and in this position run far forward, opening into the atrium some distance above the anal opening. The genital opening in Ciona is colored red, due to the presence in its walls of red vesicles, supposed to have an excretory function. In Molgula and other members of the family Molgulidae there is a large saclike organ on the right side, believed to be excretory in nature, but the presence of excretory organs in the tunicates is more or less uncertain.

In the dorsal part of the mantle, between the two siphons, will be found an elongated mass, the ganglion, which constitutes the entire central nervous system of the adult tunicate. Nerves may be seen extending from its ends to the siphons.

Draw the dissection.

3. The structure of the pharynx.-Make a longitudinal slit in the side of the pharynx forward through the oral siphon and spread out its walls. In the median ventral line of the pharynx there is present a conspicuous white cord extending the length of the pharynx. This is the endostyle or hypobranchial groove, whose walls are composed of glandular and ciliated cells, the former producing mucus. Directly opposite the endostyle in what may be designated the median dorsal line of the pharynx is located the dorsal lamina. This in some tunicates (Ascidia, Cynthia, Molgula) consists of a delicate membranous fold of the pharyngeal wall, while in others (Ciona, Corella) it consists of a row of processes called languets and resembles a fringe. At the anterior end of the pharynx at the bottom of the oral siphon there generally occurs a circlet of tentacles or irregular processes of the wall, and posterior to this is a grooved ridge, the peripharyngeal band, in which the anterior ends of endostyle and dorsal lamina terminate. The lateral walls of the pharynx in some tunicates (Molgula, Cynthia) exhibit a definite number of longitudinal folds.

Cut out a small piece of the pharyngeal wall, mount in water, spread out flat, and examine under the low power of the microscope, or examine prepared slides. The wall is found divided into squares by means of longitudinal and cross bars. Within each square so formed are seen several of the elongated gill slits or visceral clefts, separated from each other by smaller bars. The gill slits are commonly parallel to the longitudinal bars but may be curved (Molgula). On the longitudinal bars there are often present finger-like processes or papillae which have been shown to aid in pushing the food-containing mucous strands toward the esophagus. Draw a portion of the pharyngeal wall.

For further details of the anatomy of tunicates consult $\mathrm{P}$ and $\mathrm{H}$, pages $\mathrm{I} 4^{-2 \mathrm{I}}$; $\mathrm{CNH}$, Vol. VII, pages $35^{-62}$; N, pages $50^{-2} 5^{2}, \mathrm{H}$, pages $5^{\circ} 5^{-6}$. 


\section{BALANOGLOSSUS}

I. External anatomy.-Obtain a specimen, place in a dish of water, and examine. (As different species differ somewhat in appearance, the following description applies only to Balanoglossus [Dolichoglossus] kowalevskii.) It is an elongated wormlike animal about five or six inches in length. The body is divided into three parts - the proboscis, the collar, and the trunk. The proboscis is the slender, conical, pointed structure at the anterior end, used by the animal in burrowing into the sand. The collar is the band encircling the body just posterior to the proboscis. The mouth is concealed within the collar. The trunk comprises the greater part of the animal and presents a more or less ruffled appearance. The median ventral line is marked by a distinct longitudinal band; the median dorsal line in a similar but less distinct manner. The sides of the trunk for about an inch and a half posterior to the collar project somewhat and are slightly different in appearance from the rest of the trunk; these projections contain the reproductive organs and hence are named the genital ridges. Between the anterior portions of the genital ridges on the dorsal side will be found two longitudinal rows of small parallel slits, the gill pores. (The true gill slits, leading from the pharynx, are $U$-shaped and each opens into a chamber, the gill pouch, which in turn communicates with the exterior by means of the gill pore, which is somewhat smaller than the gill slit. In some balanoglossids, as Ptychodera, the U-shaped gill slits open directly to the exterior, as is the case in the larva of Amphioxus. The structure and development of the branchial region of balanoglossids is strikingly similar to that of Amphioxus.) The remainder of the trunk is occupied by the digestive tract and possesses $a$ terminal anus. The species under consideration lacks the hepatic caeca, a series of outgrowths of the intestinal wall, figured in $\mathrm{P}$ and $\mathrm{H}$. Make a drawing of the animal from the dorsal side.

For further details consult $\mathrm{P}$ and $\mathrm{H}$, pages 2-7; $\mathrm{CNH}, \mathrm{Vol}$. VII, pages 3-2 I ; $\mathrm{H}$, pages 512-I4; $\mathrm{N}$, pages $60-65$.

\section{ANATOMY OF A LAMPREY}

The lampreys belong to the lowest class of true vertebrates, the class Cyclostomata, or round-mouthed fishes.

I. External anatomy of a lamprey.-Place the specimen in a dissecting tray. The body consists of a stout cylindrical head and trunk and a flattened tail. From the median dorsal line in the posterior portion of the body arise two dorsal fins, while the tail is provided with a caudal fin, continuous with the second dorsal fin. The fins are supported by numerous fin rays, slender parallel cartilages usually visible through the skin. There are no paired ventral fins such as are found in the true fishes. The skin is soft, slimy, and naked. 
The anterior end of the head presents a peculiar appearance, owing to the apparent absence of a lower jaw (the jaw is probably present in a degenerate condition) and the presence on the ventral side of the head of a large bowlshaped depression, the buccal funnel. The edges of the buccal funnel are provided with soft papillae, and its interior is studded with brown, horny teeth, definitely arranged. At the bottom of the funnel is a projection, called the tongue, also covered with teeth. The mouth is just dorsal to the tongue. Lampreys attach themselves to the bodies of fishes by means of the buccal funnel and rasp off their flesh by filing movements of the tongue.

On the dorsal surface of the head is an opening, the nasal aperture or nostril, which leads into the olfactory sac. Cyclostomes differ from other vertebrates in possessing but one nostril, although this is probably a secondary condition. On each side of the head on a level with the olfactory organ is an eye, not provided with eyelids. Posterior to the eye on each side of the head is a row of seven oval openings, the gill slits.

Along the sides of the body the boundaries of the myotomes are generally noticeable through the skin. In the median ventral line at the junction of trunk and tail will be found a pit. In the anterior end of this pit is the anal opening and immediately behind the anus a projecting papilla, the urogenital papilla, which receives the products of the kidneys and reproductive organs and discharges them to the exterior through an opening at its tip, the urogenital aperture.

2. Sagittal section of the anterior end.-Make a median sagittal section of your specimen to a point about an inch posterior to the last gill slit or study a section so prepared. Examine the cut surface and identify the following:

a) Digestive tract: Observe again the buccal funnel with its teeth and tongue. Note the large muscle masses extending posteriorly from the tongue, by means of which the rasping movements of the tongue are brought about. Find the mouth opening above the tongue and follow it into a passage, the buccal cavity which slopes ventrally. The buccal cavity opens at its posterior end into two tubes, an upper smaller one, the esophagus, and a larger ventral one, the pharynx, the wall of which is pierced by seven oval openings. A fold, the velum, is present at the entrance of the buccal cavity into the pharynx. The esophagus leads into the remainder of the digestive tract, but the pharynx, which in the embryo constituted the anterior part of the digestive tract, ends blindly in the adult.

b) Respiratory system: The seven openings in the wall of the pharynx are the internal gill slits. They open into much enlarged gill pouches which bear the gills on their walls and which communicate with the exterior through the external gill slits already noted. Probe into one of the gill pouches and note the leaves or gill lamellae borne on its walls. The dorsal portions of the gill pouches inclose the narrow esophagus between them. 
c) Notochord: The notochord is the broad, brown rod situated just dorsal to the esophagus. It is the chief axial skeleton of the animal, the vertebral column being very embryonic.

d) Nervous system: Above the notochord is a narrow canal, the neural canal, in which the spinal cord and brain are located. The spinal cord is a slender cord occupying the canal; the brain is an enlarged lobed structure situated just dorsal to the anterior extremity of the notochord.

e) Olfactory apparatus: The section should bisect the olfactory aperture. Note that a canal extends from this aperture and opens into a sac, the olfactory sac, situated just anterior to the tip of the brain. The folds in the wall of the sac bear the olfactory mucous membrane. From the anterior margin of the olfactory sac a tube arises which bends ventrally and posteriorly and widens into an elongated sac, the pituitary pouch, lying ventral to the anterior end of the notochord. The peculiar dorsal position of the olfactory sac as well as its relation to the pituitary sac (which in other vertebrates has no connection with the olfactory cavities) are due to a shifting of these organs during development from their original ventral position (see $\mathrm{P}$ and $\mathrm{H}$, Fig. 799, n. I33). The pituitary sac corresponds to the anterior lobe of the pituitary body or hypophysis of other vertebrates.

f) Pericardial cavity: Posterior to the last gill pouch is a somewhat conical cavity, the pericardial cavity, within which the heart is situated.

Make a detailed drawing of the sagittal section.

For a complete account of the anatomy of cyclostomes consult $\mathrm{P}$ and $\mathrm{H}$, pages II9-39; CNH, Vol. VII, pages I50-52, 2I6-22, 247-48, 279-82, 37I-73. 385-95; N, pages $87-94$.

\section{E. EXTERNAL ANATOMY OF THE DOGFISH}

The dogfish or dog shark is a true fish, belonging to the lowest group of fishes, the subclass Elasmobranchii. It is a very generalized vertebrate, and hence a knowledge of its structure is indispensable for an understanding of vertebrate anatomy. There are two common dogfishes usually obtainable for laboratories, the smooth dogfish, Mustelus canis, and the spiny dogfish, Acanthias vulgaris. These two species are slightly different in several respects; attention will be called to these differences where necessary.

r. Body and skin.-The body of the dogfish has the shape and proportions which we recognize as most advantageous for free-swimming animals-fusiform (spindle shaped) and pointed at each end, thus offering little resistance to the water. The body is divided into head, trunk, and tail, which are not, however, distinctly bounded from each other. Trunk and tail are provided with fins for purposes of locomotion. The body is clothed with minute scales, each of which bears a tiny spine. Pass the hand over the skin of the dogfish and note the 
rough feeling due to the spines. A whitish line, the lateral line, extends along each side of the body; it contains sensory cells whose iunction is the detection of vibration in the water. Through the skin the zigzag muscle segments or myotomes similar to those of Amphioxus can often be perceived.

2. The head.-The head is triangular and somewhat flattened; its pointed extremity is known as the rosirum. On the ventral side of the head is the narrow crescentic mouth, bounded as in all vertebrates except cyclostomes by the upper and lower jaws, both of which bear a number of teeth arranged in diagonal rows. The head further bears the three pairs of sense organs characteristic of vertebrates - the olfactory organs, the eyes, and the ears. The nostrils are a pair of openings on the ventral side of the rostrum. A little flap of skin extends over the center of each nostril, dividing the opening into two passages, by means of which a current of water circulates through the olfactory sac, a rounded sac into which each nostril leads. The oval eyes are situated on the sides of the head. They have immovable upper and lower eyelids; in the smooth dogfish the lower lid is extended into a thin membrane, the nictitating membrane, or third eyelid, which can be drawn up over the eye. Behind each eye is a slight prominence, which is best perceived by feeling with the finger; within this the ear is located. There is no external ear, ear opening, or drum membrane, but the ears are connected with the surface of the head by two canals, the endolymphatic ducts, which open by a pair of small pores in the center of the dorsal surface of the head just back of the level of the eyes. These may be difficult to find in some specimens. The rostrum and adjacent parts of the head are punctured by many pores, which are the openings of long mucous canals imbedded under the skin and of uncertain function.

3. Gill slits.- Just behind each eye is a circular opening, the first gill slit or spiracle and a short distance posterior to this a row of five elongated slits, the second to sixth gill slits. The gill slits communicate with the cavity of the pharynx. In the respiratory movements water enters through the mouth and exits through the gill slits.

4. Fins.-These are of two kinds, the unpaired or median fins, arising from the median lines of the animal, and the paired or lateral fins, located on the ventral side of the trunk near the median line. The unpaired fins consist of an anterior and a posterior dorsal fin, arising from the median dorsal line, each, in the case of the spiny dogfish, being provided with a spine in front; a ventral fin on the ventral side of the tail, present in the smooth dogfish only; and the tail fin or caudal fin surrounding the posterior end of the body. In the dogfish and other elasmobranchs the caudal fin is asymmetrical with respect to the tail, which bends upward in it, dividing the caudal fin into a narrow dorsal part and a broader ventral portion; this type of tail is known as heterocercal. There are two pairs of paired fins; they correspond to the limbs of land vertebrates. The anterior pair, just behind the gill slits, is named the pectoral fins, the posterior pair at the junction 
of trunk and tail, the pelvic fins. In male dogfishes, the medial side of each pelvic fin is modified into a stout process directed posteriorly. These processes are cailed claspers and are used by the males in mating with the females. All of the fins are supported by slender flexible rays, the dermal fin rays, imbedded in the skin of the fins.

5. Anus.-Between the two pelvic fins is a large opening, the anus or cloacal aperture. This is the opening of a chamber, the cloaca, which receives the end of the intestine and the terminations of the ducts of the kidneys and reproductive organs. The anus of the dogfish and of the majority of vertebrates does not quite correspond to the anus of man and other mammals, which receives the intestine only, but the term is commonly used to designate both classes of opening.

\section{F. EXTERNAL ANATOMY OF THE SKATE}

The skates or rays are, like the dogfish, elasmobranch fishes but highly modified as regards external form and proportions. Obtain a specimen and examine.

I. Body and skin. - The body is divided into a greatly flattened anterior portion comprising head and trunk and a slender posterior portion, the tail. The broad, flat form is characteristic of bottom-feeding fishes (see N, pp. 97-99) and results from a shortening of the dorsoventral axis and an elongation of the transverse axis. The tough skin contains scattered scales of which the spines project conspicuously. These scales are of the same type already noted in the dogfish, consisting of a basal plate imbedded in the skin and a projecting spine, directed posteriorly. The scales of the skate are much larger and fewer in number than those of the dogfish. They are definitely arranged in lines and groups, the arrangement differing somewhat in the two sexes. In females there are scales over the lateral expansions of the trunk and several rows of scales on the median dorsal part of the trunk and dorsal surface of the tail, while in males the greater part of the lateral expansions is devoid of scales, there are fewer rows along the middle of the back and tail, and the scales on the margins of the head are enlarged. In males, furthermore, there are two rows of curious erectile spines on the lateral expansions of the trunk about an inch in from the margin; these can be erected and lowered into depressions in the skin. Are there any scales on the ventral surface? Note the marked differences in color between the dorsal and ventral surfaces.

2. Fins.-Like the dogfish the skate is provided with median and paired fins, but the former are much reduced. They consist of two small dorsal fins on the dorsal side of the end of the tail. The pectoral fins are enormously enlarged, forming the lateral expansions of the trunk already mentioned several times; they are confluent anteriorly with the margins of the head. The pelvic fins are smaller and immediately posterior to the pectoral fins with which they 
are continuous in some species. They consist of two lobes, and in the males bear large stout claspers, deeply grooved along their posterior lateral margins. The claspers are employed in mating with the females.

3. Head.-The head like the trunk is greatly flattened, its margins continuous with the pectoral fins. It terminates in a pointed rostrum. It bears dorsally a pair of large projecting eyes, without lids. Behind each eye is the large spiracle or first gill slit, bearing a valve on its anterior face, marked by parallel ridges which represent a rudimentary gill. On the ventral side of the head is the mouth bounded by tooth-bearing jaws. The jaws and teeth are commonly larger in the males than in the females. In front of the mouth are the two nostrils, each provided with a fringed ear-shaped flap. Extending posteriorly from each nostril to the angle of the mouth is a flap with a fringed posterior margin. This is the nasofrontal process. On lifting up this process a deep groove, the oronasal groove, will be found extending from the nostril into the mouth cavity. This arrangement foreshadows the appearance of a closed passage from the nostrils into the mouth such as is present in higher vertebrates. Posterior to the mouth are five pairs of gill slits. In skates the pectoral fins have grown forward above the gill slits and fused with the sides of the head, thus shoving the gill slits to the ventral surface.

4. Anus.-The anus or cloacal aperture is a large opening between the bases of the pelvic fins.

\section{G. EXTERNAL ANATOMY OF A TELEOST}

For this purpose any common bony fish can be used, but the following description is based upon the perch. The perch like other common fishes is a member of the great order Teleostei. Obtain a specimen and note the following points.

I. Body and skin.-The body has the shape typical of aquatic animals, thickest in the middle and tapering to each end. It is somewhat compressed laterally. It is indistinctly divided into head, trunk, and tail. Trunk and tail are clothed with scales arranged in diagonal rows, overlapping each other. These scales are set in pockets in the deeper part of the skin (dermis), as may be determined by removing one of them, and the superficial layer of the skin (epidermis) forms a thin film over their surfaces. The head is covered by the soft epidermis and in some regions bears small scales like those on the remainder of the body. Beneath the scaleless portions of the epidermis of the head will be noted large, thin, flat bones. These bones, which are the outer bones of the skull, are in reality nothing but enlarged scales which have sunk from their original superficial position to a deeper location. A lateral line is present along each side of the body.

2. Head.-The head bears a terminal mouth bounded by well-developed jaws. The terminal position of the mouth is probably more primitive than the 
ventral position found in the elasmobranchs. On the dorsal side of the anterior end of the head are two pairs of nostrils, a pair to each olfactory sac. This arrangement permits a current of water to circulate through the olfactory sac. The large eyes are without lids. The ears, situated behind the eyes, are invisible externally. The posterior and lateral margins of the head are formed by a large flap, the gill cover or operculum, which is supported by several opercular bones, large, flat, scalelike bones, already noted. It covers a wide slit in the body wall known as the gill opening. Attached to the ventral margin of the operculum is a membrane, the branchiostegal membrane, supported by seven bony rays, the branchiostegal rays. Grasp the membrane with a forceps and spread it out to see the rays. Lift up the operculum and look within the cavity which it covers. Four curved structures, the gill arches, which should be separated from each other with a forceps, will be seen. Each bears on its outer surface a gill, consisting of a double row of soft filaments, and on its inner margin a series of short toothlike processes, the gill rakers. Thrust a probe inward between two gill arches, open the mouth of the fish, and observe that the end of the probe has entered the mouth cavity. The cavity of the pharynx is thus in communication with the exterior through the spaces between the gill arches. These spaces are gill slits corresponding to those which we saw in the dogfish and skate, but here the portions of the body wall between successive gill slits have disappeared, and all open into a common cavity covered by the operculum. This condition is characteristic of all fishes except elasmobranchs. When the fish respires, the mouth opens, the opercula move outward, the branchiostegal membrane unfolds and closes the gill opening; water is thus drawn into the mouth and bathes the gills. The mouth then closes, the opercula move inward, the branchiostegal membrane folds up, and the water passes out through the gill slits and gill opening.

3. Fins. - The body is provided with median and paired fins. Of the former there are an anterior and a posterior dorsal fin, a caudal fin, and a ventral or anal fin. (The number and position of the median fins are very variable in different fishes.) The caudal fin is apparently symmetrical with the end of the tail, forming a homocercal tail. The paired fins are the same as in the dogfish. The pectoral fins are located just behind the operculum, but the pelvic fins have moved forward from their normal position at the level of the anus to a position nearly level with the pectoral fins. Such a forward migration of the pelvic fins is very common in the teleost fishes and is often associated with diminution and degeneration of these fins. The anterior dorsal fin is supported by sharp hard spines, and these are also present in one border of some of the other fins, but the latter are supported chiefly by flexible bony fin rays.

4. Openings.-In the median ventral line just in front of the ventral fin is a large opening, the anus. Behind this is a depression into which projects a small elevation, the urogenital papilla. In the perch there is no cloaca, but the 
intestine and urogenital systems have separate openings; this is one of the marked differences between elasmobranch and teleost fishes.

\section{H. SOME GANOID FISHES}

As certain ganoid fishes are frequently referred to in the study of the skeleton, it is advisable that the student become familiar with their appearance. The ganoid fishes belong to the lower and more primitive orders of the Teleostomi and hence are placed in the scheme of classification below the teleosts, but the latter have been considered first to introduce the terminology. We shall examine briefly four common ganoids.

I. The gar pike (Lepidosteus).- The body is clothed in a complete armor of rhomboid scales set in diagonal rows. Such scales are called ganoid scales and consist of bone with an outer coat of a shiny substance named ganoin. These scales pass onto the head as enlarged plates, which constitute the bones of the roof of the skull. The jaws are very elongated, producing a snout on the tip of which are the small nostrils. The jaws bear numerous sharp teeth. There is an operculum covering the gills. A spiracle is absent. Note median and paired fins, with their stout, jointed fin rays. The tail is heterocercal but approaches the homocercal type.

2. The sturgeon (Acipenser).-There is a covering of ganoid scales arranged in five rows on the trunk with areas of apparently naked skin (which really bear small scales) between the rows. The scales lack the outer coat of ganoin. Most of them bear sharp posteriorly directed spines. They pass onto the head, transforming into skull bones. The head is broad and terminates in a large rostrum, bearing many small scales on its dorsal surface. The nostril is divided by a partition into two openings. The operculum contains a single bone. There is a slitlike spiracle above each eye. On the ventral side of the rostrum are four branched processes, the barbels, used as sense organs for the detection of food. The mouth is curious in form, distensible, and with sensory papillae on its borders. The jaws are degenerate, and there are no teeth. Note paired and median fins; the tail is heterocercal.

3. The spoonbill (Polyodon).- - In this curious fish the rostrum is expanded into a broad, thin, spatulate structure, provided with sense organs for the detection of food. At the base of the rostrum just in front of the eyes are the nostrils, each with two openings. Behind the eyes is the small spiracle. The operculum contains no opercular bones and is extended into a pointed process; the branchiostegal membrane, continuous with the opercula, has no branchiostegal rays. Lift up the operculum and note the numerous long and fine gill rakers on the gill arches, used by the fish in separating food particles from mud. The jaws open widely and are provided with minute teeth. The body is naked. Note the fins. The tail is typically heterocercal. The fish is singularly lacking in external hard parts. 
4. The bowfin or river dogfish (Amia). - The body is clothed in flexible scales, similar to those of the perch. There are large scales on the head. The operculum and branchiostegal membranes contain bones. The nostrils have two openings, the anterior one borne on the tip of the process located on the anterior end of the head, the posterior opening situated in front of the eye. There is no spiracle. There is a very long dorsal fin and a nearly homocercal tail. The fish strongly resembles the ordinary teleost type.

\section{EXTERNAL ANATOMY OF NECTURUS}

Necturus is a salamander; it belongs to the class Amphibia, order Urodela. It is an example of the lowest land vertebrates-those which left the water, acquired lungs and the air-breathing habit, and developed legs in place of the paired fins. Obtain a specimen and note the following points.

I. Body and skin.-The body is typically vertebrate in form. It is divisible into head, trunk, and tail, as in fishes. A neck is not present. The skin is naked and very slimy, without scales or other hardened parts such as are commonly found in vertebrates.

2. Head.-The head is broad and flat and has a terminal mouth provided with lips. It bears the usual three pairs of sense organs. The nostrils or external nares are a pair of widely separated openings just back of the margin of the upper lip. By probing into the nostrils determine that they communicate with the mouth cavity by means of openings known as the internal nares. This arrangement permits air to enter the mouth cavity through the nostrils, and differs greatly from the condition found in the majority of fishes where the olfactory sacs end blindly and have no connection with the mouth cavity. In some fishes, however, as the skate, there is an external groove, the oronasal groove, extending from each olfactory sac to the mouth cavity; and by the fusion of the borders of this groove a closed passage from the nostrils to the mouth cavity is produced (see K, pp. 206-8). The small eyes without eyelids are situated on the sides of the head. The ears, as in fishes, are internal only.

From each side of the posterior margin of the head spring three gills, each consisting of a fringe of filaments dependent from a dorsal process. They are external gills and do not correspond to the gills of fishes which are internal. Between the first and second, and the second and third gills are the gill slits which open, as in fishes, into the cavity of the pharynx. The animal, however, does not pass water through the gill slits but respires by means of the external gills which are kept in constant motion, through the general surface of the body, and to some extent by means of its lungs.

3. Appendages.-The trunk bears the two pairs of appendages. These correspond to the paired fins of fishes but have evolved into typical walking limbs. Each consists of the following parts: upper arm, forearm, wrist, and hand, 
in the case of the fore limb; thigh, shank, ankle, and foot, in the hind limb. Both hand and foot bear four digits (fingers, toes), although five is the typical vertebrate number; the first digit is the one which is missing. The position of the limbs with reference to the body is very primitive, especially in the case of the hind limb, and should be carefully studied. Note that the hind limb projects out at right angles to the body, all of its parts on a plane parallel to the ground. In this primitive position the limb has an anterior or preaxial border, a posterior or postaxial border, and dorsal and ventral surfaces. In the fore limb, however, the forearm is bent downward, and the hand is directed slightly forward. This alteration of position is brought about chiefly by a torsion of the upper arm, whose former preaxial surface now looks dorsally. The preaxial border of the forearm is turned medially, its postaxial border laterally. As a result of these changes the animal is able to lift itself to a slight extent above the ground.

The flattened tail is bordered by a tail fin which differs from the fins of fishes in that it contains no fin rays. The tail is diphycercal, that is, truly symmetrical with respect to the vertebral column.

4. Anus.-At the junction of the trunk and tail in the median ventral line is the large anus or cloacal aperture, with fimbriated borders. Amphibia, like the elasmobranchs, have a cloaca.

\section{J. EXTERNAL ANATOMY OF A LIZARD}

The lizards are typical reptiles, class Reptilia, order Squamata.

r. Body and skin.-The body is characteristically vertebrate in form, thickest in the middle, tapering to each end. It consists of head, neck, trunk, and a long tail. It should be recalled that a neck is absent in fishes and Amphibia; its appearance is correlated with the assumption of the land habitat. The body is completely clothed in horny scales, which are thickenings of the outer layer of the skin (epidermis) and are not homologous with the scales of fishes. The scales are larger on the head where they are known as head shields.

2. Head.--The head bears the usual three pairs of sense organs. The external nares or nostrils are located at the tip of the head and lead, as in the Amphibia, into the mouth cavity where they open by the internal nares. Posterior to the nares are the eyes, each provided with an upper and lower movable eyelid and with a third eyelid, the nictitating membrane, a thin transparent membrane concealed from view when not in use in the anterior corner of the eye where it should be sought with a forceps. Halfway between the eye and the base of the fore limb is a slight depression in the skin across the bottom of which stretches a thin membrane. This membrane is the tympanic membrane or drum membrane, and it covers a cavity, the tympanic cavity or middle ear. The depression may be regarded as the beginning of an external ear. The lizard therefore possesses a middle and an external ear in addition to the internal ear present in fishes and Necturus. 
3. Appendages.-The trunk bears two pairs of limbs, which have the same parts as those of Necturus. Five digits, the typical vertebrate number, are present on each limb; they terminate in horny claws. The student should note that the limbs depart still further from the primitive position described in connection with Necturus, thus lifting the animal above the ground to a greater extent. In the hind limb the thigh still extends out at right angles to the body, but is slightly twisted on its longitudinal axis so that the original dorsal surface is becoming anterior. The shank is directed ventrally and posteriorly, and has undergone the same sort of torsion as the thigh. The foot retains the primitive position. In the fore limb the upper arm is rotated in the opposite direction from that observed in the thigh, so that the original preaxial surface is now dorsal; the upper arm is directed posteriorly. The forearm and hand are directed downward and forward, and are so rotated that their original preaxial borders look inward instead of forward.

4. Anus.--On the ventral side of the trunk behind the bases of the hind limbs is the anus or cloacal aperture. Its shape, a transverse slit, is characteristic of lizards and snakes.

\section{K. EXTERNAL ANATOMY OF THE TURTLE}

The turtle is a reptile belonging to the order Chelonia. Obtain a specimen and examine.

I. Body and skin. - The form of the body is considerably modified from the typical vertebrate shape. It is divided into head, neck, trunk, and tail. The head is similar in form to that of other reptiles, but the neck is unusually long and flexible; the trunk is remarkably flat and broad, and the tail diminished in diameter and length. The turtle is thus one of those forms in which, as in the skate, the transverse axis is elongated while the anteroposterior and dorsoventral axes are shortened. The skin of the legs, tail, and other exposed parts of the body is provided with small horny scales or horny thickenings as is characteristic of reptiles. The trunk is inclosed in a hard shell, the outer surface of which consists of greatly enlarged horny scales, definitely arranged. The skin of the head in most of our common turtles is bare, but in some turtles is marked off into large head shields.

2. Head.-The anterior end of the head is pointed and elevated and bears at its tip the two external nares, close together; this position of the nostrils enables the animal to breathe air with only a slight exposure of the head above water. The jaws are clothed with hard, horny beaks, teeth being absent. The large eyes are provided as in the lizard with upper and lower eyelids and a nictitating membrane, located in the anterior corner of the eye. Just posterior to the angles of the mouth can be observed a circular area, covered by skin; this is the tympanic membrane beneath which is located the cavity of the middle ear. This membrane is not, however, sunk below the surface, as in the lizard. 
3. Trunk.-The trunk is incased in a strong, thick shell consisting of the following parts: the dorsal arched portion, the carapace; the ventral, flat portion, the plastron; and the lateral bridges connecting the carapace and plastron. The shell is composed of bony and horny plates which will be studied in detail later. The trunk bears two pairs of limbs, stout and strong, and composed of the same parts as in the animals already studied. There are five digits to each limb, each bearing a claw, except the fifth digits of the hind limbs. The digits are more or less united by webs. The parts of the limbs have undergone a torsion and alteration from the primitive position similar to that observed in the case of the lizard. The student may determine for himself the details of these changes.

4. Anus. - At the base of the ventral side of the tail is the rounded anus or cloacal aperture.

\section{EXTERNAL ANATOMY OF THE PIGEON}

The pigeon is a typical representative of the class Aves, or birds. Examine specimens with and without feathers.

I. Body form and skin.-The proportions of the body, as seen in the plucked specimen, bear a general resemblance to those of the turtle. The head is well developed, the neck long and flexible; the trunk is shorter and considerably plumper than normal, and the tail reduced to a stump, the uropygium. The ancestors of birds had long tails like lizards, bearing feathers along their entire length.

The body is clothed with a covering of feathers, which conceal its shape. These feathers are called contour feathers. The contour feathers are of two kinds, the large, stout feathers of the wings and tail, called quills, and the smaller feathers, the coverts, which cover the bases of the wings and tail, and the general surface of the body. On the plucked bird from which the contour feathers have been removed note the presence of hairlike processes, the hair feathers or filoplumes. In the plucked bird, also, note the delicacy of the skin and the presence in it of numerous deep pits, the feather follicles, into which the contour feathers were set.

2. Head.-The head terminates in the elongated beak, which consists of the upper and lower jaws incased in horny sheaths. Teeth are absent on all modern birds, although extinct birds possessed them. The base of the upper beak bears a cushion-like protuberance, the cere, a structure occurring only in certain families of birds. Under the anterior margins of the ceres are the slitlike external nares. The remarkably large eyes are provided with upper and lower lids and with a nictitating membrane which may be drawn across the eye from the anterior corner. The ear is behind and below the eye and is observable only on the plucked specimen. There is visible only a deep and narrow passage, the external auditory meatus. The middle ear, which is flush with the skin in the frog and most reptiles or only slightly depressed, in birds has sunk below the surface to 
such an extent that the tympanic membrane is no longer visible. The skin around the entrance to the meatus tends to elevate as a fold; this fold together with the meatus constitutes the external ear.

3. Trunk.-The trunk is very firm and inflexible, owing to a fusion of the bones of the back. Pass your fingers along the back of the plucked bird and feel the skeleton. Feel also in the median ventral line of the trunk the projecting keel of the breastbone, to which are attached the great wing muscles. It is the presence of these muscles, the "breast" of the bird, which produces the plump contour of the trunk. The trunk bears the two pairs of limbs, of which the anterior pair is remarkably modified into wings or organs of flight. The hind limbs have also undergone considerable modification as a result of the biped mode of walking used by birds.

The parts of the wing, which are homologous to those of the fore limb of other vertebrates, should be studied on the plucked bird. In the folded condition the sections of the wing make angles with each other like the letter $\mathrm{Z}$. The upper arm is short, directed posteriorly, and slightly twisted on its axis so as to bring the preaxial margin on the dorsal side. The forearm is longer and directed forward. The wrist and hand are fused together, and the whole is considerably elongated and directed caudad. There are but three digits, which are regarded as the second, third, and fourth. The second digit is the projection found just below the joint between forearm and wrist; the third digit forms the terminal point of the wing; the fourth cannot be seen externally. When the wing is extended, its parts have nearly the primitive position described under Necturus. The great quills of the wings are known as remiges: those of the hand are called primaries; of the forearm, secondaries; and of the upper arm, humerals. The primaries differ from the others in that the soft part of the feather is wider on the posterior side of the central axis than on the anterior side. The remiges are borne on the postaxial margin of the wing, and the deep large feather follicles exposed by their removal should be noted on the plucked bird.

The hind limb is partially clothed with feathers and partially with horny scales, identical with those found in reptiles. The digits, of which there are but four-the fifth being absent-terminate in claws. The position of the hind limb with reference to the body is greatly altered. The whole limb, instead of extending straight out laterally from the body, is directed ventrally, thus raising the animal high above the ground. In order to achieve this result it is obvious that the limb must have been rotated $90^{\circ}$ from the primitive position so that the original dorsal surface now faces anteriorly - that is, has become preaxial. The toes are consequently directed forward, with the exception of the first which, through a secondary modification connected with the perching habit, points posteriorly.

4. Tail.-The tail stump bears a half-circle of large quills, known as rectrices. Under the base of the tail is the anus or cloacal aperture, a transverse opening 
with protruding lips. On the dorsal side just in front of the base of the tail will be seen by lifting up the tail coverts or, on the plucked bird, a papilla, the opening of the uropygial gland, from which the bird obtains oil for preening its feathers.

\section{EXTERNAL ANATOMY OF A MAMMAL}

For this purpose either the cat or the rabbit may be used. Both are mammals belonging to the class Mammalia. The rabbit is a rodent, the cat a carnivore.

I. Body form and skin.-The body is divisible into head, neck, trunk, and tail. Its proportions depart somewhat from the typical vertebrate form in the larger size of the head and the reduction of the tail, the latter feature particularly noticeable in the rabbit. The body is clothed with closely set hairs, forming a covering of fur, characteristic of mammals. Upon the head are a number of especially long and stout hairs, the whiskers or vibrissae, which serve as important organs of touch.

2. Head.-The large size of the head is due to the great development of the brain within it. The head may consequently be divided into an anterior facial region, in front of the eyes, and an enlarged posterior cranial region. The mouth is provided with well-developed lips. The upper lip is cleft in the center, deeply so in the rabbit, exposing the incisor teeth. The exiernal nares are large and elongated, overhung by the mobile nose. The eyes have upper and lower lids and a nictitating membrane. The latter should be sought with a forceps in the anterior corner of the eye and drawn over the eyeball. The ears are provided with a long and flexible external fold, the pinna, which springs from the rim of an opening, the external auditory meatus, which descends into the interior of the skull. Pinna and meatus constitute the external ear.

3. Trunk.-The trunk is divisible into an anterior chest or thorax, supported by the ribs, and a posterior abdomen. On the ventral surface of the trunk are four or five pairs of teats or nipples in female specimens; they are the openings of the milk glands or mammary glands. The trunk bears two pairs of limbs, which consist of the same parts as other vertebrate limbs. The upper section of each limb-that is, thigh or upper arm-is more or less included in the trunk. The limbs terminate in clawed digits, five in front, four behind, the first hind toe being absent. The claws of the cat and its relatives are retractile.

The limbs have undergone a marked change from the primitive position. Instead of extending laterally from the body they project ventrally and are elongated, so that the body of the animal is carried high above the ground. This change has involved a rotation of $90^{\circ}$ in each limb. The hind limb is rotated forward so that the original dorsal surface is anterior and the toes point forward. The knee or joint between the thigh and shank is likewise directed anteriorly. The fore limb, on the contrary, has rotated posteriorly, so that the original 
dorsaı surface faces posteriorly and the preaxial surface faces laterally. Consequently, the elbow or joint between upper arm and forearm is directed caudad. As a result of the rotation of the upper arm the toes would point posteriorly, but by an additional torsion they have been brought around to the front again. This torsion involves a crossing of the two bones of the forearm, the distal end of the preaxial bone being brought internal to the distal end of the postaxial bone (see Fig. 2 and read R, p. 26). This position of the forearm is known as
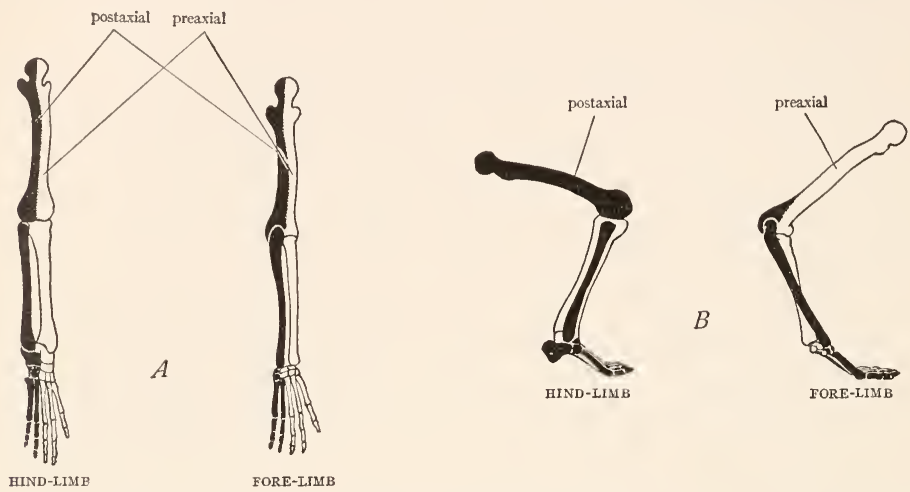

FIG. 2.-Diagrams to illustrate the torsion of the limb; preaxial surface white, postaxial surface black. $A$, primitive position of the limbs, seen from above, the limbs extending at right angles to the body, the preaxial surface facing anteriorly, the postaxial posteriorly. $B$, position of the limbs in mammals, seen from the side; the limbs extend vertically below the body; the upper arm has rotated outward and backward so that the preaxial surface now faces laterally; the forearm has rotated forward again, resulting in a crossing of its bones; the hind limb has rotated outward and forward so that the postaxial surface faces laterally. (From Flower's Osteology of the Mammalia.)

the prone position, and is imitated in the human arm when the arm hangs by the side with the back of the hand directed forward. In this position the crossing of the two long bones of the forearm can be felt. If now the arm is raised sidewise, shoulder high with the palm facing forward, the two bones return to the primitive parallel position, known as the supine position. Thus man can change his forearm from the-prone to the supine position, but in most mammals the forearm is fixed in the prone position.

The position of the parts of the foot in walking is different in different mammals. The rabbit and the cat walk on the digits, with the remainder of the hand and foot elevated. This type of gait is known as digitigrade. Man walks on the whole sole of the foot, the primitive method, known as plantigrade. Horses and cattle and other ungulates walk on their nails, which are broadened into hoofs, a mode of walking called unguligrade. 
4. Perineal region.-In mammals the region which includes both the anal and urogenital openings is designated the perineum. The anus is situated in the ventral median line just in front of the base of the tail. On each side of the anus in the rabbit is a deep, hairless depression, the inguinal or perineal space, onto which open the inguinal glands (not visible externally) whose secretion produces the odor characteristic of the animal. In the female, the urogenital opening is situated immediately anterior and ventral to the anus; it is similar in appearance to the anus. It is inclosed by a fold of skin extending around the rim; this fold is named the greater lips or labia majora. The labia together with the urogenital aperture constitute the vulva. In the male rabbit there is a hillock in front of the anus. In the center of this is usually visible a pointed projection, the end of the penis or organ of copulation; the penis bears at its tip an opening, the urogenital aperture. The hillock of skin which folds up about the penis is named the prepuce or foreskin. To each side of the prepuce and extending forward is an oval swelling, caused by the male gonad, or testis, which is inclosed within it. The double pouch of the body wall which incloses the two testes is named the scrotum or scrotal sac. In the male cat there is a pair of rounded eminences anterior to the anus; each eminence contains a testis or male gonad, and the double pouch of the body wall inclosing the testes is named the scrotum or scrotal sac. Anterior to the scrotum is a hillock of skin, the prepuce or foreskin. In the center of the prepuce is an opening, which is not, however, as might be supposed, the urogenital opening. It is merely the depression left by the withdrawal into the prepuce of the penis; the penis is generally so far withdrawn in male cats as to be invisible externally.

\section{N. SUMMARY}

Our study of the external features of representative vertebrates may be utilized to direct the student's attention to the following points.

I. The vertebrate body is typically fusiform, that is, moderately thick through the trunk, tapering toward each end. In every group of vertebrates forms may be found which deviate from this typical shape, but such deviations bear no relation to the position of the animal in the vertebrate scale, being rather adaptations to particular modes of life.

2. The body is divided into head, trunk, and tail in the lowest vertebrates. A neck is added in land vertebrates.

3. The head tends to increase relatively in size and the tail to decrease as one ascends the vertebrate series. The former change is associated with increase in the size of the brain; the latter with greater speed and agility of movement.

4. The skin of vertebrates is commonly clothed with protective structures, such as scales, feathers, or hairs. These are more complex in structure in the higher vertebrates and better fitted for keeping the body warm.

5. The head throughout bears three pairs of sense organs. Of these the eyes undergo little change throughout the series. The olfactory sacs are blind in fishes and open to the exterior only, by means of the external nares. As soon, however, as vertebrates left the aquatic environment, internal nares were developed, connecting the olfactory sacs with the 
mouth cavity. This arrangement permits the animal to breathe without opening the mouth a decided advantage in air-breathing animals, since thereby the drying of the mouth cavity is avoided. In the lower vertebrates an internal ear only is present. To this is added, beginning with the anuran Amphibia, a middle ear, closed externally by the tympanic membrane. In Amura and many reptiles the tympanic membrane is level with the surface of the head, but in some reptiles it begins to sink below the surface. In birds and mammals it has descended deeply into the head, forming a narrow passage, the external auditory meatus. Around the external rim of the meatus in mammals the skin elevates to form a sound-catching device, the pinna. Pinna and meatus constitute the external ear.

6. The gill slits and gills present in the fishes and lower Amphibia disappear in the adults of the higher Amphibia and all forms above them. This is due to the assumption of the airbreathing habit.

7. The trunk bears two pairs of appendages. These are fins in fishes, but become limbs in all vertebrates above fishes. Stages in this transformation are very imperfectly known. The parts of the limbs are the same through all of the vertebrates, although they are subject to considerable modification. The most primitive limbs, in structure, form, and position with reference to the body occur in the urodele Amphibia. In higher vertebrates the position of the limbs is altered by bending and torsion, resulting in an elevation of the body above the ground with a correspondingly more rapid progression. As a still further aid to rapid movement the digitigrade or unguligrade mode of walking has been adopted in many cases. Loss of digits is quite common among vertebrates; the missing digits are nearly always the first or last ones, rarely the middle ones.

8. In nearly all vertebrates except mammals the intestine and the urogenital ducts open into a common chamber, the cloaca, which communicates with the exterior by a single opening, the anus or cloacal aperture. In all placental mammals the intestine and the urogenital system open by separate apertures, the urogenital opening being situated always anterior to the anus. The term anus, therefore, does not have the same significance in mammals as in other vertebrates. 


\section{GENERAL FEATURES OF CHORDATE DEVELOPMENT}

Since it is impossible to understand the comparative anatomy of vertebrates, which forms the main object of study of this course, without some knowledge of the way in which a vertebrate develops, it is necessary that the student learn some of the elementary facts about vertebrate development. Every student must read either $K$, pages $7-I 6$, or $W$, pages 60-7I. He will be expected to know the contents of these pages thoroughly and to be able to answer at all times questions relating to them.

\section{A. THE CHORDATE EGG}

The manner of development of the chordate egg is dependent upon the amount of food material, or yolk, which it contains. On this basis chordate eggs are classified as follows: (I) Isolecithal eggs, with little yolk; Amphioxus and mammals. (2) Telolecithal eggs with total cleavage. There is a moderate
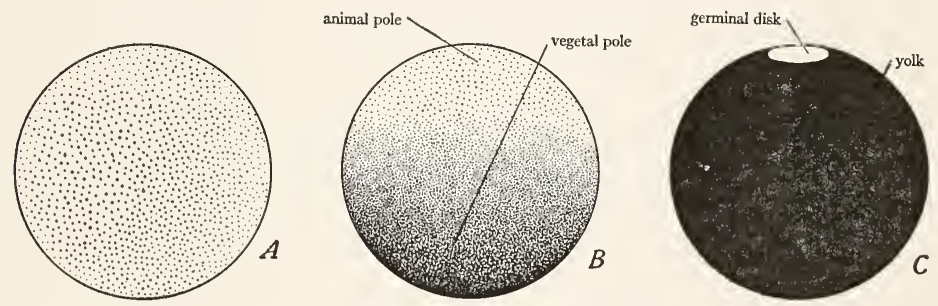

FIG. 3.-Three types of chordate eggs. The black coloring represents the yolk. $A$, isolecithal egg with small and evenly distributed yolk particles. $B$, telolecithal egg with total unequal cleavage, with the yolk more abundant in the vegetal than in the animal half. $C$, telolecithal egg with meroblastic cleavage, consisting completely of yolk except for the small germinal disk of protoplasm at one pole. The size of the germinal disk in the figure is greatly exaggerated with respect to the size of the yolk.

amount of yolk which accumulates in one-half of the egg and retards its development; Amphibia, cyclostomes, and ganoid fishes. (3) Telolecithal eggs with meroblastic cleavage. There is an enormous amount of yolk, and the protoplasm is reduced to a small disk which floats on the surface of the yolk. This spot is called the germinal disk. Such eggs are characteristic of birds, reptiles, and teleosts. The true egg in the case of birds is the yolk, the white being merely a nutritive envelope.

These three types of eggs are illustrated diagrammatically in Figure 3. (See further, H, pp. I4I-48.) 


\section{B. THE CLEAVAGE OF THE EGG AND THE FORMATION OF THE BLASTULA}

Development begins by the division of the egg into two, four, etc., cells until a large number of cells has been produced. This process of division of the egg is called cleavage, and the way in which it occurs depends on the amount of yolk which the egg contains. It should be understood that the yolk is inert material and that the process of development is carried out only by the living protoplasmic portions of the egg.

I. Holobastic equal cleavage.-In the case of isolecithal eggs the entire egg divides and produces a number of approximately equal cells. Such cleavage is said to be holoblastic and equal. The cells, as they increase in number, gradually withdraw from the center and arrange themselves in a single layer on the surface, thus producing a hollow ball of cells. This ball is called the blastula; its cavity is known as the segmentation cavity or blastocoel. Such a blastula is produced in the development of Amphioxus. Cleavage and formation of the blastula in Amphioxus are illustrated in Figure $4 A$. Similar figures will also be found in the standard textbooks, as $\mathrm{P}$ and $\mathrm{H}, \mathrm{K}, \mathrm{W}$, and $\mathrm{H}$. Study also the models of the cleavage of Amphioxus provided in the laboratory.

2. Holobastic unequal cleavage.-This type occurs in those telolecithal eggs which contain a moderate amount of yolk. The half of the egg which contains the majority of the yolk is called the vegetal hemisphere; that which contains the majority of the protoplasm is the animal hemisphere. The early cleavage planes are shifted toward the animal hemisphere, and further, the cleavage processes are delayed in the vegetal hemisphere owing to the presence of the inert yolk. In consequence of these two factors, the cells produced in the animal hemisphere are smaller and more numerous than those of the vegetal hemisphere, although the entire egg cleaves. Such cleavage is holoblastic but unequal. The cells withdraw from the center, producing a blastula with a somewhat reduced blastocoel and a wall several layers of cells thick. The cells of the blastula are of unequal sizes grading from the smallest at the animal pole to the largest at the vegetal pole.

This type of development is characteristic of Amphibia. It is illustrated in Figure ${ }_{4} B$; similar figures are presented in various textbooks, as $\mathrm{P}$ and $\mathrm{H}, \mathrm{K}$, and $N$. Study also the models of the cleavage of the amphibian egg, provided in the laboratory. Then obtain a section through an amphibian egg in the blastula stage and examine under the low power of the microscope. The blastula is a hollow sphere whose wall is composed of two or three layers of cells. The wall of the animal hemisphere is thin and consists of small cells; it is the future dorsal side of the embryo. The wall of the vegetal hemisphere is much thicker than that of the animal hemisphere and is composed of large cells, laden with yolk and with indistinct boundaries; it is the future ventral side. The blastocoel is smaller than in the blastula of Amphioxus, and is displaced dorsally, owing to the thickness of the ventral wall. Draw, showing outlines only of the cells. 


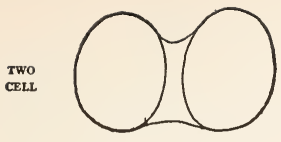

$\boldsymbol{I}$

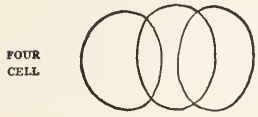

2

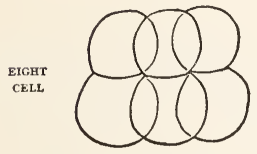

3

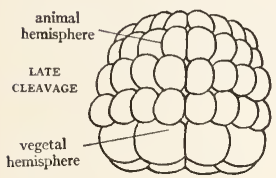

4

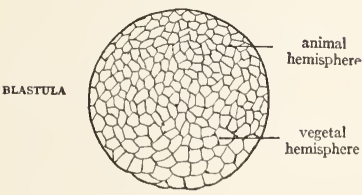

5

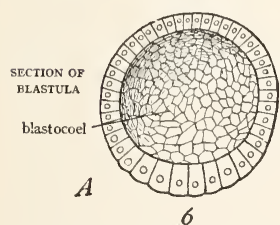

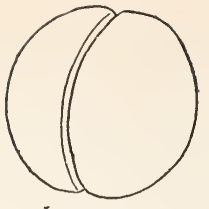
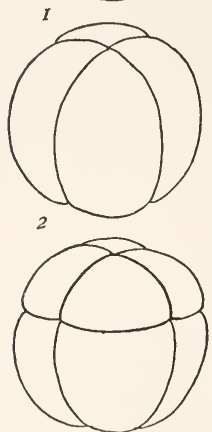

3
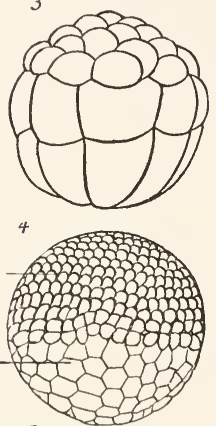

5

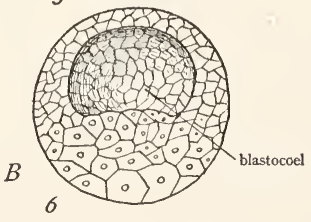

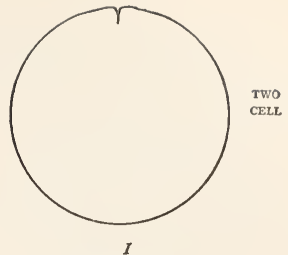
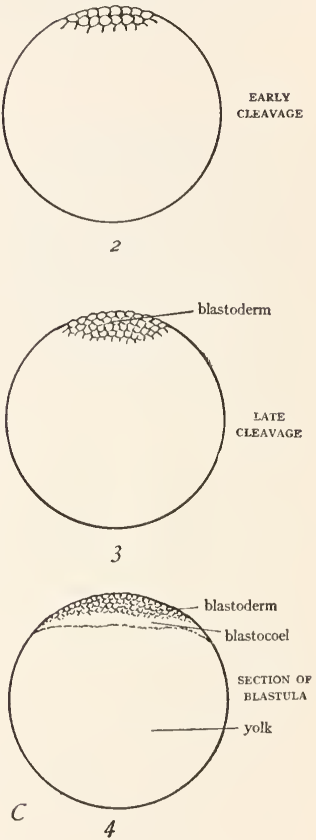

FIG. 4.-Cleavage of the three types of chordate eggs and formation of the blastula. $A$, Amphioxus; I-4, cleavage; 5, external view of the blastula; 6 , section of the blastula. Note that the cells of the vegetal hemisphere are but slightly larger than those of the animal hemisphere, the wall of the blastula is one cell-layer in thickness, and the blastocoel is large. $B$, amphibian; $I-4$, cleavage; 5 , blastula; 6 , section of the blastula. The cells of the vegetal hemisphere are considerably larger than those of the animal hemisphere, the wall of the blastula is at least two cell layers in thickness, and the blastocoel is smaller and displaced dorsally. C, reptile or bird egg with meroblastic cleavage; $I-3$, cleavage; 4 , median sagittal section of the blastula. Only the germinal disk cleaves, forming a disk of cells-the blastoderm-resting on the yolk; a slight slit between this and the yolk represents the blastocoel. ( $A$ and $B, I-5$, from Parker and Haswell's Textbook of Zoölogy, after Hatschek, courtesy of the Macmillan Company; B6 from Prentiss and Arey's Textbook of Embryology, courtesy of the W. B. Saunders Company.) 
3. Meroblastic cleavage.-In eggs containing large quantities of yolk only the small germinal disk undergoes cleavage. This kind of cleavage is called meroblastic. As a result, a minute disk of cells is produced on the surface of the relatively enormous yolk. A slight split appears between the disk and the yolk, and this corresponds to the segmentation cavity of other developing eggs; this stage is consequently the blastula stage. Meroblastic cleavage is illustrated in Figure ${ }_{4} C$. The disk of cells produced by meroblastic cleavage is called the blastoderm; in the further development it expands over the surface of the yolk which it eventually incloses.

\section{FORMATION OF THE GASTRULA}

I. In eggs of the Amphioxus type.-In such eggs the vegetal hemisphere begins to bend inward and continues this process of invagination until its wall comes in contact with the wall of the animal hemisphere. An embryo with a wall two cell-layers thick is thus produced. It is called a gastrula. The outer layer is named the ectoderm and the inner layer the entoderm. Because of their importance in the subsequent development, these layers are referred to as the first two germ layers. The hollow tube of entoderm is called the archenteron or primitive intestine; the cavity of the gastrula is the cavity of the archenteron or gastrocoel; and the opening of the archenteron to the exterior is the blastopore. Note that the blastocoel is eliminated in the production of the gastrula. The formation of the gastrula of Amphioxus is illustrated in Figure ${ }_{5} A$, and also in the various textbooks, as $\mathrm{P}$ and $\mathrm{H}, \mathrm{W}, \mathrm{K}$, and $\mathrm{N}$. Study further the models exhibited in the laboratory.

2. In eggs of the amphibian type.-In these eggs gastrulation is somewhat modified by the presence of inert yolk in the vegetal hemisphere. It is accomplished partly by the invagination of the entoderm particularly at the dorsal lip of the blastopore, and partly by the expansion of the ectoderm ventrally pushing the entoderm into the interior. The result is the same as the foregoing, a gastrula being formed. A small portion of the inclosed yolk-bearing cells commonly remains for some time protruding through the blastopore, and is called the yolk plug.

The formation of the amphibian gastrula is illustrated in Figure ${ }_{5} B$, and in $\mathrm{P}$ and $\mathrm{H}, \mathrm{N}$, and other textbooks. Study further the models of amphibian development illustrating this stage, noting especially the sagittal section of the gastrula. Then obtain a slide bearing a sagittal section of the gastrula and study with the low power of the microscope. The gastrula is slightly elongated in the anteroposterior direction. The side with the thinner wall is the dorsal side; that with the thick wall, the ventral side; the end with an opening is the posterior end; the opposite end is anterior. The wall consists of two layers each composed of more than one sheet of cells. The outer and thinner layer is the ectoderm, uniform in width over the whole embryo. The inner layer is the entoderm, separated from the ectoderm by a slight space, and very thick ventrally, where 
its cells are laden with yolk. The cavity inclosed by the entoderm is the gastrocoel. The opening of the archenteron to the exterior at the posterior end is

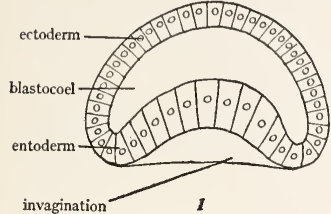

invagination

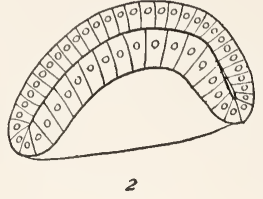

$A$

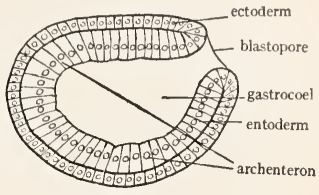

3

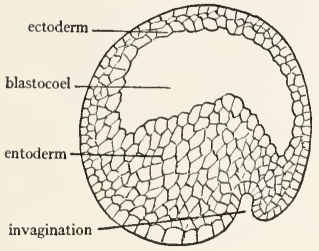

$\boldsymbol{I}$

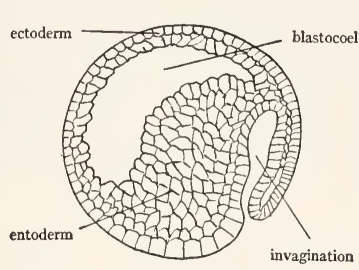

2

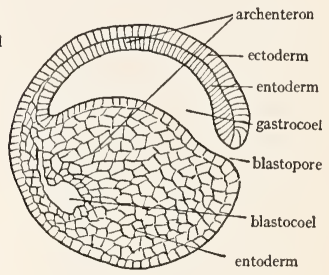

3

B
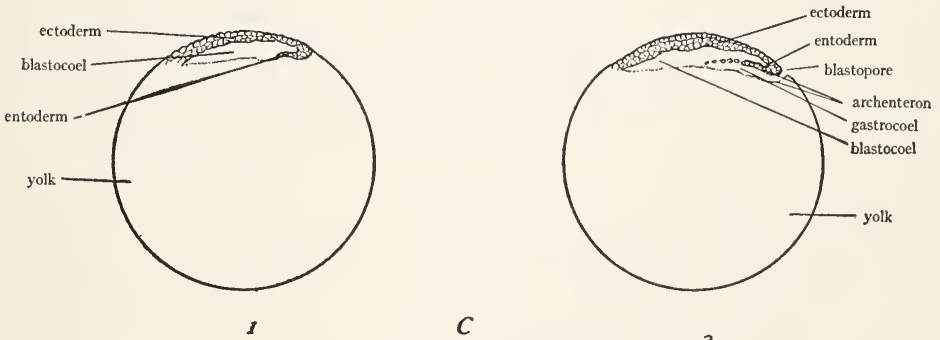

FIG. 5.-Gastrulation and formation of the entoderm in the three types of chordate eggs. All shown in median sagittal section. A, Amphioxus; I, beginning of the invagination; 2, invagination completed; 3 , completed gastrula having a wall of two layers, ectoderm and entoderm, and an internal cavity, the gastrocoel. $B$, amphibian; $I$, beginning of the invagination; 2 , progress of the invagination accompanied by downward growth of the ectoderm; 3, completed gastrula, with very thick entoderm ventrally. $C$, reptile or bird; $I$, posterior margin of the blastoderm beginning to turn under; 2 , continuation of the invagination; a slight split between entoderm and yolk constitutes the gastrocoel. In $A_{3}, B_{3}$, and $C_{2}$ the anterior end of the embryo is to the left, posterior end to the right, dorsal surface above, ventral below. ( $A$ from Parker and Haswell's Textbook of Zoölogy, after Hatschek, courtesy of the Macmillan Company; B from Kellicott's General Embryology, courtesy of Henry Holt and Company.)

the blastopore. Ectoderm and entoderm are continuous at the rim of the blastopore. A portion of the entoderm, the yolk plug, protrudes through the 
blastopore and nearly occludes the opening. Make a diagram of the section, coloring ectoderm blue and entoderm yellow.

3. In meroblastic eggs.-In such eggs the formation of the entoderm will obviously be attended by difficulties. It seems to be accomplished in two ways: (a) by a process of involution or invagination, in which the cells at the future posterior end of the blastoderm turn under and grow forward; $(b)$ by a process of delamination, in which the cells on the under side of the blastoderm arrange themselves to form a lower layer. In different forms the proportion of entoderm formed by each method varies. Eventually the two kinds of entoderm unite to form a single sheet of cells. The cavity of the archenteron is very small in meroblastic eggs, consisting of a cleft between the entoderm and the yolk, and the blastopore is reduced to a slit at the posterior end of the blastoderm. After gastrulation has occurred the blastoderm consists of two layers, an outer ectoderm and an inner entoderm; it lies on the surface of the yolk and by proliferation at its margins gradually spreads out over the yolk, eventually inclosing it.

The gastrulation of meroblastic eggs by the invagination process is illustrated diagrammatically in Figure ${ }_{5} C$.

D. FORMATION OF THE THIRD GERM LAYER, THE NEURAL TUBE, AND THE NOTOCHORD

These processes occur practically simultaneously but will be described separately.

I. In Amphioxus.-After the embryo has attained the gastrula stage it elongates and presents a flattened dorsal surface, a rounded ventral surface, and recognizable anterior and posterior ends (see Fig. $5^{A} 3$ ). From the dorsolateral regions of the entoderm, which it will be remembered forms the "inner tube" of the gastrula, hollow pouches begin to grow out in pairs. These pouches are called the coelomic sacs or mesodermal pouches. The walls of the pouches constitute the mesoderm, or third germ layer, which, unlike the ectoderm and entoderm, consists of two walls. The pouches grow laterad and ventrad, filling the space between ectoderm and entoderm. The outer wall of the pouches in contact with the ectoderm is called the somatic or parietal mesoderm; the inner wall in contact with the entoderm is the splanchnic mesoderm. The cavity of the pouches is the body cavity or coelom. Eventually the anterior and posterior walls of the pouches break down so that those of each side unite to form a tube. Thus, the coelom, originally segmented, comes to consist of a pair of continuous cavities, one on each side of the embryo.

Meantime, the ectoderm rises up on either side of the median dorsal line as a fold or ridge. The two folds meet above the median dorsal ectoderm, which is thus inclosed and becomes the neural tube, the primordium of the brain and spinal cord. 
From the median dorsal wall of the entoderm a solid rod of cells is elevated and separated off. This is the notochord or primitive skeleton.

These processes are illustrated in Figure 6, also in $\mathrm{P}$ and $\mathrm{H}$, page $56 ; \mathrm{N}$, page $45 ; \mathrm{K}$, page II; and particularly well in $\mathrm{W}$, plates between pages 62 and 63. Study also the models of the development of Amphioxus.

2. In vertebrates.--In vertebrates (with the exception of cyclostomes and possibly urodeles) the mesoderm does not arise as pouches from the entoderm. Instead it grows out as a solid sheet of cells from the median dorsal region and
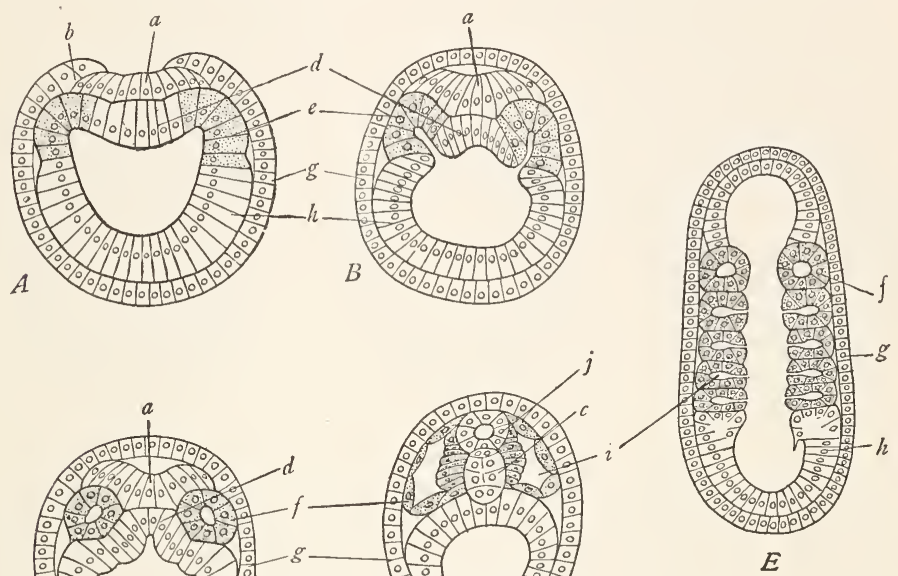

C
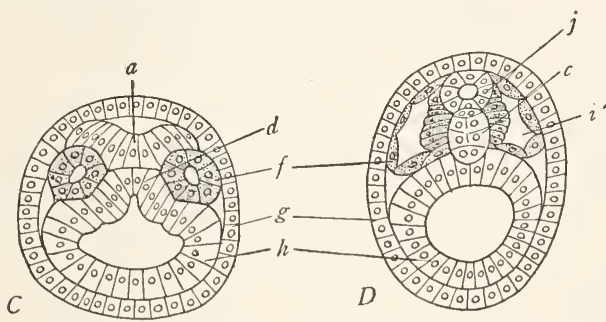

E

Fig. 6.-Formation of the neural tube, notochord, mesoderm, and coelom in Amplioxus. A-D, cross-sections; $E$, frontal section. $A$, difierentiation of the medullary plate $a$, the notochordal plate $d$, the neural folds $b$, and the mesodermal pouches $e . B$, the neural folds have closed across above the medullary plate; the mesodermal pouches are farther evaginated. $C$, the medullary and notochorda 1 plates are beginning to close; the mesodermal pouches $f$ are completely separated from the entoderm. $D$, the neural tube $j$ and the notochord $c$ are completed; the mesodermal pouches are increasing in size. $E$, frontal section to show the mesodermal pouches $f$ originating from the entoderm segmentally. $a$, medullary plate; $b$, neural fold; $c$, notochord; $d$, notochordal plate; $e$, mesoderm; $f$, mesodermal pouches; $g$, ectoderm; $h$, entoderm or archenteron; $i$, coelom. In all figures the mesoderm is stippled. (From Parker and Haswell's Textbook of Zoölogy, after Hatschek, courtesy of the Macmillan Company.)

around the blastopore and gradually spreads laterally and ventrally between ectoderm and entoderm. Its origin cannot be definitely ascribed to either ectoderm or entoderm, since at the blastopore these two germ layers are continuous. As the mesodermal sheets spread, a central split appears in them, dividing them into somatic and splanchnic walls; the split itself is the body cavity or coelom. The coelom of vertebrates thus consists from the first of a single pair of cavities 
extending along each side of the body axis from anterior to posterior end, and is never composed of a series of cavities as in Amphioxus. The end result is the same as in Amphioxus, although arrived at in a different way.

The neural tube is formed from a pair of folds, the neural folds, which appear in the median dorsal region, and fuse together to produce a tube. The notochord is separated off as a solid cord of cells from the roof of the archenteron (Amphibia, reptiles) or else arises from the somewhat indefinite region where the mesodermal sheets originate (birds).

The processes will be grasped more clearly by reference to Figure 7 , also to the figures in $\mathrm{K}$, page II; $\mathrm{P}$ and $\mathrm{H}$, page II 7 ; W, page 69. Obtain a mounted

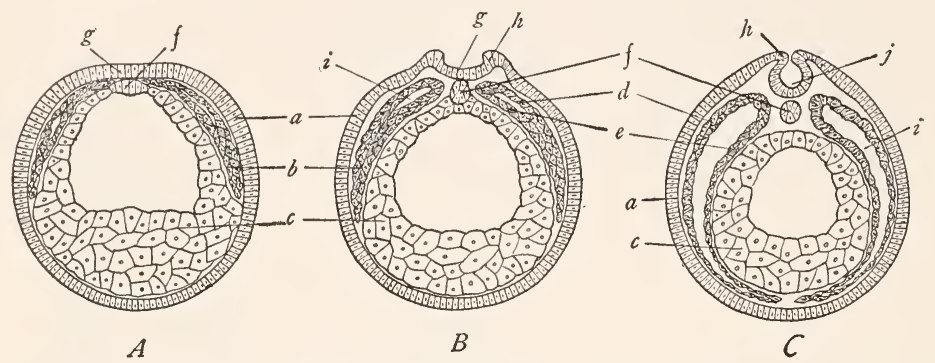

FIG. 7.-Diagrams to show the formation of the neural tube, notochord, mesoderm, and coelom in vertebrates, based on Amphibia. Cross-sections. $A$, differentiation of the notochord $f$ in the roof of the entoderm; mesodermal plates $b$ spreading ventrally. $B$, neural folds $h$ rising at the sides of the medullary plate $g$; notochord $f$ separated from the entoderm; mesodermal plates $b$ extended farther ventrally and developing a central cavity, the coelom $i$. $C$, neural folds $h$ nearly closed to form the neural tube $j$; mesodermal plates have reached the midventral line; coelomic split $i$ has extended ventrally. $a$, ectoderm; $b$, mesoderm; $c$, entoderm or archenteron; $d$, somatic mesoderm; $e$, splanchnic mesoderm; $f$, notochord; $g$, medullary plate; $h$, neural fold; $i$, coelom; $j$, neural tube. ( $A$ from Hertwig-Mark's Textbook of the Embryology of Man and Mammals, courtesy of the Macmillan Company.)

cross-section through an amphibian embryo at the stage of the formation of the mesoderm, and examine under the low power. The section is oval in form; it is in most cases still surrounded by the delicate egg membrane. The outer layer of the embryo is the ectoderm, relatively thin and of the same width over the whole surface. In the median dorsal line the ectoderm is producing or has already produced the neural tube. In the former case the ectoderm exhibits a pair of neural folds inclosing a thick plate of ectoderm between them. In the latter case the folds have fused across in the median line, forming a tube, the neural tube, which is the oval hollow mass in the median dorsal line, just beneath the ectoderm. The greater part of the section is occupied by the archenteron or primitive intestine, composed of entoderm. The archenteron has a thin dorsal wall, a thick ventral wall, whose cells contain yolk, and incloses the relatively small gastrocoe?. which occupies its dorsal part. In the median dorsal region of 
the archenteron, a mass of cells will be seen protruding dorsally; or in some slides this mass of cells may have separated from the archenteron and lies between the latter and the neural tube. This mass is the notochord. Between the ectoderm and the archenteron on each side is a narrow sheet of cells extending irom the sides of the neural tube ventrally. In some slides these sheets will extend only a short distance, while in others they reach nearly to the median veniral line. These sheets are the mesoderm. Make a diagram of the section, coloring ectoderm blue, entoderm yellow, and mesoderm red.

\section{E. FURTHER HISTORY OF THE MESODERM}

The history of the mesoderm is of the utmost importance for the understanding of vertebrate structure. We have already noted that the mesoderm splits into two layers, an outer or somatic layer, and an inner or splanchnic layer, and that the space between the two layers is the body cavity or coelom. The mesoderm grows from each side of the embryonic axis ventrally to the median ventral line, or in meroblastic eggs grows out over the yolk, pushing out between ectoderm and entoderm.

The mesoderm next becomes differentiated into three regions: a dorsal region, called the epimere, which lies to each side of the neural tube; a middle region, called the mesomere or nephrotome, situated lateral and ventral to the epimere; and a large ventral region on each side of the archenteron, called the hypomere or lateral plate. Each of these regions has of course both somatic and splanchnic walls (see Fig. $8 A$, also K, p. I4). The epimere immediately becomes segmented, that is to say, dorsoventral clefts appear in it at regular intervals, the process beginning at the anterior end of the embryo and proceeding postericrly. Consequently, the epimere becomes divided up into a longitudinal row of blocks, a row on each side of the neural tube. These blocks are epimeres, or mesoblastic somites (originally called provertebrae as it was erroneously supposed that they were primitive vertebrae). At first the epimeres are still continuous ventrally and laterally with the mesomere, but eventually they are completely cut off from the rest of the mesoderm (see Fig. $8 B$ and the figure in K, p. I5). The mesomere and the hypomere do not become segmented and remain permanently in close relation to each other. Within the mesomere little tubules appear, which open into the cavity of the hypomere; they are the tubules of the kidney (see Fig. $8 B$ ). The hypomeres of each side fold around the archenteron, their inner walls coming in contact above and below the archenteron to form double-walled membranes, the dorsal and ventral mesenteries (see Fig. 8B). The cavities of the two hypomeres become the coelom of the adult; the cavity in each epimere disappears; and that of the mesomere remains as the cavities of the tubules of the kidney.

In embryos of the amphibian type the archenteron is a closed tube and the two hypomeres are closed cavities which meet below the archenteron. In 
embryos resulting from meroblastic eggs, however, the archenteron is open below and spread out on the yolk, and the hypomeres extend out over the yolk. The differences between the two types of embryos are illustrated in Figures $8 A$ and $C$. The embryo in the case of meroblastic development is later constricted from the
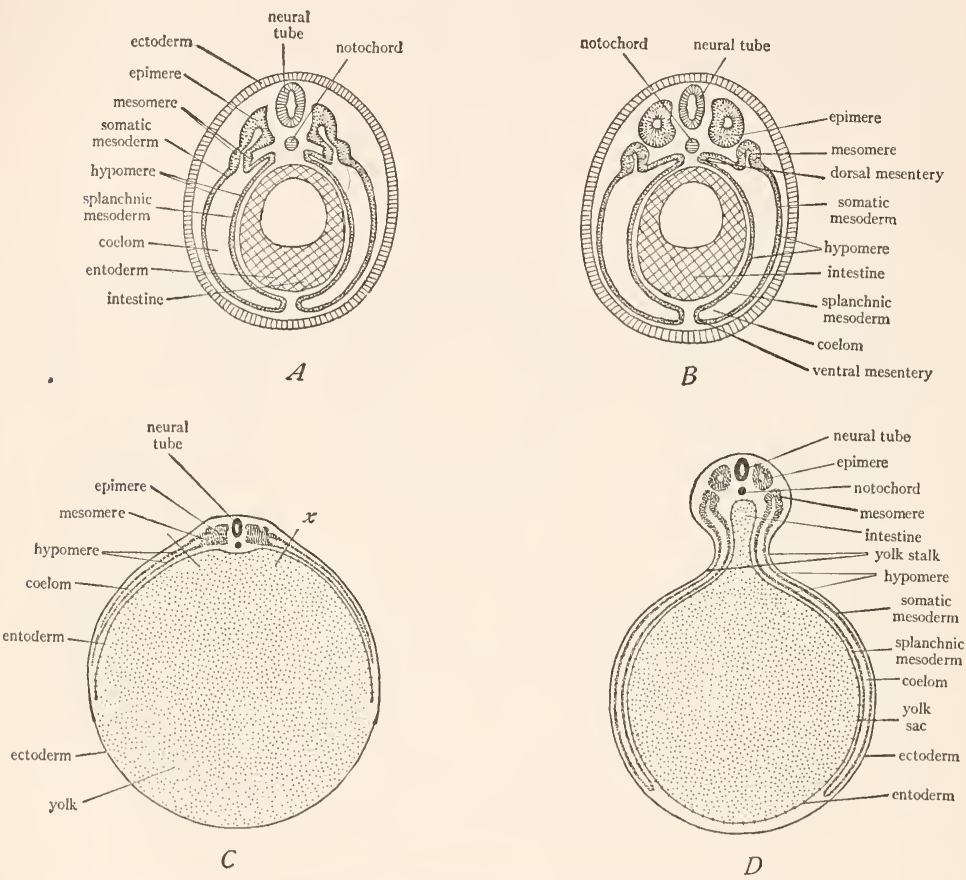

FIG. 8.-Diagrams to show the differentiation of the mesoderm in holoblastic and meroblastic types of development. $A$ and $B$, holoblastic type; $C$ and $D$, meroblastic type. $A$, differentiation of the mesoderm into epimere, mesomere, and hypomere. $B$, separation of the epimere from the mesomere, appearance of kidney tubules in the mesomere, and closure of hypomere around the intestine to form dorsal and ventral mesenteries. $C$, similar to $A$ but in the meroblastic type, showing entoderm and mesoderm growing around the yolk; note how embryo is spreac' out on the surface of the yolk; $x$, indicates lines where embryo is cut off from yolk in making sections for microscopic study. $D$, similar to $B$ but in the meroblastic type; the entoderm has completely surrounded the yolk; the mesoderm has nearly done so; the yolk sac is seen to be a part of the intestine; the embryo is partly constricted from the yolk sac, the constriction being the yolk stalk. ( $C$ from Wilder's History of the Human Body, courtesy of Henry Holt and Company.)

yolk by the formation of deep grooves on all sides. The yolk then hangs from the ventral surface of the embryo inclosed in a sac of blastoderm, the yolk sac, which is connected with the embryo by a stalk, the yolk stalk, as shown in 
Figure $8 D$. At the time of hatching, the yolk has been practically used up, and the remnant is withdrawn into the embryo, the opening in the body wall where the yolk stalk arises being then finally closed over.

Obtain a slide bearing a cross-section through the trunk of a chick embryo of two days' incubation. As explained above, the intestine of the chick embryo is open below on the yolk. In making such sections the embryo is cut off from the yolk, the lines of section being indicated in Figure $8 C$ at $x$. After understanding the relation of embryo and yolk, examine the section with the low power. The dorsal boundary of the section is a thin layer, the ectoderm, which is slightly elevated in the median dorsal line; the ventral boundary is another thin layer, the entoderm, which makes a slight upward bend in the median ventral line, indicating the future intestine. In the median dorsal line just beneath the ectoderm is the oval hollow section of the neural tube. Immediately ventral to this is a small circular mass of cells, the notochord. On each side of the neural tube is a squarish mass, its cells radiating from the center. This is the epimere or mesoblastic somite. Lateral to the epimere and continuous with it is a smaller mass, the mesomere or nephrotome, in which one or more tubules with central holes are distinguishable. Beyond the m:somere the mesoderm is observed to split into two layers. This region of the mesoderm is the hypomere or lateral plate. The outer or dorsal layer of the hypomere is the somatic mesoderm. It ascends and comes in contact with the ectoderm, the two together constituting the somatopleure or body wall. The lower or ventral wall of the hypomere is the splanchnic mesoderm; it descends and comes in contact with the entoderm and the double layer thus formed is the splanchnopleure or intestinal wall. The cavity between the somatic and splanchnic walls of the hypomere is the coelom. As already explained, the hypomere in such embryos extends far out over the yolk. Observe that the splanchnopleure contains many holes; these are the cross-sections of blood vessels, which convey the food from the yolk sac to the embryo. There is also a large artery in the embryo below each epimere. Draw the section in diagram, coloring the three germ layers as before.

\section{F. THE FATE OF THE ECTODERM}

As we have seen, the ectoderm gives rise to the neural tube, from which develop the brain, spinal cord, and nerves. The ectoderm also forms the external layer of the skin and all of its derivatives, such as hair, nails, etc. It also gives rise to the sensory part of all the sense organs, the lining membrane of the nasal cavities, the mouth, and anus, the glands and other outgrowths of the nasal and mouth cavities, the glands of the skin, the enamel of the teeth, and the lens of the eye.

\section{G. THE FATE OF THE ENTODERM}

The entoderm is, as we have seen, the primitive intestine. This intestine is the inner lining of the adult intestine. The entoderm thus forms the epithelial 
Iining of the intestine and the epithelial lining and epithelial cells of all of the outgrowths of the intestine, which include the gill pouches and gills, the larynx, windpipe, and lungs, the tonsils, the thyroid and thymus glands, the liver, the gall bladder and bile duct, the pancreas, and the urinary bladder and adjacent parts of the urogenital system. The student should note that only the epithelial cells of these structures arise from the entoderm.

H. THE FATE OF THE MESODERM AND THE FORMATION OF MESENCHYME

I. Mesenchyme.-In the further development of the mesoderm, mesenchyme plays an important rôle. Mesenchyme is not a germ layer but a particular

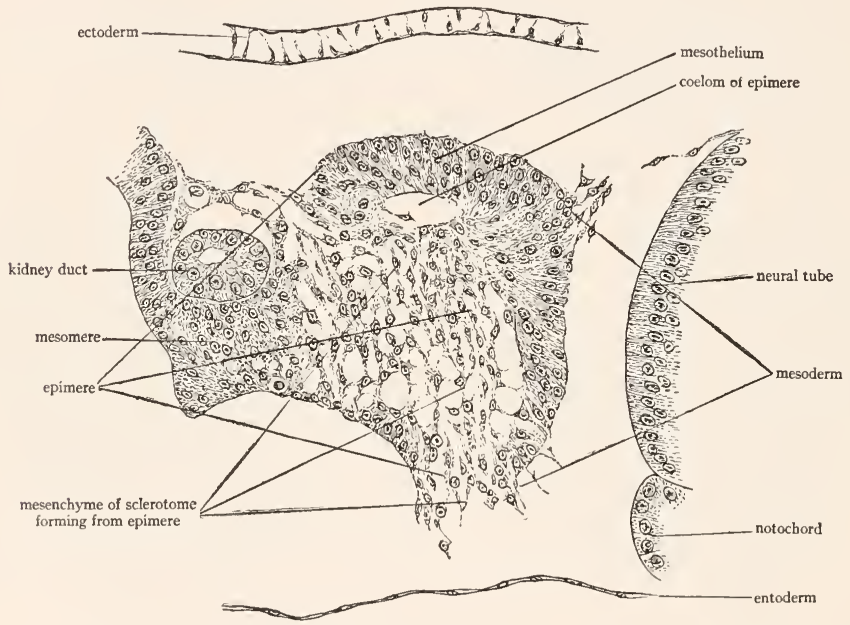

FIG. 9.-Enlarged view of the epimere of a chick embryo of two days' incubation to show the transformation of a portion of the epimere into mesenchyme. These mesenchyme cells constitute the sclerotome from which the vertebral column arises.

type of tissue. It is a primitive kind of connective tissue, consisting of branched cells, whose branches are more or less united to form a network (see Fig. 9). Nearly all of the mesenchyme comes from mesoderm; however, it may arise from the other germ layers also. Hence tissues and structures which arise from mesenchyme may owe their origin to more than one germ layer. Consequently, to avoid inaccuracy it is usually merely stated that they arise from mesenchyme, without specifying the particular germ layer or layers involved. When a germ layer is about to produce mesenchyme its cells become loose, separating from their fellows, lose their epithelial form, and taking on a branched 
irregular shape wander away by amoeboid movements to more or less definite regions where they give rise to certain tissues (see Fig. 9). Those parts of the mesoderm which do not become mesenchyme but retain their epithelial characteristics are called mesothelium.

2. The fate of the epimeres.-The medial wall of each epimere transforms into a mass of mesenchyme cells which migrate to a position around the notochord and there give rise to the vertebral column. This mass of mesenchyme is known as the sclerotome (see Fig. Io). The outer wall of each epimere transforms into mesenchyme cells which migrate to the under side of the ectoderm and there give rise to the inner layer (dermis) of the skin. This part of the epimere is called the dermatome (Fig. Io). The remainder of the epimere persists

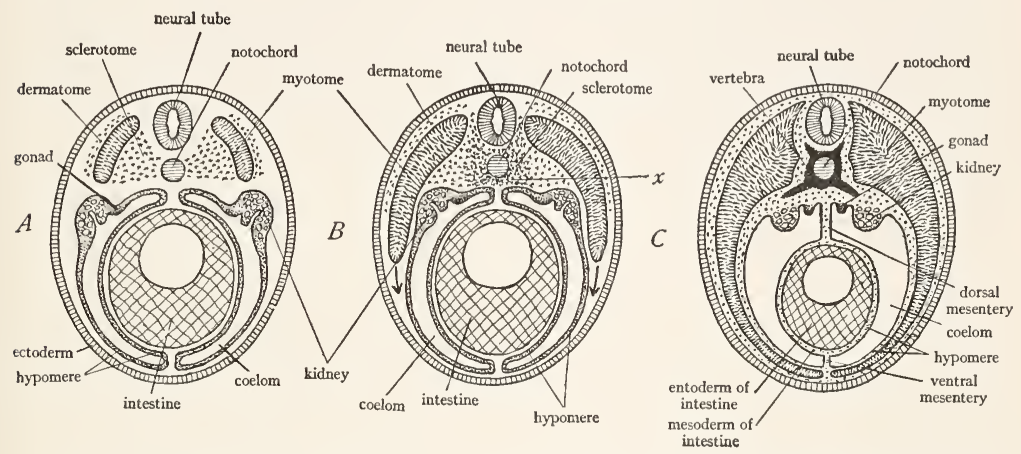

FIG. I0.-Diagram of cross-sections of vertebrate embryos to show the differentiation of the epimere into dermatome (skin-producer), myotome (muscle-producer), and sclerotome (skeleton-producer). Dermatome and sclerotome consist of mesenchyme, myotome of mesothelium. In $B$ and $C$ the dermatome is seen spreading beneath the ectoderm to form the dermis of the skin; the myotomes are growing ventrally to form the muscle layer of the body wall; the sclerotome is accumulating around the notochord at $x$ to form the vertebrae; and the hypomere incloses the intestine producing the dorsal and ventral mesenteries and the mesoderm of the intestine.

in place as mesothelium and is known as a myotome or muscle segment. Each myotome becomes separated from the adjacent ones by a connective tissue partition, the myocomma or myoseptum. The myotomes give rise to the voluntary muscles of the body (with certain exceptions). Each grows from its original dorsal position ventrally between the ectoderm and the hypomere to the median ventral line where it meets its fellow from the opposite side. There is thus produced a complete muscular coat for the body (see Fig. Io; also K, pp. I5, I 7 ; W, p. 64).

3. The fate of the mesomere.-The mesomere gives rise to the kidneys, the reproductive organs, and their ducts (the terminal portions of the urogenital ducts may have ectodermal or entodermal linings). 
4. The fate of the hypomere.-The cavity of the hypomere is the coelom of the adult. The splanchnic walls of the hypomeres of the two sides fold around the archenteron and give rise to mesenchyme, from which are pro duced the smooth muscle and connective-tissue coats of the digestive tract, and also the smooth muscle, connective tissue, cartilage, etc., as the case may be, of all the derivatives of the digestive tract mentioned above. The hypomere also gives rise to the linings of all the coelomic cavities, the serosa of the viscera, and all of the mesenteries. The splanchnic mesoderm of the hypomere produces the heart. In the region of the gill slits the hypomere produces voluntary muscles.

5. The products of the mesenchyme.-The mesenchyme gives rise to all of the connective tissues of the body, including cartilage and bone; to all of the involuntary or smooth muscles; to the blood cells, the blood vessels, the lymph vessels, and lymph glands; and to the voluntary muscles of the appendages. It has already been stated that the vast majority of the mesenchyme is of mesodermal origin, but a small part arises from the other germ layers. 


\section{THE COMPARATIVE ANATOMY OF THE INTEGUMENT AND THE EXOSKELETON}

\section{A. GENERAL CONSIDERATIONS ON THE SKELETON}

The term skeleton includes all of the hardened portions of the bodies of animals. The skeleton of the invertebrates is commonly external, forming a hard covering inclosing the body, while that of the vertebrates is both external and internal. In invertebrates, further, the skeleton is a lifeless secretion, containing no cells, while the vertebrate skeleton is almost invariably cellular, either being composed entirely of hardened cells, or consisting of cells and intercellular products. There are two distinct kinds of skeleton in vertebrates, different in origin and function: (I) The external skeleton, or exoskeleton, derived from the skin, and forming a covering and protective layer on the outside of the body. (2) The internal skeleton, or endoskeleton, derived chiefly from the inner wall of the epimere, and constituting a support and framework for the body and a place of at tachment of the voluntary muscles.

We shall study the exoskeleton first. However, certain parts of the exoskeleton have become so closely related to the endoskeleton that they will be considered with the latter.

\section{B. THE STRUCTURE OF THE SKIN}

Since the exoskeleton is derived from the skin, a thorough understanding of the structure of the skin is prerequisite to a study of the exoskeleton. The skin or integument occurs only in vertebrates and may be defined as those outer layers of the body wall which are easily separated from the inner layers. Study of the microscopic appearance and of the development of the skin reveals that it consists of two distinct parts: an outer layer, the epidermis, composed of epithelial cells, and an inner layer, the dermis or corium, composed of connective tissue (Fig. II). The epidermis originates directly from the surface ectoderm of the embryo, which by proliferation produces several strata of cells to form the epidermis. The dermis is formed by the mesenchyme of the dermatome. In the preceding section we learned that the dermatome originates from the outer part of the epimere (Fig. Io, p. 43).

I. Microscopic structure of the frog's skin.--Examine under moderate powers of the microscope a cross-section through the frog's skin and identify carefully the following parts:

a) The epidermis: The outer part of the skin consists of several strata of epithelial cells. This is the epidermis. The outermost of the cell strata consist of thin flat cells, which have become converted into a horny material. This cornified part of the epidermis is designated the stratum corneum. Beneath the stratum corneum the cells gradually change from a flattened to a rounded and finally to a columnar shape. These lavers of rounded to columnar cells constitute the stratum germinativum (also called stratum :nucosum and stratum Malpighii). In. 
the frog's skin there is no sharp demarcation between the stratum corneum and the stratum germinativum. The lowermost layer of the stratum germinativum, consisting of tall columnar cells, is the active portion of the epidermis and is continuously proliferating cells which are pushed outward, become flat and horny, and finally form part of the stratum corneum. ${ }^{\mathbf{x}}$ The stratum corneum is shed at frequent intervals and is continuously renewed from below.

b) The dermis or corium: The dermis is the inner part of the skin. It consists of connective tissue, which in the case of the frog is arranged partly in the form of a loose network located just beneath the epidermis, and partly in the form

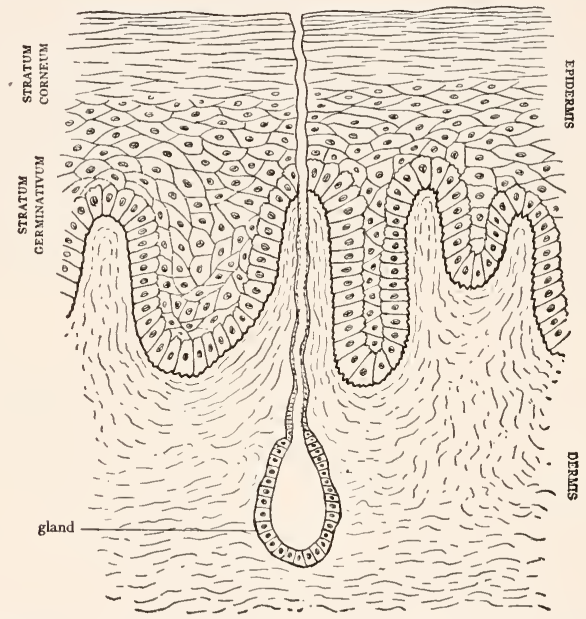

FIG. Ir.-Diagrammatic cross-section through the vertebrate skin, based on mammals.

of layers of dense, parallel, wavy fibers. In addition to the connective tissue fibers the dermis contains: pigment cells, dark, irregular, branching cells forming a thin layer just beneath the epidermis; the cutaneous glands, flask-shaped bodies, produced by an infolding of the stratum germinativum, and opening by a neck to the surface; and at intervals columns containing smooth muscle cells, blood vessels, and nerves, these columns crossing the dermis at right angles to the surface. Of these structures the cutaneous glands are the most conspicuous. They are really parts of the stratum germinativum, which have been evaginated in to the loose portion of the dermis.

Draw a small portion of the skin to show the parts named above.

${ }^{x}$ In some texts only the proliferating layer, one cell thick, is called the stratum germinativum, that portion of the epidermis between this and the stratum corneum being then designated the stratum Malpighii. We shall here regard the terms stratum germinativum and stratum Malpighii as synonymous. 


\section{THE EXOSKELETON IN GENERAL}

The exoskeleton is derived from the skin. It is produced by hardening processes in the epidermis or the dermis or in both. Exoskeleton derived from the epidermis is spoken of as epidermal; it is produced by the activity of the stratum germinativum and consists of many flat horny cells pressed firmly together to make a hard structure. It will be seen that epidermal exoskeleton structures are only special portions of the stratum corneum. Exoskeleton derived from the dermis is spoken of as dermal; it nearly always consists of bone, produced by the mesenchyme cells originating from the dermatome. From the embryological point of view epidermal exoskeleton is of ectodermal origin, while dermal exoskeleton is mesodermal. Epidermal and dermal exoskeleton are different both morphologically and embryologically. From our definition of homology it follows that exoskeletal structures of epidermal origin are homologous in different animals, and dermal structures are likewise homologous. It will be our purpose to trace the homology of the exoskeleton in the different vertebrate classes.

The student should also read the chapters on the exoskeleton in the standard texts of comparative anatomy as $\mathrm{K}, \mathrm{W}$, and $\mathrm{Wd}$.

\section{EXOSKELETON OF FISHES}

Most fishes are covered with scales, which are of four kinds.

I. The placoid scale.-This type of scale occurs in the elasmobranch fishes. It consists of a basal plate carrying a projecting spine. Good examples are obtainable from the skate. Cut out from the skate a small piece of skin containing one spine. Clean away the skin so as to expose the complete scale. It consists of a toothed basal plate, from which arises a shiny curved spine. The base of the spine is hollow, the cavity being known as the pulp cavity. This cavity can be located by probing with a needle point in the center of the under side of the basal plate. The shiny coating of the spine is composed of enamel; the basal plate and interior of the spine consist of dentine, a substance similar to bone. Draw the scale.

To understand the structure of the placoid scale it is necessary to consider its mode of development. Each scale originates from a region of cell multiplication in the dermis. The cells thus formed heap up into a cone, the dermal papilla, which pushes up against the under side of the stratum germinativum. The outer cells of the dermal papilla begin to secrete dentine, and produce the basal plate and the inside of the spine. The stratum germinativum in contact with the dermal papilla secretes on its under surface the substance enamel, ${ }^{\mathrm{I}}$ which consequently forms a coat over the dentine of the spine. The spine when formed breaks through the surface. The interior cells of the dermal papilla do not undergo any change but remain as a soft pulp occupying the pulp cavity in the interior of the spine. From this account it follows that the placoid scale is composed of both epidermal and dermal constituents. The development of the placoid scale is illustrated in Figure $\mathbf{2}$; also in W, page 82; CNH, Vol. VII, page I85; Wd, page 40; R, page 4 .

Draw an imaginary longitudinal section through a placoid scale, coloring ectodermal parts blue and mesodermal parts red.

'That this substance is really enamel, such as occurs on teeth, has been questioned, some maintaining that it is a kind of dentine (see L, p. I 20). We s'sall, however, here take the usual view of its origin. 
The dogfish is covered with placoid scales, which are much smaller and more closely set together than in the case of the skate. The spines of the scales can be felt by passing the hand over the dogfish skin. By boiling a piece of the skin with alkali, the scales may be separated out. Examine such isolated scales under the microscope. They are similar to those of the skate, with rhomboid basal plates and a projecting spine.

2. The homology of teeth and placoid scales.-The most interesting point about the placoid scale is that its structure and mode of origin are exactly the

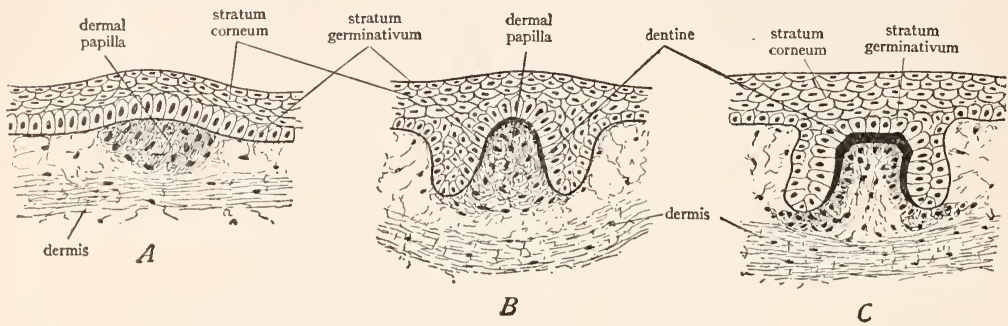

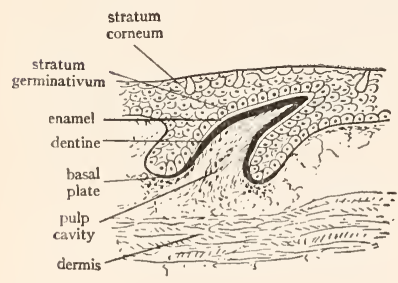

$D$

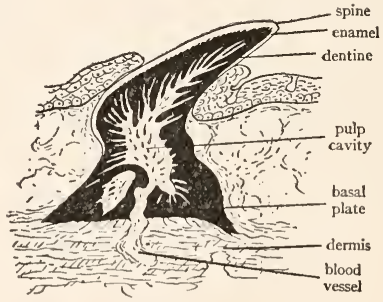

E

FIG. I 2.-Five successive stages in the development of the placoid scale of the dogfish. $A$, gathering of the cells of the dermis to form the dermal papilla. $B$, evagination of the dermal papilla and secretion of the dentine (colored black) by the outer cells of the papilla. $C$, continued secretion of dentine, thinning of the interior of the papilla to form the pulp. $D$, beginning secretion of the basal plate and formation of the enamel (left white) by the under surface of the stratum germinativum. E, eruption of the spine through the epidermis, completion of the basal plate and pulp. (From Goodrich in Part IX of Lankester's Treatise on Zoölogy, courtesy of the Macmillan Company.)

same as those of the teeth of all vertebrates. From this it follows that teeth and placoid scales are homologous structures, and teeth are merely siightly modified placoid scales. The homology is probably due to the fact that the lining of the mouth cavity is really skin turned in, and hence may be expected to give rise to structures similar to those found in the skin.

Obtain a longitudinal half of an ordinary vertebrate tooth. Identify the following parts: the crown or shiny upper part, corresponding to the spine of the 
placoid scale; the root, or dull lower part, which sets into the jaw and corresponds more or less to the basal plate of the scale; the pulp cavity, the central space, filled in life with a dermal papilla, consisting of connective tissue, blood vessels, nerves, etc.; the dentine, the bony material composing most of the tooth; the enamel, the thin shiny outer coating of the dentine of the crown. Draw the specimen, coloring ectodermal part blue and mesodermal part red.

3. The ganoid scale.-This type of scale is characteristic of many of the ganoid fishes, such as the gar pike and the sturgeon. Examine a specimen of the gar pike (Lepidosteus) and note its complete investment with hard, shiny, rhomboid plates, arranged in diagonal rows, fitting closely together. They are typical ganoid scales. Cut out a small piece of skin containing several scales. Note that each diagonal row is movable on the adjacent rows along the line of junction or hinge line, but the members of each row are immovably joined to each other by a peg-and-socket arrangement (visible only on the under side and well developed only in large specimens). Draw a few scales.

In the sturgeon note similar ganoid scales, bony rhombic plates bearing a short spine. They are arranged in five longitudinal rows, with areas of apparently naked skin between the rows. In some sturgeons (Scaphirhynchus) the ganoid scales form a complete investment for the tail.

Ganoid scales are composed of bone and are often covered with a shiny substance known as ganoin. They never bear any enamel. They are purely of dermal origin formed by the activity of the mesenchyme cells of the dermis and corresponding to the basal plates of the placoid scales. Primitively they clothed the entire body as in the case of the gar pike, but this arrangement obviously hinders movement; hence to facilitate movement they are of ten lost from some regions of the body as in the sturgeon, and in the teleost fishes are replaced by thinner, more flexible scales, overlapping each other like shingles.

4. The cycloid scale.-This is the earliest form of the thin, flexible scale and occurs in a few ganoids and some teleosts. Examine the bowfin (Amia), a ganoid fish, and note the thin, rounded scales with which the animal is clothed. These are cycloid scales. They are set in pockets in the skin, and the free projecting edges overlap like shingles, thus allowing greater freedom of movement. Remove a scale and examine under the microscope, or use mounts already prepared. Note the smooth border and the markings on the scale. Draw.

5. The ctenoid scale.-This type of scale is similar to the preceding, and occurs in the vast majority of the bony fishes. Note the arrangement of the ctenoid scales on the perch or other common fish. The scales are set in diagonal rows in pockets of the dermis, their free edges overlapping. Remove one or obtain a prepared slide of one and examine with the low power. The attached end of the scale is beautifully fluted; the free border bears several rows of small toothlike projections, which are well developed in the perch but poorly defined in some fishes; and the surface is sculptured with curved parallel ridges. It 
may generally be noticed that the epidermis forms a thin covering over the free toothed end. Draw.

Both cycloid and ctenoid scales are composed of bone and are homologous with ganoid scales. All three types of scales are produced by heaps of mesenchyme cells of the dermis. The posterior edge of each scale grows outward, carrying the epidermis with it; the latter may remain as a thin covering over the projecting part of the scale or may be rubbed off. The scales of fishes are thus of dermal origin, with the exception of the placoid scale, which contains in addition to its dermal part, a small epidermal contribution.

\section{E. EXOSKELETON OF AMPHIBIA}

The vast majority of present-day Amphibia have naked skins, that is, an exoskeleton is lacking. A few are provided with minute, concealed dermal scales. The extinct Amphibia (Stegocephala) were commonly clothed in a heavy armor of dermal plates homologous with ganoid scales and similar to them in appearance.

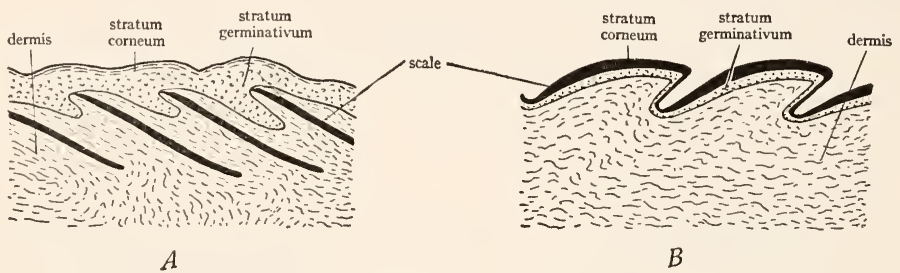

Fig. 13.-Diagrammatic longitudinal sections through the skin of $A$, a teleostome fish and $B$, a reptile, to show the locations of the scales. In $A$, the scales are in the dermis, while in $B$ they represent thickened portions of the stratum corneum. (From Wiedersheim's Comparative Anatomy of Vertebrates, courtesy of the Macmillan Company.)

\section{F. EXOSKELETON OF REPTILES}

The bodies of reptiles are characteristically clothed with an exoskeleton composed of horny scales. These scales are of epidermal origin, representing particularly dense, cornified areas of the stratum corneum. In the formation of such a horny scale a dermal papilla first appears which furnishes nutriment for the cells engaged in producing the scale. Thestratumgerminativum over thedermalpapilla begins to proliferate rapidly, producing cells which become flat and horny and compressed into a scale (see Fig. I $_{3} B$ ). In addition to the horny scales many reptiles possess bony plates of dermal origin situated beneath the epidermal scales. These bony plates originate in the same manner as the scales of fishes. In order to avoid confusion, the epidermal scales will be designated as scutes and the dermal structures as plates.

I. Exoskeleton of the lizard.--Recall the condition of the lizard, or reexamine the specimen. The body is clothed with horny scales of epidermal origin, overlapping like shingles. Note arrangement and size of the scales on 
various parts of the body. The head may bear enlarged scales or head shields. There is no dermal exoskeleton in lizards.

2. Exoskeleton of the turtle.-The exoskeleton of the turtle is somewhat complicated. For the purposes of study, carapaces and plastrons which have been separated by sawing through the bridges will be provided. It is also desirable that these parts shall have been cooked to render the sutures between the plates more distinct. The following description is based on the exoskeleton of our common pond turtles; in other families of turtles the arrangement of the scutes and plates may be slightly different.

a) The carapace: The dorsal surface of the carapace consists of large thin horny scales or scutes, whose boundaries are marked by grooves. These scutes are of epidermal origin, formed from the stratum corneum. The scutes are arranged in five longitudinal rows, one median, and two pairs of lateral rows. The median row consists of five neural scutes; on each side of this are four costal scutes and the margins of the carapace are covered by a number of small marginal scutes. The unpaired narrow marginal scute at the middle of the anterior end of the carapace is termed the muchal scute; besides this there are twelve pairs of marginal scutes of which the median posterior two, behind the fifth neural scute, are often called pygal scutes. Observe that the marginal scutes are continued over the edge of the carapace to cover the margins of the under side. Draw the dorsal surface of the carapace, showing accurately the outlines of the scutes (only half need be filled in).

Turn the carapace over and study its ventral surface. It is composed of heavy bony plates of dermal origin. The vertebral column with its ribs occupies the median line, and both are firmly fused to the carapace. The boundaries of each plate are marked by jagged sutures which should be located in identifying the plates. The plates like the scutes are arranged in five longitudinal rows. The median row of plates, fused to the dorsal sides of the vertebrae, consists of a single large anterior nuchal plate followed by eight smaller vertebral or neural plates, each attached to a vertebra, followed by two postneural or precaudal plates, not attached to vertebrae. On each side of the median row is a row of costal plates, eight pairs of elongated plates, each attached to a rib. The margins are formed of eleven pairs of marginal plates and one single unpaired pygal plate in the median posterior position. Make an accurate drawing of the under side of the carapace, showing bony plates and their relation to the vertebrae and ribs. Do the scutes and plates of the carapace correspond?

A difference of opinion exists regarding the bony plates of the turtle's carapace. According to one view, all of the plates are of dermal origin and the expanded vertebrae and ribs are fused to the under surface of these dermal plates. According to the other view, which is probably the correct one, the vertebral and costal plates are formed entirely by the expansion of the vertebrae and ribs, and are therefore not dermal exoskeleton at all but parts of the endoskeleton. This view also includes the conception that turtles originally possessed a set of real dermal plates 
external to the present vertebral and costal plates, and that these plates subsequently dissappeared. On both points of view the nuchal, marginal, postneural, and pygal plates are dermal and part of the exoskeleton.

b) The plastron: The plastron, like the carapace, consists of a set of horny epidermal scutes covering bony dermal plates. Study the external (ventral) surface of the plastron. It is covered by six pairs of scutes named from in front backward: gular, humeral, pectoral, abdominal, femoral, and anal. Irregulas inframarginal scutes cover the bridges. Study the internal (dorsal) surface, noting the large bony plates united by jagged sutures, which compose it. The small anterior pair of plates are named epiplastra. Between them is a single median plate with a posteriorly projecting point, named the entoplastron or interclavicle. Behind these are three pairs of large squarish plates named the hyoplastra, hypoplastra, and xiphiplastra, the first named being most anterior. Draw the plastron, showing outlines of scutes and plates.

c) Other exoskeletal structures: The exoskeleton also includes the claws and the horny beaks which incase the jaws. Examine these beaks in a demonstration specimen in which they have been loosened from the underlying bones. Turtles also possess scales or thickened scalelike areas on the legs and tail, and in some cases enlarged scutes over the head. All of these structures are of epidermal origin, consisting of special portions of the stratum corneum.

\section{G. EXOSKELETON OF BIRDS}

Birds are clothed in an exoskeleton consisting of feathers on the greater part of the body, scales and claws on the feet, and horny beaks. All of these structures are of epidermal origin, formed from the stratum corneum. There are no dermal elements of the exoskeleton in birds. The feathers of birds are their characteristic features and will be studied in detail. There are three kinds of feathers-down feathers, contour feathers, and hair feathers or filoplumes.

I. Structure of the down feather.-Down feathers or plumulae constitute the fluffy covering of young birds and also occur in adult birds between the bases of the contour feathers. Obtain a down feather or a prepared slide of one. Identify the short stem or quill, the soft rays or barbs which spring in a circle from the top of the quill, and the minute side rays or barbules on the barbs. Draw.

2. Development of the down feather.-A down feather arises from a papilla of the skin, the feather papilla, consisting of a dermal core, the pulp, covered by the epidermis. Later this papilla sinks into a pit in the skin, called the feather follicle. The stratum germinativum of the papilla begins to proliferate, forming a number of longitudinal columns which project into the pulp (see Fig. I4). These columns eventually separate, each being a barb, composed of cornified cells. The original stratum corneum covers the barbs like a sheath, which is called the periderm; it splits open and is shed, releasing the barbs. During this process the whole papilla has been elongating and comes to project above the surface. The lower part of the papilla does not split into barbs but remains undivided as the quill, within which the pulp dries 
up. (See further, K, p. 36; P and H, pp. 369-73; Wd, pp. 25-28.) It should be noted that the development of a feather is similar to that of a reptilian scale, involving a dermal papilla for nutritive purposes and an ectodermal thickening.

3. Structure of a contour feather.-This is the common type of feather which covers the bodies of birds. Obtain one and identify the following parts. The short, bare, hollow portion the lower end of which is inserted into the feather follicle of the skin is the calamus or quill; the remaining expanded portion, constituting most of the feather, is the vane. The quill bears two openings, the inferior umbilicus at its proximal end, which was inserted in the skin, and through which in the early stages of the feather, the dermal papilla passes; and the superior umbilicus at the junction of vane and quill on the ventral surface of the feather. From the superior umbilicus protrudes a more or less well-developed accessory

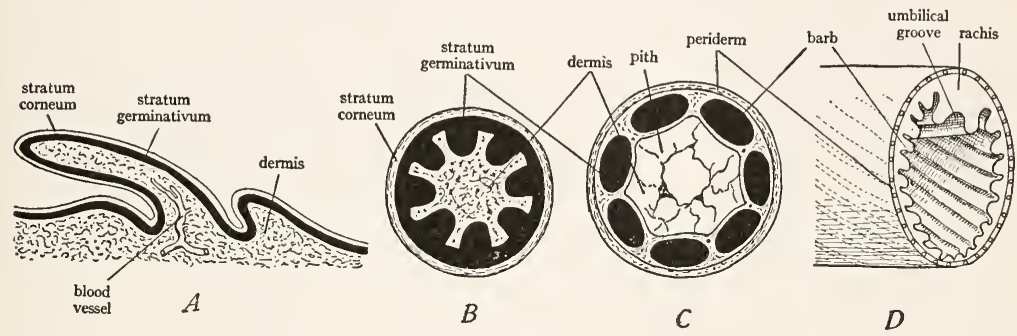

FIG. 14.-Diagrams of the development of the down and contour feathers. $A$, dermal papilla. $B$, cross-section of a later stage of the dermal papilla showing the thickenings of the stratum germinativum. $C$, cross-section of a later stage; each thickening has separated to form a barb; the dermis in the center of the papilla has degenerated into the pith; the stratum corneum forms the periderm or sheath of the down feather. $D$, section across a developing contour feather, showing the two enlarged thickenings or barbs which become the rachis and the oblique course of the other barbs. (A-C from Wiedersheim's Comparative Anatomy of Vertebrates, courtesy of the Macmillan Company; $D$ after Kingsley's Comparative Anatomy of Vertebrates, copyright by P. Blakiston's Son and Company.)

feather called the aftershaft, consisting of only a few tufts in some birds but in others of a complete feather, nearly or quite as large as the primary feather.

The vane consists of a central axis known as the shaft or rachis which is continuous with the quill and of a sort of web or membrane springing from each side of the rachis. Extending along the ventral surface of the rachis is a groove, the umbilical groove. The web or membrane of the feathers is obviously composed of a large number of parallel, obliquely placed rays, adhering to each other. These rays are the barbs, and each barb bears side rays called barbules, exactly as in the down feather. The barbules interlock, causing the barbs to adhere to produce an unbroken surface. To see the method of interlocking of the barbules it is necessary to examine a small piece of the feather under the microscope. It will then be noted that the barbules of one side of each barb (side toward the quill) 
are placed diagonally across the barbules of the other side (side toward the tip of the feather) of the adjoining barb, and that the former are provided with hooklike projections which fit over and catch flangelike extensions of the latter. The diagonal arrangement permits one hooked barbule to catch a number of flanged barbules. By pulling on the barbs one can unhook the barbules, and by stroking the barbs one can hook them up again. The latter process is the chief object of the frequent preening of the feathers habitual with birds. In the rectrices and remiges the hooklets are well developed throughout so that the barbs are interlocked over the entire feather; but in the coverts the lower barbules lack the hooklets, and the lower barbs are loose and fluffy. In some birds, as the ostrich, all of the barbules lack hooks, and the whole feather is fluffy.

Draw a contour feather, showing its parts.

4. Development of the contour feather.-Contour feathers are similar in structure and development to down feathers, except that in the latter the barbs spring directly from the quill in a circle, while in the former they spring in a row from either side of a central axis. This is due to the fact that in the development of the contour feather two of the longitudinal columns formed by the stratum germinativum become much enlarged and fuse to produce the rachis, the groove between them remaining as the umbilical groove. This enlargment of these two columns imparts an oblique position to the other columns, which become the barbs, since the rachis grows faster than the barbs. The contour feather is rolled up inside the periderm, the future dorsal or outer surface being outside next to the periderm, while the future ventral or inner surface is inside next to the dermal papilla. When the periderm splits, the feather flattens out. The quill is the base of the feather which failed to split into barbs; the white flaky material noticeable inside the quill is the dried remains of the dermal papilla (see Fig. I4; also K, p. 37, Fig. 28).

5. Feather tracts.-Birds appear to be completely covered by contour feathers, but actually the feathers are borne only by certain areas of the skin called feather tracts or pterylae, with featherless areas, apterylae, between them. Observe the feather tracts on a demonstration specimen of a young bird and consult also K, Figure 26, page 35 , or P and H, Figure 1037, page 372 .

6. Structure of the filoplume.-The filoplumes are the "hairs" visible on a plucked bird. Examine a prepared slide or remove a filoplume from a plucked bird, mount on a slide in a drop of water, and examine under the low power. It consists of a main axis bearing a few terminal barbs. It is a miniature degenerated contour feather. Draw.

\section{H. EXOSKELETON OF MAMMALS}

The exoskeleton of mammals consists primarily of hair, found in no other vertebrates. Some mammals are provided with scales in addition.

I. Structure of hair.-By examining your own skin determine that each hair springs from a pit in the skin, known as a hair follicle. Remove a fine hair from the under side of the forearm and examine with high powers of the microscope. Observe by focusing on the surface of the hair the irregular wavy 
outlines of the cells of which it is composed. Examine with the microscope a cross-section through the skin containing growing hairs, and study a hair follicle. Each is a deep pit in the skin and is lined by epidermis. At the bottom of the follicle the dermis forms a small bulb-shaped enlargement, the hair papilla. The stratum germinativum over this papilla is seen proliferating a conical heap of cells from which the hair arises. This cone extends up the follicle and after a short distance a split is seen separating a central shaft, the root of the hair, from the walls of the follicle. The transition from the ordinary epithelial cells of the proliferating area to the horny cells of the root of the hair is readily observable. The lining of the follicle is also somewhat cornified, and is known as the outer root sheath. It is the white coat which clings to the roots of hairs when they are pulled from their follicles. From this account it follows that hairs are epidermal structures produced by the activity of the stratum germinativum. Draw a hair follicle, showing its structure. Further details will be found in textbooks of histology.

2. Scales of mammals. - A number of mammals possess horny scales like those of reptiles. These may cover the body, as in the scaly anteater (Manis), figured in $\mathrm{P}$ and $\mathrm{H}$, page 485 , and $\mathrm{N}$, page 375 , but commonly occur on the tails only, as in rats, beavers, and a number of other mammals. Such scaly parts are also provided with scanty hairs. Examine a rat's tail and observe the scales and hairs upon it.

The armadillos are the only living mammals which possess, like the turtle, an armor composed of both epidermal scutes and dermal plates. Obtain a dried armadillo armor and examine the external surface. It is made up of an anterior shield of small polygonal scales, a middle region composed of nine movable bands with bare areas of skin between them, and a posterior shield similar to the anterior shield. The outer surface of the armor consists of thin horny epidermal scales or scutes, polygonal on the shields, triangular on the bands. The triangular scutes are alternately reversed in position, so that in half of them the apex points anteriorly, and in half posteriorly. The former bear hairs at their posterior margins. How many hairs to each scute? Draw some scutes and hairs to show their relation. Turn the armor over and study the internal surface. It is composed of bony plates of dermal origin, polygonal on the shields, rectangular on the bands. With a knife point scrape off some of the epidermal scutes; note relative thickness of scutes and plates, differences in the materials of which they are composed-one of horn, the other of bone - and the impressions left on the plates by the scutes.

Read W, pages $87-97$, on the relation of scales to hair and to the friction ridges.

\section{SUMMARY}

I. The vertebrate skin consists of an outer epidermis, ectodermal in origin and composed of epithelial cells, and an inner dermis, mesodermal in origin and composed of connective tissue.

2. Exoskeletal structures are produced by the skin, either by the epidermis or the dermis or both. 
3. Epidermal exoskeletal structures consist of horn and are composed of numerous flattened dead cornified cells pressed together. These cells arise by proliferation of the lower layers of the epidermis (stratum germinativum). Of such nature are the superficial scales of reptiles, birds, and mammals, feathers, hair, claws, nails, beaks, hoofs, horns such as those of cattle.

4. Dermal exoskeletal structures consist of bone secreted by the mesenchyme cells of the dermis. Of such nature are the scales of fishes-in whole or large part, the bony plates of reptiles and mammals, the horns of deer and antelope, the fin rays of fishes, the plates of recent and extinct $A$ mphibia.

5. Certain exoskeletal structures contain both dermal and epidermal constitutents inextricably fused. Such are the placoid scales of the elasmobranch fishes and the teeth of all vertebrates. By the identity of their structure and mode of development these two structures are shown to be homologous.

6. In the formation of exoskeletal parts a dermal papilla is involved, which furnishes nutrition for the proliferating or secreting cells. In the case of dermal structures the cells of the papilla give rise to the structure. In the case of epidermal exoskeleton the papilla takes no part in the formation of the structure but simply brings a blood supply to the developing part which arises solely by proliferation and subsequent cornification of epidermal cells.

7. From the foregoing account and from the definition of homology it follows that all exoskeittal structures of epidermal origin are homologous, and that similarly all exoskeletal structures of dermal origin are homologous, whatever their shape or form or function.

8. Epidermal structures exhibit more modifications and a greater complexity of structure than do dermal parts.

9. There has been no definite progressive evolution of the exoskeleton throughout the vertebrate groups, but each group is provided with exoskeletal structures correlated with its habits and mode of life. There is, however, a certain tendency for the higher vertebrates to develop complicated epidermal structures. 


\section{THE ENDOSKELETON: THE COMPARATIVE ANATOMY OF THE VERTEBRAL COLUMN AND RIBS}

\section{A. GENERAL CONSIDERATIONS ON THE ENDOSKELETON}

I. The parts of the endoskeleton.-We have already defined the endoskeleton as the internal skeleton of the body. The notochord is the first endoskeleton of the chordates and the principal endoskeleton of the lower chordates. In the vertebrates the notochord is always more or less replaced by a skull and vertebral column, and the vertebrates possess in addition other components of the endoskeleton in association with the gills and paired appendages. The parts of the endoskeleton of vertebrates are: the skull in the head; the visceral skeleton composed of gill arches supporting the gills; the vertebral column occupying the median dorsal region; the ribs, projecting from the vertebrae, one pair to each vertebra primitively; the sternum, occupying the median ventral region of the anterior part of the trunk; the pectoral girdle supporting the anterior paired appendages; the pelvic girdle, supporting the posterior paired appendages; and the skeleton of the appendages. The four parts first named constitute the axial skeleton, while the other parts constitute the appendicular skeleton.

2. The skeletogenous regions.-The endoskeleton develops from mesenchyme. The mesenchyme accumulates in certain regions known as the skeletogenous regions where skeleton is to be formed. The arrangement of these skeletogenous regions is to some extent dependent on the disposition of the myotomes. As we have already learned, the myotomes or muscle segments, which consist of those portions of the epimeres remaining after the formation of mesenchyme, grow down between the skin and the digestive tract, so as to form the muscular layer of the body wall. (Review Fig. Io, p. 43.) Each myotome is separated from the adjacent ones by a transverse partition or plate of mesenchyme, called the myoseptum or myocomma. Each myotome is further divided into a dorsal and a ventral half by a horizontal partition, the horizontal skeletogenous septum, which extends from the notochord to the level of the lateral line on the sides of the body. The notochord and neural tube are also surrounded by mesenchyme which extends from the neural tube to the median dorsal line, forming the dorsal skeletogenous septum, and from the notochord to the median ventral line (in the tail) forming the ventral skeletogenous septum. In the trunk region the ventral skeletogenous septum is naturally split into two septa, ventrolaterally situated, on account of the intervention of the coelom. The horizontal, dorsal, and ventral septa are, it is to be understood, continuous longitudinal septa, extending the length of the body. The skeletogenous septa are illustrated in Figure 15 , also in K, Figure 33, page 4I, and W, Figure 34, page 127. As their name implies, the skeletogenous septa are regions of skeleton formation. At the intersection of every myoseptum with the medially placed mesenchyme of the dorsal and ventral septa and that surrounding the notochord and neural tube a veriebra arises. As the myosepta are segmentally repeated, owing to the primary segmentation of the myotomes, it follows that the vertebrae are also segmentally arranged and that the vertebrae alternate with the myotomes.

3. Cartilage and membrane bones.-The mesenchyme in the process of forming endoskeleton first produces cartilage. All of the endoskeleton proper is first composed of cartilage, and in the lower vertebrates the endoskeleton may remain whoily or partly cartilaginous. The endoskeleton of elasmobranchs, for example, is composed entirely of cartilage. Such cartilage may be and often is stiffened by the deposition within it of calcium salts. In such cases the cartilage is said to be calcified. In most vertebrates, however, the cartilage is more 
or less replaced during development by bone, secreted by bone-forming cells. The skeleton is then said to be ossified. Bone produced in this manner by replacement of pre-existing cartilage is known as cartilage bone.

Investigation of the internal skeleton of vertebrates shows that not all of their bones arise in this manner, but some of them develop directly from the mesenchyme without passing through a cartilage stage. Such bones are called dermal, membrane, or investing bones. They are really derived from the dermis of the skin and are therefore dermal plates homologous to ganoid scales and to the plates of the turtle's armor. They are consequently parts of the exoskeleton. They have sunk inward from their original position in the skin and have attached themselves to the endoskeleton with which they are now so closely associated that they must be considered a part of it.

The student should particularly understand that cartilage and membrane bones look exactly alike; they have the same histological structure and chemical composition; they are both completely formed bone; it is not possible to distinguish them by examining them. It is only their manner of origin that is different. In order to determine which bones of the
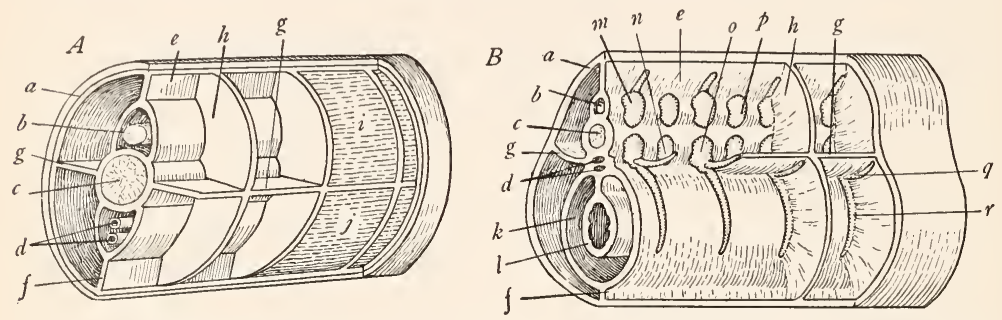

FIG. I 5.-Diagrams to show the skeleton-forming septa in $A$, the tail region, and $B$, the trunk region of a vertebrate. $a$, skin; $b$, neural tube; $c$, notochord; $d$, blood vessel; $e$, dorsal skeletogenous septum; $f$, ventral skeletogenous septum; $g$, horizontal skeletogenous septum; $h$, myoseptum; $i$, epaxial part of the myotome; $j$, hypaxial part of the myotome; $k$, coelom; $l$, intestine; $m-p$, cartilages from which the vertebrae are formed- $m$, basidorsal; $n$, interventral; $o$, basiventral; $p$, interdorsal- $q$, intermuscular rib; $r$, subperitoneal rib. In $B$ note positions of the vertebral cartilages and ribs with respect to the skeletogenous septa. (A after Kingsley's Comparative Anatomy of Vertebrates, copyright by P. Blakiston's Son and Company; B from Goodrich in Part IX of Lankester's Treatise on Zoölogy, courtesy of the Macmillan Company.)

endoskeleton are cartilage bones and which are membrane bones it is necessary to study their embryonic development. This has been done for the majority of the bones of the skeleton. In order to trace the homology of the parts of the skeleton in different vertebrates it is absolutely essential to know which bones are cartilage bones and which are dermal. This information is given in the following pages. For the present we may state that dermal bones occur in connection with the skull, jaws, and pectoral girdle (these parts also contain cartilage bones, of course). All other parts of the endoskeleton are composed wholly of cartilage bone.

\section{B. THE EMBRYONIC ORIGIN OF THE VERTEBRAE AND RIBS}

I. The development of the sclerotome.-The axis of the vertebrate skeleton is the vertebral column or backbone, which is composed of a longitudinal series of bones, each of which is called a vertebra. The vertebrae arise from the sclerotomes. We have already learned that a sclerotome is a mass of mesenchyme originating from the medial wall of each epimere (see 
Fig. Io, p. 43). The sclerotome migrates to the median region of the embryo and surrounds the notochord and neural tube. It then gives rise to cartilage, which is laid down around the notochord. The vertebrae may remain permanently in this cartilaginous state, as in elasmobranchs, being stiffened by calcification, or they later transform into bone, as in the majority of vertebrates. The student should understand clearly that the vertebral column does not come from the notochord but is formed around it. The notochord is inclosed inside the vertebral column, and is readily located in this position in the more primitive fishes; but in most vertebrates it is gradually squeezed down and disappears except for remnants between the vertebrae.

A sclerotome does not, as might be supposed, produce a vertebra as one piece. The sclerotomes are segmentally arranged, there being a sclerotome medial to each myotome. It has already been stated, however, that the vertebrae alternate with the myotomes and arise at the intersection of myosepta with the median skeletogenous region. This result is achieved as follows. Each sclerotome soon becomes divided by a vertical split into an anterior or cranial half, posterior to the plane of the preceding myoseptum, and a posterior or caudal half, anterior to the plane of the succeeding myoseptum. A vertebra arises from the fusion of the
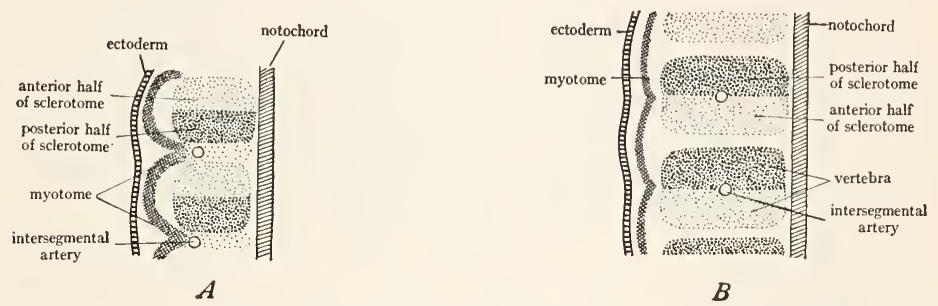

$B$

Fig. 16.-Two stages in the development of the vertebrae from the sclerotomes, only one side of the body being shown. $A$, division of the sclerotome into anterior and posterior halves. $B$, union of the posterior half of one sclerotome with the anterior half of the succeeding sclerotome to form a vertebra. (From Prentiss and Arey's Textbook of Embryology, courtesy of the W. B. Saunders Company.)

posterior half of one sclerotome with the anterior half of the succeeding sclerotome. The anterior half of one side of the vertebra comes from the posterior half of a sclerotome and the posterior half of one side of the vertebra from the anterior half of the sclerotome. (It should be understood that the sclerotomes of the two sides of the body axis co-operate in the formation of each vertebra.) It thus happens that the center of the vertebra intersects a myoseptum and that the vertebrae alternate with the myotomes (see Fig. I6).

2. The arcualia.-In the formation of a vertebra definite paired cartilages known as arcualia arise in the sclerotome. Theoretically there are four pairs of arcualia to each vertebra. The posterior halves of the two sclerotomes of each segment, which form the anterior half of a vertebra, produce two pairs of arcualia - a dorsal pair above the notochord, called the basidorsals, and a ventral pair below the notochord, called the basiventrals. Similarly the anterior halves of the two sclerotomes of each segment, which together form the posterior half of a vertebra, produce a dorsal pair of arcualia, the interdorsals, and a ventral pair, the interventrals (see Fig. I7). There may be other elements in addition above and below these, such as the supradorsals and infraventrals, but these are not of general occurrence. The members of the chief pairs of arcualia tend to fuse together medially producing arches or pieces which straddle the notochord above and below. Thus 

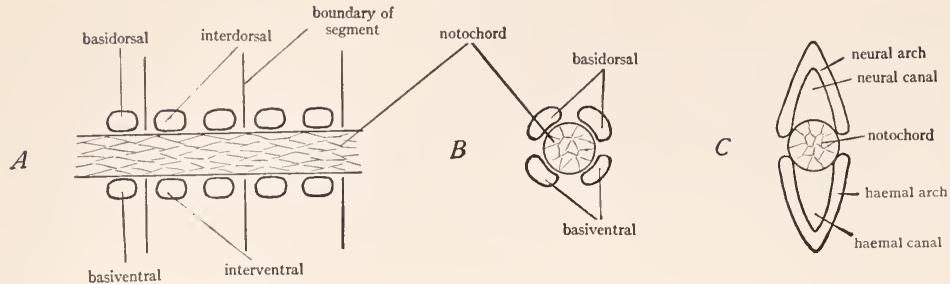

FIG. I7.-Diagrams to show the arcualia from which the vertebrae are formed. $A$, side view, showing the four arcualia of one side to each segment. $B$, cross-section through the basiventrals and basidorsals, showing their relation to the notochord. $C$, later stage of the same cross-section as in $B$, showing union of the basidorsals to form the neural arch and of basiventrals to form the haemal arch.

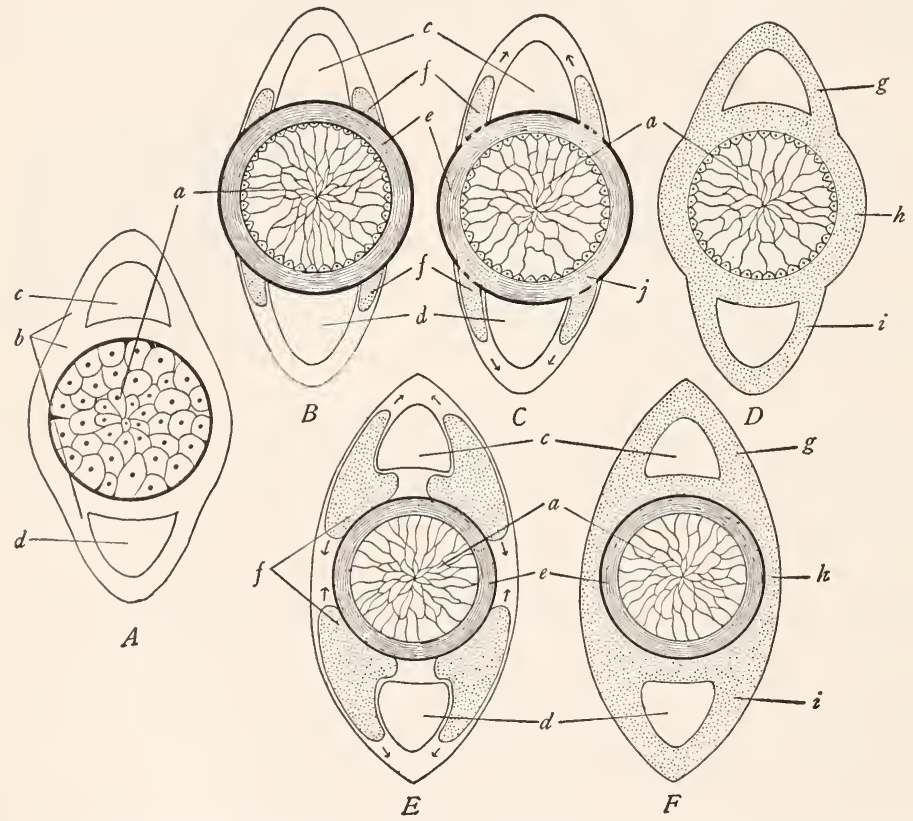

FIG. I8.-Diagrammatic sections through developing vertebrae to show formation of the arches and the centra. $A$, early stage, showing the skeletogenous regions $b$ around the notochord. $B-D$, formation of the chordal type of centrum: $B$, appearance of the arcualia $f$ and the sheath of the notochord $e ; C$, invasion of skeleton-forming cells from the arcualia into the sheath of the notochord, as at $j$; $D$, completion of the chordal centrum in the sheath of the notochord, completion of the arches by fusion of the arcualia, and union of centrum and arches. $E$ and $F$, formation of the perichordal or arch type of centrum: $E$, appearance of the arcualia $f$ and the sheath of the notochord $e ; F$, formation of the centrum and the arches by the fusion of the arcualia around and above and below the notochord. $a$, notochord; $b$, skeletogenous regions; $c$, neural canal; $d$, haemal canal; $e$, sheath of the notochord; $f$, arcualia; $g$, neural arch; $h$, centrum; $i$, haemal arch. (From Wiedersheim's Comparative Anatomy of Vertebrates, courtesy of the Macmillan Company ) 
the basidorsals extend dorsally around the neural tube and fuse to form the neural arch inclosing the neural tube; the interdorsals similarly form an intercalary arch, the basiventrals, a haemal arch, and the interventrals, an interhaemal arch (see Fig. I7). Thus primitively each segment of the vertebrate body is provided with two dorsal arches, one anterior and one posterior, and two similarly placed ventral arches. Such a condition actually occurs in the adults of some of the lower vertebrates. This tendency for the production of two vertebrae to each segment is known as diplospondyly (see $\mathrm{K}$, pp. $5 \mathrm{I}-53$ ). In most vertebrates, however, there is a single vertebra to each segment (alternating with the segment); this condition arises through the loss of some of the arcualia or their fusion. The manner of formation of the vertebrae from the arcualia is different in different vertebrate classes and is described in connection with each group.

3. The formation of the centrum.-The completed vertebra consists not only of the arches arising from the arcualia but also of a central mass, the body or centrum of the vertebra. The centrum is produced in two ways in vertebrates: (a) by developing within the sheath of
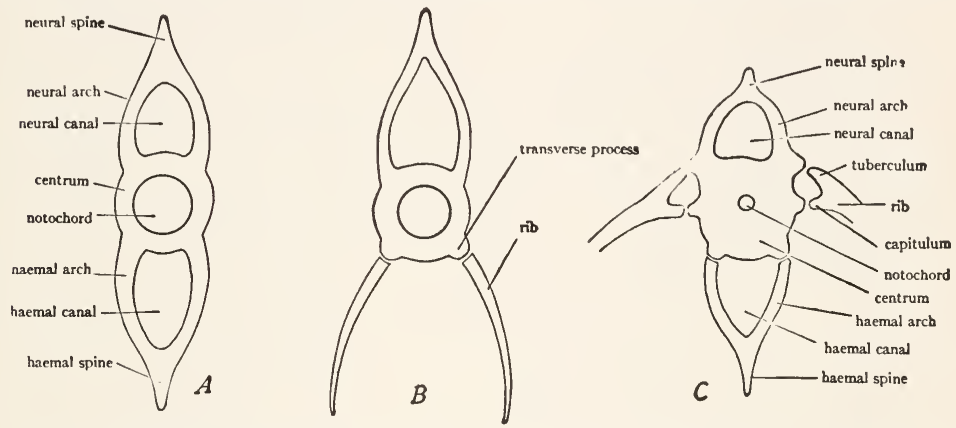

FIG. 19.-Diagrams of typical vertebrae. $A$, tail vertebra of a teleost fish. $B$, trunk vertebra of the same, showing opening of the haemal arch to form the transverse processes. $C$, vertebra of a land vertebrate, showing particularly relation of ribs to the centrum. (After Kingsley's Comparative Anatomy of Verlebrates, copyright by P. Blakiston's Son and Company.)

the notochord and becoming secondarily fused to the arcualia; (b) from the arcualia, either by fusion of the bases of the arches, or from entire arcualia (see Fig. r8). The first type of centrum is called a chordal centrum and is characteristic of elasmobranchs; the second type is named a perichordal or arch centrum and is found in practically all other vertebrates. The centrum in both cases is formed around the notochord which is inclosed within it.

In the embryonic development of a vertebra the dorsal arcualia arise first, the ventral arcualia next, and the centrum last. The same progression is observable in the phylogeny of vertebrates. In primitive vertebrates such as the cyclostomes only the dorsal arcualia are present, straddling a large persistent notochord (see Fig. 2I $A$ ). In many ganoids all of the arcualia appear surrounding the large notochord, but the centrum has not yet developed; such vertebrae are designated as acentrous (Fig. 2r $B$ ). In the higher fishes and all land vertebrates well-developed centra are present, and the notochord is reduced or absent.

4. The parts of a typical vertebra.-A typical completed vertebra consists of a centrum inclosing the notochord, a dorsal neural arch inclosing the neural tube, and a ventral haemal srch inclosing blood vessels. Neural and haemal arches are commonly prolonged dorsally: 
and ventrally, respectively, into neural and haemal spines. In addition the centrum commonly presents various projecting processes, known as apophyses, serving for articulations with adjoining vertebrae or with ribs or for the attachment of muscles. In lower forms there are not uncommonly two centra or arches, one anterior and one posterior, to each metamere, but usually only one of these structures is present to each segment, owing to the loss or fusion of the others. Figure ig illustrates a typical vertebra with its parts.

5. The origin of ribs. - Each vertebra is theoretically provided with a pair of ribs which project out from the centrum into the body wall and serve to strengthen the latter. The ribs, like other parts of the axial skeleton, arise in the skeletogenous septa. There are two kinds of ribs, both of which arise in the myosepta and consequently are segmental in arrangement. One type of rib is formed at the intersection of each myoseptum with the horizontal skeletogenous septum. Since the horizontal septum divides the myotomes into dorsal and ventral halves (see Fig. I $5 A$, p. $5^{8}$ ) such ribs lie between muscles and hence are named intermuscular ribs. They are also known as dorsal or true ribs. Intermuscular ribs are characteristic of

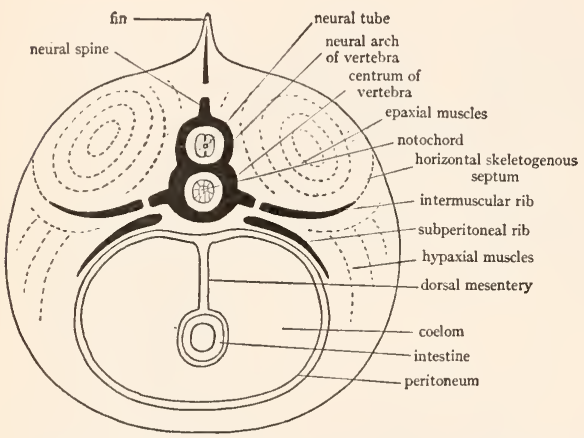

FIG. 20.-Diagrammatic cross-section through the trunk of a vertebrate showing the relation of the ribs to the skeletogenous regions and the positions of the two kinds of ribs. (From Parker and Haswell's Textbook of Zoölogy, courtesy of the Macmillan Company.) the majority of vertebrates. The second type of rib arises at the points of intersection of the myosepta with the ventral skeletogenous septum or its derivatives. It will be recalled that in the trunk region the ventral septum is split into two lateral septa, owing to the intervention of the coelom and its contents on the ventral side of the body (see Fig. ${ }_{5} B$ ). The ribs under consideration appear at the points of intersection of the myosepta with these ventrolateral septa. They lie just outside the coelomic lining, between the coelomic wall and the muscle layer. They are designated as subperitoneal ribs; also as ventral or false ribs. ${ }^{\mathrm{I}}$ Subperitoneal ribs are characteristic of the bony fishes. Both types of ribs may be regarded as extensions of the basiventral arcualia, their position in the myoseptum being shifted dorsally or ventrally in different vertebrates. In many fishes both kinds of ribs are simultaneously present on each vertebra. The two kinds of ribs and their morphological relations to the body wall are illustrated in Figure 20.

Ribs, like vertebrae, appear first as collections of mesenchyme which later transforms into cartilage. The ribs are permanently cartilaginous in some fishes but in other vertebrates are ossified, at least in part.

For the comparative anatomy of the vertebral column and ribs, the student should consult $\mathrm{K}, \mathrm{W}$, and $\mathrm{Wd}$.

I The term pleural as applied to ribs is here avoided, owing to the fact that it is differently employed in different texts. $\mathrm{K}$ and $\mathrm{W}$ use the term pleural as synonymous with intermuscular, while $\mathrm{L}$ uses it as synonymous with subperitoneal. The latter usage appears to be correct. 


\section{SOME PRIMITIVE VERTEBRAL COLUMNS}

It has already been stated that in some living and in many extinct forms the vertebrae are embryonic in structure, consisting of separate arcualia and without a centrum. In other cases diplospondyly is evident. We may glance at a few of these.

I. The vertebral column of the sturgeon.--In the sturgeon the vertebral column is very primitive-acentrous, and consisting of separate arcualia. Examine
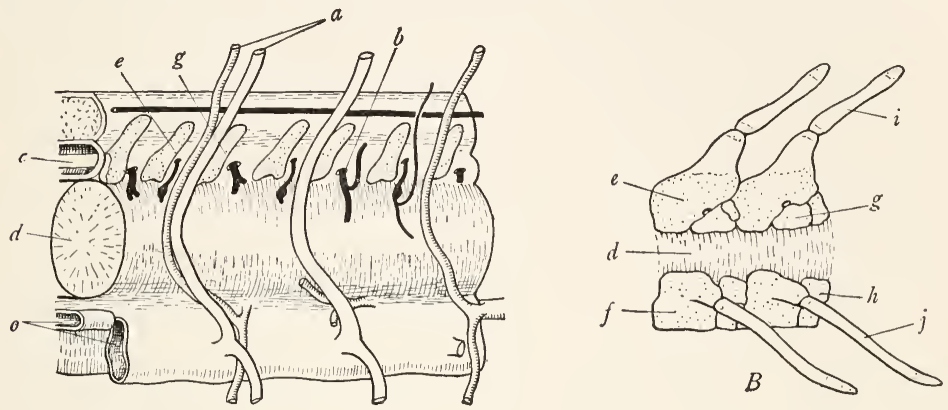

A
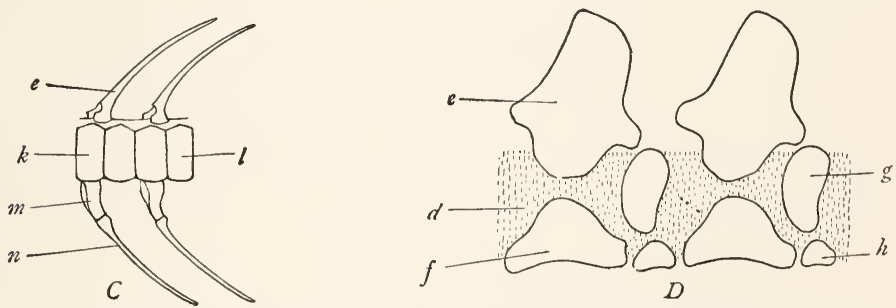

FIG. 21.-Some primitive vertebral columns. $A$, cyclostomes; the vertebral column consists of a row of arches, two arches to each segment, resting on the dorsal surface of the notochord. $B$, sturgeon; the vertebral column consists of separate arcualia partially surrounding the notochord. $C$, tail region of $A$ mia, showing the two centra, $k$ and $l$, to each segment, illustrating diplospondyly. $D$, vertebrae of an extinct amphibian belonging to the Stegocephala, showing the arcualia around the notochord. $a$, segmental blood vessels; $b$, nerve; $c$, neural tube; $d$, notochord; $e$, basidorsal or neural arch; $f$, basiventral; $g$, interdorsal; $h$, interventral; $i$, neural spine or supradorsal; $j$, rib; $k$, posterior centrum; $l$, anterior centrum, for each segment; $m$, haemal arch; $n$, haemal spine; $o$, blood vessels. ( $A$ and $B$ after Goodrich in Part IX of Lankester's Treatise on Zoölogy, courtesy of the Macmillan Company, $C$ from Zittell, $D$ from Kingsley's Comparative Anatomy of Vertebrates, after Zittell, copyright by P. Blakiston's Son and Company.)

the demonstration specimens and compare with Figure 21 $B$. The large notochord traverses the center of the vertebral column and is covered dorsally and 
ventrally by the cartilaginous arcualia. On the dorsal side are the large basidorsals, united to form a neural arch topped by a neural spine (supradorsal) Between the bases of the basidorsals are the small interdorsals. The ventrolateral regions of the notochord are covered on each side by the large basiventrals each of which bears a projecting process. Note that the basiventrals of the two sides do not meet below the notochord. Between the ventral portions of the basiventrals are the small interventrals.

2. The tail vertebrae of the bowfin.-Examine a demonstration specimen of the tail of the bowfin. Compare with Figure ${ }_{2} \mathrm{I} C$. Note that the notochord is not visible, being completely inclosed by the vertebrae. In the latter there is an alternation of a vertebra bearing dorsal and ventral arches, with one devoid of arches. The former consists of the fused basidorsals and basiventrals, forming neural and haemal arches and a central centrum. The latter consists of the fused interdorsals and interventrals. There are thus two vertebrae to each segment of the body, a condition not uncommon in primitive vertebrates.

3. Vertebrae of Stegocephala.-Study Figure $2 \mathrm{I} D$, or $\mathrm{K}$, Figure $48 B$, page 53 . These are representations of the vertebrae of extinct $A m$ phibia. Each vertebrae consists of six pieces of which four are visible from each side. There is a dorsal arch composed of the fused basidorsals, a large ventral piece composed of the fused basiventrals, and two small posterior pieces on each side, the dorsal one being the interdorsal, the ventral one, the interventral. The large notochord is inclosed by these arcualia.

\section{THE VERTEBRAL COLUMN OF THE DOGFISH}

I. Cross-section of the tail.-Obtain a cross-section through the tail of the dogfish and study the cut surface. Be sure that you have a section passing through the junction of successive vertebrae and not through the center of a vertebra. ${ }^{\mathrm{x}}$ The center of the section contains the vertebra, composed of cartilage-a clear, relatively soft material. Outside of the vertebra is the layer of voluntary muscles, consisting of a number of leaves, each separated from its neighbor by a plate of connective tissue, the myoseptum or myocomma. Each leaf is a myotome or muscle segment. The myotomes appear in whorls in the section because they are zigzag in form like those of Amphioxus. Each crosssection will consequently cut a number of myotomes. The muscle segments are somewhat indistinctly divided into dorsal and ventral portions by a partition which extends from the central region of the vertebra to the skin where it meets a line, the lateral line, running along the outer surface of the skin. This connective tissue partition is the horizontal skeletogenous septum. The muscles above it are the dorsal or epaxial muscles, those below it, the ventral or hypaxial muscles.

I When the section passes through the center of the vertebra, four rays forming a sort of cross will be seen in the vertebra. These are areas of calcification. 
Turning now to the vertebra itself, we note that it consists of a central circular concave portion, the centrum or body; dorsal to this an arch, the neural arch, which incloses a cavity, the neural canal, in which the spinal cord, a soft white body, is located; ventral to the centrum, another arch, the haemal arch, which incloses a cavity, the haemal canal, containing the caudal artery and vein. The neural arch terminates in a point, the neural spine, and the haemal arch similarly terminates in the haemal spine. Observe the connective tissue partitions which extend from the neural spine to the median dorsal line and from the haemal spine to the median ventral line. These are the dorsal and ventral skeletogenous septa and they, together with the horizontal skeletogenous septum already mentioned, mark the chief sites of skeleton formation.

Draw the section, labeling all parts.

2. Sagittal section of the tail.-Obtain or make a median sagittal section through a piece of the tail of a dogfish. The vertebrae form a row in the center of the section. Identify the centra in the section. Each consists of two somewhat triangular pieces, apparently separate, the rounded apexes of the triangles directed toward each other, the whole shaped somewhat like an hourglass. The two ends of the centra are concave, so that diamond-shaped spaces are present between successive centra. These spaces are filled with a soft, gelatinous material, the notochord, which also fills the canal which runs through the center of the centrum, between the apexes of the triangular halves of the centra. Centra of this shape, concave at each end, are known as amphicoelous centra. Above each centrum identify the neural arch, arching over the neural canal. Between successive neural arches, and lying therefore dorsal to the diamond-shaped spaces between the centra, observe an extra arch, inverted, however, with apex inclosing the neural canal. This is the intercalary arch. Below the centrum is the haemal canal, its sides formed by the haemal arches, rectangular in section. Draw the section.

The centrum of the dogfish vertebrae (and of the vertebrae of all the elasmobranch fishes) arises within the sheath of the notochord. The cells which produce the centra migrate into the sheath from the arcualia. Centra of this type are chordal centra, and vertebrae having such centra are known as chordocentrous vertebrae. The neural arch is the fused basidorsals; the intercalary arch, the fused interdorsals; the haemal arch is the fused basiventrals; interventrals are lacking (Fig. 22A). The vertebrae of elasmobranchs are permanently cartilaginous with the notochord persistent in the center of the centra.

3. Cross-section of the trunk region.-In a cross-section of the anterior part of the trunk region of the dogfish identify the following parts. The muscle segments are arranged as in the tail region, their division into dorsal and ventral masses being well marked by the horizontal skeletogenous septum. The dorsal or epaxial muscles above the septum are thick masses, but the ventral or hypaxial muscles below the septum form a thin layer inclosing a large cavity, the body cavity or coelom, lined by a smooth membrane, the pleuroperitoneum. The 
coelom incloses the viscera, some of which will be observed to be suspended by a delicate membrane, the dorsal mesentery, from the median dorsal line of the coelomic wall. Study the vertebra, which appears in the middle of the myotomes. It consists of centrum and neural arch, similar in appearance to those of tail vertebrae; but the haemal arch appears to be absent. It is represented by a pair of small cartilages at the sides of the ventral part of the centrum. These cartilages, known as transverse processes, represent the stumps of the haemal arch,

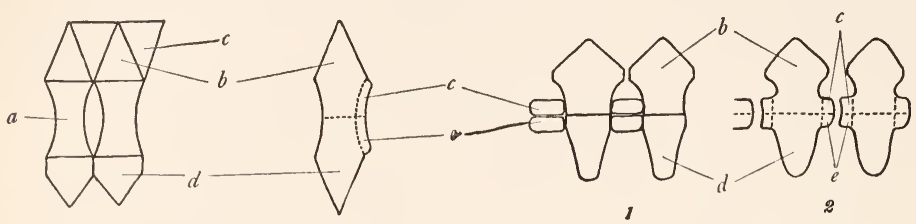

$A$

$B$

$C$
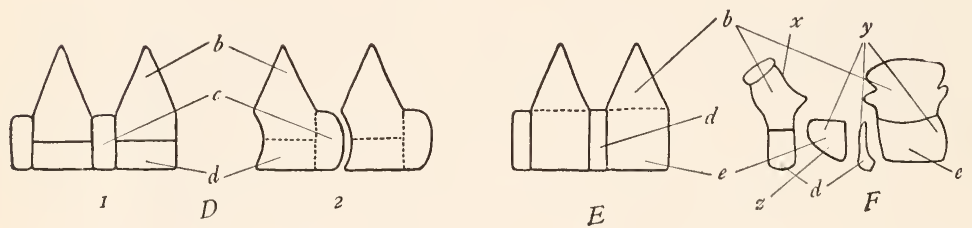

FIG. 22.-Diagrams to show the composition of the vertebrae in the different vertebrate classes, according to Gadow. $A$, elasmobranch, vertebra consisting of chordal centrum $a$, basidorsals $b$, interdorsals $c$, and basiventrals $d$. $B$, teleost, vertebra composed of all of the arcualia fused together. $C$, pseudocentrous vertebra of a urodele; $I$, early stage showing the arrangement of the arcualia; 2 , adult stage, showing fusion of the arcualia along the dotted lines; note splitting of the interdorsals and interventrals to form the ends of the vertebra. $D$, notocentrous vertebra of anurans; $r$, early stage, showing the arcualia which form the vertebra; 2, adult stage, arcualia fused along the dotted lines; note end of the procoelous centrum formed by the interdorsal. $E$, gastrocentrous vertebra of reptiles, birds, and mammals, centrum formed of the interventral $e$, the basiventral $d$ functioning as the intervertebral cartilage. $F$, composition of the atlas and axis of the alligator, according to Gadow; the centrum of the atlas $z$ functions as the odontoid process of the axis. $a$, chordal centrum; $b$, basidorsal or neural arch; $c$, interdorsal (intercalary arch in $A$ ); $d$, basiventral or haemal arch; $e$, interventral (centrum in $E$ and $F) ; x$, atlas; $y$, axis; $z$, odontoid process (interventral of atlas). ( $F$ after Gadow in the Cambridge Natural History, courtesy of the Macmillan Company.)

which may be regarded as having opened out and shifted to a more lateral position, the arch portion disappearing. Examine the horizontal skeletogenous septum carefully and find within it, by picking away the muscles if necessary, a slender cartilage on each side, which articulates with the transverse process. These cartilages are ribs, and since they are located in the horizontal skeletogenous septum they are intermuscular ribs. There is, of course, a pair of such ribs to each vertebra. Draw the section. 


\section{E. VERTEBRAL COLUMN OF TELEOSTS}

I. The tail vertebrae.-Examine a separate dried tail vertebra of any bony fish. Note that the vertebra is very much harder and more opaque than the dogfish vertebrae, owing to the fact that it is composed of bone. Identify the same parts as already seen in the dogfish vertebrae: the biconcave or amphicoelous centrum bearing a minute canal in its center for the notochord, the neural arch terminating in a very long sharp neural spine, the haemal arch terminating in a similar haemal spine. The neural canal or space inclosed within the neural arch is generally smaller than the haemal canal inclosed by the haemal arch; in this way the dorsal and ventral sides of the vertebrae may be distinguished. The spines are directed posteriorly. In some fish there are two neural spines to each vertebra, an anterior and a posterior one; the second one probably corresponds to the intercalary arch of the dogfish. Draw a vertebra.

2. The trunk vertebrae.-Obtain a separate dried trunk vertebra of a bony fish. Identify as before the centrum and the neural arch and neural spine. The haemal arch and spine appear to be missing. Instead there is a pair of projections at the sides of the base of the centrum to each of which a long slender rib is articulated. These stumps or transverse processes are the opened bases of the haemal arch. Draw a vertebra with ribs.

The centra of teleost vertebrae are arch centra formed by the fusion of the bases of the basidorsals and basiventrals chiefly, although interdorsal and interventral elements may be added to the ends of the centra (Fig. $22 B$ ). The neural arch is formed by the basidorsals, the haemal arch by the basiventrals.

3. Section through the trunk of a bony fish.-In such a section identify the parts already described for a similar cross-section of the dogfish. Note the muscle segments, the centrum and neural arch and spine of the vertebra, and the coelom with its lining. Find the ribs located just outside of the coelomic lining. The ribs are in a different position than those of the dogfish, which are in the horizontal skeletogenous septum. The ribs of teleosts are situated just outside of the coelomic walls. They are false or subperitoneal ribs. Make a diagram of the section, showing the position of the ribs.

4. Further study of ribs.-Some fishes have two (or more) pairs of ribs simultaneously on each vertebra. Examples are Polypterus, a crossopterygian ganoid from Africa, and many teleosts, including members of the salmon, herring, and pike families. Examine the skeleton of Polypterus and note two pairs of ribs attached to each vertebra. The dorsal pair attached to the transverse processes of the vertebrae are the true or intermuscular ribs; the ventral pair loosely attached to the ventral surface of the centrum are the false or subperitoneal ribs. Vertebrae of fishes like the salmon may also be examined, or sections through the trunks of such fishes. Note the ventral or subperitoneal ribs, and the dorsal or intermuscular ribs (also called epipleurals) located in the horizontal 
skeletogenous septum. Additional ribs may also be present, articulated with centrum or neural arch and extending out into the myosepta between myotomes. It appears that in teleosts ribs may be formed at almost any level of the myosepta. Make a diagram showing the vertebra and its ribs.

5. The vertebral column as a whole.-Study the entire mounted skeleton of a bony fish. Note that the axis and strongest part of the column consists of the centra, which are jointed together to form a longitudinal series. The vertebral column is divisible into two regions, a trunk region and a tail region. In the former the haemal arches are reduced to stumps, the transverse processes, to the ends of which the long slender ribs are articulated. In the tail region ribs are lacking and haemal arches are present. Observe the transition between trunk and tail regions. In the posterior part of the trunk there is a gradual elongation of the transverse process and a reduction of the ribs. At the beginning of the tail region the reduced ribs finally vanish, and the elongated transverse processes fuse to form the haemal arch. Note that the neural canals of successive vertebrae together form a continuous canal which in life contains the spinal cord. Similarly the haemal canals of the tail region form a continuous cavity inclosing in life blood vessels. At the end of the tail observe the enlargement and flattening of the haemal arches forming what are called hypural bones, which support the tail fin. The last centrum (probably consisting of several centra) forms an elongated slender bone (the urostyle) which turns sharply upward, producing an asymmetry; but the symmetry of the tail is restored by the arrangement of the hypural bones. Tails of this kind are called homocercal.

\section{F. VERTEBRAL COLUMN OF AMPHIBIA}

I. The vertebral column of urodeles.-Whole skeletons of urodeles such as Necturus and Cryptobranchus are needed for this study. With such a skeleton before you note the parts of the vertebrae of different regions. The tail vertebrae are similar to the tail vertebrae of fishes. Each possesses a low neural arch, a haemal arch, and a centrum bearing transverse processes. ${ }^{x}$ In the trunk region the haemal arch is absent, and the transverse processes bear ribs, separated from them by a suture. The vertebrae are articulated to each other by processes known as zygapophyses. These consist of a projection on each side of the neural spine which fits over a similar projection arising from the anterior end of the succeeding vertebra. Thus, each vertebra has a pair of prezygapophyses on its anterior end whose articulating surfaces face upward, and a pair of postzygapophyses on its posterior end whose articular surfaces face downward. These zygapophyses yoke the vertebrae together. ${ }^{2}$

I In fishes the transverse processes were stated to be the stumps of the haemal arches. It seems probable that the transverse processes on the vertebrae of land vertebrates are not of this kind but are lateral outgrowths of the centrum.

2 Zygapophyses are poorly developed or absent in fishes. 
The vertebral column of urodeles consists of four regions, not very sharply marked off from each other. The first region, the cervical or neck region, consists of one vertebra, the first vertebra or atlas, just behind the skull and serving as a support for the skull. The atlas lacks ribs. Following the cervical region is the long trunk region of similar rib-bearing vertebrae. There is next a region consisting of but one vertebra. This is the vertebra to whose ribs the hind legs are attached. It is called the sacral vertebra and its ribs are known as sacral ribs. This region of the vertebral column is the sacral region or sacrum. Posterior to the sacrum is the caudal or tail region, composed of vertebrae which lack ribs and bear haemal arches in most cases. By moving the vertebrae apart note that the ends of the centra are concave, that is, the centra are amphicoelous as in most fishes.

The attached ends of the ribs (Necturus) are forked into two processes or heads, a dorsal tubercular head and a ventral capitular head which articulate with similar but less marked dorsal and ventral projections of the transverse processes. The ribs are intermuscular ribs.

The vertebrae of the urodele Amphibia differ from those of all other land vertebrates in the manner in which the centra are formed. In the tail region the centra are produced by the fusion of the bases of basidorsals and basiventrals, the former giving rise also to the neural arches, the latter to the haemal arches. In the trunk region the centra and neural arches are formed from the basidorsals, the basiventrals having disappeared. Interdorsals and interventrals are absent or represented by the well-developed intervertebral cartilages, forming pads between successive centra. In some urodeles these cartilages split, half fusing to the vertebra in front and half to the vertebra behind. The vertebrae of urodeles are designated as pseudocentrous vertebrae, as the centrum does not correspond to that of the majority of land vertebrates (see Fig. ${ }_{22} C$, p. 66 ).

2. The vertebral column of an anuran amphibian, the frog.-The vertebral column of the frog is specialized in that the caudal vertebrae are all united into one piece, the urostyle or coccyx. There is, as in Necturus, one cervical vertebra, the atlas. This is followed by seven trunk vertebrae. These have low neural arches, no haemal arches, and centra bearing long transverse processes. Ribs are apparently absent, but embryological studies show that the distal part of the transverse process is a rib, which has become indistinguishably fused to the process. There is one sacral vertebra supporting the hind legs and beyond this the long urostyle already mentioned. The vertebrae are yoked together by zygapophyses as in Necturus. In single vertebrae from the frog note that the posterior end of the centrum is rounded like a ball, while the anterior end is concave. Such centra are procoelous and fit together in a ball-and-socket manner.

The centra of the vertebrae of Anura are formed by the union of the basidorsals and basiventrals as in urodeles, with the difference that the interdorsals are fused to either the anterior or posterior end of the centrum. In the frog the interdorsals are fused to the posterior end forming the ball-shaped projection. Interventrals are lacking. Vertebrae of this type are called notocentrous (see Fig. 22D, p. 66). 


\section{G. VERTEBRAL COLUMN OF REPTILES}

The vertebral column of reptiles reaches a degree of differentiation considerably in advance of that of the Amphibia and fishes.

I. The vertebral column of the alligator.-Study the whole mounted skeleton of the alligator. Observe that the column is differentiated into five regions: the cervical or neck region, much longer than in Amphibia; the thoracic region bearing long ribs; the lumbar region, without ribs; the sacral region, composed of two fused vertebrae, supporting the hind limbs; and the caudal region. The tail vertebrae are the most primitive in form and will be studied first. Each consists of a large centrum, a neural arch with a high neural spine, prominent transverse processes directed straight out from the centrum, and a haemal arch, missing on the first caudal vertebra. The haemal arches are relatively small and situated at the posterior end of the centrum. They are often referred to in texts as chevron bones. Toward the end of the tail the various processes projecting from the vertebrae tend to become reduced and finally vanish altogether, so that the last vertebrae consist of centra only. The sacral region consists of two vertebrae, each bearing a stout sacral rib, to which the supports of the hind limbs are articulated. The sacral vertebrae have high neural spines but lack the haemal arches. The lumbar vertebrae, anterior to the sacrum, are five in number, possess high neural spines and broad transverse processes. There are ten thoracic vertebrae, similar in form to the lumbar vertebrae, but bearing long ribs, which reach the median ventral line. There are nine cervical vertebrae, of which the two first, known as the atlas and the axis, are somewhat different from the other seven. These latter have strong neural arches with long neural spines and short transverse processes bearing ribs. Most of these cervical ribs are of peculiar form, being $\mathrm{V}$-shaped and attached to the cervical vertebrae by the two ends of the V. Cervical, thoracic, lumbar, and sacral vertebrae lack haemal arches, but the cervical and anterior thoracic vertebrae possess short ventral projections, the hypapophyses, on the centra. All of the vertebrae are provided with well-developed pre- and postzygapophyses. By moving the vertebrae apart note that the centra are procoelous, i.e., concave in front, convex behind.

The first two cervical vertebrae, the atlas and the axis, are very remarkable and deserve further attention. They are remarkable in that the parts which compose the primitive vertebra are retained in these two vertebrae almost unchanged (Fig. $22 F$ ). The atlas or first cervical vertebra is composed of four separate pieces, which together form a ring. The ventral side of the ring is composed of a median piece, the fused basiventrals. It bears a pair of long movable ribs. The sides of the ring are curved bones, the basidorsals. The ring is completed above by the neural spine. The atlas apparently has no centrum but the centrum is really present and has become attached to the anterior end of the second cervical vertebra or axis. This centrum is composed of the 
fused interventrals. It projects forward into the ring formed by the atlas as a pointed projection, the odontoid process. The atlas therefore possesses all of the parts of a primitive vertebra except the interdorsal. The axis (also called the epistropheus) has a large centrum, composed of the fused interventrals and bears at its anterior end the odontoid process, which, as already explained, is really the centrum of the atlas. The place of union of the odontoid process with the centrum of the axis represents the reduced basiventral of the axis. The axis has a strong neural arch (basidorsals) and broad neural spine. It bears a pair of ribs, which, curiously enough, have moved forward so that they are attached to the odontoid process. Hence it might be said that the atlas has two pairs of ribs and the axis none.

The vertebrae of the alligator are composed in general of the following arcualia. The neural arch consists of the fused basidorsals; the centrum of the fused interventrals; basiventrals are present only in the atlas, the axis, and the caudal vertebrae, where they form the chevron bones; but they are probably represented throughout by the intervertebral cartilages (absent, of course, on dried skeletons). The basiventrals are present as 'distinct bones, the intercentra, wedged in between the vertebrae in the primitive reptile Sphenodon, which is sometimes called a "living fossil" on account of its primitive characteristics, in some lizards and in many extinct reptiles. Interdorsals are absent. Vertebrae like those of the alligator in which the centrum is formed of the interventrals are named gastrocentrous vertebrae. Gastrocentrous vertebrae are characteristic of reptiles, birds, and mammals (Fig. 22E, p. 66).

2. Study of the ribs.-The ribs may be studied on the cervical or first thoracic vertebrae. Observe that each rib is attached to the vertebra by two processes, which are called heads. The upper head, or tuberculum, is attached to a short blunt transverse process arising from the neural spine. The lower head, or capitulum, is attached to the side of the anterior end of the centrum. A large opening is naturally inclosed by the two heads of the ribs. The successive openings form a canal called the vertebrarterial canal in which blood vessels to the head are located. The ribs of the third to seventh cervical vertebrae are short and blunted, those of the eight and ninth cervicals considerably longer, and those of the first eight thoracic vertebrae very long and curved, reaching to the ventral side. Observe in the series of the thoracic vertebrae that the capitular head of the rib gradually moves dorsally until it finally comes to be attached to a little projection on the transverse process. The little smooth places on the vertebrae to which the heads of the ribs are articulated are known as facets, and rib-bearing vertebrae can always be recognized by such facets.

The thoracic ribs consist of three parts. The upper third which has the heads is composed of bone and is known as the vertebral rib. The middle third is partly cartilaginous and is called the intermediate rib. The lower third is also partly cartilaginous and is the sternal rib or costal cartilage. The last two thoracic ribs consist of vertebral ribs only. 
On the ventral side of the lumbar region will be noted a series of riblike bones not attached to any other part of the skeleton. These are the so-called abdominal ribs. They are membrane bones and therefore belong to the exoskeleton, being in fact homologous with the dermal plates of the turtle's plastron. They are likely to occur in long-bellied animals.

Draw a cervical or anterior thoracic vertebra of the alligator with all of its parts, showing particularly the relation of the ribs.

3. The vertebral column of the turtle.-In our study of the carapace of the turtle we already noted certain peculiarities of the vertebral column of these animals. These peculiarities arise from the circumstance that part of the vertebral column is fused to the exoskeleton. The vertebral column of the turtle consists of cervical, trunk, sacral, and caudal regions. As usual, the caudal region is the most primitive. The caudal vertebrae have neural and haemal arches and transverse processes, of which the most anterior are ribs, as shown by the suture at their bases. The first caudal vertebra is fused to the sacrum, which consists of two sacral vertebrae bearing sacral ribs. The first caudal, the two sacral, and the ten trunk vertebrae are all fused to the carapace. As already explained in connection with the exoskeleton, the neural arches of these vertebrae are broadened to form the vertebral plates of the carapace, and the ribs of the trunk vertebrae are similarly expanded to form the costal plates of the carapace. The first and second pairs of ribs are fused distally. The ribs of the turtle possess a single head, the capitular head, which articulates at or near the boundary between two successive centra. There are eight cervical vertebra which are devoid of ribs and which are very flexibly articulated by ball-and-socket joints. The first two are differentiated as in the alligator into atlas and axis. The odontoid process, which is the centrum of the atlas, is the large mass attached to the anterior end of the axis.

\section{H. VERTEBRAL COLUMN OF BIRDS}

The vertebral column of birds, like their entire structure, is highly specialized in relation to the flight habit. With a prepared skeleton of the birdchicken or pigeon-before you, note the following points. The vertebral column is divided into cervical, thoracic, lumbar, sacral, and caudal regions, but all of these except the first are fused together in order to strengthen the back. The cervical vertebrae are numerous (sixteen in the chicken, thirteen or fourteen in the pigeon) and have very flexible articulations, birds being the only vertebrates that can turn their heads halfway round. This flexibility is due to the shape of the centra. On isolated vertebrae or by slightly separating some of the cervical vertebrae on the mounted skeleton note that the ends of the centra are saddle shaped; this type of centrum is heterocoelous. The first and second cervical vertebrae are the atlas and the axis; the former is a small ring-shaped bone, the latter bears an odontoid process as usual. Behind the axis are the 
typical cervical vertebrae. They have low neural arches and spines, welldeveloped zygapophyses, and ribs. The ribs are the masses attached to the sides of the vertebrae and bearing ventrally sharp spines directed posteriorly. The ribs, as in reptiles, articulate with the vertebrae by two heads, forming thereby a vertebrarterial canal. The ribs of the last two cervical vertebrae are longer than the others. The thoracic vertebrae are those whose ribs extend completely to the ventral side. They have high neural spines and welldeveloped transverse processes; spines, centra, transverse processes, and zygapophyses are immovably fused to one another. The last thoracic, the lumbar, the sacral, and the first few caudal vertebrae are fused into one continuous piece, the so-called sacrum or synsacrum, which is separated by a suture from the broad hip bones which extend on either side of it. By examining the ventral side of the synsacrum the individual vertebrae of which it is composed can be distinguished by their separate transverse processes. It should be noted that only certain of these vertebrae are the true sacral vertebrae, corresponding to those of reptiles, the vertebrae in front and behind these having been secondarily fused to them to furnish additional support for the hind limbs. Posterior to the synsacrum are a few free caudal vertebrae ending in an enlarged piece, the pygostyle, which represents several fused vertebrae. Haemal arches are absent from all of the vertebrae. The ribs of birds are divisible into an upper vertebral rib, composed of bone, and a lower sternal rib, or costal cartilage, partly cartilaginous. The vertebral ribs bear backwardly directed processes, the uncinate processes, characteristic of birds and serving apparently to lend greater Grmness to the ribs. The ribs of birds are true or intermuscular ribs.

The vertebrae of birds are, like those of reptiles, gastrocentrous, that is, they consist of a centrum composed of the interventrals and a neural arch composed of the basidorsals. As haemal arches are absent on the vertebrae, it is evident that the basiventral elements are lacking (except presumably in atlas and axis).

\section{VERTEBRAL COLUMN OF MAMMALS}

The vertebral column of mammals is markedly differentiated into the usual five regions: cervical, thoracic, lumbar, sacral, and caudal; and the vertebrae of these regions are so distinct from each other as to be readily identifiable when isolated. Study whole mounted skeletons of the cat or rabbit and isolated vertebrae.

I. The cervical vertebrae.-There are, as in almost all other mammals, seven cervical vertebrae. The first two are differentiated as the atlas and the axis. The atlas is very different from the others; it is ring shaped and has wide winglike transverse processes, which are perforated by a pair of openings, the vertebrarterial canals. The low, flat neural arch of the atlas is also perforated by a pair of holes for the passage of the spinal nerves. The anterior end of the atlas bears a pair of large, curved articulating surfaces which support the skull, and 
its posterior end has likewise two surfaces which articulate with the axis. As in the case of the alligator, the ventral side of the atlas ring is the basiventral; the sides and top, the basidorsals; parts of the transverse processes are ribs; and the centrum (interventral) is attached to the axis. Draw the atlas from the front.

The second cervical vertebra, the axis or epistropheus, has a very long and broad neural arch, provided with a neural spine which projects forward over the atlas. Its centrum bears at its anterior end a pointed projection, the odontoid process, which fits into the ring of the atlas, allowing the turning of the head. The odontoid process is in reality the centrum of the atlas. The axis bears laterally the so-called transverse processes, but only the dorsal part of this is the real transverse process, the lower half being a rudimentary rib. This rib, as is usually the case, is united with the vertebra and the transverse process in such a manner as to leave an opening, the vertebrarterial canal. The anterior end of the centrum of the axis has two articulating surfaces for the atlas, its posterior end, one for the next vertebra. Its neural arch has a pair of postzygapophyses. Draw the axis from the side.

The remaining cervical vertebrae are more or less similar. They have welldeveloped neural arches and spines, the latter increasing in height toward the thoracic region. All have pre- and postzygapophyses and transverse processes. The latter are in reality in part composed of a rib, resulting as before in the formation of the vertebrarterial canal. Draw a cervical vertebra from the front. ${ }^{x}$

2. The thoracic vertebrae.-These vertebrae are recognizable through the fact that they bear long ribs, most of which extend to the ventral side. In the isolated vertebrae the smooth costal facets where the ribs were attached always serve to identify them as thoraciç. There are thirteen thoracic vertebrae in the cat, generally twelve in the rabbit, various numbers in other mammals (man has twelve). The majority of the thoracic vertebrae have very tall neural spines, directed caudad, short centra, small pre- and postzygapophyses, and short, stout transverse processes, bearing at their ends a facet for articulation with the upper head of the rib. The centrum bears at its anterior and posterior ends half-facets for the lower head of the rib. The last thoracic vertebrae differ somewhat from the others. They are longer and stouter, with short neural spines, more prominent zygapophyses, and much reduced transverse processes. They bear but one facet for the ribs, a small depression near the anterior end of the centrum. Below the postzygapophysis in these last thoracic vertebrae is a posteriorly directed process, the accessory process, conspicuous in the cat.

${ }^{3}$ Note that the anterior and posterior ends of vertebrae can be recognized as follows: the smooth articulating surfaces or facets of the prezygapophyses face upward or forward while the facets of the postzygapophyses face downward or backward. 
3. The ribs.-The ribs of mammals consist of a dorsal, bony part, the vertebral rib, and a ventral cartilaginous portion, the sternal rib or costal cartilage. The vertebral rib is articulated to the vertebra by two heads, an upper tuberculum, whose facet engages the costal facet on the under surface of the transverse process; and the lower capitulum, attached between two centra to demifacets, i.e., half a facet on the posterior end of one centrum and half a facet on the anterior end of the succeeding centrum. The tuberculum is diminished on the more posterior ribs and wanting on the last three ribs which are provided with capitular heads only. The narrow part of a rib between tuberculum and capitulum is called the neck; the point of greatest curvature, just a little beyond the tuberculum, the angle; the remainder of the rib, the body. Those ribs which reach to the median ventral line and are independently attached to the median ventral structure (the breastbone) are known as true ribs; those which are not so attached to the breastbone are called false ribs; and those false ribs whose ventral ends are free are floating ribs. ${ }^{1}$ The cat has nine true ribs and four false ribs, of which the last one is floating; the rabbit has seven true ribs and five false ribs, of which the last three are floating; in man there are seven true ribs and five false ribs, the last two floating. The ribs of mammals are intermuscular ribs. Draw one of the typical thoracic vertebrae with its ribs, from the front.

4. The lumbar vertebrae.-There are seven lumbar vertebrae in the cat and rabbit. They are large and stout vertebrae with prominent neural spines, conspicuous zygapophyses, and long transverse processes directed craniad. Below the postzygapophysis is a pointed projection, directed caudally, called the accessory process. Draw a lumbar vertebra from the side.

5. The sacrum.-The sacrum is composed of a variable number of vertebrae fused together for articulation with the hind limbs. There are three sacral vertebrae in the cat, generally four in the rabbit (of which only the first two really contribute to the attachment), five in man. The boundaries between the fused sacral vertebrae are readily made out by means of the openings between them through which the spinal nerves pass out, and by means of the number of neural spines, zygapophyses, etc. The first sacral vertebra assumes the greater part of the task of transmitting the support of the hind limbs to the vertebral column; for this purpose it has large lateral expansions bearing articular surfaces for the insertion of the bony structure which supports the hind limb. These lateral expansions consist in part of transverse processes and in part of sacral ribs, indistinguishably fused to the vertebra.

6. The caudal vertebrae.- The caudal vertebrae are variable in number in mammals. Neural arches, transverse processes, and zygapophyses diminish

This use of the terms true and false should not be confused with the usage previously given in which the expression true rib refers to intermuscular ribs and false rib to subperitonea! ribs. In this comparative sense all mammalian ribs are "true" ribs. 
caudally, the last vertebrae consisting of centra only. Very small haemal arches (chevron bones) are present in the tail vertebrae of the cat, but are missing on prepared skeletons. Man has three to five caudal vertebrae fused into a single piece, the urostyle or coccyx.

7. General remarks on the whole column.-The ends of the centra of most mammalian vertebrae, as can be seen by inspection of isolated vertebrae, are more or less flat. Such centra are called amphiplatyan. Between the ends of the vertebrae are found in life cartilaginous disks, the intervertebral cartilages. On the mounted skeleton observe an opening on each side between successive centra. Through these openings, the intervertebral foramina, the spinal nerves pass out from the spinal cord. The spinal cord occupies the continuous canal formed by the neural arches. Haemal arches (basiventrals) are absent except in the atlas and axis and in the tails of some mammals, where they form chevron bones. The vertebrae of mammals are gastrocentrous.

\section{J. SUMMARY OF THE VERTEBRAL COLUMN AND RIBS}

I. The vertebrae arise at the intersection of the myosepta with the mesenchyme surrounding the notochord and neural tube. Each vertebra is produced by the union of the posterior halves of the two sclerotomes of one segment with the anterior halves of the two sclerotomes of the succeeding segment. Owing to this manner of origin the vertebrae alternate with the myotomes.

2. The vertebrae and ribs are first formed in cartilage, produced by the activity of the mesenchyme. In the elasmobranch fishes they remain permanently in the cartilage stage. In most other vertebrates they ossify during development. They belong, consequently, to the category of cartilage bones.

3. Each vertebra begins as four pairs of cartilages or arcualia surrounding the notochord: dorsally an anterior pair of basidorsals and a posterior pair of interdorsals; ventrally an anterior pair of basiventrals and a posterior pair of interventrals.

4. In primitive vertebrae (occurring in some fish, extinct Amphibia and reptiles) these pieces remain more or less separate in the adult. In the maiority of vertebrates, however, some of them are lost and the remainder fuse into a single structure.

5. The parts of a single vertebra thus formed by the fusion of originally separate pieces are: the centrum or main body of the vertebra, which incloses the notochord; a dorsally directed arch, the neural arch, inclosing the spinal cord; and a ventrally directed arch, the haemal arch, inclosing blood vessels. The neural arch consists of the fused basidorsals, the haemal arch, of the fused basiventrals; the centrum is of different origin in different groups.

6. The centrum in the elasmobranch fishes is formed within the sheath of the notochord; it is called a chordal centrum. The centra in nearly all other vertebrates are produced by the fusion of certain of the arcualia and are known as perichordal or arch centra. In different groups of vertebrates different arcualia contribute to the centra.

7. The ends of the centra are variously shaped: amphicoelous, or concave at each end (fishes, urodeles); procoelous, concave in front, convex behind (A mphibia, reptiles); opisthocoelous, convex in front, concave behind (Amphibia, reptiles); heterocoelous, saddle shaped (birds); and amphiplatyan, flat at each end (mammals).

8. In addition to centrum and arches, vertebrae commonly bear projecting processes or apophyses. The most common of these are the transverse processes and the zygapophyses. 
The former serve for the articulation of the ribs and are probably not homologous in different vertebrates. The zygapophyses yoke successive vertebrae together.

9. The haemal arches tend to disappear or become reduced in vertebrates. They persist (hiefly in the tail region and may also contribute to the axis and the atlas.

Iо. The vertebral column in fishes is divided into trunk and tail regions only. In Amphibia a very short cervical region and a sacral region are added. In reptiles, birds, and mammals, the cervical region is longer, and the trunk region is divided into an anterior thoracic region, bearing long ribs, and a posterior lumbar region with reduced or no ribs. The differences between the vertebrae of the different regions become more and more marked the higher one ascends in the vertebrate scale.

II. Originally each vertebra was provided with a pair of ribs, but in the higher vertebrates these are reduced or absent except in the trunk or thoracic regions. Reduced ribs are generally present on the cervical vertebrae and always on the sacral vertebrae.

I2. Ribs are of two kinds: those that arise at the intersection of the myosepta with the horizontal skeletogenous septum, known as true or intermuscular ribs; and those that arise at the intersection of myosepta with the ventral skeletogenous septum or its derivatives, known as false or subperitoneal ribs. The latter are characteristic of teleosts, the former of all other vertebrates. Some fishes possess both kinds of ribs simultaneously and may also develop additional ribs at other levels of the myosepta.

13. Ribs typically articulate with the vertebrae by two heads, the space between the heads forming a vertebrarterial canal for the passage of blood vessels. 


\section{THE ENDOSKELETON: THE COMPARATIVE ANATOMY OF THE GIRDLES, THE STERNUM, AND THE PAIRED APPENDAGES}

\section{A. GENERAL CONSIDERATIONS}

r. Definitions.-The girdles are crescent-shaped or arch-shaped portions of the endoskeleton which function for the support of the paired appendages. The center of the arch is directed ventrally, the points dorsally. The girdles are composed of cartilage in the lower forms; this is partly or completely ossified in the higher ones. The pectoral girdle supports the anterior appendages; the pelvic girdle the posterior appendages.

The sternum or breastbone is an elongated structure lying in the median ventral line of the anterior part of the trunk region. It is commonly composed of a chain of cartilages or bones, or
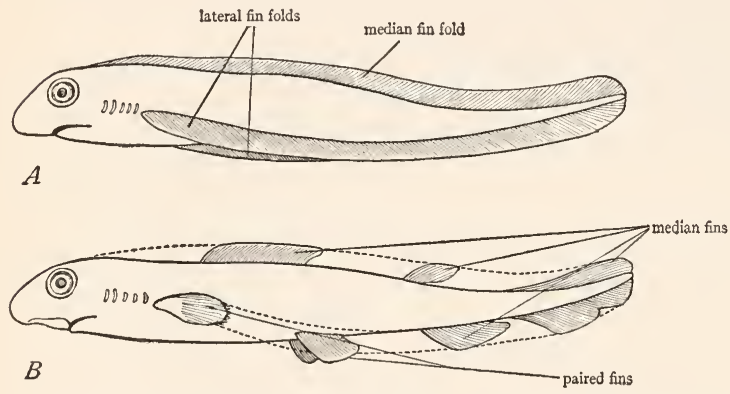

FIG. 23.-Diagrams to illustrate the theory of the origin of the median and paired fins through the persistence of certain regions of originally continuous median and lateral fin folds. $A$, early stage showing the median dorsal fin fold and the two lateral fin folds uniting at the anus. $B$, later stage illustrating persistence of certain regions of the fin folds as the median and paired fins, and disappearance of the remainder of the fin folds, as indicated by dotted lines. (From Wilder's History of the Human Body, courtesy of Henry Holt and Company.) both. The ribs and the pectoral girdle usually articulate with the sternum. Such arrangements strengthen the anterior part of the trunk in relation to the airbreathing habit and the presence of lungs.

The paired appendages consist of fins in the fishes and limbs in all of the land vertebrates. The anterior or pectoral appendages articulate with the pectoral girdle, and their support is transmitted to the body by means of this girdle. Similarly the posterior or pelvic appendages are articulated to the pelvic girdle and transmit their support through this girdle. As the support of the posterior appendages is generally the more important, the pelvic girdle is commonly stronger and more massive than the pectoral girdle. This is particularly true of biped vertebrates. The appendages possess an internal skeleton.

2. The origin of the paired appendages and the girdles. - The origin of these structures in vertebrates is obscure. According to the most probable theory, the fin-fold theory, the ancestral vertebrate possessed a pair of continuous folds, one running along each side of the trunk. These fused behind the anus to a single median fin which extended around the tail and along the median dorsal line (see Fig. 23A). This hypothetical condition resembles that actually occurring in Amphioxus, with its paired ventral metapleural folds and median caudal and dorsal 
fin. These fin folds were supported by cartilaginous fin rays. The paired fins of present fishes are supposed to have arisen through the persistence of certain regions of the paired fin folds, and the median unpaired fins through the persistence of particular regions of the median fin fold. The remaining portions of the fin folds have vanished (see Fig. $23 B$ ). This theory is supported by the facts that the unpaired and paired fins of fishes are identical in structure, that in the young stages of some forms continuous paired folds are present from which the paired fins arise, and that in the extinct shark Cladoselache (see K, Fig. I2I, p. II5) the arrangement of the fin rays in the paired fins is such as to suggest strongly the origin of the paired fins from continuous folds.

The girdles arose later than the paired fins, since extinct forms possess the latter without the former. The fins as already stated are supported by cartilaginous fin rays, which may occur in several rows. It is suggested that the most medial and anterior of these fin rays of the fins fused across the midventral line to form median plates or bars of cartilage. Such plates or bars represent primitive girdles. Later ossification occurs in the cartilage, resulting in the bony girdles composed of several bones, as found in the higher vertebrates. Read W.

In the evolution of fish into Amphibia the paired fins transformed into limbs. Stages in this transformation are very imperfectly known and difficult to conceive. It is supposed that the fin rays of the paired fins were gradually reduced in number either by loss or fusion. A few enlarged fin rays arranged similarly to the bones of the limbs persisted. Such reduction and enlargement of fin rays really occurs in the crossopterygian ganoids living and extinct, and the arrangement of the fin rays in these forms is strongly suggestive of the arrangement of the bones in the vertebrate limb. (See further, N, p. 176, Fig. 102, and p. 178, Fig. 103.)

Read further on the comparative anatomy of the sternum, girdles, and paired appendages in $\mathrm{K}, \mathrm{W}$, and $\mathrm{Wd}$.

\section{B. THE PELVIC GIRDLE AND THE POSTERIOR PAIRED APPENDAGES}

I. The parts of a typical girdle and hind limb.-Before beginning the study of the comparative anatomy of these parts it may be well to describe the generalized girdle and limb. The bony pelvic girdle arises from the ossification of a cartilaginous arch or plate. Three centers of ossification appear in each half, resulting in the production of three pairs of cartilage bones. Of these there are on each side a dorsal bone-the ilium-an anterior ventral bone-the pubis - and a posterior ventral bone-the ischium (Fig. 24). The two pubes and ischia commonly meet in the median ventral line. The ilia are articulated to the sacral ribs.

The hind limb typically consists of three regions: a proximal segment, the thigh, containing a single long bone, the femur; a middle segment, the shank, containing two long bones: a preaxial tibia and a postaxial fibula; and a distal segment, the foot or pes, including the ankle, sole, and toes, containing a number of small bones. The generalized ankle is composed of nine or ten bones in three rows: a proximal row of three bones-a preaxial tibiale, a medial intermedium, and a postaxial fibulare; a middle row of one or two centrales; and a distal row of five tarsales. The sole of the foot is composed of five bones, the metatarsals, arranged in a transverse row. The toes or digits contain a series of small bones, the phalanges, of which in primitive feet there are two to the first digit and three to all of the others. Figure 24 illustrates the bones of the typical pelvic girdle and hind limb.

2. The pelvic girdle and pelvic fin of elasmobranchs.-For this purpose study preserved skeletons of the dogfish or other elasmobranch fish. The pelvic 
girdle is the straight or slightly curved bar of cartilage placed across the ventral side at the end of the trunk region between the anterior ends of the pelvic fins. This bar is called the ischiopubic bar and represents a primitive unossified pelvic girdle. In many elasmobranchs (including the dogfish) the two ends of the bar bear slight processes projecting outward; these are the iliac processes.

Attached to the pelvic girdle, articulating to it at the base of the iliac processes, are the pelvic fins. The skeleton of the basal part of the fins, which is

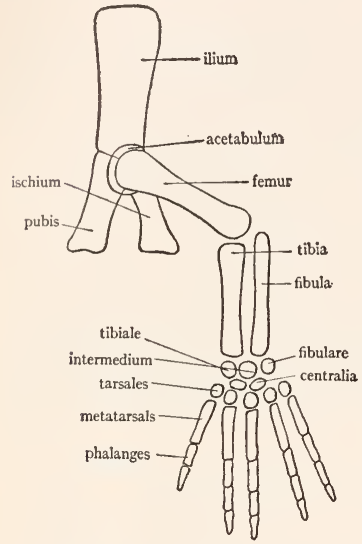

FIG. 24.-Diagram of the bones of the typical pelvic girdle and hind limb. (From Parker and Haswell's Textbook of Zoölogy, courtesy of the Macmillan Company.) imbedded in the body wall, consists of a number of cartilaginous pieces, the cartilaginous fin rays; the skeleton of the free external part of the fin is composed of a number of slender parallel dermal fin rays. As the latter are part of the exoskeleton, they will not be considered further. The cartilaginous fin rays are arranged in two series, a medial series, consisting of one or two (in some forms three or five) much enlarged cartilages, and a lateral or outer series, composed of a number of small cartilages disposed in one or more rows. The inner series of fin rays are called basalia or basals, the outer series, radialia or radials. There is usually present a single basal, the metapterygium, an elongated curved cartilage forming the whole medial border of the fin, but in some forms there is an additional basal, the propterygium, situated at the anterior end of the metapterygium. The radials usually consist of a row of rod-shaped cartilages, their long axes at right angles to the axis of the metapterygium. In male specimens the posterior radial is greatly enlarged to form the cartilage of the clasper. The basals have probably arisen through the fusion of a number of smaller cartilages.

Draw the pelvic girdle and fin of an elasmobranch.

3. The pelvic girdle and hind limb of urodeles.-Study these parts in dried or preferably preserved specimens of Necturus or Cryptobranchus. The urodele girdle is in a condition intermediate between the cartilaginous girdle of elasmobranchs and the bony girdle of reptiles. It is situated on the ventral side at the end of the trunk between the hind limbs. Its ventral portion has the form of a flattened plate, which may be designated the ischiopubic plate or pelvic plate. The anterior part of this plate is cartilaginous and is called the pubic cartilage. In the posterior part of the plate a pair of opaque (i.e., bony), rounded areas may be seen. Each of these areas is a bone, the ischium, which, as already explained, is the posterior ventral bone of typical girdles. The ischia are produced by ossification in the pelvic plate. From each side of the pelvic plate a rod of bone extends 
dorsally and is firmly articulated to the end of the sacral rib. This bone is the ilium and represents an ossification in the iliac process of elasmobranch girdles. At the point of junction of pubic cartilage, ischium, and ilium, there is a depression, the acetabulum, into which the proximal end of the hind limb is inserted by a ball-and-socket joint.

The hind limbs of urodeles like Necturus are exceedingly primitive. This matter was already discussed in Section III of this manual. The limb is divided into a proximal segment-the thigh, consisting of a single bone, the femur, a middle segment-the shank, consisting of two parallel bones, a preaxial bone, the $t i b i a$, and a postaxial bone, the fibula, and a distal segment-the pes, consisting of ankle and foot. The ankle consists of several small bones, the tarsals, which are, unfortunately, impossible to make out on most skeletons. The foot has four elongated bones, the metatarsals, which bear the toes. Each toe consists of two or three small bones, the phalanges, arranged in a row. The first toe is missing.

Draw the pelvic girdle and hind limb from above.

4. The pelvic girdle and hind limb of the turtle.-In the reptiles the ossification of the pelvic girdle is complete; very little cartilage remains, and the full number of bones characteristic of the pelvic girdle is present. The pelvic girdle of the turtle is a very generalized and representative girdle. Isolated girdles may be studied, but the position and attachment of the girdle should be noted on the entire mounted skeleton. The girdle consists of three pairs of stout bones, two pairs ventral in position, one pair lateral and dorsal. The ventral bones consist of an anterior pair - the pubes, which meet in the median ventral line forming the pubic symphysis, and a posterior pair-the ischia, united similiarly to form the ischial symphysis. These symphyses are composed of cartilage, generally missing from dried skeletons. The lateral and dorsal bones of the girdle are the ilia, which are articulated at their dorsal ends to the ends of the two sacral ribs. Note on the mounted skeleton the inverted arch or $U$ formed by the pelvic girdle. The arch is completed dorsally by the sacral vertebrae and sacral ribs. Through the arch of the pelvic girdle pass the terminal portions of digestive and urogenital systems. Between the pubis and ischium of each side is a large opening, the obturator foramen, through which in the fresh condition nerves and blood vessels pass. The two foramina are completely separated in life by a cartilage which bridges the space between the pubic and ischial symphyses and continues as the cartilage of these symphyses. Attached to the anterior extremity of the pubic symphysis is a cartilage, the epipubis. Each pubis has a prominent lateral process, the pectineal process, projecting forward. Pubis, ischium, and ilium meet at the place where the hind limb articulates, and share equally in the formation of a concave depression, the acetabulum, into which the convex head of the femur is inserted. Draw the pelvic girdle (one side is sufficient).

The parts of the hind limb are the same as in the urodeles, but the limb no longer retains its primitive orientation with regard to the body. The middle 
segment is directed ventrally, forming an angle with thigh and foot, and there has also been some slight torsion. See further on these points in Section III. The thigh consists of one large bone, the femur, which fits into the acetabulum by a prominent knob, the head of the femur. The shank is composed of tibia and fibula, the latter being the smaller. The ankle is made up of five (or in some turtles six) bones. At the bases of the tibia and fibula is a large, transversely elongated bone, which is in reality composed of four bones fused (tibiale, intermedium, centrale, fibulare). In some turtles the fibulare, at the base of the fibula, is separate. Distal to this compound bone is a row of four bones, the four tarsales, numbered from the preaxial (tibial) side to the postaxial (fibular) side. The apparent fourth tarsale, the largest of the four, is really the fused fourth and fifth tarsales. Distal to the tarsales are the five metatarsals, and beyond these the digits, composed of bony joints or phalanges, terminating in horny claws. The number of phalanges in the digits is that regarded as primitive for vertebrates, namely, two phalanges to the first digit, and three to all of the others (see Fig. 24). It is worthy of note that, in the turtles and reptiles in general, the movement of the foot upon the leg occurs between the two rows of tarsal bones, i.e., it is an intratarsal joint. Figures of the tarsal bones of turtles are given in $\mathrm{Wd}, \mathrm{K}$, and $\mathrm{R}$.

5. The pelvic girdle and hind limb of birds.-These structures, like the remainder of the skeleton, are highly modified in birds, although consisting of the same parts as in other vertebrates. Examine isolated backbones with the pelvic girdles attached, or study the whole mounted specimens. The pelvic girdle consists of three pairs of bones as in reptiles, i.e., ilium, ischium, and pubis. All three are fused on each side to form a continuous broad bone, the innominate bone. The ilium is the largest and most dorsal part of the innominate bone. It forms an elongated thin plate, concave in front, convex behind, extending from the last thoracic vertebra to the tail region. It is fused along its entire length with the synsacrum, the boundary between ilium and synsacrum generally being marked by a suture. (In the bird embryo the ilium is articulated to only two vertebrae which are the true sacral vertebrae.) The side of each innominate bone is composed of the ischium, the only boundary mark between this and the ilium being a large oval opening, the ilioischiac foramen. The pubis is the long slender bone along the ventral border of the ischium, from which it is separated by a more or less distinct suture, and the slitlike obturator foramen which may be divided into two or more openings. Ilium, ischium, and pubis contribute to the formation of the acetabulum. The anterior end of the pubis is situated anterior to the acetabulum, the normal position of the pubis, but during development the pubis turns posteriorly and comes to project beyond the posterior end of the ischium. In the embryonic development of birds, the three components of the innominate bone, ilium, ischium, and pubis, originate separately. Observe that neither pubic nor ischial symphyses are present, 
but the two innominate bones are widely separated ventrally. This is probably associated with the habit of laying large eggs.

The hind limb offers several peculiarities. The femur has a large head fitting into the acetabulum and a prominent projection lateral to the head, called the great trochanter. The distal end of the femur is shaped like a pulley, consisting of a central depression with curved ridges--the condyles-on either side. Over the joint between thigh and shank is an extra small bone, the patella or kneecap, not found in the lower vertebrates. The patella is a sesamoid bone, that is, a bone developed in a tendon. Such sesamoid bones are quite common in the limbs of higher vertebrates. The shank is composed of two bones, a medial large one and a lateral short rudimentary bone. The large bone is the tibiotarsus. It consists of the tibia fused at its distal end with the proximal tarsal bones. The proximal end of the tibiotarsus has two condyles for articulation with the condyles of the femur, and bears in front two diverging elevations or crests for muscle attachments. The small bone of the shank is the fibula, whose distal portion is atrophied. The distal end of the tibiotarsus has a pulley-like surface for articulation with the succeeding bone, the raised articular surfaces being named malleoli. Beyond the tibiotarsus is a long stout bone, the tarsometatarsus, evidently formed by the fusion of three bones, as shown by the three ridges on its distal end. The three fused bones are the metatarsals (second, third, and fourth); in addition the tarsometatarsus includes in its proximal portion the distal ankle bones. It will thus be seen that the ankle bones do not exist separately in adult birds, but the proximal ankle bones are fused to the lower end of the tibia, while the distal ones are fused to the upper ends of the metatarsals. The ankle joint is, therefore, as in reptiles, an intratarsal joint. The three metatarsals which are fused to form the tarsometatarsus are the second, third, and fourth, but a remnant of the first metatarsal is present as a small projection on the medial side of the distal end of this compound bone. Each metatarsal articulates with its respective digit, composed of phalanges and terminating in claws. Fifth metatarsal and digit are quite wanting. The gait of birds is digitigrade.

Draw the pelvic girdle and hind limb from the side.

6. The pelvic girdle and hind limb of mammals. - The pelvic girdle of mammals is very similar to that of reptiles in shape, and similar to that of birds in that the pelvic bones are fused into innominate bones. The girdle consists of the usual three pairs of bones, pubis, ischium, and ilium, the three of each side being indistinguishably fused into an innominate bone or hip bone. The ilium is the most dorsal, most anterior, and largest of the three components of the innominate bone. It articulates with the sacral vertebrae and terminates anteriorly and dorsally in a curved border, known as the crest of the ilium. The ilium extends as far posteriorly as the acetabulum; the dorsal part of the girdle posterior to the acetabulum is the ischium which is continuous with the ilium. The 
posterior ends of the two ischia form prominent projections in the rabbit or a curved rough surface in the cat, called the ischial tubcrosity, and extend toward the median line as the rami (singular, ramus) of the ischium, meeting in the median ventral line to form the ischial symphysis. The anterior ventral part of each innominate bone is formed by the pubis. Each pubis sends a projection, the ramus, toward the median ventral line, the two rami uniting as the pubic symphysis. Both ischial and pubic symphyses are in life composed of cartilage. Between the rami of the ischium and pubis is the large obturator foramen. Ilium,

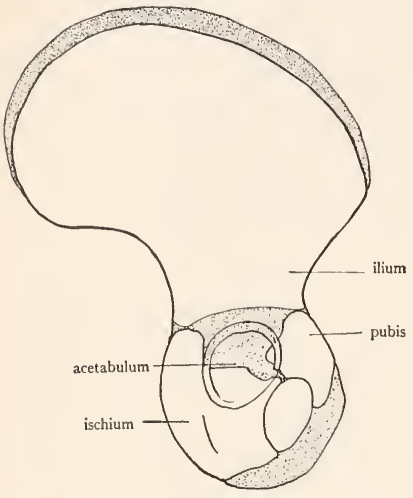

FIG. 25.-Half of the human pelvic girdle at birth, showing the three bones of which it is composed. Stippled regions represent cartilage which later ossifies, obliterating the boundaries between the bones. (From a specimen loaned by the anatomy department.) ischium, and pubis meet at the acetabulum and take part in the formation of its walls.

Draw the pelvic girdle from in front.

On a demonstration specimen of half of the girdle of a kitten note complete separation and boundaries of ilium, ischium, and pubis. Note further the presence in the acetabulum of a small bone, the acetabular bone, which forms that part of the acetabulum which would otherwise be occupied by the pubis. In the young of all mammals the three bones of the pelvic girdle are separate, as in the adults of reptiles (Fig. 25).

The hind limb is fairly typical. The femur has a head, a greater trochanter lateral to the head (which in the rabbit continues posteriorly terminating in a small projection, the third trochanter), and a lesser trochanter, situated below the head. These trochanters serve for muscle attachments. The large articulating surfaces at the distal end of the femur are condyles (medial and lateral), and they bear additional elevations or roughened areas, the epicondyles. At the knee joint a patella is present. The shank is composed of a stout tibia and slender fibula, the latter in the rabbit fused with the tibia for the greater part of its length. The anterior face of the tibia presents a crest; its proximal articulating surfaces are known as condyles; its distal ones as malleoli. The bones of the ankle are identical with those of the human ankle and are designated by the same names, which are, unfortunately, somewhat fanciful and not based upon comparative anatomy. The name derived from comparative anatomy is given in parenthesis after the name derived from human anatomy. The ankle consists of seven bones (cat), or six (rabbit). The largest and most conspicuous of these, which projects backward as the heel, is the calcaneus (fibulare). Articulating with the malleoli of the tibia and fibula is the astragalus or talus (tibiale). Directly in 
front of the astragalus is the navicular or scaphoid (centrale), a curved bone reaching to the medial side of the foot. Directly in front of the calcaneus is the cuboid (fourth and fifth tarsales fused), which articulates with the fourth and fifth metatarsals. Medial to the cuboid is the third or lateral cuneiform (third tarsale), articulating with the third metatarsal. Medial to this is the second or intermediate cuneiform (second tarsale), articulating with the second metatarsal. In the cat there is a first or medial cuneiform (first tarsale) along the medial border of the anterior part of the ankle in front of the navicular. It articulates with the small rudimentary first metatarsal which lies directly in front of it. In the rabbit the first cuneiform is fused to the proximal end of the second metatarsal. The homology of these ankle bones with those given in the primitive vertebrate plan (Fig. 24 ) is quite evident. The sole consists of four long metatarsals and one rudimentary one (the first) on the medial or ventral side of the proximal end of the second metatarsal. The terminal phalanges of the digits are curiously beak shaped, for the support of the horny claws, and in the cat have sheaths at their bases into which the bases of the horny claws fit.

The joint between foot and shank in mammals is between the ankle bones and the malleoli of the tibia and fibula, unlike the condition seen in birds and reptiles, where the joint lies between the distal and proximal ankle bones. The gait of the cat and rabbit is chiefly digitigrade, although the hind legs assume the plantigrade posture when the animal sits down.

Attention was called in Section III of the manual to the torsion which the manmalian hind limb has undergone with the result that the toes, originally pointing laterally as in $\mathrm{Nec}$ -
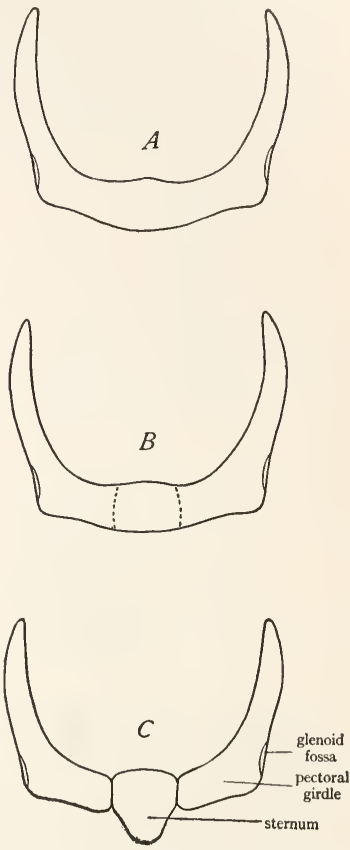

Fig. 26.-Diagrams to illustrate the theory of the origin of the sternum from the pectoral girdle. $A$, pectoral girdle of an elasmobranch. $B$, central portion of the girdle beginning to separate. $C$, central portion of the girdle completely separated, forming the sternum.

turus now point anteriorly. The statements made at that place should be reviewed here.

\section{THE PECTORAL GIRDLE, THE STERNUM, AND THE ANTERIOR} PAIRED APPENDAGES

I. The origin of the sternum.- - Since the origin of the pectoral girdle and appendages is the same as that of the pelvic girdle and appendages, it remains to discuss the formation of the 
sternum. The sternum occurs only in lung-breathing vertebrates, that is to say, it is absent in fishes. There is some doubt as to the origin of the sternum. According to the prevailing view, given in $\mathrm{K}$ and $\mathrm{W}$, the sternum of the higher vertebrates is produced by the fusion of the ventral ends of the ribs, i.e., of the costal cartilages. But since in the lower vertebrates the ribs are short and do not reach to the ventral side, this theory necessitates the invention of a different theory for the origin of the sternum of Amphibia. The sternum of Amphibia is consequently supposed to have arisen through the fusion of certain cartilages present in the ventral myosepta of urodeles. See $\mathrm{W}$, pages $\mathbf{1 4 0 - 4 3}$, for details of this idea. Recently, however, evidence has been brought forward in favor of the conception that the sternum has the same origin in all of the vertebrates and that its origin is independent of the ribs. According to this

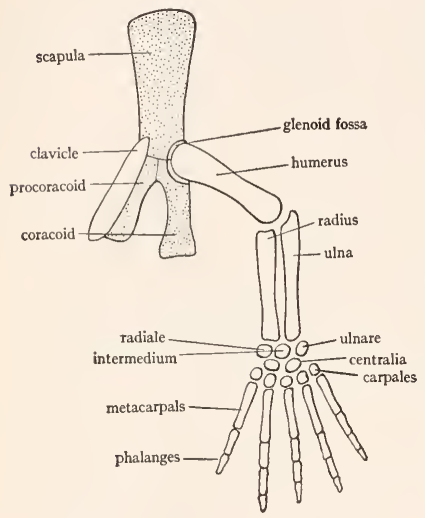

Fig. 27.-Diagram of the bones of the typical pectoral girdle and fore limb. The cartilage bones of the girdle are stippled, the membrane bone (clavicle) left blank. All of the limb bones are cartilage bones. (From Parker and Haswell's Textbook of Zoölogy, courtesy of the Macmillan Company.)

a separate ossified procoracoid. However, there is considerable evidence that the persistent element is really the procoracoid in present reptiles, and that in this group the coracoid is missing. In mammals, however, the matter is disputed, some regarding the persistent element as the procoracoid, others as the coracoid (Fig. 28). This persistent ventral cartilage bone of the pectoral girdle of reptiles, birds, and mammals is called the coracoid, and this name will be retained here, with the understanding that in reptiles it is almost certainly homologous with the procoracoid. In nearly all mammals the coracoid is rudimentary, the only cartilage bone of the girdle which retains its full importance being the scapula or shoulder blade. Diagrams illustrating the homology and parts of the girdles of various forms are given in Figure 29.

In addition to the cartilage bones present in the girdle some of the original cartilage is likely to persist unossified. The cartilages of most common occurrence are: the suprascapula,

× F. B. Hanson, Amcrican Journal of Anatomy, XXVI (1919), 4r. 
situated along the dorsal border of the scapula and sometimes partially ossified, the epicoracoids, cartilages anterior or medial to the coracoid (procoracoid) element of reptilian and mammalian pectoral girdles; and cartilages between the mcdial ends of the coracoids (also but confusingly named epicoracoids in texts, and in this manual left unnamed).

The pectoral girdle is still further complicated by the addition of membrane bones to the cartilage bones and cartilages described above. As previously stated, such membrane bones are homologous with the dermal plates of the exoskeleton and have secondarily become associated with the endoskeleton. It was stated in the introduction to the endoskeleton that such membrane bones occur in relation to certain parts of the endoskeleton, and we meet them here for the first time, in association with the pectoral girdle. In teleosts there are a number of such dermal elements added to the pectoral girdle, as shown in Figure 30 ; they form a sort of ventral incasement of the girdle. Of these there persist in the most primitive land vertebrates (extinct) five elements, a median single interclavicle, paired lateral clavicles, and lateral to the clavicles paired cleithra (singular, cleithrum) (see Fig. 29A). The interclavicle (also called episternum) is situated immediately ventral to the sternum, the clavicles invest the procoracoids which are often concealed within or dorsal to them, while the cleithra are situated along the borders of the scapulae. The cleithra are lost early in the evolution of land vertebrates and do not occur in living forms, but the interclavicles and clavicles are characteristic of many reptiles, of birds, and of monotremes. In placental mammals the interclavicle is missing, and in some of them the clavicles are also reduced or absent. There has thus occurred in the evolution of the pectoral girdle a gradual reduction in the number of membrane bones, as will be seen by reference to Figure 29.

The pectoral girdle thus consists of a complex of cartilage and membrane bones. The cartilage bones may articulate ventrally with the sternum but rarely have any articulation or fusion with the vertebral column (exceptions: skates, ptero-
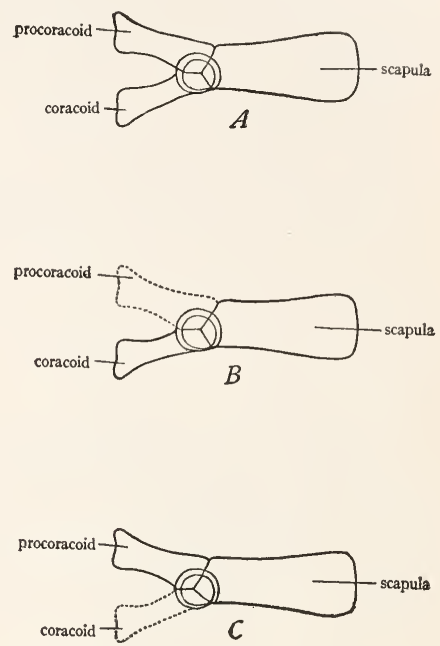

FIG. 28.-Diagrams to illustrate two theories of the homology of the cartilage bones of the pectoral girdle of amniotes. $A$, cartilage bones of the primitive pectoral girdle; there are three bones in each half of the girdle, a condition found only in extinct forms. $B$, one theory of the composition of the pectoral girdle of present amniotes, showing disappearance of the procoracoid and retention of the coracoid. $C$, alternative theory, showing retention of the procoracoid (called, however, coracoid) and disappearance of the coracoid. saurs). In this respect the pectoral girdle stands in marked contrast to the pelvic girdle, which, as we have seen, always has in land vertebrates a firm articulation with the sacrum. In teleosts the membrane bones of the girdle may articulate with the skull. The cartilage bones of the girdle always, and the membrane bones never, take part in the articulation with the fore limb. This is one of the simplest ways of distinguishing the two kinds of components in the girdle.

The skeleton of the fore limb is composed of parts similar to those already described for the hind limb. There is a proximal segment-the upper arm or brachium, consisting of a single 
bone, the humerus, a middle segment-the forearm or antibrachium, containing two bones: a preaxial radius and a postaxial ulna, and a distal segment-the hand or manus. The hand is subdivided into three parts: the wrist, the palm, and the fingers. The wrist is primitively composed of nine or ten bones in three rows: a proximal row of three, named the radiale, the intermedium, and the ulnare; a middle row of one or two centrales; and a distal row of five
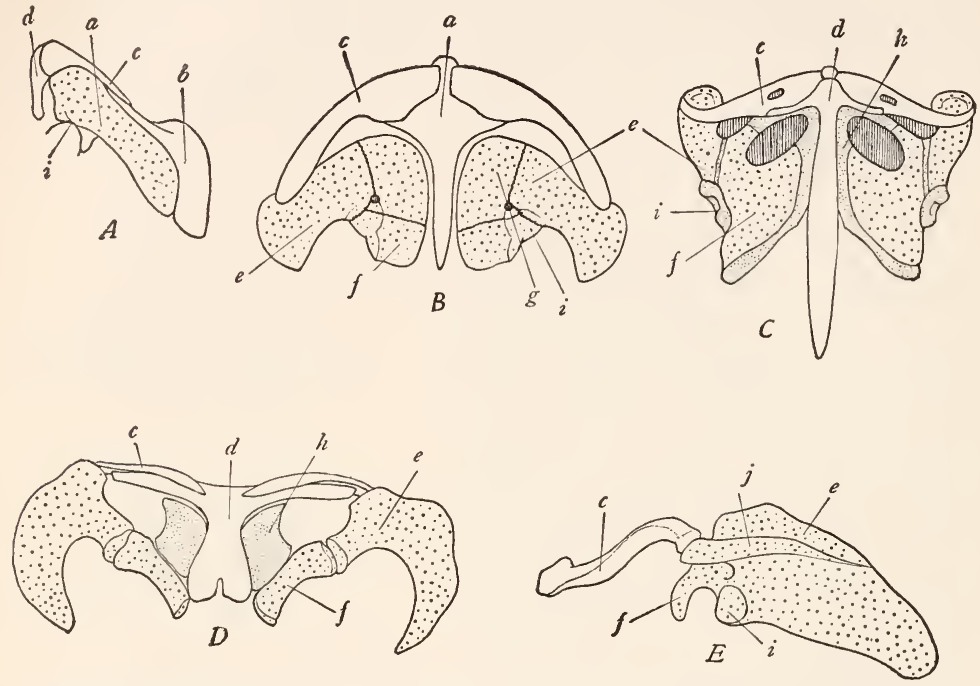

Fig. 29.-Pectoral girdles of some vertebrates. $A$, side view of half of the pectoral girdle of an extinct amphibian, Cacops, belonging to the Stegocephala; note presence of the cleithrum $b$. $B$, ventral view of both halves of the pectoral girdle of a very ancient extinct reptile, Seymouria, belonging to the Cotylosauria, illustrating the complete generalized pectoral girdle; note presence of both coracoid and procoracoid. $C$, ventral view of both halves of the pectoral girdle of a modern reptile, a lizard; note loss of one of the coracoid bones. $D$, ventral view of both halves of the pectoral girdle of a monotreme mammal, the duckbill, Ornithorhynchus, illustrating the most primitive pectoral girdle found among present mammals; note persistence of the coracoid and interclavicle, similar to reptiles. $E$, ventral view of one-half of the pectoral girdle of an ape; note absence of the interclavicle and reduction of the coracoid to the coracoid process $f$. Cartilage, close stippling; cartilage bones, open stippling; membrane bones, blank. $a$, coracoscapula; $b$, cleithrum; $c$, clavicle; $d$, interclavicle; $e$, scapula; $f$, coracoid (coracoid process in $E) ; g$, procoracoid; $h$, epicoracoid cartilage; $i$, glenoid fossa; $j$, spine of the scapula. ( $A$ from Williston's Water Reptiles of the Past and Present, University of Chicago Press; B, after Williston; $C$ from Reynolds' The Vertebrate Skeleton, courtesy of the Macmillan Company; $D$ from Wiedersheim's Comparative Anatomy of Vertebrates, courtesy of the Macmillan Company.)

carpales. The palm is composed of five elongated metacarpals and the fingers of phalanges whose numbers are primitively the same as in the case of the toes (see Fig. 27).

3. The pectoral girdle and pectoral fin of elasmobranchs.--In these fish we find the pectoral girdle in a primitive condition, resembling that of the pelvic girdle. It is a curved cartilage almost completely encircling the anterior part of 
the trunk. The median ventral portion between the bases of the two fins is called the coracoid bar; the long processes extending dorsally beyond the articulations of the fins are the scapular processes; the ends of the scapular processes commonly consist of separate pieces, the suprascapular cartilages. The pectoral fins are similar to the pelvic. They are supported at their bases by several series of cartilaginous fin rays and externally by dermal fin rays. The cartilaginous fin rays consist of a proximal row of enlarged basals and several distal rows of

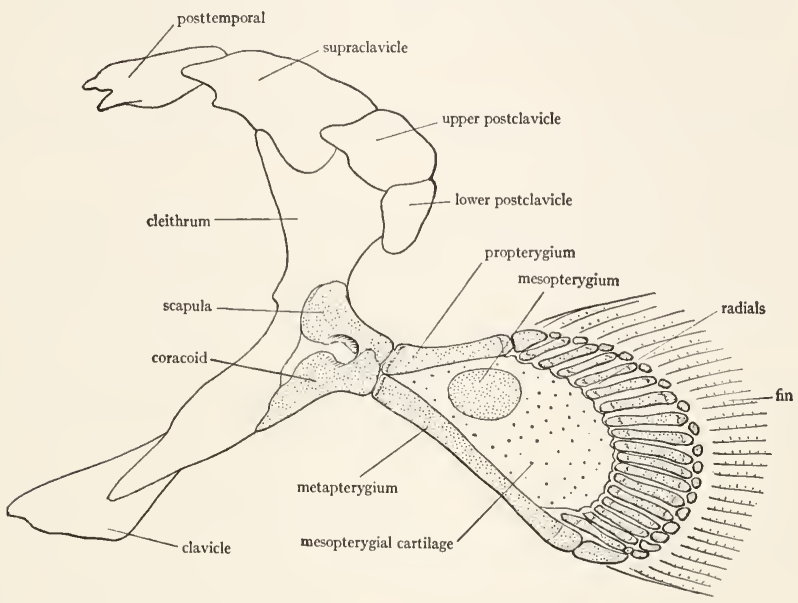

FIG. 30.-Pectoral girdle of a crossopterygian fish, Polypterus, to show the large number of membrane bones occurring in the pectoral girdle of teleostome fishes. Viewed from the inside (dorsal view) Membrane bones, blank; cartilage bones, close stippling; cartilage, open stippling. Note also the arrangement of the basals and radials in the "stalk" of the fin, the external appearance of which is shown in Figure I. (After Goodrich in Part IX of Lankester's Treatise on Zoölogy, courtesy of the Macmillan Company.)

smaller radials. There are generally three basals: an inner one, the largest, the metapterygium; a middle one, the mesopterygium; and an outer one, the propterygium. There is no trace of a sternum as such, but the median ventral portion of the coracoid bar may be regarded as the primordium of the sternum on the basis of the theory of the origin of the sternum presented at the beginning of this section (see Fig. 26, p. 85). Draw the pectoral girdle and one pectoral fin.

4. The pectoral girdle and fore limb of urodeles. - In urodeles the girdle is still in a primitive condition. Necturus or Cryptobranchus may be studied. On the ventral side the girdle forms paired, flat cartilaginous plates, which may be designated the coracoid plates. The anterior part of these plates may be regarded as a procoracoid region; this projects forward in Necturus as an 
elongated process. The posterior part is the coracoid region, but no ossification has occurred in either of the regions. Dorsally above the joint with the fore limb is a bone, the scapula - the only bone in the girdle. Attached to the dorsal border of the scapula is the suprascapular cartilage. There is no sternum (although two or three pairs of small cartilages found in the ventral wall, located in the myosepta, are regarded as the sternum by some authors). Note that the pectoral girdle has no connection with the vertebral column.

The fore limb is similar to the hind limb. The upper arm is composed of the humerus; the forearm of the preaxial radius and the postaxial ulna; the elements of the carpus or wrist are difficult to distinguish (there are six or seven cartilages in three rows); there are four metacarpals in the palm followed by phalanges. The middle segment of the limb is bent and directed ventrally, thus elevating the animal slightly above the ground.

Draw the pectoral girdle and one fore limb.

5. The pectoral girdle and sternum of the frog.-In the frog we find that the cartilages present in the urodele girdle have been partially replaced by bone. A sternum is further present in the median ventral line between the ventral ends of the halves of the pectoral girdle. Study the girdle and sternum. The sternum consists of two bones and two cartilages arranged in a longitudinal series and separated into two groups by the pectoral girdle. The anterior group, projecting cranially from the girdle, consists of a terminal rounded cartilage, the epistermum, posterior to which is a bone, the omostermum. The posterior group, projecting caudad from the girdle, is composed of an anterior bone, the stermum proper, and a terminal rounded cartilage, the xiphistermum. The ventral part of the pectoral girdle consists of two pairs of bones, their ventral ends meeting in the median ventral line between the two parts of the sternum. The anterior pair of bones is the clavicles. The clavicles are membrane bones which cover and conceal the procoracoid cartilages; these cartilages remain unossified in Anura. The posterior ventral bones of the girdle are the coracoids, ossified from the coracoid cartilages. The medial ends of the coracoids remain in the cartilaginous condition (forming cartilages which are designated in texts as the epicoracoids, but this term is confusing as it is also used in another connection). The dorsal part of the girdle consists of two bones on each side: one next to the articulation of the fore limb, the scapula, and a large, thin, flat bone medial to the scapula, the suprascapula, having a cartilaginous border. The girdle is not connected with the vertebral column. The depression which receives the head of the humerus is the glenoid fossa; scapula and coracoid take part in the formation of the glenoid fossa, but the clavicle does not. This indicates that the clavicle is of different origin from the other bones of the girdle. Draw the girdle and sternum of the frog.

6. The pectoral girdle, sternum, and fore limb of reptiles.-On the skeleton of the turtle observe that a pectoral girdle is present but a sternum is absent. 
Each half of the girdle consists of two ventral parts and one dorsal part. The anterior ventral part is not an independent bone but a projection from the scapula, and is hence named the proscapular process (erroneously referred to in some texts as the procoracoid bone). The posterior ventral bone, the larger of the two, is the coracoid (which as already explained is probably in reality the procoracoid). The dorsal bone, an elongated bone reaching to the carapace, is the scapula. All of these are cartilage bones. In addition there are certain bones of the pectoral girdle which are included in the plastron and are therefore membrane bones. These are the paired clavicles (epiplastra) and the median interclavicle (entoplastron). Refer to your drawings of the turtle plastron.

The pectoral girdle of the alligator consists of a stout dorsal bone, the scapula; a stout ventral bone, the coracoid; and a long, slender dagger-shaped bone in the median ventral line, the interclavicle. Clavicles are lacking. A sternum is present. It is composed of a plate of cartilage between the ventral ends of the coracoids and just above the interclavicle; it is drawn out posteriorly into long curved cartilages, the xiphisternal horns. Observe that the ribs are attached to the sternum by means of their sternal ribs, a condition first met with in reptiles.

The bones of the fore limbs are the same as those of Necturus in general. The bones of the carpus or wrist of the turtle are remarkably primitive in number and position, and deserve further attention. If not sufficiently clear on the specimen, expose them by cautiously picking away the dried ligaments covering them. At the base of the ulna are two bones, an outer ulnare, and an inner intermedium. The center of the carpus is occupied by a long bone which is the fused radiale (end at the base of the radius) and the centrale (larger end). Distal to this is a row of five carpales, one for each metacarpal. This arrangement is therefore very much like that of the ideal carpus. Figures of the carpus of turtles are given in $\mathrm{Wd}$, pages $16_{3}, 16_{4}$. Draw, showing the bones of the carpus.

7. The pectoral girdle, sternum, and fore limb of birds.-The pectoral girdle of birds is a very complete and generalized girdle. It is composed of the scapula, a long swordlike bone lying above the ribs; the coracoid, a stout bone reaching the sternum; and the wishbone, or furcula, in front of the coracoids and attached at the lower end by ligaments only. The furcula is membrane bone and really consists of two clavicles, the two forks of the wishbone, united to a rounded piece, the interclavicle. Coracoid and scapula take part in the formation of the glenoid fossa. The sternum of birds is highly specialized. It is an elongated bone bearing a strong ventral projection, the keel or carina. The carina serves for the attachment of the powerful wing muscles. As in reptiles, the ribs are joined to the sternum by their costal cartilages. The front end of the sternum has short costal processes, each side two long xiphisternal processes. Draw from the side, showing girdle and sternum. 
On a demonstration specimen of the sternum of an ostrich or other flightless bird note the absence of the keel or carina, the sternum presenting a convex ventral face.

The fore limb of birds is greatly modified as a wing, particularly in its distal portions. The humerus is stout, fitting into the glenoid fossa by its convex head. On either side and slightly distal to the head are prominent projections, the greater and lesser tuberosities, corresponding to the trochanters of the femur. The lesser tuberosity is continued distally into a sharp ridge, the deltoid ridge. This side of the humerus bearing the deltoid ridge is the preaxial or radial side, and it is readily observed that a rotation has occurred so that the preaxial surface faces dorsally in the folded position of the wing. The greater tuberosity is postaxial and bears on its under surface a large hole, the pneumatic foramen, leading into the air space of the humerus. The radius and ulna are typical, the former the more slender of the two, the latter exhibiting at its proximal end a projection, the olecranon process or elbow, here met for the first time. The wrist is greatly altered, consisting of but two separate bones-the radiale at the base of the radius and the ulnare at the base of the ulna. The remaining wrist bones are fused to the metacarpals to form the carpometacarpus, consisting of two elongated bones. The metacarpals contributing to these elements are the third and fourth. ${ }^{x}$ The second metacarpal is fused to the preaxial side of the proximal end of the third metacarpal where it forms a pronounced hump. From this hump projects the second digit. The third digit is the longest and consists of two phalanges, of which the proximal one is much flattened. The fourth digit is a small projection fused to the postaxial side of the proximal phalanx of the third digit. First and fifth metacarpals and digits are wanting.

8. The pectoral girdle, sternum, and fore limb of mammals.-The pectoral girdle of most mammals is reduced and somewhat modified. Only the monotremes have complete pectoral girdles, consisting of scapulae, coracoids, clavicles, and interclavicle (see Fig. $29 D$, p. 88). In all of the placental mammals, the coracoid is reduced to a small process, the coracoid process, the interclavicle is missing, and the clavicles are often reduced or wanting (Fig. 29E). Consequently the pectoral girdle of most mammals consists of the scapulae only, which are correspondingly enlarged and important as places of muscle attachment.

Study the pectoral girdle of the rabbit or cat. It consists of two pairs of bones, the clavicles and the scapulae. The clavicles are small slender bones imbedded in the muscles of the front of the shoulder, and as they are not articulated to the rest of the girdle they generally fall off in prepared skeletons. Isolated clavicles will be demonstrated and they will be seen in place later when the

' Regarded in some texts, however, as the second and third, and the metacarpal here called second as the first. According to Lillie, Development of the Chick, the embryological evidence strongly indicates that the persistent metacarpals are the second, third, and fourth. 
muscles are dissected. The scapulae or shoulder blades are the large, flat triangular bones situated dorsal to the anterior ribs. The mammalian scapula has certain characteristics which differentiate it readily from the scapulae of other vertebrates. It is triangular in form, the apex of the triangle articulating with the humerus. Its outer surface bears a prominent ridge, the spine of the scapula. The ventral end of the spine terminates ventrally in a pointed projection, the acromion process; just above this and projecting laterally and posteriorly is the metacromion process, very long in the rabbit. The apex of the scapula is concavely curved, forming the glenoid fossa. From the anterior side of the rim of the fossa a small beaklike process projects medially; this is the coracoid process, the vestige of the coracoid bone. In mammalian embryos, this process is a separate bone. For facilitating the description of muscle attachments the various surfaces and borders of the scapula are named as follows: the part of the external surface anterior to the spine is the supraspinous fossa; the part posterior to the spine, the infraspinous fossa; the whole of the internal surface is the subscapular fossa; the dorsal border is the vertebral border; the anterior margin, the anterior border; the posterior margin, the axillary border. Draw the scapula from the outer surface.

The sternum consists of a longitudinal series of pieces, the sternebrae, eight in the cat, six in the rabbit. The first sternebra is called the manubrium and articulates with the first thoracic rib at its center. The next six (cat) or four (rabbit) sternebrae constitute the body of the sternum. The last piece is called the xiphisternum and terminates in a xiphoid or ensiform cartilage. Note points of articulation of the sternebrae with the ribs.

The fore limb is fairly typical. The humerus has a large rounded head fitting into the glenoid fossa and greater and lesser tuberosities at the sides of the head. The anterior surface of the humerus below the tuberosities is slightly elevated into ridges or crests (two in the cat, one in the rabbit) which serve as points of muscle attachment. The lower end of the humerus is rounded for articulation with the bones of the forearm and is divided into two portions, an outer mass, the capitulum, and a medial mass, the trochlea. Above the capitulum is a projecting ridge, the lateral epicondyle; and a similar medial epicondyle is situated above the trochlea. Near the medial epicondyle the bone is pierced by an opening, the supracondyloid foramen, absent in the rabbit.

The forearm consists of radius and ulna, the latter the larger. The proximal end of the ulna forms a prominent projection, the elbow or olecranon. Distal to this is a deep semicircular concavity, the semilunar notch, which articulates with the trochlea of the humerus. The distal border of the notch forms another projection, the coronoid process. Observe that the proximal end of the radius is situated lateral to or in front of the proximal end of the ulna, while its distal end is medial to the distal end of the ulna. In other words, the radius crosses obliquely in front of the ulna. The cause of this crossing was explained in Section III. 
The wrist is composed of a number of small bones, arranged in two transverse rows. The proximal row consists of four pieces in the rabbit, three in the cat; the distal row of five in the rabbit, four in the cat. Articulating with the distal end of the radius is the large scapholunar in the cat, separated in the rabbit into a medial navicular (radiale) and a lateral lunate bone (intermedium). Lateral to the lunate portion or bone and articulating with the ulna is the triquetral bone (ulnare). The pisiform is the element projecting prominently lateral to the triquetral bone in the cat or behind it in the rabbit. The distal row of pieces beginning at the medial side and proceeding laterally are: the greater multangular (first carpale), the lesser multangular (second carpale), the central (centrale, missing in the cat), the capitate (third carpale), and the hamate (fourth and fifth carpales fused). These carpales are situated at or near the proximal ends of their respective metacarpals. There are five metacarpals of which the first is very much reduced, and five digits whose terminal phalanges support the horny claws.

\section{GENERAL SUMMARY OF THE GIRDLES, THE STERNUM, AND THE}

\section{PAIRED APPENDAGES}

I. The paired appendages probably represent remnants of a pair of continuous lateral fin folds, supported by cartilaginous fin rays.

2. The two girdles probably arose through the fusion in the median line of some of these fin rays.

3. The primitive girdles are bars or plates of cartilage in which subsequently ossification occurred with the formation of cartilage bones.

4. In each girdle three pairs of cartilage bones arise. These are ilium, pubis, and ischium in the pelvic girdle; scapula, procoracoid, and coracoid in the same respective positions in the pectoral girdle.

5. The pelvic girdle after the three pairs of bones have arisen undergoes little change. In birds and mammals the boundaries between the bones are lost by fusion, producing on each side an innominate bone. There are never any membrane bones associated with the pelvic girdle.

6. The pelvic girdle is free in fishes but in all other vertebrates is firmly articulated or ankylosed (i.e., immovably fused) to the sacrum by means of the sacral ribs.

7. The pectoral girdle, on the other hand, exhibits many modifications among the vertebrates and is further complicated by the addition of membrane bones.

8. In the majority of the land vertebrates only two of the three cartilage bones of the primitive girdle persist. The scapula is always present. One ventral element is generally present, called the coracoid, and believed by some to be homologous with the coracoid in the primitive girdle, by others to be homologous with the procoracoid.

9. In all of the placental mammals the coracoid element is reduced to a vestige, the coracoid process, borne on the scapula.

Io. The mammalian scapula is distinguished by the possession of a spine and of the coracoid process mentioned in paragraph 9 .

II. The membrane bones added to the pectoral girdle vary in different forms, but in living land vertebrates usually consist of paired clavicles and a median unpaired interclavicle.

12. The pectoral girdle is very rarely connnected with the vertebral column by an articulation or ankylosis. 
13. The sternum occurs first in the Amphibia and is found in most vertebrates above Amphibia. It is probably the median ventral portion of the pectoral girdle. In many reptiles, and in birds and mammals, the ribs articulate with the sternum.

14. The bones of the limbs are probably derived from the cartilaginous fin rays of the lower fishes. They are very similar in all land vertebrates, the distal portions being subject to the most modifications.

15. The limbs are divided into three segments. The bones of these segments are as follows, those of the hind limb being named first: proximal segment, one bone, femur or humerus; middle segment, two parallel bones, tibia and fibula, radius and ulna; distal segment, composed of three parts, ankle or wrist, sole or palm, and digits. Ankle or wrist consists primitively of nine or ten bones in three rows: a proximal row of three, named tibiale or radiale, intermedium, and fibulare or ulnare; a middle row of one or two centrales; and a distal row of five tarsales or carpales. Loss or fusion of any of these elements is quite common. The palm or sole is composed of five parallel bones, the metatarsals or metacarpals. The digits consist of phalanges of which the primitive numbers are, proceeding from the first to the fifth digit, $2,3,3,3,3$. Loss of digits is also common; nearly always the inner and outer digits disappear with retention of middle ones; very rarely are middle digits lost. Extra digits may also occur, usually on aquatic forms. 


\section{THE ENDOSKELETON: THE COMPARATIVE ANATOMY OF THE SKULL AND THE VISCERAL SKELETON}

The skull or cranium is that part of the endoskeleton which is found within the head where it covers and protects the brain. The skull is the most complex of all the parts of the endoskeleton, owing in part to the fact that it is derived from several different sources. To determine the homology of the bones of the skull of different vertebrates is a tremendous task which is not yet completed. The earlier investigators named bones in different skulls by the same names without any adequate evidence that they really were homologous, with
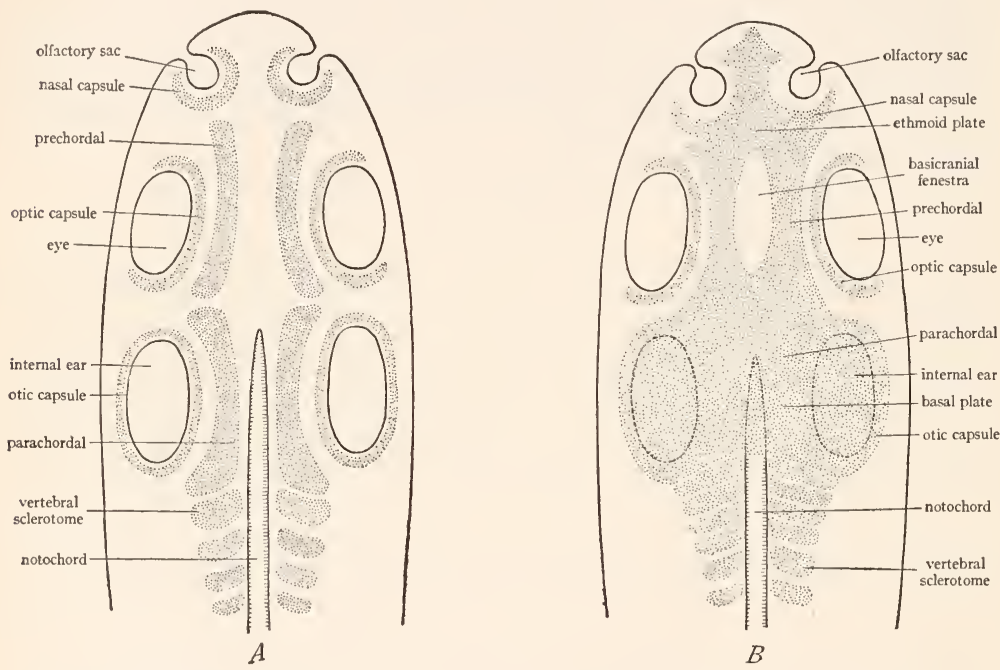

FIG. 3I.-Diagrams to illustrate the development of the chondrocranium from the prechordals parachordals, sense capsules, and sclerotomes of the vertebrae of the occipital region. $A$, early stage with the various cartilages separate. $B$, later stage, showing union of the prechordals with each other and with the olfactory capsules to form the ethmoid plate, and union of the parachordals with each other and with the otic capsules and occipital vertebrae to form the basal plate. (Combined from figures by Goodrich and Wilder.)

resulting confusion. In the following pages an attempt is made to facilitate the study of the skull by analyzing the skull into its various components and tracing in the phylogenetic scale the union of these components into the complete skull.

\section{A. THE CARTILAGE STAGE OF THE SKULL}

I. Origin of the chondrocranium. - The skull arises in the mesenchyme of the head. The first step in the formation of the skull is the production of cartilage by the mesenchyme. Five pairs of cartilages appear. One pair forms along the sides of the anterior end of the notochord; 
these are designated as the parachordal cartilages. Another pair is laid down immediately anterior to the parachordals; these are the prechordal cartilages or trabeculae. Cartilaginous capsules also develop around each of the paired sense organs of the head-olfactory sacs, eyes, and ears. These are known respectively as the nasal capsules around the olfactory sacs, the optic capsules around the eyes, and the otic capsules around the internal ears (Fig. 3I $A$ ). Fusion of these five paired cartilages then occurs. The anterior ends of the prechordals fuse together to form the ethmoid plate which in turn fuses laterally with the nasal capsules. The prechordals continue in front of the ethmoid plate as two projections, the horns or cornua trabeculae. The parachordals unite to form the basal plate which then fuses on either side with the otic capsules. The posterior ends of the prechordals unite with the basal plate (Fig. 3 I $B$ ). The optic capsules never become fused to the skull since it is necessary that the eyes remain independently movable; the optic capsules persist around the eye as a tough protective coat, the sclera. As a result of the fusion of prechordals, nasal capsules, parachordals, and otic capsules, a nearly continuous plate of cartilage is formed below the ventral side of the brain and inclosing the sense organs. In addition, it is highly probable that certain vertebrae are fused to the posterior end of the basal plate. The cartilaginous structure arising in this way is named the chondrocranium. It occurs as a stage in the development of the skull in the embryos of all vertebrates. It must be emphasized that in the majority of vertebrate embryos the chondrocranium has the form of an elongated, somewhat curved plate ventral to the brain and supporting the brain on its concave dorsal surface. Generally it has neither lateral nor dorsal walls except at the extreme pos-

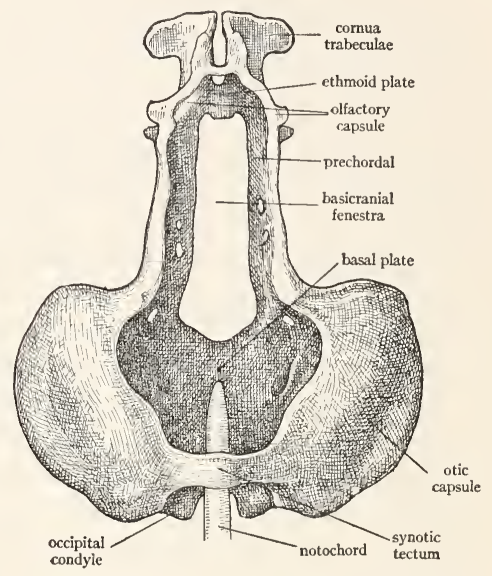

FIG. 32.-Chondrocranium of a urodele larva. (After Gaupp in Hertwig's Handbuch der vergleichenden und experimentellen Entwickelungslehre der Wirbeltiere.) terior end where it gives rise to a narrow dorsal arch, the synotic tectum. Usually there are gaps in this plate, known as fenestrae (Fig. 32). In the elasmobranch and other fishes the chondrocranium gradually extends dorsally and finally incloses the brain in a cartilaginous box. The roof of this is generally incomplete, presenting one or more gaps, the fontanelles, closed by membranes. Diagrams of the formation of the chondrocranium are given in Figure 3I; similar figures will also be found in $\mathrm{W}, \mathrm{Wd}, \mathrm{P}$ and $\mathrm{H}$, and $\mathrm{K}$.

For more complete accounts of the development and comparative anatomy of the skull, $\mathrm{K}, \mathrm{W}$, and $\mathrm{Wd}$ should be consulted.

2. The chondrocranium of the dogfish.-Study the chondrocranium of the dogfish preserved in jars. It is a cartilaginous mass inclosing the brain and without sutures or divisions. The dorsal surface is broad and flat while the ventral surface is narrower and more irregular. The anterior end of the chondrocranium is drawn out into a troughlike structure, the rostrum. Each side of 
the chondrocranium presents a large depression, the orbit, which in life holds the eye.

a) Dorsal surface of the chondrocranium: Study the dorsal surface. The rostrum opens dorsally by a large, egg-shaped cavity, the anterior fontanelle. At either side of the base of the rostrum is an olfactory capsule, a projecting structure with thin walls. Posterior to the capsule and continuous with its dorsal wall is a thick projecting shelf, the supraorbital crest, which forms the dorsal wall of the orbit. The posterior end of the crest continues into a projection, the postorbital process. Along the medial borders of the supraorbital crests runs a row of openings, which are nerve foramina, i.e., openings through which nerves pass to and from the brain. In the median line just back of the anterior fontanelle is an opening, the epiphyseal foramen, through which a portion of the brain (epiphysis or pineal body) extends. In the median line of the posterior part of the roof is a rounded depression, the endolymphatic fossa, in which are situated two pairs of openings, the terminations of the endolymph (smaller outer holes) and perilymph (larger medial holes) ducts of the internal ear. These ducts are canals which connect the fluid-filled channels of the ear with the surface of the skull. On each side of the endolymphatic fossa is a massive region which is the auditory or otic capsule, fused to the cranium. The posterior end of the cranium bears an opening, the foramen magnum, visible dorsally just back of the endolymphatic fossa. Through this opening the brain is continuous with the spinal cord. Draw the chondrocranium from the dorsal side.

b) Ventral and lateral surfaces of the chondrocranium: The ventral surface of the rostrum bears a projecting carina or keel. On either side of this medial keel is an oval opening, which may be designated the rostral fenestra. Through the rostral fenestrae one can look into the cavity of the cranium, which in life is occupied by the brain. Lateral to each rostral fenestra is the thin-walled, olfactory capsule. In most specimens part of the walls of this will probably be broken away. When complete, the nasal capsule is a nearly spherical structure with a relatively small opening to the exterior. At the bottom of the nasal capsule is a large opening which leads into the cavity of the skull. Posterior to each nasal capsule and continuous with its posterior wall is the preorbital process, which forms the anterior wall of the orbit. The walls of the orbit are pierced by nerve foramina of which the largest, located in the anteroventral region of the orbit, is the optic foramen, through which the optic nerves pass from the eye. ${ }^{x} \quad$ The ventral wall of the skull between the two orbits is rather narrow, its narrowest portion being the place where a process of the upper jaw articulates. The posterior part of the ventral surface forms a broad basal plate, whose sides are composed of the otic capsules. In the median line of the basal plate will be seen a streak of slightly different color from the chondrocranium.

${ }^{2}$ Projecting into the orbit may be present a mushroom-shaped structure, the optic pedicel, which in life supports the eyeball. 
This streak is the notochord. Its anterior end turns dorsally into the cartilage and terminates at about the level of the small median foramen lying near the anterior end of the basal plate through which the internal carotid artery passes. It will be understood that those portions of the chondrocranium lying to either side of the notochord are the products of parachordals and that the parts anterior to this up to the olfactory capsules are the products of the prechordals. The parachordals are fused laterally with the otic capsules and the prechordals with the olfactory capsules. The lateral and dorsal walls of the brain are produced by extension of these original parts. The posterior end of the ventral surface of the skull is produced at either side of the notochord into a slight process, the occipital condyle, which articulates with the first vertebra. Draw a ventral view of the chondrocranium.

\section{B. THE VISCERAL SKELETON}

The visceral skeleton or splanchnocranium is that part of the endoskeleton which supports the gills. The gills are located in the ventral and posterior part of the head. To support the gills and enable them to be moved for respiratory purposes a special skeleton is present which is called the visceral skeleton, because gills are part of the walls of the digestive tract, as will be demonstrated later. The visceral skeleton consists of a longitudinal series of crescent-shaped cartilages (or bones) situated between the gill slits in the pharyngeal wall. Each such element is designated a gill arch. There are typically seven gill arches in vertebrates, although some elasmobranchs have nine (Fig. 33). The gill arches in the lower vertebrates are closely associated with the chondrocranium, and in the course of evolution some of them take part in the production of the skull.

I. The visceral skeleton of the dogfish.-Obtain a specimen in which the visceral skeleton has been left attached to the chondrocranium, and study it carefully. The seven gill arches form a series of curved cartilages ventral to the posterior part of the chondrocranium and extending posteriorly to the pectoral girdle. The first gill arch, the mandibular arch, is the largest and most modified of the series. It is seen when viewed from below to consist of dorsal and ventral halves. Each side of the dorsal half is called the palatoquadrate or pterygoquadrate cartilage; in profile view it will be seen that this cartilage is closely applied to the ventral surface of the chondrocranium, to which in life it is attached by a ligament. It sends up a well-developed palatal process into the orbit. The pterygoquadrate cartilages bear teeth and, in fact, constitute the upper jaw of the animal. The ventral half of the mandibular arch consists of two halves, each of which is known as Meckel's cartilage. These bear teeth and together constitute the lower jaw of the dogfish. The wide gap between the two jaws is the mouth opening. At their posterior ends the pterygoquadrate and Meckel's cartilages join at an acute angle called the angle of the jaw, forming a hinge joint, permitting opening and closing of the mouth. 
The second or hyoid arch is more slender than the mandibular arch to the posterior face of which it is closely applied. It consists of a ventral median piece, the basihyal; a slender bar, the ceratohyal, on each side of the basihyal; and a stout piece, the hyomandibular, dorsal to each ceratohyal. The hyomandibular articulates to the otic region of the skull and thus acts as a suspensor of the lower jaw. The hyomandibular bears on its posterior margin some slender projections, the gill rays, which in life support the gills.

The remaining arches, known simply as gill or branchial arches, are similar to each other. Each consists typically of five pieces, named from the dorsal side ventrally: pharyngobranchial, the most dorsal piece, elongated and directed posteriorly; epibranchial, the succeeding much shorter piece; ceratobranchial,

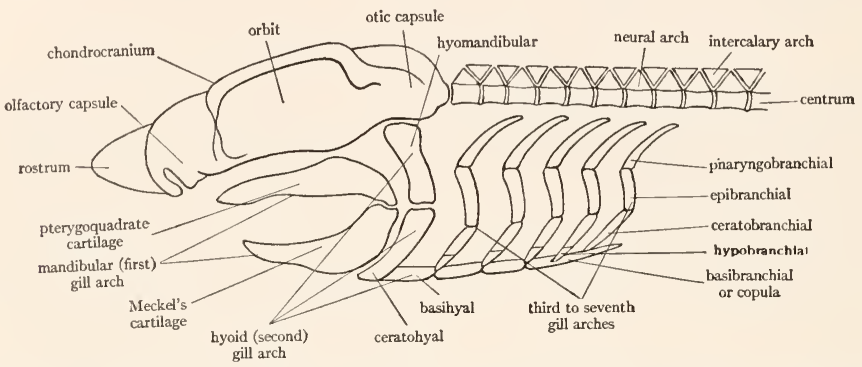

FIG. 33.- Diagram of the chondrocranium, vertebral column, and gill arches of an elasmobranch to show particularly the parts and relations of the seven gill arches. (Slightly modified from Vialleton's Éléments de Morphologie des Vertébrés.)

another elongated piece; hypobranchial, curved ventral pieces, of which there are but three pairs to the five branchial arches; and the basibranchials, two in number-an anterior small one situated between the medial ends of the first and second pairs of hypobranchials and a large posterior piece between the bases of the fifth ceratobranchials and terminating in a caudally directed point. Epi- and ceratobranchials bear gill rays. Note that the gill arches are not attached to the vertebral column. A diagram of the gill arches is given in Figure 33 .

Draw from the side, showing chondrocranium and visceral skeleton.

\section{THE FORMATION OF THE MEMBRANE BONES OF THE SKULL}

I. Origin of the membrane bones of the skull.-In addition to the chondrocranium and gill arches, still another component enters into the formation of the skull. This component consists of the dermal or membrane bones of the skull. These elements first appear in the ganoid fishes. In these forms it can be observed that the ganoid scales have become fused on the head to form large bony plates incasing the head. These scales, which as we have already learned, are dermal in origin, sink into the head and apply themselves closely to the chondrocranium and mandibular arch. It has already been pointed out that in the majority 
of vertebrates the chondrocranium consists chiefly of a ventrally located plate and lacks side walls and roof. Walls and roof are completed by these dermal scales, which thereupon become the superficial bones of the skull. Similarly dermal scales incase the pterygoquadrate and Meckelian cartilages, which are the primitive upper and lower jaws, and become the superficial bones of the jaws. In this fashion the entire skull becomes sheathed in dermal scales, originating in the skin and in reality belonging to the exoskeleton. These incasing bones of the skull and jaws are known as dermal, membrane, or investing bones, because of their manner of origin. The membrane bones of the skull are present in their most complete and generalized condition in the earliest Amphibia (Stegocephala) and the earliest reptiles (Cotylosauria); but in the course of evolution gradually decrease in number owing to processes of loss and fusion (see Fig. 36, p. 106).

2. Membrane bones of some typical ganoids. - Study the ganoid scales on the head of any one of the following ganoids: Polypterus, sturgeon (Acipenser), the gar pike (Lepidosteus), or the bowfin (Amia). Some of these enlarged scales on the heads of ganoid fishes correspond to the superficial bones of the skull of higher vertebrates, and have received the same names; others, especially those supporting the operculum, have been lost subsequently. The student should understand that these dermal scales are on the outside of the animal's head, imbedded in the skin, and that a typical cartilaginous or partially ossified chondrocranium, like that of the dogfish, is present inside of the covering of scales.

a) Membrane bones of Polypterus: (see Fig. $34 B$ ) The dermal scales on the head of Polypterus are very much like the bones on the dorsal side of the skull of higher forms. Identify on the dorsal side of the head the two small nasal openings near the tip and the larger oval orbits posterior to them. In front of the nasal openings is a pair of bones bearing teeth, the premaxillae; behind the nasal openings is a pair of nasals; between the two nasals is situated a small triangular membrane bone, the dermal mesethmoid. Posterior to the nasals are two large frontal bones, and posterior to them, two smaller parietal bones. The several small bones posterior to the parietals are called temporals. A row of small bones extends from each orbit posteriorly; these are postorbitals. Below the orbit is an elongated bone bearing teeth, the maxilla. The sides of the head behind the orbit are covered by large flat bones, the operculars. The lower jaw is similarly clothed in dermal bones, consisting of a tooth-bearing dentary in front and a toothless angular behind.

b) Membrane bones of the sturgeon: (Fig. $34 \mathrm{C}$ ) The anterior end of the sturgeon's head is extended into a snout or rostrum, covered by many small rostral scales. At the sides of the base of the snout are the two pairs of nasal openings, and just posterior to them, the orbit. On the dorsal side between the two orbits are two large scales, the frontals, and posterior to them, two parietals. Numerous other small bones need not be considered. The jaws and visceral skeleton of the sturgeon are degenerate on account of its method of feeding. 

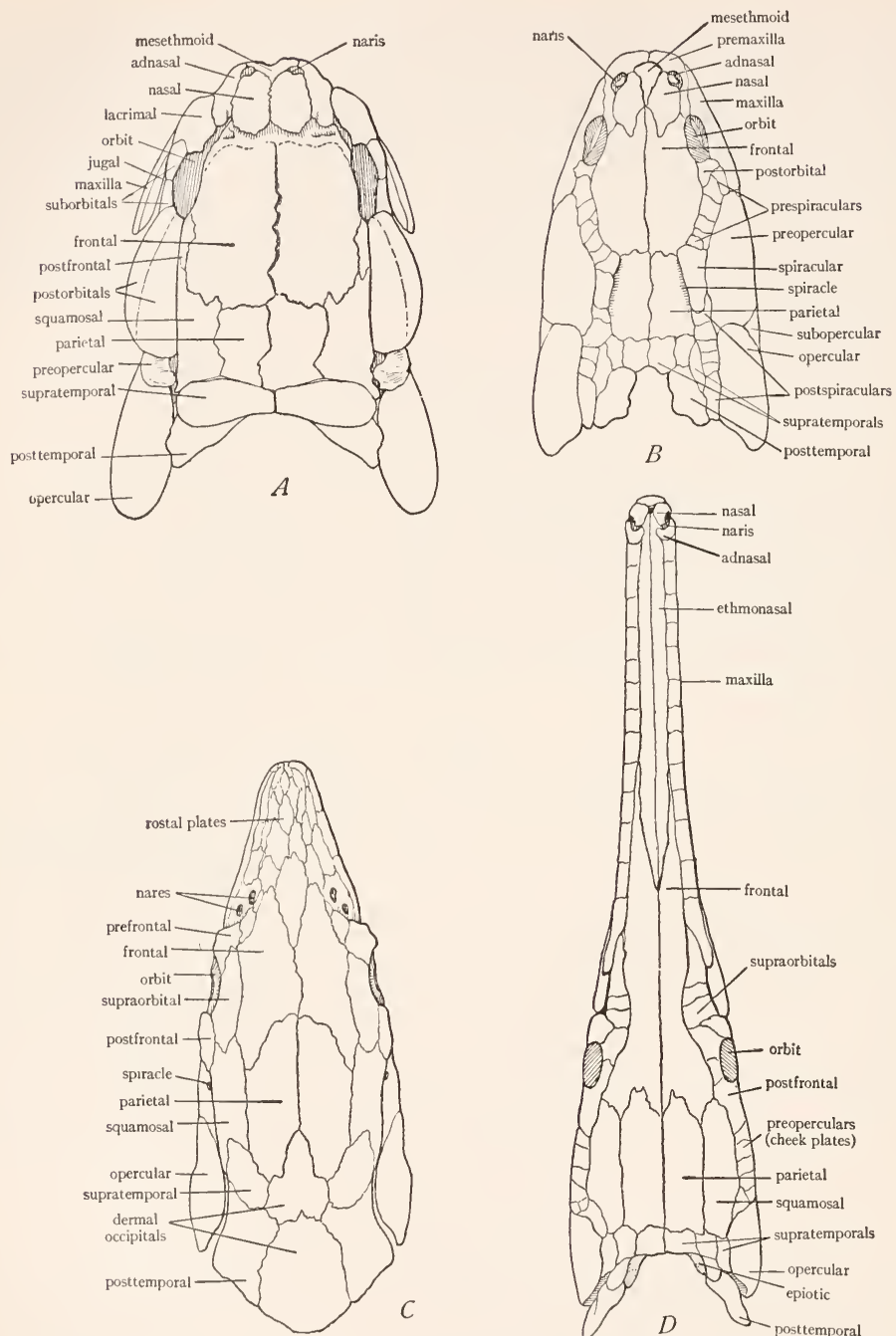

C

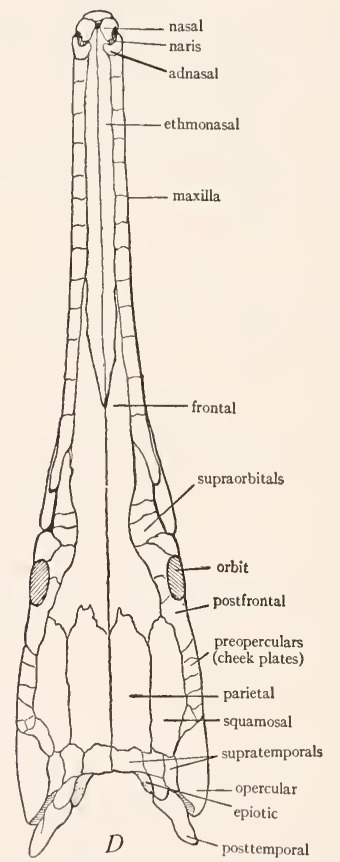

FIG. 34.-Dermal scales (superficial bones of the skull) of the head of some ganoid fishes. $A$, Amia. B, Polypterus. C, sturgeon (Acipenser). D, gar pike (Lepidosteus). The arrangement of some of these scales, which in teleosts become the bones of the dorsal surface of the skull, greatly resembles that of the dorsal skull bones of the land vertebrates, and these scales have received the same names as the skull bones, although the strict homology is doubtful. (After Goodrich in Part IX of Lankester's Treatise on Zoölogy, cnurtesy of the Macmillan Company.) 
c) Membrane bones of the gar pike: (Fig. $34 \mathrm{D}$ ) The head is prolonged into a long snout having the nasal openings at its extremity. The small nasal bones surround the nasal openings. Posterior to these occupying the center of the dorsal surface of the snout are the elongated ethmonasal bones. Posterior to them are the frontals, whose anterior ends inclose the posterior ends of the ethmonasals. Posterior to the frontals are the large parietals and behind them a number of temporals. The edges of the upper jaw are composed of the maxillae, bearing teeth and each consisting of a series of squarish bones. The lower jaws consist chiefly of the long dentary bones, bearing teeth. The sides of the head behind the orbit are covered by a large number of small cheek plates and, posterior to them, by the larger operculars.

d) Membrane bones of the bowfin: The skull of Amia is "perhaps less specialized than in any other living teleostome," according to L (Fig. 34A). Identify on the skull anterior stalked and posterior nostrils and the orbits. Between the anterior nostrils projects the small triangular dermal mesethmoid, a membrane bone. Anterior to this are the premaxillae, bearing teeth. Covering the space between the four nostrils are the nasals, posterior to them in the median line the large frontals, posterior to them the parietals, and at the posterior end of the skull the four temporals. Below and anterior to the orbit is the large lacrimal, below the orbits the two suborbitals, and posterior to the orbit two large postorbitals. Forming the sides of the upper jaw are the tooth-bearing maxillae. Between the dorsal, postorbital, and the parietal is the squamosal. The operculum is supported by several opercular bones.

e) Demonstration of the separate origin of chondrocranium and membrane bones: Examine the demonstration specimen of the head of a ganoid fish in which the incasing membrane bones have been loosened from the underlying chondrocranium. Remove the sheath of membrane bones, noting that they are situated in the skin, and note the chondrocranium, similar to that of the dogfish, lying within the sheath. Such a specimen shows clearly the origin of the skull from two separate sources, the cartilaginous chondrocranium and the dermal scales.

\section{THE FORMATION OF THE CARTILAGE BONES OF THE SKULL AND THE COMPOSITION OF THE COMPLETE SKULL}

The next and last step in the formation of the skull is the production of cartilage bones in the chondrocranium, including the sense capsules, and in the mandibular and hyoid arches. In definite regions of these structures centers of ossification-that is, centers of bone formation-arise. Each of these centers transforms a certain area of cartilage into bone, and each such bone is primitively a cartilage bone of the skull. It has already been stated that in the majority of vertebrates the chondrocranium, present in the embryonic stage only, consists of a ventrally situated plate with neither lateral nor dorsal walls, except at the extreme posterior end (Fig. 32). Consequently, the cartilage bones of the skull, formed in the chondrocranium, are likewise limited to the ventral and extreme posterior parts of the skull, where thev form a floor for the brain. The roof and sides of the skull are completed by membrane 
bones, derived, as we have already seen, from the dermal scales of the ganoid fishes. In a similar way certain cartilage bones arise in the mandibular and hyoid arches and become sheathed in membrane bones. The complete vertebrate skull, therefore, consists of cartilage bones derived from the chondrocranium and its sense capsules, and the first and second gill arches, and of membrane bones covering the cartilage bones everywhere except on the ventral surface and posterior end of the skull. We may now state in detail the bones derived in these ways to form the skull.

I. Cartilage bones derived from the pre- and parachordals.-There are four groups of such cartilage bones: one occipital, two sphenoid, and one ethmoid group (see Fig. 35).

a) Occipital group: This consists of four occipital

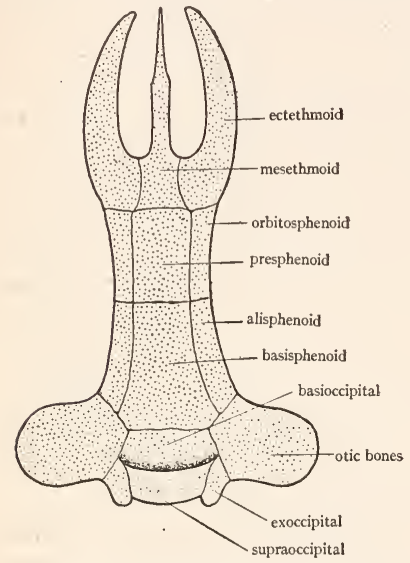

FIG. 35.-Diagram to show the arrangement of the cartilage bones of the skull, seen from above. The separate otic bones, which may be as many as five in number, are not represented. The supraoccipital forms an arch above the basioccipital. (Modified from Kingsley's Comparative Anatomy of Vertebrates.) bones situated at the posterior end of the skull, forming a ring around the foramen magnum. They are one supraoccipital, two exoccipitals, and one basioccipital. They arise from the parachordal cartilages, together with their dorsal extension-the synotic tectum-and the vertebral elements which are fused to the posterior end of the parachordals (Fig. 35).

b) Posterior sphenoid group: This comprises three sphenoid bones, on the ventral side of the skull, in front of the basioccipital. They are the median unpaired basisphenoid and the paired alisphenoids, one on each side extending up into the back wall of the orbit. These bones come from the posterior part of the prechordals.

c) Antcrior sphenoid group: This includes three bones on the ventral side anterior to the preceding and also derived from the prechordal cartilages. They are a median unpaired presphenoid and paired orbitosphenoids, one on each side forming part of the walls of the orbit.

d) Ethmoid group: This group consists of three bones at the anterior end of the ventral side of the skull, just behind and fused with the olfactory capsules. The bones are a median mescthmoid and paired ectethmoids, one on each side of the preceding. The mesethmoid forms a median partition or septum between the two olfactory capsules, while the ectethmoids contribute to the posterior walls of these capsules. The ethmoids arise in the ethmoid plate, which represents the fused anterior ends of the prechordals.

2. Cartilage bones derived from the sense capsules. -

a) From the otic or auditory capsules: A number of otic bones arise in the walls of the otic capsules. Since the latter are fused with the parachordal region of the skull, the otic bones are naturally closely associated with the occipital group of bones and often fused with them. There may be as many as five otic bones (in teleosts), the proötic, epiotic, opisthotic, pterotic, and sphenotic, but they are commonly fused together or fused with nearby bones. The three first named are the ones most constant in the higher vertebrates. When fused into one bone they are designated as the periotic or petromastoid bone.

b) From the optic capsules: As already explained, the optic capsules do not fuse with the skull, owing to the necessity for retaining free movement in the eyes. The optic capsule 
generally forms in vertebrates a tough membrane, the sclera or sclerotic coat, which is the outer coat of the eyeball. In some vertebrates, however, particularly birds and reptiles, a ring of sclerotic bones arises in the sclera, but these always remain free from the skull.

c) From the olfactory capsules: Additional cartilage bones, closely fused to and almost indistinguishable from the ethmoid bones already mentioned may arise in the walls of the olfactory capsules. These are the lateral ethmoids and the turbinals or conchae in part. The latter are curiously scrolled or grooved bones situated on the walls of the nasal cavities.

\section{Cartilage bones derived from the gill arches. -}

a) From the pterygoquadrate cartilage: These cartilages form, as we have seen, the primitive upper jaw and the dorsal half of the first or mandibular gill arch. The anterior part of these cartilages may form the palatines, the middle part one or more paired pterygoids, while the posterior part invariably gives rise to the paired quadrate bones to which the lower jaw is generally articulated. It should be noted that the bones designated in various vertebrates as palatines and pterygoids are in some cases cartilage bones derived from the pterygoquadrate cartilage and in many cases are membrane bones. The quadrates are therefore the only constant cartilage bones originating from the upper jaw (Fig. 37, p. 107).

b) From Meckel's cariliage: These two cartilages constitute the two halves of the lower jaw and the ventral half of the first gill arch. The greater part of Meckel's cartilages fails to ossify, and either remains as a cartilaginous core of the lower jaw or disappears. The posterior end of each cartilage commonly ossifies to form the articuiar bone which articulates with the quadrate, forming a hinge joint for the lower jaw. The anterior tip of the cartilage may rarely produce a cartilage bone (mentomeckelian bone). The articular bones are thus the chief contribution of the lower jaw to the skull.

c) From the second or hyoid gill arch: The dorsal portion of the hyoid arch forms the hyomandibular cartilage as we saw in elasmobranchs. This ossifies into a hyomandibular bone, which in many fishes suspends the lower jaw. The remainder of the hyoid arch together with parts of the succeeding gill arches forms the hyoid apparatus. This is a plate or bar of cartilage or bone, situated in the floor of the mouth cavity and throat, from which processes extend posteriorly on each side to the otic region. It is variable in form and composition in different vertebrates.

d) From the remaining gill arches: These arches ossify in fishes, but above fishes gradually retrogress and become much modified, taking part in the formation of the hyoid apparatus and transforming into the cartilages of the larynx. The larynx is the Adam's apple, so-called, of man, and is the chamber at the top of the windpipe from which the voice comes. The walls of this chamber are supported by cartilages which are the remains of the cartilages of the gill arches. Wd, page 374 , gives a figure to show the origin of the larynx of man from the gill arches.

4. Membrane bones added to the dorsal surface of the skull.-As has been said, the chondrocranium is open dorsally (except in fishes) and no cartilage bones are ever formed there in any vertebrates, except at the extreme posterior end. Instead, the roof of the skull consists of membrane bones, evolved from the dermal scales of the ganoid fishes. The chief membrane bones covering the olfactory capsules and roof of the skull are: nasals, lacrimals, brefrontals, frontals, posifrontals, and parietals. A number of others are present in fishes, such as orbitals, temporals, operculars, etc., but as these do not persist, we shall not consider them further. The earliest Amphibia and reptiles also had a considerably larger number of membrane bones in the roof of the skull than do any living land vertebrates. These are represented in Figure $36 A$ and $B$. From such a generalized condition the dorsal aspect of the skull of present-day land vertebrates has resulted through the loss of certain bones (see Iig $36 C$ and $D$ ). 

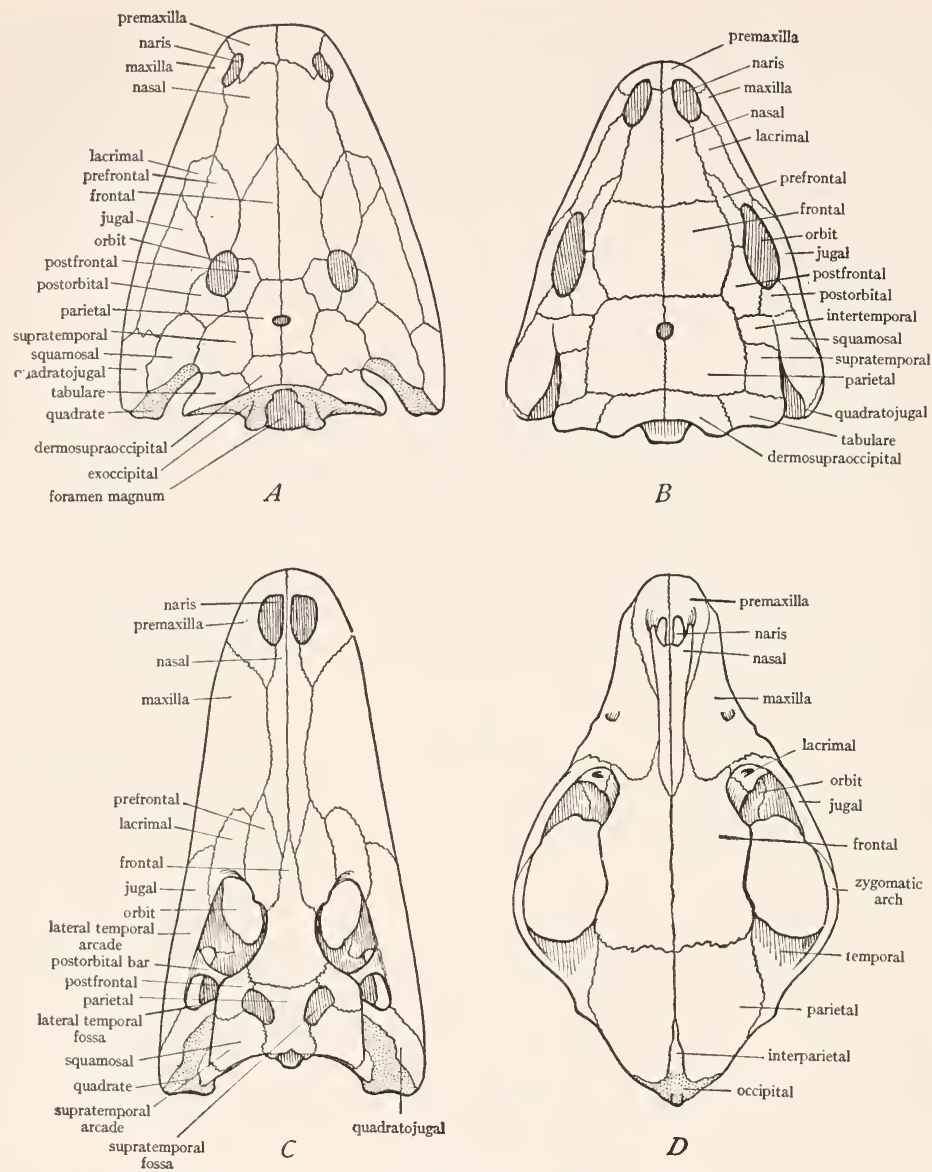

FIG. 36.-Dorsal view of the skulls of four representative vertebrates to show the reduction of the membrane bones of the roof in the course of evolution. A, skull of an extinct amphibian, Capitosaurus, belonging to the Stegocephala; note the large number of membrane bones completely roofing the skull. $B$, skull of one of the most ancient reptiles, Seymouria, belonging to the Cotylosauria; the membrane bones are nearly as numerous as in the amphibian, are similarly arranged, and completely roof the skull. $C$, skull of a modern reptile, the alligator; several of the membrane bones present in the extinct forms have been lost, and the roof bears several openings. $D$, skull of a modern mammal, the dog, showing still greater loss of membrane bones. Membrane bones blank; cartilage bones stippled. (A from Reynolds' The Vertebrate Skeleton, courtesy of the Macmillan Company; B from Williston's Water Reptiles of the Past and Present, University of Chicago Press.) 
5. Membrane bones added to the ventral surface of the skull.-These are from the anterior end, posteriorly, chiefly: vomers, palatines, pterygoids (Fig. 37). It should be stated again that palatines and pterygoids are membrane bones in some animals and cartilage bones in others. The parasphenoid is a membrane bone which in Amplibia typically forms the chief bone on the ventral surface of the skull, but it does not persist in higher forms. These membrane bones are situated ventral to the ethmoid and part of the sphenoid bones, which they conceal from surface view, but the posterior part of the ventral side of the skull is composed of cartilage bones, sphenoids and occipitals.

6. Membrane bones added to the jaws, and other gill arches.-

a) Upper jaw: The upper jaw (pterygoquadrate cartilages) becomes incased in membrane bones. The chief ones from the tip of the jaw posteriorly are, on each side: premaxilla, maxilla, jugal (malar), quadratojugal, and squamosal (see Fig. 37). The upper jaw in elasmobranchs is separate from the chondrocranium, to which it is generally attached by ligaments, but in all of the land vertebrates the upper jaw is inseparably fused to the ventral side of the chondrocranium and becomes an integral part of the skull.

b) Lower jaw: The lower jaw (Meckel's cartilages) is similarly sheathed in membrane bones, Meckel's cartilage often persisting within them. The chief membrane bones of the lower jaw are the dentary, the splenial, the angular, the surangular (also given as supra-angular), the coronoid, the gonial, and two or three others occurring only in extinct forms. In the evolution of the lower jaw there has been a continual decrease in the number of membrane bones (see Fig. 38).

c) Other gill arches: No membrane bones occur in connection with the hyoid

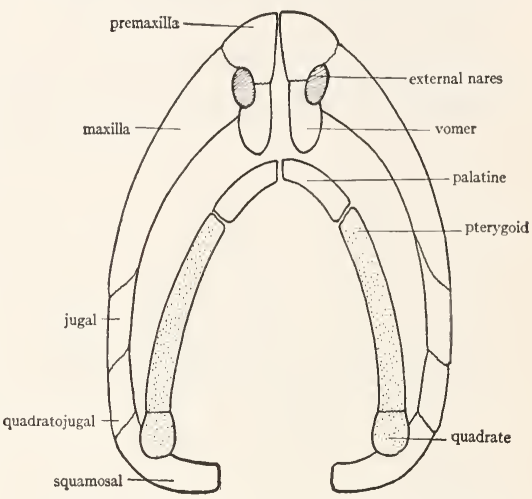

FIG. 37.-Diagram of the cartilage and membrane bones of the upper jaw and floor of the skull. Membrane bones blank; cartilage bones stippled. The cartilage bones come from the pterygoquadrate cartilage. (Modified from Kingsley's Comparative Anatomy of Vertebrates.) or other gill arches.

With this account of the composition of the skull in mind we may now turn to the study of some vertebrate skulls.

\section{E. THE SKULL OF NECTURUS, A PARTIALLY OSSIFIED SKULL}

In the skull of Amplibia the ossification of the chondrocranium has proceeded to but a limited extent so that a partially cartilaginous chondrocranium is present from which the incasing membrane bones can be readily separated as in fishes. The pterygoquadrate cartilages are inseparably fused to the ventral and lateral sides of the skull proper and are partially ossified. In the lower jaws Meckel's cartilages are persistent as cores inclosed by membrane bones. The number of membrane bones in the skulls of present-day Amphibia is considerably less than that of extinct forms, such as shown in Figure $36 \mathrm{~A}$. 
For the study of the skull of Necturus, complete skulls, preferably preserved in fluid, and chondrocrania, from which the membrane bones have been removed, should be at hand.

I. General regions of the skull.-The skull is partly bony, partly cartilaginous. The bony part exists in the form of distinct areas or bones, separated from
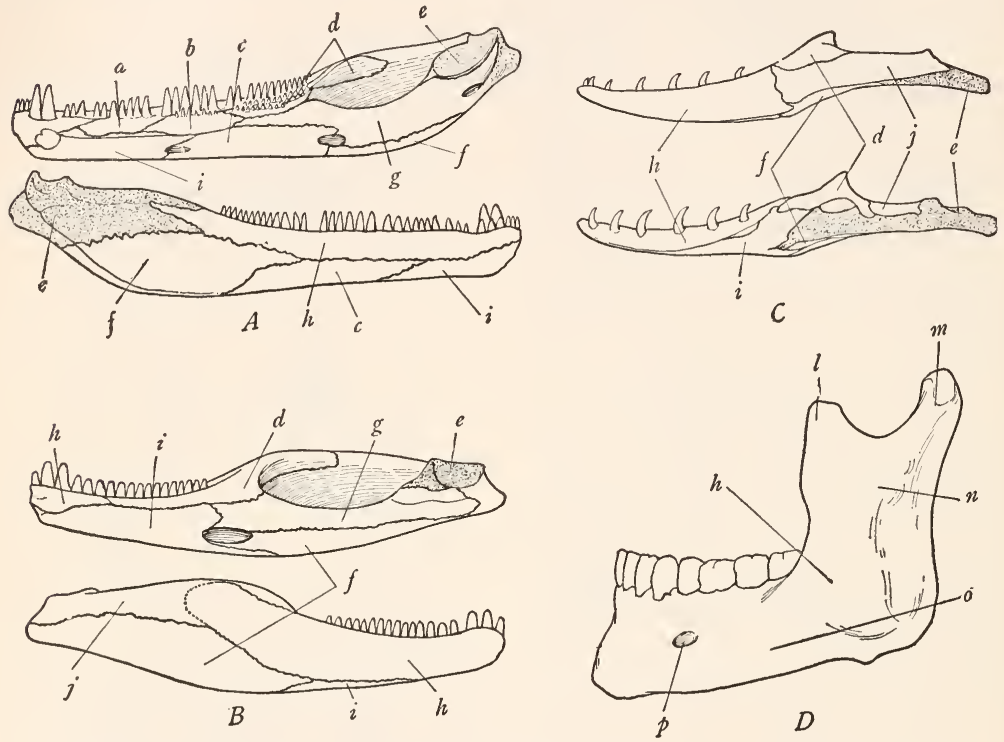

FIG. 38.- Lower jaws of four vertebrates to show the reduction in the number of bones in the course of evolution. A, lower jaw of an extinct amphibian, Trimerorhachis, belonging to the Stegocephala; inner surface above, outer surface below; note large number of membrane bones. $B$, lower jaw of an extinct reptile, Labidosaurus, belonging to the Cotylosauria; inner surface above, outer surface below; note reduction in the number of membrane bones and increased size of the dentary $h$ and the splenial $i . \quad C$, lower jaw of a modern reptile, a lizard, Varamus, showing still farther reduction in the number of bones; outer surface above, inner surface below. $D$, half of the lower jaw of man, seen from the outer surface; it consists of but one bone, the dentary, all other bones having vanished. Membrane bones blank; cartilage bones stippled. $a$, precoronoid; $b$, intercoronoid; $c$, postsplenial; $d$, coronoid; $e$, articular; $f$, angular; $g$, prearticular; $h$, dentary; $i$, splenial; $j$, supra-angular or surangular; $l$, coronoid process; $m$, condyloid process; $n$, ramus; $o$, body; $p$, mental foramen. ( $A$ and $B$ from Williston's Water Reptiles of the Past and Present, University of Chicago Press; $C$ from Reynolds' The Vertcbrate Skeleton, courtesy of the Macmillan Company; $D$ from a specimen loaned by the anatomy department.)

each other along wavy or jagged lines, the sutures. The membrane bones are somewhat distinguishable from the cartilage bones by their more superficial positions. The skull is divisible into a median portion, the skull proper, and 
lateral regions, which constitute the two halves of the upper jaw. The upper jaw forms the margins of the skull and consists in part of tooth-bearing bones. The teeth are arranged in two rows. About the middle of the margin is a projecting process; on examining the ventral surface of this it will be seen to bear a fossa for articulation with the lower jaw. At the posterior end of the skull is an opening, the foramen magnum, through which the brain is continuous with the spinal cord. On either side ventral to the foramen magnum is a projection, the occipital condyle, bearing a smooth face for articulation with the atlas. On each side of the foramen magnum and extending anteriorly is an expanded region with partially cartilaginous walls. This is the otic capsule fused on each side to the occipital region of the skull. At the anterior end of each otic capsule is a depression for the passage of nerves. Anterior to this depression is another, the orbit, in which the optic nerve runs to the eyeball. Anterior to the orbit is a slitlike cavity, the base of the cavity of the olfactory capsules.

\section{Bones of the skull proper.-}

a) Membrane bones of the roof of the skull: The roof of the skull is formed by two pairs of membrane bones, in the median region-a posterior pair of parietals and an anterior pair of frontals. The parietals extend from the dorsal rim of the foramen magnum to a little beyond the middle of the roof where they terminate in a median point. The frontals lie on each side of and partially cover the anterior end of the parietals and extend forward nearly to the tip of the skull.

b) Membrane bones of the floor of the skull: Examine the ventral surface. It is composed almost entirely of the very large parasphenoid or parabasal bone extending from the occipital condyles forward nearly to the tip of the skull. It is shaped somewhat like a short-necked bottle, the neck of the bottle lying between a pair of bones, the vomers, which complete the sides of the anterior part of the ventral surface. Each vomer bears teeth on its margin and extends to the dorsal side, forming the floor of the nasal capsule. On the ventral side between the anterior parts of the vomers and in front of the termination of the parasphenoid is a cartilage, the ethmoid plate, which is the anterior part of the chondrocranium.

c) Cartilage bones of the chondrocranium and otic capsules: The chondrocranium is ossified only at its posterior end, all the rest of it remaining in the cartilage stage. The ossification consists of a pair of exoccipital bones which bear the occipital condyles. The exoccipitals, besides bearing the occipital condyles, form the lateral walls of the foramen magnum; the dorsal rim of the foramen magnum is formed by a strip of cartilage, the synotic tectum, which is partially covered by the posterior ends of the parietals.

The otic capsules are partially ossified each containing two cartilage bones, the opisthotic and the proötic. The opisthotic is lateral to the exoccipital and is a cone-shaped bone composing the projecting angle of the skull noticeable on each side of the occipital region. The dorsal portion of the opisthotic articulates 
with the parietal. In front of the dorsal portion of the opisthotic is an area of cartilage, and in front of this is the proötic bone. Extending from the opisthotic obliquely forward and laterally is a slender membrane bone, the squamosal. The posterior part of the squamosal covers a part of the opisthotic and lies above the lateral border of the otic capsule. The proötic bone is wedged in between the squamosal and the parietal. On turning the skull laterally so as to obtain a lateral view of the otic capsule a small rounded bone will be found situated in the lateral wall of the otic capsule. It articulates behind with the opisthotic and bears a dorsally projecting process which meets a ventrally directed process from the middle of the squamosal. This rounded bone is called the columella and is believed to be equivalent to the hyomandibular bone of fishes, that is, the dorsal portion of the hyoid gill arch. The internal ear is located inside of the proötic bone, and the columella fits into an opening in the proötic bone, called the oval window or fenestra ovalis. The columella, when the middle ear appears (first in Anura), becomes a bone of the middle ear, and it, or at least that portion of it which fits into the fenestra ovalis, is probably homologous with the stapes of the mammalian middle ear. The homologies of the middle ear bones are, however, in some doubt (see K, pp. 8o-82).

3. Bones of the upper jaw.-The upper jaw, composed originally of the two pterygoquadrate cartilages, is fused to the skull proper and forms its lateral portions. It consists, in Necturus, in part of the persistent portions of the pterygoquadrate cartilages, in part, of the cartilage bones formed in these cartilages, and in part of membrane bones ensheathing and replacing the cartilage.

a) Membrane bones of the upper jaw: The anterior tip of the jaw is formed of the premaxillae, $\mathrm{V}$-shaped bones, the angle of the $\mathrm{V}$ directed forward and constituting the tip of the snout. One limb of the $V$ is a slender process situated dorsally on top of the anterior end of the frontal bone. The other limb of the $\mathrm{V}$ is a tooth-bearing process forming the margin of the anterior end of the skull. Posterior to the premaxilla is the tooth-bearing vomer already mentioned and not regarded as a bone of the jaw. Posterior to the vomer is the palatopterygoid bone, the anterior portion of which bears teeth. From the projecting angle to which the lower jaw articulates to the posterior angle of the otic capsule extends the slender squamosal bone.

b) Cartilage bones of the upper jaw: The quadrate bone forms the fossa into which the end of the lower jaw articulates and extends posteriorly from the fossa for a short distance in contact with the inner side of the squamosal. Medial to the quadrate bone is a considerable area of cartilage, the quadrate cartilage, representing unossified portions of the pterygoquadrate cartilage. Besides the quadrate bone, a portion of the palatopterygoid bone is likewise a cartilage bone ossified in the pterygoquadrate cartilage.

Draw dorsal and ventral views of the skull, outlining the bones accurately. 
4. The chondrocranium.-When the skull of Necturus is soaked in warm soap solution, the membrane bones can easily be lifted off, revealing the chondrocranium beneath. The chondrocranium (including the olfactory and otic capsules) consists in large part of cartilage with a few cartilage bones, which have already been identified.

Study prepared chondrocrania. The form of the chondrocranium is similar to that illustrated in Figure 32, p. 97. The posterior part of the chondrocranium consists chiefly of the rounded, hollow otic capsules. These are connected dorsally by a narrow arch of cartilage, the synotic tectum, and ventrally by the broader basal plate, formed by the fusion of the two parachordals. The two exoccipital bones are ossified in the basal plate. From the anterior end of each otic capsule a slender curved prechordal cartilage extends forward. Between these is a large space, the basicranial fenestra, which in the complete skull is covered above by frontals and parietals and below by the parasphenoid. Near the anterior end in front of the point where in the complete skull the parasphenoid ends, the prechordals are fused across to form the ethmoid plate, already noted. From the ethmoid plate a slender process continues forward on each side, the cormua or horns of the prechordals. In front of the otic capsule is the quadrate bone and quadrate cartilage. The latter sends processes to the otic capsule and the prechordal. In the walls of the otic capsule, opisthotic, proötic, and columella may be identified and their boundaries more clearly distinguished than in the study of the entire skull. The olfactory capsules are so delicate as to be lost in preparing the chondrocranium.

5. The lower jaw.-The lower jaw consists of a pair of Meckel's cartilages united in front and sheathed for the greater part of their course in membrane bones. The outer surface of each half of the lower jaw consists of the dentary, a membrane bone bearing teeth. The inner surface is formed of two membrane bones, the splenial and the angular. The former is a small bone situated at about the middle of the inner surface, bearing the last group of teeth, consisting of five or six teeth. The angular covers the remainder of the inner surface and passes onto the outer surface at the extreme posterior end of the jaw, below the posterior end of the dentary. The articulating surfaces of the lower jaw are composed of cartilage, which is the posterior end of Meckel's cartilage. This articulates with the quadrate of the upper jaw. Meckel's cartilage runs almost the entire length of the jaw concealed between the dentary and the angular. It can be revealed by removing these membrane bones.

6. The remaining gill arches.-The hyoid and three succeeding gill arches are present in Necturus and are almost completely cartilaginous. Preserved material is necessary for their study. The hyoid arch is a broad somewhat V-shaped cartilage situated just posterior to the lower jaw in the floor of the mouth cavity. On each side it is divisible into two cartilages-a small anterior hypohyal and a much larger posterior ceratohyal. The third gill arch (first true 
gill-bearing arch) is more elongated and slender than the hyoid arch and is likewise divisible into two pieces, an anterior ceratobranchial and a posterior, slightly longer, epibranchial. Between the median ventral portions of the hyoid and third arch is a triangular copula representing a basibranchial piece. The fourth and fifth gill arches are short curved rods of cartilage on each side, composed of epibranchials. At the anterior end of the epibranchial of the fourth arch is a small ceratobranchial. In the median ventral line is a slender bone, the second basibranchial. It will be seen that the gill arches of Necturus are reduced both in number and as regards the pieces of which they are composed as compared with the gill arches of the elasmobranchs. For the typical condition of the gill arches in elasmobranchs see Figure 33, page 100, and compare with the condition in Necturus.

\section{F. THE SKULL OF THE ALligator, A TYPiCAL MODERN SKULI}

The skull of the alligator is a nearly completely ossified skull in which the various components of which the skull is composed are so closely knit into a single structure as to be inseparable. The skull of the alligator is probably as typical and generalized a skull as is to be found among living land vertebrates and has further the advantage of large size. The student must learn the bones of the skull and jaw of the alligator and be able to distinguish the cartilage from the membrane bones. Figures of the alligator skull will be found in R, pages 252, 254, 256, 26 I P and H, page 342; CNA, Vol. VIII, page 458; K, page ro4.

I. General regions and cavities of the skull.-Obtain a skull and study its structure. It is composed of a number of separate bones closely jointed to each other along somewhat jagged lines known as sutures. The various components of which we learned the skull is constructed are here morphologically indistinguishable from each other but will be pointed out later. The bones of the roof of the skull are pitted and roughened. At the anterior end of the dorsal surface are the two nasal openings or anterior nares. Posterior to the middle of the roof are the two large orbits, the largest openings in the roof of the skull. Posterior to each orbit is the temporal region in which are several holes, known as fossae or vacuities. The development of these temporal fossae is characteristic of reptiles and has occurred during their evolution, since the early reptiles, as in Figure $36 B$, possessed completely roofed skulls. (See further on this point, R, pp. $285-87$, and Fig. 44, p. r76.) The alligator skull has two pairs of these openings, a dorsal pair-the supratemporal fossae, on the dorsal side of the posterior end of the skull-and a lateral pair - the infratemporal fossae, just posterior to the orbits from which each is separated by a rod of bone, the postorbital bar. Lateral to each supratemporal fossa is a projecting ledge of bone, the supratemporal arcade. Underneath this overhanging ledge is an opening, the external auditory meatus, which leads into the cavity of the middle ear or tympanic cavity. Numerous canals and passages will be found entering the tympanic cavity, and 
the cavities of the two sides are connected by a passage in the roof of the skull The bones surrounding the tympanic cavity are bones ossified in the otic capsules, which are thus seen to be closely knit into the structure of the skull. At the posterior end of the skull in the median line is a rounded opening, the foramen magnum, surrounded by the occipital group of bones. Below the foramen is a rounded projection, the occipital condyle. At either side of the posterior end of the skull is a very large cavity, the pterygoid fossa, continuous dorsally with the orbits and infratemporal fossae. The lateral margins of the posterior part of the skull are formed by a bar of bone, which constitutes the lateral boundaries of the orbits and infratemporal fossae; this is called the infratemporal arcade.

On the ventral surface of the skull identify: a small opening near the tip, the anterior palatine vacuity; a pair of larger oval openings ventral to the orbits, the posterior palatine vacuities; and the posterior nares, a pair of small openings in the median ventral line posterior to the preceding.

The smaller holes in the skull are nerve foramina through which nerves enter and leave the brain. The brain in life occupies the very small cavity which may be seen by looking into the foramen magnum. The elongated cavities occupying the interior of the remainder of the skull and extending from the anterior to the posterior nares are the nasal passages. It will be noted that the posterior nares have been moved far posteriorly from the position in which they are found in Amphibia.

2. Membrane bones of the roof of the skull.-At the anterior end of the dorsal surface just behind the anterior nares is a pair of long bones, the nasals. At each side of their posterior ends is a prefrontal bone, and at each side of that a small lacrimal bone. Prefrontal and lacrimal bones form the anterior rim of the orbit. The adlacrimal or supraorbital is a small bone lying loose in the eyelid (and hence missing in many skulls) lateral to the prefrontal. Between the two orbits dorsally is the frontal bone, single in the adult but double in the embryo. In the median line posterior to the frontal is the parietal, also a single bone in the adult but paired in the embryo. The postfrontal bone includes the anterior part of the supratemporal fossa and sends down a process which forms the upper half of the postorbital bar. Directly behind the postfrontal is the squamosal which overhangs the external auditory meatus.

3. The bones of the upper jaw.-The upper jaw consists of the original cartilaginous half of the mandibular arch (pterygoquadrate cartilages) plus a number of membrane bones. Although separate in elasmobranchs, it is inseparably fused to the skull in land vertebrates. It consists of two parts, a maxillary arch forming the lateral parts of the skull both above and below and composed entirely of membrane bones, and a median region developed from the pterygoquadrate cartilage, and consisting partly of cartilage bones and partly of membrane bones. From the dorsal view identify the bones of the maxillary arch as follows: premaxillae, in front of and at the sides of the anterior nares and bearing 
teeth; maxillae, the large bones at the sides of the nasal bones, and also bearing teeth; jugal or malar, the elongated bones forming the lower boundaries of the orbit and meeting the postfrontals at the middle of the postorbital bar; and quadratojugal, a slender, obliquely placed bone forming the posterior boundaries of the lateral temporal fossae. The quadrate is a stout bone obliquely placed between the quadratojugal and the exoccipital. The posterior end of the quadrate has a concave surface for articulation with the lower jaw.

Turn the skull over and identify the same bones of the maxillary arch from below. Premaxillae and maxillae form the anterior half of the ventral surface, meeting in the median line. Between the two premaxillae is the small anterior palatine vacuity. Posterior to the maxillae in the median region and forming the inner boundaries of the posterior palatine vacuities are the palatines. Posterior to the palatines are the broad pterygoids which inclose the posterior nares. Extending from the sides of the pterygoids to the posterior end of the maxillae are the ectopterygoids or transpalatines.

All of these bones of the upper jaw except the quadrate are membrane bones. The quadrate represents the ossified posterior end of the pterygoquadrate cartilage. The quadrate articulates with the lower jaw, as in the majority of vertebrates.

4. The occipital region.-This region forms the posterior end of the skull and consists of four cartilage bones derived from the parachordal cartilages. Turn the skull so that its posterior end faces you. The foramen magnum is bounded by three bones, one on each side, the exoccipitals, and one below, the basioccipital. .The basioccipital bears most of the large rounded occipital condyle but both exoccipitals send down processes which take part in the condyle. Above and between the two exoccipitals is the triangular supraoccipital, which articulates with the posterior end of the parietal.

5. The bones of the otic capsule.-There are three of these surrounding the external auditory meatus and inclosing the ear. They are named the epiotic, the opisthotic, and the proötic. The first of these is fused with the supraoccipital; the second with the exoccipital; the third alone remains distinct in the adult. They are difficult or impossible to see in external view. To find the proötic, turn the skull sidewise so as to obtain a profile view, and identify a large foramen, the foramen ovale, just below the supratemporal fossa. The posterior wall of this foramen is formed by the proötic bone, which articulates with the quadrate bone behind, the suture between the two occurring at the external rim of the foramen. The otic bones are of course cartilage bones, formed in the otic capsule.

6. The posterior sphenoid region.-This region is anterior to the occipital region and forms the floor of the cavity occupied by the brain. Turn the posterior end of the skull toward you and identify the V-shaped end of the basisphenoid, between the basioccipital and the pterygoids. Most of the basisphenoid is concealed from view by the pterygoids and the quadrates, but its 
anterior end projects as a thin blunt process, the rostrum, into the space between the two orbits. Find it in side view of the skull. Above the rostrum on each side is an alisplienoid bone, extending upward to the roof of the skull and forming part of the walls of the foramen ovale and the supratemporal fossa. In the interior of the skull is a cavity extending from the foramen magnum to the alisphenoid bones; this cavity contains the brain in life, and the ventral surface of the brain therefore rests upon the occipital and sphenoid bones, derived from the parachordals and prechordals.

7. The anterior sphenoid and ethmoid regions.- These regions remain in the cartilage stage in the alligator, forming a cartilaginous partition between the two nasal passages and between the orbits. The cartilage is of course wanting in the dried skull so that the nasal passages and orbits appear to be connected.

Draw a ventral view of the skull, showing the sutures accurately.

8. The lower jaw.-The lower jaw or mandible is composed of two halves or rami united in front by a symphysis. Each ramus consists of six separate bones. Near the posterior end of the jaw is a large vacuity, the external mandibular foramen, and in front of this on the inner side a smaller internal mandibular foramen. The bones of each ramus are: the dentary, bearing teeth and forming the outer surface of the anterior two-thirds of the ramus; the splenial, of about the same shape and size as the dentary and in the same position on the inner surface; the angular, below the external mandibular foramen, and separated from the splenial by the internal mandibular foramen; the supra-angular or surangular, above the external mandibular foramen; the coronoid, a small bone on the inner surface, between the anterior ends of the angular and the supraangular; and the articular, above the posterior end of the angular and bearing a concavity for articulation with the quadrate. A cavity exists in the interior of the ramus between the dentary and the splenial. This cavity is occupied in life by Meckel's cartilage, which it may be recalled is the original lower half of the first gill arch. The posterior end of this cartilage has ossified into the articular, which is therefore the only cartilage bone in the lower jaw. The articulation between upper and lower jaw is by way of the articular and the quadrate, a condition found in the majority of land vertebrates. Draw the lower jaw from both inner and outer views.

9. The hyoid apparatus.-As has already been explained, this is derived from the hyoid arch and remaining gill arches. The hyoid apparatus is generally missing on dried skeletons, and for its study preserved specimens are necessary. It consists of a broad cartilaginous plate, the body of the hyoid, and a pair of processes or horns (cormua) extending posteriorly and dorsally from the body, one on either side. The cornua are partially ossified. The body of the hyoid apparatus is derived from the bases of the second and other arches while the horns are remnants of the third arch. In lieu of alligator material, the hyoid apparatus may also be studied on turtle skeletons. In the turtles there is a 
median ventral plate, the body of the hyoid, located in the floor of the mouth it represents the ventral ends of the hyoid and other gill arches. From it pro. ject posteriorly two pairs of slender processes, known as the anterior and posterior horns of the hyoid. These are portions of the third and fourth gill arches.

Io. The teeth.-The teeth of the alligator occur in a single row on the premaxillae, maxillae, and dentary bones. Their structure is the same as that already described under the exoskeleton. The pulp cavity of these teeth is widely open at the base, and the teeth can therefore be replaced an indefinite number of times, each successive tooth having the same pulp cavity as its

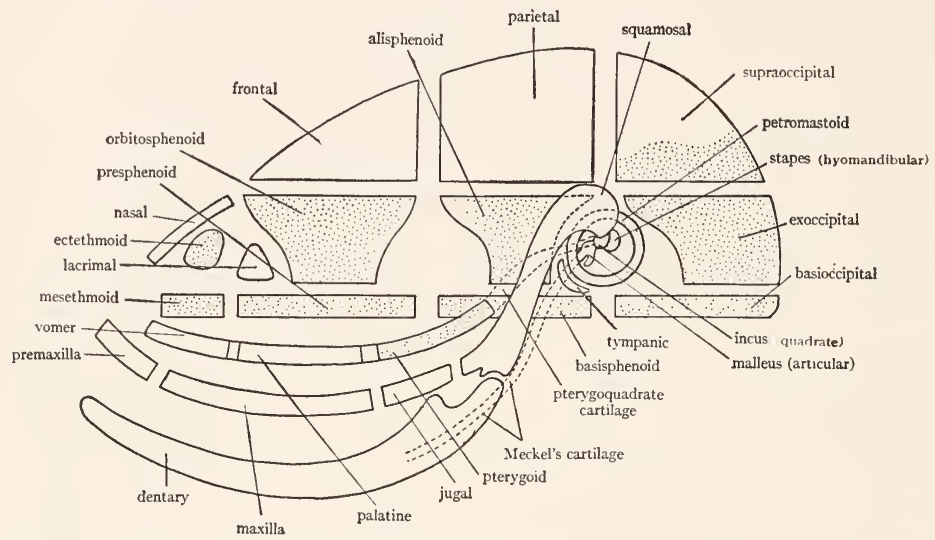

FIG. 39.-Diagram of the bones of the mammalian skull. Membrane bones blank; cartilage bones stippled. (After Kingsley's Comparative Anatomy of Vertebrates, copyright by P. Blakiston's Son and Company.)

predecessor. The teeth are set into sockets or alveoli in the jaw; teeth so placed are said to be thecodont. The teeth are all alike in size and form; hence they are said to be homodont.

\section{G. THE BONES OF THE MAMMALIAN SKULL}

The mammalian skull is completely ossified with the exception of a small part of the ethmoid region. The number of bones which it contains is, however, considerably less than that found in reptile skulls. This is due in part to a loss of bones and in part to a fusion of persisting bones. This fusion is most marked in the human skull. Thus, as Williston says, "The most primitive reptiles had no less than seventy-two separate bones in the skull; the human skull has but twenty-eight inclusive of the (six) ear bones." The accompanying diagram of the mammalian skull, Figure 39, should be studied carefully and will aid in acquiring an understanding of the construction of the mammalian skull. The 
reduction in the membrane bones of the roof of the skull is illustrated in Figure 36 , page 106.

The following description applies to the skull of the cat and rabbit. That of the dog is so similar to that of the cat that the same description applies to both. The cat skull is described and figured in $\mathrm{R}$ and $\mathrm{J}$, and $\mathrm{D}$, the rabbit skull in $\mathrm{B}$, and $\mathrm{P}$ and $\mathrm{H}$, and the dog skull in $\mathrm{R}$. The student must learn the bones of the mammalian skull and be able to state which are membrane bones and which cartilage bones. In studying the bones locate carefully the sutures between the bones and by this means determine accurately the extent of each bone.

I. General regions and features of the skull.-The skull is a hard, bony case, in which the limits of the separate bones are marked by wavy or jagged lines, the sutures. Along these lines the bones dovetail into each other, forming firm, immovable joints. The skull may be divided into an anterior facial portion supporting the nose and eyes and a posterior cranial portion inclosing the brain. At the anterior end of the facial portion are the two nasal openings or anterior nares separated in life by a cartilaginous partition, the anterior part of the septum of the nose. At the side of the facial portion is a large cavity, partially separated by projecting bony processes into two cavities: an anterior, large, nearly circular one, the orbit or orbital fossa, which contains the eye in life; and a posterior one, the temporal fossa, filled in life by muscles. The temporal fossa is very small in the rabbit. The lower boundary of these fossae is formed by a projecting arch of bone, the zygomatic arch, a feature very characteristic of the mammalian skull. In the cat an orbital process extends dorsally from the middle of the zygomatic arch and nearly meets a zygomatic process descending from the roof of the skull. These two processes form the posterior boundary of the orbit. In the rabbit the zygomatic process extends backward and downward from the roof of the orbit; in life this process is connected to the zygomatic arch by a ligament, thus marking off a small temporal fossa posterior to the ligament. Dorsal to the orbit is a projecting margin of bone, the supraorbital arch, the posterior end of which projects as the zygomatic process already mentioned. In the rabbit the anterior end of this arch also bears a projecting process.

The cranial portion of the skull presents the following features. At the posterior end is the large foramen magnum; on each side of this is a projection, the occipital condyle, which articulates with the atlas. Lateral and slightly anterior to each occipital condyle is a conspicuous hollow expansion, the tympanic bulla, which contains the middle ear. On the posterior surface of each bulla are two processes, an anterior mastoid process and a posterior jugular process. The mastoid process will be seen to be part of a bone, differing in its rough and pitted surface from the other bones of the skull; this bone, the petromastoid bone, contains the internal ear. The tympanic bulla opens laterally by a large opening, the external auditory meatus, which in the rabbit is bounded by a bony 
tube. The meatus leads into the cavity of the bulla, which is the tympanic cavity or cavity of the middle ear. From the dorsal side of the bulla a ridge begins which proceeds across the posterior part of the skull to the other bulla; this ridge is the superior muchal line (or lambdoidal ridge) and is the most anterior point of attachment of the muscles of the vertebral column. From the middle of this ridge there projects posteriorly the external occipital protuberance, slight in the cat but forming a rectangular prominence in the rabbit.

On the ventral surface the anterior part of the skull is occupied by the hard palate. Dorsal to this are the nasal passages which open at the posterior end of the hard palate by the posterior nares or choanae. The hard palate contains a pair of openings, the incisive foramina or anterior palatine foramina; these are small and at the anterior extremity of the palate in the cat but much longer and more prominent in the rabbit. They lead into the nasal cavities. At the posterior end of the zygomatic arch on its ventral side is a depression, the mandibular fossa, for the reception of the lower jaw. Medial to this is the pterygoid fossa for the attachment of certain muscles. The pterygoid fossa in the cat is continuous with the temporal and orbital fossae, while in the rabbit it is included between two projecting plates of bone which point toward the tympanic bulla.

2. Membrane bones of the roof of the skull.-Beginning just behind the anterior nares are the paired nasals, roofing the nasal cavities; next posterior, the paired frontals; and last, the paired parietals, terminating at the superior nuchal line. Between the posterior ends of the parietals is generally a small triangular interparietal bone, the boundaries of which are not always distinct. Each frontal bone forms the dorsal part of the orbit and projects out above the orbit as the supraorbital arch which terminates posteriorly in the zygomatic process of the frontal bone, already noted. In the anterior wall of the orbit is the small lacrimal bone (probably homologous with the prefrontal bone of reptiles). At the anterior end of the lacrimal bone is an opening, the posterior end of the nasolacrimal duct, by means of which the tears drain into the nasal cavity.

3. The bones of the upper jaw.-These as in the alligator comprise a lateral maxillary arch on each side and a median series of bones. The maxillary arch consists on each side of the following elements: premaxilla, in front of the anterior nares and bearing teeth; maxilla, forming the side of the facial region of the skull and also bearing teeth; malar or jugal, forming most of the zygomatic arch; and temporal, completing the zygomatic arch and covering the side of the cranial part of the skull including the tympanic bulla. Further details concerning these bones may be noted. The premaxillae send frontal processes dorsally alongside the nasal bones; these are very pronounced in the rabbit. The premaxillae also form the anterior part of the hard palate by means of their palatine processes, which meet in the median ventral line and include the incisive foramina. The maxilla is the main bone of the facial region; in the rabbit it is much fenes- 
trated. It forms part of the anterior wall of the orbit, ventral to the lacrimal bone, by its orbital process; it extends to the frontal bone above by its frontal process; its palatine process meets its fellow in the median ventral line continuing the hard palate; its alveolar process bears teeth; and its zygomatic process constitutes the beginning of the zygomatic arch. The malar or jugal bone is distinct in the cat but in the adult rabbit is fused to the zygomatic process of the maxilla. The temporal bone is a compound bone characteristic of mammals. It consists of a squamous portion which by its zygomatic process completes the zygomatic arch and which also contributes to the cranial wall, ventral to the parietal; of the tympanic bulla composed of a tympanic bone of uncertain homology; and of the periotic or petromastoid bone only slightly visible on the surface and consisting of the fused otic bones of lower vertebrates. The squamous part of the temporal bone is homologous with the squamosal bone of the lower vertebrates and is the most posterior bone of the maxillary arch. All of the bones of the maxillary arch are membrane bones.

The median portion of the upper jaw forms part of the ventral surface of the skull. We have already noted that the anterior portion of the hard palate is composed of the palatine processes of the premaxillae and maxillae. Posterior to the latter are the palatines, membrane bones which include the posterior nares. Dorsal to the palatines the roof of the nasal passages is completed by the vomer of which only the extreme posterior tip is visible in the roof of the choanae. The vomer forms the floor of the nasal cavities and is best seen in a sagittal section of the skull. The posterior parts of the palatines extend up into the orbit, there being in the rabbit a very deep cleft between them in the midventral line.

Observe that the quadrate is wanting. The lower jaw consequently articulates with the squamosal (temporal) by means of a depression, the mandibular fossa, on the under surface of the zygomatic process of the temporal bone. This feature distinguishes mammalian skulls from those of all other vertebrates. Owing to the absence of the quadrate all of the bones of the upper jaw are membrane bones.

4. The occipital region.-This region surrounds the foramen magnum and consists of a single occipital bone extending from the superior nuchal line to a point between the anterior ends of the tympanic bullae. It is really composed of the four occipital bones present in reptiles; these are distinct in embryonic and young mammals, as shown in Figure $40 A$, page 127 , but are fused in the adults. The occipital bone bears the two occipital condyles, whose number serves to distinguish mammalian skulls from those of all other vertebrates except Amphibia, where there are also two condyles. The dorsal part of the occipital may appear to extend anteriorly between the posterior ends of the two parietals; this is due to the fact that interparietal bone is commonly fused to the occipital. This is the case in man. The occipital bone bears the superior nuchal line and the jugular process resting on the bulla. 
5. The otic capsules.-As already explained, the bones of the otic capsules are all fused together and fused with the squamosal and the tympanic bulla to form the temporal bone, a bone very characteristic of mammals (Fig. $40 \mathrm{C}$, p. I 27). That part of the temporal bones which is composed of the otic bones is named the periotic or petromastoid bone. The separate otic bones of which the petromastoid is composed can be seen only in early embryonic stages. The mastoid portion of this bone is visible on the external surface of the skull between the bulla and the occipital; it projects over the bulla as the mastoid process, very prominent in man as the bump behind the pinna. The petrous portion of the petromastoid bone incloses the internal ear and is visible only from the inside of the skull. The tympanic bulla consists of one or two membrane bones of uncertain homology; it incloses the middle ear and the three middle ear bones. These can be seen in well-cleaned cat skulls as a delicate chain of bones extending across the anterior part of the tympanic cavity. If separate specimens are available, identify them as follows: the malleus or hammer, a slender bone terminating in a knob; the incus or anvil, a smaller bone with two pointed processes; and the stapes or stirrup, shaped like a stirrup. There is good reason to believe that the malleus represents a very much reduced articular; that the incus is the reduced quadrate; and the stapes is the hyomandibular.

6. The posterior sphenoid region.-On the ventral surface of the skull in front of the ventral part of the occipital bone is the basisphenoid. The basisphenoid extends laterally in front of the tympanic bullae as processes, the wings of the basisphenoid. These wings are in reality the alisphenoids and are separate in young stages (see Fig. 40B). The alisphenoids meet the squamous part of the temporal bones dorsally. The alisphenoids extend forward as the pterygoid processes which meet the posterior ends of the palatines. In the rabbit the pterygoid process presents two backwardly projecting thin plates of bone, the lateral and medial lamellae. These inclose between them the pterygoid fossa. The medial lamella in the rabbit and the pterygoid process itself in the cat bear a pointed process, the hamulus. The pterygoid processes correspond to the pterygoid bones of lower forms.

7. The anterior sphenoid region.-In the median ventral line in front of the basisphenoid is the presphenoid, a slender bone. In the rabbit this is at the bottom of the deep cleft between the palatines. The presphenoid sends up wings into the orbit which meet the frontal bones above and contain the large optic foramen for the passage of the optic nerve. In the cat this foramen is the most anterior of a row of four foramina. To see the wings of the presphenoid turn the skull to obtain a lateral view. The wings are in reality the orbitosphenoid bones of lower vertebrates and are separate in young stages (Fig. 4o $B$, p. 127)

Draw a ventral view of the skull, showing the sutures accurately.

8. The ethmoid region and the sagittal section of the skull.-The ethmoid is nearly completely ossified in mammals but can be studied only in sagittal 
sections, since it is in the interior of the nose. Obtain a sagittal section of the skull. These sections are cut slightly to one side of the median line so that the septum of the nose is included in one half and missing on the other half. Students should see both halves. The section shows that the interior of the cranial portion of the skull is occupied by a large cranial cavity, divisible into three regions of unequal size. The most posterior region inclosed within the occipital and temporal bones is the posterior or cerebellar fossa of the skull. Its anterior boundary is marked by a prominent (cat) or slight (rabbit) ridge or shelf of bone, the tentor$i u m$, which in life is completed by a membrane. In the lateroventral wall of the cerebellar fossa is a rounded area of very hard compact bone bearing two openings; this is the petrous part of the petromastoid bone and incloses the internal ear. The greater part of the cranial cavity comprises the middle or cerebral fossa, extending forward from the tentorium. Its roof and walls are formed by the frontal, parietal, and temporal bones, its floor by the sphenoids. In the floor of the cerebral fossa, located in the basisphenoid bone, is a marked saddle-shaped depression, the sella turcica, in which in life the pituitary body is lodged. The presphenoid bone contains a cavity, the sphenoidal simus. In the anterior part of the frontal bone, cavities, the frontal simuses, are also present. The anterior end of the cranial cavity is the small anterior or olfactory fossa located between the anterior parts of the two frontal bones. The olfactory fossa is separated from the nasal cavities which lie in front of it by a nearly vertical plate of bone, perforated by numerous holes, the cribriform plate of the ethmoid. This plate is best seen in the intact skull of the cat by looking through the foramen magnum. The plate pierced by holes like a sieve is then seen closing the anterior end of the cranial cavity. Our study of the sagittal section shows that the floor of the skull is composed of a chain of cartilage bones, occipital, sphenoids, and ethmoid, on which the brain rests. These bones as has already been explained are derived from the chondrocranium.

The nasal cavities or nasal fossae are inclosed partly in cartilage bonesderived from the ethmoid plate-and partly by membrane bones. The roof of the cavities consists of the nasal bones and a small part of the frontals. The two cavities are separated by a median, vertical, bony partition, the perpendicular plate of the ethmoid; in the living state this is continued to the anterior nares by a cartilaginous plate. The two together constitute the septum of the nose. The bony part of the septum, that is, the perpendicular plate of the ethmoid, is small in the rabbit. Dorsally the septum meets the nasal and (cat) frontal bones; ventrally it meets the vomer, an elongated bone dorsal to the maxillae and palatines. The posterior end of the septum meets the cribriform plate.

On the half of the skull where the septum is missing the turbinated bones or conchae may be studied. They are peculiar, delicate, grooved and folded bones which occupy the lateral walls of the nasal cavities and fill most of the interior. 
The most posterior of these bones is the ethmoturbinal or ethmoid labyrinth situated just in front of the cribriform plate. It is a greatly folded structure, the folds inclosing spaces known as the ethmoid cells. In front of the ethmoturbinal is another but smaller mass, the maxilloturbinal, borne on the inner surface of the maxilla. Each nasal bears on its inner surface a single elongated ledge of bone, which constitutes the nasoturbinal, and which lies above the uppermost scrolls of the ethmoturbinals. The function of the turbinals is to increase the respiratory and olfactory surface of the nose. The ethmoturbinals are covered by the olfactory epithelium, while the maxilloturbinals serve to strain and moisten the air. The latter will be seen to project into the passage from the anterior to the posterior nares.

From the comparative standpoint the septum of the nose is the mesethmoid, the perpendicular plate of the ethmoid being its ossified portion, while the ethmoid labyrinths are the ectethmoids. The cribriform plate is produced by the extension of ossification processes between the mesethmoid and ectethmoids. The perforations in the plate are for the passage of the olfactory nerve. Thus, the three ethmoid bones of lower vertebrates are fused into the single ethmoid bone of mammals.

Draw the sagittal section.

9. The foramina of the skull.-The skull is pierced by numerous openings for the passage of nerves and blood vessels and sometimes other structures. These are listed below for convenient reference.

Cat:

a) Incisive foramina. Anterior end of ventral side of maxillae; connect roof of mouth with nasal cavities.

b) Infraorbital foramen. Large opening in the maxilla at the beginning of the zygomatic arch; for the passage of certain branches of the fifth cranial nerve and blood vessels.

c) Nasolacrimal canal. Anterior end of the lacrimal bone and passing through the maxilla into the nasal cavities; for the draining of the tears.

d) Sphenopalatine foramen. In that part of the palatine bone which extends into the orbit, posterior to the lacrimal; for the passage of branches of the fifth nerve into the nasal cavity.

e) Posterior palatine canal. The posterior end of this is immediately ventral to the sphenopalatine foramen; its anterior end is in about the middle of the palatine process of the maxilla; for the passage of a branch of the fifth nerve to the palate.

f) Optic foramen. In the orbitosphenoid part of the presphenoid, in the posterior part of the orbit, most anterior of a row of four foramina; for the passage of the optic nerve.

g) Orbital fissure. Second and largest of the row of four; through it pass the third, fourth, and sixth nerves to the muscles of the eyeball, and a part of the fifth. 
h) Foramen rotundum. Third of the row; in the alisphenoid bone; transmits part of the fifth nerve.

i) Foramen ovale. Last of the four; in the alisphenoid; transmits part of the fifth nerve.

j) Canal for the Eustachian tube. In the anterior wall of the bulla, its roof formed by the alisphenoid; for the passage of the Eustachian tube from the pharynx into the bulla.

k) Pterygoid canal. Each bulla terminates anteriorly in a point (styliform process) lying on the basisphenoid; this point is directed to a minute opening, the pterygoid canal, lying in the suture between the basisphenoid and the pterygoid process; for the passage of a branch of the fifth nerve into the bulla.

l) Jugular foramen. Large foramen on the medial side of the posterior end of the bulla, for the passage of the ninth, tenth, and eleventh nerves.

m) Hypoglossal foramen. In the medial side of the preceding foramen for the passage of the twelfth nerve.

n) Stylomastoid foramen. At the ventral tip of the mastoid process for the passage of the seventh nerve.

\section{Rabbit:}

a) Incisive foramina. As in the cat but larger.

b) Infraborbital foramen. As in the cat but more slitlike and elongated, forming an infraorbital canal, opening into the orbit above the expanded part of the maxilla.

c) Nasolacrimal canal. As in the cat, situated under the pointed anterior end of the supraorbital arch.

d) Posterior palatine foramen. On the ventral side, in the suture between the palatine process of the maxilla and the palatines; forms the anterior opening of the palatine canal. The posterior opening is at the posterior end of the expanded mass of the maxilla located in the orbit. This canal is for the passage of a branch of the fifth nerve.

e) Sphenopalatine foramen. In common with the posterior end of the palatine canal just described; for the passage of branches of the fifth nerve.

f) Anterior and posterior supraorbital foramina. The projecting anterior and posterior ends of the supraorbital arch are continued in life by ligaments, thus forming foramina, through which branches of the fifth nerve pass.

g) Optic foramen. Large opening in the center of the orbit, for the passage of the optic nerve.

h) Orbital fissure. Posterior and ventral to the preceding, and including the foramen rotundum of other mammals. For the third, fourth, and sixth nerves to the eyeball, and the greater part of the fifth.

i) Anterior, middle, and posterior sphenoidal foramina. Three foramina in a row in the lateral lamella of the pterygoid process at the place where this is continuous with the alisphenoid. For the passage of part of the fifth nerve. 
j) Foramen lacerum. In front of the tympanic bulla on the ventral surface and including the foramen ovale of other mammals; for the passage of part of the fifth nerve and an artery.

k) External carotid foramen. A small foramen in the middle of the medial surface of the bulla; for the passage of the internal carotid artery, the other end of the canal lying in the foramen lacerum.

l) Jugular foramen. Just posterior to the preceding, in the depression between occipital condyle and bulla; for the ninth, tenth, and eleventh cranial nerves, and a vein.

m) Hypoglossal canal. Including small apertures posterior to the preceding for the passage of the twelfth (hypoglossal) nerve.

n) Stylomastoid foramen. In front of the middle of the mastoid process, for the passage of the seventh nerve.

I0. The lower jaw.-The lower jaw or mandible consists of a single pair of bones, the dentaries, fused in front by a symphysis. All other bones seen in the lower jaw of the alligator have vanished (except the articular which is supposed to be the malleus of the middle ear). The horizontal part of the mandible is named the body, the vertical part, the ramus. (In the lower vertebrates each half of the lower jaw is also named ramus.) The posterior end of the mandible (cat) extends dorsally into a strong coronoid process, which in the natural position projects into the temporal fossa. In the rabbit this is reduced to a slight projection which forms the lateral boundary of a deep groove. The articulating surface of the mandible is borne on the condyloid process. The depressed areas in the posterior part of the mandible are for the insertion of the muscles of mastication. Near the anterior tip of the mandible on the outer surface is the mental foramen (or two in the cat) through which the nerve of the lower jaw exits. Near the caudal end of the inner surface is the mandibular foramen through which the nerve enters and pursues a course in the interior of the mandible to the mental foramen. In the rabbit there is an additional foramen just above the mandibular foramen, for the passage of a vein.

Owing to the absence of the quadrate and of all of the bones of the lower jaw except the dentary, the articulation of the lower jaw to the skull is between the dentary and the squamosal. This feature distinguishes mammals from all other vertebrates, for in the latter the articulation is between the articular and the quadrate. The condition found in mammals is, however, approached by those reptiles directly ancestral to mammals.

II. The teeth.-The teeth of mammals possess several characteristics: they are set in sockets or alveoli in the jaws, a condition known as thecodont; they are heterodont, that is, differentiated into several different kinds; they can generally be replaced only once; and some of them are complicated in form. The teeth of mammals furnish important taxonomic characters. Those of the cat and rabbit are very different, the former having teeth characteristic of 
carnivores, the latter with the chisel-like front teeth and grinding back teeth common to rodents.

Cat: At the tip of the jaws are six small simple teetl, named incisors. On either side of the incisors is a canine, a long, sharp but simple tooth. Back of the canine on each side are four teeth in the upper jaw, three in the lower. These teeth are mostly more complicated than the preceding, having more than one cusp or pointed projection and more than one root. These teeth are known as premolars and molars. In the upper jaw the first three on each side are premolars, and the first two in the lower jaw; the last tooth on each side in each jaw is a molar. Note that the upper incisors are borne on the premaxillae; the other teeth of the upper jaw on the maxilla. Between the canines and the premolars is more or less of a gap, known as a diastema.

Rabbit: At the tip of the premaxillae are borne four chisel-like incisor teeth, a small pair behind a larger anterior pair. The chisel-like form of the incisors is characteristic of rodents and is due to the fact that the enamel is present on the front face of the tooth only; the posterior face being composed of the softer dentine wears away, leaving a sharp edge to the enamel. The incisors of rodents further continue to grow indefinitely so that the loss from use at the tip is replaced by growth at the root. At the tip of the lower jaw are two similar incisors. Posterior to the incisors is a very wide gap or diastema. The canine teeth, found in most mammals posterior to the incisors, are missing in the rabbit and rodents in general. On the alveolar process of the maxilla are borne six teeth in the upper jaw and five in the lower, on each side. Of these the first three in the upper and first two in the lower jaw, are premolars, the last three molars. The teeth are ridged crosswise, an adaptation for the grinding of regetable food; the ridges consist of enamel with dentine between them.

The teeth of mammals are generally designated by a dental formula, which expresses the number of each different kind of tooth in each half-jaw from the anterior to the posterior extremity of the jaw. The teeth of the upper jaw are placed in the numerator of the formula, those of the lower jaw in the denominator. The complete dentition for mammals consists of three incisors, one canine, four premolars, and three molars in each half of each jaw; the dental formula is then $3 / 3, I / I, 4 / 4,3 / 3$. In the cat the formula is $3 / 3, I / I, 3 / 2, I / I$; in the rabbit $2 / I, O / O, 3 / 2,3 / 3$; in $\operatorname{man} 2 / 2, I / I, 2 / 2,3 / 3$. There is no structural difference between premolars and molars, but those teeth with complicated crowns which occur in both the "milk" and the permanent dentitions are designated premolars, while those which are permanent from the first and never replaced are designated as molars. It is not known whether the complicated, apparently compound structure of premolars and molars is due to their having arisen through the fusion of several simple teeth or to the subdivision of the original simple conical teeth. The structure of teeth and their origin from placoid scales was discussed in Section V. 
12. The hyoid apparatus. - This is, as already explained, the remnant of the hyoid and other gill arches. It is generally absent on prepared skeletons and isolated specimens will be provided for its study.

The hyoid of the cat consists of a bony bar placed in the root of the tongue just in front of the larynx; this bar is called the body of the hyoid. From it extend two pairs of processes or horns, an anterior pair and a posterior pair. The anterior horns consist of four separate pieces, of which the terminal piece is attached to the tympanic bulla just ventral to the stylomastoid foramen of the skull. The groove which it occupies can generally be seen on the side of the bulla. The posterior horns consist each of a single piece which is united to the larynx. The body and anterior horns belong to the hyoid arch; the posterior horns to the third gill arch. The remaining gill arches are represented in the larynx which will be studied later.

The hyoid apparatus of the rabbit consists of a stout bone, the body of the hyoid, situated at the base of the tongue in front of the larynx. It bears two pairs of processes or horns. The anterior horn is a short piece connected by a muscle with the jugular process of the skull. The posterior horn is a longer piece connected by ligament with the larynx and by muscle with the jugular process. The body and anterior horn are remnants of the hyoid arch, the posterior horn of the third gill arch.

\section{H. GENERAL SUMMARY OF THE SKULL AND VISCERAL SKELETON}

I. The skull begins as a cartilaginous case, the chondrocranium, whose method of formation has already been described. This case includes the olfactory and otic capsules.

2. The gill arches become closely associated with the chondrocranium. There are usually seven of them, the first forming the upper and lower jaws, the second the hyoid arch. The arches are reduced in number and altered in function in land vertebrates.

3. Bones derived from the chondrocranium and gill arches are the cartilage bones of the skull.

4. In addition to these there are membrane bones added. They come from the dermis of the skin and were originally dermal scales.

5. The cartilage bones of the skull and sense capsules are the occipitals, sphenoids, ethmoids, otics, and turbinals. There are four occipitals (supraoccipital, two exoccipitals, basioccipital); a posterior group of three sphenoids (basisphenoid, two alisphenoids); an anterior group of three sphenoids (presphenoid, two orbitosphenoids); and a group of three ethmoids (mesethmoid, two ectethmoids). The otic bones ossified in the ear capsule are generally three in number but may be as many as five; in mammals they are fused to form a periotic or petromastoid bone. The nasal capsules may furnish additional cartilage bones. The occipitals and sphenoids form the posterior end and floor of the brain cavity, while the ethmoids inclose the nasal cavities.

6. The first gill arch generally gives rise to two cartilage bones, a quadrate derived from the posterior end of the upper jaw cartilages or pterygoquadrate cartilages; and an articular derived from the posterior end of the lower jaw cartilages or Meckel's cartilages. In some forms the pterygoquadrate cartilages may give rise to palatines and pterygoids in addition. 
7. The second or hyoid gill arch gives rise to a cartilage bone, the hyomandibular, and the remainder of it takes part in the formation of the hyoid apparatus.

8. All other bones of the skull and jaws are membrane bones.

9. The number of membrane bones of the skull is greatest in the lower vertebrates, and the membrane bones present the most typical arrangement in extinct and primitive groups of Amphibia and reptiles. The number of membrane bones diminishes in the higher vertebrates, chiefly by loss but also by fusion. Thus, we may note that several membrane bones present in the alligator are missing in the cat and rabbit. This loss of membrane bones is illustrated in Figure 36, page го6.

Io. The number of cartilage bones is likewise greatly reduced in the higher forms by a process of fusion of originally separate bones. Thus, the occipital bone of mammals consists of four originally separate bones; the sphenoids of mammals are more or less fused and in man unite to a single sphenoid bone which consists of six originally separate cartilage bones
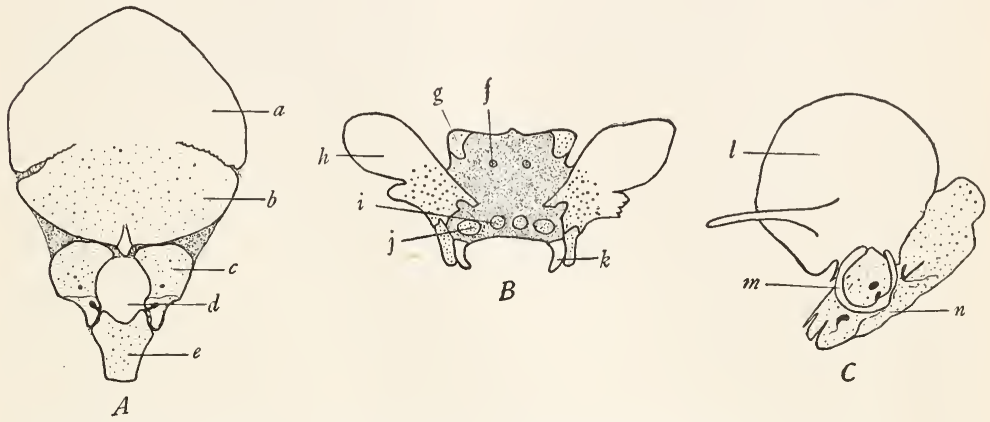

FIG. 40.- Some bones of the human skull at an early age, showing their compound nature. $A$, the occipital bone at birth, showing the five elements of which it is composed; $a$, interparietal; $b$, supraoccipital; $c$, exoccipital; $d$, is the foramen magnum; $e$, basioccipital. $B$, the sphenoid bone in an embryo of four months, showing its components; $f$, center for the presphenoid; $g$, center for the orbitosphenoid; $h$, alisphenoid; $i$, center for the basisphenoid; $j$, center for the lingula; $k$, center for the pterygoid. $C$, the temporal bone at birth, showing its three components; $l$, squamosal; $m$, tympanic; $n$, petromastoid or periotic. Membrane bones blank; cartilage bones open stippling; cartilage, close stippling. The subsequent ossification of the cartilage obliterates the boundaries between the components. ( $A$ and $C$ from specimens loaned by the anatomy department; $B$ after McMurrich's Development of the Human Body, copyright by P. Blakiston's Son and Company.)

and includes some membrane bones in addition; the ethmoid of mammals is composed of three ethmoid bones; the petromastoid of several otic bones, etc. (Fig. 40).

I I. The lower jaw undergoes reduction to the mammalian condition, where it consists of but a single pair of bones. In most vertebrates the articulation of the lower jaw is between the quadrate and the articular bones, while in mammals it is between the dentary and the squamosal bones.

I 2. The quadrate, the articular, and the hyomandibular are believed to be represented in mammals in the tiny bones found in the middle ear. The remnant of the quadrate forms the incus, of the articular, the malleus, and of the hyomandibular, the stapes.

I3. The hyoid and other gill arches are gradually reduced in land vertebrates but persist in part in the hyoid apparatus and the cartilages of the larynx. 


\section{THE COMPARATIVE ANATOMY OF THE MUSCULAR SYSTEM}

\section{A. GENERAL CONSIDERATIONS}

I. The kinds and origin of muscle.-The muscles of the vertebrate body may be divided into two general classes, the involuntary and the voluntary. The involuntary or smooth muscles occur in the walls of the digestive tract and other viscera, and in the skin and certain derivatives thereof. They originate through the transformation of mesenchyme cells, which may be of various origins. The majority of the smooth musculature, however, is produced by the mesenchyme of the hypomere, since in development the hypomere closes around the archenteron and its derivatives (see Fig. Io, p. 43). The voluntary or striated muscles, on the other hand, with certain exceptions specified below, arise from the myotomes. The myotomes, it will be recalled, are those portions of the epimeres remaining after the epimeres have given rise to the sclerotomes and dermatomes. From their original dorsal positions the myotomes grow down between the hypomere and the skin and those of opposite sides meet in the median ventral line. See Figure ro and re-read Section IV of the manual. In this way there is produced a complete coat of voluntary muscles, lying beneath the skin. This muscle coat is divided into dorsal and ventral parts by the horizontal skeletogenous partition which intersects the skin at the lateral line. The muscles dorsal to this septum are called the epaxial muscles, those below the septum, the hypaxial muscles (Figs. I $5 A$, p. $5^{8}, 20$, p. 62).

All of the muscles originating from the myotomes are voluntary muscles and are designated as parietal or somatic muscles. Not all of the voluntary muscles are, however, of this kind. In the gill region of vertebrates a system of voluntary muscles is developed for moving the gill arches. Since the gills and related parts are of entodermal origin, the muscles in the walls in the gill region are homologous with the muscles of the rest of the digestive tract and are, in fact, derived from the mesenchyme of the hypomeres. These gill arch muscles are consequently designated as visceral muscles, although, unlike the muscles of the viscera, they are striated and voluntary. There are consequently two kinds of voluntary muscles, identical in structure but different in origin-the parietal or somatic muscles derived from the myotomes and distributed widely over the body and the visceral muscles derived from the hypomeres and found only in the gill region.

The student should note that the terms muscle and muscular system, when used without further qualification, refer only to the voluntary muscles. In dissecting a vertebrate only the voluntary muscles are studied, as the study of the involuntary muscles properly belongs to histology. It should further be always borne in mind that when the expression visceral muscles is employed this refers not to the involuntary muscles of the viscera but to the voluntary muscles of the gill arches. It is assumed that the student understands the histological difference between smooth and striated muscle.

Consult also the accounts of the comparative anatomy of the muscular system in $\mathrm{K}$, $W$, and $W d$.

\section{B. THE MUSCLES OF THE DOGFISH}

I. The parietal or somatic muscles.-Strip off the skin from the dogfish at the base of the tail, in the neighborhood of the pelvic fins. In doing this make a cut through the skin, grasp the cut edge with the fingers, and strip off a piece of 
skin without the further use of the knife. If you attempt to cut the skin from the body by means of a scalpel you will slash into the muscles. After removing a considerable area of skin note that the body wall under the skin is composed of a coat of muscles completely sheathing the body. These are the parietal muscles. Observe that the parietal muscles consist of a series of zigzag myotomes, each separated from its neighbor by a white sheet of connective tissue, the myocomma or myoseptum. In the middle of the side of the body observe a white line running lengthwise. This is the outer edge of the horizontal skeletogenous septum, which intersects the skin at the lateral line. This septum divides the myotomes into dorsal portions-the epaxial muscles-and ventral portions - the hypaxial muscles. On the ventral side note that there is a white partition in the median ventral line. This is the linea alba; it separates the myotomes of the two sides of the body. No muscle ever crosses the dorsal or ventral median lines, and hence all of the muscles are paired.

Draw from the side a portion of the body to show the myotomes.

2. The modification of the parietal muscles by the presence of appendages.Strip off the skin from the bases of the pelvic fins and observe that additional muscles are present in the fins at their bases for the purpose of moving the fins. A mass of muscle springs from the myotomes and is inserted on the fin on both dorsal and ventral sides. Cut through the middle of the dorsal mass, carefully separate the cut ends from the body wall, and note that the myotomes are revealed underneath the fin muscles. The dorsal ends of the fin muscles will be seen to spring from the myotomes with which they are continuous, and it is important to note that several myotomes contribute in this way to the fin muscles. We thus learn that in the neighborhood of an appendage the myotomes bud off muscle slips for the appendages. In this manner the muscles of the appendages originally arose. It should be stated, however, that in the higher vertebrates the intrinsic appendicular muscles can no longer be seen to originate in this fashion but are formed in place in the limbs.

3. The visceral muscles of the dogfish.-Make a median ventral incision in the skin of the ventral side of the head between the gill slits and strip off the skin in an upward direction over the gill slits and up to the median dorsal line. Note that dorsally above the gill slits the myotomes are present and as typical in form and arrangement as in more posterior parts of the body. In the region of the gill slits and on the ventral surface of the head, however, entirely new sets of muscles are found which serve to move the gill arches and the jaw. These muscles are the visceral muscles, and they are derived from the hypomeres. There are a great many of them, and it is not our purpose to identify them in detail, but the following may be noted: trapezius, the long muscle above the gill slits, and below the myotomes; the dorsal constrictors, between the dorsal portions of the gill arches, and with their fibers directed obliquely forward; the ventral constrictors, between the ventral portions of the gill arches, and covering most of 
the ventral surface, extending forward to the mouth, with fibers directed backward; and the adductor mandibularis, the thick muscle at the angle of the jaws, used to close the lower jaw. The constrictors open the gill slits by diminishing the distance between the gill arches by their contraction. These muscles of the dogfish persist in higher vertebrates after the gills have been lost in association with those structures which are derived from the gill arches, that is, the jaws, the hyoid, and the cartilages of the larynx.

\section{THE MUSCLES OF NECTURUS}

Animals that have been preserved for some time in formalin are preferable for the study of the muscles. Make a median dorsal incision extending the length of the head and trunk. Loosen the cut edges of skin with the fingers, noting in the cut surfaces the flask-shaped cutaneous glands which secrete slime. Then with the fingers separate the skin from the muscles in a ventral direction until you have removed the skin from the head, trunk, and appendages. The gills are to be left in place. The white fibrous material between the skin and muscles is the subcutaneous connective tissue or superficial fascia.

I. The muscles of the trunk and tail.- - These muscles preserve the generalized arrangement typical of primitive and embryonic vertebrates. They consist, as in the dogfish, of a series of myotomes, separated by myosepta. The myotomes are long, nearly rectangular blocks extending from the mid-dorsal to the midventral line. Their narrowed dorsal ends slant forward. Note their division into epaxial and hypaxial portions by the horizontal septum. Although the trunk muscles appear to be unmodified, they are in reality already separating into layers. On cutting into the epaxial muscles they will be seen to constitute a mass whose fibers are all directed forward; this mass corresponds to the longissimus dorsi muscle of higher forms. On cutting into the hypaxial muscles, on the other hand, they will be found divisible into three distinct layers. The outer layer, or external oblique muscle, is composed of fibers directed obliquely ventrad and caudad. The middle layer, or internal oblique muscle, is composed of fibers directed craniad and ventrad. On cutting through the internal oblique a third layer - the transverse - next to the body cavity will be found. On either side of the median ventral line the fibers of the myotomes are directed parallel to the longitudinal axis of the body and form a narrow band, the rectus abdominis muscle, which is rather distinct just anterior to the pelvic girdle.

2. The muscles of the pelvic girdle and hind limb.-In this region of the body, as in the case of the dogfish, the series of myotomes is interrupted by the presence of limb muscles, extending from the trunk into the limb. Examine the ventral face of the pelvic girdle. It is covered by muscles which may be separated into two muscles. The anterior muscle covering the pubic cartilage extends from the median line of the girdle to the femur; it may be named the pubofemoralis externus. The posterior muscle extends from the ischial region of the girdle 
to the tibia and is called the ischiotibialis. From the anterolateral margin of the girdle a muscle, partly concealed under the pubofemoralis externus, projects forward, and its fibers become continuous with the ventral portions of the myotomes. This is the posterior end of the rectus abdominis noted above. Around the anus is a large mass, the anal gland. On removing this gland two muscles are revealed on each side. The medial one is the ischiocaudalis, extending from the posterior margin of the ischium to the tail, and the lateral one is the pyriform, its anterior end fastened to the ischiotibialis, its posterior end proceeding into the tail. Follow these two muscles into the tail. Turn the animal sidewise, bend the leg forward, and note that the pyriform splits near its anterior end and gives off another muscle which passes to the femur. This muscle is the femorocaudalis. The three muscles just mentioned are tail or caudal muscles.

Certain definitions may now be made. Each muscle is attached at its two ends and more or less free in the middle. The attached end which is fixed and immovable is named the origin; the attached end which moves when the muscle contracts is the insertion. The best method of naming muscles is to combine the origin and insertion into a compound word, the origin preceding. The names of the majority of the muscles given above are of this kind, but muscles are also frequently named from their shapes, positions, etc. In the case of many muscles either end may serve as the origin or insertion, depending on which end is held fixed; thus, the caudal muscles of Necturus will move the tail if the pelvic girdle is fixed or will move the girdle if the tail remains stationary. The muscles of the girdle already named serve chiefly to bend the limb toward the median ventral line, a movement known as adduction.

On the ventral surface of the thigh four muscles may be identified. These are: the pubofemoralis internus, the most anterior one, originating on the anterior rim of the acetabulum and inserted on the distal end of the femur; the pubotibialis, next posterior to the preceding, extending from the acetabulum to the proximal end of the tibia; next, the distal portion of the ischiotibialis, this part being sometimes designated the gracilis muscle; and most posteriorly, a slender muscle, the femorofibularis, originating on the preceding muscle and inserted on the fibula. Some of these muscles act to bend the shank toward the thigh, an act known as flexion.

On the dorsal side of the thigh in addition to some of the muscles already mentioned, which appear also in dorsal view, there is found chiefly the ilioextensorius, a broad band originating on the ilium (which may readily be found as a curved bone between the myotomes) and inserted on the distal end of the femur by a tendon (sheet of connective tissue) which also passes onto the shank. This muscle abducts the thigh, that is, draws it dorsally, and straightens or extends the shank. Between the ilioextensorius and the pubofemoralis internus, partly concealed by the latter, is the iliofemoralis, extending from the ilium to the femur. 
The homology of these muscles with those of mammals is somewhat doubtful. Some suggestions are given by $\mathrm{W}$, page 229. A figure which may aid in identifying the muscles will be found in W, page 228 .

3. The muscles of the pectoral girdle and fore limb.-Study the ventral surface of the pectoral girdle. Covering the coracoid cartilage is a fan-shaped muscle, the supracoracoideus, inserted on the humerus. On either side, overlying the elongated procoracoid cartilage, is the procoracohumeralis, inserted on the humerus just anterior to the preceding. Behind the supracoracoideus and practically continuous with it is the large fan-shaped pectoral muscle, which originates on the linea alba and some of the myosepta. Covering the ventral surface between the two procoracohumerales, is the broad sternohyoid muscle, differentiated out of the ventral portions of the most anterior myotomes. At its anterior end the two halves of the sternohyoid muscle fork, another pair of muscles (geniohyoids) being inserted between the two parts of the fork. The sternohyoid should be traced posteriorly; it has some attachments to the pectoral girdle, passes dorsal to the pectoral muscles which should be lifted to see it, and becomes continuous with the rectus abdominis. The sternohyoid and rectus abdominis preserve almost perfectly the segmented condition.

On the dorsal side of the pectoral girdle identify the latissimus dorsi, the most posterior and largest of the dorsal girdle muscles. It originates by about five separate slips from the myosepta of adjacent myotomes, and these slips converge to the humerus. This muscle illustrates very well the compound origin of limb muscles by slips or buds from several myotomes. Anterior to the latissimus dorsi and covering the surface of the scapula is the dorsalis scapulae, also inserted on the humerus. Anterior to this is the trapezius (or cucullaris) originating by two slips or heads and inserted on the scapula. Between the trapezius and the procoracohumeralis is the omohyoid muscle, running parallel to the latter, from the hyoid to the girdle. Cut through the middle of the latissimus dorsi and find beneath it the typical myotomes. These become in higher forms the serratus muscle.

In the upper arm identify on the ventral side two muscles: an anterior one, the biceps, extending from the humerus to radius; and a posterior coracobrachialis originating from the coracoid cartilage and passing to the distal end of the humerus. The dorsal side of the upper arm is occupied by the large triceps muscle, with origins on coracoid, scapula, and humerus, and insertion on the ulna. The muscles of the forearm are in two chief masses, a dorsal extensor which extends the hand and a ventral flexor which bends the hand.

4. The visceral and head muscles. - The visceral muscles or muscles of the gill arches are considerably modified in relation to the greater separation of head from trunk and the degeneration of the gill arches. Skin the head completely. Across the ventral surface from one half of the lower jaw to the other passes the mylohyoid muscle. It originates on the jaws and is inserted on the median 
connective tissue partition (raphe). Slit up the raphe and find beneath it two elongated parallel muscles, the geniohyoids, arising from the tip of the mandible and inserted on the fascia of the sternohyoid. The sternohyoid parts on either side of this place and passes around and dorsal to the posterior ends of the geniohyoids to be inserted on the hyoid arch. On each side of the geniohyoid a large muscle, the external ceratohyoid, originates from the hyoid arch and passes posteriorly around the angle of the jaw. The posterior end of the ceratohyoid is covered by the posterior part of the mylohyoid, which is inserted on the surface fascia of the ceratohyoid and on the gill region, on which the ceratohyoid is also inserted. At the posterior end of the ceratohyoid is a fan-shaped muscle which is inserted on the gill arches. This muscle, the levator branchiarum, serves to lift the gills, while the ceratohyoid draws them forward.

On the dorsal side of the head on either side of the median line is the temporal muscle. Lateral to this is a large mass, the masseter muscle. Both are inserted on the lower jaw at the angle of the jaw (the insertion of the temporal being concealed by the masseter) and serve as elevators of the lower jaw. Separate the masseter and ceratohyoid widely and find between and beneath them a smaller muscle, the digastric, which extends from the skull to the angle of the lower jaw. It is the depressor or opener of the lower jaw. Behind the digastric under the posterior end of the ceratohyoid is a muscle slightly smaller than the digastric, the levator arcuum, which passes to the gill arches and serves to lift them.

Draw dorsal and ventral views of the head, shoulder girdle and gill arch muscles. This region of urodeles is illustrated in K, page 137 , Figure 146 , and $W$, page 247 , Figure 58 .

\section{THE MUSCLES OF THE CAT AND RABBIT}

In mammals those processes of change in the muscular system which we saw beginning in the dogfish and progressing in Necturus reach a maximum. Owing to the increase in size and strength of the limbs and the adoption of the habit of elevating the body above the ground, the muscles associated with the limbs become correspondingly increased in size and importance. We have already learned that the girdle and limb muscles originate as small buds from the myotomes. In mammals they have so increased in size and so spread from their original locations that the original myotomes are scarcely recognizable in the adult, although present in the embryo in the typical primitive arrangement. Further, owing to the transformation of the gill arches the visceral muscles have undergone great changes. We shall attempt to point out to some extent the homology between the muscles of the mammals and those of the lower forms. Muscles become altered from the primitive segmented condition by processes of splitting, fusion, and extension.

For more complete details concerning the muscles than are given below see, for the cat, R and J, and for the rabbit, B. The directions which follow are 
applicable to either animal, differences between them being specifically noted wherever necessary.

I. The dermal or integumental muscles.-Skin the animal and in doing so note the structures named in the following paragraph. (If the animal has already been skinned, this part of the work will have to be omitted.) In skinning, make a median dorsal incision from the base of the tail to the back of the head. Be sure that this and following incisions cut through the skin only. Make an incision through the skin around the throat, around ankles and wrists, and incisions along the outer surface of the limbs. Connect these with the median incision. Loosen the skin along the incisions and gradually work the skin loose from the muscles, using fingers and back of the scalpel. Avoid as far as possible the method of cutting the skin from the body, as one is liable to cut into the muscles by this procedure. Work from the dorsal side toward the ventral side. Leave the skin on the head and on the perineal region for the present.

The following points should be noted during the skinning. The skin is connected with the underlying muscles by a loose weblike material, the subcutaneous connective tissue or superficial fascia, often impregnated with fat. Below this is the much firmer and tougher connective tissue on the surface of the muscles, forming the deep fascia. Passing from among the muscles into the skin will be seen at regular intervals, which represent the segments of the body, a slender cord, composed of an artery, a vein, and a sensory nerve. These may be severed. Other blood vessels, not segmentally arranged, will also be seen passing onto the under surface of the skin, from anterior and posterior regions toward the middle. The arteries are colored by an injection mass and are readily recognized. The veins are usually of a very dark reddish-brown color. All vessels to the skin should be severed.

When the skin has been loosened to the sides of the animal, there will be noted a thin layer of muscle fibers on its under surface, appearing like a fine striping. Toward the chest and shoulder region this assumes the form of a thin sheet. This muscle is a dermal or skin muscle, the panniculus carnosus or cutaneous maximus. It covers the entire lateral surface of the thorax and abdomen, being more prominent anteriorly. On continuing to skin forward and ventrally the muscle will be found to take its origin from the outer surface of a muscle (latissimus dorsi) situated posterior to the shoulder, and from the axilla in the cat, the medial side of the humerus (rabbit), and from the linea alba and various points on the ventral side of the thorax in both animals. These points of origin should be cut through and the cutaneous maximus removed with the skin to which it generally adheres. The muscle is inserted on the skin and serves to shake the skin. In man this muscle is degenerate. It is an outgrowth of the latissimus dorsi muscle.

There is one other dermal muscle, the platysma. This will be found on the under surface of the skin of the neck and head, and consists of many different 
parts, which have received separate names. Some of these will be seen later, but the study of the parts of the platysma is almost impossible in any but freshly killed specimens. The platysma muscle is inserted on the skin of the ears, eyelids, lips, etc., and serves to move them. In man it constitutes the muscles of facial expression. The platysma is a visceral muscle, derived from the muscles of the hyoid arch by extension.

In females on the under surface of the skin of the ventral side the mammary or milk glands will be noted spread out as a thin irregular layer.

The skin having been removed and discarded, clean away fascia and fat from the surface of the muscles. There is generally a large mass of fat at the base of the hind legs. It will now be seen that the exposed surface in part consists of muscles, pinkish masses composed of parallel fibers, and in part of the deep fascia which forms very strong white sheets. The posterior half of the back is covered by such a sheet, known as the lumbodorsal fascia. In the median ventral line is the linea alba. The angle between the base of the thigh and the abdominal wall is known as the inguinal region. At the bottom of this will be found in the rabbit a stout white shining cord, the inguinal ligament, which stretches from the pubic symphysis to the crest of the ilium. It is absent in the cat. The angle between the upper arm and chest is called the axilla, or axillary fossa.

In studying the muscles it is necessary to separate each muscle from its neighbors. This is done by searching carefully for the white lines of connective tissue which mark the boundaries of muscles and slitting along these lines with the point of the scalpel. Observing the direction in which the fibers run will also aid in separating muscles, since the fibers in one muscle generally run in the same direction, which is usually different from that of the neighboring muscles. After freeing the margins of a muscle the fingers should be worked under the muscle until it is separated from its fellows. As each muscle is inclosed in a connective tissue sheath, it will separate smoothly from its neighbors; the presence of rough edges indicates that the muscle itself has been cut. Avoid the use of sharp instruments in freeing the muscles. In case it is necessary to cut through a muscle in order to reveal another muscle beneath it, always cut through the center of the muscle.

2. The parts of a muscle.-We shall first study the parts of some convenient muscle in order to become acquainted with the terminology applied to muscles. For this purpose we shall select the external oblique. The external oblique is the large muscle covering the sides of the abdomen. The superficial fascia should be cleaned away from its surface. On closely inspecting the cleaned surface of the external oblique, the muscle will be observed to consist of numerous pinkish stripes directed obliquely ventrad and caudad.

The pinkish stripes are bundles of muscle fibers, called fasciculi. The white material between the fasciculi which binds them together into a muscle and 
which dips down between them is part of the deep fascia. It forms a sheath, the perimysium, for each fasciculus. The part of the muscle which is composed of fasciculi with their perimysia, is known as the fleshy part of the muscle, or the belly. The fasciculi do not extend completely to the ends of a muscle, but the deep fascia does. Consequently the ends of muscles are non-fleshy, composed of connective tissue only. These connective tissue ends of muscles commonly form tough shining bands or cords, known as tendons. When the tendon is very broad and flat, it is often called an aponeurosis, or the name fascia may be retained for such broad tendons. The attachments of muscles are always by means of tendons, aponeuroses or fasciae, never by the muscle fibers. Muscles are attached to bones or to tendons, aponeuroses, or fasciae, which themselves are attached to bones. The purpose of the voluntary muscles is to move the bones of the skeleton.

The anterior part of the external oblique is concealed under a large flat muscle, the latissimus dorsi, which covers the anterior part of the back and slopes toward the upper arm. The posterior boundary of this muscle should be located and slit, and it should then be lifted from the surface of the external oblique by thrusting the fingers between. If fat is present between these two muscles, it must be cleaned away. The most posterior chest muscles also cover the anterior part of the external oblique and should be lifted off in a similar manner. The external oblique will then be found to be attached to the posterior ribs by separate slips. The fixed points of attachment of a muscle are called its origin. When there is more than one origin, each one is known as a head. When there are a number of points of attachments segmentally arranged, they are generally designated as slips. The origin of the external oblique is from the posterior ribs by separate slips and from the lumbodorsal fascia. Its fibers (fasciculi) pass obliquely downward and backward, and in the rabbit the more dorsal ones pass nearly straight caudad. The movable points of attachment of a muscle on which it exerts its effect are called its insertion. The insertion of the external oblique is by way of an extensive aponeurosis which passes to the median ventral line. The insertion is: rabbit, on the linea alba by its aponeurosis and on the inguinal ligament, which is in turn attached to the ilium and the pubic symphysis; cat, on the linea alba and the pubis by its aponeuroses, and on the median ventral line (raphe) of the thorax. The function of a muscle is called its action. The action of the external oblique is constrictor of the abdomen.

In the following dissection of the muscles the dissection is to be confined strictly to the left side, leaving the right side intact for the dissection of other systems.

3. The muscles of the abdominal wall.-The abdominal wall is composed of three layers of muscles with their aponeuroses. The aponeuroses of these muscles are auite extensive. The three layers are: an external layer, the 
external oblique; a middle internal oblique; and an internal layer, the transversus abdominus.

a) External oblique: This is the outermost of the muscle layers of the abdominal wall. It was described above.

b) Internal oblique: Very carefully cut through the middle of the belly of the external oblique, in a longitudinal direction, and separate it from the underlying muscle, which is the internal oblique. This separation is often difficult The internal oblique is a short muscle lying beneath the more dorsal portion of the external oblique. Its fibers are directed obliquely downward and forward, and are continued by a very broad aponeurosis. Origin: rabbit-second sheet of the lumbodorsal fascia, posterior ribs and inguinal ligament; cat-second sheet of the lumbodorsal fascia and border of the pelvic girdle. Insertion, on the linea alba by the extensive aponeurosis; action, compressor of the abdomen.

c) Transverse: On cutting through the preceding and separating the edges, a third muscle layer, very thin, will be found. This is the transversus abdominis. Its fibers are directed ventrally and slightly posteriorly. Origin, insertion, and function similar to the preceding.

d) Rectus abdominis: This is a long slender muscle on each side of the linea alba, extending from the pubic symphysis to the anterior part of the thorax. It is found inside of and between the aponeuroses of the preceding muscles. Slit open these aponeuroses along each side of the linea alba and expose the rectus abdominis. Its fibers run longitudinally and in the cat are crossed at regular intervals by transverse white lines. Origin, anterior end of the pubic symphysis; insertion, sternum and costal cartilages; action, retracts ribs and sternum and constricts the abdomen. Underneath the transverse and the rectus abdominis is the peritoneal membrane, or lining of the coelom.

The foregoing muscles are hypaxial muscles, formed into sheets by the sidewise fusion of myotomes. The rectus abdominis represents the ventral ends of the myotomes. The three layers on the sides of the abdomen have resulted from a splitting process.

4. The epaxial muscles.-Remove the lumbodorsal fascia over the posterior part of the back, finding beneath it a great thick mass of muscle inclosed in a tough shining fascia. Note attachment of the lumbodorsal fascia in the median line to the neural spines of the vertebrae. The mass of muscle is divisible into a slender narrow median portion, the multifidus, next to the median dorsal line, and a very thick lateral portion, the sacrospinalis. The latter is in the cat readily divisible into three longitudinal portions. These muscles continue up into the thoracic region, where they will be seen later, to the back of the head, their various portions receiving different names. They are the epaxial muscles and are sharply separated from the hypaxial muscles in the abdominal region by a furrow which corresponds to the position of the horizontal skeletogenous septum of fishes. These muscles are the most powerful muscles in the body. 
They are attached to the sacrum, the ilium, the vertebrae and ribs at various points, and to the back of the skull, and serve to move the vertebral column as a whole or in part, raise the head, etc. All parietal muscles not included in this mass are hypaxial muscles, which have in some cases spread dorsally so as to cover the epaxial muscles.

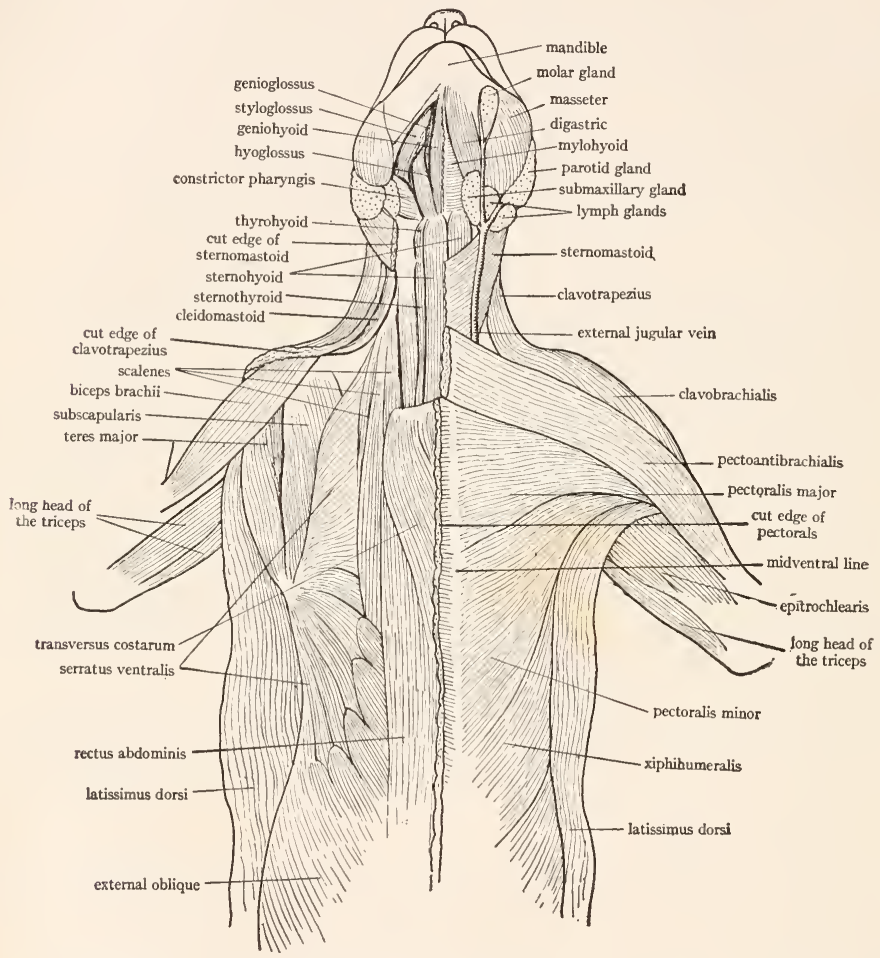

FIG. 4I.-Ventral view of the anterior part of the cat to show the muscles. All dermal muscles have been removed. Superficial muscles on the right side, deeper layer of muscles on the left side, after removal of the pectoral muscles, sternomastoid, mylohyoid, and digastric. The nerves and blood vessels which cross the axilla have been omitted. The view of the axilla is different from that revealed in the dissection given in the text. The epitrochlearis is called extensor antibrachii in the text.

5. The muscles of the chest.-(Fig. 4r.) Turn the animal on its back and expose the chest by spreading and fastening the fore limbs. The great muscles covering the chest or ventral side of the thorax are the pectoral muscles. They are divisible into several portions which are not very definitely separable from 
each other. According to $\mathrm{B}$ there are five parts in the rabbit, and according to $\mathrm{R}$ and $\mathrm{J}$, four parts in the cat. Of these we may consider the following.

\section{Rabbit:}

a) Pectoralis major. This is a large muscle originating from the whole length of the sternum and inserted on the humerus. It covers most of the surface of the chest, but the insertion is concealed by a muscle coming down from the head (clavodeltoid). Action, draws the arm toward the chest.

b) Pectoralis primus. A slender muscle at the anterior end of the preceding and covering its anterior fibers. Origin, manubrium of the sternum; insertion, humerus; action, like the preceding. Its anterior border is in contact with the clavodeltoid, which also partly covers its insertion.

c) Pectoralis minor. Cut through the middle of the belly of the pectoralis major, and upon deflecting the cut edges note internal to it a similar muscle, the pectoralis minor. Origin, manubrium; insertion, clavicle, and spine of the scapula. To find the insertion loosen up the clavodeltoid and locate in its fibers at the shoulder a small slender bone, the clavicle. The clavicle is on the inner surface of the muscle. Then loosen the muscle next lateral to the clavodeltoid, a long muscle coming down from the back of the neck (anterior trapezius). The pectoralis minor is inserted by some fibers on the clavicle, but most of its fibers sweep over the shoulder internal to the clavodeltoid and anterior trapezius and are inserted on the spine of the scapula. Action, draws arm and shoulder toward the chest.

Cat:

a) Pectoantibrachialis. Anterior and most superficial of the chest muscles. Origin, manubrium; insertion, by a flat tendon on the fascia of the forearm; action, draws the arm toward the chest.

b) Pectoralis major. Next posterior to the preceding and extending anteriorly dorsal to the preceding which should be cut across; originating on the sternum and median ventral raphe and inserted on the humerus. Action, like the preceding.

c) Pectoralis minor. Next posterior to the preceding and covered in large part by the pectoralis major. The latter should be cut through and the extent of the pectoralis minor noted. The pectoralis minor is divisible into several parts. Origin, sternum; insertion, humerus; action, like the preceding. The insertion cannot be fully traced at the present stage of the dissection.

d) Xiphihumeralis. The last of the chest muscles, a thin flat long muscle, passing from the xiphoid process of the sternum, its anterior part passing dorsal to the posterior part of the pectoralis minor, and inserted on the humerus. The insertion is covered by a mass of fat in the axilla. Remains of the cutaneous maximus are probably present on its surface. Action, like the preceding.

6. The muscles of the neck and throat.-(Figs. 4I or 42.) Slit the skin up the center of the throat to the tip of the lower jaw and loosen it so as to expose 
fully the lower jaw. Note in doing so parts of the platysma muscle on the under side of the skin. It sweeps from the median dorsal line of the neck around the sides of the head to face and ears, and portions of it generally are attached near the anterior end of the sternum. In dissecting the throat muscles work on one side only, leaving the other intact for the study of other parts. Avoid cutting any blood vessels. A large vein, the external jugular vein, runs in the superficial muscles of the throat. At the angle of the jaw is a rounded pinkish body, the submaxillary gland, one of the salivary glands. Other small bodies are lymph glands.

Rabbit:

a) Special portion of the platysma. A broad thin sheet of dermal muscle extends from the manubrium of the sternum forward, forking like the letter $\mathrm{V}$, each half inserting at the base of the ear. This is the depressor conchae posterior and is the most superficial muscle on the ventral surface of the neck. The external jugular vein runs in it. It is a part of the platysma. It should be well separated from the underlying muscles and the posterior end severed and turned forward, without, however, injuring the vein.

b) Sternohyoid. This is the long muscle in the median line of the neck, the two members of the pair being closely fused in the median ventral line. Origin, manubrium of the sternum; insertion, anterior horn of the hyoid. Follow the muscle up to the throat and feel with the fingers the bony hyoid on which the muscle is inserted. Action, draws the hyoid posteriorly or raises the sternum.

c) Sternomastoid. The long muscle on each side of the preceding, the two members of the pair converging toward the manubrium of the sternum from which they originate ventral to the origin of the preceding. Insertion, mastoid process of the skull; action, singly turn the head, together depress the head on the neck.

d) Cleidomastoid and basioclavicularis. These two long strap-shaped muscles are next lateral to the preceding and unite at the clavicle with the clavodeltoid. The cleidomastoid is the more medial one and lies lateral and somewhat dorsal to the sternomastoid. Origin, mastoid region of the skull; insertion, clavicle; action, elevates clavicle or turns the head. The basioclavicularis is slightly lateral to the preceding at its cranial end but crosses ventral to it caudally so that its insertion on the clavicle is medial to that of the cleidomastoid. Origin, occipital bone; insertion, clavicle; function, like preceding. The origins of these muscles cannot be followed out conveniently.

e) Clavodeltoid. Continuation of the two preceding muscles. Origin, clavicle; insertion, humerus; action, raises the humerus.

f) Masseter. The great mass of muscle covering the angle of the jaws, its outer surface with a very tough shining fascia. Origin, zygomatic arch; insertion, outer surface of the posterior end of the mandible; action, closes the lower iaw (elevator of the jaw.) 
g) Digastric. The muscle along the ventral surface of each half of the jaw bone, terminating in a slender tendon. Origin, occipital bone; insertion, ventral surface of the mandible; action, opens the jaw (depressor of the jaw).

h) Mylohyoid. The thin sheet of muscle crossing transversely between and dorsal to the two digastrics. Origin, mandible; insertion, median ventral line (raphe) and the hyoid; action, raises the floor of the mouth and brings the hyoid forward.

i) Sternothyroid. Divide the two sternohyoids in the median ventral line. This exposes the trachea or windpipe, a tube stiffened by rings of cartilage. At the top of the trachea at a level about between the two submaxillary glands there is an enlarged chamber, the larynx or Adam's apple, whose walls are supported by cartilage. The chief cartilage of the larnyx is the large shield-shaped cartilage, which forms the ventral wall; this is called the thyroid cartilage. The sternothyroid muscle will be found, one on each side of the trachea, dorsal to the sternohyoid, originating on the sternum and inserted on the thyroid cartilage. Action, pulls the larynx posteriorly.

j) Thyrohyoid. A thin muscle at each side of the larynx, extending from the thyroid cartilage to the hyoid. Action, raises the larynx.

\section{Cat:}

a) Sternomastoid. This is the superficial muscle of the ventral side of the neck. A large vein, the external jugular vein, crosses its surface at an angle to the direction of its fibers. Origin by two parts, from the median raphe and the manubrium of the sternum, the first-named origin lying ventral to the second, so that the muscle appears divisible into two muscles. From the origins the muscle passes obliquely away from the median ventral line around the sides of the neck and is inserted on the skull from the lambdoidal ridge onto the mastoid process. The muscle passes internal to the submaxillary gland and the parotid gland; the latter is a mass at the base of the ear. The insertion on the mastoid process is by means of a thick tendon. Action, singly turn the head, together depress head on neck.

b) Sternohyoid. The anterior ends of these muscles are visible between the two sternomastoids, as the latter diverge from the median raphe. Slit the raphe of the sternomastoids to the manubrium of the sternum, thus exposing the full length of the sternohyoids. They extend in the median ventral line from the first costal cartilage to the body of the hyoid bone, the two being closely united in the median line. Action, draw the hyoid posteriorly.

c) Cleidomastoid. Lateral to the sternomastoid is a long muscle passing from the head to the upper arm. Loosen this up and find internal to it a narrow flat muscle, the cleidomastoid. Origin, ciavicle, which will be found as a slender bone on the internal surface of the long muscle just mentioned at the level of the shoulder; insertion, mastoid process, dorsal to the insertion of the 
sternomastoid. Action, pulls clavicle craniad or turns head, acting singly, or lowers head on neck.

d) Clavotrapezius and clavobrachial. The long muscle on the side of the neck and passing over the ventral surface of the shoulder to the forearm is sometimes considered as one muscle, the cephalobrachial, or as two. In the latter case the upper part is known as the clavotrapezius and extends from the skull to the clavicle. It will be considered later. The lower part from the clavicle to the forearm is the clavobrachialis. Origin, clavicle and fibers of the clavotrapezius; insertion, ulna; action, flexor of the forearm. The clavicle will be found on the inner surface of the muscle in the shoulder region, imbedded in the muscle.

e) Masseter. The great thick muscle covering the angle of the jaws, situated in front of the submaxillary and parotid glands. It is covered by a very tough shining fascia. Origin, zygomatic arch; insertion, posterior half of the lateral surface of the mandible; action, elevator of the lower jaw.

f) Temporal. Remove the skin from the side of the head up to the median dorsal line. A great mass of muscle covered by a strong shining fascia will be seen occupying the temporal fossa of the skull, dorsal to the ear. Origin, from the side of the skull from the superior nuchal line to the zygomatic process of the frontal bone, and from part of the zygomatic arch; insertion, coronoid process of the mandible; action, elevator of the jaw, in common with the masseter.

g) Digastric. The muscle lying along the medial surface of each half of the mandible. It extends posteriorly internal to the submaxillary gland. Origin, jugular and mastoid processes of the skull; insertion, mandible; action, depressor of the lower jaw.

h) Mylohyoid. The thin transverse sheet passing across between the two digastrics from one half of the mandible to the other. Origin, mandible, the origin concealed by the digastrics; insertion, median raphe; action, raises floor of the mouth and brings hyoid forward.

i) Geniohyoid. Cut through the median raphe of the mylohyoid. This exposes a pair of long slender muscles, the geniohyoids, lying in the median line. Origin, mandible near the symphysis; insertion, body of the hyoid; action, draws the hyoid forward.

j) Sternothyroid. Separate the two sternohyoids in the median line. This exposes the trachea or windpipe, a tube stiffened by rings of cartilage. At the top of the trachea is a chamber with cartilaginous walls, the larynx. The chief cartilage of the larynx is the large shield-shaped thyroid cartilage, forming the ventral walls of the larynx. Just in front of the thyroid cartilage the body of the hyoid is felt as a bony bar. The sternothyroid muscles are located, one on each side of the trachea, dorsal to the sternohyoids. Origin, sternum in common with the sternohyoid; insertion, thyroid cartilage of the larynx; action pulls the larynx posteriorly. 
k) Thyrohyoid. Short narrow muscle on each side of the thyroid cartilage from which it takes its origin; insertion, posterior horn of the hyoid; action, raises the larynx.

The majority of the muscles mentioned in this section are the visceral muscles. They correspond to the muscles found in the dogfish associated with the

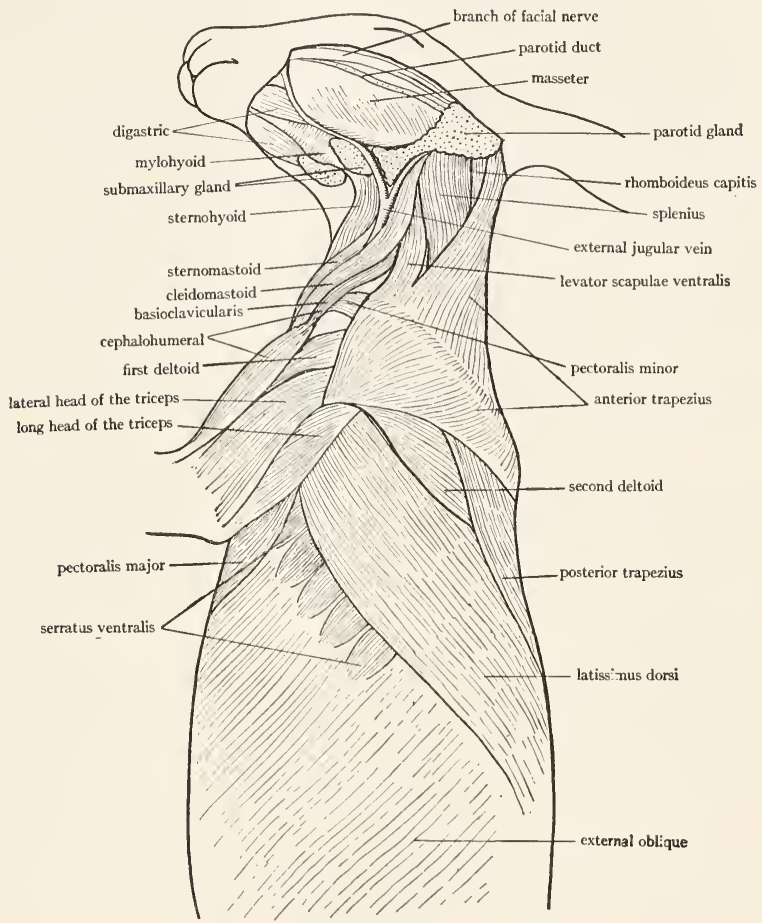

FIG. 42.- Lateral view of the anterior part of the rabbit to show the muscles. The head is turned slightly to give a ventral view of the throat. All dermal muscles have been removed.

gill arches. They are the muscles of the jaws, the hyoid, and the cartilages of the larynx, all of which structures are, as learned in the study of the skull, homologous with the gill arches of fishes. The masseter, the temporal, the mylohyoid, and part of the digastric are muscles of the mandibular arch; the platysma and the rest of the digastric are muscles of the hyoid arch; while the muscles of the remaining arches become muscles of the pharynx and larynx, which were not studied. 


\section{(Fig. 42.)}

7. The muscles of the upper back and shoulder and back of the neck.-

\section{Rabbit:}

a) Latissimus dorsi. Turn the animal on one side, so that the side on which the muscles have already been dissected will be uppermost. The large flat muscle extending obliquely from the middle of the back to the fore limb is the latissimus dorsi. Origin, lumbodorsal fascia and posterior ribs; insertion, on the crest on the medial side of the humerus, the insertion covered by the chest muscles; action, draws the arm caudad and dorsad.

b) Anterior and posterior trapezius. These two muscles are the flat, thin muscles covering the upper back and back of the neck anterior to the latissimus dorsi. The posterior trapezius originates from the lumbodorsal fascia and the neural spines of the thoracic vertebrae, and is inserted on the spine of the scapula. Action, draws the scapula dorsally. The anterior trapezius originates on the external occipital protuberance of the skull and ligament in the mid-dorsal line and is inserted on the metacromion process (which is very long in the rabbit) and nearby muscles and fascia; action, draws the scapula and limb upward and forward. The space between the two trapezius muscles is filled by a stout fascia.

c) Levator scapulae ventralis. This long slender muscle runs along the ventral border of the anterior trapezius near its insertion, then diverges to its origin from the ventral surface of the skull at the suture between occipital and basisphenoid; insertion, metacromion process in common with the anterior trapezius; action, pulls the scapula anteriorly.

d) Rhomboideus. Cut across the middle of the bellies of the two trapezius muscles and the latissimus dorsi. The large thick muscle extending from the vertebral border of the scapula to the mid-dorsal line is the rhomboideus. Origin, mid-dorsal ligament of the neck and succeeding neural spines; insertion, vertebral border of the scapula; action, draws scapula toward vertebral column.

e) Splenius. A fairly broad but thin muscle on the back of the anterior part of the neck under the anterior trapezius. (Running along its external surface is a narrow straplike muscle, the rhomboideus capitis, see below.) Origin of splenius, mid-dorsal line of neck and adjacent fascia; insertion, occipital region of the skull and atlas; action, singly turns the head, together raise the head.

Under the splenius are the epaxial muscles, continuations of those already noted in the lumbar region.

f) Supraspinatus. The superficial muscular layer of that part of the scapula anterior to the spine consists of the pectoralis minor, which sweeps over the scapula to be inserted on the spine and vertebral border. Lift up its anterior border and separate it from the muscle beneath it. This muscle is the supraspinatus, filling the supraspinous fossa of the scapula. Origin, supraspinous fossa; insertion, greater tuberosity of the humerus; action, extends the humerus. 
g) Deltoids. There are three deltoids in the rabbit, of which one, the clavodeltoid, has already been considered. The second deltoid is a small triangular muscle lateral to the clavodeltoid. Origin, acromion process; insertion, humerus; action, raises the humerus. The third deltoid is lateral to the second and is a longer muscle. It passes under the long metacromion process and takes its origin from the fascia of the muscle which fills the infraspinous fossa. Insertion and action like the preceding.

h) Infraspinatus. The muscle partly covered by the third deltoid which is attached to its surface. The deltoid may be removed to see it. Origin, infraspinous fossa and spine; insertion and action like the supraspinatus.

i) Teres major. The stout muscle along the axillary border of the scapula behind the preceding. Origin, dorsal half of the axillary border of the scapula; insertion, on the humerus in common with the latissimus dorsi; action, draws humerus against body and rotates it.

j) Teres minor. Separate the teres major well from the infraspinatus and look in between them. On the inner surface of the latter will be found a small but stout muscle. Origin, ventral half of the axillary border of the scapula; insertion, greater tuberosity of the humerus; action, like the preceding.

k) Rhomboideus capitis (levator scapulae minor of $\mathrm{B}$ ). Cut through the rhomboideus. A slender bandlike muscle lies in contact with the inner surface of the rhomboideus and passes along the external surface of the splenius to be connected with the skull. Origin, side of the skull above the tympanic bulla; insertion, posterior end of the vertebral border on the medial side; action, draws scapula craniad and rotates it.

l) Subscapularis. Lift the scapula. A large muscle, the subscapularis, completely covers the medial or inner surface of the scapula, its fibers disposed in several directions. The muscle has a shining fascia; its posterior end is more or less continuous with teres major. Origin, medial surface of the scapula; insertion, lesser tuberosity of the humerus; action, pulls the humerus toward the median ventral line.

$m$ ) Serratus ventralis (anterior). On raising with the finger the vertebral border of the scapula a large fan-shaped muscle will be seen extending anteriorly and posteriorly from the scapula to the walls of the thorax. This is the serratus ventralis; it is readily divisible into anterior and posterior portions. The anterior or cervical portion originates on the transverse processes of the cervical vertebrae by separate slips and on the first two ribs. The posterior or thoracic portion takes its origin by seven slips from the ribs. Insertion, vertebral border of the scapula above the subscapularis; action, draws scapula forward, backward, or against the body.

n) Scalenes. The scalenes are several long flat muscles extending from the transverse processes of the cervical vertebrae to the ribs. They will be seen by lifting up the scapula and looking on the ventral side of the origin of the 
serratus ventralis. They lie internal to the sternomastoid, sleidomastoid, etc., previously identified and farther posteriorly they form the layer next internal to the pectoralis muscles. Action, raise the ribs and bend the neck.

o) Serratus dorsalis (posterior). The dorsal half of the thorax underneath the latissimus dorsi, trapezius, and rhomboideus muscles is covered by a strong aponeurosis (part of the lumbodorsal fascia), in the ventral part of which muscle fibers are present which are inserted on the ribs by slips. The foremost slips are quite fleshy and take their origin by a tendon from the median dorsal line of the neck. This muscle is the serratus dorsalis. Action, raises the ribs craniad.

p) Intercostals. On the sides of the chest a series of muscles will be seen running from one rib to the next one. They are the external intercostals. They extend on the chest wall ventral to the insertion of the serratus dorsalis, which muscle in fact covers their most dorsal portions. Origin, posterior margins of the vertebral ribs; insertion, anterior margins of the succeeding vertebral ribs; action, pull the ribs forward. Observe that the fibers of the external intercostals are directed obliquely backward. On carefully cutting through any of the external intercostals, a layer of internal intercostals will be found inside of them, their fibers being directed obliquely forward. The internal intercostals are best seen in the ventral thoracic wall, internal to the scalenes, which may be cut through. Here between the costal cartilages the internal intercostals are not covered by the external intercostals. Origin and insertion, margins of the vertebral and sternal ribs; action, lower the ribs. The intercostals are the chief muscles concerned in the respiratory movements of the thorax. The scalenes, serratus, and other muscles assist.

q) Epaxial muscles of the thorax. The mass of epaxial muscles is conspicuous running along the dorsal part of the thorax. This mass passes internal to the serratus dorsalis which should be cut through; it lies upon the dorsal portions of the ribs and thus conceals the dorsal portions of the intercostal muscles. It is covered by the tough shining lumbodorsal fascia, which should be removed. The epaxial mass is easily divisible into a narrow median portion next to the median dorsal line, the semispinalis dorsi, and a very thick lateral portion, the longissimus. The latter gives off on its ventral margin the narrow iliocostalis lying on the ribs to which it is inserted. The longissimus is the continuation of the sacrospinalis and is inserted on the ribs. The epaxial mass may be followed along the neck by cutting the splenius.

The attention of the student is directed to the fact that the intercostal muscles represent the original layer of myotomes, all of the muscles external to them having been derived by processes of budding and splitting. The serratus muscles beautifully illustrate the method of origin of a muscle by buds from a number of myotomes, since the slips by which they arose from the intercostals are still present. 
Cat:

a) Latissimus dorsi. Turn the animal on one side so that the side on which the muscles have already been dissected will be uppermost. The large flat muscle extending obliquely forward from the middle of the back to the upper arm is the latissimus dorsi. Origin, from the neural spines of the last thoracic and most of the lumbar vertebrae and from the lumbodorsal fascia; insertion, by a tendon on the medial surface of the humerus; action, pulls the fore limb dorsally and caudally.

b) Trapezius muscles. There are three trapezius muscles in the cat; they are the thin flat muscles covering the back and neck anterior to the preceding. The posterior trapezius or spinotrapezius takes origin from the spines of the thoracic vertebrae and passes obliquely forward, covering part of the latissimus to be inserted on the fascia of the scapula; action, draws the scapula dorsad and caudad. In front of this is the middle trapezius or acromiotrapezius. Origin, neural spines of cervical and first thoracic vertebrae; insertion, metacromion process and spine of the scapula and fascia of the preceding muscle; action, draws scapula dorsad and holds the two scapulae together. The anterior trapezius or clavotrapezius is the anterior part of the long muscle already described as cephalobrachial. Origin, superior nuchal line and median dorsal line of neck; passes obliquely ventrally to be inserted on the clavicle which is imbedded on its inside surface; it is continuous with the clavobrachial muscle. Action, draws the clavicle dorsad and craniad.

c) Levator scapulae ventralis. Carefully free the three trapezius muscles. Along the ventral border of the acromiotrapezius and apparently continuous with it is seen a flat bandlike muscle which passes anteriorly diverging from the acromiotrapezius and passing internal to the clavotrapezius which should be cut across. Origin, transverse process of the atlas and occipital bone; insertion, metacromion process and neighboring fascia; action, draws the scapula craniad.

d) Rhomboideus. Cut across the middle of the bellies of the spino- and acromiotrapezius muscles. A thick muscle will be seen beneath them extending from the vertebral border of the scapula to the mid-dorsal line; this is the rhomboideus. Origin, neural spines of the vertebrae and adjacent ligaments; insertion, vertebral border of the scapula; action, draws scapula dorsad. The most ventral portion of this muscle is a practically separate muscle, the rhomboideus capitis, which extends as a slender band forward to originate from the superior nuchal line; insertion, scapula; action, draws the scapula craniad and rotates it.

e) Splenius. This is the large sheet of muscle covering the back of the neck in front of the rhomboideus, internal to the trapezii and crossed by the rhomboideus capitis. Origin, from the mid-dorsal line and fascia; insertion, superior nuchal line; action, raises or turns the head. 
f) Supraspinatus. On turning back the ventral half of the cut acromiotrapezius a stout muscle is seen occupying the supraspinous fossa of the scapula. Origin, whole surface of the supraspinous fossa; insertion, greater tuberosity of the humerus, next to the insertion of pectoralis minor; action, extends the humerus.

g) Deltoids. There are two deltoids in the cat. The clavobrachialis already described is sometimes also considered as a deltoid and is called the clavodeltoid by some authorities. The acromiodeltoid is a short thick muscle passing ventrally from the acromion process; it is inserted on the surface of other muscles of the shoulder. It lies lateral to the clavobrachial. Action, with the next. Turn the flap of the acromiotrapezius forward again. Posterior to the line marking the insertion of the acromiotrapezius and the levator scapulae ventralis is a muscle which passes to the upper arm, across the upper ends of the muscles of the upper arm. This is the spinodeltoid. Origin, spine of the scapula; insertion, ridge (deltoid ridge) of the humerus, the insertion being concealed by the acromiodeltoid; action, the deltoids raise and rotate the humerus.

h) Infraspinatus. Cut across the belly of the latissimus dorsi. Bring the anterior parts of the latissimus and the spinotrapezius forward so as to expose the posterior part of the scapula. Two large muscles are here seen. The anterior one fills the infraspinous fossa of the scapula, from whose surface it takes its origin and is inserted on the greater tuberosity of the humerus, the insertion being concealed by the deltoids which may be cut across to see it. This muscle is the infraspinatus. Action, rotates the humerus.

i) Teres major. The stout muscle immediately behind the preceding, its fibers running in the same direction. Origin, axillary border of the scapula and fascia of neighboring muscles; insertion, in common with latissimus dorsi on the medial surface of the humerus; action, rotates the humerus, and lowers it.

j) Teres minor. Carefully separate the infraspinatus from teres major and separate the former from the deltoids and the muscles of the upper arm. On the posterior border of the infraspinatus and somewhat covered by it is the small teres minor. Origin, axillary border of the scapula; insertion, greater tuberosity; action, assists the infraspinatus.

k) Subscapularis. Place the finger under the anterior border of the scapula and clear away connective tissue from the under surface of the scapula. The subscapular fossa is seen to be occupied by a muscle, the subscapularis, which covers the inner or medial surface of the scapula. Origin, subscapular fossa; insertion, lesser tuberosity of the humerus; action, pulls the humerus medially. Posterior to the subscapularis will be found part of teres major which extends to the medial surface of the scapula.

l) Serratus ventralis (anterior). Cut through the rhomboideus close to the vertebral column. The scapula then swings loose. On raising the scapula, a large fan-shaped muscle is seen extending from the vertebral border of the 
scapula to the sides of the thorax and neck. Origin, by slips from the first nine or ten ribs and the anterior part (called by $\mathrm{R}$ and $\mathrm{J}$ levator scapulae) from the transverse processes of the last five cervical vertebrae; insertion, scapula near the vertebral border; action, draws the scapula craniad, ventrad, and against the thoracic wall.

$m$ ) Serratus dorsalis (posterior). The anterior part of this muscle arises by a number of fleshy slips from the ribs near their angles. The short slips soon pass into a thin aponeurosis which overlies the epaxial muscles of the thorax, and which is fastened to the median dorsal line. The posterior part of this muscle consists of a few slips lying under the latissimus dorsi and appearing like a forward continuation of the internal oblique. These slips originate on the last ribs and are inserted by means of an aponeurosis onto the median dorsal line. Action, draw the ribs forward.

n) Scalenes. Raise up the pectoralis muscles from the chest wall by passing the fingers under them. If necessary their posterior parts may be cut into. Several long muscles will be seen in the chest wall ventral to the origin of the serratus ventralis and in front of the anterior boundary of the external oblique. These muscles are scalenes; they originate on the ribs and pass forward in a nearly straight course to be inserted on the transverse processes of the cervical vertebrae, uniting anteriorly into one band which will readily be seen by looking immediately ventral to the origin of the anterior part of the serratus ventralis. Insertion, transverse processes of the cervical vertebrae; action, draw the ribs forward and bend the neck.

o) Intercostals. The intercostals are a set of muscles extending from one rib to the next. The external layer is called the external intercostals and will be seen in part by looking at the chest wall between the origins of the serratus ventralis and dorsalis. Their fibers run obliquely backward and downward. On cutting through some of them another layer, the internal intercostals, will be seen inside of the external layer. The fibers of the internal intercostals run obliquely forward and downward. Near the median ventral line the external intercostals are lacking, so that the internal ones are exposed by cutting through the scalenes. Action, external intercostals bring the ribs forward, internal intercostals draw them back again. The intercostals are the chief respiratory muscles of the thoracic wall.

p) Epaxial muscles of the thorax. On cutting through the aponeurosis of the serratus dorsalis, the thick mass of epaxial muscle is exposed. This may be followed up into the neck by cutting through the splenius. In the thoracic region the muscle is divisible into three parts of about equal width, a dorsal part next the median dorsal line, the semispinalis dorsi; a median part, the longissimus; and a ventral part, the iliocostalis. The latter is composed of a number of separate bundles with prominent tendons between them, and lies on the ribs to which it is attached. The longissimus may be traced forward to its 
insertion on the transverse processes of the vertebrae in contact with the origin of the serratus ventralis. The mass in the neck is divisible into a medial oiventer cervicis and a lateral complexus. All of the epaxial muscles are very complex consisting of numerous bundles. They are attached to various parts of the vertebrae, ribs, and head, and have the general function of moving the vertebral column and related parts.

8. The muscles of the upper arm.- Separate these muscles from each other and identify them.

Rabbit:

a) Triceps brachii. The triceps is the great muscle on the back of the upper arm. It consists of three heads which are practically distinct muscles. Free the heads. The long head is the large mass on the back of the upper arm; origin, scapula, from the axillary border; insertion, olecranon. The lateral head is on the lateral surface of the upper arm, ventral to the preceding; origin, greater tuberosity of the humerus. The medial head is in contact with the humerus. To see it spread the other two heads apart and look deep between them or cut through the middle of the belly of the lateral head; origin, along the dorsal surface of the humerus. All three heads insert on the olecranon and are the great extensors of the forearm.

b) Extensor antibrachii. On the medial surface of the long head of the triceps is a fascia from the lower part of which this muscle originates. Insertion, olecranon; action, in common with the triceps.

c) Biceps brachii. On the anterior surface of the upper arm found by removing the insertions of the pectoral muscles is a spindle-shaped muscle, the biceps. Origin, glenoid fossa; insertion, ulna and radius; action, flexor of the forearm.

d) Brachialis. This muscle is next lateral to the biceps and in contact with the humerus. Origin, ventral and lateral surface of the humerus; insertion and action, in common with the biceps.

Cat:

a) Triceps brachii. As above under rabbit with three heads.

3) Extensor antibrachii. On the medial side of the long head, a thin sheet of muscle, taking origin from the latissimus dorsi and inserted on the olecranon; action, in common with the triceps, tending also to rotate the ulna.

c) Biceps brachii. As in the rabbit; visible only after cutting the insertions of the pectoral muscles. Origin, glenoid fossa; insertion, radius; action, flexor of the forearm.

d) Brachialis. Lateral to the biceps, in contact with the lateral head of the triceps. Origin, lateral surface of the humerus; insertion, ulna; action, with the biceps.

e) Anconeus. A small triangular muscle at the elbow joint, covered by the distal end of the lateral head of the triceps, which should be deflected. 
Origin, distal end of humerus; insertion, lateral surface of the ulna; action, strengthens the elbow joint, which it covers, and possibly rotates the ulna.

9. The muscles of the thigh.--(Fig. 43.)

\section{Rabbit:}

a) Tensor fasciae latae. The anterior half of the lateral surface of the thigh is covered by a tough fascia or aponeurosis called the fascia lata. In the dorsal part of this will be found a short muscle, the tensor fasciae latae, which is more or less continuous with adjacent muscles. Origin, ilium; insertion, in the fascia; action, tightens the fascia.
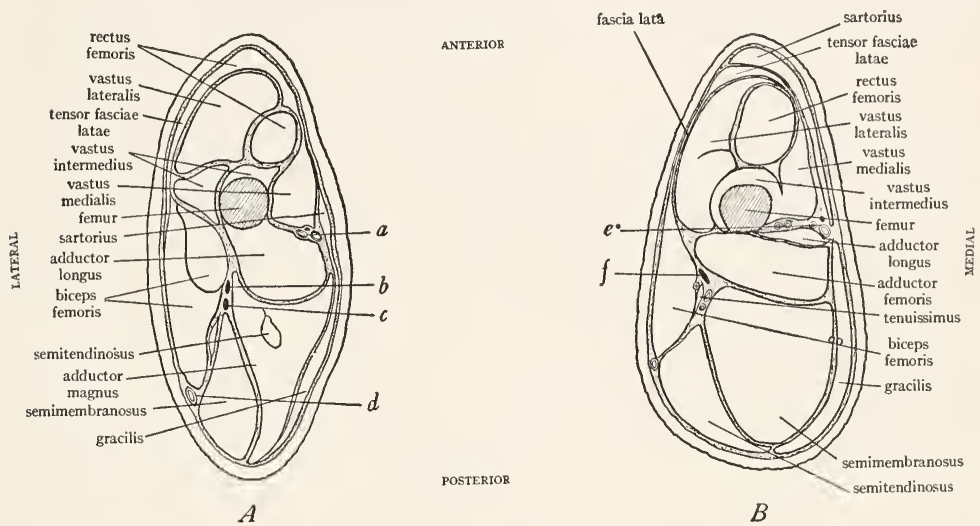

F1G. 43.-Cross-sections through the thigh of $A$, rabbit, and $B$, cat to show the location of the muscles. Black spots are nerves, small circles, blood vessels. $a$, greater saphencus nerve, artery, and vein; $b$, peroneal nerve; $c$, tibial nerve; $d$, sciatic vein; $e$, femoral nerve, artery, and vein; $f$, sciatic nerve. (A from Bensley's Practical Anatomy of the Rabbit, University of Toronto Press.)

b) Biceps femoris. Slit open the fascia lata. Posterior to the fascia occupying the middle portion of the lateral surface of the thigh is the large biceps femoris. Separate it from the muscle posterior to it which forms the posterior margin of the thigh (semimembranosus). On removing the surface fascia from the biceps it will be found divisible into two heads. Follow these heads dorsally to their origins, clearing away the tough surface fascia. Origin of the smaller anterior head, neural spines of adjacent vertebrae, of the larger posterior head, ischial tuberosity; insertion, by a tendon on the kneecap and on the fascia of the shank; action, abductor of the thigh and flexor of the shank.

c) Gluteus maximus. Remove the fascia over the sacral region. The gluteus maximus is under the fascia, a thin muscle whose posterior part is partly covered by the biceps femoris. The muscle also has an anterior part which is continuous with the tensor fasciae latae. The two parts of the gluteus maximus 
are united by an aponeurosis. Origin, fascia of the sacrum and part of the ilium; insertion, third trochanter, the small projection below the great trochanter; action, abducts the thigh.

d) Gluteus medius. The larger, thicker muscle anterior to the preceding and partly covered by it. Origin, crest and anterior part of the ilium; insertion, greater trochanter; action, like the preceding. When the two gluteus muscles have been well separated, the origin of the tensor fasciae latae will be found on the ventral edge of the gluteus medius dipping deep down to the ilium.

e) Vastus lateralis. Cut through the fascia lata and its tensor by a longitudinal slit extending to the knee. Under the fascia will now be seen the stout vastus lateralis. Origin, greater trochanter dorsal to the insertion of the gluteus medius.

f) Vastus intermedius. This is the name given to what appears to be the posterior part of the preceding muscle. It is partly separable from the vastus lateralis and lies between this and the anterior head of the biceps femoris. Origin, greater trochanter and surface of the femur.

g) Rectus femoris. This is the thin muscle which is folded over the anterior margin of the thigh; it extends on both lateral and medial surfaces of the thigh. It originates on the ilium and the fascia lata and is fused with the fibers of the tensor fasciae latae. The rectus femoris consists of two parts, the part just described and a second part, which may be located as follows. Separate the rectus femoris from the vastus lateralis to which it will be found slightly fused, continuing this separation to the medial side of the thigh, spreading the legs apart. The first part of the rectus femoris may be cut through at the middle. On the medial side of the thigh internal to the first part of the rectus femoris and ventral to the vastus lateralis is the second part of the rectus femoris, a cylindrical muscle. Origin, ilium in front of the acetabulum.

h) Vastus medialis. On the medial side of the thigh posterior to the first part of the rectus femoris and not very well separable from it. Origin, femur.

The rectus femoris and the three vastus muscles together constitute the great quadriceps femoris muscle. The origins of its several parts have already been given. Insertion, on the tibia and the patella and the tendon which extends over the patella; action, powerful extensor of the shank.

i) Sartorius. The very long narrow muscle extending like a band along the middle of the medial surface of the thigh. Origin, inguinal ligament; insertion, tibia; action, adductor of the thigh, rotator of the thigh, extensor of the shank. The sartorius lies between the vastus medialis and the next muscle to be described, and covers some large blood vessels.

j) Gracilis. A large thin muscle over the posterior half of the medial surface of the thigh. Origin, pubic symphysis; insertion, fascia of the distal portion of the thigh and proximal portion of the shank: action. adductor of the leg. 
k) Adductor longus and adductor magnus. Cut through the middle of the gracilis and find beneath it two stout muscles, their fibers running from the median ventral line to the femur. The anterior muscle is the adductor longus; the posterior one, the adductor magnus. Origin, various parts of the ischium; insertion, femur; action, adductors of the thigh.

l) Semitendinosus. Split open the adductor magnus and find inside of it a cylindrical muscle, the semitendinosus. Origin, ischial tuberosity; insertion, medial condyle of the tibia; action, flexor of the shank.

$m$ ) Semimembranosus. This is the muscle which forms the posterior margin of the thigh, between the biceps femoris and the adductor magnus. Origin, fascia over the biceps, and ischial tuberosity; insertion, with the gracilis in the fascia of the shank; action, flexor of the shank.

\section{Cat:}

a) Tensor fasciae latae. Examine the lateral (outer) surface of the thigh. The anterior part of this is covered by a tough fascia, the fascia lata. In the dorsal part of this is a muscle, the tensor fasciae latae, a thick triangular muscle. Origin, ilium and neighboring fascia; insertion, fascia lata; action, tightens the fascia lata.

b) Biceps femoris. This is the large muscle on the lateral surface of the thigh posterior to the fascia lata and covering more than half of the surface of the thigh. It has but one head in the cat. Origin, tuberosity of the ischium; insertion, patella and tibia by a tendon, and the fascia of the shank; action, abductor of the thigh and flexor of the shank.

c) Caudofemoralis. Clean away the fascia from the back in front of the base of the tail, as far forward as the anterior end of the pelvic girdle. Muscles will be found between the median dorsal line and the thigh. The most posterior of these is the narrow flat caudofemoralis, passing from the side of the root of the tail toward the dorsal end of the biceps femoris. Origin, transverse processes of the second and third caudal vertebrae; insertion, the muscle passes ventrally concealed by the anterior margin of the biceps femoris. This should be lifted up and the caudofemoralis followed to its tendon; the latter is very long and passes to the patella on which it is inserted. Action, abductor of the thigh, extensor of the shank.

d) Gluteus maximus. A rather thin flat muscle immediately anterior to the preceding. It is imbedded in the fascia and is continuous with the tensor fasciae latae anteriorly. Origin, from the fascia and from the transverse processes of the last sacral and first caudal vertebrae; insertion, fascia lata and to a slight extent on the greater trochanter; action, in common with the next.

e) Gluteus medius. The very large triangular muscle immediately in front of the preceding and partly covered by it. The gluteus maximus should be cut across to see it. Origin, adjacent fascia, crest of the ilium, and lateral surface of the ilium, and transverse processes of the last sacral and first caudal vertebrae; 
insertion, by a strong tendon on the greater trochanter of the femur; action, abductor of the thigh. Along the anterior border of this muscle the origin of the tensor fasciae latae passes internally toward the ilium.

f) Sartorius. This muscle forms the anterior margin from the lateral view of the thigh. It is folded over the margin and on following it to the medial or inner surface of the thigh will be found to cover the anterior half of the medial surface. Origin, crest, and ventral border of the ilium; insertion, proximal end of the tibia and the patella and the fascia and ligaments between; action, adductor and rotator of the thigh and extensor of the shank.

g) Vastus lateralis. Cut through the fascia lata by a longitudinal slit extending to the patella. Separate well the sartorius from underlying parts. The tensor fasciae latae is now well exposed. The large stout muscle which was covered by the fascia lata is the vastus lateralis. Origin, greater trochanter and surface of the femur.

h) Rectus femoris. At its anterior margin the vastus lateralis will be found partly separable from a stout muscle lying on its medial side and covered externally by the sartorius. The sartorius may be cut across the middle. The muscle in question is the rectus femoris. Origin, ilium near the acetabulum.

i) Vastus medialis. This is on the medial side of the thigh posterior to the rectus femoris which its anterior margin partly covers. It also is covered externally by the sartorius. Origin, femur.

j) Vastus intermedius. On widely separating the rectus femoris from the vastus lateralis a muscle will be seen deep down next to the shaft of the femur; Origin, surface of the femur.

The rectus femoris and the three vastus muscles are more or less united to each other and constitute the great quadriceps femoris muscle. Its origins have been described; all its parts are inserted on the patella and adjacent ligaments. action, extensor of the shank.

k) Gracilis. This is the large flat muscle forming the posterior half of the medial surface of the thigh. Origin, ischial and pubic symphyses; insertion by an aponeurosis which passes to the tibia; action, adductor of the leg.

l) Adductor longus and adductor femoris. Cut through the middle of the gracilis and separate each half from the underlying muscles. The latter consist of three muscles passing from the median ventral line to the femur. The most anterior of the three is quite small; this is the adductor longus. Origin, pubis; insertion, femur; action, with the next. The middle muscular mass is the large adductor femoris (corresponding to adductor magnus and brevis of other mammals). Origin, pubis; insertion, femur; action, adductor of the thigh.

$m$ ) Semimembranosus. The large posterior part of the mass which was covered by the gracilis. Origin, ischium; insertion, medial epicondyle of the femur and proximal end of the tibia; action, extensor of the thigh. The muscle is more or less divisible into two parts. 
n) Semitendinosus. The most posterior muscle of the thigh, posterior to the preceding. Origin, ischial tuberosity; insertion, tibia; action, flexor of the shank.

o) Tenuissimus. Turn to the lateral surface of the thigh. Cut through the middle of the biceps femoris. Beneath it will be noted a very narrow long muscle, the tenuissimus. Origin, transverse process of the second caudal vertebrae, in common with the caudofemoralis; insertion, on the same fascia as the insertion of the biceps.

On separating the biceps from the underlying muscles they will be revealed as extensions of muscles already identified on the medial surface. The adductor femoris is seen in contact with the femur posterior to the vastus lateralis; the semimembranosus comes next, and the semitendinosus is again the most caudal of the thigh muscles.

\section{I0. The muscles of the shank.-}

\section{Rabbit:}

a) Tibialis anterior. The lateral (outer, dorsal) surface of the shank is covered by the distal end of the biceps femoris and fascia. These should be removed. The most anterior of the muscles of the lateral surface is the tibialis anterior. Origin, lateral condyle and tuberosity of the tibia; insertion, second metatarsal; action, flexor of the foot.

b) Peroneus. Next dorsal to the preceding on the lateral surface, consisting of a group of several more or less fused muscles. Origin, tibia and fibula; insertion, metatarsals; action, flexor of the foot.

c) Gastrocnemius. This is the thin but broad muscle forming the caudal surface of the shank, divisible into two nearly separate portions, one of which is on the lateral, one on the medial surface of the shank. Origin, lateral and medial condyles of the femur and tibia; insertion, by a strong tendon, the tendon of Achilles, which passes over the heel (calcaneus) on which it is inserted; action, extensor of the foot.

d) Soleus. This is the muscle just internal to that part of the gastrocnemius which is on the lateral surface of the thigh. Origin, head of the fibula; insertion and action with the preceding.

e) Plantaris. This is situated internal to that part of the gastrocnemius which is medial. Origin, lateral condyle of the femur; insertion and action with the preceding.

f) Other muscles of the shank (optional). There are three more muscles of the shank; they lie in contact with the tibia. They are: the extensor hallucis longus exposed on the medial surface of the tibia; extensor digitorum longus, covered by the tibialis anterior; and flexor digitorum longus, between the tibia and the soleus and plantaris. As their names imply, these muscles are inserted by long slender tendons on the digits and act to flex and extend the digits.

It should be noted that the muscles named extensor in the shank and foot are really flexor in their action and those named flexor are extensor in their 
action, following a custom borrowed from human anatomy. The custom arises from a desire to retain the names applied to the muscles of the fore limb for the muscles in the corresponding positions in the hind limb. Thus in the supine position of the forearm, the extensors face anteriorly and the flexors posteriorly. Similarly the muscles on the anterior side of the leg are designated extensors, although they really flex the foot, and those on the posterior side, flexors, although they extend the foot. In describing the action, the terms flexor and extensor are used with reference to the movement produced and not with reference to the position of the muscle on the limb.

Cat:

a) Tibialis anterior. Clean away the tough fascia of the shank and also the insertions of the biceps and the gracilis. Examine the lateral (outer) surface of the shank. The most ventral muscle whose ventral border is in contact with the tibia is the tibialis anterior. Origin, proximal parts of tibia and fibula; insertion by a strong tendon which should be traced into the foot where it will be found to pass obliquely to the medial side of the foot to be inserted on the first metatarsal; action, flexor of the foot.

b) Extensor digitorum longus. This is the muscle next dorsal to the preceding on the lateral surface of the shank. It is so closely placed to the preceding as to appear as part of it, but the line of separation will be found by a little searching. Origin, lateral epicondyle of the femur; insertion, by a stout tendon which if followed into the foot is found to diverge into four tendons, one of which is inserted on each digit; action, extensors of the digits.

c) Peroneus muscles. These are next dorsal to the preceding, originating on the fibula. There are three of them more or less fused to each other. The three end each in a tendon; the three tendons pass over the lateral surface of the lateral malleolus of the tibia and over the calcaneus, and are inserted on the metatarsals and digits. Action, extensors and flexors of the foot.

d) Gastrocnemius. This is the large muscle forming the posterior or caudal surface of the shank. It is divisible into two large portions, one on the medial surface, the other on the lateral surface of the shank. The lateral head is subdivisible into four heads. Origins, from the surface fascia, the femur, and the tendon and fascia of the plantaris muscle (see below); insertion, by a strong tendon, which passes to the heel bone (calcaneus) on which it is inserted. Action, extensor of the foot.

e) Soleus. On carefully separating the lateral head of the gastrocnemius, a muscle, the soleus, will be found internal to it. It is a flat muscle in contact with the peroneus muscles ventrally; it tapers abruptly to a tendon which joins the tendon of the gastrocnemius. Origin, fibula; insertion, calcaneus; action, with the gastrocnemius, of which it is sometimes considered a part.

f) Plantaris. On carefully separating the medial head of the gastrocnemius a large muscle will be found internal to it, lying between the two heads of the gastrocnemius which practically inclose it. It is fused to a considerable extent 
to the lateral head, but quite separable from the medial head, being covered on the medial side by a shining aponeurosis. Origin, patella and femur; insertion, by a thick tendon which passes in the middle of a sort of tube formed by the tendon of the gastrocnemius and soleus onto the ventral surface of the calcaneus. Here it broadens and finally divides into four slips, each attached to a digit. Action, flexor of the digits.

g) Flexor digitorum longus. On turning to the medial side of the shank and clearing away the surface fascia, the following may be identified. Most ventrally will be seen the tibialis anterior; next comes the exposed surface of the tibia. Immediately dorsal to the bone is the flexor digitorum longus which consists of two parts, somewhat separated. The other part is more lateral in contact with the peroneus muscles. Separate the part of the flexor which appears on the medial surface from the tibia by a cut, and lift it up. Internal to it is seen a long tendon, and on the other side of this tendon is the other part of the flexor, this part corresponding to the flexor hallucis longus of man. Both parts of the flexor terminate in slender tendons which unite distally into a broad tendon, which eventually divides into four tendons inserted on the digits. Origin, tibia, fibula, and adjacent fascia; action, flexor of the digits.

h) Tibalis posterior. The long tendon between the two parts of the preceding muscle was noted above. It is the tendon of the tibialis posterior and on following this tendon proximally the belly of the muscle will be located. Origin, fibula, tibia, and fascia; insertion, scaphoid and medial cuneiform of the ankle; action, extensor of the foot.

\section{E. SUMMARY}

I. The voluntary muscles are of two kinds-the somatic or parietal muscles which are produced by the epimeres and the visceral muscles which arise from the hypomeres. Voluntary muscles of hypomeral origin occur only in connection with the gill arches, from which, however, they may spread to cover considerable areas.

2. In primitive vertebrates the somatic muscles exist in the form of muscle segments or myotomes which are repeated at regular intervals along the longitudinal axis. Each myotome extends from the mid-dorsal to the mid-ventral line. The myotomes are bounded by connective tissue partitions, the myosepta.

3. The myotomes are divided into dorsal or epaxial halves and ventral or hypaxial halves by the horizontal skeletogenous septum.

4. The girdles and paired appendages interrupt the series of myotomes. The myotomes adjacent to the appendages send out muscle buds which produce the muscles of the girdles and paired appendages.

5. In higher animals these muscles of the girdles and appendages increase in size and importance and spread over the segmented musculature until the latter is scarcely recognizable. The myotomes are further altered by processes of splitting and fusion so that the primitive arrangement no longer exists in the adults of higher land vertebrates.

6. The derivatives of the hypaxial muscles form the larger part of the mammalian musculature. The epaxial muscles remain as a thick mass on either side of the vertebral column, concealed from surface view by dorsal extensions of the hypaxial muscles.

7. With the adoption of the air-breathing habit the visceral or gill musculature is greatly changed and is found in mammals associated with the larynx, pharynx, hyoid apparatus, upper and lower jaws, and the skin of the neck and face. The muscles of the head are practically all visceral muscles (except the muscles of the eyeball). 


\section{THE COMPARATIVE ANATOMY OF THE COELOM, THE DIGESTIVE, AND RESPIRATORY SYSTEMS}

\section{A. THE ORIGIN AND PARTS OF THE COELOM AND THE MESENTERIES}

I. Origin.-The coelom or body cavity of vertebrates is the cavity of the hypomere. It is never at any stage segmented. The outer wall of the hypomere comes in contact with the inner surface of the layer of voluntary muscles and forms the lining of the body wall. This lining is known as the parietal peritoneum. The inner walls of the hypomeres of the two sides come in contact in the median plane folding around the intestine and inclosing the intestine between their two walls. The inner walls of the hypomere thus become the covering layer of the intestine and other viscera, and are then named the visceral peritoneum or serosa. Above

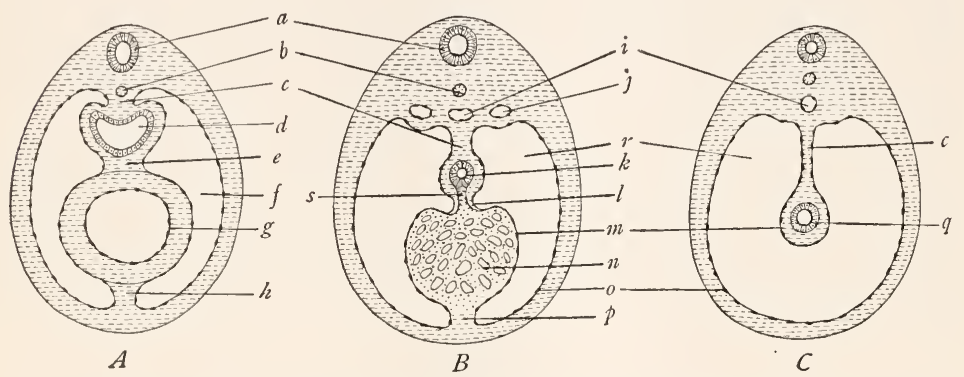

FIG. 44.-Diagrams to show the relations of certain viscera to the mesenteries. $A$, showing intestine $d$, supported by the dorsal mesentery $c$, and the heart $g$, inclosed in the ventral mesentery $e$ and $h$. $B$, showing the liver $n$, inclosed in the ventral mesentery, part of which, the lesser omentum $l$, extends between the intestine $k$ and the liver, and part of which, the falciform ligament $p$, extends between the liver and the ventral body wall. $C$, showing relation of the intestine $q$ to the dorsal mesentery $c$. $a$, neural tube; $b$, notochord; $c$, dorsal mesentery of the digestive tract; $d$, esophagus; $e$, dorsal mesentery of the heart or dorsal mesocardium; $f$, pericardial cavity; $g$, heart; $h$, ventral mesentery of the heart or ventral mesocardium; $i$, dorsal aorta; $j$, posterior cardinal vein; $k$, duodenum; $l$, lesser omentum or hepatoduodenal ligament; $m$, serosa or visceral peritoneum; $n$, liver; $o$, parietal peritoneum; $p$, falciform ligament of the liver; $q$, small intestine; $r$, peritoneal cavity; $s$, bile duct. In $A, e$ and $h$, and in $B, l$ and $p$, form the ventral mesentery of the digestive tract which incloses the heart and liver; in $C$ the ventral mesentery is absent. (From Prentiss and Arey's Textbook of Embryology, courtesy of the W. B. Saunders Company.)

and below the intestine the two walls of the hypomere are in contact and form a double-walled membrane, which is designated as a mesentery. That portion of the mesentery b tween the dorsal wall of the coelom and the intestine is called the dorsal mesentery; that between the ventral wall and the intestine is the ventral mesentery. Different portions of these mesenteries receive special names, which will be given in the cours of the dissection. The dorsal mesentery is intact for its entire length in most vertebrates, but the ventral mesentery very early disappears except in certain regions which will be noted later. These matters have already been 
described in connection with the section on chordate development, Section IV. This should be re-read and Figures 7, 8, and ro studied. (See also Fig. ${ }_{44} C$; K, Figs. 8 and 9, pp. I 4 and 15; W, Fig. 15, p. 65.)

2. Divisions of the coelom.-At first the coelom consists of a continuous cavity extending the entire length of the trunk region, divided into two longitudinal halves by the dorsal and ventral mesenteries. With the partial disappearance of the ventral mesentery, the two halves of the coelom are connected ventral to the intestine (Fig. ${ }_{44} C$ ). In the adults of all vertebrates the coelom is divided into at least two r.ompartments by the formation of a partition. This partition, called the transverse septum, develops at the posterior end of the heart and cuts off the heart from all of the other viscera. The transverse septum thus divides the coelom into a small anterior compartment, the pericardial cavity, which contains only the heart, and a very large posterior compartment, the pleuroperitoneal cavity, which contains all 0 ' the other viscera (see Fig. $45 A$ ). The pericardial cavity in fishes and urodeles is anterior to the pleuroperitoneal cavity, and the transverse septum in those groups passes transversely across the body (Fig. $45 A$ and $B$ ). In the Anura and all vertebrates above Anura the pericardial cavity has descended posteriorly so that it comes to lie ventral to the anterior part of the pleuroperitoneal cavity; the transverse septum then assumes an oblique position (Fig. ${ }_{45} \mathrm{C}$ ). In that portion of the pleuroperitoneal cavity which in consequence of the descent of the pericardial cavity lies dorsal to the pericardial cavity, the lungs are situated. This condition of the coelom, as in Figure ${ }_{45} C$, is found in Anura and reptiles. In birds and mammals, the pleuroperitoneal cavity is divided into anterior and posterior parts by the formation of a partition which descends from the dorsal body wall and unites with the transverse septum (Fig. $45 D$ and $E$ ). This partition is known as the oblique septum in birds and as the diaphragm in mammals. In birds it is non-muscular while in mammals it is infiltrated with striated muscles derived from adjacent myotomes of the body wall.

The oblique septum or diaphragm forms immediately posterior to the lungs. That portion of the pleuroperitoneal cavity which is cut off anterior to the oblique septum or diaphragm consequently contains the lungs. It consists of the two pleural cavities or pleural sacs, each inclosing a lung. The two pleural cavities are completely separated from each other, the pericardial cavity containing the heart being situated in the median line between their ventral portions. That part of the pleuroperitoneal cavity cut off posterior to the oblique septum or diaphragm is called the peritoneal or abdominal cavity; it incloses the greater part of the digestive tract and the urogenital system. It will be seen from this account that in birds and mammals the coelom is divided into four compartments-the pericardial cavity, the two pleural cavities, and the peritoneal cavity.

It is convenient to speak of the viscera as being inclosed in or contained in the coelomic cavities. This is not, however, a correct expression, since, owing to the fact that the viscera are covered by the visceral peritoneum or serosa, they are not really inside of the coelom in the same sense that a chair could be said to be inside of a room. They are outside of it and have the same relations to it as if they were pushed into the coelom carrying the coelomic wall before them. To illustrate farther, one cannot get into the inside of a tent or a balloon by pushing against the wall; one carries the tent or balloon wall before him and always remains in reality on the outside of the tent or ba!loon. Similarly, the viscera are outside of the coelom although they appear to be contained within its cavity.

\section{B. THE DIGESTIVE TRACT AND ITS DERIVATIVES}

1. The origin of the digestive tract.-The primitive intestine or archenteron, as we learned in the section on development, is produced by the process of invagination or other processes in the gastrula stage of the embryo. It is at first a simple tube of entoderm with one 
opening to the exterior, the blastopore, which is situated at the future posterior end of the embryo. This entodermal tube persists as the lining of the adult digestive tract and of all of its derivatives; to it there are added other layers (connective tissue and muscular layers) derived from the splanchnic mesoderm of the hypomere, which, it will be recalled, folds around the archenteron. The adult digestive tract thus consists of a thick-walled tube, composed of
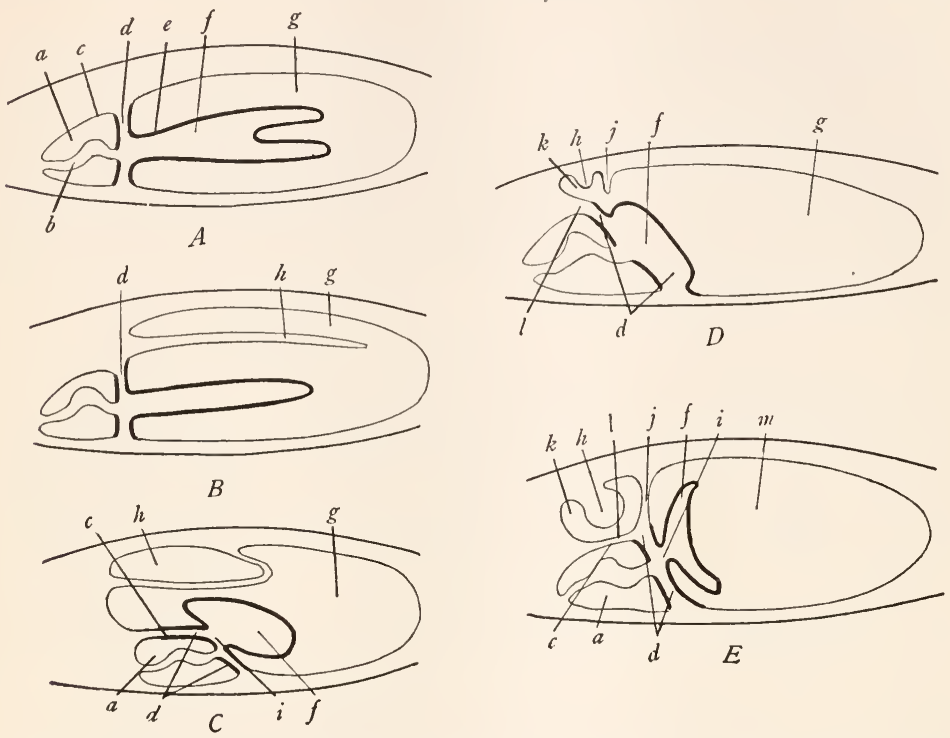

$E$

FIG. 45.-Diagrams to illustrate the divisions of the coelom in the various vertebrate classes. The transverse septum and its derivatives are indicated by thick lines. $A$, fishes, showing the division of the coelom into pericardial cavity $a$ and pleuroperitoneal cavity $g$ by means of the transverse septum $d$. $B$, urodeles, similar to fishes with the addition of the lung $h$ which projects into the pleuroperitoneal cavity g. $C$, turtle; the pericardial cavity $a$ has descended posteriorly until it lies ventral to the anterior part of the pleuroperitoneal cavity $g$; the anterior face of the transverse septum $d$ has now become part of the wall of the pericardial sac; the lung $h$ is retroperitoneal. $D$, early stage of mammals, showing the beginning of the coelomic fold (pleuroperitoneal membrane) $j$ descending from the dorsal body wall, and the liver $f$ inclosed within the transverse septum $d$. $E$, later stage of mammals, showing union of the coelomic fold $j$ with the transverse septum $d$, the two together forming the diaphragm which separates the pleural cavity $k$ from the peritoneal cavity $m$; the liver has constricted from the main part of the transverse septum, the constriction becoming the coronary ligament $i$. $a$, pericardial cavity; $b$, heart; $c$, parietal pericardium or pericardial sac; $d$, transverse septum; $e$, serosa of the liver, this being a part of the transverse septum originally; $f$, liver; $g$, pleuroperitoneal cavity; $h$, lung; $i$, coronary ligament of the liver; $j$, coelomic fold which forms part of the diaphragm; $k$, pleural cavity; $l$, pleuropericardial membrane or anterior continuation of the transverse septum; $m$, peritoneal cavity.

both entodermal and mesodermal elements, the latter predominating. The anterior and posterior ends of the digestive tube are formed by the invagination of the surface ectoderm, that at the anterior end which becomes the lining of the mouth cavity being called the stomodaeum, that at the posterior end, lining the anus, the proctodaeum. 
2. The differentiation of the digestive tube.-Along its course, the tube soon differentiates into various regions with different functions. These regions, beginning at the anterior end, are: mouth or oral cavity, pharynx, esophagus, stomach, small intestine, large intestine or colon, cloaca. The esophagus may be provided with an enlargement, the crop, as in birds. The stomach may be subdivided into two or more compartments, each with special functions, as in birds and cud-chewing mammals. The first part of the small intestine is named the duodenum; the remainder, if of sufficient length, is subdivided into jejunum and ileum. There is generally a blind pouch, the caecum (sometimes more than one), at the junction of small and large intestine. The colon may be divided into several regions. Its terminal portion is often named the rectum. The rectum passes into the cloaca which opens to the exterior by the anus. The cloaca receives not only the intestine but also the genital and urinary ducts.

3. The outgrowths of the digestive tube.-A number of outgrowths arise from the tube at various levels (see Fig. 46).

a) The oral glands: The mouth cavity is commonly provided with glands which consist of evaginations of the lining epithelium. These glands are of two kinds: mucous glands, which secrete a slimy fluid used to moisten the mouth cavity and the food and in some forms as an aid in capturing prey; and salivary glands, characteristic of mammals, in which the secretion contains, not only mucus, but also digestive enzymes. The names of the oral glands indicate their positions in the walls of the mouth cavity.

b) The anterior lobe of the hypoph-

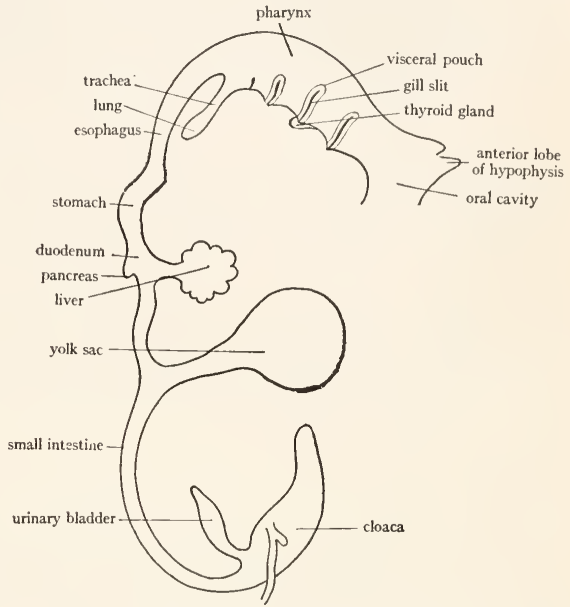

FIG. 46.-Diagram to illustrate the chief derivatives of the digestive tract. (From McMurrich's Development of the Human Body, after His, copyright by P. Blakiston's Son and Company.) ysis: From the roof of the mouth cavity an evagination occurs in the embryo, producing a blind pouch which extends toward and comes in contact with the floor of the brain in a certain region. This pouch, known as the anterior lobe of the hypophysis, fuses with a portion of the brain wall, the compound structure thus formed being designated the hypophysis or pituitary body. It is one of the glands of internal secretion.

c) The thyroid gland: This is an outgrowth from the floor of the pharynx midway between the second gill slits. It is homologous with the endostyle of lower chordates and is a gland of internal secretion, producing a secretion necessary for health and growth.

d) The visceral pouches and gills: The visceral pouches are paired evaginations from the wall of the pharynx, typically five or six in number in vertebrates. Opposite each visceral pouch, the ectoderm invaginates, forming the visceral furrow. Visceral pouches and furrows meet at their extremities and the point of fusion breaks through, the opening being known as a gill slit or visceral cleft (see Fig. 47). The entoderm lining the visceral pouch grows out into thin plates or delicate filaments, the gill, used for respiration. The tissue between successive visceral pouches and slits is called the visceral arch. In the center of each visceral arch is a 
cartilaginous or bony gill arch, already described in connection with the skeleton. Certain blood vessels, the aortic arches, also traverse the visceral arches. The visceral muscles, already described, develop from the tissue of the visceral arches. During the change from the aquatic to the terrestrial habit of life the visceral clefts, pouches, arches, and their derivatives, undergo profound alterations.

e) The tympanic cavity and the external auditory meatus: Beginning with Amphibia the first visceral pouch forms a pouchlike outgrowth in the direction of the internal ear. The end of this outgrowth expands into a chamber, the tympanic cavity or cavity of the middle ear. The stalk of the outgrowth forms the auditory or Eustachian tube, connecting the pharyngeal cavity with the tympanic cavity. In some reptiles, in birds, and mammals an invagination occurs corresponding to the position of the first external gill slit. The bottom of this invagination meets the wall of the tympanic cavity forming at the place of contact a membrane of double

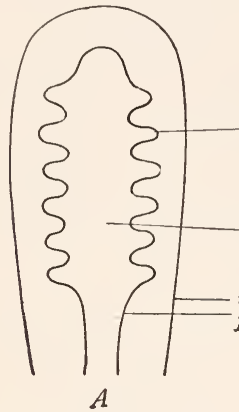

A

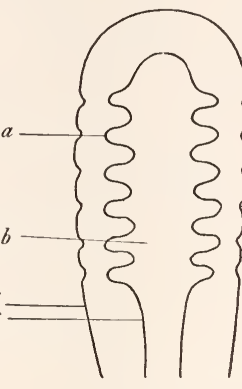

$B$

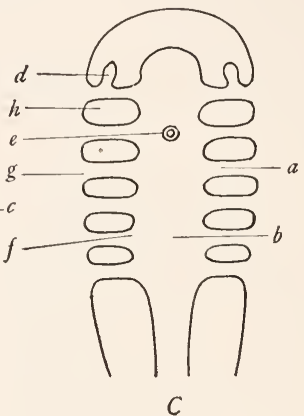

C

FIG. 47.-Diagrams to illustrate the formation of the visceral pouches, furrows, arches, and clefts. $A$, early stage, showing the evaginations $a$ from the wall of the pharynx $b$, which form the visceral pouches. $B$, later stage, illustrating formation of the visceral furrows $c$ by invagination of the surface ectoderm. $C$, later stage showing formation of the external gill slits $g$ by the union of the visceral pouches and furrows and breaking through of the points of union. $a$, visceral pouch; $b$, pharyngeal cavity; $c$, visceral furrow; $d$, tympanic cavity; $e$, thyroid gland; $f$, internal gill slit; $g$, external gill slit; $h$, visceral arch; $i$, ectoderm; $j$, entoderm.

origin, the tympanic membrane (ear drum). The passage formed by the invagination is the external auditory meatus.

f) Glandlike derivatives of the visceral pouches: In the adults of land vertebrates the visceral pouches and clefts disappear, leaving, however, certain bodies which are produced by proliferation of the lining epithelium of the visceral pouches. These glandlike bodies, remnants of the visceral pouches, are very variable in number and mode of origin in different vertebrates. Among them may be mentioned: the true or palatine tonsils, from the second visceral pouch, and the thymus, parathyroids, and the postbranchial or epithelial bodies, from a variable number of visceral pouches. The three last named are glandlike bodies which persist in the neck region and appear to belong to the category of the glands of internal secretion, although their function is uncertain. These glands are present although imperfectly developed in fishes.

g) The trachea and lungs: When the vertebrates adopted the land habitat, the gill slits and gills disappeared in the adult, and their physiological rôle was taken over by a new outgrowth from the pharynx. This outgrowth is a tube which evaginates from the midventral 
line of the pharynx. At its distal end it divides into two sacs. The tube is the trachea or windpipe, and the two sacs at its end are the lungs. Both trachea and lungs subsequently enlarge and become subdivided, and their structure is further complicated by the addition of mesodermal tissues (connective tissue, smooth muscle, cartilage) to the original simple entodermal layer.

h) The swim bladder: This is an outgrowth from the digestive tract, occurring only in the teleostome fishes. It is at first connected with the digestive tract, generally with the esophagus, on either dorsal or ventral side by a duct, which persists throughout life in ganoids. The swim bladder lies just internal to the dorsal body wall of teleostomes and is supposed to have hydrostatic functions. It is not improbable that the swim bladder is the forerunner of lungs.

i) The liver: The liver is a very large gland which arises by outgrowth from the intestine. The stalk of the outgrowth becomes the bile duct, which enlarges on the surface of the liver to form the gall bladder. The liver is situated between the two layers of the ventral mesentery and is also located in the transverse septum but has grown so large that it projects extensively out of these structures (see Figs. $44 B$ and 48 ).

j) The pancreas: This gland arises by one to four, generally three, outgrowths, from the intestine slightly posterior to the origin of the liver. The stalks of the outgrowths become the pancreatic ducts. The outgrowths combine to form one gland of various form in different vertebrates. The pancreas may lie between the two layers of the dorsal mesentery, the ventral mesentery, or both.

k) The yolk sac: In the embryos of most vertebrates a yolk-filled sac projects from the intestine. The wall of this sac is part of the intestinal wall, which may be regarded as having been stretched out into a sac. The yolk in the sac is used for the growth of the embryo, and at the completion of the embryonic stage the yolk sac is greatly reduced in size and is drawn into the intestinal wall.

l) The urinary bladder: This is a large sac growing out from the ventral wall near the posterior termination of the intestine. In the embryos of reptiles, birds, and mammals it is represented by a large sac, the allantois, which projects beyond the limits of the body. The urinary bladder is inclosed between the two layers of the ventral mesentery.

The structures discussed in this introduction will be better understood after they have been studied in the dissections.

Read also the accounts of the comparative anatomy of the coelem, digestive, and respiratory systems in $\mathrm{K}, \mathrm{W}$, or $\mathrm{Wd}$.

\section{THE COELOM, DIGESTIVE, AND RESPIRATORY SYSTEMS OF ELASMOBRANCHS}

The following directions apply chiefly to the spiny dogfish but may also be used for the smooth dogfish and skate, the minor differences between these animals being specified where necessary.

I. The body wall and the pleuroperitoneal cavity.-Make an incision from the left side of the cloaca forward through the pelvic girdle slightly to the left of the midventral line up to the pectoral girdle. The incision will probably cut through the skin first and should then be extended through the muscle layer. To assist in exposing the interior a transverse incision may be made in the middle of the lateral body wall on each side. In the skate cut along the left side of the cloaca and then along both lateral borders of the body cavity but not anteriorly, leaving the flap of body wall adhering to the pectoral girdle. The large internal cavity is the pleuroperitoneal cavity and constitutes the greater 
part of the coelom. It is lined by a smooth shining membrane, the parietal peritoneum, which adheres closely to the inside of the body wall. The body wall is seen to be composed of three layers: skin, muscles, and parietal peritoneum.

2. The viscera of the pleuroperitoneal cavity.-Within ${ }^{\mathrm{x}}$ the cavity are a number of organs or viscera, most of which belong to the digestive tract. At the anterior end of the cavity is the large brownish or grayish liver. This consists in the spiny dogfish of long left and right lobes and a small median lobe in which is located the long greenish gall bladder. In the smooth dogfish, the liver is subdivided into right and left lobes, the median lobe being absent; the gall bladder is imbedded in the anterior part of the left lobe and is visible as a thin place in the liver. In the skate the liver is composed of right, median, and left lobes of equal length and size, and the gall bladder is situated in the angles between the right and median lobes. Dorsal to the liver on the left side is the large J-shaped stomach, often distended with food. (In some specimens the stomach is everted into the mouth cavity and should be pulled back into the pleuroperitoneal cavity by exerting a gentle traction on it.) The greater part of the stomach consists of a large straight tube extending from the anterior end of the pleuroperitoneal cavity to a point somewhat posterior to the ends of the liver lobes. It then makes a sharp bend, decreasing in diameter considerably, and extends anteriorly, terminating in a constriction, the pylorus. Along the posterior margin of the bend of the stomach (or in the skate on the dorsal side of the bend) is a dark-colored organ, the spleen, a part of the lymphatic system. From the pylorus the short intestine extends to the anus. The first part of the intestine beyond the pylorus is called the duodenum. It extends for a short distance to the right and then curves posteriorly. The bile duct, a long stout duct, is easily seen descending from the gall bladder to enter the duodenum shortly caudad of the bend. The bile duct accompanied by some blood vessels runs in a strip of mesentery. It passes to the dorsal side of the duodenal wall and runs for a short distance caudad imbedded in the wall before it penetrates into the cavity of the duodenum. In the curve of the duodenum reposes the ventral lobe of a white gland, the pancreas. The dorsal lobe of the pancreas, which is long and slender in the spiny dogfish, reaching to the spleen, should be located by raising the stomach and duodenum and looking dorsal to them. The duct of the pancreas is somewhat difficult to find in the dogfishes, less difficult in the skate; it lies imbedded in the tissue of the pancreas near the posterior margin of the ventral lobe and may be exposed by picking away the pancreas tissue in this region. Beyond the duodenum the intestine widens considerably, and its surface is marked by parallel rings. These rings are the lines of attachment of a spiral fold, the spiral valve, which occupies the interior of the intestine. (A portion of the intestine often protrudes through the anus and should be pulled back into the coelom by grasping the portion in the cavity and exerting a gentle

I It has already been explained that the organs are not in reality inside of the coelom. 
pull.) At the posterior end of the intestine is a small cylindrical body, the rectal gland, attached to the intestine by a duct. It is possibly excretory in function. The rectal gland marks the division between large and small intestine, that part of the intestine anterior to its attachment being the small intestine, that posterior to it, the large intestine. The latter is so short as to be almost absent and opens at once into a terminal chamber, the cloaca, which opens to the exterior through the anus.

Cut open the stomach and wash out its contents. Partly disintegrated fish and squids are commonly found in the stomach. Observe the folds or rugae in the walls of the posterior part of the stomach and the papillae projecting from the walls of the anterior part. Cut open the small intestine along one side midway between the large blood vessels which traverse its walls longitudinally. Observe the spiral valve in its interior. It consists of a fold of the intestinal wall spirally coiled so as to make a series of overlapping cones. The purpose of the spiral valve is to increase the digestive and absorptive surface of the intestine.

The reproductive organs and their ducts in part may also be identified at this time. In the spiny dogfish and the skate the gonads are a pair of soft bodies located dorsal to the anterior part of the stomach. The lobes of the liver must be raised to see them. In the smooth dogfish they are long slender bodies extending the entire length of the cavity dorsal to the digestive tract and terminating at the rectal gland. In mature females the oviducts are noticeable as stout white tubes, one on each side, in contact with the dorsal walls of the coelom.

3. The mesenteries. - The viscera are held in place by delicate membranes, the mesenteries, whose mode of origin was explained in the introduction to this section. In studying them lift and spread each organ as it is mentioned. The dorsal mesentery extends from the median dorsal line of the coelom to the digestive tract but is not complete in the animals under consideration, a gap being present in the region of the small intestine. That part of the dorsal mesentery supporting the stomach is called the mesogaster; in the skate it is limited to the anterior part of the stomach. The mesogaster incloses the spleen between its two walls, and that portion of the mesogaster from the spleen to the stomach is the gastrosplenic ligament. That portion of the dorsal mesentery which supports the small intestine is called the mesentery, in the limited sense. This is absent in the skate. In the spiny dogfish there is a fusion between the mesentery and the mesogaster so that a sort of pocket is formed dorsal to the bend of the stomach. In the dorsal wall of this pocket is located the greater part of the pancreas which is thus in the dorsal mesentery. The dorsal mesentery begins again in the region of the rectal gland, this portion of the mesentery being named the mesorectum.

The ventral mesentery is represented in these animals, as in all vertebrates, by remnants only. Such a remnant is the gastro-hepato-duodenal ligament extending from the right side of the stomach to the liver and duodenum. It is 
also called the lesser omentum. It may be roughly divided into two portions, the hepatoduodenal ligament extending from the liver to the duodenum and containing the bile duct and blood vessels, and the gastrohepatic ligament extending from the stomach to the liver and duodenum and in the dogfishes occupying also the angle formed by the bend of the stomach. Another remnant of the ventral mesentery is the suspensory or falciform ligament of the liver. This will be found at the anterior end of the liver, extending from the midventral surface of the liver to the midventral line of the body wall. In mature females the mouth of the oviduct will be noticed in the falciform ligament as a funnel-shaped aperture. After seeing the falciform ligament the flap of body wall left in the skate may be cut off.

Each gonad has a mesentery which is a special fold arising from the dorsal wall very near the origin of the dorsal mesentery. The mesentery of the ovary is called the mesovarium, of the testis, the mesorchium. In the case of mature females, each oviduct has also a mesentery, the mesotubarium.

The anterior end of the pleuroperitoneal cavity will be found closed by a partition, the transverse septum, the posterior face of which is clothed by the parietal peritoneum. The liver is attached to the septum by the strong coronary ligament which is, in fact, a portion of the septum. In its early development the liver is inclosed in the transverse septum, and subsequently, because of increased size, projects posteriorly from the septum which then narrows around the anterior end of the liver and forms the coronary ligament (see Fig. 48, p. I95).

The pleuroperitoneal cavity communicates with the exterior by means of the abdominal pores. These will be found one on each side of the anal opening (in the skate posterior to the anus) somewhat concealed by a fold of skin. Probe into them and note that they lead into the pleuroperitoneal cavity. Their purpose is obscure.

The pleuroperitoneum is the lining of the hypomere and the pleuroperitoneal cavity is the cavity of the hypomere. The pleuroperitoneal membrane is divided into three regions, according to its relations to other structures: first, the parietal peritoneum, that portion of the membrane lining the inner surface of the body wall; second, the visceral peritoneum, forming the thin outer covering of all of the viscera; and third, the mesenteries or ligaments, portions of the membrane extending from the body wall to the viscera or from one viscus to another.

Draw the contents of the pleuroperitoneal cavity. Make a diagram of an imaginary section through the anterior end of the pleuroperitoneal cavity, showing gonads, stomach, and liver, and the relations of the pleuroperitoneum to them and to the body wall.

4. The pericardial cavity.-Make an incision through the skin in the median ventral line from the pectoral girdle up to the lower jaw. Leave the girdle intact. Gently strip off with a forceps the layers of visceral muscle until you have exposed a membrane. This membrane is the parietal pericardium. Slit open this membrane and see that it incloses a cavity, the pericardial cavity, in 
which the heart is situated. To reveal this cavity more fully cut laterally along the anterior face of the girdle keeping your instrument in contact with the girdle. Portions of the girdle may be sliced away, but the heart must not be injured. The pericardial cavity is thus revealed as a conical cavity lined by the parietal pericardium and containing the heart. By gently lifting up the heart note that it is attached only at its anterior and posterior ends. At these places the pericardial lining is deflected from the walls of the pericardial cavity and passes over the surface of the heart as a covering layer, the visceral pericardium, which is indistinguishably fused with the heart wall. With the heart lifted note that the posterior end of the heart is a fan-shaped chamber, the sinus venosus, whose walls are continuous with the partition that forms the posterior wall of the pericardial cavity. This partition is the transverse septum, whose posterior face we have already seen. The septum is thus seen to be a partition whose anterior wall is composed of the parietal pericardium and whose postericr wall, of the parietal peritoneum. The wings of the sinus venosus are buried in the transverse septum; they constitute large venous channels through which the venous blood is returned to the heart.

We may now explain the formation of the transverse septum. Since the heart is situated in the ventral part of the body it is necessary in order that the blood from the dorsal body wall may reach the heart that a bridge be formed passing from the dorsal to the ventral side. In early embryonic stages a bridge or cylinder of mesoderm develops on each side of the posterior end of the heart connecting the splanchnic mesoderm surrounding the sinus venosus with the somatic mesoderm of the dorsal body wall. In these bridges the main venous channels pass from the dorsal body wall into the sinus venosus. Later, the bridges enlarge and finally fuse with each other and with the body wall laterally, forming a partition, the transverse septum, which thus cuts the heart off from the remainder of the coelom. In elasmobranchs the fusion is not quite complete, leaving an opening, the pericardio-peritoneal canal, dorsal to the sinus venosus, extending from the pericardial cavity into the pleuroperitoneal cavity. This opening will be seen at a later time.

5. The mouth and pharyngeal cavities and the respiratory system.-Insert one blade of a scissors into the left corner of the mouth and make a cut through the angle of the jaws back across the ventral parts of the gill slits through the pectoral girdle so that you emerge to the left side of the stomach. A flap is thus formed which should be turned over to the right. A large cavity is revealed which at its posterior end converges into the extremely short esophagus which passes at once into the stomach. The esophagus may be slightly slit to aid in opening the flap.

The anterior part of the cavity inclosed by the jaws and gill arches is the mouth or oral cavity. It is bounded in front by the upper and lower jaws, provided with teeth. The upper and lower jaws are the two halves of the first or 
mandibular gill arch; the section of the arch should be identified in the cut surface. On the floor of the mouth back of the teeth is the tongue; it forms a flat, slight projection, which is practically immovable. It is supported by the second or hyoid gill arch which should be felt within it and identified in section at the cut surface. (The tongue is absent in the skate, owing to the reduction of the hyoid arch.)

The posterior and greater part of the cavity under consideration is the pharynx. Its wall is pierced by six internal gill slits. The first of these, the spiracle, is a rounded opening in the roof of the mouth immediately posterior to the mandibular arch. The remaining five gill slits are elongated. The internal gill slits communicate with large cavities-the visceral pouches-which in turn open to the exterior by way of the external gill slits. The tissue between successive visceral pouches is a visceral arch. The parts of a visceral arch should be examined in section on the left side where the arches have been cut through. The center of the section of each visceral arch is formed by the interbranchial septum which extends to the outer surface of the body, where the spaces between successive septa form the external gill slits. On each face of the interbranchial septum is borne a series of low, thin folds or plates, the branchial or gill filaments. The set of filaments on one face of the septum ronstitutes a half-gill or demibranch and the demibranchs on the two sides of a septum together constitute a whole branchia or gill. The gills are sutgrowths of the walls of the visceral pouches and are covered with entoderm. By examining all of the septa determine how many demibranchs are present and where they are missing. In the inner end of the section of each visceral arch locate the cross-section of the cartilaginous gill arch and external to this, lying in the septum, the cartilages of the gill rays. Just external to the section of the gill arch is the section of a blood vessel-the afferent branchial vesselwhich brings venous blood to the gills. At each side of the gill arch is a section of another vessel, which is injected with a colored solution; these are the efferent branchial vessels, which carry the aerated blood away from the gills. Note the fine branches of these vessels in the gill filaments. The gills are the respiratory mechanism of the animal in which the blood obtains oxygen and gives off carbondioxide. Water is kept flowing over the gills by movements of the gill arches.

Draw the mouth cavity and pharynx. Draw one visceral arch and all of its parts in cross-section.

D. THE COELOM, DIGESTIVE, AND RESPIRATORY SYSTEMS OF NECTURUS

Obtain a specimen and place in a wax-bottomed dissecting pan, fastening it ventral side up by pins through the legs.

I. The viscera of the pleuroperitoneal cavity.-Make a longitudinal incision through the body wall a little to the left of the median ventral line from the left side of the anus through the pelvic girdle to the pectoral girdle. Spread apart the two flaps of the body wall and look within. The large cavity is, as in fishes, 
the pleuroperitoneal cavity, lined by the pleuroperitoneum. The body wall consists of skin, muscle, and peritoneum as can be seen on the cut surface. In the median ventral line on the inside of the abdominal wall runs a large vein, the ventral abdominal vein.

Examine the viscera. The liver is the large greenish or brownish organ occupying the anterior half of the pleuroperitoneal cavity. Its margins are divided into several scallop-like lobes by shallow indentations. It is united to the median ventral line by a mesentery which should not be disturbed at present. On raising the left side of the liver, the elongated stomach will be seen dorsal to the liver. Along the left side of the stomach is situated the dark-colored spleen. On raising the spleen there will be seen dorsal to it and lying along the left side of the stomach, the left lung, a very long slender tubular structure which terminates some distance posterior to the liver. Follow the stomach posteriorly. It is a straight tube, somewhat shorter than the liver, terminating at a constriction, the pylorus. From the pylorus the small intestine begins and makes an abrupt right-angled bend to the right. In this bend rests a white gland, the pancreas, which also extends onto the dorsal surface of the liver. That part of the small intestine in contact with the pancreas is known as the duodenum. On raising the duodenum and looking on its dorsal side the pancreas will be seen to send one tail toward the spleen and another posteriorly along the small intestine. The small intestine proceeds posteriorly somewhat coiled. In the case of females it will be found coiled on the ventral surface of the large ovaries, on the surface of which the eggs will be noted. (The size of the ovaries varies with the sexual state of the animal.) To each side and dorsal to the ovaries is a large, white, much-coiled tube, the oviduct. Trace the intestine, posteriorly pressing the ovaries away from the median line. The small intestine widens near the anus into the short large intestine. It lies in female specimens between the posterior terminations of the two oviducts. At the posterior end of the pleuroperitoneal cavity ventral to the large intestine will be found a sac, generally collapsed and shriveled, the urinary bladder. Note the stalk by which it is attached to the ventral side of the intestine. That part of the intestine to which the urinary bladder is attached (and into which the ducts of the kidneys and gonads also open) is the cloaca. It terminates at the anus.

The female gonads and ducts have already been noted. The left male gonad will be found dorsal to the intestine and posterior to the spleen. Dorsal to the gonad will be seen the left kidney with its duct attached to its left margin.

2. The mesenteries.-The digestive tract is attached for most of its length to the median dorsal line of the coelom by the dorsal mesentery. This should be noted by pressing other organs away from the median line. It is missing in the pyloric region of the stomach. That portion of the dorsal mesentery supporting the stomach is the mesogaster. The spleen is inclosed in the mesogaster, that portion of the mesogaster which extends from the spleen to the stomach 
being designated the gastrosplenic ligament. The lung is also attached to the mesogaster by a short mesentery. That part of the dorsal mesentery supporting the small intestine is the mesentery, in the limited sense; that part supporting the large intestine is the mesorectum.

The ventral mesentery is present only in the region of the liver and urinary bladder. One part of it forms the long mesentery extending between the median ventral line of the body wall and the median line of the ventral face of the liver. This is the falciform ligament of the liver. It contains a number of blood vessels which pass from the ventral body wall into the substance of the liver (where they join the hepatic portal vein). In the free posterior margin of the falciform ligament the ventral abdominal vein crosses from the body wall to the liver. On raising the liver the gastrohepatic ligament will be seen extending from the anterior part of the stomach to the dorsal face of the liver. In the region of the pancreas the hepatoduodenal ligament joins the duodenum and liver and incloses the greater part of the pancreas. The tails of the pancreas, however, are situated in the mesentery of the small intestine. Both of the ligaments just mentioned are parts of the ventral mesentery. The last part of this mesentery is found extending from the urinary bladder to the midventral line of the body wall; this is the median ligament of the bladder.

Each gonad has a mesentery: mesovarium in the female, mesorchium in the male. The mesentery of the oviduct is the mesotubarium. These should be located by lifting up the structures in question.

The falciform ligament should now be severed without, however, cutting through the ventral abdominal vein. The numerous lobes of the liver appearing as scallops of the margin may now be seen more clearly. On raising the right side of the liver, the right lung may be identified dorsal to it. Is it of the same length as the left lung? The small gall bladder will be seen on the dorsal surface of the right side of the liver. Its duct surrounded by pancreas tissue may be readily traced to the duodenum. The pancreas is said to open into the duodenum by a number of fine ducts.

The anterior end of the pleuroperitoneal cavity is closed by a membrane, the transverse septum. The liver is attached to this by the coronary ligament, which is continuous posteriorly with the falciform ligament. The mode of formation of the septum and the coronary ligament was described in connection with the dogfish.

3. The pericardial cavity.-Make a median ventral incision through the skin from the level of the fore limbs forward to the level of the gills. Remove the underlying muscles bit by bit until you have exposed a membrane, the parietal pericardium. Cut through this membrane. The pericardial cavity in which the heart is situated is thus exposed. Widen the opening into the cavity by cutting laterally along the anterior margin of the pectoral girdle. The muscles between the pericardial cavity and the fore limbs may also be split. 
The pericardial cavity is a conical cavity lined by the parietal pericardium. On gently raising the heart the posterior wall of the cavity is seen to be formed by the transverse septum. The transverse septum is pierced by two veins (hepatic sinuses) which extend forward and enter the sinus venosus, the most dorsal chamber of the heart.

4. The oral cavity and the pharynx.-Open the mouth and cut through the angle of the jaws on each side so that the jaws can be spread widely. Carry your cuts back to the gill arches. The cavity thus exposed consists of an anterior oral cavity and a posterior pharynx.

The oral cavity is bounded externally by the well-developed lips. Internal to the lips are the small conical teeth. There are two rows of teeth on the roof of the mouth, the posterior row being the longer. External to the last teeth of the posterior row on each side is a slit, one of the posterior nares or internal openings of the nasal passages. Probe into one of the anterior nares and note emergence of the probe through the posterior naris. The floor of the mouth cavity bears a single row of teeth which on closing the mouth will be found to fit between the two rows on the roof. Posterior to the teeth is the tongue supported by the strongly developed hyoid arch which should be felt within the tongue.

The walls of the pharynx are pierced by two pairs of gill slits. Probe through them and note emergence of the probe between the external gills. Note the cartilaginous gill arches supporting the bars (visceral arches) between and on each side of the gill slits. The walls of the gill slits are the visceral pouches. Unlike the dogfish they bear no gills, the gills being external. The pharyngeal cavity narrows posteriorly into a tube, the esophagus. By passing a probe into the esophagus determine that it extends dorsal to the pericardial cavity and is continuous with the stomach.

5. The larynx and the lungs.-In the floor of the pharynx midway between the second gills slits will be found a short slit, the glottis. The walls of the glottis, as should be determined by feeling them with a fine forceps, are stiffened by a pair of delicate cartilages, the arytenoid cartilages. These probably represent reduced gill arches. They are the first of the laryngeal cartilages to appear in the phylogenetic series. The small cavity into which the glottis leads and which is inclosed between the two arytenoid cartilages is the $\operatorname{larynx}$. Cut across the gill slits of the left side so that the pharyngeal cavity can be opened more widely. Slit the glottis posteriorly. The larynx is thus seen to lead into a narrow flattened passage, the trachea. The posterior end of this is widened and receives two openings.' Probe into each with a slender probe and note emergence of the probe into a lung. The trachea is thus seen to communicate with the lungs. The air passage in primitive air-breathing vertebrates takes the following course: anterior nares, nasal cavities, posterior nares, oral cavity, pharyngeal cavity, glottis, larynx, trachea, lungs. The lungs have already been noted. Slit open one of them and note the smooth interior, not provided with air sacs. 


\section{E. THE COELOM, Digestive, AND RESPiRATORY SYSTEMS OF THE TURTLE}

Obtain a specimen and place in a dissecting pan. Specimens which have not been injected should be employed. Remove the plastron. This is done by sawing through the bridges on each side, lifting up the plastron and separating it with a scalpel from the surrounding skin and underlying membrane.

I. The divisions and relations of the coelom.-The removal of the plastron exposes a membrane, the parietal peritoneum, which covers and conceals the viscera. Note that the muscle layer which is normally present between the skin and the peritoneum is completely lacking in the ventral body wall of the turtle, owing to the presence of the plastron. The ventral body wall in turtles therefore consists of but two layers, the skin with its contained exoskeleton, and the peritoneum. Owing to this circumstance the parietal peritoneum can be easily separated from the inside of the body wall, a procedure which is difficult or impossible in other vertebrates. Note, however, the usual muscles in connection with the girdles and limbs.

In the median line in the anterior part of the parietal peritoneum shortly posterior to the pectoral girdle is situated a triangular membranous sac, the pericardial sac, which incloses the heart. It will be noticed that the heart is much more posterior in position than is the case in the fishes and Necturus; in fact, there has occurred a posterior descent of the heart (and of other viscera as well). The membranous sac covering the heart is, as in the dogfish, the parietal pericardium. Here it takes the form of an isolated sac, the pericardial sac, while in fishes and Necturus it formed the lining of a chamber surrounded by. the body wall. The space between the pericardial sac and the heart is the pericardial cavity, a portion of the coelom. The ventral face of the pericardial sac rests in the natural position against the internal surface of the plastron, while its dorsal face is fused, as we shall see, to the parietal peritoneum. Cut into the ventral wall of the pericardial sac, thus exposing the pericardial cavity and the contained heart.

Two conspicuous veins, the ventral abdominal veins, run longitudinally in the parietal peritoneum between the pericardial sac and the pelvic girdle. Cut through the peritoneum halfway between the heart and pelvic girdle by a transverse cut which severs both of the abdominal veins. The large cavity thus exposed is the pleuroperitoneal cavity, whose walls are lined by the parietal peritoneum.

The coelom of the turtle, like that of the fishes and Necturus, consists of two parts, a small pericardial cavity and a much larger pleuroperitoneal cavity. We note, however, that whereas in the low er forms the pericardial cavity is anterior to the pleuroperitoneal cavity and separated from the latter by the transverse septum, in the turtle the pericardial cavity is ventral to the pleuroperitoneal cavity, and the transverse septum seems to have disappeared. We may explain this change as follows. (See also Fig. 45, p. I60.) In its posterior descent the heart must necessarily carry with it the transverse septum and the parietal pericardium. The latter in order to move posteriorly must separate from the body wall to which it is attached in lower 
vertebrates. It does this and so becomes an independent sac, the pericardial sac. This process of the splitting of the pericardial sac from the body wall is aided by the invasion forward of the pleuroperitoneal cavity. The heart contained in the pericardial sac descends posteriorly, the pleuroperitoneal cavity at the same time advancing anteriorly. The pericardial sac may be thought of as sliding posteriorly ventral to the ventral wall of the pleuroperitoneal cavity. The pericardial sac thus comes to lie ventral to the anterior part of the pleuroperitoneal cavity. The posterior wall of the pericardial sac is still the anterior face of the transverse septum, the posterior face of the latter as in lower forms being placed between the pericardial sac and the liver. The transverse septum thus in the turtle forms part of the partition between the pericardial and the pleuroperitoneal cavities, the remainder of the partition being composed of the rest of the parietal pericardium, which is now the pericardial sac. These matters will be better understood by reference to Figure $45^{A} A$ and $C$.

2. The viscera and their mesenteries.-With the bone scissors cut away the margins of the carapace on each side between fore and hind limbs so as to gain easy access to the pleuroperitoneal cavity. Masses of fat, greenish-yellow material, will be found in various places and may be removed. Lift up the edges of the cut already made in the peritoneum, widening this if necessary, and look inside. Identify in the anterior part of the pleuroperitoneal cavity the large brown liver lying on each side of the heart. Posterior to the liver are the coils of the intestine. In female specimens the ovaries containing eggs of various sizes are conspicuous objects in the lateral and posterior part of the pleuroperitoneal cavity. Running alongside each ovary is the coiled oviduct. Just in front of the pelvic girdle is the large bilobed urinary bladder.

The liver consists of right and left lobes whose lateral margins curve dorsally to fit the curves of the carapace. The pericardial sac rests in a depression between the two lobes. The latter are united by a narrow bridge passing dorsal to the heart. Posterior to the heart the liver is united to the parietal peritoneum by very short mesenteries corresponding to the falciform ligament of other vertebrates. In these mesenteries the ventral abdominal veins leave the peritoneum and pass into the liver. Trace the parietal peritoneum anteriorly from this region. It passes along the dorsal face of the pericardial sac, to which it is inseparably fused. This compound membrane between the heart and liver is the transverse septum, which has assumed an oblique position, owing to the descent of the heart (Fig. 45). The ventral (original anterior) face of the septum is as before, part of the wall of the pericardial cavity; the dorsal (original posterior) face forms part of the parietal peritoneum. The liver is as usual attached to the transverse septum by the coronary ligament. Continue to trace the parietal peritoneum to the anterior end of the pleuroperitoneal cavity. On the posterior face of the pectoral girdle it turns dorsally and passes to the carapace of which it forms the inner lining. Similarly trace the parietal peritoneum posteriorly by lifting the posterior cut edge of the membrane. It curves dorsally, following along the anterior surface of the pelvic girdle, and passes to the inner surface of the carapace. 
Press both lobes of the liver forward against the pectoral girdle and look on the dorsal surface of the liver. The elongated stomach will be found curving dorsal to the lateral border of the left liver lobe. On following the stomach anteriorly, the narrow esophagus will be found entering the stomach. The stomach passes along the dorsal surface of the left lobe of the liver to the middle of which it is attached along its entire length by the short gastrohepatic ligament. About opposite the bridge connecting the two lobes of the liver, the stomach passes insensibly into the small intestine, the first part of which is the duodenum. The duodenum is united to the middle of the dorsal surface of the right lobe of the liver by the hepatoduodenal ligament. In this ligament is situated a long white gland, the pancreas. About one-quarter of an inch back of the right end of the pancreas, a pancreatic duct passes from the pancreas into the duodenum and may be revealed by picking away the substance of the pancreas at this point. On the dorsal surface of the right lobe of the liver near its lateral border is the large gall bladder, which is connected to the duodenum by a short but stout bile duct. Beyond the entrance of the bile duct the small intestine turns sharply posteriorly and is then thrown into a number of coils. In the case of female specimens it will generally be necessary to remove one of the large egg-bearing ovaries at this point before the intestine can be conveniently traced farther. By lifting the coils of the small intestine note the dorsal mesentery which attaches it to the median dorsal line of the coelom; this part of the dorsal mesentery is the mesentery proper. Follow the dorsal mesentery forward and note the portions of it which support the duodenum and the stomach, named mesoduodenum and mesogaster, respectively. The mesoduodenum is fused to the hepatoduodenal ligament so that the two appear as one, but the mesogaster is distinct from the gastrohepatic ligament. Trace the small intestine posteriorly, noting the coiling of the mesentery corresponding to the coils of the intestine. Find on the right side the entrance of the small intestine into the large intestine or colon. At the junction of the small and large intestine is a slight projection, the caecum. The colon generally crosses the pleuroperitoneal cavity transversely and then turns posteriorly and runs straight caudad to the cloaca. Note the mesocolon supporting the colon. In the transverse part of the colon it is fused to the mesogaster. In the mesocolon on the dorsal side of the colon shortly beyond the caecum is a rounded red body, the spleen. Trace the colon to the place where it disappears dorsal to the pelvic girdle. At this point ventral to the colon will be found the large, thin-walled, bilobed urinary bladder. It is generally greatly distended with urine but in some specimens may be contracted to a small mass. The bladder has no ligaments, the peritoneum leaving the body wall around the stalk of the bladder and passing over its surface to form its visceral investment.

Cut away the pelvic girdle by making a cut through each side of it with the bone scissors and removing a median piece. The large intestıne will be traced into 
a tube, the cloaca, which proceeds dorsal to the girdle to the anus. At the point of entrance of the large intestine into the cloaca the urinary bladder will be found attached to its ventral surface by a stalk. On each side of the stalk of the bladder, in females, a large white oviduct will also be seen entering the cloaca.

The female reproductive system may be noted at this time; that of the male is so inconspicuous that it will not be described at this point. The ovaries are a pair of large saclike bodies containing in their walls eggs of various sizes. Each ovary is attached by its mesentery, the mesovarium, to the dorsal body wall. Lateral to each ovary runs the oviduct, a large coiled white tube. It is supported by the mesotubarium.

3. The respiratory system.-Pry open the jaws of the turtle and cut through the angles of the jaws, cutting nearer the lower than the upper jaw. The anterior portion of the cavity thus revealed is the oral or mouth cavity; the posterior portion, the pharynx. The oral cavity is bounded by the jaws which have no teeth but are clothed with horny beaks of epidermal origin. These beaks extend as plates into the mouth cavity. In the roof of the mouth cavity posterior to the plate is a pair of elongated openings, the posterior nares. Probe them and determine that they connect with the anterior nares by passages which run through the nasal cavities. The floor of the mouth cavity is occupied by the fleshy pointed tongue.

In the pharynx note that neither gills nor gill slits are present, although, as we shall see shortly, the gill arches are represented. Behind the base of the tongue is an elevation, the laryngeal prominence, in the center of which is an elongated slit, the glottis. Feel the pair of small arytenoid cartilages, one on each side of the glottis; they are derived from one of the gill arches. On each side of the roof of the pharynx posterior to the muscles which connect the skull and lower jaw is the opening of the auditory or Eustachian tube, a canal which leads from the pharynx to the cavity of the middle ear. (The opening may have been destroyed in cutting the jaws apart.) The auditory tube and also the cavity of the middle ear are outgrowths from the first visceral pouch. Posteriorly the pharynx narrows into the esophagus.

Cut through the skin in the median ventral line of the neck and peel away the skin from neck and throat. Separate the muscles in the median line of the neck and find a tube stiffened by rings of cartilage. This is the trachea or windpipe. Trace it forward until it disappears into the pharynx. In front of this place note the hard body of the hyoid, and by cleaning away muscles find also two pairs of horns of the hyoid extending posteriorly. The hyoid and its horns are derivatives of the second, third, and fourth gill arches. Open the mouth and make a cut around the laryngeal prominence, freeing it from its position on the dorsal surface of the body of the hyoid. The structure thus freed is the $\operatorname{larynx}$, an expanded chamber at the anterior end of the trachea. Find in the 
lateral walls of the larynx the two arytenoid cartilages, small cartilages supporting the two triangular flaps which inclose the glottis between them. Posterior to the glottis is a ring-shaped cartilage, the cricoid, which is much wider on the ventral than on the dorsal side. The arytenoids probably are remnants of the fifth gill arches while the cricoid is the enlarged first cartilage of the series of rings in the trachea.

Now trace the trachea posteriorly. Note the esophagus, a soft tube, lying dorsal to or to one side of the trachea. Find just anterior to the heart the point where the trachea bifurcates into the two bronchi which proceed to the lungs. Raise the right and left lobes of the liver and the stomach and find dorsal to them against the carapace a large spongy organ, the lung, on each side. Trace a bronchus into each lung; it is accompanied by a pulmonary artery and a pulmonary vein. Study the relation of the lung to the pleuroperitoneal cavity. Note that the lung is in contact with the inner surface of the carapace, and that the parietal peritoneum passes over the ventral surface of the lung leaving the lung outside of the membrane. Such a relation to the peritoneum is spoken of as retroperitoneal. The posterior end of the lung, however, projects into the pleuroperitoneal cavity and is clothed with the peritoneum. Cut open the lung and observe its extremely spongy texture; cords of connective tissue divide the interior into air spaces or alveoli.

The path followed by the air in respiration is: external nares, nasal cavities, internal nares, mouth cavity, pharyngeal cavity, glottis, larynx, trachea, bronchi, and lungs. In the pharyngeal cavity the paths of food and air cross.

Make drawings to show the parts of the digestive and respiratory systems. Make a diagram of a cross-section through the body at the level of the heart to show the pericardial and pleuroperitoneal cavities and membranes and their relation to the viscera.

\section{F. THE COELOM, DIGESTIVE, AND RESPIRATORY SYSTEMS OF THE PIGEON}

Obtain a specimen and place in a dissecting pan. The feathers must be removed. It is desirable that the air sacs should have been inflated through the trachea.

I. The oral cavity and the pharynx.-Open the mouth widely by cutting through the angles of the jaws. An anterior oral cavity and a posterior pharynx are thus revealed.

a) Oral cavity: Roof and floor of the oral cavity are bounded laterally by horny beaks of epidermal origin which incase the jaws. Teeth are absent, as in all living birds. The roof of the mouth cavity bears a pair of elongated palatal folds with free fimbriated margins. These palatal folds correspond to the hard palate of mammals, but differ in that they do not meet in the median line, leaving here a deep palatal fissure. The hard palate of many birds is therefore a split palate and is normally in the condition which in mammals is the result 
of imperfect development. In the roof of the mouth cavity, dorsal to the palatal folds and concealed by them, are the posterior nares. Locate them by bending aside or cutting away the palatal folds. Probe into the anterior nares and note that the probe emerges through the posterior nares. The floor of the mouth cavity is occupied by the pointed tongue, whose posterior free border is fimbriated and terminates in a point on each side. The tongue of birds is not very muscular. Numerous glands open into the oral cavity in birds but are too small to study in gross dissection.

b) Pharynx: Note that, as in all adult vertebrates above urodeles, gill slits are absent from the lateral walls of the pharynx. In the roof of the pharyngeal cavity just posterior to the caudal ends of the palatal folds is a median aperture, the opening of the paired auditory tubes. Each auditory tube extends from this opening to the cavity of the middle ear; tube and cavity represent in part an evagination from the first visceral pouch. In birds unlike other vertebrates the two auditory tubes unite to one at the point of communication with the pharynx. Posterior to this opening the roof of the pharynx bears a pair of folds with fimbriated borders, which hang down like a curtain into the pharyngeal cavity. These folds constitute the soft palate. In the floor of the pharynx, immediately posterior to the caudal end of the tongue, is a hardened elevation, the laryngeal prominence, bearing in its center an elongated opening, the glottis. The margins of the glottis are also fimbriated, and immediately posterior to the glottis on each side is a fringed fold. In the walls of the glottis the supporting laryngeal cartilages are readily felt.

Make a drawing of the oral and pharyngeal cavities.

2. The hyoid apparatus, the larynx, the trachea, and the esophagus.Make a median ventral longitudinal incision in the skin of the neck from the throat to the anterior end of the sternum. Deflect the skin on each side of the incision. The trachea or windpipe, a tube with walls stiffened by rings of cartilage, is immediately exposed. Dorsal to it or to one side of it is the soft esophagus.

Trace the trachea forward to the glottis, cleaning away the muscles which cover its anterior end. At the same time a cut may be made to the sides of the tongue so that the tongue may be pulled down ventrally from the mouth cavity. The hyoid apparatus may now be studied. It consists of remnants of the hyoid (second) and third gill arches. It is composed of three median elements, arranged in a longitudinal series, and two pairs of horns or cormua. The most anterior of the three median pieces is the entoglossal cartilage. It is situated inside of the tongue and may be revealed by dissecting off the covering membrane of the tongue. It represents the two fused ceratohyals. From its posterior end projects posteriorly on each side a small cartilage which occupies the caudal point of the tongue already noted. These two cartilages constitute the anterior horns of the hyoid and consist of the free ends of the two ceratohyals whose 
anterior portions fused to form the entoglossal cartilage. Posterior to the entoglossal cartilage is a median bony piece, the basihyal. Posterior to this is the basibranchial of the third gill arch. From the point of junction of basihyal and basibranchial projects on each side the long posterior horn of the hyoid, consisting of portions of the third gill arch. On following the posterior horns they will be found to extend toward the ears and to be divided into a proximal longer portion, the ceratobranchial, and a distal shorter rod, the epibranchial.

The cartilages of the larynx may next be identified. Cut around the laryngeal prominence freeing it so that it can be drawn ventrally. Also free the hyoid apparatus from the ventral surface of the larynx. The larynx is the expanded chamber thus revealed at the top of the trachea and opening into the pharyngeal cavity by way of the glottis. By dissecting in the margins of the glottis on each side, expose a slender, curved, partially ossified arytenoid cartilage. On the ventral side of the larynx note the enlarged triangular cricoid cartilage. Follow this around to the dorsal side where it terminates by much narrowed ends. Between the two dorsal ends of the cricoid cartilage is another median cartilage, the procricoid, which is in contact with the posterior ends of the arytenoids. The arytenoids are derived from certain of the gill arches while the cricoid and procricoid cartilages are the most anterior modified rings of the trachea. Although the larynx of birds is morphologically the same as the larynx of other vertebrates from which sounds issue, in birds the voice is not produced in the larynx but in another part of the trachea which will be seen later.

Examine the cartilages of the trachea. They are broad, hard, and bony ventrally, but narrower, softer, and cartilaginous in composition dorsally. There is consequently a somewhat soft strip along the dorsal side of the trachea which lies against the cervical vertebrae.

Trace the esophagus posteriorly. Shortly in front of the sternum it widens into an enormous bilobed sac, the crop. Birds swallow their food whole; the food collects in the crop which is capable of great distension and is passed on into the stomach in small quantities. The crop should be carefully loosened on all sides. Not all birds possess a crop.

3. The anterior air sacs and the pectoral muscles.-The respiratory system of birds is the most remarkable among vertebrates. It consists, not only of the lungs, but also of a number of air sacs located among the viscera and of air spaces in the bones. These air sacs and air spaces communicate with the lungs by means of branches of the bronchi. This system not only aids in decreasing the specific gravity of the bird but also insures a more complete exposure of the lung tissue to the air; for the residual air is retained in the air sacs and not in the lungs as in other vertebrates, and the air in the lungs is consequently completely renewed at each inspiration. Owing to the delicacy of the air sacs the student 
may not be able to locate all of those mentioned below, but some of them will be seen. They are best studied in freshly killed specimens in which they have been inflated through the trachea.

Dorsal to the crop in the angle formed by the two halves of the furcula or wishbone is situated the interclavicular air sac. Its delicate ventral wall is in contact with the dorsal wall of the crop. It consists of two lobes, one on each side of the median line; in the embryo these lobes are separate. Puncture the interclavicular air sac and find, dorsal to it on each side, another sac, the cervical air sac.

Extend the median ventral incision in the skin to the anus. Separate the skin from the underlying muscles on each side of chest and abdomen. The great pectoral muscles are revealed immediately internal to the skin and occupying the angle between the keel and the body of the sternum. The pectoralis major is the great muscle covering the entire sternum and extending to the humerus. It takes origin from the keel of the sternum, the surface of the body of the sternum, and the furcula which will be found imbedded in its anterior border; its fibers converge toward the humerus, and passing over the shoulder are inserted on the outer and dorsal surface of the humerus. The muscle should be followed to its insertion. Action, depresses the wing. Now carefully cut through the pectoralis major slightly to the right of the keel of the sternum and along the posterior margin of the furcula. The muscle can then be deflected and separates easily from the underlying pectoralis minor. The large pectoral arteries and veins will probably be noticed emerging between the pectoral muscles which they supply. The pectoralis minor originates from the body of the sternum and converges toward the humerus. On following the muscle laterally there will be found between it and the pectoralis major another air sac, the axillary sac. Cut into the axillary sac. The anterior wall of this sac is in contact with the coracoid bone, and laterally on looking into the sac the tuberosities of the humerus will be seen. A large opening into the humerus, the pneumatic foramen, is readily noticed; on probing this it will be found to lead into the interior of the humerus. It is the entrance to the air space of the humerus which communicates with the axillary air sac. The axillary air sac communicates in front with the interclavicular air sac. The pectoralis minor may now be followed to its insertion. It converges to a tendon which passes ventral to the posterior end of the cervical air sac and beneath the shoulder to the dorsal side of the humerus on which it is inserted. To see the insertion turn the bird dorsal side up and dissect away the superficial muscles of the dorsal side of the shoulder. The tendon of the pectoralis muscle, owing to its mode of insertion, has a pulley-like action which enables the muscle to raise the wing. Whereas in mammals all of the pectoral muscles act together to adduct the fore limb, in birds the actions of the pectoralis major and minor are opposed to each other, the one depressing, 
the other raising the limb. This arrangement eliminates all powerful muscles from the back and enables all of the wing muscles to take their origin from the firm and strong sternum.

4. The divisions of the coelom and the posterior air sacs.-Cut through the ventral abdominal wall to the right of the median line. Beneath the skin are the thin layers of abdominal muscles corresponding to those of mammals, and internal to this the parietal peritoneum generally impregnated with streaks of fat. Cut through this and extend the incision anteriorly cutting through the sternum slightly to the right of the keel, keeping the scissors in contact with the bone so as to avoid injuring internal parts. Spread apart the cut edges and look within.

The small cavity posterior to the sternum is the peritoneal cavity. Note in it the liver dorsal to the posterior end of the sternum, the closely coiled intestine, and to the left the large firm gizzard. From the gizzard a mesentery extends to the ventral body wall to the left of the median line. This may be designated the ventral ligament of the gizzard. ${ }^{\mathbf{I}}$ It is continuous anteriorly with the falciform ligament of the liver which extends from the median ventral region of the liver to the midventral line of the body wall and inner surface of the sternum. The falciform ligament and ventral ligament of the gizzard together constitute a partition which divides the peritoneal cavity into a large right portion and smaller left portion. This division is not found in other vertebrates. In the partition courses a small vein ${ }^{2}$ extending from the mesenteries in question to the liver.

Deflect the pectoralis major muscle on the left side of the sternum and make a cut through the left side of the sternum slightly to the left of the keel. Remove and discard the median piece of sternum containing the keel.

Immediately dorsal to the sternum is situated the delicate pericardial sac containing the heart. The ventral wall of the pericardial sac will probably have been opened in cutting through the sternum. The heart, as in the turtle, has descended posteriorly, and a pericardial sac has been formed of the anterior face of the transverse septum and the parietal pericardium as described in connection with the turtle. The space between the pericardial sac and the heart is, as before, the pericardial cavity, a portion of the coelom. The pericardial sac is in contact on its ventral surface with the inner surface of the sternum, and anteriorly and laterally is also in contact with the inner surface of the body wall. Hence, only the posterior part of the pericardial sac is freed from the body wall.

s This is commonly called the greater omentum in texts and manuals, but since it is not at all nomologous with the structure so named in mammals, it is desirable that the name be dropped. The ligament of the gizzard is a mesentery peculiar to birds, and arises as a secondary outgrowth from the serosa of the gizzard to the ventral body wall. It is probably due to the need for additional support for the heavy gizzard.

${ }^{2}$ This vein is named in manuals the ventral abdominal vein but it does not appear to be homologous with the vein of that name in other vertebrates. 
From the points where the pericardial sac meets the lateral body wall a membranous partition extends obliquely posteriorly on each side. This partition is called the oblique septum. It contains a large air sac. It stretches across from the lateral body wall to that part of the pericardial sac which is derived from the transverse septum, and thus divides the pleuroperitoneal cavity into anterior and posterior portions. That part of the original pleuroperitoneal cavity left anterior to the oblique septum consists of the two pleural cavities, one on each side of the pericardial cavity. That part of the pleuroperitoneal cavity posterior to the oblique septum is the peritoneal cavity, already mentioned. The oblique septum is produced by a pair of mesenterial folds which arise one on each side of the esophagus and grow ventrally, eventually fusing with the transverse septum and with each other (Fig. $45^{D}$ and $E$, p I60).

Inside of the oblique septum inclosed between its anterior and posterior walls is a large air sac, the posterior intermediate air sac. Immediately anterior to this, lying to each side of the heart, is the small anterior intermediate air sac.

In the peritoneal cavity cut through the falciform ligament and ligament of the gizzard at their line of attachment to the ventral body wall. On either side of the viscera and slightly dorsal to them find the large abdominal air sac.

From the foregoing account it is seen that the coelom of birds is divided into four compartments, the pericardial cavity, the two pleural cavities, and the peritoneal cavity.

5. The peritoneal cavity and its contents. - This cavity has already been mentioned. As in other vertebrates it is lined by the parietal peritoneum which is deflected at certain points to form mesenteries and which continues over the surface of the viscera as the visceral peritoneum.

The viscera of the peritoneal cavity may now be studied in more detail. At the anterior end is the large liver, consisting of right and left lobes, the former the larger. The pericardial sac rests between the two lobes of the liver. The liver is attached to the pericardial sac (that portion of it derived from the transverse septum) by the coronary ligament. The falciform ligament of the liver was already noted and severed. To the left and slightly covered by the left lobe of the liver is the gizzard. On raising the left lobe of the liver the gastrohepatic ligament will be noted passing between the gizzard and the liver. The mesogaster connects the gizzard with the dorsal body wall. The ventral ligament of the gizzard was already noted and cut. On breaking through the gastrohepatic ligament the soft proventriculus will be found extending anteriorly from the gizzard dorsal to the liver. Proventriculus and gizzard together correspond to the stomach of other vertebrates; they are specialized regions of the stomach correlated with the absence of teeth. From the stomach, at the place where proventriculus and gizzard join, the small intestine arises. The first portion of this, the duodenum, makes a long U-shaped loop posteriorly. The beginning of the duodenum is attached to the right lobe of the liver by the hepatoduodenal 
ligament. Between the two sides of the duodenal loop stretches the mesoduodenum, a portion of the mesentery of the intestine. In this is situated the pancreas, lying between the two limbs of the loop. From a deep depression in the dorsal surface of the right lobe of the liver, the two bile ducts (there is no gall bladder) emerge and pass into the duodenum. The left bile duct is the shorter and stouter of the two and enters the left limb of the duodenum about half an inch beyond the gizzard. The more slender right bile duct passes to the right limb of the duodenal loop. There are three pancreatic ducts, all of which pass from the right side of the pancreas into the right limb of the duodenal loop. One of these arises from the anterior part of the pancreas and passes obliquely forward, entering the duodenum near the anterior termination of the right limb of the loop. The other two ducts emerge from the middle of the pancreas and pass across to the right limb of the duodenum. The ducts are generally easily seen by spreading out the mesentery.

Trace the small intestine posteriorly from the duodenum. It is much coiled and supported by the mesentery, which, owing to the small space into which the intestine is packed, is fused in many places. Near its termination the small intestine turns toward the median line, widens slightly, and then runs straight caudad in the median line. At about the middle of the peritoneal cavity it passes without enlargement into the large intestine. The point of junction of large and small intestine is marked by a pair of small lateral diverticula, the caeca. The large intestine is so short as to constitute little more than a rectum which soon passes into the cloaca. Owing to the absence of pubic and ischial symphyses in birds, the cloaca in birds does not pass through the ring of the pelvic girdle but may be traced directly to the anus. There is no urinary bladder. In female specimens the single left oviduct will probably be noted entering the left side of the cloaca. The single ovary (left one) is situated in the anterior part of the peritoneal cavity, dorsal to the gizzard.

Make a drawing of the digestive tract.

The gizzard and proventriculus may now be freed from the adjacent air sacs and the mesenteries. On turning the gizzard far forward there will be found between the proventriculus and the anterior end of the right limb of the duodenal loop a rounded red body, the spleen. The gizzard may now be cut open along its posterior margin. The interior contains small stones and probably partially digested food. Note the extremely thick muscular walls and the hard horny lining of the gizzard. Cut from the gizzard into the proventriculus and note the soft glandular walls of the latter. The gizzard grinds up the food into small pieces, thus taking the place of teeth, and the proventriculus digests the food by means of the digestive fluid secreted by the glands in its walls.

6. The pleural cavities and their contents.-The posterior intermediate air sac situated in the oblique septum may now be punctured if this has not already been done. The two walls of the septum are now more clearly observable. The 
anterior intermediate air sac may also be punctured. Against the dorsal wall of the pleural cavity on each side will be found a reddish, spongy flattened organ, the lung. The openings of some of the air sacs into the lungs will probably be noted on some specimens. On cutting into the lung the organ will be found to be solid, not hollow, as in the preceding animals.

The cavity in which each lung is contained is, as already explained, a pleural cavity. It is lined by a coleomic membrane, the pleura. As the lungs are flattened against the dorsal wall of the pleural cavity, the pleura passes over their ventral faces, leaving them outside, so to speak. The pleura, furthermore, passes over the surface of the pericardial sac and lines the inner surface of the body wall.

7. The syrinx.-Examine the posterior part of the trachea. Two slender muscles, the sternotracheal muscles, diverge from their insertion on the ventral surface of the trachea to their origin on the sternum. These muscles should be severed. The trachea disappears dorsal to the heart and the great blood vessels which enter and leave the heart. These blood vessels must not be injured. Loosen the trachea and pull it forward. The bifurcation of the trachea into the two bronchi can then be seen dorsal to the heart. Cut across the bronchi with a fine scissors and draw the trachea forward. At the point where the trachea forks into the two bronchi an expanded chamber, the syrinx, is present. The voice of birds issues from the syrinx, not from the larynx. Along each side of the trachea extending from the point of insertion of the sternotracheal muscles to the lateral walls of the syrinx is a muscle, the intrinsic syringeal muscle. The walls of the syrinx are supported by the last tracheal rings and the first bronchial half-rings. The last two tracheal rings are widely separated from each other but are connected in the median ventral line by median processes. Make a slit in the ventral wall of the syrinx and spread apart the cut edges. The cavity of the syrinx is named the tympanum. In the dorsal wall of the tympanum a slight vertical fold is present in the median dorsal line, extending forward from the level of the bifurcation of the trachea. This fold is called the semilunar membrane, and its vibrations are said to produce the voice. There are also large thickenings in the lateral walls of the tympanum which may have some function in the production of the voice. The sternotracheal and syringeal muscles doubtless aid by changing the size and shape of the tympanum.

G. THE COElom, Digestive, AND RESPiratory SYSTEMS OF A MAMMAL

The following directions apply to both the rabbit and the cat. Whenever the differences between the two animals warrant, a separate description of each will be given; otherwise they will be described together.

I. The mouth cavity and the pharynx.-

a) The salivary glands: The salivary glands are masses of gland tissue which are outgrowths of the lining of the mouth cavity: the stalk of the outgrowth 
remains as the salivary duct. The glands are situated among the muscles of the head and throat. They should be located according to the following descriptions and their ducts followed as far as practicable. There are four pairs of salivary glands in the rabbit, five in the cat. The dissection should be carried out on the same side of the head as that on which the muscles were dissected.

The parotid gland is located ventrad and craniad of the base of the pinna of the ear, just under the skin. Remove the skin from this region and find the pinkish gland spread out under the skin anterior and ventral to the ear. Its duct passes across the external surface of the masseter muscle and penetrates the upper lip. The submaxillary gland has already been noted as a roundish mass at the angle of the jaw near the posterior margin of the masseter. Loosen it and find the duct springing from the internal surface. In the cat the beginning of this duct is surrounded by the elongated sublingual gland. Trace the submaxillary duct forward; it is accompanied in the cat by the sublingual duct. The duct will be found to pass internal to the digastric muscle. This muscle should be severed. The duct (or two ducts in the cat) will then be seen to pass internal to the mylohyoid muscle. This in turn should be cut and the duct traced forward. In the rabbit the small flattened sublingual gland will soon be noted lying in the path of the submaxillary duct. The submaxillary duct (accompanied by the sublingual duct in the cat), situated just external to the lining of the mouth cavity, runs forward nearly to the symphysis of the mandible and then penetrates the lining. In the rabbit the sublingual gland opens into the mouth cavity by several short ducts which are impractical to find. The molar gland, present in the cat only, is situated between the skin and the external surface of the mandible, just in front of the masseter muscle. It will be found by deflecting the skin at this place. It opens onto the inside of the cheek by several small ducts, impractical to locate. The infraorbital gland in both cat and rabbit lies in the floor of the orbit and will be seen later when the eye is dissected.

b) The mouth cavity: Cut through the skin at the corners of the mouth and see that the skin is well cleared away over the angles of the jaws. Cut through the masseter and other muscles attached to the lower jaw at the angle of the jaws. It should then be possible to pull the lower jaw down. Pry open the mouth, grasp the lower jaw, and exert a strong traction. The jaw will generally yield, but if it does not, the ramus of the mandible may be cut through with the bone scissors. The anterior part of the cavity thus revealed is the mouth or oral cavity. It is bounded by the lips and cheeks. That part of the oral cavity lying between the teeth and lips is called the vestibule of the mouth. The teeth were described in connection with the skull.

The anterior portion of the roof of the oral cavity is occupied by the hard palate, the posterior part by the soft palate which is very long in the rabbit. The difference between the hard and soft palate should be determined by feeling. 
The hard palate is supported by the premaxillary, maxillary, and palatine bones, as should be recalled from the study of the skull. The soft palate lacks bony support. The mucous membrane of the hard palate is thrown into a number of roughened transverse ridges. At the anterior end of the hard palate just behind the incisor teeth will be found a pair of openings, the openings of the nasopalatine ducts which connect the mouth and nasal cavities by way of the incisive foramina of the maxillary bones. The opening of the duct of the parotid gland may be sought for on the inside of the cheek opposite the second upper premolar tooth in the rabbit, opposite the last cusp of the third upper premolar of the cat, in which animal it is situated on a slight ridge: the openings are difficult to identify with certainty and not much time should be spent in looking for them.

The floor of the oral cavity is occupied by the tongue, a fleshy muscular organ, more mobile in mammals than in most other vertebrates. The anterior margin of the attachment of the tongue to the floor of the mouth has the form of a vertical fold, the frenulum. Halfway between the lower incisors and the frenulum will be found in the rabbit the two small slitlike openings of the ducts of the submaxillary glands, the two being about an eighth of an inch apart. In the cat a fold runs forward from the frenulum on each side just within the teeth, and terminates anteriorly in a well-marked flattened papilla which bears the openings of the ducts of the submaxillary and sublingual glands.

Cut through the floor of the mouth on each side, keeping the scalpel next to the mandible. The tongue can now be pulled down and out between the two halves of the lower jaw. The cuts may be continued on each side at the base of the tongue back to the level of the submaxillary glands so that the tongue can be pulled well down. The surface of the tongue may now be examined in detail. In the rabbit the tongue is divisible into two portions, an anterior softer portion, covered with minute pointed elevations, the fungiform papillae; and a posterior, elevated, smoother, and harder portion. At the posterior end of the latter on each side is situated a vallate papilla, consisting of a round elevation set into a pit. In front of each vallate papilla on the side of the tongue is an oval area of considerable size marked by numerous fine parallel ridges, the foliate papilla. In the cat the anterior part of the tongue is covered with the filiform papillae, many of which are hard and spinelike, pointed posteriorly; the remainder of the tongue is provided with fungiform papillae; among the fungiform papillae are four to six vallate papillae arranged in a V-shaped row, each consisting of a round elevation set into a pit. At the sides of the vallate papillae are some very large fungiform papillae. The papillae are provided with microscopic taste buds.

c) The pharynx: The pharynx is that portion of the cavity lying posterior and dorsal to the soft palate. Pull the tongue well forward and examine the soft palate. It descends like a curtain across the posterior end of the oral cavity. Find its free posterior margin, arching above the base of the tongue.

(The 
margin may be concealed by a leaf-shaped structure, the epiglottis, which projects from the base of the tongue. If so, the epiglottis should be pressed out of the way.) The opening formed by the free border of the palate is known as the isthmus of the fauces. This opening leads into the cavity of the pharynx. Shortly anterior to the free border of the soft palate on each side is a pit, the tonsillar fossa, which contains a small mass of lymphoid tissue, the palatine tonsil. The tonsillar fossa is bounded in front and behind by low folds, an anterior glossopalatine arch and a posterior pharyngopalatine arch. Now slit the soft palate forward along its median line. A cavity, the nasopharynx, a part of the pharynx, is revealed dorsal to the soft palate. At the anterior end of the nasopharynx are the two posterior nares or choanae, the internal ends of the nasal passages. Posterior to them on the lateral wall of the nasopharynx will be noted a pair of oblique slits; they are the openings of the auditory or Eustachian tubes, canals which connect the pharynx with the cavity of the middle ear.

The pharynx narrows posteriorly into the esophagus. Anteriorly to the entrance into the esophagus is situated the entrance into the respiratory tract. This entrance is guarded by a projecting process, the epiglottis, which if not already identified will be seen on pulling the tongue well forward. In the pharynx the paths for food and air are crossed (as is the case in all of the airbreathing vertebrates). It will be noted, however, that owing to the formation of the palate and the consequent posterior migration of the posterior nares, the air no longer enters the oral cavity as is the case in Amphibia and most reptiles but proceeds directly into the pharynx.

2. The hyoid apparatus, the larynx, the trachea, and the esophagus.Press the tongue dorsally against the lower jaw and find on its external surface at its base a bone, the body of the hyoid. This is a stout bone in the rabbit, a narrow bar in the cat. Clear away muscles from its surface so as to reveal it and the two horns or cormua which extend from its sides. In the rabbit the horns are short processes which are connected by slender tendinous muscles with the jugular process of the occipital bone. In the cat the anterior horn is long and slender and consists of a chain of four bony pieces, the last of which articulates with the tympanic bulla; the posterior horn is short and is united to the larynx. The hyoid and its horns are derived in mammals from the second and third gill arches. The hyoid supports the base of the tongue and serves for the origin and insertion of muscles.

In the median ventral line posterior to the body of the hyoid is a chamber with cartilaginous walls, the larynx or voice box, which constitutes the projection in the throat popularly known as Adam's apple. By making a cut through the base of the tongue and gently severing the muscle attachments, the larynx may be freed and lifted forward. At the top of the larynx is a large opening, the glottis, from whose ventral margin the epiglottis projects. Dorsal to the glottis and bound with it by muscles is another opening, generally collapsed and 
concealed from view by portions of the larynx. This opening should be located by probing; the probe will be found to enter a soft tube which proceeds posteriorly dorsal to the larynx. This tube is the esophagus.

The structure of the larynx should now be examined in detail. The ventral wall of the larynx is supported by a large shield-shaped cartilage, the thyroid cartilage. A short distance posterior to this is the cricoid cartilage, which forms a ring around the larynx. The dorsal rim of the glottis between the glottis and the opening to the esophagus is supported by a pair of projecting cartilages, the arytenoids. On looking into the glottis a pair of folds, the vocal cords, will be seen extending from the arytenoid cartilages to the thyroid cartilage. They nearly occlude the opening. In the cat, in addition to these true vocal cords, there is a pair of false vocal cords, situated lateral to the former and extending from the tips of the arytenoid cartilages to the base of the epiglottis. It will be noted that the vocal cords are not cords but folds of the lateral wall of the larynx. On dissecting away the esophagus from the dorsal side of the larynx the dorsal side of the cricoid cartilage will be exposed. It is much broader than the ventral side. By cleaning away the mucous membrane covering it, the two arytenoid cartilages which rest on the anterior extremity of the dorsal part of the cricoid will be exposed.

From the larynx the trachea or windpipe proceeds posteriorly. Its walls are stiffened by cartilaginous rings, which are incomplete dorsally, leaving a soft strip in the dorsal wall of the trachea into which the esophagus fits. On each side of the trachea lying against the trachea and internal to the muscles is a flattened elongated body, one of the lobes of the thyroid gland. The anterior end of each lobe is at a level with the cricoid cartilage. The caudal ends of the two lobes are connected by a median portion, the isthmus, which crosses the ventral side of the trachea. The trachea is not to be traced farther posteriorly at this time.

3. The pleural and pericardial cavities.-The trunk of mammals is divided into an anterior thoracic region and a posterior abdominal region. Each of these regions contains cavities which are portions of the coelom. The thoracic region has three coelomic cavities, the two pleural cavities, laterally located, and the median pericardial cavity, situated between the two pleural cavities.

With the bone scissors make a cut through the ribs one-half inch to the left of the sternum, extending the cut the length of the sternum. At each end of this, cut laterally and dorsally between two adjacent ribs at right angles to the first cut. In this way a flap is formed in the chest wall. Open the flap and bend it dorsally so that you can look within. The cavity thus revealed is the left pleural cavity or pleural sac, as it is often called; a similar sac exists on the right side. The pleural sac contains the soft spongy lung. In the median region under the sternum lies the large heart. Note the delicate partition which stretches from the heart to the ventral median line. This partition is called the 
mediastinal septum. It consists of the two medial walls of the right and left pleural sacs in contact with each other. At the level of the heart the two walls separate so that the heart and its pericardial sac are inclosed between them. This space between the two walls of the mediastinal septum is called the mediastimum. The posterior wall of the pleural sac is formed by a muscular domeshaped partition, the diaphragm. The pleural sac is lined by a smooth moist membrane, the pleura. The pleura is divided into parietal and visceral parts. The parietal pleura lines the inside of the pleural cavity, covers the anterior face of the diaphragm, and together with the medial wall of the other pleural sac forms the mediastinal septum. The visceral pleura is that part of the pleura which passes over the surface of the lung to which it is indistinguishably fused. Examine the left lung. It is a soft spongy organ divided into three lobes, a smaller anterior, and larger middle, and posterior lobes. The anterior lobe is quite small in the rabbit. The large posterior lobe fits very neatly on the convex surface of the diaphragm. Cut into the lung; it appears solid but is really composed of innumerable minute air-cells.

Now carefully cut through the mediastinal septum ventral to the heart and look into the right pleural cavity. The diaphragm may be slit along its left side so as to facilitate the spreading apart of the thoracic walls. The right pleural cavity is similar to the left cavity. It contains the right lung. The right lung is somewhat larger than the left lung. It is divided into anterior, middle, and posterior lobes. The large posterior lobe is subdivided into two lobules, a medial and a lateral. The medial lobule projects into a pocket formed by a special dorsally directed fold of the mediastinal septum. This fold, the caval fold, has the function of supporting a large vein, the postcaval vein, which ascends from the liver to the heart and will be found inclosed in the free dorsal margin of the caval fold.

Examine the heart and the pericardial sac. The pericardial sac or parietal pericardium is a sac of thin tissue inclosing the heart but not attached to it except at the anterior end where the great vessels enter and leave the heart. The heart is freely movable inside of the pericardial sac. The narrow space between the pericardial sac and the heart is the pericardial cavity, a portion of the coelom. Cut through the pericardial sac so as to expose the heart. The surface of the heart is invested by a thin membrane, the visceral pericardium, inseparably adherent to the heart wall. The visceral pericardium is continuous with the pericardial sac at the anterior end where the blood vessels enter and leave the heart. As the heart with its pericardial sac is situated in the mediastinum, it is evident that there are three coelomic layers surrounding the heart: the visceral pericardium closely adherent to the heart wall, the parietal pericardium or pericardial sac separated from the heart by the pericardial cavity, and the parietal pleura of the mediastinal septum, which is closely fused to the pericardial sac (Fig. $49 B$, p. 197). 
In the mediastinum in the median line ventral to the anterior part of the heart and extending forward will be found a mass of gland tissue, the thymus. It is larger the younger the specimen. In searching for it do not injure the large blood vessels occurring in this region. The thymus is possibly one of the glands of internal secretion and is derived from the entodermal lining of certain of the visceral pouches of the embryo.

Now press the heart and the left lung over to the right. The lung will be found attached by a narrow region, the radix or root of the lung. An artery, a vein, and a bronchus or air tube pass to the lung and veins from the lung in the root, but these structures are better investigated at a later time. In the cat, furthermore, the lung is attached along most of its length to the dorsal thoracic wall by the pulmonary ligament, a fold of the pleura. Note that dorsal to the root of the lung the pleura continues onto the dorsal and lateral surfaces of the pleural cavity and that certain structures can be seen that lie internal to the pleura. These structures lie between the dorsal portions of the two walls of the mediastinal septum and consequently are situated in the mediastinum. The most conspicuous of these structures lying in the mediastinum is the dorsal aorta, a very large vessel injected with a colored solution which arches away from the heart to the left and descends toward the diaphragm. About one-half an inch ventral to the aorta is another tube, the esophagus, also lying in the mediastinum. Trace it posteriorly to the place where it penetrates the diaphragm.

The diaphragm is a curved sheet forming the posterior wall of the thoracic cavity and completely separating it from the abdominal cavity. The center of the diaphragm is seen to consist of connective tissue forming a circular tendon, the central tendon of the diaphragm. The remainder of the diaphragm is muscular. The diaphragm takes origin from the ribs, sternum, and vertebrae, and is inserted on the central tendon. It is an important respiratory muscle. When contracted, it flattens, thus lengthening the pleural cavities posteriorly and causing air to rush into the lungs. The diaphragm is pierced at several points to allow important structures to pass through; the chief ones which penetrate the diaphragm were already noted, i.e., the esophagus, the aorta, and the postcaval vein. The diaphragm is a structure peculiar to mammals. It is formed in part of the transverse septum and in part of other coelomic membranes; it then becomes invaded by muscle buds from the adjacent cervical myotomes.

Make a diagram of a cross-section through the thorax showing the pleural and pericardial cavities and the relation of their linings to the thoracic wall, lungs, and heart.

4. The peritoneal cavity and its contents.-Make a longitudinal slit through the abdominal wall, a little to the left of the median ventral line from the inguinal region up to the diaphragm. Widen the opening by a transverse sit in the middle of the left abdominal wall. A large cavity, the abdominal or peritoneal 
cavity, is exposed. Its anterior wall is formed by the concavely arched diaphragm which completely separates the peritoneal from the pleural cavities. Posterior to the diaphragm and shaped so as to fit the concave surface of the diaphragm is the large, lobed liver, generally grayish brown in preserved specimens. Posterior to the liver the peritoneal cavity is filled by the coils of the intestine. In the cat the intestine is covered ventrally by a thin membrane impregnated with streaks of fat, the greater omentum. This membrane is present also in the rabbit, but is very much smaller and less conspicuous. At the posterior end of the peritoneal cavity may be noted the pear-shaped urinary bladder, generally distended with fluid. On raising the liver and looking dorsally and to the left of it will be found the stomach with the spleen attached to its left border. On the dorsal wall of the peritoneal cavity at about the level of the posterior ends of the liver lobes are the kidneys, round organs; to see them, gently lift the coils of the intestine. In female specimens, especially those which are pregnant, the horns of the uterus will be noted as a tube on each side in the posterior part of the peritoneal cavity.

The peritoneal cavity is lined by a membrane, the peritoneum. As in all coelomate animals, that portion of the membrane on the inside of the body wall is the parietal peritoneum. In both dorsal and ventral regions the peritoneum is deflected from the body wall and passes over the surface of the viscera, forming a covering layer, the visceral peritoneum or serosa, for all of the viscera. In passing to and from the body wall to the viscera, the peritoneum forms doublewalled membranes, the mesenteries or ligaments. The dorsal mesentery is present intact in mammals, and the ventral mesentery persists in the region of the liver and urinary bladder as in other vertebrates.

Examine the stomach first, by raising the liver and pressing it craniad. The exposure of the stomach is facilitated by slitting the diaphragm on the left side. The stomach is a large and rounded organ in the rabbit, smaller and more elongated in the cat. Find where the esophagus emerges from the diaphragm and enters the anterior surface of the stomach. The area of junction of the stomach and esophagus is called the cardia; and the region of the stomach adjacent to the junction, the cardiac end of the stomach. The shorter, slightly concave anterior surface of the stomach from the cardia to the pylorus is the lesser curvature; the larger convex posterior surface, the greater curvature. The saclike bulge of the stomach to the left of the cardia is known as the fundus; the remainder of the stomach, the body. At the right the stomach passes into the small intestine, the point of junction, known as the pylorus, being marked by a constriction, beyond which the small intestine makes an abrupt bend. Along the left side of the stomach lies the spleen, a rather large organ in the cat, but smaller in the rabbit.

The relations of the stomach to the peritoneum are somewhat complicated. Raise the fundus and note the mesogaster extending from the dorsal wall to the stomach. Only a small portion of the mesogaster passes directly to the stomach; 
the greater part of it first descends posteriorly, forming a bag, the greater omentum. This is a very large and extensive sheet in the cat, covering the intestine ventrally as noted above. In the rabbit it is a short membrane dependent from the greater curvature of the stomach. The greater omentum is to be thought of as formed in the following way. Suppose one should grasp the mesogaster and pull it posteriorly, drawing it into a sac. Such a sac would have two walls, each double, i.e., composed of the two layers of the mesogaster; the sac would contain a cavity which is known as the lesser peritoneal sac and would open anteriorly (this opening will be seen later) (see Fig. 50, p I98.). By manipulating the omentum determine that it consists of two separate walls. Having formed the greater omentum the mesogaster returns to the stomach wall and passes onto the stomach along the greater curvature. The spleen is inclosed in the ventral wall of the great omentum just before the latter passes to the stomach. The portion of the great omentum between the spleen and the stomach is called the gastrosplenic (or gastrolienal) ligament. Posterior to the spleen, near the left kidney, a secondary fusion, the gastrocolic ligament, has formed between the mesogaster and the mesentery of the intestine (see Fig. 50).

The greater omentum owes its origin in part to the rotation of the stomach. The line of attachment of the omentum to the greater curvature is the original dorsal surface of the stomach, while the lesser curvature is the original ventral surface. The mesogaster passes over the stomach, forming the visceral peritoneum of the stomach, and inclosing the stomach between its walls, and at the lesser curvature is continued by a strong ligament, the lesser omentum, which passes to the liver.

The liver may be studied next. It presents a convex anterior surface, fitting against the posterior surface of the diaphragm, and a concave posterior surface, fitting over the stomach and first part of the small intestine. The liver is divided into right and left lobes, each of which is subdivided into two lobes, a median and a lateral. The left lateral and right median lobes are larger than the others. In the cat the right lateral lobe is deeply cleft into two lobules. The large elongated gall bladder is imbedded in the right median lobe, on its dorsal surface in the rabbit, in a cleft in this lobe in the cat. On raising the liver and looking between the liver and the stomach another small lobe, the caudate lobe, will be seen. It is situated between the two layers of the lesser omentum. The lesser omentum, or gastro-hepato-duodenal ligament, is the ligament passing from the lesser curvature of the stomach to the posterior surface of the liver. It is divisible into two portions: the gastrohepatic ligament from the lesser curvature to the liver, and the hepatoduodenal ligament from the liver to the first part of the small intestine. That portion of the gastrohepatic ligament which contains the caudate lobe of the liver forms a sac which continues anteriorly the cavity of the greater omentum. In the hepatoduodenal ligament runs the bile duct which should be traced from the gall bladder by gently dissecting in the ligament. Note the cystic duct 
from the gall bladder and the hepatic ducts from the lobes of the liver, that from the right lateral lobe being especially large. The cystic and hepatic ducts unite to form the common bile duct which passes to the intestine in the hepatoduodenal ligament. It should be traced to the duodenum by cleaning away the connective tissue around it. Note to the right and dorsal to the bile duct, lying also in the hepatoduodenal ligament, the large hepatic portal vein. This must not be injured. Immediately dorsal to this vein, posterior to its branch into the right lateral lobe of the liver, the hepatoduodenal ligament has a free border which forms the ventral rim of an opening or slit of some size, the foramen epiploicum or entrance into the cavity of the omentum. It can be identified with certainty by making a slit into the cavity of the omentum and probing through the slit toward the right, toward the spot just described.

The lesser omentum extends to the middle of the posterior face of the liver, where it becomes the serosa of the liver; here its two walls part and inclosing the liver between them pass to the anterior face of the liver, where they again unite to form ligaments. The falciform ligament extends from between the two median lobes of the liver to the median ventral line; it is a thin sheet with a concave posterior border. Anteriorly and dorsally it is continuous with the coronary ligament, a stout ligament which attaches the liver to the central tendon of the diaphragm. The coronary ligament is circular in form, and its ring of attachment to the liver bounds a small space on the anterior face of the liver which is free from serosa.

Now trace the intestine from the pylorus. Its first portion, the duodenum, is bound to the liver by the hepatoduodenal ligament. The duodenum curves abruptly caudad. In the rabbit it is very long and forms a loop. The part of this loop which descends posteriorly is named the descending limb; the short turn at the most posterior part of the loop is the transverse limb; and the part which ascends anteriorly toward the stomach is called the ascending limb. The duodenum of the cat descends caudad for about two inches and then turns to the left. The duodenum is supported by a part of the dorsal mesentery, the mesoduodenum. It is also attached to the right kidney by a mesenterial fold, the duodenorenal ligament. Located in the mesoduodenum is the pancreas. It will be seen by spreading the mesentery. In the rabbit the pancreas consists of streaks of gland tissue scattered in the mesentery and situated chiefly along the courses of blood vessels. In the cat the pancreas is a definite compact white gland which extends to the left into the dorsal wall of the greater omentum, dorsal to the greater curvature of the stomach. In the rabbit the pancreatic duct enters the duodenum about an inch or an inch and one-half anterior to the beginning of the ascending limb of the duodenum. This location of the pancreatic duct is unusual in mammals. In the cat there are two pancreatic ducts. The principal one joins the common bile duct at the point where the latter enters the duodenum. On picking away the substance of the pancreas at this point the duct is readily 
located. The common slightly swollen chamber where bile and pancreatic ducts unite is known as the ampulla of Vater. The second or accessory pancreatic duct in the cat enters the duodenum about three-quarters of an inch caudad of the principal duct but is not easy to find.

From the duodenum trace the coils of the remainder of the small intestine. It is supported by a part of the dorsal mesentery, the mesentery proper. The first portion of the small intestine beyond the duodenum is called the jejunum, and the remainder, the ileum, but there is no definite boundary between the two. Note the coils of the mesentery accompanying the intestine and the frequent fusions which occur (especially in the rabbit) between these coils. In the cat it will be necessary to withdraw the greater omentum from the coils of the intestine; the omentum may then be cut across near the spleen and discarded. Follow the small intestine to its enlargement into the large intestine. In doing this it may be necessary to tear slightly the fusions of the mesentery, but the structures should be kept as intact as practicable.

Rabbit: At the point of juncture of the large and small intestine there is an enlargement, the sacculus rotundus. From the sacculus rotundus extends an enormous blind sac, about a foot and a half in length. The first foot of this is very large and is known as the caecum; the last five or six inches is reduced in diameter and constitutes the vermiform appendix. Both caecum and appendix are very much longer in the rabbit than in most mammals, owing probably to the habit of the animal of ingesting large quantities of vegetable food. The wall of the caecum is marked by a spiral line which denotes the position of an internal spiral fold of the lining membrane of the caecum. From the sacculus rotundus trace the large intestine after it has given off the caecum. The large intestine beyond the caecum is named the colon. The colon is supported by a part of the dorsal mesentery called the mesocolon. The first part of the colon, the ascending colon, is rather long and pursues a winding course. At first its wall bears three longitudinal muscular bands, the bands or taeniae of the colon. Between the taeniae the wall of the colon is greatly puckered, forming little sacculations, the haustra. Beyond the ascending colon, the colon runs for a short distance transversely across the peritoneal cavity from right to left and is then named the transverse colon. At the left it turns abruptly posteriorly as the $d e$ scending colon. At this turn the mesocolon is fused to the mesogaster. The descending colon passes straight posteriorly and disappears dorsal to the urogenital organs.

Cat: The junction of small and large intestine is marked by a slight projection, the caecum, a vermiform appendix being practically absent. The large intestine or colon passes forward as the ascending colon; then turns and extends across the peritoneal cavity from right to left as the transverse colon; and turns abruptly at the left and proceeds straight posteriorly as the descending colon. The mesentery of the colon is named the mesocolon. At the left where the 
transverse colon turns caudad the mesocolon is fused secondarily to the mesogaster.

The urinary bladder is a sac occupying the posterior end of the peritoneal cavity, immediately internal to the body wall and ventral to the large intestine. From the ventral surface of the bladder a mesentery, the median ligament of the bladder (median umbilical fold of human anatomy) extends to the median ventral line and here continues forward for some distance. Near the exit of the bladder from the peritoneal cavity there is on each side a slightly developed ligament, the lateral ligament of the bladder.

The terminal portion of the descending colon is the rectum. Both the rectum and the duct of the bladder pass to the exterior through the ring formed by the pelvic girdle and vertebral column. They will be followed at a later time in connection with the urogenital system. For the present it may be stated that the rectum and urogenital ducts are completely separated from each other. A cloaca is therefore absent.

The small bodies which may have been noted in the mesentery, usually buried in fat, are lymph glands, parts of the lymphatic system. Small portions of lymphatic tissue called lymph nodules are also abundantly present in the wall of the intestine. Aggregations of lymph nodules are known as Peyer's patches. Peyer's patches are thickened oval spots on the surface of the intestine, of slightly different color from the rest of the intestinal wall. Look for them. They occur in the rabbit along the entire small intestine on the side opposite that attached to the mesentery; there is a larger patch at the place of junction of sacculus rotundus and caecum. The walls of the sacculus rotundus, caecum, and vermiform appendix are composed almost entirely of lymph nodules. In the cat, Peyer's patches are readily found as oval light-colored spots along the colon.

Slit open various parts of the digestive tract along the side opposite the at tachment of the mesentery. Wash out the interior. In the cat note the marked ridges or rugae in the wall of the stomach; these are very slight in the rabbit. Cut through the pylorus and note the thickened ridge or pyloric valve at this place. In the wall of the small intestine observe the velvety appearance due to the villi (finger-like projections of the mucous membrane). Find also the depressions marking the positions of the lymph nodules. In the rabbit slit open the sacculus rotundus, caecum, and appendix, and note the spotted appearance of the interior owing to the lymph nodules composing the walls. In the interior of the caecum is a spiral ridge. Cut through the junction of large and small intestine and note in both animals an elevation, the ileocolic valve, projecting into the ileum.

\section{H. THE COMPARATIVE ANATOMY OF THE COELOM AND THE MESENTERIES}

Since the development and comparative anatomy of the coelom and mesenteries are extremely difficult and complicated subjects, it seems advisable at the risk of repetition to 
make some concluding general remarks concerning them. Only a simplified account can be given here.

The coelom of fishes and urodeles is divided into two compartments, a small anterior pericardial cavity and a much larger posterior pleuroperitoneal cavity. The former is situated entirely anterior to the latter and far anterior in the body, at the level of the pharynx. The partition between the two coelomic cavities in these groups is the transverse septum. This septum is formed, as previously explained, for the purpose of conveying the great veins from the dorsal body wall into the posterior end of the heart. It arises first as a column of mesenchyme on each side, conveying a vein into the heart; later these columns enlarge and fuse to form a partition. The transverse septum is a rather thick membrane with anterior and posterior walls, which inclose between them the great veins and the posterior end of the heart. The septum in fishes and urodeles lies as the name implies in a plane transverse to the body axis (Fig. $45 . A$ and B, p. 160 ).

The lining of the pericardial cavity is named the parietal pericardium. In fishes and urodeles it also constitutes the lining of the body wall in the region under consideration. The
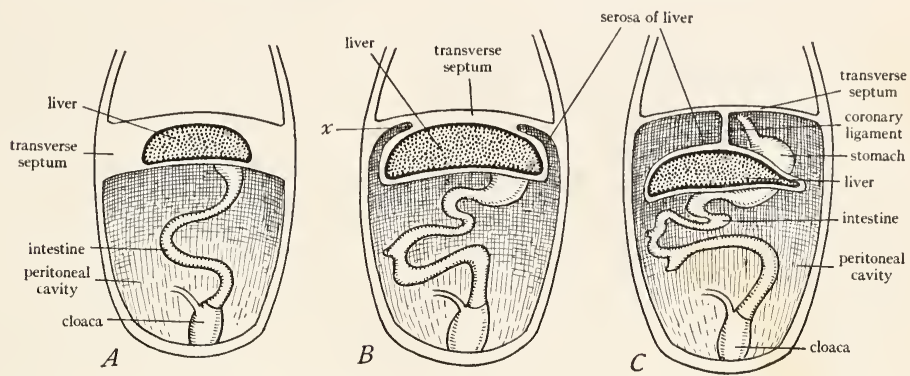

FIG. 48.-Diagrams to show the separation of the liver from the transverse septum and formation of the coronary ligament. $A$, liver inclosed in the transverse septum. $B$, beginning of constriction of the liver from the septum at $x$. C , completion of the constriction, leaving the liver suspended from the septum by the coronary ligament. The falciform ligament is also formed at the same time by the same constriction. (B and C after McMurrich's Development of the Human Body, copyright by P. Blakiston's Son and Company.)

parietal pericardium is continuous with the anterior face of the transverse septum, which, in fact, forms the posterior part of the parietal pericardium and the posterior wall of the pericardial cavity. The lining of the pleuroperitoneal cavity is the pleuroperitoneum. This is continuous anteriorly with the posterior face of the transverse septum, which constitutes the anterior part of the pleuroperitoneum and forms the anterior wall of the pleuroperitoneal cavity (see Fig. $45^{A}$ and $\left.B\right)$.

The relations of the transverse septum to the liver are somewhat complicated and require explanation. The liver is a diverticulum from the small intestine. It happens that at the point where the liver diverticulum grows out ventrally from the intestine, the transverse septum is situated. Consequently, the liver is compelled to grow out into the septum. It lies at first within the mesenchyme of the septum, between the two walls of the septum. Owing to the fact as already explained that the great veins enter the heart by way of the septum, the liver also acquires important relations to these veins, as will be discussed in the section on the circulatory system. The liver rapidly increases in size so that it can no longer be contained within the limits of the septum. It consequently bulges posteriorly, carrying with it the 
posterior wall of the septum, which thus becomes the peritoneal covering or serosa of the liver. Later, the region where the liver bulges from the septum narrows down on dorsal and lateral sides, leaving anterior and ventral connections between the liver and the septum (Fig. 48). The anterior connection is named the coronary ligament and is, as the name indicates, a circular ligament, by means of which the liver is permanently in all vertebrates suspended from the transverse septum or its derivative. The ventral partition formed by the constriction of the liver from the septum is the falciform ligament of the liver, which is also by virtue of its location a part of the ventral mesentery (Fig. $44 B$, p. 158 ). We thus see that the transverse septum has the following parts: the posterior wall of the pericardial cavity, the anterior wall of the pleuroperitoneal cavity, the falciform and coronary ligaments of the liver, the serosa of the liver, and the mesoderm tissue of the liver.

In Anura and reptiles the coelom is divided in the same way as in fishes and urodeles into two compartments, the pericardial and pleuroperitoneal cavities. In these groups, however, the pericardial cavity is no longer situated anterior to the pleuroperitoneal cavity but has descended posteriorly until it lies ventral to the anterior part of the latter cavity. This descent occurs during embryonic development. In this descent the pericardial cavity necessarily carries with it the transverse septum, since this septum formed the posterior wall of the cavity. Furthermore, since the parietal pericardium or wall of the pericardial cavity in the lower vertebrates lined the inside of the body wall, it is necessary that this membrane become split, at least in part, from the body wall before the descent can take place. This happens mainly by the invasion of the pleuroperitoneal cavity forward. As a result of these processes, the parietal pericardium is separated from the body wall, at least in part, and forms a delicate sac about the heart, the pericardial sac. The separation of the pericardial sac from the body wall is complete in the Anura but in the turtle is incomplete ventrally so that the ventral wall of the pericardial cavity is still in contact ventrally with the body wall (see Fig. ${ }_{45}$ C, p. I60).

In the groups under consideration the relations of the transverse septum remain the same as before, but its position is now oblique, indeed almost frontal, while formerly it was transverse (Fig. ${ }_{45} \mathrm{C}$ ). It still presents anterior and posterior walls (which are now nearly ventral and dorsal in position) and still incloses the great veins and the posterior end of the heart between its walls. Its anterior (ventral) face forms part of the pericardial sac, while its posterior (dorsal) face forms part of the lining of the pleuroperitoneal cavity as previously. To this latter portion the liver is attached as before by the coronary and falciform ligaments.

The posterior descent of the pericardial cavity brings a portion of the pleuroperitoneal cavity dorsal to the pericardial cavity. In this portion of the pleuroperitoneal cavity which lies above the heart, the lungs grow out.

In birds the relations of pericardial cavity and sac and of the transverse septum are the same as just described. The pericardial sac is, however, only partially separated from the body wall. The remainder of the coelom, corresponding to the pleuroperitoneal cavity of fishes, amphibians, and reptiles is in birds further subdivided. This is accomplished by means of a pair of mesenterial folds which grow ventrally from the sides of the esophagus and fuse with the transverse septum (Fig. ${ }_{5} D$ and $E$, p. r60). The pleuroperitoneal cavity is thus divided into anterior and posterior compartments. The anterior compartment consists of the two pleural cavities, one on each side of the pericardial cavity and each containing a lung. The posterior compartment is named the peritoneal cavity. The lining of the pleural cavities is known as the pleura; that of the peritoneal cavity as the peritoneum. The partition between pleural and peritoneal cavities is called the oblique septum. Its median portion is composed of the transverse septum; its lateral portions, of the two folds already mentioned which are known as the pleuroperitoneal membranes.

In mammals the coelom is divided as in birds into four compartments: the pericardial, the two Dieuia', and the peritoneal cavities. The pericardial cavity has descended posteriorly 
as described above, and the pericardial sac is free on all sides from the body wall. That part of the former pleuroperitoneal cavity which lies dorsal and lateral to the heart is separated from the posterior part of the same cavity by a muscular partition, the diaphragm, which extends across the body cavity in a transverse plane. The two pleural cavities lie anterior to the diaphragm, one on either side of the pericardial cavity which is inclosed between their medial walls. The pericardial cavity is free on all sides, owing to the extension of the two pleural cavities ventrally (Fig. 49). The two pleural cavities, at first dorsal in position in mammals. grow ventrally and split the pericardium from the ventral body wall. They push in between the pericardial sac and the ventral body wall and their medial walls meet ventral to the pericardial sac to form the mediastinal septum seen in the dissection. The cavity posterior to the diaphragm is the peritoneal cavity.

The diaphragm corresponds in large part to the oblique septum of birds but contains additional components. It is a structure peculiar to mammals. It is formed in part by mesenterial folds, the pleuroperitoneal membranes, which grow down from the dorsal body wall and fuse
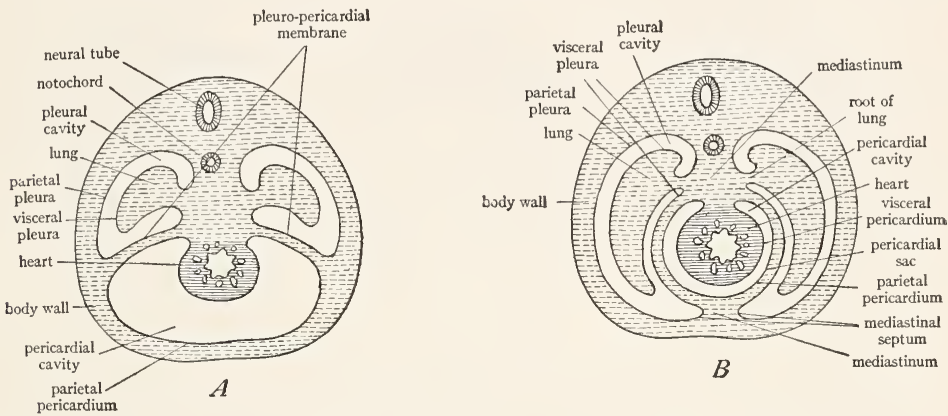

FIG. 49.-Diagrams to show the separation of the pericardial sac in mammals. $A$, early stage in which the parietal pericardium forms the lining of the body wall; the pericardial and pleural cavities are separated by the pleuropericardial membrane, which is the anterior continuation of the transverse septum. $B$, later stage, showing how the ventral extension of the two pleural cavities splits the parietal pericardium from the body wall, and gives rise to the mediastinal septum; the parietal pericardium then becomes the pericardial sac. ( $B$ from Prentiss and Arey's Textbook of Embryology, courtesy of the W. B. Saunders Company.)

with the posterior wall of the transverse septum, much as in birds (Fig. $45 D$ and $E$, p. r6o). To these components there are also added some portions of the dorsal body wall and a portion of the dorsal mesentery. Finally the diaphragm is penetrated by buds from adjacent myotomes and thus becomes in large part muscular.

The derivatives and relations of the transverse septum in mammals are similar to those previously described. Its anterior face forms part of the pericardial sac. The great veins have, however, gradually been drawn out of it (as also in birds) and now all of the veins enter the anterior end of the heart. The posterior face of the septum forms part of the diaphragm, that part to which the liver is attached by the coronary ligament. Coronary and falciform ligaments of the liver are present as previously and originate in the same way.

The coelomic linings, pericardium, pleuroperitoneum, or pleura and peritoneum, present the same relations in all of the vertebrates. Each has a parietal portion lining the body wall, a visceral portion covering the viscera, and mesenteries or ligaments connecting the parietal and visceral portions. 
The relations of the mesenteries are very similar in all of the vertebrates. The dorsal mesentery extends, usually unbroken, from the dorsal median line to the digestive tract. Each portion of it bears a special name, according to the part of the digestive tract which it supports. In mammals the mesentery of the stomach is drawn out posteriorly into a bag, the greater omentum, which apparently serves to protect the abdominal viscera (Fig. 50). The ventral mesentery extends from the digestive tract to the ventral body wall but is reduced to remnants in the adults of all vertebrates. These remnants are found in the region of the liver and the bladder where they form the gastrohepatic and hepatoduodenal ligaments, the falciform ligament, and the median ligament of the bladder. All viscera of vertebrates either are retroperitoneal, i.e., situated external to the coelomic lining, or they are situated between the two layers of a mesentery. The liver, the heart, and the urinary bladder are situated in the ventral mesentery; the pancreas is generally in the dorsal mesentery but may be in the ventral mesentery as well; the digestive tube may be regarded as situated in the dorsal mesentery.
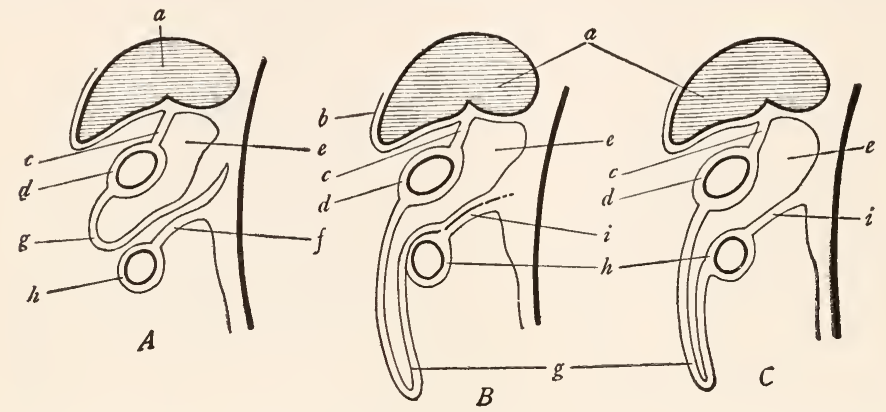

FIG. 50.-Diagrams to show the formation of the greater omentum in mammals and the fusion of the mesogaster and the mesocolon. A, early stage, in which the mesogaster is beginning to form a bag at $g$. $B$, the mesogaster is drawn posteriorly into a long bag $g$, which is the greater omentum; the mesogaster and mesocolon are fusing at $i$. $C$, completion of the fusion of mesogaster and mesocolon at $i$. $a$, liver; $b$, serosa of the liver; $c$, lesser omentum or gastro-hepa to-duodenal ligament; $d$, stomach; $e$, lesser peritoneal sac or cavity of the greater omentum; $f$, mesocolon; $g$, portion of the mesogaster which forms the greater omentum; $h$, intestine; $i$, fusion of the mesogaster and mesocolon. (From Prentiss and Arey's Textbook of Embryology, after Hertwig, courtesy of the W. B. Saunders Company.)

\section{SUMMARY}

I. The parts of the digestive tract are much the same in all of the vertebrates. There has been little evolution of this system. In general, the intestine is longer and more coiled in the higher forms.

2. The mouth cavity is provided with glands in land vertebrates.

3. The nasal cavities are blind sacs in fishes, but beginning with Amphibia they communicate with the mouth and pharyngeal cavities for respiratory purposes. At first these respiratory passages through the nose open into the anterior end of the mouth cavity but later a hard palate, a shelf of bone, is developed ventral to the nasal passages. By this means the internal nares are moved posteriorly. In birds and mammals the nasal passages are extended still farther posteriorly by the development of the soft palate; the air then passes directly into the pharyngeal cavity without first passing the mouth cavity.

4. The wall of the pharynx is pierced by gill slits provided with gills in fishes and without gills in urodeles. Above the urodeles the gill slits vanish, with the exception of the first which contributes to the external auditory meatus. The first visceral pouch evaginates to form the 
auditory tube and tympanic cavity. The entodermal lining of the visceral pouches persists as certain glandlike bodies, such as the tonsils, the thymus, and the epithelial bodies. The thyroid gland is an evagination from the floor of the pharynx between the second visceral pouches.

5. The pharynx in fishes leads into a single tube, the esophagus. Above fishes it leads into two tubes, the esophagus and the trachea. At the caudal end of the trachea are the paired lungs.

6. The upper end of the trachea is expanded into a chamber, the larynx, the walls of which are supported by the modified gill arches.

7. Both esophagus and trachea become more and more elongated in higher forms, owing to a descent of the viscera posteriorly. This descent leaves a space in the anterior dorsal part of the body cavity into which the lungs grow out from the lower end of the trachea.

8. Lungs are present in all vertebrates above fishes (they have secondarily disappeared in some urodeles). They are probably homologous with the swim bladder of fishes. Their walls become more and more complicated and divided into air spaces, the higher one ascends in the phylogentic series. In birds the lungs connect with air sacs in the viscera and air spaces in the bones.

9. The intestine of vertebrates is provided with two glands, the liver and the pancreas, which are attached by ducts to the duodenum (the bile duct is generally and the pancreatic duct sometimes lacking in cyclostomes).

Io. The intestine terminates in all vertebrates except placental mammals (cyclostomes and teleostomes) in a chamber, the cloaca, into which the urinary and genital ducts also open. In mammals (and cyclostomes and teleostomes) the cloaca is lacking and the urinary and genital ducts open separately from the intestine.

II. In most vertebrates there is a saclike outgrowth, the urinary bladder, from the ventral wall of the cloaca.

I2. In all vertebrates except birds and mammals the coelom is divided into two compartments, the pericardial cavity containing only the heart and the pleuroperitoneal cavity containing the other viscera. The two cavities are separated by a partition, the transverse septum.

13. In cyclostomes, fishes, and urodeles the pericardial cavity is anterior to the pleuroperitoneal cavity. From Anura on the pericardial cavity is ventral to the anterior part of the pleuroperitoneal cavity. This change is due to a posterior descent of the heart and pericardial cavity carrying the transverse septum with them. As a consequence of the descent the wall of the pericardial cavity together with the transverse septum forms a sac, the pericardial sac, around the heart. That portion of the pleuroperitoneal cavity dorsal to the heart is destined to form the pleural cavities.

I4. In birds and mammals the pleuroperitoneal cavity is divided into anterior and posterior portions by the formation of a partition which descends from the dorsal body wall and unites with the transverse septum. This partition is called the oblique septum in birds and the diaphragm in mammals. The diaphragm is more complex in origin than the oblique septum and unlike the latter contains a considerable amount of striated muscle. Anterior to the oblique septum or diaphragm are the two pleural cavities, each of which contains a lung; posterior to these partitions is the peritoneal cavity, containing the digestive and reproductive systems. Thus, in birds and mammals the coelom is divided into four compartments-the pericardial, the two pleural, and the peritoneal cavities.

I5. A dorsal mesentery supports the digestive tract in all vertebrates and remains practically complete throughout. The ventral mesentery of the digestive tract is absent in the adult except in the regions of the liver and the bladder. There are special mesenteries for the gonads and their ducts. In mammals the mesentery of the stomach develops a special posterior prolongation called the greater omentum. 


\section{THE COMPARATIVE ANATOMY OF THE CIRCULATORY SYSTEM}

\section{A. GENERAL CONSIDERATIONS}

1. The parts of the circulatory system.-The circulatory system of vertebrates comprises two systems of branching tubes inclosing circulating fluids, the blood-vascular system and the lymphatic system. The former is the larger and more conspicuous of the two and is the one referred to when the expression circulatory system is employed without qualification.

The blood-vascular system is a closed system, that is, it consists of a set of branching tubes, the blood vessels, which are continuous with each other, unconnected with other systems (except the lymphatic system), and in which the inclosed fluid travels in a circuit. The parts of the blood-vascular system are the heart, the arteries, the veins, and the capillaries. The heart is a contractile muscular organ situated in the median ventral region in the anterior part of the body. It has essentially the form of an S-shaped tube subdivided into chambers which will be named in connection with the dissection. The arteries are the vessels which leave the heart and in which the contained fluid flows away from the heart. The veins are vessels in which the contained fluid flows toward the heart. The student should particularly note that arteries and veins cannot be defined on the basis of the kind of blood which they contain. The veins of the vertebrate body fall into three classes: the systemic veins, which flow directly into the right side of the heart; the pulmonary veins, which flow from the lungs into the left side of the heart; and the portal veins or portal systems, in which the blood does not return directly to the heart but passes into a system of capillaries from which it is re-collected into systemic veins. The capillaries are the minute microscopic vessels which connect the ends of the arteries with the beginnings of the veins and through which the circulation is completed. All the tissues of the body are permeated with networks of capillaries through the walls of which the gaseous and other exchange between the blood and the body cells takes place.

The blood-vascular system incloses a fluid, the blood, which in vertebrates is colored red. The blood consists of a colorless fluid, the plasma, in which float microscopic cells, the corpuscles. The latter are of two general kinds, red and white, the former giving the red color to the blood. Study of the blood lies outside of the limits of this course.

The lymphatic system is an open system, that is, it consists not only of branching tubes, the lymph vessels, but of large spaces, the lymph sinuses; and it is further in communication with the coelomic spaces of the body. Lymph sinuses occur beneath the skin (the student may recall the large subcutaneous lymph sinuses in the frog), between the muscles, in the mesenteries, in the walls of the digestive tract, around the central nervous system, etc. From these sinuses the fluid passes into more or less definite lymph vessels, which eventually open into the veins of the blood-vascular system. In the lower vertebrates contractile lymph hearts are placed in the course of the lymphatic vessels, to aid the flow, but these are absent in mammals. The lymphatic system further differs from the blood-vascular system in that nodules of tissue, the lymph glands, are placed in the path of the lymph vessels. The lymph glands consist of a network of connective tissue in which are imbedded masses of cells, known as lymphocytes. Lymphocytes are a variety of white blood corpuscles. The function of the lymph glands appears to be to destroy foreign particles, bacteria, etc., and to add white blood corpuscies to the circulation. The tonsils, the thymus, and the spleen belong to the category of lymph glands. 
The lymphatic system contains a colorless fluid, the lymph, whose composition is similar to the plasma of the blood. The lymph contains white blood corpuscles but red ones are absent. The lymph fills all of the spaces of the body and bathes all of the cells, and all exchange between the tissues and the blood must occur by way of the lymph.

Owing to the delicate nature of the lymph vessels and the general diffuse character of the lymphatic system, very little of this system can be made out in an ordinary dissection. Our study of the circulatory system will therefore be confined almost entirely to the blood-vascular system.

2. The origin of the blood and of the blood vessels. - The blood vessels and the blood arise from the mesoderm, from mesenchyme cells. In the mesenchyme little patches of cells form; the central cells of these patches become modified into blood corpuscles; the peripheral
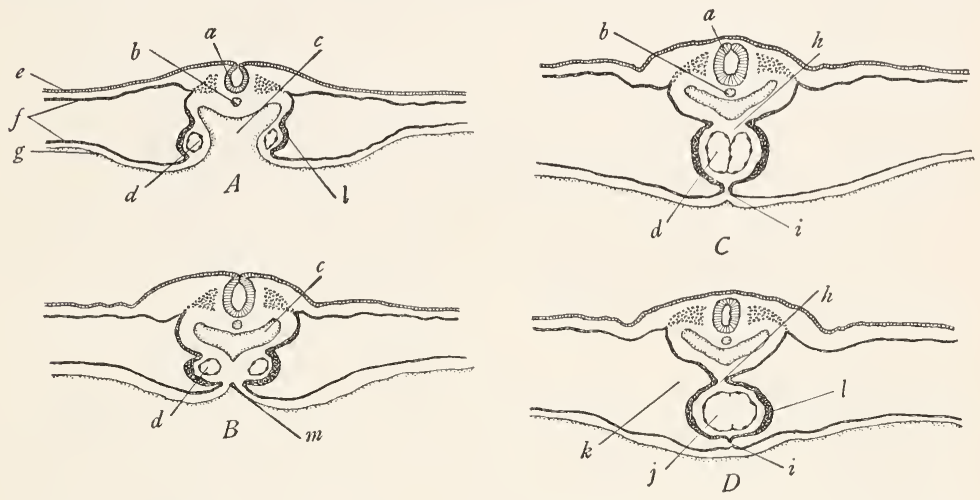

FIG. 51.-Drawings of cross-sections through four successive stages of development of the chick embryo, between 25 and 29 hours of incubation, to show the formation of the heart. $A$, early stage showing the open intestine at $c$, the vitelline veins $d$ in the splanchnic mesoderm $f$, and the thickening $l$ in the splanchnic mesoderm covering the veins. $B$, next stage in which the splanchnopleure has fused together at $m$, closing the intestine $c$ and the bringing the two vitelline veins $d$ closer together. $C$, later, showing the two vitelline veins $d$ in contact. $D$, completion of the heart by the fusion of the vitelline veins; the thickened mesoderm $l$ becomes the muscular wall of the heart. $a$, neural tube; $b$, notochord; $c$, anterior part of the digestive tract; $d$, vitelline vein; $e$, ectoderm; $f$, somatic and splanchnic layers of the mesoderm; $g$, entoderm; $h$, dorsal mesentery of the heart or dorsal mesocardium; $i$, ventral mesentery of the heart, or ventral mesocardium (it is disappearing in $D$ ); $j$, lining of the heart formed by the union of the vitelline veins; $k$, pericardial cavity; $l$, muscle layer of the heart formed by the thickening of the splanchnic mesoderm; $m$, point of fusion of the splanchnopleure of the two sides. (After Patten's Early Embryology of the Chick, copyright by P. Blakiston's Son and Company.)

cells arrange themselves to form tubes, the blood vessels. The blood vessels which arise in the somatic mesoderm are somatic vessels; those in the splanchnic mesoderm are visceral or splanchnic vessels.

3. The origin of the heart.-The heart arises in the ventral mesentery in the anterior part of the embryo. In cases where the embryo is closed below from the beginning, a tubular cavity appears in the ventral mesentery and in the walls of this cavity the heart tissue differentiates. In the majority of vertebrates, with meroblastic development, the embryo is at first open below on the yolk sac (see Fig. $8 C$ and $D$, p. 40). In the splanchnic mesoderm of the 
hypomere there arises on each side a tubular cavity, of the same nature as the blood vessels. As the hypomere closes below in the median ventral line the two cavities are brought together and fuse to form the heart (Fig. 5I). By either method of formation the heart necessarily lies in the ventral mesentery of the gut and is therefore provided with dorsal and ventral mesenteries, known as the dorsal and ventral mesocardia (see Fig. $44 A$ ). These, however, very rapidly disappear, the ventral mesocardium vanishing as soon as formed. The heart then swings free in the coelom, attached only at its two ends. The portion of the coelom in which the heart lies is at first continuous with the general cavity of the hypomeres, but is soon cut off from the rest of the coelom by the formation of the transverse septum and is thereupon named the pericardial cavity.
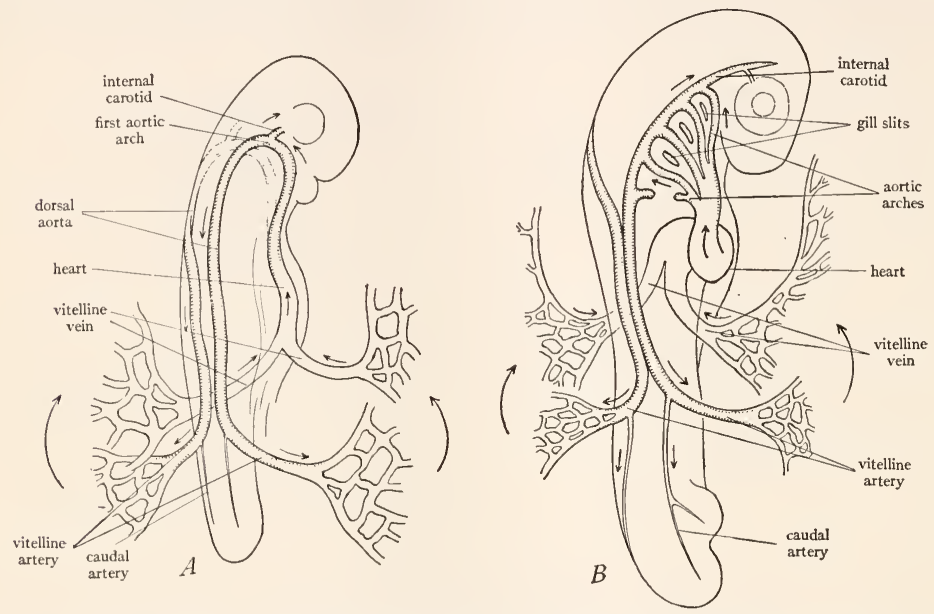

FIG. 52.-Diagrams of early vertebrate embryos to show the development of the main blood vessels. $A$, earliest stage, in which the circulatory system consists of the vitelline veins, heart, ventral aorta, first aortic arch, dorsal aortae, and vitelline arteries. $B$, later stage showing the development of successive aortic arches following the first one. The method of formation of the aortic arches by buds from the ventral and dorsal aortae is illustrated by the last aortic arch in B. (From Wilder's History of the Human Body, courtesy of Henry Holt and Company.)

4. The chief embryonic blood vessels. - The earliest vessels to form in the vertebrate embryo are the vitelline veins. These veins develop on the surface of the yolk sac (or in the absence of a yolk sac, along the intestine) in the splanchnic mesoderm of the hypomere. They pass to the embryo in the mesentery of the gut and enter the posterior end of the heart. At the anterior end of the heart a blood vessel, the ventral aorta, arises and connects with the heart. The ventral aorta extends forward to the anterior end of the pharynx. Here it divides in two, and the two branches turn dorsally, one on either side of the pharynx. This pair of branches encircling the pharynx is the first pair of aortic arches. Each vessel lies in the center of the first or mandibular visceral arch. On reaching the dorsal side of the pharynx the two vessels turn posteriorly and as the dorsal aortae proceed backward, situated in the median dorsal region of the body wall. Each dorsal aorta on reaching the region of the yolk sac sends out a vitelline artery over the surface of the yolk sac. The early embryonic circulation is thus completed (Fig. 52A). 
There subsequently develop around the pharynx additional aortic arches connecting the ventral and dorsal aortae. In typical vertebrates six such pairs of aortic arches appear, one pair to each pair of visceral arches (Fig. $52 B$ ). The aortic arch runs in the center of the visceral arch. The aortic arches develop in order, the second after the first, then the third, and so on. Posterior to the pharyngeal region, the two dorsal aortae soon fuse to form a single dorsal aorta which continues into the tail as the caudal artery. From the aorta, as development proceeds, branches arise for the various parts of the body. In fishes the middle part of each aortic arch later breaks up into a system of capillaries which occupies the gill filaments, but in non-aquatic forms the arches remain unbroken.

It will now be perceived that provision must be made for the return of the venous blood of the embryo to the heart. For this purpose two pairs of veins arise in the somatic mesoderm of the body wall. These are a pair of anterior cardinal veins returning blood from the anterior part of the body and a pair of posterior cardinal veins returning blood from the posterior part

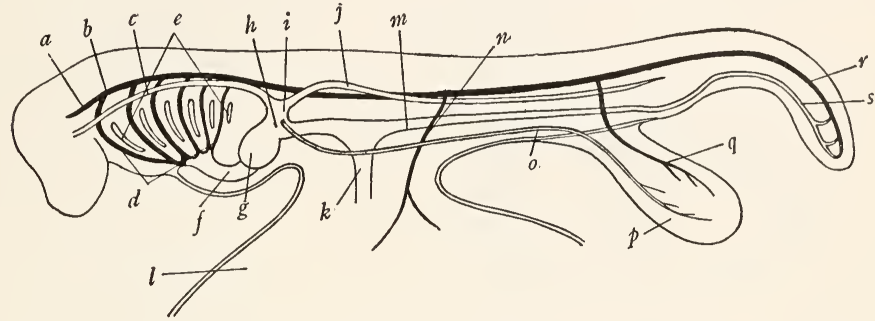

FIG. 53. - Later stage of the development of the circulatory system, showing the chief veins. Veins blank, arteries black. $a$, internal carotid; $b$, dorsal aorta; $c$, anterior cardinal vein; $d$, the six aortic arches; $e$, the six gill slits; $f$, the conus arteriosus of the heart continuing into the ventral aorta; $g$, main part of the heart; $h$, sinus venosus of the heart; $i$, duct of Cuvier or common cardinal vein; $j$, posterior cardinal vein; $k$, vitelline vein; $l$, yolk sac; $m$, subintestinal vein; $n$, vitelline artery; $o$, lateral abdominal or umbilical vein; $p$, allantois (urinary bladder); $q$, allantoic or umbilical artery; $r$, caudal artery; $s$, caudal vein. (Slightly modified from Vialleton's Éléments de Morphologie des Vertébrés.)

of the body. In order for these veins, which are situated in the dorsal body wall, to reach the heart, a bridge of mesoderm is formed on each side extending between the somatic and splanchnic mesoderm. We have already learned that this pair of bridges is the beginning of the transverse septum; and by the union and extension of the bridges the septum is completed. At the level of the posterior end of the heart the anterior and posterior cardinal veins on each side unite to a common vessel, the duct of Cuvier or common cardinal vein. The common cardinal vein then passes to the heart from each side by way of the transverse septum between the two walls of which this vein is inclosed. In addition to the cardinal veins there appears in vertebrate embryos a pair of veins in the lateral or ventral abdominal walls, known as the lateral or ventral abdominal veins, which enter the heart along with the common cardinal veins. They are also called the umbilical or allantoic veins in the embryos of amniotes. The main veins of the embryo at this time are illustrated in Figure 53.

The two vitelline veins are soon extended posteriorly in the embryo by means of a tributary, the subintestinal vein, which courses in the mesentery of the gut and constitutes the chief vein of the digestive tract. It is shown in Figure 53. It continues into the tail as the caudal vein, making a loop around the anus. In embryos without a yolk sac the subintestinal and vitelline veins appear as one continuous vein. 
The-arteries and veins thus far described are the chief longitudinal trunks of the embryo. From them, branches, usually segmentally arranged, extend to various parts of the body. The branches of the main longitudinal vessels are classified into three kinds: the median visceral or splanchnic branches, unpaired branches to and from the digestive tract; the lateral visceral branches, paired branches to and from the urogenital organs; and the parietal or somatic branches, paired vessels to and from the body wall. This arrangement is most obvious in the arteries (Fig. 54). It is more or less persistent in the adult, chiefly in the posterior part of the body. The vessels to the paired appendages are simply enlarged somatic branches.

5. The origin of the portal systems. - It has already been explained that a portal system is a portion of the venous system, the constituent veins of which instead of entering the heart pass into a network of capillaries from which the blood is re-collected by a systemic vein. In other words, in a portal system, a network of capillaries is interposed in the path of a vein or veins. There are two portal systems, the hepatic portal system, in which the interposed capillaries are located in the liver, and the renal portal system, in which they are in the kidney.

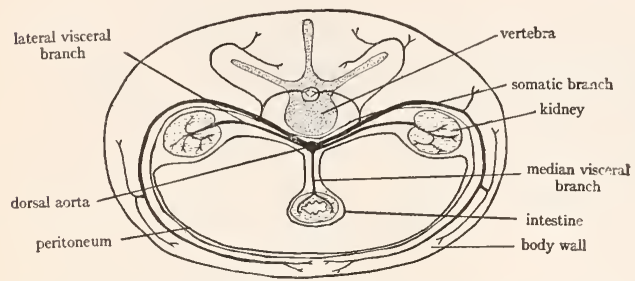

FIG. 54.-Diagram of a cross-section through a vertebrate embryo, to show the segmental branches of the aorta. (After McMurrich's Development of the Human Body, copyright by P. Blakiston's Son and Company.)
The origin of each of these systems may be given briefly.

a) The hepatic portal system: This arises as follows. When the transverse septum develops at the posterior end of the heart, the two vitelline veins must naturally pierce the septum on their way to the heart. At this region the liver buds out from the small intestine and extends into the transverse septum. As it grows, the liver gradually uses up the substance of the transverse septum and fills the available space in the septum. The liver substance thus comes to surround the proximal ends of the two vitelline veins (Fig. $55^{A}$ and $B$ ). At first the vitelline veins pass through the liver into the heart, but soon they begin to break up into smaller and smaller vessels (Fig. ${ }_{55} \mathrm{C}$ ) in the liver until the liver is occupied by a network of capillaries (of the kind known as sinusoids) which permeate the liver substance. Thus, the circulation in the vitelline veins passes from the yolk sac and digestive tract to the liver, passes through a capillary network in the liver, and from the liver to the heart in the remaining proximal portions of the vitelline veins (Fig. $55 \mathrm{D}$ ). The latter are now called the hepatic veins. Posterior to the liver by ringlike unions between the two vitelline veins (Fig. ${ }_{5} C$ and $D$ ), a single vessel, the hepatic portal vein, is formed, and caudad of these unions the right vein disappears, leaving the left vein with its tributary, the subintestinal vein, now named the mesenteric vein, to form the chief vein of the digestive tract. This arrangement insures that all of the venous blood from the digestive tract caudad of the cardia must pass through a capillary network in the liver before it can reach the heart.

b) The renal portal system: This develops as follows. At first the caudal vein opens into the subintestinal vein, forming a loop around the anus as in Figure $55 C$ and $D$. Later the posterior cardinal veins grow posteriorly and connect with the caudal vein. The union of the caudal vein with the subintestinal vein is then broken as in Figure ${ }_{55} E$, the latter vein then becoming a tributary of the hepatic portal system. There next develops between the kidneys a vein, at first single, later paired-the subcardinal vein (Fig. $55 F$ ). This connects with the caudal vein and posterior ends of the posterior cardinal veins. The blood flows from the tail 


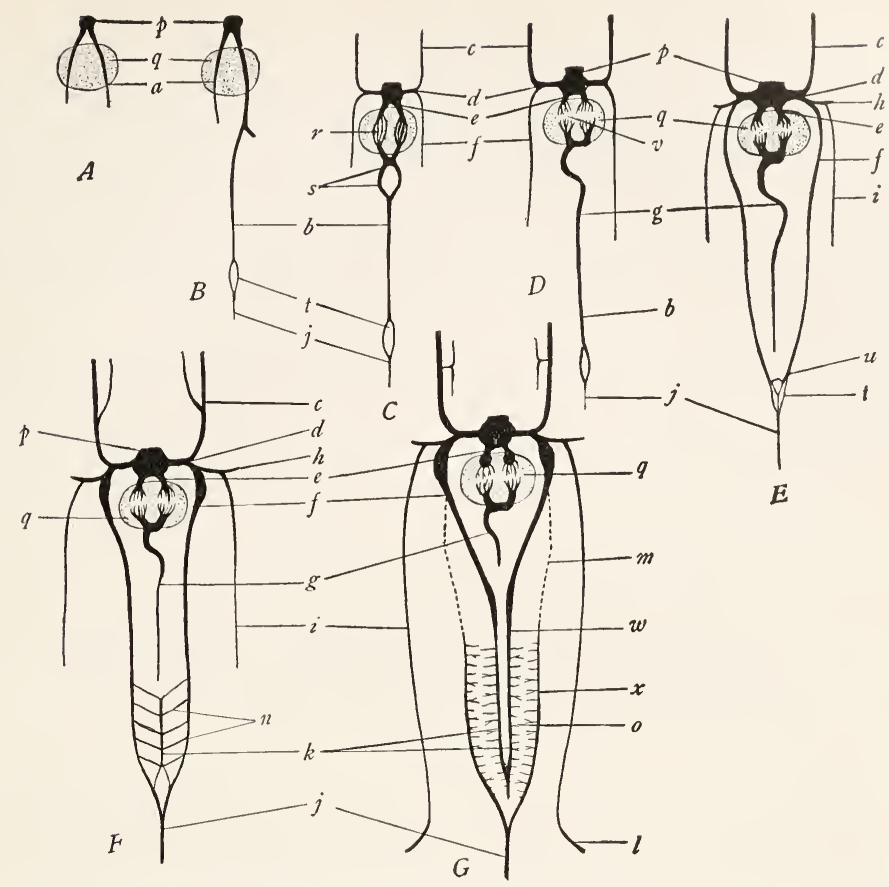

FIG. 55.-Diagrams to show the development of the veins in elasmobranchs. $A$, earliest stage, showing the two vitelline veins $a$ passing through the liver $q$ into the sinus venosus $p$. $B$, the subintestinal vein $b$ has appeared; it connects with one of the vitellines, makes a loop $t$ around the anus, and continues into the tail as the caudal vein $j . \quad C$, the anterior and posterior cardinal veins, $c$ and $f$, have appeared and connect with the sinus by way of the common cardinal vein $d$; the vitelline veins are breaking up in the liver at $r$ and caudal to the liver are connected by ring-shaped anastomoses at $s$. $D$, the vitelline veins have broken up into a network of capillaries in the liver at $v$; their proximal portions remain as the hepatic veins $e$; their distal portions have formed the hepatic portal vein $g$, which is continuous with the subintestinal $b$. $E$, the posterior cardinals $f$ have extended posteriorly and at $u$ have joined the loop $t$ formed by the caudal vein $j$ around the anus; the subintestinal has severed its connection with the caudal; the lateral vein $i$ and its tributary, the subclavian vein $h$, have appeared. $F$, a new vein, the subcardinal vein $k$, has appeared between the kidneys and connects with the posterior parts of the posterior cardinals $f$ by means of cross-vessels $n$ and also connects with the caudal vein $j$. $G$, the posterior cardinal veins have joined the subcardinals $k$ at $w$, their intermediate portions $m$ disappearing; the posterior parts of the posterior cardinals persist as the renal portal veins $x$, which flow into a network of capillaries $o$ in the kidneys; the lateral abdominal veins have grown posteriorly and developed iliac tributaries $l$ from the pelvic fins. $a$, vitelline vein; $b$, subintestinal vein; $c$, anterior cardinal vein; $d$, duct of Cuvier or common cardinal vein; $e$, hepatic vein; $f$, posterior cardinal vein; $g$, hepatic portal vein; $h$, subclavian vein; $i$, lateral abdominal vein; $j$, caudal vein; $k$, subcardinal vein; $l$, iliac vein; $m$, obliterated portion of the posterior cardinals; $n$, communications between subcardinals and renal portals; $o$, capillary network in kidneys; $p$, sinus venosus; $q$, liver; $r$, branching of vitelline veins in the liver; $s$, rings between the two vitellines; $t$, loop around the anus; $u$, union of posterior cardinals with the caudal vein; $v$, capillary network between hepatic portal and hepatic veins; $w$, union of posterior cardinals with subcardinals; $x$, renal portal veins. (Slightly modified after Hochstetter in Hertwig's Handbuch der vergleichenden und experimentellen Entwickelungslehre der Wirbeltiere.) 
into the subcardinal veins and through the kidneys into the posterior cardinal veins. There next occurs a break between the anterior and posterior parts of the posterior cardinal veins. The anterior parts form a connection with the subcardinal veins. The posterior parts retain their connection with the caudal vein. There is then a reversal of flow through the kidneys, since the blood now passes from the caudal vein into the posterior parts of the posterior cardinal veins, now called the renal portal veins, through the kidneys into the subcardinal veins, and from them into the anterior portions of the posterior cardinal veins (Fig. $55 G$ ). At first the channels through the kidneys are direct connections between the renal portal and subcardinal veins, but later they break up into a capillary network. In this way the renal portal system is established. The blood from the tail must pass through a capillary network in the kidneys.

We have now carried the circulatory system up to the stage of development in which it occurs in adult elasmobranchs. The further evolution of the circulatory system will best be followed on the specimens.

6. The segmental character of the circulatory system.-Like most systems of vertebrates, the circulatory system exhibits marked traces of an originally highly segmented condition.

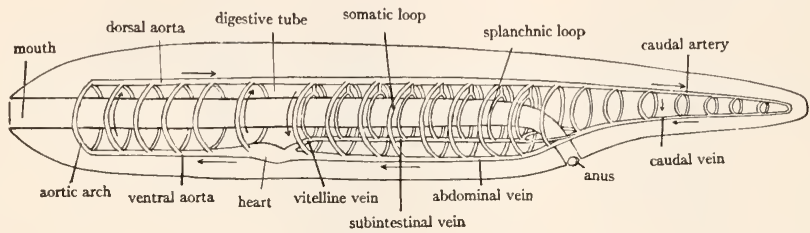

FIG. 56.-Diagram of the hypothetical primitive vertebrate circulation from which that of present vertebrates was probably derived, emphasizing the markedly segmental arrangement of the primitive vessels. (After Kingsley's Comparative Anatomy of Vertebrates, copyright by P. Blakiston's Son and Company.)

However, even in vertebrate embryos the segmental arrangement of the blood vessels is somewhat imperfect. It is helpful to an understanding of the cirulatory system to imagine that it is derived from a highly segmented condition such as that present in annelids. In this hypothetical ancestral state, as illustrated in Figure 56 , there are three main longitudinal vessels: a dorsai somatic vessel, corresponding to the dorsal aorta; a ventral somatic vessel, corresponding to the abdominal vein; and a splanchnic vessel, corresponding to the subintestinal and vitelline veins, and the ventral aorta. In each segment these longitudinal vessels are connected by loops, which are of two kinds, somatic and splanchnic. In the anterior part of the body only the splanchnic connecting loops are present, represented by the aortic arches connecting the dorsal and ventral aortae. Posterior to the heart each segment is provided with a somatic and a splanchnic loop, the former passing in the body wall from the aorta to the ventral abdominal vein, and the latter passing in the intestinal wall from the aorta to the subintestinal vein. These transverse loops pictured as continuous are in reality interrupted by capillaries in the body and intestinal walls. In vertebrates all of the connecting loops are lost except the aortic arches; but segmentally arranged transverse vessels corresponding to the loops are plainly evident in vertebrate embryos, and persist in certain regions of the adult, as in Figure 54.

For more extensive accounts of the comparative anatomy of the circulatory system, $\mathrm{K}$ or W should be consulted. 


\section{B. THE CIRCULATORY SYSTEM OF ELASMOBRANCHS}

The following account applies to both species of dogfish and to the skate.

I. The chambers of the heart.-The heart when first formed is a simple straight tube, but it soon becomes bent upon itself in the shape of the letter S, and its wall becomes differentiated into a number of chambers. The heart of the elasmobranchs is in this condition, consisting of four chambers. The pericardial cavity has already been exposed in the preceding dissection. Spread its walls apart. Identify the chambers of the heart as follows. On raising the heart a triangular chamber will be seen extending from the heart to the transverse septum, its two corners buried in the septum. This is the sinus venosus, the most posterior chamber of the heart. Each corner of the sinus venosus is continuous with a large vein, the duct of Cuvier or common cardinal vein, which is inclosed in the transverse septum and will be seen later. Anterior to the sinus venosus is the atrium or auricle, a large thin-walled chamber expanded on each side of the heart and appearing as if paired. Between the two sides of the auricle rests the ventricle, a thick-walled, heart-shaped chamber, the most conspicuous portion of the heart from ventral view. The pointed posterior end of the ventricle is known as the $a p e x$, the broad anterior end, the base. From the base of the ventricle a thick-walled tube runs forward and penetrates the anterior wall of the pericardial cavity. This is the conus arteriosus, tine fourth and most anterior chamber of the heart. The blood circulates through the chambers of the heart in the following order: sinus venosus, auricle, ventricle, conus arteriosus.

2. The systemic veins.-Systemic veins have already been defined as those veins which enter the heart. All systemic veins in vertebrates open into the sinus venosus or its equivalent, that is, they enter the phylogenetically posterior end of the heart. Owing to differences between them, the dogfishes and skate will be described separately.

In dissecting the veins, they are followed away from the heart, and it is often convenient to speak of them as if they proceeded from the heart to body structures. The student must, however, always bear in mind the fact that they convey the blood from the parts of the body to the heart.

Dogfish: Insert one blade of a fine scissors in the sinus wall and slit the ventral wall of the sinus venosus open in a crosswise direction. The cavity of the sinus is thus exposed and should be washed out thoroughly under a stream of running water. All of the systemic veins open into the cavity of the sinus and the openings may now be identified, with the cut edges of the sinus wall spread well apart. Each lateral wing of the sinus which lies buried in the transverse septum receives a very large opening, the entrance of the duct of Cuvier or common cardinal vein. The natural relations of this entrance are best observed on the intact right side. On the left side carry the slit in the sinus laterally to 
meet the incision previously made across the gill slits. The entrance of the common cardinal vein into the sinus is thus slit open. The following maythen be noted. In the spiny dogfish just medial to the main opening of the common cardinal vein into the sinus are several small apertures, most of which appear to be subdivisions of the chief opening. The most anterior of these small apertures is, however, the opening of the inferior jugular vein. In the smooth dogfish the accessory openings are lacking, and the entrance of the inferior jugular vein is situated just anterior to the opening of the common cardinal vein. Probe into the opening and note that the vein comes from the floor of the mouth and pharyngeal cavities where it runs alongside the ventral ends of the gill arches. By turning back the flap, previously formed, of the floor of the mouth and pharyngeal cavities, the course of the vein will be more readily followed. Now look into the posterior part of the wall of the sinus, putting this on a stretch. In the median line of the posterior wall will be noted a white fold, and on each side of this is an opening. Probe into both openings and note that your probe passes internal to the coronary ligament and into the liver. Fol'ow your probe into the liver by slitting the substance of the liver, and note the cavity in the right and left lobes of the liver thus revealed. These cavities which extend nearly the entire length of the liver lobes are the hepatic sinuses. ${ }^{x}$ The two hepatic sinuses are the persistent proximal parts of the vitelline veins of the embryo.

Now probe posteriorly into one of the common cardinal veins. Raise the viscera in the anterior part of the pleuroperitoneal cavity and observe that your probe has entered a large bluish sac located in the dorsolateral wall of the pleuroperitoneal cavity. Follow this sac and its fellow of the opposite side posteriorly. Each bends toward the median region, narrowing considerably, and on pressing the viscera to one side, each may be traced posteriorly as a narrow tube lying immediately to each side of the attachment of the dorsal mesentery to the median dorsal line. These two vessels are the posterior cardinal sinuses; they are the chief somatic veins of the trunk. At the level of the anterior part of the liver the two posterior cardinal sinuses communicate with each other by a broad connection which may be found by probing into the left vein and directing the probe toward the right one. In this same region each vein has on its ventral surface an extensive communication with a large blood sinus, the genital sinus, surrounding each gonad. Each posterior cardinal sinus also receives numerous segmentally arranged branches from the body wall (parietal veins) and from the kidneys (renal veins). The kidneys are the long, slender, flat organs, brownish in color, which lie immediately lateral to the posterior cardinal sinuses, extending the entire length of the pleuroperitoneal cavity. The parietal and renal veins are readily identifiable in those specimens in which they happen to be filled with blood, but are impossible to see when empty.

${ }^{1}$ Owing to the fact that the veins of elasmobranchs are not definite vessels but spaces in the tissues without definite walls, they are more correctly designated sinuses. 
Now turn the animal dorsal side up and locate the lateral line. Make a longitudinal incision above the gill slits on the left side along the lateral line in the spiny dogfish and about one-third of the way between the lateral line and the ends of the gill slits in the smooth dogfish. Deepen the incision until you break into a large cavity with a smooth lining. This cavity is the anterior cardinal sinus. Probe it anteriorly and follow your probe by an incision. The anterior cardinal sinus can thus be traced forward above the spiracle to the eye, where it connects with an orbital simus surrounding the eyeball. At the level of the posterior end of the eyeball is situated an opening in the ventral wall of the anterior cardinal sinus. On probing into this it will be found to extend medially into the skull. It is the opening of the interorbital sinus, which connects the two orbital sinuses. Locate the hyoid arch. In the floor of the anterior cardinal sinus, between the hyoid and third gill arches, is an opening. On probing this it will be found to lead into a vessel which extends ventrally along the outer surface of the hyoid arch. This vessel is the hyoidean sinus and it connects with the inferior jugular vein. Next trace the anterior cardinal sinus posteriorly. It turns abruptly ventrally and joins the posterior cardinal sinus. On probing into the anterior cardinal sinus at the turn the probe will be found to emerge into the posterior cardinal sinus. The union of the two sinuses forms the common cardinal vein already described.

Running along the lateral walls of the pleuroperitoneal cavity, immediately external to the pleuroperitoneum, on each side is a conspicuous vein, the lateral abdominal (often called simply lateral) vein. Note the parietal branches which it receives segmentally from between the myotomes. Trace the right vein anteriorly and find where it enters the common cardinal vein just in front of the baglike expansion of the posterior cardinal sinus. Slit open the lateral vein at its point of entrance into the common cardinal vein and find at the same place immediately posterior to the pectoral girdle the opening of the subclavian vein which drains the pectoral fin. The subclavian vein passes along the posterior surface of the pectoral girdle in contact with the cartilage, and may be picked up easily on the left side where the girdle has been cut across. The vein may also be found by cutting across the base of the fin; it will then be seen in the section of the fin as an opening dorsal to the fin rays in the posterior half of the fin. Next trace the lateral vein posteriorly. It passes internal to the inner surface of the pelvic girdle. In the spiny dogfish it then continues posteriorly along the latera] margin of the cloacal aperture as the cloacal vein. At about the middle of the base of the pelvic fin the lateral vein receives an iliac vein from the fin. The opening of the iliac vein into the lateral vein will be found by slitting open the latter vein. The iliac vein is a short vessel situated just under the skin of the dorsal surface of the fin. In the smooth dogfish the two lateral veins connect with each other by a cross vein which runs along the internal surface of the pelvic girdle. Beyond this connection each vein continues as the cloacal vein along the 
lateral margin of the cloacal aperture, and near the anterior part of the base of the pelvic fin receives the iliac vein from the fin. Trace this according to the directions for the spiny species.

In the median ventral line are traces of a ventral cutaneous vein which seems, however, to dwindle away anteriorly.

Draw the systemic veins.

Skate: The sinus venosus consists of a tube on each side, the central portion being reduced in size and attached to the transverse septum by a sheet of connective tissue which may be broken. Each side of the sinus is buried in the transverse septum. Follow out the right side to the point where it disappears dorsal to the cartilage of the pectoral girdle. Carefully shave away the cartilage and surrounding tissues until the sinus venosus can be followed laterally. Insert one blade of a fine scissors in the ventral wall of the sinus and slit it open in a crosswise direction. The sinus is seen to be continuous on each side with a tube or chamber, the common cardinal vein or duct of Cuvier, which turns dorsally. All of the systemic veins open into the common cardinal vein, and their openings may now be identified. The junction of common cardinal vein with the sinus venosus is marked by a slight fold. In the anterior wall of the common cardinal vein concealed by this fold is the small opening of the inferior jugular vein. This opening is so small that the probe can probably not be passed into it. The inferior jugular vein drains the walls of the pericardial cavity and the floor of the mouth and pharyngeal cavities. In the posterior wall of the common cardinal vein, at its junction with the sinus venosus, is the opening of the hepatic sinus. Probe posteriorly into this. It leads into the hepatic sinus, a large space situated between the anterior margin of the liver and the transverse septum and inclosed by the coronary ligament. In females the hepatic sinus lies dorsal to the mouth of the oviduct which is inclosed in the falciform ligament. In locating the hepatic sinus press the liver caudad away from the transverse septum; the sinus forms a bag between liver and septum ventral to the esophagus. Cut into the hepatic sinus. A tube, the pericardio-peritoneal canal, extends through the center of its cavity. Look in the posterior wall of the sinus for the small openings of the hepatic veins which drain the liver. The sinus opens on each side into the common cardinal vein.

Now probe into the main cavity of the common cardinal vein in a dorsal and posterior direction. The probe enters the posterior cardinal sinus which is a broad, thin-walled tube lying against the dorsal wall of the pleuroperitoneal cavity. In females it is on the dorsal side of the oviduct; in males, dorsal to the testis. Follow the posteror cardinal sinus posteriorly. It swerves toward the median line where it soon meets its fellow of the opposite side to form a single large median sinus. This sinus communicates on its ventral surface with the large genital sinus surrounding each gonad. More posteriorly the posterior cardinal sinus separates again into two veins which proceed caudad on the medial 
side of the kidneys. The kidneys are rounded lobes at the posterior end of the pleuroperitoneal cavity against the dorsal wall. To see them, remove the pleuroperitoneum from the dorsal wall on each side of the cloaca. Do not injure the ducts from the kidneys. After exposure of the kidneys the posterior cardinal veins will be found on the medial side of the kidneys. They connect with each other between the kidneys.

Return to the common cardinal vein and probe into it in a dorsal direction. Turn the animal dorsal side up and locate the end of your probe. Make an incision into the spot indicated by the probe and extend the incision longitudinally forward to the eye. On carefully deepening the incision an elongated cavity with smooth walls, the anterior cardinal sinus, is exposed. It is situated just medial to the dorsal ends of the gill arches and visceral pouches. It may be followed forward with the aid of the probe. It turns laterally in front of the first visceral pouch, follows along the anterior border of this pouch, and then turns anteriorly again.

With the dorsal side of the animal still facing you, locate by feeling the chief anterior cartilage (propterygium) of the pectoral fin. It forms a crescentic ridge lateral to the gill region nearly halfway from the mid-dorsal line to the margin. Make an incision along the medial face of this cartilage on the left side. A vein will be exposed running along the cartilage here. It is one of the brachial veins. Follow it posteriorly. It will be found to enter the common cardinal vein.

Turn the animal ventral side up again. Along the lateral wall of the pleuroperitoneal cavity runs the lateral abdominal vein. Note the parietal branches which it receives from the body wall at each myoseptum. Trace the lateral abdominal vein anteriorly. It passes along the internal surface of the cartilages of the pectoral fin and pectoral girdle, and enters the common cardinal vein. Cut into the vein where it passes the cartilages. Immediately on the posterior side of the cartilage of the pectoral girdle a brachial vein will be found entering the lateral vein. Immediately posterior to this is another cartilage, and on the caudal side of that another brachial vein joins the lateral vein. A third brachial vein was mentioned in the preceding paragraph. Trace the lateral vein posteriorly. It originates in a network of small vessels on the sides of the large intestine and cloaca. It passes on the inner surface of the cartilages of the pelvic girdle and pelvic fin. Slit the vein open along the surface of the cartilages. Iliac veins will be found emerging from between the cartilages and entering the lateral vein. The largest of the iliac veins is located along the posterior side of the cartilage of the pelvic girdle. Probe into the iliac veins and note their distribution in the pelvic fin.

Draw the systemic veins.

3. The hepatic portal system.-A portal system is a system of veins which tlows into a network of rapillaries in some organ. The hepatic portal system 
consists of veins which collect the venous blood from the digestive tract and spleen, and pour it into a network of capillaries in the liver. Locate the bile duct. Lying in the hepatoduodenal ligament alongside the bile duct is a large vein, the hepatic portal vein. Trace it posteriorly and identify the branches which it receives from the digestive tract. ${ }^{x} \quad$ These branches differ slightly in the three forms under consideration.

Spiny dogfish: On tracing the hepatic portal vein posteriorly, it will be found to receive first a very small branch, which may be called the duodenal branch, which runs along the bile duct and collects from the duodenum and anterior portion of the spiral valve. Posterior to this the hepatic portal vein is seen to be formed by the union of three large branches. The left branch, the gastric vein, passes at once to the stomach, where it is formed by the union of the dorsal and ventral gastric veins, which branch on the dorsal and ventral surfaces of the stomach. The middle of the three main branches of the hepatic portal is the lienomesenteric vein. It passes posteriorly dorsal to the duodenum and is imbedded in the substance of the dorsal lobe of the pancreas, from which it receives branches. At the posterior end of the pancreas the vein is seen to be formed by the union of two branches: one, the posterior splenic vein, from the spleen; and the other the posterior intestinal vein, which comes from the left side of the small intestine. Note its numerous branches from the intestinal wall along the lines of attachment of the turns of the spiral valve. The right branch of the three that form the hepatic portal vein is the pancreatico-mesenteric. It passes dorsal to the pylorus and is imbedded in the substance of the ventral lobe of the pancreas. Here it receives the anterior splenic vein from the anterior part of the spleen and several veins from the pancreas and duodenum. Its main trunk, the anterior mesenteric vein, is situated along the right side of the intestine from which it receives branches in the same manner as the posterior intestinal vein.

Smooth dogfish: The branches are similar to those of the spiny species. The hepatic portal first receives the very small duodenal branch from along the bile duct and shortly beyond this point is formed by the union of three large veins: the gastric vein on the left, the lienomesenteric in the middle, and the pancreaticomesenteric to the right. The gastric vein passes toward the stomach and receives an anterior ventral gastric vein. It is situated in the mesentery between the two limbs of the stomach and in its course receives branches from both limbs. The lienomesenteric vein passes dorsal to the pylorus lying imbedded in the dorsal lobe of the pancreas. At the anterior margin of this lobe of the pancreas it receives the anterior dorsal gastric vein from the corresponding part of the stomach. It receives numerous pancreatic veins from the pancreas. At the

x These branches are generally filled with blood and therefore easily traced. If they are empty, they may be readily injected through the hepatic portal vein, even in specimens which have been oreserved for a long time. 
posterior margin of the pancreas it is found to be formed by the union of two veins, a left-hand lienogastric vein and a right-hand posterior mesenteric vein. The lienogastric vein has a posterior dorsal gastric vein from the stomach and the posterior splenic vein from the posterior end of the spleen, and adjacent walls of the stomach. The posterior mesenteric vein runs along the left side of the small intestine, where it receives branches on both sides along the lines of insertion of the turns of the spiral valve. The pancreatico-mesenteric vein passes dorsal to the pylorus, receiving pancreatic veins from both lobes of the pancreas and the anterior splenic vein from the anterior part of the spleen and adjacent stomach wall. At about the region of the pylorus the vein is formed by the union of two large branches from the intestinal wall. One of these, the intraintestinal vein, is a short branch from the anterior part of the spiral valve. The other branch, the anterior mesenteric, lies along the right side of the intestine corresponding in position and branches to the posterior mesenteric.

Skate: The hepatic portal vein is soon seen to be formed by the union of three tributaries: a gastric vein from the left, a lienomesenteric vein from the middle, and a pancreatico-mesenteric from the right. Follow each of these. The gastric vein passes to the right margin of the stomach and there receives the dorsal and ventral gastric veins from the dorsal and ventral surfaces of the stomach. The dorsal gastric vein receives tributaries from the spleen. The lienomesenteric vein receives a splenic branch from the spleen. Its main tributary, the posterior mesenteric vein, runs along the left side of the intestine beginning in the tip of the rectal gland; in its course along the intestine it receives branches along the lines of attachment of the spiral valve. It also collects from the pancreas. The pancreatico-mesenteric vein collects from the pancreas and, as the anterior mesenteric vein, from the duodenal region. It also receives a posterior gastric vein from the narrow portion of the stomach between the pylorus and the bend.

Trace the hepatic portal vein anteriorly in all three forms. It reaches the dorsal surface of the liver and here divides into branches which penetrate the substance of the liver. In the liver the branches fork into smaller and smaller veins and finally pass into capillaries. From these capillaries originate other veins which empty into the hepatic sinuses which as already seen open in the sinus venosus (dogfish) or common cardinal vein (skate). As already explained (see Fig. 55, p. 205) the hepatic sinuses are the persistent proximal portions of the vitelline veins of the embryo, while the hepatic portal vein and its branches develop from one of the vitelline veins and the subintestinal vein of the embryo.

Draw an outline of the digestive tract and place on this outline the hepatic portal vein and its tributaries from the various parts of the digestive tract.

4. The renal portal system.-In the renal portal system the venous blood passes into a network of capillaries in the kidneys. Cut across the tail just posterior to the anal opening. In the cross-section locate the caudal blood 
vessels, inclosed in the haemal arch. The caudal artery is dorsal, the caudal vein immediately ventral to the artery.

Dogfish: Probe into the caudal vein. Observe that the probe can be passed either to the right or left, showing that the vein forks at the anus. The two forks are the renal portal veins. Leaving your probe in one of the renal portal veins, locate the kidney in the pleuroperitoneal cavity. It is a long brown organ situated against the dorsal body wall, one on each side of the mid-dorsal line external to the pleuroperitoneum. Slit the pleuroperitoneum along the lateral border near the posterior end of the kidney on the side where your probe is inserted and gently lift the kidney away from the body wall. A space will be found between the kidney and the body wall; into this space your probe has passed. This space is the renal portal vein or sinus. It branches into the kidney and also receives tributaries from the body wall.

Skate: The kidney has already been exposed. Look along the medial side of the posterior part of the kidney for a vein coming from the vertebral column. Do not injure any ducts on the ventral surface of the kidney. In males the vein in question lies immediately to the dorsal side of the male duct, which will be seen passing along the ventral surface of the kidney to the cloaca. The duct may be lifted from the kidney surface and bent to one side. The vein in question is the renal portal vein. At first it lies along the medial border of the kidney, but soon turns onto the ventral surface of that organ, giving off branches into its substance and receiving tributaries from the body wall lateral to the kidney. The renal portal veins are continuations of the caudal vein; the latter forks at the anus giving rise to the two renal portal veins. The forking is, however, difficult to trace in the skate.

Reference to Figure 55, page 205, will show that the renal portal veins are the posterior parts of the posterior cardinal veins and that the apparent posterior parts of the posterior cardinal veins of the adult are in reality the subcardinal veins. Whereas in the embryo the blood flows from the subcardinals into the posterior cardinals, in the adult the direction of flow is reversed. The renal portal system provides that the blood from the tail must pass into a capillary system in the kidneys from which the blood is re-collected into the subcardinal veins. The purpose of this arrangement is obscure; it seems to have been disadvantageous, for the vertebrates later shunted part of this blood into another system and finally abandoned the renal portal system altogether.

Draw the renal portal system.

5. The ventral aorta and the afferent branchial vessels.-Turn once more to the pericardial cavity of the specimen. The conus arteriosus runs forward and penetrates the anterior wall of the pericardial cavity. Carefully pick away muscles and connective tissue from the region extending from the anterior end of the pericardial cavity to the lower jaw. In the median ventral line will be 
revealed a large vessel, the ventral aorta, which continues torward from the conus arteriosus. By dissecting carefully to the left side find the branches of the ventral aorta. They are as follows:

Dogfishes: There are three main pairs of branches, two of which subdivide into two. The most posterior pair of branches arises just at the point where the conus arteriosus passes into the ventral aorta. (At this point note the coronary artery, paired in the spiny dogfish, single in the smooth species, passing along the conus arteriosus onto the surface of the ventricle and to the walls of the pericardial cavity. This artery should be preserved as far as possible.) Follow the most posterior branch of the ventral aorta. It very shortly divides in two, the posterior branch penetrating the interbranchial septum of the sixth visceral arch, the anterior branch the septum of the fifth. The middle brarch of the ventral aorta arises shortly in front of the third branch and passes without division into the interbranchial septum of the fourth visceral arch. After giving off this branch the ventral aorta proceeds forward without branching to a point just posterior to the lower jaw. Here it forks to form its anterior pair of branches. Trace the left branch laterally. After some distance it forks, supplying the second and third visceral arches. Trace any one of the branches of the ventral aorta out into the interbranchial septum, slitting the septum. Note the small branches from the artery into the gill filaments. The five pairs of branches of the ventral aorta are named the afferent branchial arteries. How many gills does each supply?

Skate: There are two main pairs of branches from the ventral aorta. The posterior pair arises where the conus arterious passes into the ventral aorta. (At this point note the coronary artery, paired, passing to the conus arteriosus and to the visceral muscles. Preserve it as well as possible.) Follow out the posterior branch of the ventral aorta. After some distance it subdivides into three branches which pass to the fourth, fifth, and sixth visceral arches, penetrating the interbranchial septa. Trace them into the septum by slitting the septum, and note the branches from each to the gill filaments of both demibranchs of the septum. Follow the ventral aorta forward beyond the pair of posterior branches. It passes without branching for some distance and then forks into right and left branches. Follow the left branch. After a considerable distance it forks into two vessels, which penetrate the interbranchial septa of the second and third visceral arches. The five pairs of branches of the ventral aorta are named the afferent branchial arteries. How many demibranchs does each supply?

In front of the anterior fork of the ventral aorta will be found some soft brownish diffuse material, composing the thyroid gland.

The afferent branchial arteries are the ventral halves of the aortic arches which were previously described as present in the embryo. We noted that there are six such arches in vertebrate embryos. Since the adult elasmobranch 
has but five pairs, one pair must be missing. The missing pair is the first, which should supply the first visceral arch; it has disappeared during development, probably because this arch bears no gills.

Draw the ventral aorta and the afferent branchial arteries showing their relation to the visceral arches.

6. The efferent branchial arteries and the dorsal aorta.-Open the flap already formed of the floor of the mouth and pharyngeal cavities; turn it outward and fasten it in that position. The esophagus may be cut to facilitate this procedure. With a forceps strip off the mucous membrane from the roof of the mouth and pharyngeal cavities. In the roof there will now be seen four pairs (dogfishes) or three pairs (skate) of large blood vessels extending from the angles of the gill slits obliquely caudad. They are the efferent branchial arteries and represent the dorsal halves of the aortic arches. Clean away the connective tissue from these arteries so that they are clearly exposed. Trace them to the left and note that they disappear dorsal to the cartilages of the gill arches. Remove these cartilages carefully. This is best done by cutting across them in the visceral arch, grasping the cut end, and loosening the cartilage toward the median dorsal line. After the cartilages are removed trace each of the efferent branchial arteries toward the gills. Note that each is formed at the dorsal angle of the gill slit by the union of two vessels, a smaller pretrematic branch which comes from the demibranch on the anterior face of the visceral pouch and a much larger post-trematic branch which comes from the demibranch on the posterior wall of the visceral pouch. Note the small vessel which runs from each gill filament into the pre- and post-trematic branches. In the skate the first two efferent branchial arteries unite to one so that there are but three pairs of main vessels in the roof of the pharyngeal cavity.

Next, dissect on the right side, which has been kept intact, in order to see the full course of the efferent branchial arteries. Remove the mucous membrane from the floor of the mouth and pharyngeal cavities, thus exposing the ventral portions of the gill arches. Remove these cartilages carefully without disturbing any of the arteries, and also remove the cartilage from the full length of the visceral arches. It will now be seen that the pre- and post-trematic branches are united at their ventral ends so that they form a complete loop around each gill cleft. Note further that the post-trematic branch on the anterior wall of each visceral arch is connected with the pretrematic branch on the posterior face of the same arch by means of cross-branches, about three in number in the spiny dogfish, one in the smooth dogfish and skate. Thus each efferent branchial artery collects from several demibranchs.

From the ventral ends of some of the loops formed by the pre- and posttrematic branches vessels arise on each side and proceed posteriorly in the floor of the pharyngeal cavity, ventral to the cartilages of the gill arches, to supply the pericardial cavity, wall of the heart, and visceral muscles. These vessels 
constitute the coronary arteries. In the spiny dogfish, the coronary artery arises on each side from the ventral end of the loop around the third gill cleft (counting the spiracle as the first). It gives off a branch anteriorly and then proceeds posteriorly to the anterior end of the pericardial chamber. Here it forks, giving one branch to the conus arteriosus and other chambers of the heart and the other to the walls of the pericardial cavity. The latter branch after a short distance again passes to the floor of the pharyngeal cavity and is distributed to the esophagus. In the smooth dogfish the coronary artery is formed by paired branches from the ventral ends of the loops around the third and fourth gill clefts. These branches pass to the median line and join to form a single vessel which penetrates the pericardial cavity and runs along the ventral surface of the conus arteriosus where it branches to the conus and ventricle. From the branches arising from the ends of the loops of the fourth gill clefts a vessel runs posteriorly on each side in the dorsal wall of the pericardial cavity and into the walls of the esophagus. In the skate the disposition of the coronary arteries is somewhat irregular. Vessels arise from or near the ventral ends of the loops around the fourth and fifth gill slits. The former passes forward along the floor of the mouth cavity; the latter gives rise to the anterior coronary arteries which run along the conus arteriosus to the heart walls. In the posterior part of the pericardial cavity are the posterior coronary arteries, running along the sinus venosus. These originate from the subclavian artery which will be identified later.

Turn again to the roof of the mouth. From the dorsal end of the loop around the second gill slit a vessel runs forward. It is the common carotid artery. After a short distance it bends toward the median line. At this bend it gives off a branch, the external carotid artery, which will be found by gently shaving away the cartilage at this point. Beyond this branch the main artery, now known as the internal carotid artery, passes to the median line where it joins its fellow of the opposite side; the vessel thus formed penetrates the cartilage of the skull. In the dogfishes slender vessels connect the first efferent branchial arteries with the common carotid artery; in the spiny dogfish these connecting branches are paired all of the way, while in the smooth species the vessel arises unpaired from the median line and subsequently forks.

Clear away all tissue from the pretrematic branch of the first efferent branchial artery. From about the middle of the pretrematic branch a vessel arises and passing forward very soon turns sharply dorsally and disappears. This is the hyoidean artery. Turn the animal dorsal side up and remove the skin around and posterior to the spiracle. Pick up the hyoidean artery again below the spiracle. It runs on the inner side of a white band (hyomandibular nerve) which is located just posterior to the spiracle. Follow the hyoidean artery to the walls of the spiracle and note its branches to the rudimentary gill in the spiracle. (In the skate there are numerous branches to adjacent muscles 
and only very small branches to the walls of the spiracle.) Now turn the animal ventral side up, remove the mucous membrane between the spiracle and the upper jaw, and shave away the cartilage about halfway between the upper jaw and the common carotid artery An artery of moderate size will be revealed. Trace it toward the spiracle and note that it is the continuation of the hyoidean artery already seen, formed by the reunion of the branches in the spiracular gill. It passes into the skull ventral to the external carotid artery, and is then known as the ventral carotid artery. On tracing it into the skull by scraping away the cartilage, the ventral carotid artery will be found to join the internal carotid.

The efferent branchial arteries pass to the median dorsal line of the roof of the pharyngeal cavity. In the skate the first and second join to one, and shortly posterior to this junction a vertebral artery arises on each side and passes into the cartilage of the skull, where it is distributed to the brain and spinal cord. The efferent branchial arteries join in pairs in the median dorsal line and form a large trunk, the dorsal aorta, which passes into the pleuroperitoneal cavity.

Draw, showing efferent branchial arteries, their branches, and their distribution to the gills.

We are now in a position to compare the head arteries of the elasmobranch with the primitive plan explained at the beginning of this section. The afferent branchial arteries are the ventral halves, the efferent branchial arteries the dorsal halves of the aortic arches. These arches connect the ventral aorta springing from the heart with the dorsal aorta, and run in the walls of the pharynx, one to each visceral arch. In elasmobranch and other fishes the central part of each aortic arch breaks up into a number of smaller vessels and capillaries running in the gills. Theoretically and in vertebrate embryos there are six aortic arches. In elasmobranchs there are five ventrally and four dorsally. The first is lacking on the ventral side and also absent on the dorsal side; but the second aortic arch is imperfectly represented by the hyoidean artery which supplies the spiracle. The four efferent branchial arteries present in their full development in elasmobranchs are, then, the third, the fourth, fifth, and sixth aortic arches. It should be noted that the carotid artery springs from the third aortic arch, a condition universal among vertebrates. The first aortic arch is missing in all adult vertebrates (except cyclostomes), and above fishes the second has also vanished.

7. The dorsal aorta and its branches.-Separate the esophagus from the body wall on the left side and follow the dorsal aorta posteriorly. From the dorsal aorta between the points where the third and fourth pairs of efferent branchial arteries unite with it a subclavian artery is given off on each side. Trace the left one into the pectoral fin. It proceeds obliquely caudad and laterad passing on the dorsal wall of the large bag formed by the posterior cardinal sinus. (In the skate it gives off the posterior coronary artery and passes internal to a large white band, the nerve of the pectoral fin. This may be cut through.) At the lateral boundary of the posterior cardinal sinus, the subclavian artery gives rise to the small lateral artery which branches into the body wall and usually proceeds posteriorly along the body wall in a position on a level with the lateral line. Farther 
laterally at the point where the lateral vein enters the common cardinal vein, the subclavian artery gives rise to the ventral abdominal artery which proceeds posteriorly halfway between the lateral vein and the midventral line. This artery is somewhat irregular in the smooth dogfish but conspicuous in the spiny dogfish and skate. It gives off branches segmentally into the body wall and at the posterior end of the pleuroperitoneal cavity anastomoses with the vessels supplying the pelvic fins. After giving off these branches into the body wall the subclavian artery, now named the brachial artery, proceeds into the pectoral fin.

The dorsal aorta is a very large vessel situated in the mid-dorsal line of the pleuroperitoneal cavity. It has median unpaired visceral or splanchnic branches to the viscera, lateral visceral branches to the urogenital system, and somatic branches to the body wall. The median visceral branches are as follows. Work on the left side turning the viscera to the right.

Spiny dogfish: Just after it has penetrated the pleuroperitoneal cavity, the dorsal aorta gives rise to the large coeliac artery which distributes blood to the gonads, stomach, and liver. Near its origin the coeliac artery gives off small branches into the adjacent gonads, esophagus, and cardiac end of the stomach. It then runs posteriorly for a considerable distance without branching; it enters the gastrohepatic ligament and gives rise to three branches: the gastric, the hepatic, and the pancreatico-mesenteric artery. The gastric artery passes to the stomach and divides into dorsal and ventral gastric arteries which branch on the surface of the stomach and penetrate its walls. The hepatic artery turns anteriorly, runs alongside the bile duct, and enters the substance of the liver. The pancreatico-mesenteric artery passes dorsal to the pylorus, gives off small branches into the pyloric portion of the stomach and the ventral lobe of the pancreas, a moderately large duodenal artery into the duodenum, and a large anterior mesenteric artery along the right side of the small intestine to which it gives off branches at the rings of attachment of the spiral valve. The dorsal aorta after giving rise to the coeliac artery runs without further visceral branches to the free edge of the dorsal mesentery. Here it gives off two arteries which course in the border of the mesentery. One of these, the gastrosplenic artery, passes to the spleen and bend of the stomach. The other vessel is the superior mesenteric artery. It passes to the small intestine and, as the posterior mesenteric artery, runs posteriorly on the left side of the intestine with branches corresponding to those of the anterior mesenteric. Beyond the gap in the dorsal mesentery, the dorsal aorta gives off the inferior mesenteric artery which passes along the free anterior border of the mesorectum into the rectal gland.

Smooth dogfish: Shortly after entering the pleuroperitoneal cavity, the dorsal aorta gives rise to the large coeliac artery. This has small branches into the adjacent gonads and soon divides into gastric and pancreatico-mesenteric arteries. The gastric artery immediately forks into a smaller anterior gastric 
artery which passes to the walls of the anterior part of the stomach and a posterior gastric artery which runs posteriorly in the gastruhepatic ligament where it branches to both limbs of the stomach. The posterior gastric artery near its origin from the coeliac sends about two hepatic arteries to the liver. The pancreatico-mesenteric artery sends pancreatic branches into the pancreas, a large duodenal branch into the duodenum, a posterior gastrosplenic artery which runs posteriorly along the narrowed part of the spleen and adjacent stomach wall, and a large anterior mesenteric artery which passes along the right side of the small intestine and branches at the lines of attachment of the spiral valve. Shortly posterior to the point of origin of the coeliac artery two arteries arise from the dorsal aorta. They are the gastrosplenic and the superior mesenteric. The gastrosplenic proceeds to the thick portion of the spleen and the adjacent stomach wall. The superior mesenteric supplies branches to the gonads and then, as the posterior mesenteric artery, passes along the left side of the small intestine, branching at the lines of attachment of the coils of the spiral valve. The dorsal aorta proceeds unbranched for some distance and then gives off the inferior mesenteric artery. This passes into the adjacent gonads to which it supplies some branches and then emerging from the gonad proceeds to the rectal gland where it forms a network of branches.

Skate: Shortly after entering the pleuroperitoneal cavity, the dorsal aorta gives off the coeliac artery, which supplies a number of organs. It has: a hepatic branch to the liver; an anterior gastric branch which divides into dorsal and ventral gastric arteries to the stomach wall; splenic branches to the spleen; and a gastroduodenal branch, from which arise a posternor gastric artery to the posterior part of the stomach, pancreatic branches to the pancreas, and a duodenal branch to the pylorus and duodenum. Shortly posterior to the origin of the coeliac artery, the dorsal aorta gives rise to the superior mesenteric artery, which after some small branches to the pancreas and spleen proceeds posteriorly along the small intestine to which it supplies branches at the lines of attachment of the turns of the spiral valve. Shortly caudad of the origin of the superior mesenteric artery, the inferior mesenteric artery branches from the dorsal aorta. It sends genital arteries to the gonads and their ducts and then passes in the mesentery to the rectal gland.

The lateral visceral and somatic branches of the dorsal aorta are similar in the three forms under consideration. The former consist of the genital arteries already noted (but completely developed only in mature specimens) and the renal arteries into the kidneys. The latter are seen by loosening the kidney from the dorsal body wall and looking on the dorsal surface of the organ. The somatic branches consist of paired parietal arteries to the body wall, passing out along the myosepta. The arteries to the paired fins are merely enlarged parietal vessels. The subclavian to the pectoral fin was already seen. The paired iliac arteries to the pelvic fins arise from the dorsal aorta shortly in front of 
the cloaca. They course along the body wall, resembling the parietal arteries, and after giving off a network of branches into the walls of the cloaca and anastomising anteriorly with the posterior end of the ventral abdominal artery, they enter the pelvic fins. The dorsal aorta continues into the tail as the caudal artery which is situated in the haemal canal immediately ventral to the centra of the vertebrae.

Draw the dorsal aorta and its branches.

8. The structure of the heart.-The heart of elasmobranchs is a tube bent into an S-shape and differentiated into four chambers. These chambers have already been named. They were originally arranged in a straight line, but the bending of the heart tube brings the ventricle in contact with the sinus venosus and the auricle in contact with the conus arteriosus. The sinus venosus has already been examined. It is a thin-walled chamber, triangular in form in the dogfishes, tubular in the skate. Cut across the connections of the sinus venosus with the transverse septum and also across the base of the ventral aorta, and remove the heart from the body. Look into the previously opened sinus venosus and find the large sin-auricular aperture which leads into the auricle. It is guarded by a pair of valves formed of the smooth free edges of the sinus wall. Note the shape of the auricle. It is a broad thin-walled chamber with large lateral expansions on each side of the ventricle. Slit open the auricle and wash out the contained blood clots. Note the folds in its wall. Find the auriculo-ventricular opening into the ventricle. It is guarded by two valves. Each of these is a pocket of thin tissue, the opening of the pocket being directed into the ventricle. Cut off the ventral half of the ventricle and also slit open the conus arteriosus by a longitudinal ventral incision. Note the small U-shaped cavity of the ventricle and its very thick, spongy walls forming numerous cavities and crevices in which the blood is held. Examine the auriculo-ventricular valve from the ventricular side, and note the two pockets of which it is composed and the attachment of the pockets to the ventricular wall. On the wall of the conus arteriosus note the pocket-shaped semilunar valves, the pockets opening anteriorly. In the spiny dogfish there are nine valves in three circles of three each; two circles are near the posterior end of the conus, while the third circle composed of larger and stronger valves is near the junction of the conus with the ventral aorta. In the smooth dogfish there are two circles of valves of three each, situated at the posterior end of the conus. The conus arteriosus of the skate bears three longitudinal rows of valves with five valves in each row. To distinguish the valves, run the point of a probe along the conus wall from the anterior end backward, thus opening the pockets.

Make a drawing to show the structure of the heart.

The heart of elasmobranchs contains only venous blood. This enters the sinus venosus from the systemic veins and passes in turn through the auricle, ventricle, and conus arteriosus, and out into the ventral aorta which distributes the blood to the gills by way of the afferent 
branchial arteries. On passing through the gill filaments the blood is aerated and becomes arterial blood. It is then collected by the efferent branchial arteries and passed into the dorsal aorta which distributes it to all parts of the body. The venous blood is returned to the heart by means of the systemic veins and the two portal systems. There is thus in the elasmobranchs (and all fishes) a single circulation through the heart. Since the heart contrins only venous blood, the heart must obtain arterial blood from some outside source. This is accomplished by means of the coronary artery which arises from the efferent branchial vessels and returns to the heart. As we shall see the coronary artery originates in a different manner in fish than in other vertebrates.

9. The pericardio-peritoneal canals.-Inspect the posterior wall of the pericardial cavity after the removal of the heart. In the dogfishes, a large opening will be found in the posterior wall dorsal to the previous attachment of the sinus venosus. On probing into it, it will be found to lead into a canal, the pericardio-peritoneal canal, situated along the ventral wall of the esophagus internal to the visceral peritoneum of the esophagus. Eventually the canal opens into the pleuroperitoneal cavity by a small slit. In the skate there is an opening of moderate size in the center of the posterior wall of the sinus venosus. On probing into this it will be found to lead into a canal, the pericardio-peritoneal canal, which passes through the center of the hepatic sinus. On probing into this it will be found to fork into two canals lying on the ventral wall of the esophagus internal to its serosa. They open into the pleuroperitoneal cavity by minute slits. The pericardio-peritoneal canals serve to connect the pericardial and pleuroperitoneal cavities, and arise through the failure of the transverse septum to close completely across the coelom.

\section{THE CIRCULATORY SYSTEM OF NECTURUS}

I. The chambers of the heart.-The pericardial cavity has already been exposed; if a new specimen is provided, the pericardial and pleuroperitoneal cavities are to be opened as before. The parts of the heart visible in ventral view are the ventricle, the auricles, and the conus arteriosus. The ventricle is thick walled and conical in form. Anterior to the ventricle on either side is a thin-walled auricle. Springing from the base of the ventricle and passing forward between the two auricles is the tubular conus arteriosus. Anteriorly the conus passes into the enlarged beginning of the ventral aorta; this muscular expansion of the base of the ventral aorta is named the bulbus arteriosus. Lift the apex of the ventricle and note the sinus venosus situated dorsad to the ventricle. The sinus venosus receives two large venous channels lying in the dorsal wall of the pericardial cavity. These are formed by the union of the two hepatic sinuses with the two common cardinal veins. The hepatic sinuses are the two large veins which emerge from the transverse septum and pass into the sinus. The common cardinal veins join the hepatic sinuses on their lateral surfaces. 
2. The hepatic portal system and the ventral abdominal vein.- In the median ventral line of the body wall, posterior to the liver, inclosed in the falciform ligament is situated the ventral abdominal vein. It is homologous with the lateral abdominal veins of the elasmobranchs. It receives parietal branches from the body wall. At the level of the posterior end of the liver it leaves the body wall and passes into the liver where it is situated on the dorsal surface. After a short course it joins the hepatic portal vein at the place of attachment of the hepatoduodenal ligament.

The hepatic portal vein is the vein which collects the blood from the digestive tract. It is formed by the union of branches from the intestine, pancreas, spleen, and stomach. Stretch out the dorsal mesentery of the small intestine. In this mesentery about halfway between the body wall and the intestine runs a conspicuous vein, the mesenteric vein. Trace it posteriorly and note its beginning in the wall of the large intestine. As it passes forward in the mesentery it receives numerous intestinal veins from the small intestine. It then passes into the substance of the pancreas receiving small pancreatic veins from that organ. At the level of the pancreas the large gastrosplenic vein also joins the mesenteric from the left. On following the gastrosplenic to the spleen it will be found to be formed of splenic branches from the spleen and gastric veins from the stomach. The union of the mesenteric, gastrosplenic, and pancreatic veins produces a large vessel, the hepatic portal vein, which lies along the center of the dorsal face of the liver. It also receives the ventral abdominal vein as already noted. Follow it along the surface of the liver. It branches into the liver substance and in its course also receives additional gastric veins from the stomach and also veins from the ventral body wall which pass into the liver by way of the falciform ligament. (These have probably been destroyed in the study of the digestive tract.) The hepatic portal vein subdivides in the substance of the liver and eventually passes into capillaries.

Draw the hepatic portal system.

3. The renal portal system.-Trace the ventral abdominal vein posteriorly. It soon receives some vesical veins from the bladder. Shortly anterior to the hind limbs the abdominal vein is seen to be formed by the union of the two pelvic veins which run along the inner surface of the lateral body wall just in front of the pelvic girdle. Follow one of the pelvic veins. It is joined by the femoral vein from the hind limb. The vein formed by this union is the renal portal vein. It passes at once to the dorsal surface of the adjacent kidney. In male specimens the kidney is a brownish organ of considerable size situated at the side of the intestine. In female specimens the kidney is much smaller and more slender, and is situated at the common point of attachment of the mesovarium and mesotubarium. It will be located by laying the ovary to one side and the oviduct to the other side. At the posterior end of the pleuroperitoneal cavity the kidney in the female lies between the intestine and the oviduct. Having 
located the kidney, identify the renal portal vein near the lateral margin of its dorsal surface. At the place where the renal portal vein passes from the body wall to the surface of the kidney, the vein receives the caudal vein, which ascends from the tail, forks, and passes to the surface of the posterior end of each kidney.
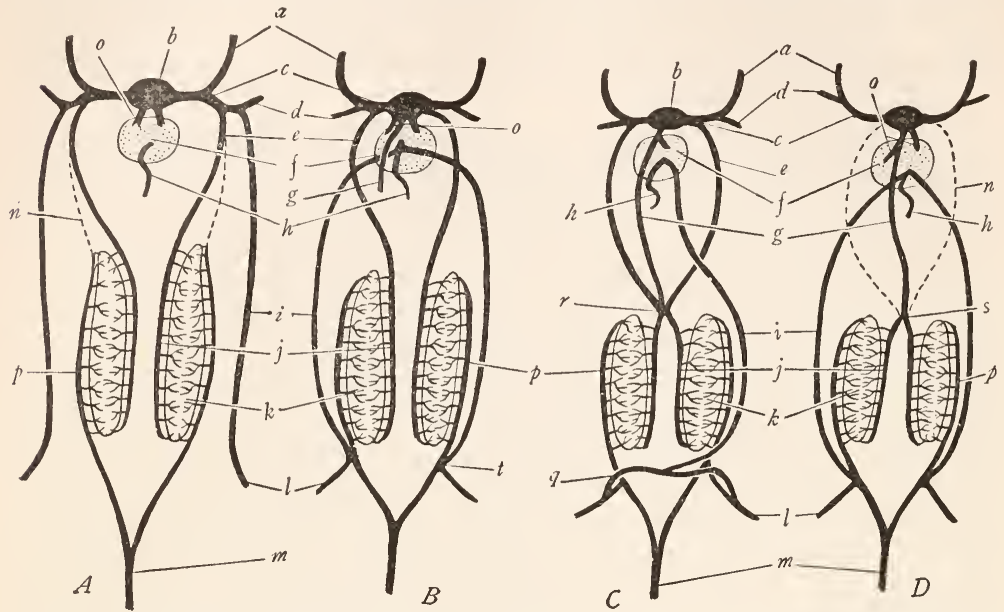

Fig. 57.-Diagrams to show the origin of the postcaval vein and the changes in the abdominal vein in amphibians and reptiles. $A$, elasmobranch stage, same as in Figure $55 G$; the lateral abdominal veins $i$ enter the common cardinal veins $c$ and are not connected with the renal portal veins $p$. $B$, the lateral abdominals $i$ have joined the renal portals at $t$ posteriorly, and anteriorly pass into the liver $f$, where they unite with the hepatic portal vein $h$; a new vein, the postcaval vein $g$, is seen growing caudad from the liver $f$ where it arises from the hepatic veins $o$. C , condition in the adults of urodele amphibians; the postcaval vein $g$ has reached and fused with the posterior cardinals $e$ and the subcardinals $j$ at the point $r$; the two lateral abdominal veins have united to form the ventral abdominal vein $i$ which empties into the hepatic portal $h . \quad D$, condition in adult reptiles; the anterior portions of the posterior cardinal veins $n$ are obliterated, leaving the postcaval vein $g$ as the sole drainage for the subcardinals $j$ and the kidneys $k$; the two lateral abdominal veins remain separate as in elasmobranchs. $a$, anterior cardinal vein; $b$, sinus venosus; $c$, common cardinal vein; $d$, subclavian vein; $e$, posterior cardinal vein; $f$, liver; $g$, postcaval vein; $h$, hepatic portal vein; $i$, lateral (or in $C$ ventral) abdominal vein; $j$, subcardinal vein; $k$, kidney; $l$, iliac or femoral vein; $m$, caudal vein; $n$, obliterated part of the posterior cardinals; $o$, hepatic veins; $p$, renal portal veins; $q$, pelvic veins; $r$, union of postcaval, posterior cardinals, and subcardinals; $s$, union of postcaval and subcardinals; $t$, union of abdominal vein with renal portal system.

The renal portal vein runs forward along the surface of the kidney, into the substance of which it sends numerous branches. It also receives branches from the body wall.

Draw the renal portal system.

It will be seen that the ventral abdominal vein forms a connection between the renal portal and hepatic portal systems. Blood from the hind legs and tail 
may pass into the abdominal vein and so into the hepatic portal vein, or may pass into the renal portal vein. This arrangement appears to be a device to assist the return of the blood from the posterior regions of the body. In the elasmobranchs there is no such connection between the two portal systems (although connections have been reported in some specimens as individual variations). Further, in elasmobranchs the lateral abdominal veins enter the cardinal system of veins, while in Necturus their homologue, the ventral abdominal vein, empties into the hepatic portal system (Fig. 57).

4. The systemic veins.-

a) The anterior systemic veins: It has already been noted that the common cardinal vein joins the hepatic sinus on each side in the pericardial cavity. Turn to the pericardial cavity and locate the common cardinal veins. Trace one of them laterally, removing the muscles between the pericardial cavity and the base of the fore limb. Just outside of the pericardial cavity the common cardinal receives the jugular and subclavian veins. The latter lies ventral and anterior to the former. Trace the subclavian into the fore limb by removing the skin from the outer surface of the limb. The subclavian is seen to be formed at the shoulder by the union of the cutaneous vein from the skin and the brachial vein which runs along the surface of the limb muscles. The jugular vein is homologous with the anterior cardinal vein of the elasmobranchs. Follow it forward. It is formed by the union of the external and internal jugular veins. The external jugular vein first receives branches from the floor of the mouth. The main vein then passes dorsally immediately behind the gills. It may be picked up here by removing the skin behind the last gill. The external jugular may then be traced forward above the gills, where it enlarges, forming the jugular sinus. This sinus receives tributaries from the head and jaws. The internal jugular vein is a small vein which joins the external jugular posterior to the jugular sinus. It is difficult to find, and part of it will be seen later in the roof of the mouth.

The common cardinal vein also receives a lateral vein from the body wall. Remove the skin from the lateral line shortly posterior to the fore limb. Cut through the shoulder muscles so as to reveal the partition (horizontal skeletogenous septum) between the epaxial and hypaxial muscles. The lateral vein will be found situated along this partition and can be followed forward into the common cardinal vein.

b) The postcaval vein: Turn to the pleuroperitoneal cavity. Examine the dorsal mesentery of the small intestine at its junction with the dorsal body wall (in female specimens spread the ovaries apart, laying one to each side). In the mesentery runs a large vein, the postcaval vein. It passes forward, receiving numerous genital veins from the adjacent gonads and renal veins from the kidneys. Trace it forward. At about the level of the spleen it turns ventrally and enters the dorsal surface of the right side of the liver. It is best seen by laying the 
stomach to the left and the liver to the right. It passes forward imbedded in the liver substance and should be followed by picking away the liver tissue. It receives several hepatic veins from the liver; one of the larger of these lies along the midventral line of the liver and joins the postcaval near the anterior end of the liver. At the anterior end of the liver the postcaval vein emerges as a very large vessel situated in the coronary ligament. It pierces the transverse septum and forks into the two hepatic sinuses which, after being joined by the common cardinal veins, enter the sinus venosus. The origin of the postcaval vein is discussed below.

c) The posterior cardinal veins: At the place where the postcaval vein turns ventrally toward the liver it is connected with a pair of veins, the posterior cardinal veins. Trace these anteriorly. They lie very near the mid-dorsal line of the anterior half of the pleuroperitoneal cavity, one to either side of the dorsal aorta. In females they are situated in the mesotubarium, along the line where this unites with the dorsal wall. Trace the posterior cardinals posteriorly and note connections between them and the renal portal veins (which are of course the original posterior ends of the posterior cardinals, as shown in Fig. 57). Note also the parietal veins which enter the posterior cardinals in their course along the body wall. The posterior cardinals may be traced to the transverse septum. Shortly before reaching this they diverge from each other and penetrating the lateral portions of the septum enter the common cardinal vein practically at the same point as the entrance of the jugular and the subclavian.

5. The pulmonary veins.-The pulmonary vein is a large vessel situated along the ventral side of each lung, i.e., the side opposite that which is attached to the dorsal wall. The two pulmonary veins run forward in the walls of the lungs and shortly caudad of the transverse septum converge and at the septum unite to one vessel. This passes through the transverse sept" $1 \mathrm{~m}$ and running forward in the dorsal wall of the left hepatic sinus enters the left auricle.

6. The ventral aorta and the aortic arches.-The conus arteriosus passes anteriorly into the ventral aorta. The greater part of the ventral aorta lies within the pericardial cavity and owing to the fact that this portion of the aorta is expanded and possesses thickened muscular walls, it is named the bulbus arteriosus. ${ }^{x}$ Trace the ventral aorta forward out of the pericardial chamber by dissecting away the anterior wall of the chamber. The ventral aorta very soon forks into two vessels which pass to the right and left. Trace the right one, since the visceral arches have been left intact on that side. Follow it toward the gill arches. It soon divides into two vessels and subsequently the posterior

- The term bulbus arteriosus is very ambiguously used in many texts of vertebrate anatomy and embryology. The term should be applied only to the expanded muscular base of the ventral aorta. The bulbus arteriosus does not take part in the heart beat and is not a chamber of the heart but a portion of the ventral aorta. This definition does not correspond with the one given in $\mathrm{K}$. Very few groups of vertebrates have a bulbus arteriosus; the chief group possessing it is the Teleostei. The term truncus arteriosus is another ambiguous name. It should probably be used as synonymous with ventral aorta. 
one again divides in two, making a total of three afferent branchial arteries, one to each of the gills. Trace each one into the gill, removing the skin from the gill. At the entrance into the gill the first afferent branchial artery gives off an external carotid artery which turns medially, running beside the branchial artery, and then branches into the floor of the mouth. Within the gill each branchial artery sends up a loop which branches among the gill filaments, from which other branches collect into a loop on the other side of the gill, this loop joining the branchial artery again. In addition to the two loops, a short connecting branch runs through the base of each gill.

Next turn back the flap, previously formed, of the floor of the mouth and pharyngeal cavities. Extend the incision posteriorly along the left side of the esophagus. Strip off the mucous membrane from the roof of the mouth and pharyngeal cavities. In the roof will be seen a pair of large vessels, the roots or radices of the aorta, which pass obliquely posteriorly and unite. Trace the right root laterally into the visceral arches dissecting off the mucous membrane from the latter. Locate from the inside the branchial arteries exposed in the preceding paragraph and note their emergence from the dorsal ends of the visceral arches as the efferent branchial arteries. The second and third efferent branchial arteries unite as they emerge from the visceral arches, thus forming two efferent branchial arteries on each side. From the common vessel formed by the union of the second and third efferent branchial arteries an artery arises which passes posteriorly. Trace it into the pleuroperitoneal cavity and note that it courses along the dorsal or attached side of the lung. It is the pulmonary artery. From the first efferent branchial artery very near the place where it joins the second and third arises the internal carotid artery which passes forward on the roof of the mouth. Accompanying the internal carotid artery is the internal jugular (anterior cardinal) vein. The efferent branchial arteries medial to the origin of the pulmonary and internal carotid arteries unite to form the root of the aorta on each side. From this springs the vertebral artery which passes at once dorsally into the skull. The two roots of the aorta then pass obliquely caudad and unite in the mid-dorsal line to form the large dorsal aorta.

Draw the afferent and efferent branchial arteries and their branches.

The afferent and efferent branchial arteries are as in the elasmobranchs, the ventral and dorsal halves, respectively, of the aortic arches. Besides the network in the gills, each arch forms a complete semicircle by means of the direct connecting branch in the base of the gill. The three aortic arches present in Necturus correspond to the third, fourth, and sixth arches of the original six. Note the origin of the external carotid from the ventral part, of the internal carotid from the dorsal part of the third aortic arch, and of a new vessel, the pulmonary artery, from the last or sixth arch (see Fig. $5^{8 C}$, p. ${ }^{267}$ ).

7. The branches of the dorsal aorta.-Trace the dorsal aorta posteriorly into the pleuroperitoneal cavity. It runs in the median dorsal line. Immediately beyond its origin it gives off a subclavian artery on each side. Trace one 
of them. It passes laterally. It gives off a conspicuous cutaneous artery which lies on the inner surface of the pectoral girdle. It branches to nearby muscles and the skin. The subclavian then gives off an artery to the shoulder and as the brachial artery passes into the fore limb where it branches extensively.

In its course along the pleuroperitoneal cavity the dorsal aorta gives off both visceral and somatic branches. The median visceral branches will be described first.

The first visceral branch of the dorsal aorta is the gastric artery. It passes to the stomach and forks into the dorsal and ventral gastric arteries supplying the corresponding walls of the stomach. The ventral gastric artery also furnishes a few small branches to the spleen. Some distance posterior to the origin of the gastric artery, the coeliaco-mesenteric artery springs from the aorta. It passes ventrally in the mesentery giving rise to some mesenteric branches to the beginning of the small intestine; it then proceeds to the region of the hepatoduodenal ligament where it branches into a splenic artery to the spleen, a pancreatico-duodenal artery to the pancreas, duodenum and pyloric region of the stomach, and a hepatic artery, which runs along the dorsal surface of the liver in contact with the hepatic portal vein and supplies numerous branches to the liver substance. Posterior to the point of origin of the coeliacomesenteric vessel, the dorsal aorta gives off a number of mesenteric arteries into the intestine.

The lateral visceral branches of the dorsal aorta consist of numerous genital arteries to the testes in the male, and ovaries and oviducts in the female, and of renal arteries to the kidneys. The somatic branches of the aorta consist of the parietal or intercostal arteries. These arise from the dorsal side of the aorta at segmental intervals; they pass dorsally and divide in two, one branch going to each side of the body. These branches pass laterally along the internal surface of the body wall and supply the body musculature.

Near the posterior end of the pleuroperitoneal cavity, the aorta gives off on each side an iliac artery, which passes laterally alongside the femoral vein toward the hind limb. It gives off an epigastric artery which runs anteriorly along the body wall, a hypogastric branch to the urinary bladder and cloaca, and as the femoral enters the hind limb, into which it should be traced. It runs along the medial side of the leg and at the knee gives rise to a number of branches. The dorsal aorta proceeds into the tail as the caudal artery, giving off a pair of cloacal arteries into the cloaca as it passes that region.

Draw the branches of the dorsal aorta.

8. The chambers of the heart.--Remove the heart from the pericardial cavity by cutting across both ends. The chambers of heart were previously named. The sinus venosus is a chamber with very thin, delicate walls. It receives from behind the two large trunks formed by the union of the common cardinal vein and hepatic sinus on each side. Anteriorly the sinus passes into 
the auricle. The sinus connects chiefly with the right auricle and is slightly displaced to the right. By cutting open the sinus locate the sin-auricular opening guarded by a pair of valves. Cut into one of the auricles and wash out its contents. Looking into the auricle note the interauricular septum which separates the two auricles; the septum is very incomplete, being perforated by a number of openings. Remove the ventral half of the ventricle and also slit open the conus and bulbus arteriosus by a longitudinal incision. Note the thick spongy walls of the ventricle and the numerous muscle strands in the interior. Locate the single auriculo-ventricular opening between the auricles and ventricle. It is on the left side. It is guarded by a pair of valves. In the base of the opened conus arteriosus note the transverse row of three semilunar valves. In the bulbus arteriosus is a longitudinal partition, dividing the interior in to right and left channels.

The heart of Necturus and of all Amphibia receives both arterial and venous blood, and consequently there is a double circulation through the heart. The blood from the systemic veins enters the sinus venosus and is passed on chiefly to the right auricle. The blood from the pulmonary veins, which is aerated blood, returns to the left auricle. Owing to the incomplete nature of the interauricular septum there is some mixing of arterial and venous blood. The blood from both auricles passes into the single ventricle where it is further mixed, and the mixed blood exits by way of the conus arteriosus and ventral aorta. Passing to the gills the blood is aerated and as arterial blood enters the dorsal aorta and pulmonary artery. As the pulmonary artery already contains aerated blood before it reaches the lungs, it is evident that the lungs are but slightly functional in the gilled urodeles.

9. Comparison of the circulatory system of Necturus and the elasmobranchs. - It is evident that the circulatory system of Necturus has undergone some modifications from the condition seen in elasmobranchs. In the arterial system the chief changes concern the aortic arches. Of the five arches present in elasmobranchs but three have persisted in Necturus. The persistent arches are the third, fourth, and sixth, the second and fifth having vanished. In addition we note the origin of a new vessel, the pulmonary artery, as a branch from the last (sixth) aortic arch. The aortic arches of Necturus have still the form of an arch which passes through the substance of a visceral arch. In the venous system the changes are more pronour sed. The anterior cardinal vein still remains the chief vein of the head, but the posterior carcinal vein is much decreased in importance. The functions of the posterior cardinal vein are taken over by a new vein, the postcaval vein, which is absent in fishes (except in the Dipnoi). The origin of the postcaval vein is somewhat complicated but from a study of its relations in Necturus it is evident that the anterior part is derived from the hepatic veins or sinuses of the elasmobranchs (and these in turn are the proximal portions of the vitelline veins), and that the posterior part of the postcaval is formed from the subcardinal veins, chiefly the right one (Fig. 57, p. 224). The posterior portions of the posterior cardinal veins are functioning as the renal portal veins as in fishes. The anterior portions of the posterior cardinals are diminished in importance but have the same relations as in fishes; posteriorly they are connected with the subcardinals (postcaval) as also in elasmobranchs (Fig. 55, p. 205). It will also be noted that the renal portal veins (posterior cardinals) have increased their posterior connections. Whereas in fishes they collect only from the tail, in Necturus they collect from both tail and posterior appendages. This is due to a union between the renal portal and abdominal veins (Fig. 57). Meantime the ventral abdominal vein (same as the lateral abdominal veins of elasmobranchs) 
has shifted its anterior connections. Whereas in fishes it opens into the common cardinai vein, in Amphibia it passes into the hepatic portal system. The abdominal vein thus becomes a connection between the renal and hepatic portal systems and provides two outlets for the blood from legs and tail. The pulmonary veins are new formations.

\section{THE CIRCULATORY SYSTEM OF THE TURTLE}

Specimens for the study of this system should have been doubly injected, that is, into the arterial system, and in both directions into one ventral abdominal vein. Remove the plastron. With the bone scissors cut away the sides of the carapace between fore and hind limbs.

I. The chambers of the heart.-The heart of the turtle possesses but three different chambers, in contrast to the four chambers present in the forms already considered; one of the chambers is divided into two completely separate halves. Examine the heart in the pericardial cavity, removing the ventral wall of the pericardial sac if this has not already been done. From the ventral view the visible parts of the heart are the ventricle-the thick-walled, conical posterior part-and the auricles-thin-walled chambers, one on each side anterior to the ventricle. The two auricles are entirely separate from each other, as will be seen later. The ventricle is attached to the posterior pericardial wall by a ligament, which is apparently a remnant of the ventral mesentery or ventral mesocardium of the heart, a structure which was considered in the general discussion of the coelom. Cut through this ligament, raise the ventricle, and press it forward. A large chamber, the sinus venosus, is revealed dorsal to the auricles and attached to the right auricle. The large bases of the systemic veins will be seen entering the sinus. Put the ventricle back in place. Observe the large vessels, arteries, which spring directly from the base of the ventricle without the intervention of a conus arteriosus. The latter is lacking, or to be very accurate, is so shortened down as to be invisible. The bases of the arteries which spring from the ventricle correspond to the ventral aorta of elasmobranchs. The ventral aorta is thus seen to have split into several separate trunks. The arteries will be investigated later and should be left undisturbed at present.

2. The ventral abdominal veins and the renal portal system.-Running in the ventral pleuroperitoneum from the pelvic girdle up to the heart are two large veins, the ventral abdominal veins. They are homologous with the lateral abdominal veins of elasmobranchs. The two veins are generally connected just anterior to the pelvic girdle by a cross-branch. Trace the veins forward. They receive pericardial veins from the pericardial sac and then each turns dorsally to enter the liver. Just at this turn each vein receives a pectoral branch from the pectoral muscles of that side. Trace the pectoral vein into the muscle. Slit the pleuroperitoneum alongside each abdominal vein and by lifting the cut edges find the places where the vein of each side penetrates the lobe of the liver.

Trace the ventral abdominal veins posteriorly. Make a longitudinal slit in the pleuroperitoneum midway between the two veins, and separating the cut 
edges, look within and locate the urinary bladder. Note the small vesical vein passing from the bladder into each abdominal vein. Continue to trace the abdominal veins posteriorly. Each passes to one side of the pointed anterior extremity of the pelvic girdle and at the same time gradually turns laterally. As it turns it receives a pelvic vein which runs over the ventral surface of the muscles of the pelvic girdle. The left pelvic vein seems to be usually larger than the right one.

In their course between the heart and pelvic girdle each vein gives off laterally one or more small branches which pass to the borders of the carapace where they join the margino-costal vein to be described later.

Draw the two abdominal veins with their branches thus far noted.

Continue to trace the abdominal veins in the posterior direction. As both have identical branches it is necessary to follow only one, selecting the one which has been most successfully injected. It passes along the dorsal surface of the pelvic girdle near the anterior margin of the latter; the girdle should be pulled toward the student in order to follow the vein. Grasp the hind leg on the side on which you are dissecting and work it back and forth until it is freely movable. Press the leg away from the carapace of that side and cut through the skin between the leg and the carapace back to the end of the tail. Remove the skin from leg and tail. Now trace the abdominal vein laterally along the base of the leg. Just beyond the pelvic vein a small crural from thigh muscles and a larger vein from fat enter the abdominal. About an inch and one-half lateral to this the large femoral vein emerges from the leg and joins the abdominal vein, now designated the iliac vein. The femoral vein should be followed into the leg by separating the muscles. The iliac vein is now situated alongside a conspicuous artery, the epigastric artery, both being imbedded in the abdominal wall from which small veins pass into the iliac vein. After a short distance the iliac vein receives the epigastric vein which accompanies the artery of the same name anteriorly along the curve of the carapace. The iliac vein now turns abruptly posteriorly and runs between the base of the leg and the carapace, deeply imbedded in some loose tissue. This tissue should be cleared away and the vein followed. It receives branches from the carapace and near the posterior part of the thigh a well-marked sciatic vein from the thigh. Posterior to this point it receives several small branches from the leg and as the caudal vein passes along the side of the tail, receiving at the base of the tail a cloacal branch from the anal region.

Return to the point where the epigastric vein enters the iliac vein. At this place a large vein continues forward from the anterior and dorsal surface of the iliac. This vein, the renal portal vein, runs forward and dorsally, penetrating the pleuroperitoneum. Cut the pleuroperitoneum transversely halfway between the heart and pelvic girdle, cutting across both abdominal veins. Cut also into the pleuroperitoneum at the place where the renal portal vein passes through it. A layer of muscle will be found outside the peritoneum at this place. Both 
muscles and membrane should be slit ventrally to meet the transverse incision across the pleuroperitoneum. In this way free access is gained to the pleuroperitoneal cavity. With the left hand carefully press all of the viscera forward. It is usually necessary to detach the lung from the dorsal wall and push it forward also. With the right hand press the pelvic girdle caudad. A space cleared of viscera is thus left dorsal to and in front of the pelvic girdle. Look into this place near the median dorsal line for a somewhat flattened organ, the kidney, situated against the median dorsal wall. The kidney is retroperitoneal, that is, dorsal to the pleuroperitoneum. This latter membrane should be stripped off from the ventral face of the kidney. (In male specimens the rounded yellow testis and black coiled epididymis will be noted attached to the ventral surface of the kidney.) The renal portal vein may now be followed from the point where it leaves the iliac through the pleuroperitoneum toward the kidney. Before reaching the kidney it receives a vein from the carapace. At about the middle of the lateral border of the kidney is a fissure; the renal portal vein enters this fissure and passes onto the ventral face of the kidney where it immediately forks. One of its branches, the vertebral vein, runs forward and may be traced in well-injected specimens by separating the lung from the carapace and raising the lung and also stripping off the pleuroperitoneum from the dorsal wall. The vertebral vein passes anteriorly dorsal to the arches of the ribs and receives laterally an intercostal branch at each suture between the costal plates of the carapace. The intercostal veins anastomose with each other in the curve of the carapace by means of a longitudinal vessel, the margino-costal vein, which is the anterior continuation of the epigastric vein previously noted. The margino-costal vein also has connections with the abdominal veins. The posterior branch of the renal portal vein passes posteriorly over the ventral face of the kidney and as the internal iliac or hypogastric vein receives branches from the reproductive organs (male), bladder, cloaca, etc. The renal portal vein in its passage along the ventral face of the kidney gives off branches into that organ. The renal portal vein is the posterior part of the posterior cardinal vein (Figs. 55 and 57 ). The vertebral vein is formed by the longitudinal fusion of segmental branches of the posterior cardinal vein of the embryo.

Draw these veins as far as you have seen them, adding them to the drawing of the abdominal veins already made.

The student should consider at this point the differences between the connections of the ventral abdominal veins of the turtle and their homologues, the lateral abdominal veins, of elasmobranchs. In the turtle these veins have formed a connection with the renal portal system posteriorly while anteriorly they enter the liver instead of the cardinal system.

3. The hepatic portal system.- - Lift up the lobes of the liver separating them gently from the stomach and duodenum, and find on their dorsal surfaces at the place where the gastro-hepato-duodenal ligament is attached to the liver a large 
rein, the hepatic portal vein. It runs completely across the liver imbedded in its wall, and at the right, at the point where the bile duct enters the duodenum, turns abruptly posteriorly, penetrating the mesentery. On the left note the numerous gastric veins entering the hepatic portal vein from the stomach. Just to the right of the bridge connecting the two lobes of the liver, two or three anterior pancreatic veins pass from the pancreas into the hepatic portal vein. Near the bile duct it receives cystic veins from the bile duct, posterior pancreatic veins from the right end of the pancreas, and a long duodenal branch from the first part of the small intestine. The hepatic portal vein should be followed posteriorly; it is imbedded in the pancreas and at the bend of the duodenum penetrates the mesentery and emerges to the left of the duodenum. Liver and duodenum must be pressed forward to follow it. The vein next passes to the posterior side of the adjacent loop of the small intestine which should also be pressed forward. The vein will then be found to pass on the left side of the spleen in contact with that organ and to receive numerous splenic tributaries from it. Shortly posterior to the spleen the hepatic portal vein reaches the central point of the mesentery where the mesentery is thrown into a coil. At this place the numerous mesenteric veins, accompanied by arteries, will be seen passing in the mesentery from all parts of the intestine into the hepatic portal vein.

Draw the hepatic portal vein and its branches.

Now by dissecting away the liver substance trace the anterior portions of the ventral abdominal veins into the liver and find their union with the hepatic portal vein. Note how the hepatic portal vein breaks up into many branches in the liver substance. As in other vertebrates the direction of flow in the hepatic portal vein is from the digestive tract into the liver.

Add to the drawing the connections of the ventral abdominal vein in the liver.

4. The systemic veins.-Four large systemic veins enter the sinus venosus. Turn the ventricle forward so as to obtain a clear view of the sinus. As already noted, it is not symmetrically placed but is displaced slightly to the right, connecting with the right auricle. A large vein enters the left wall of the sinus, passing around the border of the left auricle. This is the left precaval vein (also called anterior vena cava and descending vena cava). Another vein, the left hepatic vein, emerges from the bridge of the liver and enters the left angle of the posterior wall of the sinus. The very large vein which passes into the right angle of the posterior wall of the sinus is the postcaval vein (also named posterior vena cava and ascending vena cava); it emerges from the right lobe of the liver. Just in front of the entrance of the postcaval vein and best seen by pressing the heart to the left, the right precaval vein passes into the right anterior angle of the sinus venosus.

a) The branches of the precavals: Each precaval enters the pericardial cavity by passing through the anterior wall of the pericardial sac. From this point 
it may be followed forward. As both have identical branches, it is necessary to follow only one. The one whose branches appear to be filled with blood should be selected; the left one is usually easier to follow. In specimens which have been preserved for a long time the dissection of the branches of the precaval veins is generally unsatisfactory because the branches are often empty, but, of those named below, as many as the condition of the specimen permits should be identified. Be very careful not to injure the adjacent arteries springing from the ventricle. Trace the precaval forward out of the pericardial sac. Shortly anterior to the place where the precaval penetrates the pericardial sac the vein receives practically simultaneously four tributaries, three small and one large one. The most medial branch is the small thyreo-scapular vein which collects a branch from the thyroid gland (the gland situated in the fork of the large arteries) and then passes to the inner surface of the shoulder where it collects from several muscles. Lateral to this vein is the slightly larger internal jugular vein. This runs anteriorly along the side of the neck in contact with a white nerve (vagus or tenth cranial nerve). It receives medially an extensive network of branches from the esophagus. It may be traced anteriorly to the base of the skull from which it issues, making an anastomosis with the external jugular vein, soon to be described. The third tributary of the precaval vein is the large subclavian vein, by far the largest of the four branches which enter the precaval. It passes along the side of the neck and as the axillary vein turns toward the shoulder. Here it is seen to be formed by the union of two large branches, the external jugular vein from the neck and the brachial from the fore limb. The external jugular lies along the side of the neck, lateral and dorsal to the internal jugular. It collects from the head, and in its passage posteriorly along the neck has at regular intervals vertebral veins passing into it from between the vertebrae. Near its junction with the brachial it receives the last of the vertebral veins which descends from the junction between last cervical and first trunk vertebra, where it connects with the anterior end of the vertebral vein described with the renal portal system. The external jugular vein also receives branches from the skin and muscles of the shoulder region. The fourth and most lateral and dorsal of the tributaries of the precaval is a small scapular vein which comes from the muscles covering the scapula.

Draw the branches of the precaval as far as you have found them.

b) The left hepatic vein: The left hepatic vein should be traced into the left lobe of the liver from which it collects venous blood. To do this, clear away the intervening posterior wall of the pericardial sac and pleuroperitoneum.

c) The postcaval vein: Trace the postcaval vein posteriorly into the right lobe of the liver. Its course may be followed by making a slight hole in the vein where it enters the sinus and probing posteriorly in to the hole, dissecting away the liver substance along the probe. Note the numerous hepatic veins which enter the postcaval during its passage through the liver. Find where the post- 
caval enters the liver from behind to the right of the hepatic portal vein. At this point the serosa of the liver is fused to the pleuroperitoneal membrane over the ventral face of the lung. This fusion should be broken and the postcaval vein freed. On following it posteriorly it will be found to swerve toward the median line where it runs alongside a large artery (dorsal aorta). The postcaval may be traced to the posterior end of the pleuroperitoneal cavity. Its relations there will be described later.

Add the left hepatic and postcaval veins to your drawing of the precaval vein.

5. The pulmonary veins.-A pulmonary vein passes from each lung to the left auricle of the heart. It is situated posterior to the bronchus where it should be identified. Follow it toward the heart. It passes dorsal to the precaval vein. The right pulmonary runs in the dorsal wall of the pericardial sac anterior to the sinus venosus and joins the left vein at the entrance of both into the left auricle. The point of entrance is near the left precaval vein.

6. The aortic arches and their branches.-From the ventricle three large arterial trunks extend forward. Together they constitute the ventral aorta which must be conceived of as having split into three trunks. Clean away the connective tissue from these arteries and separate them from each other. The trunk farthest to the left is the pulmonary artery; the vessel next to it is the left aorta; the third and right-hand trunk is the right aorta, but it is concealed from view by the large branch, the brachiocephalic (innominate) artery which it gives off immediately on leaving the heart. Note the small coronary arteries springing from the base of the brachiocephalic artery and branching over the surface of the heart. The brachiocephalic artery lies in the median line and forks at once into large branches. In the angle of the fork lies a reddish body, the thyroid gland.

a) The branches of the brachiocephalic artery: We shall follow this vessel first; it divides at once into four trunks; the large medial ones are the right and left subclavian arteries, the much smaller lateral ones are the right and left carotid arteries. Clean away connective tissue from these vessels and follow their courses. The two subclavians embrace the thyroid gland between their bases and supply small thyroid arteries into this gland. Each subclavian next gives off branches to the ventral side of the neck and to the trachea, of which the chief one is the ventral cervical artery, a vessel arising from the subclavian about onehalf inch beyond the thyroid gland and branching profusely into the esophagus, trachea, muscles of the neck, and thymus gland. The thymus gland is a yellowish mass lateral to the ventral cervical artery and receiving branches from it. The subclavian artery, now named the axillary, turns laterally and passes to the inner surface of the pectoral girdle, where a large branch arises and branches extensively into the pectoral and shoulder muscles. The axillary then turns abruptly posteriorly and about an inch beyond the turn gives off the small dorsal cervical into the neck, the first intercostal laterally, and the vertebral caudally. The first inter- 
costal runs laterally and then turns posteriorly, joining the margino-costal artery, which courses along the curve of the carapace. The vertebral passes backward along the vertebral column dorsal to the ribs alongside the vertebral vein and gives off at the sutures of the costal plates the intercostal arteries, which run laterally into the margino-costal artery. At the point where the first intercostal and vertebral arise, the axillary bends sharply laterally and as the brachial artery passes into the fore limb alongside the brachial vein.

Each carotid artery passes forward along the ventral side of the neck, soon crossing dorsal to the subclavian and then coming to lie medial to the subclavian. In specimens in which the neck is drawn into the shell the carotids usually make loops in the neck region. As the carotid artery passes the thymus gland it gives branches into the gland. It then proceeds, without branching, the entire length of the neck in contact with the internal jugular vein and the vagus nerve, and enters the skull by a foramen in front of the auditory region.

Draw the brachiocephalic artery and its branches.

b) The pulmonary arteries: The pulmonary artery is the one farthest to the left of the three arterial trunks which spring from the ventricle. It divides immediately into right and left pulmonary arteries. To see this division lift the pulmonary trunk and look on its dorsal side. Trace the left pulmonary first. It proceeds laterally posterior to the left aorta to which it is more or less bound by connective tissue, forming the arterial ligament or ligament of Botallus. (The significance of this ligament will be explained later.) The pulmonary proceeds directly to the left lung in company with the left bronchus and left pulmonary vein. Trace the right pulmonary in the same way. It is bound to the right aorta by the right arterial ligament.

Add these to the preceding drawing.

c) The right and left aortae: Trace both of these arteries away from the heart. Each makes a curve as it leaves the heart and turns posteriorly, passing dorsal to the precaval vein, the bronchi, and the pulmonary vessels, and disappearing dorsal to the lobes of the liver. Vessels already studied may be cut to follow the aortae posteriorly. Trace the left aorta first. Grasp the stomach and left lobe of the liver and press them to the right, separating the cardiac end of the stomach from the lung. The left aorta will be found passing to the left of the esophagus and dorsal to the stomach. It gives off simultaneously three large branches. One of these is the gastric artery which passes to the stomach in the cardiac region and follows the curve of the stomach along the length of this organ. After a short distance it forks into anterior and posterior gastric arteries which supply the lesser and greater curvatures of the stomach respectively. Another branch from the left aorta is the cocliac artery. It soon forks into anterior and posterior pancreatico-duodenal arteries. The anterior pancreatico-duodenal artery passes to the left end of the pancreas, gives off there branches into the pvloric end of the stomach and to the liver, then turns to the right and runs 
along the pancreas supplying the liver, pancreas, and duodenum with many small branches. The posterior pancreatico-duodenal artery enters the right end of the pancreas and passing along the pancreas supplies branches to the liver, pancreas, duodenum, and gall bladder. The third branch of the left aorta is the superior mesenteric artery. It runs posteriorly in the mesentery; trace it, tearing the mesentery, to the center of the coils of the mesentery. At this point the artery breaks up in a fanlike manner into many radiating branches which traverse the mesentery to all parts of the small intestine. One branch, the inferior mesenteric, passes to the large intestine and accompanies it to the cloaca.

Now follow the left aorta posterior to the point where it gives rise to the superior mesenteric artery. It becomes smaller and very soon meets another vessel coming from the right. The two join in a V-shaped manner and form one vessel, the dorsal aorta, which continues posteriorly in the median dorsal line. Follow the vessel, which meets the left aorta, anteriorly, to discover its identity. Separate the right lobe of the liver from the right lung, and turn the liver and duodenum to the left. The vessel in question can then be traced anteriorly dorsal to the right bronchus and pulmonary vessels to the heart. It is therefore the right aorta. Immediately beyond its origin from the heart the right aorta gives rise to the large brachiocephalic artery whose branches were followed above. It has no other branches.

Draw the right and left aortae and the branches of the latter.

7. The dorsal aorta and the postcaval vein.-The digestive tract may now be removed, except the large intestine which is to be left in place. Follow the dorsal aorta posteriorly. It runs in the median line ventral to some long muscles and in company with the postcaval vein which courses at first to its right and later comes to lie ventral to the aorta. We shall study the branches of both vessels. The postcaval vein is seen to be formed by two vessels running along the medial side of the kidneys. Each of these receives numerous renal and genital veins from the kidneys and reproductive organs respectively. The postcaval vein is thus seen to originate between the kidneys. After adding these branches to your drawing of the postcaval vein, the vein may be removed and the dorsal aorta studied. The aorta gives off a number of small branches into the muscles on which it rests and then passes between the two kidneys. Hold the large intestine backward and clear away the connective tissue from between the two kidneys. The dorsal aorta is seen to give numerous renal arteries into the kidneys and genital arteries to the reproductive system. At the posterior end of the kidneys it forks into the right and left common iliac arteries.

Separate one kidney from the carapace and press it and the reproductive organs to the other side. Two large arteries will be seen emerging dorsal to the kidney. The anterior one is the epigastric artery; the posterior one, the common iliac mentioned in the preceding paragraph. Follow the epigastric. (If it was 
injured in the dissection of the renal portal system, try the other side.) It runs laterally to the point where the renal portal vein enters the pleuroperitoneal cavity. At this point it divides. The anterior branch continues to the carapace and runs forward along the curve of the carapace, supplying the fat bodies and becoming continuous with the margino-costal artery described above. The posterior branch turns and passes medially parallel to the ventral abdominal vein. It supplies the base of the leg and the pelvic muscles and terminates on the ventral surface of the pelvis.

Next follow the common iliac artery of the same side. It divides at once before it has emerged from above the kidney into an internal iliac and an external iliac artery. The external iliac forks after a short distance. The medial and larger branch supplies the muscles of the pelvis and as the femoral artery enters the thigh. The smaller and lateral branch passes deep dorsally to the point where the ilium is articulated to the sacral ribs; here it passes dorsal to a nerve and turns ventrally as the sciatic artery into the hind leg, running along the medial surface of the ilium. The internal iliac is best followed by replacing the kidney against the dorsal wall, pulling the large intestine backward and locating the point of origin of the internal iliacs from the common iliac. The chief branch of the internal iliac is the hemorrhoidal artery which passes forward along the side of the large intestine; in addition there are branches to the bladder, the reproductive organs, and the pelvic region in general.

Draw the dorsal aorta and its branches.

8. The structure of the heart.- Separate the heart of the turtle by cutting across the great vessels and remove it from the body. The posterior chamber of the heart is the simus venosus which receives the four great systemic veins. Clean out the blood from the sinus. It is a thin-walled chamber attached to the right auricle, into which it opens by the sin-auricular opening guarded by a pair of thin valves. Open each auricle by making a slit in the margin and washing out the blood clots. The walls of the auricles are somewhat spongy. Look into the left auricle and note the thin interauricular septum which completely separates the cavity of the left auricle from that of the right one. Find the opening of the pulmonary veins into the dorsal wall of the left auricle near the septum. Find on each side the large auriculo-ventricular opening between each auricle and the ventricle. Make a cut all of the way around the margin of the ventricle so as to make dorsal and ventral flaps of the ventricle. Spread apart the two flaps cautiously extending your cut inward until the two flaps are attached only along the base of the ventricle. Note the exceedingly thick walls of the ventricle and the muscular columns projecting into the interior. The cavity of the ventricle is a broad but flattened cavity usually containing a spongy network which may be cleaned out. Spreading the two flaps widely, note in the base of the ventricle a band passing across from one side to the other. On each side of this band is an auriculo-ventricular opening. The band is a continuation of the interauricular 
septum and forms a fold or valve on each side, which partially occludes the auriculo-ventricular opening. The right valve continues ventrally into a ridge which is on the ventral flap of the specimen. This ridge is the incomplete interventricular septum. On bringing the two flaps of the specimen together, it will be seen that the interventricular septum was connected with the muscular wall of the dorsal flap and that a space is left dorsal to the septum by which the right and left ventricles communicate with each other. The right ventricle to the right of the septum is very small, while the left ventricle is much larger and communicates with the cavity of both auricles owing to the incomplete character of the interventricular septum. Spread the flaps of the specimen again and pass a probe ventral to the interventricular septum. The probe emerges in the pulmonary artery. Probe into the other arterial trunks and find their openings into the ventricle. The opening of the left aorta is to the right of the interventricular septum, into the small right ventricle, while that of the right aorta is to the left of the septum, into the left ventricle; however, owing to the gap dorsal to the septum, the left aorta can also obtain blood from the left ventricle. By slitting open the arterial trunks find the little pocket-like semilunar valves which guard their exits from the ventricle. They represent the remains of the conus arteriosus.

We may now attempt to explain the course of the circulation through the turtle's heart. The matter is somewhat complicated and further details will be found in $\mathrm{P}$ and $\mathrm{H}$, page 359 . We have noted that all of the venous blood returns to the sinus venosus and that this in turn connects with the right auricle which passes it on into the right side of the ventricle. Although this is imperfectly separated from the left side of the ventricle, the venous blood is well retained in the right side owing to the spongy nature of the ventricular walls. Meantime, the two pulmonary veins have returned the blood from the lungs to the left auricle. Since the function of the lungs is to aerate the blood, this blood is arterial. From the structure and relations of the heart, the right auricle always contains venous blood, and the left auricle arterial blood. The left auricle passes the arterial blood into the left side of the ventricle. There is some slight mixture of venous and arterial blood in the ventricle. As the ventricle contracts both kinds of blood are moved toward the arterial trunks. We have noted that the pulmonary artery springs from the small right ventricle and that the opening into this artery is to the right of and somewhat concealed by the interventricular septum. When the ventricle contracts, the pressure practically closes the septum so that most of the venous blood passes out into the pulmonary artery. Simultaneously, the nearly pure arterial blood in the left ventricle passes into the base of the right aorta, since that is connected with the left ventricle and since the communication with the left aorta is closed temporarily by the interventricular septum. Toward the end of the contraction the remaining blood in both ventricles passes into the left aorta, as the diminished pressure again opens up the gap in the septum. It thus happens that the brachiocephalic artery passing to the anterior part of the body contains nearly pure arterial blood, while the left aorta contains mixed blood. On account of the function of right and left aortae, the dorsal aorta also carries mixed blood. It is universally true among vertebrates that the arrangement of the circulatory system is such that the purest blood is received by the head; and this is no doubt due to the greater oxygen requirements of the nervous parts of the head. 
We now see that whereas in fishes there is a single circulation through the heart, which carries only venous blood, there is in the turtle a double circulation, one half of the heart (always the left) conveying arterial blood and one half (always the right) venous blood. This change is due to the development of the lung method of breathing with the consequent shifting of the sinus venosus to the right side and the division of the auricle into two separate chambers. The blood could not go through the lungs and then to the body because all of the force of the heart beat would be lost in the passage of the blood through the capillaries of the lungs, and the circulation would stagnate in the lungs. The aerated blood returns to the heart to take advantage of the driving action of the heart. Thus, the double circulation arose. We note, however, that the method employed by the turtle (and other reptiles) is imperfect in that the arterial and the venous blood are mixed in the heart. A little consideration will show that this situation cannot be remedied merely be completing the interventricular septum (which has indeed happened in the crocodiles and alligators) because the left aorta opens into the right ventricle and would still continue to receive venous blood. The difficulty is in reality due to the presence of the three arterial trunks formed by the splitting of the ventral aorta. We can see that if the ventral aorta would split into but two trunks, one of which (pulmonary) is connected with the right side of the heart and the other (aorta) with the left side and if, further, the interventricular septum would be completed, the difficulty would be overcome and no venous blood could get into the arterial system. This is precisely what has happened in birds and mammals in which the double circulation is complete and perfect. It follows from this that birds and mammals could not have evolved from any living groups of reptiles but must have arisen far back in the reptilian line before the splitting of the ventral aorta occurred.

9. Comparison of the circulatory system of the turtle with preceding forms. -The student can hardly fail to have noted marked differences between the circulatory system of the turtle and of the elasmobranchs and Amphibia. These changes are associated with the adoption of the air-breathing habit. In the arterial system profound changes have occurred in the aortic arches (Fig. 58, p. 267). We have learned that in vertebrate embryos there are six aortic arches; in elasmobranchs the first is missing, the second incomplete, and four complete ones persist; in Necturus the first, second, and fifth have vanished, only the third, fourth, and sixth remaining. In the turtle we note that but one pair of aortic arches has persisted; these unite dorsally to form the dorsal aorta (Fig. $58 \mathrm{E}$ ). This surviving pair of arches represents the fourth pair, and is usually referred to as the aortic arch, since there is but one. The third pair of arches is quite disconnected from the dorsal aorta and is represented by the bases of the carotids. The fifth pair is absent. The bases of the sixth pair persist as the bases of the pulmonary arteries (Fig. 58E). The connection of the sixth arches with the dorsal aorta is present in vertebrate embryos as the duct of Botallus or arterial duct, but after birth or hatching this closes up and degenerates into a band of connective tissue, the arterial ligament noted in the dissection (Fig. 58). The branches of the dorsal aorta are similar to those of the animals already considered. The splitting of the ventral aorta into three trunks has been emphasized during the dissection, and the relation of this to the double circulation through the heart explained above.

In the venous system, likewise, changes have occurred. The precaval vein is the anterior cardinal vein of lower forms; its base connecting with the sinus venosus is the common cardinal vein. The renal portal veins are the persistent posterior parts of the posterior cardinal veins (Figs. 55, p. 205, and 57, p. 224). The anterior part of the posterior cardinal vein is missing (Fig. ${ }_{57} D$ ) and is replaced functionally by the vertebral vein, which is formed by a longitudinal anastomosis between the segmental branches of the embryonic posterior cardinal. It is important to note that the posterior cardinal (renal portal) has extended its posterior connections, as in $\mathrm{Am}$ phibia. Whereas in the elasmobranch the renal portal vein collects only from the tail, in Amphibia and reptiles it collects from the hind limb as well (Fig. 57). This is due to a union between 
the renal portal and abdominal veins as shown in Figure ${ }_{57} B$. We note further that the ventral abdominal vein (which is homologous with the lateral abdominal vein of elasmobranchs) has shifted its anterior connections; in elasmobranchs it returns to the common cardinal vein as in Figure $57 A$, but in Amphibia and reptiles it passes to the liver where it joins the hepatic portal vein, as in Figure $57 B$. It thus happens that in Amphibia and reptiles the blood from the hind limbs and tail can pass either into the renal portal system or into the ventral abdominal veins. This arrangement appears to be an attempt to prevent the stagnation of the blood from the posterior part of the body in the kidneys. It will be noted that the ventral abdominal veins act as connections between the renal portal and hepatic portal systems. The renal portal vein passes into a capillary system in the kidneys from which the blood is re-collected into the postcaval. There is, however, some evidence that already in the turtle there exist direct channels through kidneys by which the renal portal veins empty directly into the postcaval. This is the beginning of the retrogression of the renal portal system as a portal system.

The postcaval vein with the loss of the anterior parts of the posterior cardinal veins becomes the chief vein of the posterior part of the body. Its mode of origin in Amphibia and reptiles is shown in Figure $57 B$ and $C$. Its anterior part arises from the hepatic veins which are the proximal portions of the vitelline veins. Its posterior part between the kidneys consists of the two subcardinal veins, which in elasmobranchs are continuous with the posterior cardinals. The middle part of the postcaval between these two regions is formed by an outgrowth from the hepatic portion. In some urodeles the posterior cardinals persist, and they, together with the postcaval, connect with the subcardinals, as in Figure ${ }_{57} C$, but in reptiles these parts of the posterior cardinals vanish, leaving only the postcaval to collect from the kidneys (Fig. $57 D$ ).

\section{E. THE CIRCULATORY SYSTEM OF THE PIGEON}

In case a fresh specimen is provided for this work it should be opened as before by deflecting the pectoral muscles from either side of the keel of the sternum, then cutting through the sternum on each side of the keel and removing a median portion of the sternum including the keel. The peritoneal cavity is to be opened as before by a longitudinal incision. The specimen should have been injected through the pectoral artery.

I. The chambers of the heart.-The heart is relatively large and more compact than in the forms previously studied. The chambers are more closely knit together than in the lower vertebrates. The major portion of the heart is formed of the right and left ventricles, which together constitute a muscular thick-walled cone, having a pointed apex directed posteriorly and a broad base directed anteriorly. The two ventricles are completely separated from each other, but the division between them is indistinct externally. This division passes obliquely from the left side of the base to about the middle of the right side of the heart; the left ventricle is therefore much the larger of the two and includes the whole of the apex of the heart. Anterior to the ventricles are the two much smaller auricles, thin-walled chambers. The division between auricles and ventricles is generally concealed by a line of fat which should be removed. From the anterior end of the heart between the auricles the great arteries spring without the intervention of a conus arteriosus. On raising the ventricles the dorsal nortions of the auricles become visible. There is no sinus venosus, the great 
veins opening directly into the right auricle. There are three of these veins, the two precavals and the postcaval. The postcaval enters the right auricle from behind, emerging from the liver. The precavals come from the anterior part of the body, one on each side, and curving toward the heart at the level of the auricles enter the right auricle. The pulmonary veins may be noticed opening into the left auricle.

2. The hepatic portal system.-Turn to the peritoneal cavity. Cut across the falciform ligament of the liver near the gizzard, noting first the small vein passing from the ventral ligament of the gizzard in the falciform ligament to the liver. The lobes of the liver may now be turned forward. Running along the dorsal surface of the liver and branching into its substance is the large hepatic portal vein. The main part of the vein enters the right lobe of the liver, coursing between the two bile ducts. The remainder of it lies along the dorsal surface of the left lobe of the liver, sending branches into the liver, and at the left receives the left and median gastric veins from the margin and left side of the gizzard and from the proventriculus. Follow posteriorly that part of the hepatic portal which lies between the two bile ducts. It is soon seen to be formed by the union of three veins, a superior mesenteric, a gastroduodenal, and an inferior mesenteric. The superior mesenteric collects from the greater part of the small intestine. The gastroduodenal receives the right gastric vein from the right side of the gizzard; the pancreatico-duodenal vein, which runs along the duodenal loop collecting from duodenum and pancreas; and the mesenteric vein from the last loop of the small intestine. The inferior mesenteric vein runs along the large intestine from which it collects many branches. At its posterior end it turns dorsally and joins the renal portal system, where it will be followed later.

Draw the branches of the hepatic portal system.

3. The systemic veins.-As already stated, these consist of two precavals and one postcaval.

a) The branches of the precaval veins: As both veins have identical branches, only one need be followed. Find the vein on each side lateral to the auricle and trace each into the right auricle, lifting the heart. The left precaval passes around the left auricle to enter the right auricle. The right precaval is much shorter and enters the right auricle directly.

Follow one precaval forward. It lies just posterior to a large artery and is there seen to be formed by the union of three large veins, laterally the pectoral vein, slightly anterior and dorsal to this the subclavian vein, and anteriorly the jugular vein. Each of these veins should be followed. The pectoral vein at its union with the others receives the internal mammary vein, ascending from the inner surface of the ribs, and has also a tributary from the sternum and coracoid. The main vein is formed laterally by the union of two veins emerging from the pectoral muscles. These may be followed into the muscles from which they are seen to collect many branches. The subclavian vein passes deep dorsally ventral 
to a group of nerves (brachial plexus) and somewhat concealed by arteries which should not be injured. As the brachial vein it emerges from the wing and then receives a branch from the shoulder muscles. The jugular vein passes anteriorly on the dorsal side of the large arteries. On tracing the jugular forward it will be found to receive the following veins, named in order from the heart forward: on the medial side some small and then a large branch from the crop (at the point of entrance of these into the jugular is situated a small reddish body, the cervical lymph gland); on the lateral side a vein from the shoulder, and at the same level, the vertebral vein from the vertebral column; medially, another branch from the crop; laterally a large vein from a plexus of blood vessels in the skin of the neck; then small veins from the esophagus and trachea. On freeing the anterior end of the esophagus (also trachea) and cutting across it, the jugular vein can be followed to the soft palate, where it joins its fellow of the opposite side. Posterior to this union each receives a plexus of veins from the skin of the face. On dissecting away the soft palate from the anastomosis of the two jugular veins, branches from the skull will be found passing into the anastomosis.

Draw the branches of the precaval vein as far as found.

b) The postcaval vein: Raise the ventricles of the heart and note once more the large postcaval vein emerging from the liver and entering the right auricle between the two precaval veins. Note the large hepatic veins which it receives from the liver. The left one of these hepatic veins receives the small vein of the falciform ligament mentioned previously. Follow the postcaval vein into the peritoneal cavity, turning all of the viscera to the left. The postcaval will be picked up again at the posterior margin of the right lobe of the liver in contact with the dorsal body wall. The postcaval is here seen to be formed by the union of two veins, the iliac veins. In males the two oval testes will be noted at this point of junction. In females the single ovary and oviduct will be noted to the left, concealing the left iliac vein. Each iliac runs along the ventral face of a three-lobed organ, the kidney, which is set close against the dorsal body wall. Follow the right iliac vein. From between the first and second lobes of the kidney it receives the large femoral vein, emerging from the leg. The femoral vein receives a small branch from the body wall. Posterior to the entrance of the femoral vein the iliac vein corresponds to the renal portal vein of reptiles and Amphibia and may be so named. From between the second and third lobes of the kidney it receives the sciatic vein which also comes from the thigh. At the posterior end of the kidneys the two renal portal veins have an anastomosis with each other. From this anastomosis rises the inferior mesenteric vein, already noted as a branch of the hepatic portal system. It runs in the mesorectum. The anastomosis of the renal portals also receives in the median line a small caudal vein from the tail and on each side an internal iliac vein from the roof of the pelvic region. The left iliac and renal portal veins are the same as the right, except that in the female the left veins receive, genital veins from the ovary and 
oviduct. These may be seen by turning the oviduct to the right. In their course over the kidneys the renal portal veins give off branches into the kidney as in lower forms. There is probably some portal circulation in the kidneys, but most of the blood from the renal portals passes directly into the iliac veins. The iliac vein receives renal veins from the kidney of which there is one chief renal vein, which runs along the medial side of the kidney but is so imbedded in the kidney substance as to be difficult to identify. Between the two renal portal and iliac veins runs the dorsal aorta.

Turn the animal dorsal side up and remove the skin over the thigh. By separating the muscles pick up the femoral and sciatic veins and trace them into the leg. The sciatic vein accompanies the large sciatic nerve and soon turns forward to run parallel to the femoral vein. Both veins are accompanied by arteries of the same name.

Draw the branches of the postcaval vein and the renal portal system.

4. The pulmonary veins.-The pulmonary veins emerge on each side from the lung and pass toward the heart immediately posterior to the precaval veins. There is usually one pulmonary vein from each lung but there may be two. Note the branches collected by each vein from the lung. The veins pass to the dorsal side of the bases of the precavals and enter the left auricle. Their entrance into the auricle is best seen later when the heart is dissected.

5. The arterial system.- It has already been noted that the great arteries spring directly from the ventricle. They are situated between the two auricles. Separate their bases from the auricles. It will be then be found that there are two arterial trunks. The larger, medially located one is the aorta. The smaller one, passing to the left and dorsal to the aorta, is the pulmonary artery. The ventral aorta in birds is split into these two vessels.

a) The anterior branches of the aorta: Follow the aorta away from the heart. The aorta immediately gives rise in the median line to two large arteries, the brachiocephalic (innominate) arteries. The aorta then turns to the right and disappears dorsally. It will be followed at a later time. Identify the branches of the brachiocephalic arteries; as both have identical branches follow only one. Each proceeds laterally and slightly anteriorly and forks into two branches, an anterior common carotid artery and a lateral subclavian artery. The subclavian artery soon gives rise to a number of branches: the small internal mammary artery passing posteriorly along the inner surface of the ribs, the two pectoral arteries to the pectoral muscles along with the veins of the same name, and the axillary artery to the wing. The axillary artery runs anteriorly and after giving off a branch into the shoulder enters the wing as the brachial artery. The two common carotid arteries pass forward and at the level of the cervical lymph gland each gives rise to a vertebral artery, which passes dorsally into the vertebrarterial canal of the vertebral column. The common carotid arteries then approach the median line and penetrate the muscles on the ventral sur- 
face of the vertebral column. On separating these muscles in the midventral line, the two arteries may be followed forward. They pass anteriorly side by side. Shortly before they reach the head, they diverge, and at the angle of the jaws each divides into an external carotid, from which branches may be traced to the esophagus, palate, and head generally, and into a more deeply situated internal carotid which passes through the skull to the brain.

Draw the branches of the brachiocephalic artery.

b) The pulmonary arteries: The pulmonary artery passes to the left side of aorta and immediately forks into right and left pulmonary arteries. The left artery goes directly to the left lung. The right artery turns and passing on the dorsal side of the brachiocephalic arteries and posterior to the turn of the aorta enters the right lung. It will be better seen in the next paragraph.

c) The aorta: The aorta turns to the right, forming what is called the arch of the aorta. This may be followed by cutting across the right precaval vein and the right brachiocephalic artery. The arch of the aorta curves to the dorsal side of the right pulmonary artery, which can now be traced into the lung, and turns caudad. Follow it by dissecting away the tissue between the heart and the right lung and by breaking through the oblique septum. Turn the viscera to the left. Cut through the postcaval vein. The aorta, now called the dorsal aorta, lies in the median dorsal line between the two lungs. It gives off small branches to the esophagus and body wall in its passage along the pleural cavities. At the entrance to the peritoneal cavity the large coeliac artery arises from the aorta. This runs posteriorly along the proventriculus to which it branches. The coeliac artery then gives rise to the relatively small left gastric artery, which passes to the left side of the gizzard branching to this side and the edge of the gizzard. The coeliac artery then passes by the spleen to which it gives small splenic arteries and just beyond the spleen gives rise to the hepatoduodenal branch. This sends a hepatic branch into the liver and then as the anterior pancreatico-duodenal artery runs along the duodenal loop supplying duodenum and pancreas. The coeliac artery continues as the right gastric, which spreads out over the right surface of the gizzard. The right gastric sends a large posterior pancreatico-duodenal branch to the duodenal loop and pancreas and a mesenteric branch to a loop of the small intestine.

Very shortly posterior to the origin of the coeliac artery, the superior mesenteric artery arises from the dorsal aorta and branches to the small intestine. One of these branches passes along the large intestine and anastomoses with the inferior mesenteric artery described below.

The dorsal aorta now passes between the two kidneys. It gives off on each side the renolumbar artery which supplies the anterior lobe of the kidney and then passes to the body wall and some muscles of the thigh. In female specimens genital arteries are given off to the ovary and oviduct from the renolumbar artery and the renofemoral artery. The renofemoral artery is the large vessel arising 
next from the dorsal aorta; it supplies the middle and posterior lobes of the kidney, then proceeds to the lateral body wall, and as the femoral artery supplies the leg. It may be followed by turning the animal dorsal side up and looking between the muscles of the thigh along the course of the femoral vein previously identified. The femoral artery accompanies the large sciatic nerve and branches into the leg muscles. Returning to the peritoneal cavity, trace the dorsal aorta further. As it passes between the kidneys it gives off lumbar arteries into the dorsal body wall. At the posterior end of the kidneys it forks. At the point of forking arise the inferior mesenteric artery, which runs anteriorly in the mesorectum and anastomoses with a branch of the superior mesenteric artery, and the caudal artery, which proceeds straight posteriorly in the median line to the tail. The two forks of the dorsal aorta are named the internal iliac arteries. They pass posteriorly along the roof of the pelvic region. The left one gives off branches into the oviduct.

Draw the branches of the dorsal aorta.

6. The structure of the heart.-Free the heart by cutting across the great vessels. Note that all veins enter the apparently anterior end of the heart, having shifted forward out of the transverse septum. As already stated, there is neither sinus venosus nor conus arteriosus in the bird's heart. Only two of the original four chambers of the heart have persisted, namely, auricle and ventricle, but each of these is subdivided into two completely separate halves, the right and left auricles and ventricles. The auricles are small thin-walled chambers anterior to the ventricles. In the walls of the right auricle identify the openings of the systemic veins and in the left auricle of the pulmonary veins. Slit open the right auricle and looking within note the thin interauricular septum separating it from the left auricle. A fold extends from this septum to the entrance of the postcaval vein partly concealing the entrance. Note the deep cleft, the auriculoventricular opening, through which the right auricle opens into the right ventricle. Similarly open the left auricle and find the left auriculo-ventricular opening. Cut across the apex of the ventricles. Observe the crescentic form and relatively thin walls of the right ventricle and circular section and enormously thickened wall of the left ventricle. What appears to be the internal wall of the ventricles is the interventricular septum, completely separating the cavities of the two ventricles. Open the right ventricle by a slit extending from the previously cut apex to the base. Note the single valve which guards the right auriculo-ventricular opening; it is a muscular band extending from the ventricular wall to the auriculo-ventricular opening. In the left side of the anterior end of the right ventricle find the opening of the pulmonary artery, or probe into the base of the pulmonary artery and note emergence of the probe into the right ventricle. At the base of the pulmonary artery are three pocket-like semilunar valves. Cut into the left ventricle and note the two thin membranous valves which guard the auriculo-ventricular opening. They are called the mitral valve. Each is 
attached by delicate cords, the chordae tendinae, to the wall of the ventricle. The wall of the ventricle has several muscular ridges which project into the cavity; they are called the columnae carnae. Find, to the medial side of the mitral valve, the opening of the left ventricle into the aorta. Probe into this and satisfy yourself that it leads into the aorta. Note the three semilunar valves at the beginning of the aorta.

The removal of the heart permits the tracing of the esophagus into the proventriculus and of the bronchi into the lungs. The form and extent of the lungs can also be observed to advantage at this time.

7. The circulation through the heart and the comparison of the circulatory system of bird and reptile.--In birds the heart is completely divided into right and left auricles and ventricles. The venous blood enters the right auricle from the systemic veins, passes into the right ventricle, and out into the pulmonary arteries which convey it to the lungs. After aeration in the lungs the blood returns by way of the pulmonary veins to the left auricle, from which it flows into the left ventricle and out of the aorta. Thus, the right side of the heart contains only venous blood and the left side only arterial blood. There is a perfect double circulation, both kinds of blood flowing simultaneously through the heart, the two streams completely separated from each other. Since the aorta is connected only with left ventricle, the arterial system receives pure arterial blood. The perfection of the double circulation is achieved by the splitting of the ventral aorta into two trunks-aorta and pulmonary-which are connected with the left and right sides of the heart, respectively. The sinus venosus is apparently absent, but in reality is reduced and incorporated into the right auricle. The conus arteriosus is represented by the semilunar valves of the aorta and pulmonary artery.

The aortic arches are further modified from the condition seen in reptiles. As in the turtle, the bases of the common carotid arteries represent the third aortic arches. The union of the subclavians with these is secondary. The arch of the aorta is the right fourth aortic arch, the left fourth arch having vanished during embryonic development. There is consequently in birds no complete aortic arch as in the preceding forms but only, so to speak, one-half an arch, the persistent half being the right one. The pulmonary arteries represent the sixth aortic arch, separated as in reptiles from the aorta (Fig. $58 F$, p. 267).

The venous system is reptilian in character. The two precaval veins are similar to those of the turtle and are homologous with the anterior cardinal veins of lower forms (the internal jugular branch being the original anterior cardinal). The bases of the precavals entering the sinus venosus are the common cardinal veins. The posterior cardinal veins are as in the turtle represented only by their posterior portions, which are named in the adult the renal portal veins. These veins have, as in reptiles and Amphibia, absorbed the veins of the legs and tail. In birds it is very interesting to note the union which is in progress between the renal portal system (posterior cardinals) and the postcaval vein. As we shall see, this union is complete in mammals. In birds the renal portal system is probably to a slight degree functional as a portal system through the kidneys, blood passing from the renal portal vein into the kidneys and re-collecting into the renal veins tributary to the postcaval vein. Most of the blood, however, passes directly from the renal portal veins into the postcaval.

The origin of the postcaval vein in birds is the same as that given for reptiles. That part of it which is situated between the kidneys is formeo of the subcardinal veins, chiefly the right one. The part through and anterior to the liver comes from the vitelline veins. The middle region of the vein is a new formation. The postcaval is seen to be usurping the renal portal system and thus extending itself posteriorly. This process is completed in mammals. 
The hepatic portal system is similar to that in the forms already discussed. The renal portal system is identical with that of reptiles. The inferior mesenteric vein which connects the two portal systems is probably homologous with the ventral abdominal veins of reptiles. It is, however, of decreased importance as a channel between the two portal systems, owing to the junction of the renal portal system with the postcaval vein.

\section{F. THE CIRCULATORY SYSTEM OF THE MAMMAL}

The specimen should have been injected in the arterial system.

I. The chambers of the heart.-The heart is relatively large and compact, the chambers closely united with each other. The pericardial sac should be removed if this has not been done previously. In case the thymus gland is well developed, it will be necessary to dissect this away from the anterior part of the heart. The greater portion of the heart consists of the two ventricles. These constitute a firm thick-walled cone, having a posterior pointed $a p e x$ and a broad anterior base. This cone consists of two completely separated ventricles, the right and left ventricles; the division between them is marked externally by an indistinct line or groove extending from the left side of the base obliquely to the right, and terminating to the right of the apex. The groove contains branches of the coronary artery and vein which will be found ramifying over the surface of the ventricles. The left ventricle is much the larger of the two and includes the apex. Anterior to the base of each ventricle is a much smaller, thin-walled, generally dark-colored chamber, the auricle or atrium. Each auricle in the contracted state presents a lobe, the auricular appendage, projecting medially and slightly posteriorly over the ventricle; in the cat (and man) this lobe has a scalloped margin and is shaped something like the human ear (hence the name auricle, meaning little ear). ${ }^{x}$ Extending anteriorly from the middle of the base of the ventricles forward between the two auricles is a large artery, the pulmonary artery. This makes an arch to the left and disappears. Dorsal to the pulmonary is another arterial trunk, the aorta. These two trunks are generally imbedded in fat which should be removed. They represent the split ventral aorta. A conus arteriosus is lacking, the arteries springing directly from the ventricles. Grasp the apex of the heart and turn the heart forward and note the bases of the great veins (pulmonary and systemic veins) entering the auricles. There is no sinus venosus present as a distinct chamber; it is greatly reduced and absorbed into the right auricle where it may be located by physiological experiments as a small spot at the point where the systemic veins enter the right auricle. From the morphological point of view, we may say that the mammalian heart consists of but two different chambers (each of which is, however, double) in contrast with the fish and amphibian heart, in which there

'In human anatomy only the ear-shaped lobe is named the auricle, the whole chamber being termed atrium. In comparative anatomy, however, the terms atrium and auricle are regarded as synonymous. 
are four chambers and the reptilian heart where the number of different chambers is reduced to three.

2. The hepatic portal system.-Turn to the peritoneal cavity. Press the lobes of the liver forward and the other viscera to the left. Put the hepatoduodenal ligament on a stretch by widely separating the stomach and liver, without, however, tearing the ligament. In the ligament lying dorsal to the common bile duct is the large hepatic portal vein (commonly called simply the portal vein in mammals, since mammals have but one portal system). Free it by carefully cleaning connective tissue from its surface. Follow it anteriorly and note how it branches into the liver substance. Follow it posteriorly, ripping away fat and connective tissue from its surface with the dull point of a probe. Note the large branch it sends into the right lateral lobe of the liver. The branches received by the portal vein from the digestive tract are slightly different in the rabbit and cat. In preserved specimens the branches are not always easy to follow, and the student should identify as many as possible. The arteries accompanying the veins must not be injured.

Rabbit: Immediately posterior to the branch into the right lateral lobe of the liver, the portal vein receives on the right side the gastroduodenal vein. This vein is soon seen to be formed by the union of two veins, a larger anterior pancreatico-duodenal vein, which appears as a continuation of the main vein and the smaller right gastro-epiploic vein. The first-named vessel runs in the tissue of the pancreas alongside the first part of the duodenal loop, collecting tributaries from both pancreas and duodenum. The right gastro-epiploic vein comes from the pyloric region of the stomach and receives also branches from the great omentum. Shortly posterior to the entrance of the gastroduodenal vein into the portal, the portal receives on the left side the larger gastrosplenic vein. This vein is seen to be formed a short distance from the portal by the union of the splenic and coronary veins. The latter comes from the lesser curvature of the stomach where it is seen to be formed by numerous branches collecting from both surfaces of the stomach. The splenic vein is a large vessel running in the great omentum past the spleen and extending as far as the left end of the stomach. In its course it collects numerous splenic branches from the spleen and the left gastro-epiploic veins from the stomach and omentum. Some distance posterior to the entrance of the gastrosplenic vein, the portal receives the posterior pancreatico-duodenal vein, which runs in the mesentery of the duodenal loop, collecting from pancreas, and duodenum, and anastomosing with the anterior pancreatico-duodenal vein. At the same level as the entrance of this vein, the portal receives on the opposite side the inferior mesenteric vein. This may be traced alongside the descending colon and rectum, from which it receives many branches as well as some from part of the transverse colon. The main trunk of the hepatic portal posterior to this point is now named the superior mesenteric vein. It collects from all parts of the intestine not already mentioned. In 
tracing its branches, tear the mesenteries which bind together the coils of the intestine as far as necessary and also strip off fat and lymph glands. The intestinal vein is the large vessel collecting from the greater part of the small intestine. It runs in the middle of the mesentery, receiving many tributaries in its course. The branches from the jejunum immediately beyond the duodenum, however, enter the posterior pancreatico-duodenal vein. The very large ileocaecocolic vein collects from the ileum, appendix, caecum, and ascending and transverse colons. Chief among its tributaries are: the appendicular vein from the appendix; the anterior ileocaecal vein from the sacculus rotundus, proximal part of the caecum, adjacent ileum, and ascending colon; and the posterior ileocaecal vein from the distal part of the caecum, adjacent ileum, and ascending colon.

Draw, showing the portal system.

Cat: The first tributary of the portal, on following the portal away from the liver, is the coronary vein from the stomach. This lies in the curve between pylorus and stomach and at the lesser curvature is formed by the union of many branches from both sides of the stomach. At about the same level as the entrance of the coronary vein, the anterior pancreatico-duodenal vein enters the portal; it collects from the pancreas and duodenum. At the same level as the two preceding, the right gastro-epiploic vein en ters, coming from the pyloric region and greater curvature of the stomach and adjacent greater omentum. The three veins just described may enter the portal separately or may unite with each other in any combination before entering the portal. Beyond the entrance of these small veins the hepatic portal receives a large tributary, the gastrosplenic vein. This passes to the left in the substance of the pancreas receiving one or more small middle gastro-epiploic veins from the stomach wall and omentum and a pancreatic vein from the pancreas. Beyond these tributaries the gastrosplenic is formed by the union of two main branches, the right and left splenic veins. The left splenic vein passes in the gastrosplenic ligament along the spleen, receiving branches from the spleen, the greater omentum, and several left gastro-epiploic veins from the omentum and stomach. The right splenic vein comes from the right end of the spleen, receiving also tributaries from the omentum and stomach wall. Beyond the entrance of the gastrosplenic vein the portal is known as the superior mesenteric vein. This soon receives a small posterior pancreatico-duodenal vein from the pancreas and distal part of the duodenum; next, the inferior mesenteric vein from the descending colon and rectum; and then is seen to be formed by numerous converging intestinal branches from the small intestine, caecum, and ascending colon. The lymph glands lying along the superior mesenteric vein as well as fat, should be removed in tracing the branches.

Draw the hepatic portal system.

It will be seen that the relations of the hepatic portal system are the same in mammals as in all other vertebrates. The system conveys all of the venous blood from the digestive 
tract into the capillaries of the liver. The purpose of this arrangement is that the liver cells may remove from the blood the digested food materials. There is no renal portal system in mammals, it having been completely usurped by the postcaval vein.

3. The systemic veins.-There are three systemic veins in the rabbit-two precavals and one postcaval-and two in the cat-one precaval and one postcaval. The condition in the cat is due simply to the union of the two precavals anterior to the heart (Fig. 59, p. 268.). Although the branches are similar in the two animals, they will be described separately.

a) The branches of the precaval vein: This vein is also called the anterior vena cava and descending vena cava.

Rabbit: Turn the apex of the heart forward and examine the great veins which enter the right auricle. The left precaval vein comes from the left, and passing around the left auricle enters the left side of the right auricle. It receives small coronary veins from the heart wall. The right precaval passes directly into the right anterior part of the right auricle. Note additional coronary ${ }^{\mathbf{I}}$ veins $^{2}$ entering the right auricle directly.

Carefully trace the right precaval forward, clearing away connective tissue and muscle from about its course, and follow it away from the heart. At the point of entrance into the right auricle it receives from behind the azygos vein. Press the lungs to the left and follow the azygos posteriorly along the dorsal thoracic wall near the median line. Note the intercostal veins which enter it at segmental intervals; they course along the posterior margin of each rib. Entering the precaval immediately anterior to the entrance of the azygos is the supreme intercostal vein, the first of the series of intercostal veins. Shortly anterior to this the internal mammary vein enters the precaval. This vein ascends on the internal surface of the chest very near the midventral line. Trace it posteriorly, noting branches from the intercostal muscles. It continues posteriorly on the abdominal wall as the superior epigastric vein. The next tributary of the precaval is the vertebral vein. It enters the medial side of the precaval at about the same level as the internal mammary joins the lateral side. It may be traced deep dorsally to the cervical vertebrae from which it emerges receiving a costocervical tributary from the neck. Beyond this point the precaval receives the large subclavian vein from the fore limb. Follow this laterally. It passes between the first and second ribs into the axilla and is then known as the axillary vein. Expose the axilla by cutting down through the pectoral muscles near the midventral line and at their insertion on the humerus. The pectoral muscles should then be separated from the underlying serratus ventralis but should not be removed. The large, stout white cords seen crossing the axilla are the nerves of the brachial plexus and are not to be injured. Lymph glands-small rounded masses-will also be noted in the axilla.

I The term coronary (meaning literally a crown or wreath) is in mammals unfortunately applied to two vessels, those of the heart wall and a vessel of the stomach wall having radiating branches. To avoid confusion the latter is referred to as the coronary vessel of the stomach (coronaria ventriculi). 
In the axilla the axillary vein receives the following branches: the long thoracic vein, the subscapular vein, and the cephalic vein. The long thoracic vein runs caudad on the thoracic wall in the serratus muscle; it then passes to the inner surface of the skin and extends the entire length of the abdominal wall, being especially prominent in females, where as the external mammary it collects from the mammary glands. (The greater part of this vein was probably removed with the skin.) The subscapular vein enters the axillary dorsal to the preceding. It collects a conspicuous branch (thoracodorsal vein) from the latissimus dorsi and cutaneous maximus muscles; it then passes through the teres major muscle to the external surface of the shoulder where it collects from various muscles. The cephalic $^{\mathrm{I}}$ vein is the chief superficial vein of the arm. It can best be picked up on the outer surface of the upper arm; near the distal end of the upper arm it penetrates deep between muscles and passing between the teres major and subscapularis muscles emerges on the internal surface of the shoulder and enters the axillary vein at the same place as or in common with the subscapular vein. Immediately beyond the entrance of these tributaries, the axillary vein becomes the brachial vein of the arm. This proceeds along the inner surface of the upper arm in company with an artery and a nerve.

Return to the precaval vein. At the point of entrance of the subclavian vein the precaval vein receives from the neck the external and internal jugular veins. The external jugular vein is the large vein which extends forward in the depressor conchae posterior muscle (most superficial muscle of the ventral surface of the neck). It appears as the anterior portion of the precaval. The internal jugular vein is a very small vein which runs alongside the trachea, passing the thyroid gland, and accompanying the carotid artery and the vagus nerve. The place of entrance of the internal jugular as well as its general relations are highly variable; it may enter the precaval after the latter has received the subclavian, but it usually enters with the external jugular. The precaval vein may thus be said to be formed by the union of the subclavian, external jugular, and internal jugular veins. Follow the external jugular. Shortly anterior to its union with the subclavian it receives the transverse scapular vein from the ventral end of the shoulder and near the same level has a cross-connection (transverse jugular vein) with its fellow of the opposite side (this union was probably destroyed in the previous dissection). Along the neck it receives various small tributaries from muscles and about one inch posterior to the angle of the jaws is seen to be formed by the union of two veins, the anterior and posterior facial veins. The anterior facial vein proceeds to the angle of the jaws where it is seen to be formed by the union of veins from the anterior part of the face and jaws. Its main tributaries are the angular vein, which passes over the ventral part of the masseter muscle and then turns to the region in front of the eye, and the deep facial vein which emerges between the masseter and digastric muscles and passes along the

${ }^{x}$ So named because the corresponding vein in man was formerly thought to connect with the head. 
surface of the masseter. Other tributaries of the anterior facial vein come from the nearby lymph and salivary glands. The posterior facial vein may next be followed. It passes to the parotid gland where it receives a superficial vein, the posterior auricular vein, from the back of the ear and head. The main vein beyond the entrance of this branch lies imbedded in the parotid gland which may be dissected from it. The vein is accompanied by the facial nerve. At the base of the ear it is formed by the inferior ophthalmic vein from the orbit, the temporal veins from the temporal region, and the anterior auricular vein from the region in front of the ear.

The internal jugular vein extends the length of the neck, receiving but few small branches, of which the chief ones are those from the thyroid gland. It may be traced to the occipital region of the skull, from which it emerges by way of the jugular foramen; it collects part of the blood from the brain. As already stated, its size and place of junction with the external jugular are highly variable.

The left precaval vein is identical in its tributaries with the right, except that there is no azygos vein on the left side.

Draw the branches of the precaval as far as found.

Cat: Turn the apex of the heart to the left and note the large vein which enters the anterior margin of the right auricle. This is the precaval vein. Note that there is no such vein on the left side. Instead there is a vein called the coronary sinus, which runs along the dorsal surface of the heart in the groove between the auricles and ventricles. The coronary sinus will be found by cleaning out the fat from this groove. Note the numerous coronary veins which come from the heart wall and enter the sinus. The sinus itself opens into the left posterior corner of the right auricle. It represents the reduced proximal part of a former left precaval vein. The distal part of this vein is still present and, as we shall see shortly, is united with the right precaval. Again pressing the heart to the left, clean the base of the precaval and note the large vein which passes in front of the root of the right lung and joins the precaval as the latter enters the auricle. This tributary of the precaval is the azygos vein. Trace it posteriorly, pressing the right lung to the left. It passes along the dorsal thoracic wall near the mid-dorsal line and receives at regular intervals the intercostal veins. These course along the posterior borders of the ribs. The most anterior of the intercostal veins join into a common trunk which enters the azygos shortly caudad to the entrance of the latter into the precaval. The azygos also receives small branches from the esophagus and bronchi.

Trace the precaval anteriorly. It receives small branches from the thymus gland and then receives a tributary of moderate size, the common stem of the internal mammary veins, which comes from the midventral wall of the chest. On following this posteriorly it is soon seen to be formed by the union of two veins, the internal mammary veins, which run posteriorly in the chest wall one to each side of the midventral line, and are extended onto the abdomen as the superior 
epigastric veins. In their course the two internal mammary veins receive branches from the diaphragm, chest wall, pericardium, etc. The precaval vein next receives small branches from the thymus glands and adjacent muscles, and at a level between the first and second ribs is seen to be formed by the union of two large veins. These are the brachiocephalic or innominate veins. They are the two precaval veins of embryonic stages which later unite to form the single precaval vein of adult anatomy by the crossing over of the left vein to join the right one (Fig. 59, p. 268). The branches of the two brachiocephalic veins are identical, and only one need be followed, preferably the right one, since the right side has not been touched in the previous dissection. The places of entrance of the various tributaries are, however, somewhat variable.

Immediately anterior to the junction of the two brachiocephalics, opposite the first rib, each of them receives on the dorsal side a large tributary. This is located by dissecting on the dorsal side of the vein and lifting the vein. The main part of the tributary can be traced into the cervical vertebrae; it is the vertebral vein and courses in the vertebrarterial canal, collecting from the brain and spinal cord. Before it enters the brachiocephalic the vertebral is joined by the costocervical vein, which comes from the muscles of the back, and receives branches also from the chest wall on the inner surface of the first two ribs. The costocervical vein may be picked up by turning the animal dorsal side up and, on the side where the muscles were dissected, dissecting in the serratus ventralis and the epaxial muscles. The communication of the vertebral and costocervical veins with the brachiocephalic and with each other is variable and may not be as described here.

The brachiocephalic at the same place as the entrance of the veins just described is seen to be formed by the union of two large veins, a lateral subclavian and an anterior external jugular. The subclavian will be followed first. It passes laterally in front of the first rib into the axilla, where it is known as the axillary vein. Expose the axilla by cutting through the pectoral muscles near the midventral line and at their insertion on the humerus. The pectoral muscles should then be separated from the underlying serratus ventralis but should not be removed. The stout white cords crossing the axilla are the nerves of the brachial plexus and are not to be injured. Lymph glands will also be noted in the axilla. The most medial tributary of the axillary vein is the large subscapular vein which passes through the proximal part of the upper arm to the dorsal side of the humerus and collects from various muscles of the upper arm and shoulder, receiving also the posterior circumflex vein from the external surface of the upper arm. The beginnings of the subscapular vein will be found in the trapezius muscles. The axillary vein lateral to the entrance of the subscapular receives the small ventral thoracic vein from the medial portions of the pectoral muscles. Lateral to this it receives the long thoracic vein, which runs caudad along the inner surface of the pectoral muscles; and the thoracodorsal vein, which 
courses parallel to the preceding but dorsal to it and collects chiefly from the latissimus dorsi muscle. There is a broad connection between the thoracodorsal and subscapular veins. Lateral to these branches the axillary vein is known as the brachial vein. It runs along the inner surface of the upper arm in company with nerves and the brachial artery. These structures will be found by separating the muscles on this surface of the upper arm.

Return now to the external jugular vein. It soons receives on its medial side the very small internal jugular vein which passes forward in the neck alongside the trachea in company with the carotid artery and vagus nerve. The much larger external jugular vein assumes a more superficial position and in addition to small branches from adjacent muscles receives the large transverse scapular vein from the shoulder. This passes laterally in front of the shoulder and anastomoses with the cephalic vein of the arm. The cephalic vein is the superficial vein of the fore limb and will be found on the external or lateral surface of the upper arm. It also connects with the posterior circumflex vein described above. The external jugular anterior to the entrance of the transverse scapular vein is situated in the sternomastoid muscle. On following it forward it is seen to be formed at the angle of the jaw by the union of the anterior and posterior facial veins. At their point of union they are connected across the ventral side of the throat by the transverse vein which has probably been destroyed. The anterior facial vein collects from the face and jaws and submaxillary and lymph glands, its main tributary being the angular vein from the region of the eye. The posterior facial vein emerges from the parotid gland and at the place of emergence receives the posterior auricular vein from the pinna and back of the head. The main vein then lies imbedded in the parotid gland and may be followed by dissecting away the gland. It is then seen to be formed by the union of veins from the temporal region and region anterior to the ear.

Draw the branches of the precaval as far as found.

b) The branches of the postcaval: The following description applies to both the rabbit and the cat. Turn the apex of the heart forward and note the large vein which enters the right auricle from behind. This is the postcaval vein (also called vena cava posterior or inferior and ascending vena cava). It passes posteriorly in the thorax, lying slightly to the right of the median line, inclosed in the free dorsal border of the caval fold of the pleura. Follow it caudad. It passes through the diaphragm from which it receives several phrenic veins. In the rabbit it then lies against the dorsal wall of the peritoneal cavity slightly to the right of the median line, dorsal to the right median lobe of the liver and in contact with the hepatic portal vein. It then passes into the right lateral lobe of the liver from which it emerges near the right kidney. In the cat the postcaval vein passes into the right median lobe of the liver and inclosed in the liver substance traverses the length of the liver emerging from the posterior lobule of the right lateral lobe. Note the large hepatic veins which flow from the 
liver into the postcaval vein. These are best seen by dissecting in the substance of the liver. Follow the postcaval posteriorly carefully cleaning away connective tissue and fat from it and its tributaries. It runs slightly to the right of the mid-dorsal line of the peritoneal cavity alongside the dorsal aorta which must not be injured. The first tributary of the postcaval is the right adrenolumbar vein. This passes along the posterior surface of a small gland, the adrenal gland, which lies anterior to the kidney in contact with the postcaval vein and will be found by dissecting in the fat in this location. The adrenolumbar vein receives branches for the adrenal gland (which is one of the glands of internal secretion) and also collects from the adjacent body wall. Immediately posterior to this vein the large right renal vein passes from the kidney into the postcaval. Next, by turning the viscera to the right locate the left adrenal gland and kidney and find the left adrenolumbar and renal veins. They are situated posterior to the right ones. The left adrenolumbar and renal veins generally unite to a common stem before they enter the postcaval. Into the left renal vein opens the vein of the left gonad. In male specimens this is the left internal spermatic vein; it may be traced posteriorly (in contact with the postcaval in the rabbit) to the scrotum. In female specimens it is the left ovarian vein which comes from the ovary, a small oval body lying about the middle of the lateral wall of the peritoneal cavity. The right internal spermatic or ovarian vein enters the postcaval directly, in the cat shortly posterior to the right kidney, in the rabbit much farther caudad The postcaval vein in its course along the body wall receives at regular intervals the paired lumbar veins from the wall; these are seen by loosening the vein, raising it slightly, and looking on its dorsal surface. The lumbar veins are then seen passing ventrally in the median groove between muscle masses. Near the posterior end of the peritoneal cavity the postcaval receives a pair of iliolumbar veins. Each of these in company with an artery extends laterally along the body wall and receives an anterior branch from the neighborhood of the kidney. Sometimes the left ovarian vein enters the left iliolumbar. Posterior to this point the dorsal aorta comes to lie ventral to the postcaval, concealing the latter. The dissection of the remainder of the postcaval will therefore be deferred until the aorta is studied.

It is very common for the postcaval and its branches to vary considerably from the foregoing account. An apparent splitting of the postcaval into two main trunks posterior to the kidneys is a common variation; others are mentioned in $\mathrm{R}$ and $\mathrm{J}$, page $328 .^{\mathrm{x}}$

Draw the postcaval and its branches as far as followed.

4. The pulmonary veins.-Examine the roots of the lungs and note numerous veins, several on each side, entering the left auricle from the lungs. These are the pulmonary veins. They lie to either side of the postcaval vein, those of the

s For a more detailed account of the occurrence and origin of these variations of the postcaval in the cat and man see Huntington and McClure, Analomical Record, December, I92o. 
right side passing dorsal to the postcaval, and in the rabbit those of the left side to the dorsal side of the left precaval. They convey the aerated blood from the lungs into the left auricle.

5. The pulmonary artery.-The pulmonary artery is the conspicuous vessel extending from the base of the right ventricle forward between the auricles; it soon curves to the left. Its base is generally surrounded by fat, which should be cleaned away. It divides in two at the turn into right and left pulmonary arteries. The division may be found by dissecting along the pulmonary artery immediately in front of the left auricle. Press the heart to the right and follow the left pulmonary artery into the left lung. In the rabbit it passes to the dorsal side of the left precaval vein which may now be severed. The left pulmonary artery courses parallel to and anterior to the most anterior of the pulmonary veins. Now turn the heart to the left and similarly find the right pulmonary artery proceeding to the right lung; to trace it sever the precaval vein. It lies immediately anterior to the foremost pulmonary vein. Dorsal to the right pulmonary artery lies the trachea.

6. The aorta and its branches.- Springing from the base of the left ventricle to the left of and dorsal to the pulmonary artery is a very large trunk, the aorta. Right and left coronary arteries spring from the base of the aorta where it leaves the ventricle. The left coronary artery lies between the pulmonary artery and the left auricle, and branches over the ventral and left side of the heart. The right coronary artery lies along the groove between the right auricle and right ventricle and branches to the right and dorsal surfaces of the heart.

Follow the aorta forward, cleaning away tissue from its surface. It soon describes a curve, known as the arch of the aorta, to the left. From the arch of the aorta spring the large arteries of the neck, head, and fore limbs. These are two in number in the cat, three in the rabbit. Beginning at the right they are in the rabbit: the brachiocephalic or innominate artery; the left common carotid; and the left subclavian. In the cat the branches are the brachiocephalic or innominate artery to the right and the left subclavian to the left. The difference is due to the fact that in the cat, not in man, the left common carotid branches from the brachiocephalic, and this may also occur in the rabbit as a variation.

Trace the brachiocephalic artery forward. The precaval vein and its branches may be removed. The artery gives off small branches into the thymus gland and trachea lying dorsal to it and then divides into two branches in the rabbit and three in the cat. These are: right subclavian and right common carotid in the rabbit, and right subclavian, right and left common carotids in the cat. Each of these will be traced separately.

a) Subclavian artery: Trace the right subclavian; both have identical branches.

Rabbit: From the posterior surface of the subclavian arises the internal mammary artery which follows the vein previously described along the ventral 
chest wall and continues on the abdomen as the superior epigastric artery. At the same level from the posterior surface of the subclavian, practically in common with the preceding, the supreme intercostal artery arises. It runs posteriorly on the dorsal wall of the thorax and receives the first intercostal arteries. On its anterior surface at about the same level as these the subclavian artery gives rise to the vertebral artery which passes immediately dorsad toward the cervical vertebrae where it enters the vertebrarterial canal; and to the superficial cervical artery which ascends in the lateral part of the neck, supplying various muscles, its main branch (ascending cervical) accompanying the external jugular vein. The transverse artery of the neck leaves the subclavian at the same place or in common with the supreme intercostal artery. It passes dorsally in front of the first rib through a loop formed by two nerves, and emerges on the medial side of the serratus ventralis muscle. It is best found by looking on this muscle and then tracing the artery toward the subclavian. After giving off the foregoing branches the subclavian passes in front of the first rib into the axilla where it is named the axillary artery. This lies between two of the stout nerves belonging to the brachial plexus. Its branches are similar to those of the axillary vein and accompany the veins. After giving rise to the small thoracoacromial artery to the pectoral and deltoid muscles, the axillary gives off the long thoracic and subscapular arteries, accompanying the veins previously described. The former runs posteriorly along the serratus muscle and then as the external mammary artery passes to the under surface of the skin of the lateral abdominal wall, being especially conspicuous in females. (Most of this vessel was destroyed in removing the skin.) The subscapular has a conspicuous branch (thoracodorsal artery) passing caudad to the latissimus dorsi and cutaneous maximus muscles; it then turns dorsally and perforating the teres major emerges on the outer surface of the shoulder, supplying various muscles. Near the point of origin of the subscapular the deep artery of the arm arises, and after giving off branches into the subscapular muscle passes between this muscle and the teres major to the dorsal part of the arm where it runs in company with one branch of the cephalic vein and a nerve, all three situated internal to the lateral head of the triceps which should be deflected. The axillary artery now passes to the upper arm, where as the brachial artery it courses along the inner surface of the limb in company with the brachial vein and nerves.

Draw the branches of the subclavian.

Cat: At the level of the first rib the subclavian has four branches: internal mammary, vertebral, costocervical axis, and thyrocervical axis. The internal mammary springs from the ventral surface of the subclavian, accompanies the corresponding vein along the chest wall, and passes on to the abdominal wall as the superior epigastric artery. The vertebral artery arises from the dorsal surface of the subclavian and passes dorsally into the vertebraterial canal, giving off small branches into the neck muscles. The costocervical axis divides in two almost at once. One branch, the supreme intercostal artery, passes posteriorly 
near the mid-dorsal line of the thorax, giving off intercostal branches and then supplying the deep muscles of the back. The other branch of the costocervical axis leaves the thoracic cavity, passing deep dorsally in front of the first rib, and divides into the transverse artery of the neck, supplying the serratus ventralis and rhomboideus muscles, and the deep cervical artery to the epaxial muscles of the neck. These branches are best found by looking among the muscles in question and tracing the vessels toward the subclavian. The thyrocervical axis generally arises anterior to the other branches. It passes forward near the carotid artery and, after branching to the muscles of the dorsal side of the neck, turns laterally in front of the shoulder, being then named the transverse scapular artery; it accompanies the external jugular vein for a short distance and supplies many muscles of the shoulder and neck.

The subclavian artery now passes in front of the first rib into the axilla, where it is named the axillary artery. This gives off: the ventral thoracic artery, passing medially to the medial ends of the pectoral muscles; the long thoracic artery, passing posteriorly along the middle region of the pectoral muscles and then to the latissimus dorsi; and, near the arm, the large subscapular artery. This gives off the thoracodorsal artery, lying parallel but more dorsal to the long thoracic artery and supplying the latissimus dorsi; the subscapular then turns dorsally, passes through the proximal part of the upper arm dorsal to the humerus, and branches to the muscles of the upper arm and muscles of the back and shoulder.

The axillary artery then proceeds as the brachial to the medial surface of the fore limb, where it accompanies the brachial vein and some nerves, and branches into the limb.

Draw the branches of the subclavian.

b) Common carotid artery: The two common carotid arteries arise in the cat from the brachiocephalic and immediately diverge; in the rabbit the right one arises in common with the right subclavian, while the left usually springs independently from the arch of the aorta. Trace the common carotids forward. Their branches are similar in the two animals. They pass anteriorly in the neck, one to each side of the trachea, to which they give small branches. At the level of the anterior end of the thyroid gland each supplies a superior thyroid artery to the gland. At the level of the larynx there are branches into the larynx and adjacent parts (probably destroyed) and an occipital branch into the dorsal muscles of the neck. The common carotid at about this same level gives off the internal carotid artery. In the rabbit this artery arises at the place where the carotid passes to the dorsal side of the shining ligament of the digastric. In the cat it is much smaller and arises at the same level as the occipital artery. In both animals the internal carotid passes dorsally in company with nerves and enters the skull by a foramen through the tympanic bulla. It need not be followed. The artery beyond this point is called the external carotid artery. 
At the angle of the jaw it branches to all parts of the head. Its chief branches are: the lingual artery into the tongue and the external maxillary running along the ventral border of the masseter muscle and branching to the upper and lower lips and jaws. The main artery then passes along the posterior border of the masseter muscle. It receives auricular and temporal branches from the pinna and temporal regions and then, as the internal maxillary artery, turns internal to the masseter muscle and is lost to view. It need not be followed farther.

Draw the branches of the common carotid artery.

c) The thoracic aorta: After having given rise to the subclavians and the carotids, the aorta arches to the left. Note as it passes the left pulmonary, the strong fibrous band which connects the two vessels. This is the arterial ligament or ligament of Botallus and is the remnant of the embryonic connection between the aorta and the pulmonary (Fig. 58, p. 267). Follow the aorta posteriorly, pressing the left lung to the right. It descends posteriorly lying against the dorsal wall of the thorax to the left of the median line. It is situated within the mediastinum; the mediastinal wall may be cleared away. The aorta in its course along the thorax is named the thoracic aorta. Its chief branches are the paired intercostal arteries which arise from the aorta at segmental intervals and run along the thoracic wall along the posterior margin of the ribs. The aorta also has small bronchial arteries to the bronchi and esophageal arteries to the esophagus. Along the dorsal surface of the aorta on its left side runs a delicate tube, resembling a streak of fat. This is the thoracic duct, the main lymphatic channel for the posterior part of the body. Trace it forward; its connection with the jugular vein, generally at the point of union with the subclavian, may be found.

The aorta penetrates the diaphragm to which in the rabbit it gives superior phrenic arteries and passes into the peritoneal cavity where it is known as the abdominal aorta.

d) The abdominal aorta: Turn the digestive tract to the right and locate the dorsal aorta after it has passed the diaphragm. It will be found against the dorsal wall in the median dorsal line. Clear away the mesogaster and clean the surface of the aorta. Follow it identifying its branches. These branches consist of unpaired median visceral branches to the digestive tract, paired lateral visceral branches to the kidneys and reproductive organs, and paired somatic branches to the body wall.

Shortly posterior to the diaphragm the aorta gives rise to two large unpaired visceral arteries, the coeliac and the superior mesenteric arteries. In the cat the second is shortly posterior to the first, while in the rabbit the superior mesenteric artery lies one-half inch posterior to the coeliac. As the branches of these two vessels are different in the two animals owing to the differences in their digestive tracts, it will be necessary to describe them separately.

Rabbit: The coeliac artery near its origin from the aorta gives rise to the small inferior phrenic arteries to the diaphragm. Beyond this point the splenic 
artery arises from its posterior surface. This vessel passes in the mesogaster to the spleen, where it runs in the gastrosplenic ligament. In its course to the spleen it provides the short gastric arteries to the left end of the stomach; along the spleen it supplies splenic branches to the spleen; beyond the spleen it branches into the omentum; at about the middle of the spleen a large branch, the left gastro-epiploic artery, arises from the splenic and passes to the greater curvature of the stomach. The coeliac artery beyond the splenic passes to the lesser curvature of the stomach where it may best be followed by turning the stomach forward. Here it gives off a group of vessels, the left gastric (or coronary) arteries which radiate to the stomach wall on both sides of the lesser curvature and also send small branches to the esophagus. Shortly beyond this point the coeliac artery is known as the hepatic artery, which passes along the right end of the lesser curvature, very shortly giving rise to the gastroduodenal artery. This runs to the pyloric region and there branches into the anterior pancreaticoduodenal artery to the pancreas and first part of the duodenum and the right gastro-epiploic artery which returns to the stomach wall by way of the great omentum. The hepatic artery now passes to the dorsal side of the pylorus and enters the hepatoduodenal ligament. After giving off the small right gastric artery to the pylorus it proceeds to the liver, lying to the right of the bile duct.

The superior mesenteric artery is the chief artery of the intestine and has many and complicated branches in the rabbit, these branches following for the most part the branches of the hepatic portal vein. Clean the surface of the vessel and follow it; it runs alongside the superior mesenteric vein. The first branch is the small middle colic artery, arising from the ventral wall of the superior mesenteric and passing to the transverse colon and beginning of the descending colon. At the same level but from the dorsal side arises the posterior pancreaticoduodenal artery which passes to the duodenal loop and pancreas. The superior mesenteric artery then forks into the intestinal artery, which runs in the mesentery of the small intestine and gives off numerous branches ventrally into the intestine, and into the large ileocaecocolic artery. This last has many branches to the ileum, the caecum, the appendix, and the ascending colon. Its branches are: small arteries to the terminal part of the ascending colon; the anterior right colic artery which forks several times, supplying the greater part of that portion of the ascending colon which bears the haustra; the posterior right colic artery arising near the preceding and supplying the remainder of the haustra-bearing region of the ascending colon, its end joining one end of the preceding; the appendicular artery arising with the preceding and running along the appendix and that part of the ileum adjacent to the appendix; the large posterior ileocaecal artery passing to the greater part of the caecum and to that portion of the ileum lying between the caecum and the ascending colon; the much smaller anterior ileocaecal artery to the more distal part of the caecum and adjacent ileum, and running toward the preceding with which it anastomoses; and the caecal artery or arteries to that portion of the caecum which adjoins the appendix. 
Cat: The coeliac artery passes toward the stomach and very soon divides, at about the same level, into three branches. The most cranial one is the hepatic, the next one, the left gastric, and most caudal and largest is the splenic. Trace the splenic artery. It courses in the great omentum toward the spleen and forks. One branch goes to the left end of the spleen and sends also branches into the pancreas and the short gastric arteries to the stomach. The other branch passes to the right end of the spleen and supplies also branches to the pancreas, the omentum, and the left gastro-epiploic arteries to the greater curvature. The left gastric or coronary artery passes to the lesser curvature where it splits into many branches, supplying both sides of the stomach. The hepatic artery passes along the border of the left end of the pancreas and to the dorsal side of the lesser curvature and enters the hepatoduodenal ligament. It is best found by separating the stomach and liver. It lies to the left side of the hepatic portal vein. As it passes the pylorus it gives off the large gastroduodenal branch. This branches into the anterior pancreatico-duodenal artery descending along the beginning of the duodenum and supplying also the pancreas; the right gastro-epiploic passing from the pylorus along the greater curvature of the stomach to the left; and the small pyloric artery to the pyloric region (this may also arise independently from the hepatic). The hepatic artery proceeds into the liver sending a cystic artery to the gall bladder.

The superior mesenteric artery supplies the greater part of the intestine. It passes toward the intestine. Follow it, cleaning away fat and lymph glands from its surface. It first gives rise to the middle colic artery which passes to the transverse and descending parts of the colon. A little farther on the superior mesenteric gives rise simultaneously to the posterior pancreatico-duodenal artery which ascends along the duodenum, supplying it and the pancreas and anastomosing with the anterior pancreatico-duodenal; and to the ileocolic artery to the caecum and terminal portion of the ileum and sending also a right colic branch to the ascending colon (this last may arise independently from the superior mesenteric). The superior mesenteric then divides into numerous intestinal branches to the small intestine.

Draw the branches of the coeliac and superior mesenteric.

Return now to the dorsal aorta. Its next branches are the paired adrenolumbar and renal arteries. In the rabbit the adrenolumbars are branches of the renals, but in the cat they arise independently. They pass close to the adrenal gland to which they give an adrenal branch and then course along the dorsal body wall. In the cat each sends a phrenic artery anteriorly to the diaphragm. The renal arteries are large vessels passing into the kidneys. The aorta posterior to the kidneys gives rise to the paired arteries to the gonads (these may, however, branch from the renals). They are the internal spermatic arteries in the case of the male and run posteriorly on the dorsal wall to the scrotum. In the female the corresponding ovarian arteries are larger, and in the cat con- 
voluted, and pass laterally to the ovaries. In its passage along the mid-dorsal line the aorta gives off paired lumbar arteries at segmental intervals. These are found by loosening the aorta and looking on its dorsal surface. Posterior to the genital arteries the inferior mesenteric arises as an unpaired visceral branch and passes to the descending colon and rectum, running in the mesocolon. In the mesocolon it forks into the left colic artery passing craniad along the descending colon and the superior hemorrhoidal artery passing caudad to the posterior part of the descending colon and the rectum.

Add these vessels to the drawing of the aorta.

The digestive tract may now be removed and discarded, leaving the end of the large intestine in place. Hold the stump of the colon together with the urinary bladder and in female specimens the uterus (the forked coiled tube at the posterior end of the peritoneal cavity) back against the pubes and follow the aorta farther. Near the end of the peritoneal cavity it forks into the two common iliac arteries in the rabbit; in the cat it gives off a pair of external iliac arteries followed shortly by a pair of internal iliac arteries. Anterior to this place in the cat, or in the rabbit at the level of the fork or from the common iliac arteries, a pair of iliolumbar arteries arises and passes laterally along the body wall. The iliolumbar artery divides into an anterior branch, which passes forward toward the kidney, and a posterior branch, which extends to the thigh.

The two common iliac arteries in the rabbit soon fork into an anterior external iliac and a posterior internal iliac. In the cat the external and internal iliacs arise separately from the aorta, the latter immediately posterior to the former. After giving rise to the iliacs the aorta continues in the mid-dorsal line as the small median sacral or caudal artery, lying halfway between the two internal iliacs. In the cat this vessel arises from the fork of the internal iliacs. In the rabbit it springs anterior to the forking of the aorta, from the dorsal surface of the latter; its origin is concealed by the postcaval vein and will be seen later. The sacral artery supplies the sacral region and the tail.

Follow the external iliac. It passes laterocaudad out of the peritoneal cavity, in the rabbit to the dorsal side of the inguinal ligament. As it passes through the abdominal wall or shortly beyond the wall it gives rise to the deep femoral artery (cat) or the inferior epigasiric (rabbit). In the cat this vessel gives off branches into the thigh, while these are lacking in the rabbit. In both animals the following branches are present: branches into the mass of fat between the thighs and into the external genital organs, of which one branch, in male specimens, constitutes the external spermatic artery; and the main vessel then, as the inferior epigastric artery, turns craniad and ascends in the abdominal wall, running along the inner surface of the rectus abdominis muscle. It anastomoses with the superior epigastric artery. In the rabbit there arises, either from the inferior epigastric at the origin of the latter from the external iliac or from the external iliac itself near by, the superficial epigastric artery which extends 
forward on the inner surface of the skin of the abdominal wall and anastomoses with the external mammary, a branch of the long thoracic. These vessels are particularly prominent in females, but the greater part of their course is destroyed in removing the skin. The external iliac, now named the femoral, proceeds along the center of the medial surface of the thigh, giving branches into the leg muscles. For these branches consult $\mathrm{R}$ and $\mathrm{J}$, and $\mathrm{B}$.

Follow the internal iliacs, being careful not to injure the end of the postcaval vein lying in contact with them nor any parts of the urogenital system (in males do not injure the male ducts curving around the base of the urinary bladder). The internal iliacs lie against the dorsul wall. At their origin from the common iliac (rabbit) or posterior to their origin from the dorsal aorta (cat) each gives rise to an umbilical artery which passes to the bladder or in female rabbits to the uterus first with a branch to the bladder. The internal iliacs then pass to the dorsal side of the postcaval vein. To follow them dissect as deeply as possible between the rectum and the base of the thigh. The internal iliacs give some branches to the pelvis and then each gives off a middle haemorrhoidal artery to the rectum. This accompanies the rectum to the anus but cannot be followed at this time. In female cats the uterine artery arises from the middle haemorrhoidal and passes anteriorly again to the uterus. The internal iliacs cannot be followed farther conveniently. They give branches to the tail and thigh.

Draw the branches of the iliacs adding them to the drawing of the dorsal aorta already made.

7. The posterior portion of the postcaval vein.-The postcaval may now be followed caudad from the point where it was previously left by removing the arteries which cover it. Its tributaries should be traced as far as practicable to the posterior end of the peritoneal cavity, dissecting deeply dorsally as before.

Rabbit: The postcaval receives, at the same level as the forking of the dorsal aorta, the two large external iliac veins. It then continues in the mid-dorsal line for a short distance caudad to this point, this portion often receiving the name of common internal liac vein, and is then seen to be formed by the union of the two internal iliac or hypogastric veins. Trace the external iliac; its branches are similar to and accompany those of the artery of the same name. It soon receives the vesical vein from the bladder: this vein in females also collects from the uterus. At the place where it passes through the abdominal wall, the external iliac receives the inferior epigastric vein. The main part of this runs forward along the internal surface of the rectus abdominis muscle and anastomoses anteriorly with the superior epigastric. The inferior epigastric near its place of entrance into the external iliac also receives tributaries from the fat between the bases of the thighs and the external genital region, and sends a superficial epigastric vein along the inner surface of the skin of the lateral abdominal wall. This last-named vessel is particularly conspicuous in females, but is 
destroyed in removing the skin; it anastomoses with the external mammary, a tributary of the long thoracic. The external iliac passes to the dorsal side of the inguinal ligament, and as the femoral vein continues along the medial side of the leg in company with the femoral artery. Follow the internal iliacs. After a short distance the sacral or caudal vein enters one of them, usually the left one; it accompanies the caudal artery. Caudad of this, each internal iliac receives the middle haemorrhoidal vein which ascends from the anus and lies along the side of the rectum. The tributaries of the internal iliac cannot be followed farther conveniently; they come chiefly from the gluteal region.

Draw the branches of the postcaval, adding them to the drawing previously made. The iliacs and postcaval may then be removed and the origin of the caudal artery from the aorta traced.

Cat: The postcaval is formed dorsal to the forking of the aorta by the union of the two large common iliac veins. One of them, usually the left one, receives the small sacral or caudal vein which lies parallel to the artery of the same name. About one inch posterior to its junction with the postcaval each common iliac is formed by the union of the internal iliac (hypogastric) and the external iliac. The former receives branches from the gluteal region and receives the middle haemorrhoidal vein, which runs along the sides of the rectum from the anus forward, and also collects from the bladder. The external iliac passes out of the abdominal cavity. At the place of exit it receives on its medial side the deep femoral vein, which collects from the thigh, from the fat between the thighs, from the external genital region (receiving in males the external spermatic vein from the testes), and also receives the inferior epigastric vein from the inner surface of the rectus abdominis muscle. The branches from the thigh may enter the external iliac separately. The external iliac, now known as the femoral vein, passes along the thigh, receiving branches from the leg muscles. For these consult $\mathrm{R}$ and $\mathrm{J}$.

Draw these branches as far as found, adding them to the drawing of the postcaval vein previously made.

8. The structure of the heart.-Remove the heart from the body, cutting across the bases of the great vessels. Identify the systemic veins entering the right auricle (three in the rabbit, two in the cat) and the pulmonary veins entering the left auricle. Cut into the wall of each auricle by a transverse slit and wash out the clotted blood which generally fills the interior. Note the thick ridged walls of the auricular appendages and the thinner smoother walls of the remainder of the auricle. Note the interauricular septum extending dorsally between the two auricles and completely separating them. Find the large auriculo-ventricular openings in the floor of the auricles. In the cat find near the dorsal edge of the interauricular septum the opening of the coronary sinus into the right auricle, noting the valve - the valve of the coronary sinus-which guards the opening. The coronary sinus is the remnant of the original left 
precaval vein. Cut off the apex of the heart and note the thick walls and rounded form of the left ventricle, and smaller size, thinner walls, and crescentic form of the right ventricle. Cut open the right ventricle by an oblique cut beginning at the cut surface already made and extending out through the pulmonary artery, slitting open this artery. Wash out the right ventricle. Its cavity is rather small, the walls being deeply cleft by muscular ridges, the trabeculae carnae. From the walls project a number of pointed finger-like muscles, the papillary muscles, which are connected by slender fibers, the chordae tendinae, to a thin membrane. This membrane consists of three flaps and is called the tricuspid valve. Two of the flaps can be stretched by pulling on the cut surfaces of the ventricle while the third lies collapsed against the interventricular septum, to which it is fastened without the intervention of the papillary muscles. The tricuspid valve guards the right auriculo-ventricular opening and prevents the blood from flowing back from the ventricle into the auricle. In the base of the pulmonary artery note the three pocket-shaped semilunar valves. The pulmonary artery is the sole exit from the right ventricle. Similarly cut open the left ventricle by a longitudinal slit from apex to base. Wash out the interior. The cavity of the left ventricle is considerably larger than that of the right, and its walls thicker. The two ventricles are completely separated by the interventricular septum, which appears as the common internal wall of both ventricles. Note in the left ventricle the trabeculae carnae, the papillary muscles, and the chordae tendinae. The latter are attached to the membranous bicuspid or mitral valve, which consist of but two flaps. This guards the left auriculoventricular opening and prevents the regurgitation of the blood from the ventricle back into the auricle. By probing, find the sole exit of the left ventricle, its opening into the aorta. Follow the probe by a cut and note the three semilunar valves at the base of the aorta.

Make drawings to illustrate the structure of the heart.

The removal of the heart permits a clearer view of some of the structures of the pleural cavity. The student should examine carefully the forking of the trachea into the bronchi, the form of the lungs and their relation to the pleural cavity, and the pulmonary arteries and veins.

9. Comparison of the mammalian heart and circulatory system with those of the preceding animals. - The chambers of the mammalian heart, like those of birds, are but of two kinds namely, auricles and ventricles, in contrast to the four different chambers of the fish and amphibian heart and the three of the reptilian heart. Each is, however, completely divided into two compartments, right and left, by the formation of septa in the center of the originally single chamber. The division of the auricle into two chambers begins with the Amphibia, while that of the ventricle begins in reptiles and is completed in the crocodiles, birds, and mammals. In mammals, as in reptiles and birds, the conus arteriosus has vanished, leaving as remnants the semilunar valves at the bases of the great arteries. The sinus venosus still persistent in reptiles has disappeared in birds and mammals, or, more correctly speaking, is represented by a small spot in the wall of the right auricle; this spot is not detectable by gross examination. 
The ventral aorta in mammals as in birds is split into two trunks, in contrast with the three trunks common to reptiles. These two are the pulmonary artery and the aorta. The pulmonary artery leaves the right ventricle, the aorta the left ventricle. All of the systemic veins open into the right auricle and the pulmonary veins into the left auricle. The right half of the heart is venous, and the left arterial, and owing to the completion of the interventricular septum there is no mixing of arterial and venous blood in the heart, but a complete and perfect double circulation is maintained through the heart. The venous blood passes from the

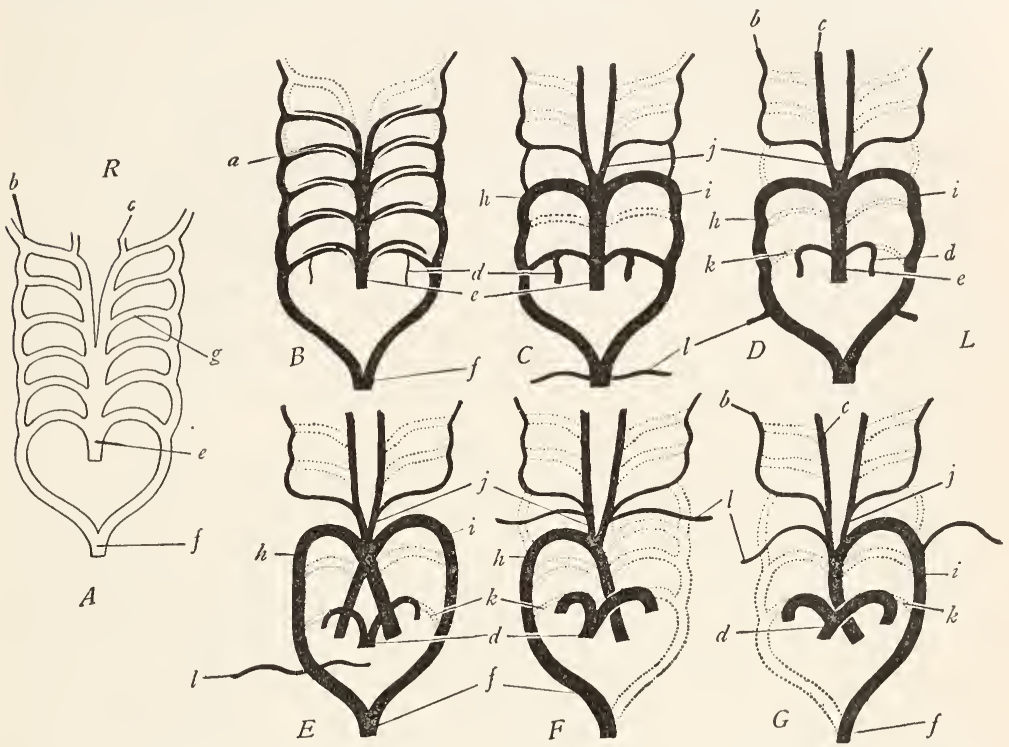

FIG. 58.-Diagrams to show the evolution of the aortic arches. $A$, primitive condition with six aortic arches. $B$, fishes, the first aortic arch missing. $C$, some urodeles, the first, second, and fifth arches missing. $D$, anurans, with the connection $k$ between the pulmonary arteries $d$ and the aorta obliterated. $E$, reptiles, showing the ventral aorta split into three trunks and the fourth aortic arch $h$ and $i$, persistent on both sides. $F$, birds, the ventral aorta split into two trunks, and the fourth aortic arch $h$ persistent on the right side only. $G$, mammals, the ventral aorta split into two trunks and the fourth aortic arch $i$ persistent on the left side only. $a$, interruption of the aortic arches by the gill capillaries in fishes; $b$, internal carotid; $c$, external carotid; $d$, pulmonary, developed from the sixth arch; $e$, ventral aorta; $f$, dorsal aorta; $g$, aortic arch; $h$, right fourth aortic arch, called the right aorta above urodeles; $i$, left fourth aortic arch, called the left aorta above urodeles; $j$, common carotid; $k$, arterial ligament or obliterated vessel originally connecting pulmonary and aorta; $l$, subclavian. (Slightly modified from Wilder's History of the Human Body, courtesy of Henry Holt and Company.)

right auricle into the right ventricle and out through the pulmonary artery to the lungs, where it is aerated. The arterial blood returns by way of the pulmonary veins to the left auricle, passes into the left ventricle, and out of the aorta.

Little trace is left in the adult mammal of the original system of aortic arches passing around the pharynx. As in all of the land vertebrates, the bases of the common carotid arteries represent the remains of the third aortic arches; the arch of the aorta is the left fourth aortic 

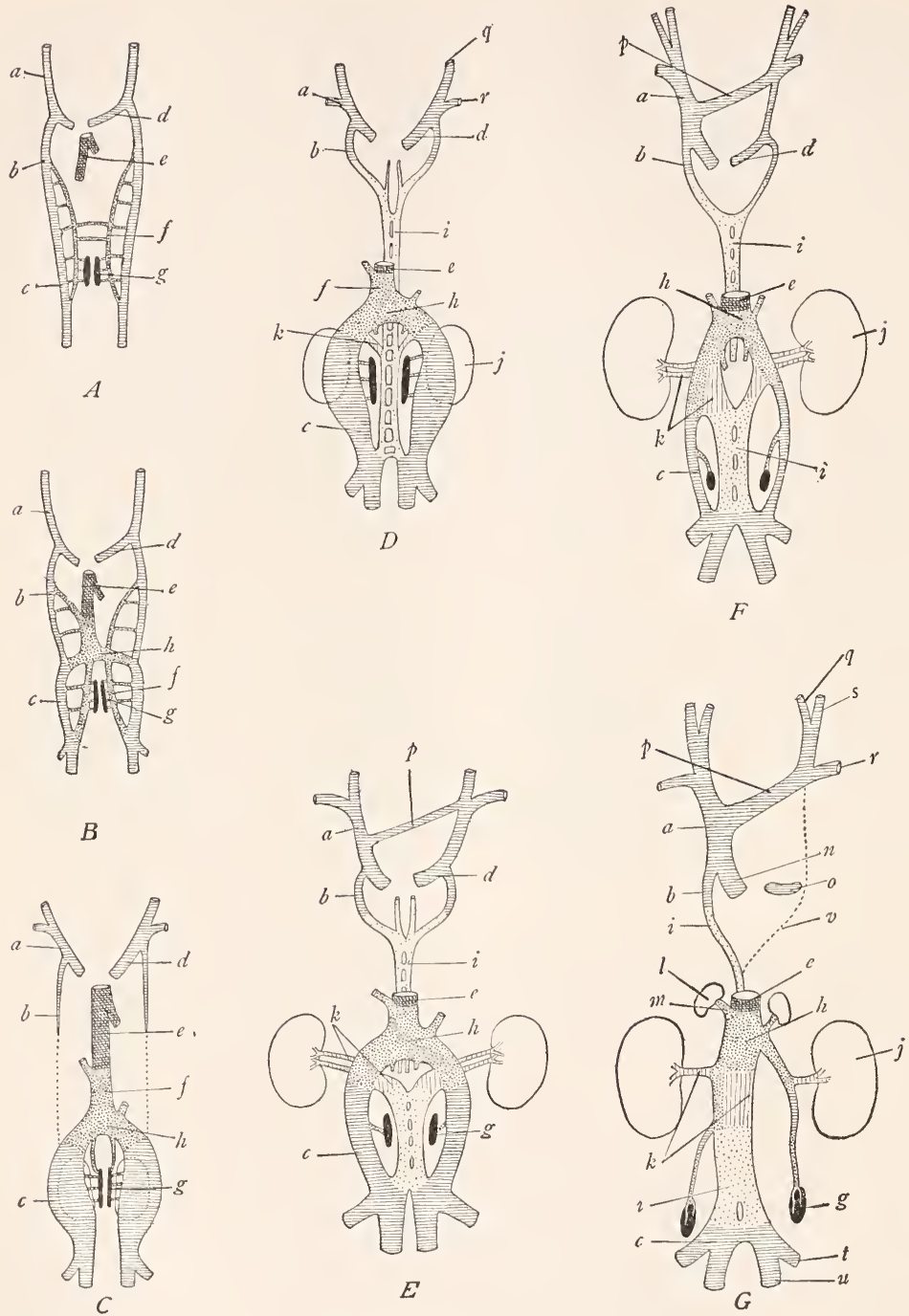

FIG. 59.-Diagrams to show the development of the postcaval vein in the cat. The cardinal system of veins is crosshatched, the subcardinal veins closely stippled, the hepatic veins indicated by cross, vertical, and oblique hatching combined, the supracardinal veins by open stippling, and the 
arch, the right side having disappeared; the fifth aortic arches are absent; the bases of the sixth arches are the right and left pulmonary arteries (Fig. $5^{8 G}$ ). The embryonic connection of the aorta and the pulmonary (Fig. 58) persists in the adult as the arterial ligament. The branches of the dorsal aorta are similar to those of the other animals studied.

The venous system is not markedly different from that of reptiles. There are two precaval veins as in the turtle, or in some mammals, the left precaval joins the right precaval by a crossconnection in front of the heart forming a single precaval (Fig. 59). The proximal end of the left precaval then becomes the coronary sinus of the heart. The coronary sinus and bases of the precavals are the common cardinal veins of the dogfish and of the embryo. The internal jugular branch is the original anterior cardinal vein, but in the adult mammal is often exceeded in importance by its branch, the external jugular. The proximal portions of the posterior cardinal veins are absent as in the turtle, except that the azygos vein, at least in part, is the remnant of the right posterior cardinal (Fig. 59). Usually, however, other veins also contribute to the formation of the azygos. The postcaval vein has the same relations as in the turtle, but has now extended itself posteriorly. Whereas in the turtle it extends no farther caudad than the posterior ends of the kidneys, it is now seen to collect from the entire posterior part of the body. This change has been accomplished very simply; the postcaval vein unites with that part of the renal portal system posterior to the kidneys. The renal portal system then passes out of existence and there is no longer in the adult any trace of a portal circulation through the kidneys. It will be remembered that it was explained in connection with the turtle that

renal collar by vertical hatching. $A$, early stage, showing the anterior and posterior cardinal veins $a, b, c$, the common cardinal vein $d$, the subcardinal veins $f$, and the outgrowth $e$ from the hepatic veins of the liver. $B$, next stage, showing the union of the hepatic outgrowth $e$ with the subcardinal veins $f$, to form the proximal part of the postcaval vein; the two subcardinals have united with each other at $h$. $C$, the anterior part of the posterior cardinal vein $b$ has separated from the posterior part $c, c$ now being the renal portal vein; the postcaval vein is seen to be formed of the hepatic vein $e$, the right subcardinal $f$, and to be united by means of the two subcardinals below $h$ with the renal portals $c . \quad D$, the supracardinal system of veins $i$, represented by open stippling, has appeared and has united anteriorly with the anterior parts of the posterior cardinals $b$, medially with the subcardinals by an anastomosis $k$, named the renal collar, and posteriorly with the renal portals $c$. $E$, union of the two anterior cardinals by a crossconnection $p$, and development of the renal veins from the renal collar $k$; the supracardinal veins have separated into anterior parts connected with the posterior cardinals $b$ and posterior parts connected with the subcardinals and renal portals $c . F$, continuation of $E$. $G$, adult stage; the left anterior cardinal joins the right by means of the cross-vein $p$, which is the left innominate vein; the common stem $a$, which is the right anterior cardinal, enters the heart by way of $n$, which is the right common cardinal vein; the left common cardinal vein persists as the coronary sinus $o$; the right anterior parts of the posterior cardinal vein and supracardinal form the azygos vein, $b$ and $i$, while on the left side these are obliterated at $v$; the postcaval vein is now complete and is seen to be composed of the hepatic vein $e$, the right subcardinal, the anastomosis between the two subcardinals at $h$, the right renal collar $k$, the posterior part of the supracardinal vein $i$, and the posterior parts of the renal portals (posterior cardinals) $c$ : the left subcardinal and posterior cardinal contribute to the vein of the left gonad, hence the asymmetrical arrangement of the genital veins of mammals. $a$, anterior cardinal; $b$, anterior part of the posterior cardinal; $c$, posterior part of posterior cardinal or renal portal; $d$, common cardinal; $e$, hepatic portion of the postcaval (this is partly removed in Figs. $D-G$ ); $f$, subcardinal; $g$, gonad; $h$, union between the two subcardinals; $i$, supracardinal; $j$, kidney (metanephros); $k$, renal collar or union between subcardinals and supracardinals; $l$, adrenal gland; $m$, vein to adrenal gland; $n$, base of the precaval vein or right common cardinal; $o$, coronary sinus or left common cardinal; $p$, left innominate or connection between the two anterior cardinals; $q$, internal jugular; $r$, subclavian; $s$, external jugular; $t$, external iliac; $u$, internal iliac. (After Huntington and McClure in the Anatomical Record, Vol. XX.) 
the renal portal veins are the posterior part of the posterior cardinal veins, and that the postcaval vein between the kidneys is formed by the subcardinal veins, chiefly the right subcardinal. Similarly in mammals the postcaval vein is formed of the distal ends of the posterior cardinals, of the right subcardinal, of the hepatic veins (vitelline) in the region of and anterior to the liver, and in the region between the kidneys and the hind limbs of other subordinate veins. This will be clearer by reference to Figure 59. The manner of origin of the postcaval from so many different sources explains the numerous variations common to this vein, such as the frequent finding of a double postcaval, and further explains the asymmetrical origin of the veins to the reproductive organs.

In conclusion it may be stated that the embryology of the mammalian circulatory system furnishes a beautiful and striking example of the repetition of evolutionary stages. In its development the mammalian circulatory system passes successively through each of the stages which we have found to persist as the adult condition in the types we have studied, and the evolution of this system can be determined equally well either by studying its development in the mammal or by studying and comparing its form in the adults of the different classes of vertebrates, which were ancestral to the mammal.

\section{G. SUMMARY OF THE CIRCULATORY SYSTEM}

I. The entire circulatory system is derived from the mesoderm.

2. The first blood vessels are the vitelline (omphalomesenteric) veins. These course along the intestine and are continued posteriorly as the subintestinal vein. In forms with yolk sacs they are the veins of the yolk sac.

3. As the walls of the hypomere fuse on the ventral side of the embryo the two vitelline veins unite to form the heart. The heart lies in the median ventral part of the body inclosed in the ventral mesentery.

4. The anterior end of the heart continues forward as the ventral aorta.

5. The ventral aorta forms a series of loops, the aortic arches, around the pharynx. These unite dorsally to form the dorsal aortae, at first double, but subsequently fusing to a single vessel. In typical vertebrates there are six pairs of aortic arches.

6. The dorsal aorta proceeds posteriorly along the mid-dorsal line of the coelom, supplying branches to all parts of the body below the heart.

7. The chief somatic veins at first are the anterior and posterior cardinal veins uniting at the level of the heart to a common cardinal vein on each side which enters the sinus venosus; the subcardinal veins extending along the kidneys; and the vein of the lateral body wall, the abdominal or umbilical vein, opening into the common cardinal.

8. Both arteries and veins are provided with paired segmental and unpaired non-segmental branches. The former are of two kinds: the somatic vessels to the products of the epimere, and the lateral visceral branches to the products of the mesomere. The unpaired branches consist of visceral vessels to the digestive tube. The vessels of the appendages-subclavian to the anterior appendages, iliac to the posterior appendages-consist simply of enlarged somatic vessels.

9. In all vertebrates the vitelline and subintestinal veins become converted into the hepatic portal system, as explained in the introduction of this section. The proximal portions between the liver and the heart form the hepatic veins; within the liver a network of capillaries is produced; posterior to the liver one of the vitellines with the subintestinal becomes the hepatic portal vein.

10. The renal portal system develops from the posterior parts of the posterior cardinal veins which detach from the anterior portions and are then known as the renal portal veins. They collect from the tail and pour their blood into the kidneys from which the blood passes into 
the subcardinal veins which establish connections with the anterior portions of the posterior cardinals.

II. The venous system of fishes is in essentially the stage reached to this point, with a renal portal and a hepatic portal system, and the cardinal veins as the chief somatic veins. In fishes there is also an inferior jugular vein in the head region.

1 2. The arterial system of fishes is also in a primitive condition. There is a ventral aorta connected by aortic arches with a dorsal aorta which supplies the body below the heart. Of the original six pairs of aortic arches the first is missing, the second imperfect, and only the last four are retained entire. In fishes the central part of each aortic arch is secondarily broken up into capillaries in the gill region; the ventral halves of the arches then become the afferent branchial arteries, the dorsal halves the efferent branchial arteries.

13. The primitive vertebrate heart is a bent tube consisting of four chambers, named from the posterior end forward: sinus venosus, auricle, ventricle, and conus arteriosus. There is a single circulation, composed of venous blood, through the heart; the blood enters the sinus and passes through the chambers of the heart in the order named. This condition is permanent in fishes (except Dipnoi).

14. The anterior cardinal veins persist in all vertebrates as the internal jugular veins. These unite with other veins of the anterior part of the body to form large trunks, the precaval veins. The bases of the precavals are the common cardinal veins. In some mammals the left precaval joins the right precaval in front of the heart, forming a single trunk. In such cases the reduced base of the left precaval (left common cardinal) remains as the coronary sinus, a vein of the heart wall.

I5. The anterior portions of the posterior cardinal veins lose their importance in land vertebrates and disappear above urodeles, except that in mammals the proximal portion of the right one contributes to the azygos. The posterior portions of the posterior cardinals become the renal portal veins in all vertebrates. In fishes they collect only from the tail, while above fishes they also usurp the veins from the leg which at first open into the abdominal veins. The renal portal veins in fishes, Amphibia, and reptiles flow into a capillary system in the kidneys, from which the blood is re-collected into the subcardinal veins which run between the two kidneys.

I6. Simultaneously with these changes in the posterior cardinals there appears a new vein, the postcaval vein. It is found in a few fishes and is present continuously from the Amphibia onward. It is formed by the union of a hepatic vein in the liver (which is in turn derived from the vitellines) with the subcardinal veins, chiefly the right subcardinal. The subcardinals then become parts of the postcaval vein.

I7. Simultaneously with these changes the abdominal veins, which become connected with the renal portal veins when the latter usurp the veins from the legs, change their anterior connections. Whereas they originally entered the common cardinal veins, they now enter the hepatic portal vein. The abdominal veins thus come to constitute a connection between the renal portal and hepatic portal systems.

18. In Amphibia and reptiles the postcaval vein extends only to the posterior end of the kidneys.

I9. In birds the postcaval vein establishes direct connections with the renal portal veins; the renal portal circulation is thus greatly reduced. In mammals the connection is completed so that the veins from the legs, tail, and adjacent regions pass directly into the postcaval. The renal portal circulation then vanishes.

20. With the changes outlined in paragraph $\mathrm{i} 8$ the abdominal vein loses its function. It is probably present in birds but has established different connections and is lacking in mammals except monotremes. In embryonic stages, however, this vein (or veins) is of great importance as the veins of the allantois or embryonic respiratory organ. 
21. The hepatic portal system remains unchanged throughout.

22. The ventral aorta persists as a single vessel in fishes and Amphibia. In reptiles it splits into three trunks, right and left aortae and pulmonary; in birds and mammals into two trunks, the aorta and the pulmonary.

23. The aortic arches are more or less modified in land vertebrates. In all of these the first two have vanished; and in all but certain Amphibia the fifth has likewise disappeared. In these Amphibia (urodeles and Gymnophiona) the three (or four) remaining arches still form complete arches, connecting with the dorsal aorta. In Anura, reptiles, birds, and mammals the third and sixth arches lose their connection with the dorsal aorta, only the fourth arches retaining this connection. The third arches persist as the bases of the carotid arteries, the fourth arches form the aorta, and the bases of the sixth arches become the right and left pulmonary arteries. In Anura and reptiles both fourth arches persist, forming right and left aortic arches which unite dorsally to produce the dorsal aorta; but in birds the left fourth arch and in mammals the right fourth arch disappear. The right fourth in birds and the left fourth in mammals then remain as a single arch connecting the dorsal aorta with the left ventricle. The dorsal aorta in all vertebrates is the main artery of the body posterior to the heart.

24. The pulmonary veins appear as new structures in the air-breathing vertebrates. They enter the left auricle. Simultaneously there occurs a change in the heart and the double circulation is initiated.

25. In Amphibia the four chambers of the heart are retained as in fishes, but the auricle is partially or completely divided into right and left auricles. The sinus venosus is then attached to the right auricle, while the pulmonary veins enter the left auricle. The right side of the heart consequently contains venous blood and the left side arterial blood. There is then a double circulation through the heart, but the two kinds of blood are imperfectly separated.

26. Above Amphibia the conus arteriosus is reduced to valves (the semilunar valves) at the bases of the great arteries which thereupon spring directly from the ventricles.

27. In reptiles the sinus venosus is retained, the two auricles are completely separated, the ventricle is usually incompletely separated into right and left chambers. The double circulation is present but imperfect as in Amphibia.

28. In birds and mammals the sinus venosus is reduced to a mere node in the wall of the right auricle, the systemic veins then entering the right auricle directly. The ventricle is completely divided by a partition into right and left ventricles; consequently the two circulations through the heart are wholly separated. The right side of the heart is venous, the left arterial. 


\section{THE COMPARATIVE ANATOMY OF THE UROGENITAL SYSTEM}

The excretory organs of vertebrates consist of a pair of kidneys and their ducts and the reproductive system of a pair of gonads or sexual glands and their ducts. These two systems are generally closely united, since the reproductive organs use the ducts of the kidneys to convey their products to the exterior. Hence it is customary to consider the two systems together as the urogenital (urinogenital) system.

\section{A. EMBRYONIC ORIGIN AND EVOLUTION OF THE UROGENITAL SYSTEM}

I. Development of the kidneys.-The kidneys or excretory organs arise from the mesomere; this it will be recalled is the small middle region of the mesoderm. Within the mesomere there are first produced the tubules of the kidneys. These tubules arise through a hollowing out of the tissue of the mesomere. The tubules of the kidney belong to the category of ducts known as coelomoducts..$^{.}$Each excretory tubule opens into the coelom (cavity of the hypomere) by means of a ciliated funnel-shaped opening called a nephrostome, pursues a more or less winding course in the mesomere and finally terminates in the collecting duct. At first there is a pair of tubules to each segment, but later they increase greatly in number and become compacted together into a definite body which is called a kidney. In connection with the kidneys there are developed peculiar networks of capillaries which are known as glomeruli. Each glomerulus consists of a knot of capillaries into which blood is brought by an artery and conveyed away by a vein. This arrangement evidently serves to bring the blood into closer contact with the cells of the kidneys. The glomeruli come into close relationship with the kidney tubules.

2. The kidneys and their ducts. - We have thus far spoken of the kidneys as if the kidneys of all vertebrates were homologous organs. This is not, however, the case. On the contrary, in the evolution of the vertebrates there have been present three more or less distinct excretory organs, which are similar in structure and arise in a similar manner from the mesomere but are nevertheless not identical (Fig. 60).

a) The pronephros: The first kidneys to appear in the chordate phylum are the paired pronephroi (singular, pronephros). The pronephroi arise from the anterior end of the mesomere, far forward in the body and extend over several segments (Fig. 60A). They are composed of tubules, each pronephros possessing one tubule to each segment of the body. Each tubule consists of a simple curved tube, opening at one end into the coelom by a nephrostome and at the other end into a common duct, the pronephric duct, which collects from all of the tubules. The pronephroi are present in the adult stage of only cyclostomes and a very few fishes; they appear, however, as transient structures during the development of all other vertebrates. In most fishes and Amphibia they are probably functional as excretory organs during embryonic stages. In reptiles, birds, and mammals they appear during early embryonic development, but do not develop completely, do not function, and soon disintegrate and disappear. Glomeruli are generally present in connection with the pronephroi but may be absent even in

${ }^{1}$ It was formerly supposed and is commonly taught that the tubules are homologous with the nephridia of the earthworm and other oligochaetes. It has been shown, however, that the nephridia are of ectodermal origin, while the tubules of the vertebrate kidney are of mesodermal origin. It is therefore desirable to adopt the terminology of Goodrich, designating as nephridia such excretory or genital ducts as are homologous with the nephridia of the earthworm; while those genital or excretory ducts or tubules which arise in the coelomic wall are named coelomoducts. 
functional cases. The glomeruli of the pronephros are generally of the type known as external glomeruli, that is, they project into the coelom adjacent to the nephrostome and are not directly associated with the tubule (Fig. 6I). In some cases, however, internal glomeruli which project into the tubule occur.

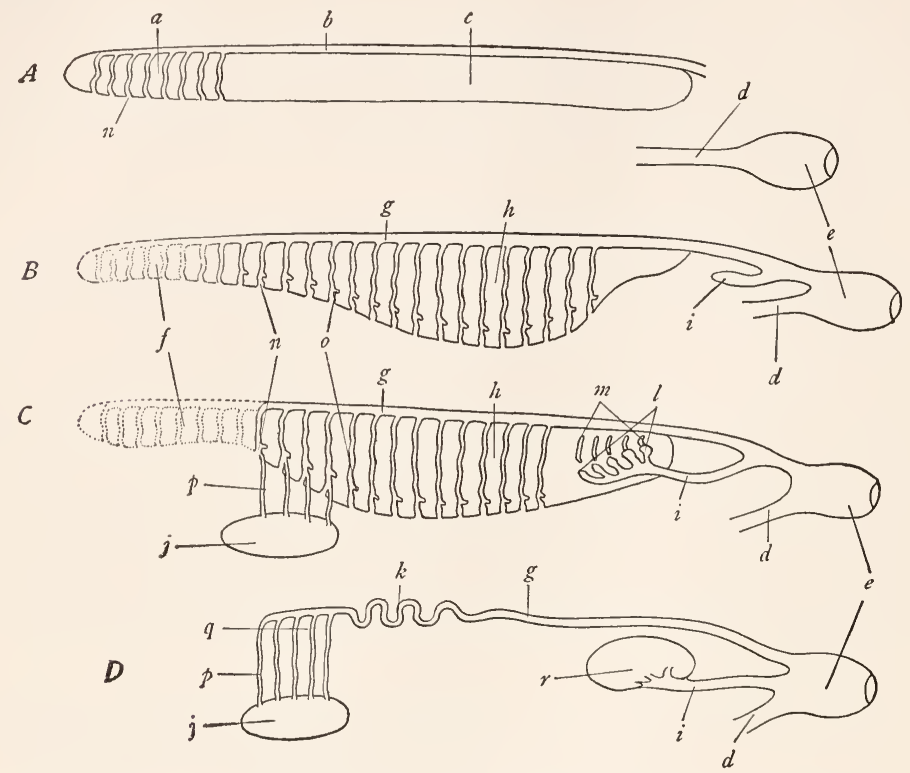

FIg. 60.-Diagrams to show the development of the three kidneys and their ducts and their relation to the male gonad. $A$, early stage showing the pronephros $a$ developing from the anterior end of the mesomere $c$ and the pronephric duct $b$ which has not yet reached the cloaca $e . B$, next stage, illustrating the degeneration of the pronephros at $f$, the development of the mesonephros $h$ from the middle portion of the mesomere, the junction of the pronephric duct, now the mesonephric duct $g$, with the cloaca and the beginning of the metanephric evagination $i$ from the mesonephric duct. $C$, later stage, showing connection between certain tubules of the mesonephros and the testis $j$ by means of tubules, the vasa efferentia $p$, which grow out from the mesonephros; and the penetration of the metanephric evagination into the posterior end of the mesomere where it is subdividing to form the collecting apparatus $l$, which becomes associated with secretory metanephric tubules $m$, developed in the mesomere. $D$, final stage, in which the mesonephros has disappeared except for the remnant $q$ which connects with the testis $j$ by means of the vasa efferentia $p$; the mesonephric duct $g$ persists as the vas deferens; the two parts of the metanephros shown in $C$ have united to form a single organ $r$. $a$, pronephros; $b$, pronephric duct; $c$, mesomere or nephrotome; $d$, intestine; $e$, cloaca; $f$, degenerating pronephros; $g$, mesonephric or Wolffian duct; $h$, mesonephros or Wolffian body; $i$, metanephric evagination from the Wolffian duct in $B$, ureter in $C$ and $D ; j$, testis; $k$, coiled portion of the vas deferens forming part of the epididymis; $l$, collecting part of the metanephros derived from the Wolffian duct; $m$, execretory tubules of the metanephros derived from the mesomere; $n$, nephrostome; $o$, renal corpuscle or Malpighian body; $p$, vasa efferentia; $q$, remnant of the mesonephros, forming part of the epididymis; $r$, metanephros.

b) Mesonephros: The second vertebrate kidneys are the paired mesonephroi (singular, mesonephros), also known as the Wolffian bodies, after the embryologist Wolff, who first 
described them. These develop in the mesomere immediately posterior to the pronephroi, even overlapping the posterior ends of the pronephroi. They extend over a number of segments, and are much larger than the pronephroi. As they develop, the pronephroi generally degenerate. The mesonephros consists of many tubules, a number to each segment; these, like the pronephric tubules, open at one end into the coelom by nephrostomes and at the other end terminate in the pronephric duct (Fig. 6oB). Although the pronephroi vanish, the pronephric ducts persist; they grow posteriorly alongside the region where the mesonephroi are developing, and become the ducts of the latter. They are then known as the mesonephric or Wolffian ducts. The mesonephric ducts grow posteriorly until they reach the caudal end of the intestine. To this they become connected, pouring their contents into the terminal chamber of the intestine, which consequently receives the name of cloaca (Latin, "a sewer"). The structure

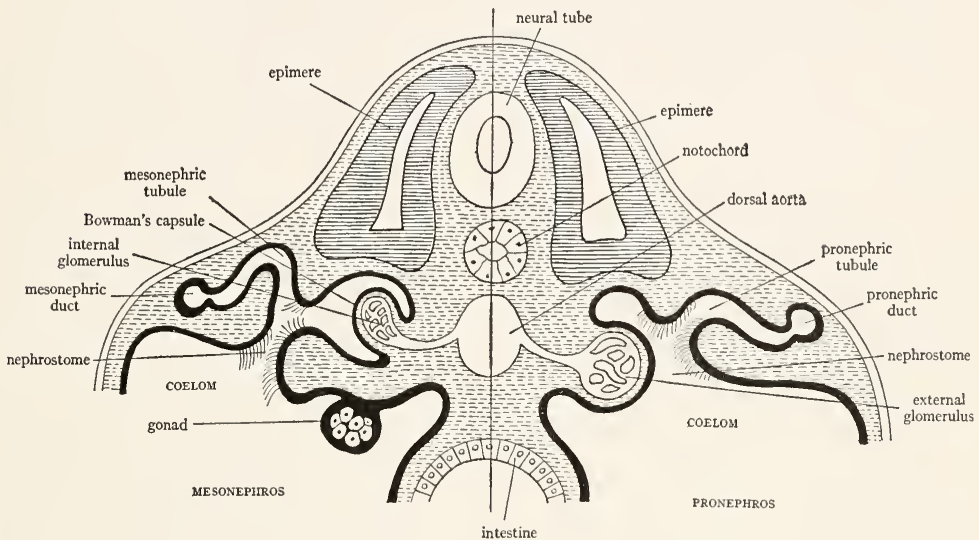

FIG. 6r.-Diagram to show the structure of the pronephros and mesonephros. Pronephros on the right, mesonephros on the left. The chief difference is in the relation of the glomerulus; in the pronephros it projects into the coelom; in the mesonephros it projects into the tubule, which forms a Bowman's capsule about it. (From Wiedersheim's Comparative Anatomy of Vertebrates, courtesy of the Macmillan Company.)

of the mesonephros differs from that of the pronephros. The tubules are more numerous, longer, and more convoluted. The glomeruli are always of the internal kind (Fig. 6I). Each glomerulus projects into the mesonephros, carrying the wall of the tubule before it to form a flask-shaped capsule in which the glomerulus rests. The capsule is named Bormman's capsule, and this, together with the glomerulus, forms a rounded body known as the Malpighian body or renal corpuscle. The mesonephric tubules generally open into the coelom by nephrostomes which are situated near the renal corpuscles.

The mesonephroi are the kidneys of the adults of fishes and Amphibia, and are also the chief functional kidneys of cyclostomes, although the latter possess in addition persistent pronephroi. The mesonephric or Wolffian ducts are the excretory ducts in these vertebrates, opening into the cloaca. The mesonephroi appear in the embryos of reptiles, birds, and mammals, and are in most cases functional in the embryos, often becoming of large size. They generally, however, lack nephrostomes. They degenerate before hatching or birth, or very shortly after. 
c) The metanephroi: The third kidneys of vertebrates are the paired metanephroi. The metanephroi arise in part from the remainder of the mesomere, posterior to the mesonephroi; and in part as an outgrowth from the Wolffian ducts. This outgrowth occurs as an evagination from the Wolffian duct near its connection with the cloaca; it pushes anteriorly and fuses with that portion of the metanephros arising from the mesomere (Fig. $6 \circ B$ and $C$ ). The metanephros is thus an organ of double origin. That part which comes from the mesomere develops into the tubules of the kidney and the renal corpuscles; that part derived from the Wolffian duct becomes the collecting tubules and the spaces (calices) into which they empty. The stalk of the outgrowth from the Wolffian duct becomes the metanephric duct or ureter, ${ }^{\mathrm{r}}$ which conveys the excretion of the metanephros to the cloaca. The structure of the metanephros is similar to that of the mesonephros, except that nephrostomes are wanting. The metanephric tubules are very long and complicated, with several loops and convolutions. The metanephroi are the kidneys of the adults of all reptiles, birds, and mammals.

From the foregoing account it will be seen that the three pairs of vertebrate kidneys arise successively from the mesomere, each situated caudad to the preceding one. For this reason it seems probable that they represent persisting parts of a kidney which originally extended the entire length of the mesomere. The anterior part of this hypothetical kidney has gradually degenerated so that the kidney appears to descend posteriorly. This disappearance of organs from anterior segments is common in heteronomously segmented animals.

3. The urinary bladder.-The urinary bladder is in all forms above fishes a saclike evagination from the ventral wall of the cloaca. In fishes, the bladder when present is formed in part by the enlargement of the terminal portions of the Wolffian ducts as well as by a cloacal evagination. In the embryos of reptiles, birds, and mammals, there is an enormous cloacal evagination, the allantois, which may be regarded as a greatly expanded urinary bladder. The allantois serves respiratory as well as urinary functions. The adult bladder develops at the base of the allantoic stalk. Adult birds lack a urinary bladder. The excretory ducts do not generally open into the urinary bladder directly, except in mammals.

4. The gonads and their ducts.-The gonads or reproductive organs consist of a pair of lestes in the male, ovaries in the female. These organs arise as swellings, the genital ridges, on the ventral wall of the mesomere, immediately to the medial side of the kidneys. They project into the coelom and in the mature condition are generally provided with mesenteries.

a) Male: The testis consists of a mass of tubules, the seminiferous tubules, which empty into a network of tubules called the rete, which is situated usually along the attached part of the testis. Tubular connections are established during embryonic stages between the rete and the Bowman's capsules of the anterior part of the adjacent mesonephros (Fig. 60C). These connections are called the vasa efferentia, and they traverse the mesorchium into the mesonephros. The vasa efferentia are then continuous within the mesonephros with certain of the mesonephric tubules and through them find exit into the Wolffian duct. It thus happens that in nearly all vertebrates the Wolffian duct serves as the male duct. In elasmobranchs and Amphibia, in which the mesonephros is the functional adult kidney, the Wolffian duct in males serves as both excretory and male duct (Fig. 62A). In male reptiles, birds, and mammals, where the mesonephros has disappeared, the Wolffian duct remains as the male duct, and is then named the vas deferens (Fig. ${ }_{6} \mathrm{C}$ ). In these groups the mesonephros disappears, except those mesonephric tubules which have established connections with the rete testis. These tubules constitute the vasa efferentia and a part of the epididymis. Epididymis is the name given to that part of the male duct which is convoluted.

I A great deal of confusion will be avoided by reserving the term ureter for the duct of the metanephros. 

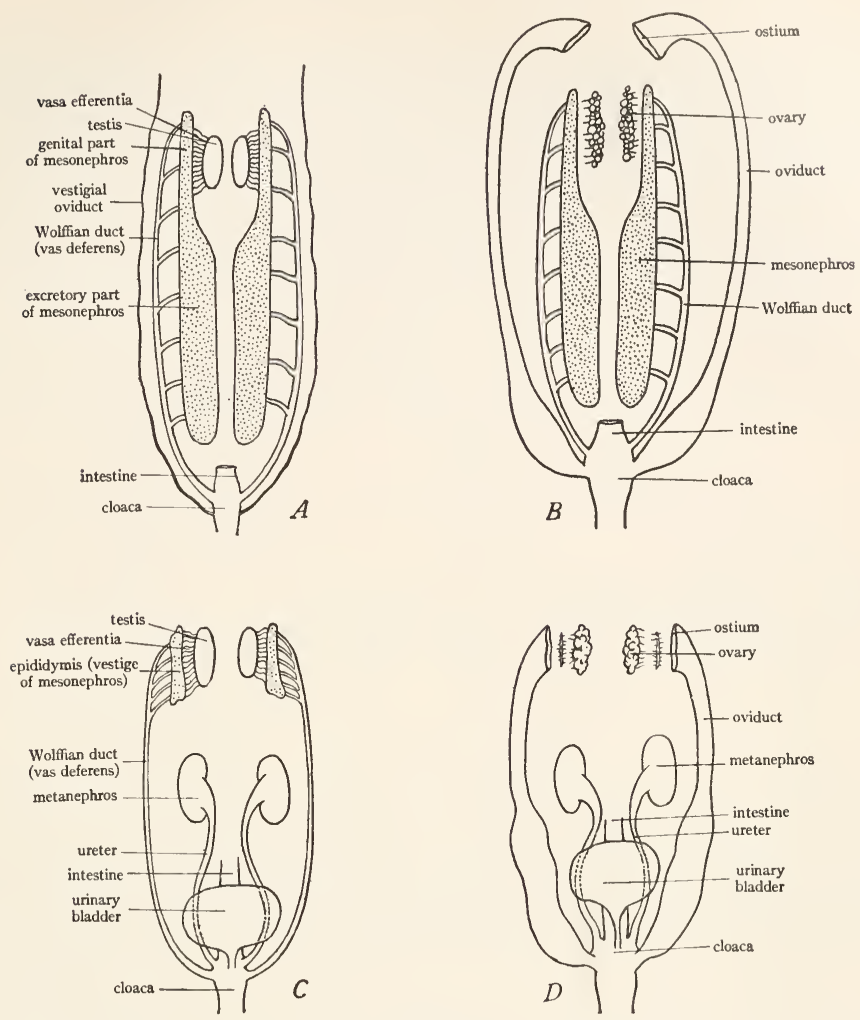

Fig. 62.-Diagrams to illustrate the urogenital systems of male and female anamniotes and amniotes. A, male elasmobranch or amphibian; the mesonephros is differentiated into anterior genital and posterior excretory portions; the genital part is connected with the testis by means of the vasa efferentia which are outgrowths from the mesonephros; the mesonephric or Wolffian duct serves as both genital and excretory duct; the oviduct or Müllerian duct is vestigial. $B$, female elasmobranch or amphibian; the ovary is not connected with the mesonephros; the mesonephros and mesonephric duct serve only excretory functions; the oviduct is well developed and opens into the coelom by the ostium near the ovary. $C$, male reptile, bird, or mammal; the excretory part of the mesonephros has disappeared but the genital part persists as the epididymis (in part) which is connected as in anamniotes with the testis by means of the vasa efferentia; the Wolffian duct is purely genital and is renamed the vas deferens; the excretory function is served by metanephroi and ureters. $D$, female reptile, bird, or mammal; the mesonephros and Wolffian duct have entirely vanished; the condition of the ovary and oviduct is the same as in anamniotes; the excretory function is served by the metanephroi and ureters exactly as in the male. (The changes in the relation of the urogenital ducts and cloaca which occur in mammals are not indicated in these figures but are shown in Figs. 63 and 64.) (Slightly modified from Wilder's History of the Human Body, courtesy of Henry Holt and Company.) 
b) Female: The ovaries are masses of connective tissue containing the developing eggs, each egg surrounded by a capsule of nutritive cells forming a follicle. The ovaries, unlike the testes, never have any connection with the kidneys. The ducts of the ovaries are named the oviducts or Millerian ducts. The origin of the Müllerian ducts is somewhat problematical. In elasmobranchs they arise by a splitting of the pronephric duct; half of the pronephric duct then becomes the oviduct, and the other half becomes the mesonephric duct. Although this mode of origin is the one commonly accepted, it cannot be demonstrated for other vertebrates; in them the oviducts arise independently in the mesomere. The oviducts are never directly connected with the ovaries. They open into the coelom near the ovaries

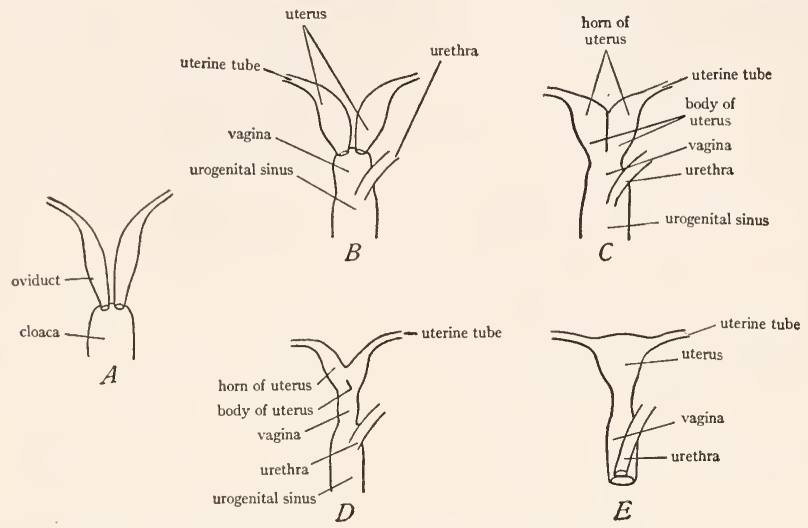

FIG. 63.-Diagrams to show the various types of mammalian oviducts. $A$, condition found in the majority of female vertebrates; the two oviducts are completely separate and open independently into the cloaca. $B-E$, various conditions found in mammals, showing differentiation of the oviducts into uterine tube, uterus, and vagina, and progressive fusion of the lower parts of the oviducts: $B, d u p l e x$ type found in rodents, in which the two vaginae are united to one; $C$, bipartite type occurring in carnivores; not only are the vaginae fused but the lower parts of the two uteri are fused to form a single body, divided in two by a partition which represents the fused walls of the two uteri; the upper parts of the two uteri remain separate as the horns; $D$, bicornuate type, found in many ungulates, similar to $C$ except that the partition has disappeared; $E$, simplex type, occurring in man and the apes, in which both vaginae and uteri are fused along their entire lengths leaving only the uterine tubes separate. Note further that in $B-D$ the urethra joins the vagina to form the urogenital sinus which opens to the exterior, while in $E$ the urethra and vagina are wholly separate and open independently to the exterior. (From Wiedersheim's Comparative Anatomy of Vertebrates, courtesy of the Macmillan Company.)

by a funnel-shaped opening, the ostium, which probably represents one or more of the nephrostomes of the pronephros (Fig. $6{ }_{2} B$ and $D$ ). The eggs escape from the ovary by rupture of the ovarian wall, pass into the ostium of the oviducts by methods which are not always understood, and are conveyed down the oviducts.

The oviducts in the majority of vertebrates remain as two separate tubes opening into the cloaca (Fig. 63A). In mammals each oviduct is differentiated into a narrower anterior portion called the uterine or Fallopian tube, which bears the ostium, and a wider more muscular posterior portion, the uterus. In the monotremes, or egg-laying mammals, each uterus opens separately into the cloaca. In the marsupials the terminal portion of the uterus is differentiated 
as a vagina to receive the penis. In mammals above marsupials the two vaginae fuse to a single vagina (hence the name Monodelphia). There is also generally more or less fusion of the two uteri (Fig. 63). When only the posterior portions of uteri are fused, the fused portion is called the body of the uterus and the separate portions the horns of the uterus. In man and other primates the uteri are fused along their entire length producing the single uterus or womb. The young of the placental mammals develop only in the uterine part of the oviducts; in those forms with partially fused uteri, only in the horns.

5. The evolution of the cloaca. - The cloaca is found in all vertebrates except cyclostomes, teleostomes, and the placental mammals. It receives the termination of the intestine and the urinary and genital ducts. From the preceding account it will be evident that in the males of elasmobranchs and Amphibia the cloaca receives only the Wolffian ducts, while in the females both oviducts and Wolffian ducts enter it (Fig. $62 A$ and $B$ ). It commonly happens, however, that in the males of these groups vestiges of the oviducts are present. In the males
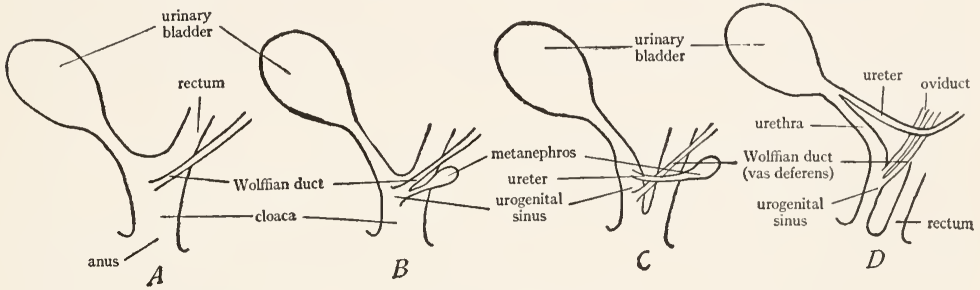

FIG. 64.-Diagrams to illustrate the changes in the cloaca in mammals during development. $A$, early embryonic stage, showing the cloaca receiving the urinary bladder, the rectum, and the Wolffian duct, as in the lower vertebrates. $B$, later stage, showing the beginning of the fold which divides the cloaca into a ventral urogenital sinus which receives the urinary bladder, Wolffian ducts, and ureters, and into a dorsal part which receives the rectum. $C$, further progress of the fold, dividing the cloaca into urogenital sinus and rectum; the ureter has separated from the Wolfian duct and is shifting anteriorly. $D$, completion of the fold, showing complete separation of the cloaca into ventral urogenital sinus and dorsal rectum. Note in $D$ that the ureter has shifted farther so that it opens into the urinary bladder.

of reptiles and birds the cloaca receives the Wolffian ducts (vasa deferentia) and the ureters; in the females the oviducts and the ureters (Fig. $6{ }_{2} C$ and $D$ ). In addition, in many fishes, Amphibia, reptiles, and the embryos of birds and mammals the urinary bladder opens into the ventral wall of the cloaca. Adult birds have no urinary bladder; mammals have one, but it is no longer attached to the digestive tract.

In placental mammals marked changes occur in the relations of the terminal portions of the urogenital ducts. In the embryo the cloaca becomes divided by a fold which extends posteriorly to the body wall and separates the cloaca into two parts, each with its own opening to the exterior (Fig. 64). The dorsal part includes the intestine only; this terminal portion of the intestine is called the rectum and opens to the exterior by the anus. The ventral part separated from the cloaca is called the urogenital sinus. It receives the stalk of the bladder and the excretory and genital ducts. The excretory ducts (either Wolffian ducts or ureters) at first open into the urogenital sinus, but subsequently the ureters shift so as to open into the bladder, in all of the placental mammals (Fig. 64). Thus, the ureters pass into the bladder while the Wolffian ducts (vasa deferentia) in males or the vagina in females unite with the duct of the bladder, named the urethra, forming a common tube or chamber, the urogenital sinus, which opens externally in front of the anus by a urogenital aperture. In the females only of the 
highest placental mammals there is no urogenital sinus, but the urethra and the vagina are separate and open separately to the exterior, the former anterior to the latter. In such cases there are three openings in the perineum: the anus, the mouth of the vagina, and the mouth of the urethra.

For more complete accounts of the comparative anatomy of the urogenital system, the appropriate chapters of $\mathrm{K}, \mathrm{W}$, or $\mathrm{Wd}$ should be consulted.

\section{B. THE UROGENITAL SYSTEM OF ELASMOBRANCHS}

The following outline applies to the smooth and spiny dogfish and the skate. the latter being described separately. The majority of the dogfish used for dissection are immature, and it is therefore difficult or impossible to locate in them all of the parts of the urogenital system. At least a few mature males and females will be on hand for demonstration. The skates are sexually mature when still relatively small, and most of the specimens used have fully developed reproductive systems.

Remove the digestive tract except cloaca and liver.

I. The female urogenital system.-

Dogfish: The ovaries are a pair of soft bodies, situated dorsally. In the spiny dogfish they are oval in form and located dorsal to the liver, each with a mesentery, the mesovarium. In the smooth species they are long and slender, extending the whole length of the coelom, more or less fused, and attached posteriorly to the mesentery of the rectal gland. Their posterior ends are toothed. There is single mesovarium for the two ovaries. In both species when mature, the large eggs, consisting chiefly of yolk, are readily noted in the ovaries.

Locate the kidneys lying against the dorsal body wall, one to each side of the dorsal aorta. They are long, slender, brown bodies. Their posterior portions are broader and thicker than the anterior portions and probably perform most of the work of excretion. The kidneys are retroperitoneal. Free their lateral borders by slitting the pleuroperitoneum and note thickness of the organ at different levels. The kidneys are mesonephroi; the posterior thicker part may be named the caudal mesonephros, the anterior more slender part the cranial mesonephros. ${ }^{\text {I }}$ Between the two kidneys is a tough shining ligament which should not be mistaken for. a duct.

The oviducts in immature females are slender tubes running along the ventral face of the kidneys, without mesenteries. In mature females they are very large tubes which spring free from the kidneys by means of well-developed mesenteries, the mesotubaria. Trace the oviducts forward. They pass forward along the dorsal coelomic wall, curve around the anterior border of the liver, and enter the falciform ligament. Here the two oviducts are united to a common opening,

I The caudal mesonephros is often regarded as a metanephros and its duct as a ureter. Professor Kingsley has kindly expressed his opinion that this usage is unjustifiable. 
the ostium. This is a wide, funnel-shaped opening lying in the falciform ligament, the opening directed posteriorly. To find the opening it is generally necessary to separate the walls of the ostium, as these tend to adhere. Trace the oviducts posteriorly. They are narrow tubes at first, soon presenting in mature specimens a slight enlargement, the shell gland. Posterior to this they narrow again and then in mature females enlarge greatly to form the uterus, which swings free by means of the mesotubarium. In immature females there is no such enlargement, nor is there any mesotubarium, but the oviducts widen slightly as they proceed posteriorly along the ventral faces of the kidneys.

Trace the oviducts to the cloaca. Cut open the cloaca by a median ventral slit which opens up the intestine. Note the opening of the intestine into the ventral part of the cloaca and the slight fold which separates this from the dorsal urogenital region of the cloaca. Note the urinary papilla in the median dorsal wall of the cloaca; it is a conical papilla in the spiny dogfish, a low elongated ridge in the smooth species. In mature specimens the large openings of the oviducts are readily seen to each side of the urinary papilla. In immature specimens they are in the same position but quite small. To find them it is best to cut into the posterior ends of the oviducts and probe toward the cloaca.

The ducts of the kidneys are more difficult to locate in females. The cranial mesonephros is provided with a Wolffian duct. This lies along the ventral face of the kidney exactly dorsal to the oviduct in immature specimens, or in mature ones along the line of attachment of the mesotubarium. Locate it in immature specimens by carefully stripping off the oviduct and also freeing the pleuroperitoneum from the ventral face of the kidney. The Wolffian duct is a slender duct proceeding posteriorly to the cloaca. The caudal mesonephros (posterior third) is also provided with a duct, the accessory mesonephric duct. In the smooth dogfish this lies alongside of the Wolffian duct and has probably been noted already. In the spiny species it is more deeply located imbedded in the kidney tissue; it will be found by dissecting in this tissue near the median line. The accessory ducts are delicate tubes receiving ducts from the kidney at intervals. Both Wolffian ducts and accessory ducts proceed to the cloaca lying in contact with and on the dorsal surface of the oviducts. To follow them cut along one side of the cloaca, separating the cloaca from the body wall. They all terminate by a single median pore in the urinary papilla.

Skate: The ovaries are a pair of elongated soft bodies containing large yellow eggs; they are situated dorsally in the anterior half of the pleuroperitoneal cavity. The large oviducts pass dorsal to them. Follow one oviduct forward. Its narrow anterior portion passes along the dorsal coelomic wall, curves around the anterior margin of the liver, and passing into the falciform ligament unites with its fellow to a single common opening or ostium. The ostium is a wide funnel-like aperture situated in the ligament and facing posteriorly. Trace the oviducts caudad. After a short distance they widen greatly to a uterus, the 
beginning of which bears a conspicuous bilobed swelling, the oviducal gland: which secretes the horny case in which the eggs are laid (N, p. I64, Fig. 96). The uteri proceed to the cloaca supported by the thickened mesotubaria. Cut open the cloaca in the median ventral line, slitting open the intestine. Note the opening of the intestine into the ventral part of the cloaca and the conspicuous horizontal fold which separates this from the dorsal urogenital part. Cut into the latter by cutting forward through this fold. The cloaca is greatly extended and thickened in the anterior direction. Find the openings of the oviducts, one to each side of this thickened part of the cloaca. Halfway between the openings of the oviducts is the urogenital opening in the median dorsal wall.

The main part of the kidneys in female skates consists of a thick rounded lobe lying against the dorsal wall at each side of the cloaca. These lobes are revealed by stripping off the thick pleuroperitoneum which covers their ventral surfaces. These lobes may be named the caudal mesonephros. The anterior part of the mesonephros or cranial mesonephros is nearly degenerate in females but will be found as diffuse brownish tissue extending forward ventral to the dorsal aorta. From the median surface of the caudal mesonephros several ducts, the accessory mesonephric ducts, pass anteriorly and medially in contact with the posterior cardinal vein and open into a small chamber, the urinary sinus, situated on the dorsal surface of the anterior end of the cloaca. The two urinary sinuses of the two sides unite into a common chamber, which is sometimes called the urinary bladder. It does not correspond to the bladder of higher forms, since it consists of the enlarged terminations of the mesonephric ducts. Cut into this, note the entrance into it of the two urinary sinuses, and find the opening in its mid-dorsal wall by which it opens into the cloaca. The Wolffian ducts or ducts of the cranial mesonephros are slender tubes extending anteriorly from the urinary bladder, lying on the dorsal surface of the strong white portions of the mesotubaria.

Draw, showing kidneys, gonads, and their ducts, and the opened cloaca.

2. The male urogenital system.-The testes are a pair of soft bodies dorsally situated. In the spiny dogfish they are located in the anterior part of the pleuroperitoneal cavity, dorsal to the liver; each has a mesorchium. In the smooth dogfish they are long and slender bodies extending the length of the pleuroperitoneal cavity, their toothed posterior ends attached to the mesentery of the rectal gland. They are more or less fused and are supported by a single mesorchium. In the skate the testes are broad, flat bodies against the dorsal wall; each is provided with a mesorchium.

The kidneys are identical with those of the females and should be next examined according to the directions given under females. In the male skate, however, the cranial part of the kidney is very much better developed than in the female and extends forward as a firm cylindrical body on either side of the mid-dorsal line. 
As explained in the introduction the male ducts in the majority of vertebrates are the mesonephric or Wolffian ducts. In mature males these ducts are consequently much larger than in females. The Wolffian ducts run posteriorly along the ventral face of the kidneys. In immature specimens each is a slender, straight tube, similar to that of the female, but in mature males, it is greatly coiled. The testis is connected with the cranial mesonephros by means of delicate ducts, the vasa efferentia, which run in the mesorchium and can sometimes be seen by holding the mesorchium up to the light. The vasa efferentia connect with the tubules of the mesonephros. The greater part of the cranial mesonephros apparently serves in male elasmobranchs for transmitting the sperm, and is sometimes called the epididymis, since it corresponds to the head of the epididymis of mammals. The sperm-bearing tubules of the niesonephros then connect with the Wolffian duct. Trace this duct posteriorly. Its anterior part is greatly coiled in mature males, but in dogfishes (not in skate) straightens as it approaches the caudal mesonephros and in all three animals enlarges upon the surface of the latter to form the seminal vesicle. Trace it by removing the pleuroperitoneum from the ventral face of the caudal mesonephros. At its posterior ends on the sides of the cloaca, the seminal vesicle terminates in a sac, the sperm sac, which projects forward as a blind sac lying against the ventral surface of the seminal vesicle.

Cut open the cloaca as directed under the female and identify its parts as directed there. There is no difference in the cloaca of the dogfish between the males and females, but in the male skate the cloaca is very much smaller than in the female and is not divided into intestinal and urogenital parts. In the median dorsal line there is in the male skate a urogenital papilla.

The sperm sacs should now be cut open and the papillae, where the seminal vesicles open into them, identified. The two sperm sacs unite at their posterior ends to form a urogenital sinus which opens at the tip of the urinary papilla.

The accessory mesonephric ducts are similar to those of the females; see the description under females. In male dogfishes each runs along the medial side of the seminal vesicle and enters the sperm sac near the opening of the vesicle. In the male skate several accessory ducts pass from the caudal mesonephros into the sperm sac.

Draw, showing gonads, kidneys, and their ducts, and the opened cloaca.

Look in male dogfishes for remnants of the ostium and oviducts in the region of the liver.

\section{THE UROGENITAL SYSTEM OF NECTURUS}

I. The female urogenital system.-The ovaries have already been noted as elongated saclike bodies bearing eggs of various sizes. Note the mesovarium. Lateral to each ovary running along the dorsal body wall is the oviduct, a thick, white, coiled tube supported by the mesotubarium. Follow it anteriorly. 
At the anterior end of the pleuroperitoneal cavity it becomes of a more delicate texture and is fastened to the lateral wall. Here it opens by a funnel-shaped opening, the ostium; the dorsal rim of this is fastened to the body wall, but the ventral rim is free and can be lifted to expose the opening. Trace the oviducts posteriorly to the cloaca. They enter this one to each side of the large intestine. Cut the cloaca open by a lateral slit extending up into the intestine. Note the papillae by which the oviducts open into the cloaca.

The kidneys are long slender organs extending from the cloaca forward along the dorsal surfaces of the oviducts. They are retroperitoneal. They are mesonephroi. The duct, the Wolfian or mesonephric duct, lies along the lateral border of each kidney. It is very delicate in the female and difficult to locate. It proceeds to the cloaca into which it opens to the dorsal side of the oviduct. In tracing it make a cut along one side of the cloaca, freeing the cloaca from the body wall.

Note the urinary bladder extending from the midventral region of the cloaca. Find its opening into the cloaca.

Draw, showing ovaries, kidneys, their ducts, and the opened cloaca.

2. The male urogenital system.-The testes are a pair of elongated bodies situated to the sides of the small intestine. Each has a mesentery, the mesorchium. Dorsal and lateral to each testis is the long brown kidney, larger than in the female. The kidney is a mesonephros. Along the lateral border of the kidney is a conspicuous coiled duct, the Wolffian or mesonephric duct. This also, as in vertebrates in general, acts as the sperm duct. By holding up the mesorchium to the light note the delicate ducts, the vasa efferentia, which cross it into the kidney. The sperm pass through the tubules of the kidney and into the Wolffian duct. Trace the latter to the cloaca. Open the cloaca by a slit to one side of the median line carrying the slit into the large intestine. The openings of the Wolffian ducts into the cloaca are generally difficult to locate, owing to their small size. Note the urinary bladder and its opening into the ventral cloacal wall.

Draw, showing testes, kidneys, Wolffian ducts, and opened cloaca.

\section{THE UROGENITAL SYSTEM OF THE TURTLE}

Remove the digestive tract, if not already done, leaving the large intestine in place.

I. The female urogenital system.-This consists as usual of a pair of ovaries and a pair of Müllerian ducts or oviducts. The ovaries have already been noted as large baglike bodies in the posterior part of the pleuroperitoneal cavity. They usually contain yellow eggs in various states of development. Each ovary is supported by a mesentery, the mesovarium. Along the posterior border of each ovary runs the oviduct, a large white coiled tube, supported by the mesotubarium. Trace the ovjduct forward and find the ostium; this lies in the mesen- 
tery and has winglike borders which are generally closed together and should be spread apart to see the opening. Trace each oviduct to the cloaca. Each opens into the side of the anterior end of the cloaca, ventral to the opening of the intestine. The stalk of the large bilobed urinary bladder joins the cloaca midway between the two oviducts.

The cloaca has already been exposed. (If not, do so by cutting through the pelvic girdle on each side and removing the median portion of the girdle.) Clear away connective tissue from around the cloaca. Attached to each side of the cloaca posterior to the oviducts are two elongated sacs, the accessory urinary bladders. Their function is unknown but is possibly respiratory or hydrostatic. A dark structure visible through the wall of the cloaca is the clitoris, homologous with the penis of the male. It is of no use in the female.

Now cut open the cloaca to one side of the clitoris, extending the cut in the median ventral line up to the stalk of the bladder. Look into the cloaca. Observe that the clitoris consists simply of thickenings in the ventral wall. Find the large openings of the accessory bladders. Next note the opening of the large intestine. This is the most dorsal of the openings and is somewhat separated by a fold from the urogenital openings. Ventral to the opening of the intestine are the openings of the oviducts on thickened papillae. They are best found by cutting into the oviduct and probing posteriorly into the cloaca. Between and ventral to the oviducal openings is the opening of the urinary bladder.

The kidneys of the turtle are metanephroi. They have already been identified as flattened lobed organs fitting snugly against the posterior end of the pleuroperitoneal cavity. The renal portal vein and its tributary, the internal iliac, run along the ventral face of each kidney. Dissect off this vein; directly dorsal to it is a tube, the metanephric duct or ureter, extending from the middle of the kidney to the cloaca. It enters the cloaca at the base of the oviduct. By making a slit in it and passing a probe into it its opening into the cloaca will be found just anterior to the thickening caused by the oviducal entrance.

Draw the female urogenital system with opened cloaca.

2. The male urogenital system.-The male system consists of the paired testes and their ducts. The ducts of the testes are the Wolffian ducts, now called the vasa deferentia.

Expose the cloaca as directed in the female and find the two accessory bladders attached to its lateral walls. Note the place of attachment of the rectum to the cloaca and ventral to this the attachment of the urinary bladder. The dark mass seen through the ventral wall of the cloaca is the penis or organ of copulation. A rounded mass projects from the anterior wall of the cloaca to either side of the stalk of the bladder; they are parts of the penis and are called the bulbs of the corpora cavernosa. Muscles which retract the penis will be seen attached to the ventral wall of the cloaca. 
The kidneys were previously identified as flattened lobed bodies fitting against the posterior wall of the pleuroperitoneal cavity. Each testis is a yellow spherical body attached to the ventral face of the kidney by the mesorchium. I ateral and posterior to the testis is an elongated, dark-colored coiled body, the epididymis. The testis is connected to the anterior part of the epididymis by the minute vasa efferentia which run in the mesorchium. The vasa efferentia and this portion of the epididymis are the remnants of the mesonephros. The remainder of the epididymis constitutes the male duct or vas deferens (Wolffian $d u c t)$. Remove the peritoneal covering of the epididymis and uncoil the vas deferens. Trace it to the cloaca. It enters anterior to and at the base of the bulb of the penis.

Next cut open the cloaca, inserting the blade of the scissors into one corner of the anus and cutting far to one side to avoid injuring the penis. Spread apart the cloacal walls and study the penis. It consists of two spongy ridges, the corpora cavernosa or cavernous bodies, in the ventral wall of the cloaca. Between these folds in the midventral line is a deep groove, the urethral groove, which in the natural condition is practically converted into a tube by the approximation of the cavernous bodies. The urethral groove terminates caudad at the base of a heart-shaped projection, the glans of the penis. The anterior ends of the cavernous bodies form the bulbs already noted, which project forward into the coelom at the sides of the stalk of the bladder. The bulbs are filled with blood which they receive from the internal iliac vein. All parts of the penis are highly spongy and vascular. In the sexual act the blood from the bulbs rushes into the spongy spaces of the cavernous bodies and the glans, erecting them, and causing the cavernous bodies to come in contact above the urethral groove, converting the latter into a canal for the passage of the sperm.

The kidneys are metanephroi, and their ducts the metanephric ducts or ureters. The ureter will be found immediately to the dorsal side of the epididymis which should be removed. The ureter is a short straight tube proceeding to the cloaca, into which it opens just anterior to the opening of the vas deferens. The two openings will be found at the sides of the anterior beginning of the urethral groove.

Find the openings of the accessory bladders, the urinary bladder, and the rectum into the cloaca. The latter is dorsal to the urogenital openings.

Draw the male urogenital system with open cloaca.

\section{E. THE UROGENITAL SYSTEM OF THE PIGEON}

I. The female urogenital system.--Remove the digestive tract, leaving the large intestine in place. In adult birds there is a single ovary and oviduct on the left side. The right ovary and duct are present in the embryo but almost entirely disappear before hatching. The ovary is a mass containing eggs of 
various sizes, situated at the anterior end of the left kidney. It is attached by a short mesovarium. Posterior to the ovary the coiled left oviduct proceeds to the cloaca, being supported by the mesotubarium. The ostium is situated in the mesotubarium near the ovary; it is a wide opening with winglike borders fastened to the mesotubarium. A small remnant of the right oviduct is attached to the right side of the cloaca.

The kidneys are metanephroi. Each is a flattened, three-lobed organ situated against the dorsal wall. The ureters or metanephric ducts are located just dorsal to the renal portal veins which should be stripped from the face of the kidney. The ureter begins on each side at the groove between the anterior and middle lobes of the kidney and extends straight posteriorly to the cloaca. The left ureter is concealed by the oviduct.

The cloaca is an expanded chamber receiving the rectum on its median ventral surface, the left oviduct to the left, the very small right oviduct to the right, and the ureters dorsal to the oviducts. Cut into the cloaca to the right of the rectum. Note that the cavity of the cloaca is subdivided. There is a large ventral portion (coprodaeum) into which the rectum opens. Dorsal to this and separated from it by a fold is the urodaeum into which open the oviducts and ureters. The opening of the left oviduct is readily found here; the openings of the ureters are more medial and smaller. The most dorsal compartment of the cloaca is the proctodaeum, a small chamber with a raised rim, which opens to the anus. In the anterior wall of the proctodaeum dorsal to the rim an opening may be noted; it leads into a small pouch which seems to be functional in young birds but degenerates with maturity. It is called the bursa of Fabricius.

Draw urogenital system and cloaca.

2. The male urogenital system.-The testes are a pair of oval organs at the anterior ends of the kidneys; their size varies considerably with the season. The kidneys are the same as in the female, and the description given under the female should be read and the ureters identified. The male ducts, vasa deferentia (Wolffian ducts), spring from the medial border near the posterior end of the testes and pass posteriorly parallel to the ureters. They are slender convoluted tubes. Trace both ureters and vasa deferentia to the cloaca.

The cloaca is smaller than in the female and the lips of the anus more protruding. The rectum enters medially and ventrally, the urogenital ducts laterally. Cut into the cloaca as directed under female and identify its chambers as there described. They are the same in the two sexes, except that the urodaeum is smaller and receives the two vasa deferentia instead of the oviduct. Ureters and vasa deferentia open on small papillae in the lateral walls of the urodaeum. There is no penis in most birds.

Draw 


\section{F. THE UROGENITAL SYSTEM OF THE MAMMAL}

- Remove the digestive tract, leaving the rectum in place.

I. The kidneys and ureters.-The kidneys of mammals are metanephroi and their ducts the metanephric ducts or ureters. The kidneys are large oval organs situated on the dorsal body wall of the peritoneal cavity. As in all vertebrates they are retroperitoneal. The right kidney is generally considerably anterior to the left one. Clear away fat and connective tissue from about the kidneys. The medial face of each kidney is concave; this concavity is called the hilus. From the hilus a white tube, the ureter, passes out and turns posteriorly. Follow the ureters caudad, clearing away the fat from about them and note their entrance into the bladder. In females the ureters pass dorsal to the horns of the uterus. In males each passes dorsal to a white cord, the male duct or vas deferens, which loops over the ureter and disappears dorsal to the bladder.

Remove by a cut the ventral half of a kidney. A cavity, the sinus, is revealed within the hilus; this sinus is occupied chiefly by the renal pelvis, or expanded beginning of the ureter, and also by the renal artery and vein. Into the pelvis the substance of the kidney projects as the renal papilla on which are situated the microscopic openings of the collecting tubules. The kidney substance is readily divided into two areas, a peripheral region, the cortex, and a central region, the medulla. The cortex contains the renal corpuscles and the convoluted and looped portions of the kidney tubules. The medulla is marked by lines which converge to the renal papilla; these lines are the collecting tubules. It will be recalled that the collecting tubules, the pelvis, and the ureter are outgrowths of the Wolffian duct. The collecting tubules and renal papilla together form a pyramid, of which there is but one in the rabbit and cat but about twelve in man.

Draw.

The urinary bladder is a pear-shaped sac at the posterior end of the peritoneal cavity. It is ventral to the rectum in the male, ventral to both rectum and uterus in the female. The free anterior end of the bladder is named the apex or vertex, the posterior portion the fundus. The fundus continues posteriorly as a narrowed stalk, the urethra ${ }^{\mathbf{x}}$ (also called neck of the bladder). The bladder is covered by the peritoneum, which is continuous with that of the abdominal wall by means of the median and lateral ligaments previously noted. The pouch between the bladder and rectum (male) or bladder and uterus (female) is named the rectovesical or vesicouterine pouch, respectively.

Draw the excretory system.

${ }^{x}$ The term urethra is in much confusion in comparative anatomy, owing to the differences between the urogenital systems of various mammals. Although in the embryo the urethra is the same as the urogenital sinus, this is not the case in the adults of most mammals, and consequently the use of urethra as synonymous with urogenital sinus appears to be inadvisable. Urethra is therefore here employed in the same sense as in human anatomy, that is, as the name of the duct leading from the bladder to the exterior. 
2. The female reproductive system. - This consists of a pair of ovaries and their ducts. The ovaries are very small oval bodies located at the sides of the peritoneal cavity at the anterior end of the coils of the uterus. Each will be seen to bear little clear vesicles, the Graafian follicles, each of which contains an egg or ovum; in pregnant females the ovary also bears little hard lumps, the corpora lutea, which represent follicles from which the eggs of the pregnancy were discharged. The ovary is suspended by the mesovarium, which extends forward to the kidney and is continuous posteriorly with the ligament of the uterus.

The ducts of the ovaries are, as in other vertebrates, the Millerian ducts or oviducts, but they are differentiated into several distinct parts in mammals. The uppermost portion of the oviducts is a slender convoluted tube which passes lateral to the ovary and curves over its anterior end; its mesentery, the mesosalpinx, forms a sort of hood, partly inclosing the ovary. This portion of the oviduct is the uterine or Fallopian tube. It opens in front of the ovary (rabbit) or to the lateral side of it (cat) by the ostium having fringed borders, the fimbriae. On tracing the uterine tube posteriorly it is found to widen suddenly into a thick-walled tube, the uterus (rabbit) or horn of the uterus (cat). The size of this depends on whether the animal is pregnant or not; in pregnant animals the uteri or horns are greatly enlarged and exhibit a series of swellings, each of which contains an embryo (these will be examined later). The strong fold of peritoneum supporting the uteri or horns is the mesometrium. Mesovarium, mesosalpinx, and mesometrium together are called the broad ligament of the uterus in mammals. The round ligament of the uterus is the fold extending from the beginning of the uterus or horn posteriorly to the body wall; it is continuous with, but at right angles to, the broad ligament. In the cat the two horns of the uterus unite in the median line, dorsal to the bladder, to a single tube, the body of the uterus. Body and horns together constitute the uterus or womb, but the young develop only in the horns. In the rabbit the two uteri are separate along their entire lengths, and consequently there is no division into body and horns. In the cat the body of the uterus continues posteriorly as the vagina; in the rabbit the two uteri join the vagina; the vagina is a tube situated in the median line between the bladder and the rectum. It exits through the ring formed by the pelvic girdle and vertebral column.

The external genital parts or external genitalia were described with the external anatomy. Review this (p. 29). Then in the rabbit make an incision through the skin forward from the vulva. In the median line beneath the skin is a hardened body, the clitoris, homologous with the penis of the male. Its anterior end is attached by ligaments to the ischium and pubic symphysis. Cut across the clitoris and note the two cavernous bodies of which it is composed. In the cat the clitoris is minute. 
Now cut through the pubic and ischial symphyses and spread the legs well apart. Trace the urethra, the vagina, and the rectum posteriorly. The urethra lies at first on the ventral face of the vagina to which it is bound by tissue; it then unites with the vagina to form a common tube, the urogenital canal or sinus. Dissect this free and lift it out and follow it to the urogenital aperture. Cut open the urogenital aperture and note the free posterior end, or glans, of the clitoris, projecting into the cavity in the rabbit. Free the rectum from the urogenital canal and follow it to the anus. Along its sides in the rabbit are a pair of elongated glands, the anal glands. In the cat the rounded anal glands or sacs are situated one at each side of the rectum, just internal to the anus.

Draw the female urogenital system.

Cut open the vagina. In the rabbit note the external uterine orifice with raised fringed lips by means of which each uterus opens into the vagina. The rabbit uterus is of the duplex type (Fig. $6_{3} B$, p. 278 ). In the cat the body is divided into lateral halves by a median partition, a horn opening to each side of the partition. The cat uterus is of the bipartite type (Fig. $6{ }_{3} C$ ). The lower end of the uterus, called the cervix, projects into the vagina by a fold. The opening of the body of the uterus into the vagina is the external uterine orifice.

3. The male reproductive system.-The external parts or external genitalia were described with the external anatomy. Review this (p. 29). The two testes are lodged in the scrotal sac which is divided into two compartments by an internal partition. Cut through the skin over and in front of one testis, exposing the testis as an oval white body. Clear away the connective tissue anterior to the testis and find a white cord, the spermatic cord, passing forward and entering the peritoneal cavity through a canal called the inguinal canal. The external end of this canal is the external inguinal ring; the internal end, the internal inguinal ring. Trace the spermatic cord by cutting open the canal.

The spermatic cord contains a white duct, the male duct or vas deferens, and the blood vessels and nerves of the testis. The two vasa deferentia turn toward the median line, loop over the ventral surfaces of the ureters, and disappear on the dorsal surface of the urethra.

It is now necessary to explain these relations of the testes and their ducts. In the vertebrates previously studied the testes lie within the peritoneal cavity, but in most adult mammals they are situated posterior to this cavity. In the embryos of male mammals, however, the testes lie within the peritoneal cavity; subsequently they descend caudad. There is first formed a sac of the body wall, the scrotal sac, which contains all of the layers of the body wallskin, muscles, and peritoneum-and incloses a portion of the coelom, the vaginal sac. The testes then descend into the scrotal sac, as shown in Figure 65 , carrying with them their ducts, the nerves, blood vessels, etc. The canal along which the descent occurs later narrows and eventually is completely obliterated in the higher mammals but in some remains open. (See $\mathrm{K}$, p. 373.) A new canal, the inguinal canal, is later secondarily formed around the spermatic cord. The descent of the testes explains the peculiar looping of the vasa deferentia over the ureters and the course of the internal spermatic vein. The scrotum is now believed to serve as a temperature regulating mechanism for the testes. 
Now cut through the pubic and ischial symphyses and spread the legs apart. Trace the vasa deferentia and the urethra caudad, separating them from the rectum. The ureters may be cut through and the urinary bladder held posteriorly. The vasa deferentia pass along the dorsal surface of the urethra. In the rabbit they enlarge and enter an expanded sac, the seminal vesicle, which pouches forward between the rectum and the bladder. Cut into the seminal vesicle and note the openings into its ventral wall of the two vasa deferentia, and in its posterior dorsal wall the thickening caused by the prostate gland. Find the union of the seminal vesicle and urethra to form a common tube, the urogenital simus. In the cat the two vasa deferentia join the urethra without the formation of a seminal vesicle, the point of junction being surrounded by a slight enlargement,
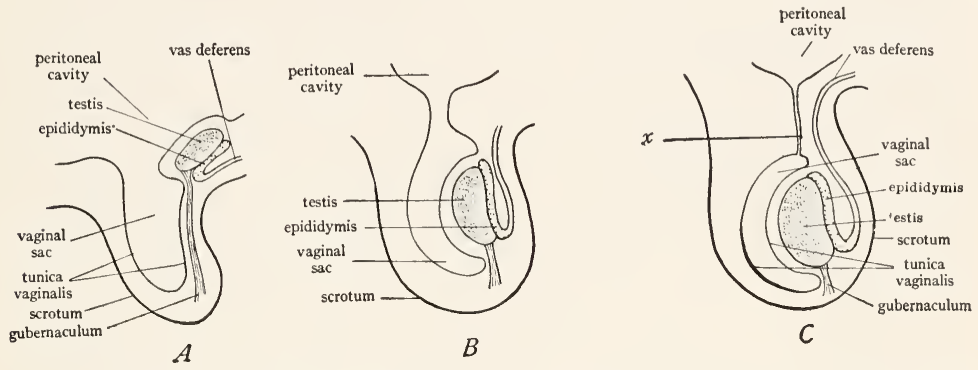

FIG. 65.-Diagrams to illustrate the descent of the testis in the male mammal. The testis descends into the scrotum which is a sac of the body wall containing a portion of the peritoneal cavity called the vaginal sac; in the descent the gubernaculum or ligament of the testis shortens; the passage $x$ along which the descent occurs and which at first forms a connection between the vaginal sac and the peritoneal cavity is later completely obliterated in the higher mammals; it does not correspond to the inguinal canal. (From Prentiss and Arey's Textbook of Embryology, courtesy of the W. B. Saunder Company.)

the prostate gland. The common tube thus formed is the urogenital canal or simus. The bulbourcthral glands or Cowper's glands are small swellings situated on the urogenital canal shortly posterior to the prostate gland in the rabbit, about an inch posterior in the cat. The terminal inch of the urogenital canal is inclosed in the penis. Cut into the prepuce and note the pointed projection within it, called the glans of the penis. Note that the prepuce is simply a fold of skin around the glans. At the tip of the glans is the urogenital opening. The glans in the cat bears a number of minute spines. Dissect anteriorly from the glans exposing the remainder of the penis as a hardened cylindrical structure. Find where the urogenital canal enters its anterior end. Note also the strong attachments of the penis to the pelvic region. Cut across the middle of the penis and note that it is composed of two cylindrical bodies, the corpora cavernosa or cavernous bodies closely placed. The urogenital canal, here called the cavernous urethra, lies on the dorsal side of the penis resting in a depression 
between the two cavernous bodies. At the anterior end of the penis the two cavernous bodies diverge, forming the crura of the penis, which are attached to the ischia. The cavernous bodies are spongy structures and in the sexual act become distended with blood so that the penis is caused to project out of its sheath, the prepuce.

Draw the parts of the male genital system.

Trace the rectum to the anus following directions given for the female.

The structure of the testis may now be investigated. Each testis is inclosed in a white fibrous sac, the parietal portion of the tunica vaginalis, which is in reality the peritoneal pouch made by the descent of the testis (Fig. 65). Cut open this sac, exposing the cavity in which the testis lies, this being a part of the peritoneal cavity. The tunica vaginalis which lines this cavity is reflected over the surface of the testis as the visceral portion of the tunica vaginalis, which forms the outer thin coat of the testis. The point of deflection lies along the mid-dorsal line of the scrotal sac, and a mesentery is formed along this line between the sac and the testis. This mesentery is the mesorchium, corresponding to the broad ligament of the uterus. The posterior end of the testis is a ttached to the posterior wall of the scrotum by a short but stout ligament, the gubernaculum, continuous with the mesorchium. The gubernaculum corresponds to the round ligament of the uterus. The duct of the testis is the vas deferens or Wolffian duct. It lies along the dorsal surface of the testis much coiled, the coiled portion being named the epididymis. The epididymis begins at the anterior end of the testis as a coil, the head of the epididymis. It then passes down the dorsal surface of the testis as a coiled tube, the body of the epididymis. At the posterior end of the testis it forms another coiled mass, the tail of the epididymis, to which the gubernaculum is attached. From this the vas deferens proceeds anteriorly, much convoluted, and passes into the inguinal canal where it becomes a straight tube. The head of the epididymis is derived from the mesonephros and is connected with the tubules of the testis by minute vasa efferentia. The remainder of the epididymis and the vas deferens are the Wolffian duct.

Draw, showing contents of the scrotum.

\section{G. THE EMBRYONIC MEMBRANES}

I. General.-There are four embryonic membranes in vertebrates. They are: the $y$ Jlk sac, the allantois, the amnion, and the chorion (Fig. 66).

The yolk sac is simply an evagination from the ventral wall of the intestine. Its connection with the intestine forms a narrow yolk stalk. The yolk sac occurs in the embryos of all vertebrates having meroblastic eggs and also in mammals, for they are descended from forms which have meroblastic eggs. The yolk sac is filled with yolk, except in mammalian embryos where it is empty.

The allantois is a large evagination from the floor of the cloaca. It is primarily respiratory in function, but also probably serves to hold embryonic excretory materials. The adult bladder develops at the base of the allantoic stalk. 
The amnion and the chorion are formed by a fold of the body wall (somatopleure) which rises up around the embryo. The folds of the two sides meet above the embryo and fuse across. The outer limb of the fold becomes the chorion, the inner limb the amnion (see Fig. $66 A$ and $B$ ). The amnion forms a sac inclosing the embryo. The chorion is the outermost
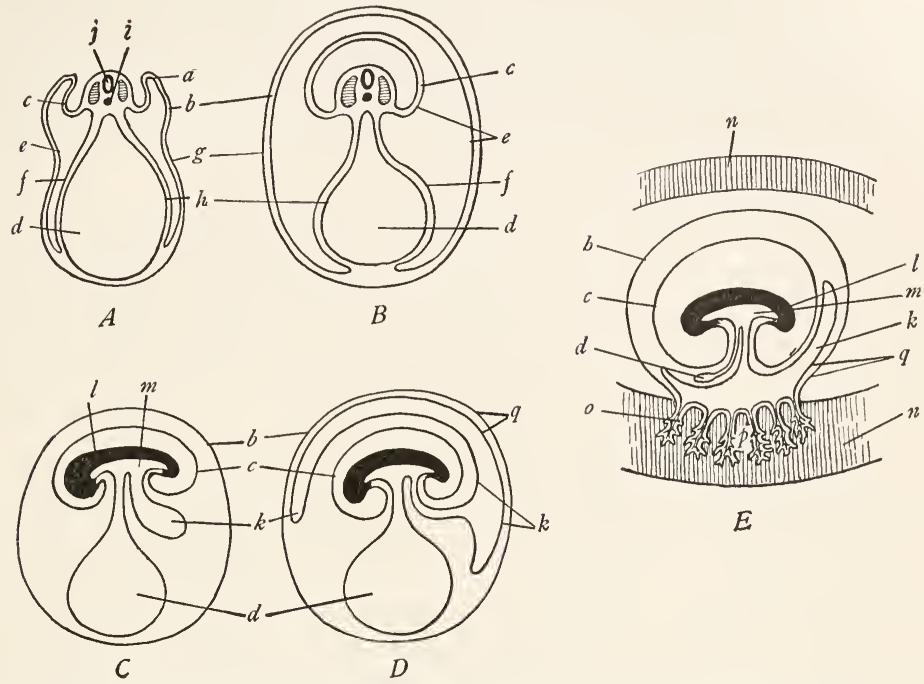

$E$

Fig. 66.-Diagrams to illustrate the mode of formation of the embryonic membranes of amniotes and of the placenta. A, cross-section through an early stage of the formation of the amnion and the chorion; a fold of the somatopleure is seen rising up at $a$; the outer wall of the fold $b$ becomes the chorion, the inner wall $c$, the amnion. $B$, later stage after completion of the process of formation of the amnion and chorion; the fold fuses across above the dorsal surface of the embryo forming two membranes, an outer chorion $b$ which incloses embryo and yolk sac, and an inner amnion $c$, which incloses the embryo. $C$, sagittal section of a later stage of the embryo to show the origin of the allantois $k$ as an evagination from the digestive tract $m$. $D$, later stage following $C$, showing the spreading of the allantois between the chorion and the amnion and yolk sac; note that the outer wall of the allantois is in contact with the chorion, the two together forming the chorio-allantoic membrane $q$. $E$, formation of the placenta in mammals by the penetration of the chorio-allantoic membrane $q$ into the wall of the uterus $n$; the penetration takes the form of treelike ingrowths which are called the chorionic villi $o$; note small size of the mammalian yolk sac $d$; the placenta consists of the inner part of the uterine wall and the chorionic villi; the latter are generally restricted to certain areas of the chorio-allantoic membrane. In $C, D$, and $E$ the two layers of which the chorion, amnion, allantois, and yolk sac are each composed are omitted for simplicity. $a$, amniotic fold of the somatopleure; $b$, chorion; $c$, amnion; $d$, yolk sac; $e$, somatic mesoderm; $f$, splanchnic mesoderm; $g$, ectoderm; $h$, entoderm; $i$, notochord; $j$, neural tube; $k$, allantois; $l$, body of embryo, head to the left; $m$, digestive tract of embryo; $n$, wall of uterus; $o$, chorionic villi; $p$, placenta; $q$, chorio-allantoic membrane. (Suggested by figures in Hertwig.)

membrane of the embryo. The yolk sac and allantois are between chorion and amnion on the ventral side of the embryo (Fig. 66C).

The yolk sac and allantois are highly vascular, their blood vessels being named the vitelline and umbilical (allantoic) vessels. Much has already been said of the vitelline veins. The 
umbilical veins are the abdominal veins of lower forms. The amnion and the chorion never contain any blood vessels.

The yolk sac may occur in the embryos of any group of vertebrates. The allantois, amnion, and chorion occur only in reptiles, birds, and mammals, which are hence designated amniotes. The allantois spreads out inside of the chorion and becomes fused to the chorion forming a chorio-allantoic membrane (Fig. 66D). In the placental mammals this chorioallantoic membrane comes into close contact with the internal wall of the uterus and in the highest mammals fuses inseparably with the uterine wall (Fig. 66E). The compound structure thus produced by approximation or fusion of the chorio-allantoic membrane with the uterine wall is designated the placenta. It is of various shapes in various mammals, the name placenta being derived from the disk shape of the human placenta.

2. Anamniote embryo of the dogfish.-Cut open the pregnant uterus of a dogfish and remove an embryo or examine embryos provided. Note that the embryo is naked. From the middle of its ventral wall hangs the large yolk sac, filled with yolk and attached to the body by the narrowed yolk stalk. The yolk sac is covered externally by a layer of the body wall and internally consists of the intestinal wall inclosing the yolk. As the yolk is used up the yolk sac is gradually drawn into the body. Draw.

3. Amniote embryo of the cat.-If pregnant females are available, open one of the enlargements in the horns of the uterus. The enlargement contains an embryo. Note that the embryo is inclosed in a thin membrane, the amnion. On the inner surface of the uterine wall at the enlargement note a thickened vascular ring of tissue. This is the placenta; it will probably peel off from the uterine wall, especially in advanced stages of pregnancy. Cut into the amnion and note the umbilical cord extending from the ventral side of the abdomen of the embryo to the inner surface of the amnion. The umbilical cord is a connection between the embryo and its own membranes and not, as popularly supposed, a connection between the embryo and the mother. There is no direct connection between embryo and mother.

\section{H. SUMMARY OF THE UROGENITAL SYSTEM}

I. The urogenital system is derived from the mesomere of the embryo.

2. The urinary or excretory system consists of the paired kidneys and their ducts. The kidneys are composed of tubules opening at one end into the coelom by a nephrostome and at the other end into the collecting duct.

3. In the evolution of the vertebrates there have been three successive kidneys, each situated more posteriorly than its predecessor.

4. The first kidney of vertebrates is called the pronephros. Its duct is the pronephric duct. It appears in the embryos of all vertebrates but functions in the adults of only cyclostomes and a few fishes.

5. The second kidney is the mesonephros or Wolffian body. Its duct is the mesonephric or Wolffian duct; this is a continuation of the pronephric duct. It enters the cloaca. The mesonephros is the functional kidney of most adult fishes and all Amphibia.

6. The third kidney is the metanephros. Its duct is the metanephric duct or ureter. The ureter and part of the metanephros arise by evagination from the Wolffian duct. The metanephros is the functional kidney of the adults of all the amniotes. 
7. A urinary bladder is generally present as an evagination of the ventral wall of the cloaca. It receives the ducts of the kidneys in mammals only. It opens into the cloaca in all forms except adult placental mammals.

8. The ovaries and testes are paired bodies developed from the ventral surface of the mesomere and projecting into the coelom.

9. The ovaries are always located within the peritoneal or pleuroperitoneal cavity in all vertebrates. The ducts of the ovaries are the Müllerian ducts or oviducts. They are supposed to be derived from the pronephric duct by splitting, but arise in this way only in elasmobranchs.

ı. The oviducts never have any direct connection with the ovaries. They open into the coelom near the ovaries by an ostium which is believed to represent one or more nephrostomes.

I I. The oviducts enter the cloaca separately except in the placental mammals. In these latter the oviducts are more or less united and differentiated into regions. This union proceeds from their posterior ends anteriorly, forming in the lowest placental mammals first a common vagina, in higher forms a partially fused uterus with separate horns, and in the primates a single uterus produced by the fusion of two originally separate uteri. The upper portions of the oviducts form narrow uterine tubes which always remain separate.

I2. The testes are located internally except in the higher mammals, where they descend temporarily or permanently into pouches of the body wall located externally in the inguinal region.

13. The ducts of the testes in all vertebrates (except cyclostomes and teleostomes) are the Wolffian or mesonephric ducts, also called the vasa deferentia. In the males of groups where the mesonephros is functional the Wolffian ducts have both genital and excretory functions. In groups where a metanephros is present the Wolffian ducts have only genital functions.

14. The Wolffian duct, vas deferens, is always directly connected to the testis by the intervention of a portion of the mesonephros. This is differentiated into the vasa efferentia and the epididymis in part. The remainder of the epididymis is part of the Wolffian duct. In male amniotes a portion of the mesonephros thus always persists as part of the male system; in female amniotes the mesonephros takes no part in the functional female apparatus.

15. A cloaca which receives the intestine and urogenital ducts is present in most vertebrates (cyclostomes, teleostomes and placental mammals excepted). In placental mammals the cloaca splits into a dorsal portion which receives the intestine and opens to the exterior by the anus, and a ventral portion-the urogenital canal or sinus-which receives the bladder and the urogenital ducts. The bladder in mammals develops a stalk, the urethra, which leads to the exterior. The ureters shift so as to open directly into the bladder. In all male placental mammals the vasa deferentia (Wolffian ducts) join the urethra to form a urogenital canal; in most female placental mammals the vagina similarly unites with the urethra forming a urogenital canal; but in primates the urethra and vagina open separately to the exterior, a urogenital canal being absent. The male urogenital canal is generally provided in mammals with various glands, whose secretion is believed to be of importance for the sperm; its terminal portion traverses the penis or organ of copulation and forms the glans of the penis. The penis is formed by the union of two spongy bodies, the cavernous bodies. It begins in reptiles, where the cavırnous bodies are separate. 


\section{THE COMPARATIVE ANATOMY OF THE NERVOUS SYSTEM AND THE SENSE ORGANS}

\section{A. GENERAL CONSIDERATIONS}

In this section of the manual we shall include both the nervous system proper and the sense organs. The former functions for the conduction, co-ordination, and correlation of stimuli, while the latter is differentiated for the reception of stimuli.

I. The parts of the nervous system. - The nervous system consists of three parts: the central nervous system, the peripheral nervous system, and the sympathetic system. The central nervous system is composed of the brain, situated within the skull, and of the spinal cord, situated within the neural canal formed by the neural arches of the vertebrae. Brain and spinal cord are made up of both nerve-cell bodies (gray matter) and of nerve-cell processes (white matter). The peripheral nervous system consists of the cranial nerves springing from the brain, and the spinal nerves, springing from the spinal cord. The nerves are markedly metameric in arrangement, a pair being typically present for each segment. Nerves are composed of nerve-cell processes only. The sympathetic nervous system controls and regulates in general the involuntary activities and the organs which subserve those functions, as the heart, the digestive tract, the smooth musculature in general, the secreting glands, blood vessels, respiratory and reproductive systems. It consists chiefly of a paired cord lying against the dorsal wall of the coelom from which branches and networks extend into the viscera. The sympathetic system is connected with the peripheral nervous system of which indeed it is an outgrowth. Associated with both of these systems are numerous ganglia, of ten segmentally arranged. A ganglion is a collection of nerve-cell bodies, situated outside of the central nervous system. Within the central nervous system a similar collection is called a nucleus or center, although a few of these are designated ganglia, as retentions from an older terminology.

2. The development of the nervous system. - The central nervous system is formed as previously learned by the infolding of the ectoderm in the median dorsal line of the embryo. A tube extending the length of the embryo is thus produced. That portion of the tube situated within the head develops into the brain, while that portion posterior to the head becomes the spinal cord. The originally single layer of enrolled ectoderm cells proliferates to form a thick zone of cells around the central cavity. Most of these become nerve cells, while the remainder give rise to supporting cells. In the spinal cord of all vertebrates and in the brains of the lower ones the zone of nerve cells retains its primitive position around the central cavity, but in the higher vertebrates there is considerable migration of nerve cells to the periphery of the brain. Axones and dendrites arise from the nerve cells by outgrowth; part of these remain in the central nervous system forming a peripheral zone of fibers, called the white matter. Part of the processes of the nerve cells grow out from the central nervous system (or grow into it from sense organs and ganglia) forming the nerves or peripheral nervous system. The ganglia of the peripheral nervous system arise chiefly from the neural crests; these are a pair of longitudinal cords of ectodermal cells which are left outside of the neural tube at the time of its closure. The sympathetic ganglia arise by the migration of cells from the neural tube or from the ganglia of the peripheral nervous system.

In the development of the brain the original simple tube is first marked off into three vesicles, the primary brain vesicles, by two constrictions (Fig. 67). These visicles are named 
the forebrain or prosencephalon, the midbrain or mesencephalon, and the hindbrain or rhombencephalon. Subsequently the first and third vesicles subdivide into two. There thus arise the five principal lobes of the brain, arranged in a longitudinal series (Fig. 67). These are named, beginning anteriorly: the telencephalon, the diencephalon or thalamencephalon, the mesencephalon or midbrain, the metencephalon or cerebellum, and the myelencephalon or medulla oblongata. Each of these five divisions becomes further complicated by additional evaginations, foldings, thickenings, etc., but nevertheless remains as a clearly marked region of the adult brain. The parts derived from each of these divisions will be studied in connection with the specimens. The cavity of the brain becomes the ventricles of the adult brain.

The spinal cord develops chiefly by thickening of the lateral walls of the original neural tube. The central cavity is reduced to a small canal, the central canal. The cord consists of an internal zone of gray matter and external zone of white matter formed as described above.
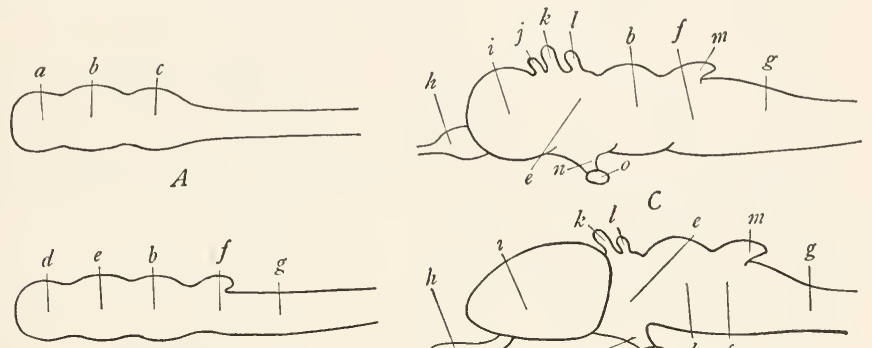

$B$

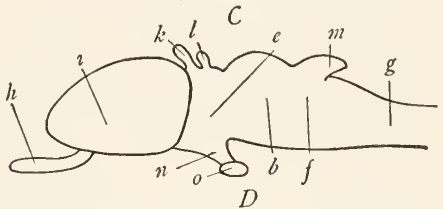

FIG. 67.-Diagrams to show the development of the vertebrate brain. $A$, early stage, showing the three primary brain vesicles, prosencephalon $a$, mesencephalon $b$, and rhombencephalon $c$. $B$, later stage, showing division of the prosencephalon into telencephalon $d$ and diencephalon $e$ and of the rhombencephalon into metencephalon $f$ and myelencephalon $g . \quad C$, adult stage of a lower vertebrate, showing enlargement of the telencephalon to form the cerebral hemisphere $i$, differentiation of the olfactory lobe $h$, and development of outgrowths $j, k, l$ from the roof of the telencephalon and diencephalon and of the infundibulum $n$ from the floor of the diencephalon. $D$, adult stage of a higher vertebrate, illustrating further enlargement of the cerebral hemisphere $i$. $a$, prosencephalon; $b$, mesencephalon; $c$, rhombencephalon; $d$, telencephalon; $e$, diencephalon; $f$, metencephalon; $g$, myelencephalon or medulla oblongata; $h$, olfactory lobe or bulb; $i$, cerebral hemisphere; $j$, paraphysis or evagination of roof of telencephalon; $k$, parietal eye and $l$, pineal body, evaginations from the roof of the diencephalon; $m$, cerebellum; $n$, infundibulum; 0 , pituitary body. (From Parker and Haswell's Textbook of Zoölogy, courtesy of the Macmillan Company.)

The dorsal portions of the gray matter are named the dorsal columns, the ventral portions the ventral columns, and in some regions of the cord there are distinct lateral columns (Fig. 68).

3. The functional composition of the nervous system.- The nervous functions are divisible into two great classes, sensory and motor. The term sensory applies to those impulses which come into the nervous system from the sense organs, to the nerves which conduct such impulses, and to the tracts, nuclei, or areas of the central nervous system which are concerned with these impulses. The dorsal half of the central nervous system is sensory. The term motor is similarly applied to the nerves, tracts, areas, etc., which are concerned with the initiation and conduction from the central nervous system of impulses which excite parts such as the muscles and glands to activity. The ventral half of the central nervous system is motor. The sensory and motor functions are each subdivisible into somatic and 
visceral components, the former dealing with structures in the body wall, the latter with those situated in the viscera. The visceral impulses both motor and sensory are always transmitted by way of the sympathetic system, except in the case of the visceral muscles of the gill region. The spinal nerves transmit all four classes of impulses, while the cranial nerves are irregular in this regard. The somatic sensory impulses arise in the sense organs peripherally located in the body wall and pass along nerves whose cell bodies are nearly always situated in the ganglia of the peripheral nervous system; the visceral sensory impulses arise in nerve endings in the viscera; both pass into the central nervous system. The somatic motor impulses arise from motor cells within the central nervous system and pass out in the nerves to the voluntary muscles; the visceral motor impulses arise in the brain and cord, nearly always

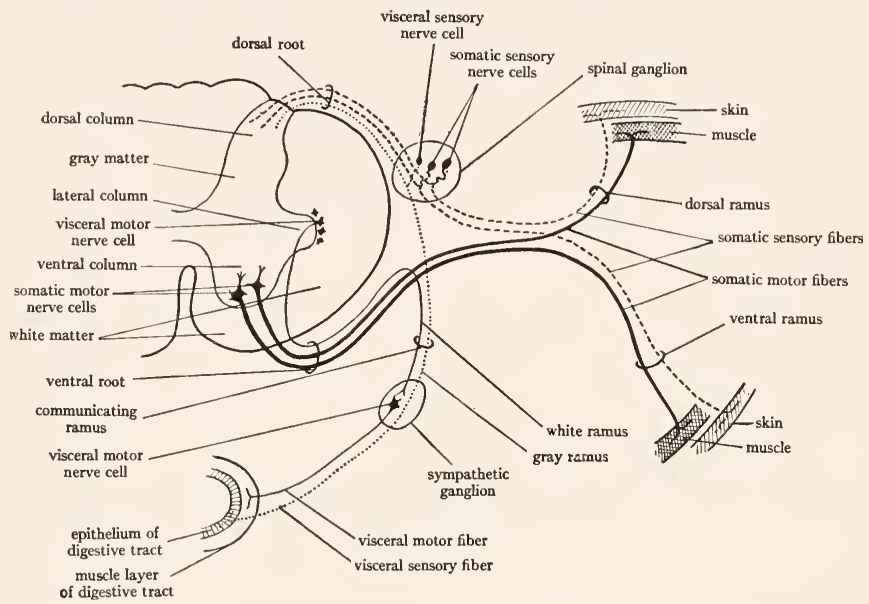

FIG. 68.-Diagram of a cross-section through the spinal cord and a spinal nerve to show the functional components of the spinal nerve and their relation to the spinal cord and sympathetic system. Somatic motor fibers, heavy continuous lines; visceral motor fibers, light continuous lines; somatic sensory fibers, broken lines; visceral sensory fibers, dotted lines. (Slightly altered from Herrick's Introduction to Neurology, courtesy of the W. B. Saunders Company.)

make a relay in the sympathetic ganglia or arise in those ganglia, and pass to the involuntary muscles, glands, etc. (Fig. 68).

In addition to the foregoing, it will naturally be understood that a considerable part of the central nervous system is concerned with the correlation and co-ordination of the foregoing four classes of functions.

4. The peripheral nervous system.-The spinal nerves will serve as examples, as they are more typical. Each spinal nerve is connected with the spinal cord by two roots or bundles of fibers, a dorsal and a ventral. The dorsal root bears a ganglion, the dorsal or spinal ganglion, which consists of a collection of sensory nerve cells. The fibers springing from these cells make up most of the dorsal root. This root enters the spinal cord and connects with the dorsal gray column. The ventral root has no ganglion; its fibers arise from the somatic motor cells of the ventral column and the visceral motor cells of the lateral column of the gray matter of the spinal cord (Fig. 68). Beyond the ganglion both roots unite to form a 
single spinal nerve which passes out through the intervertebral foramen and almost immediately divides into three branches or rami-the dorsal, ventral, and visceral rami. Although, as intimated above, the dorsal root is almost purely sensory, entirely so in the higher vertebrates, and the ventral root purely motor, the rami are mixed. The dorsal and ventral rami both contain somatic motor and sensory fibers passing to and from the body wall and also include a few visceral fibers. The somatic motor fibers of the dorsal rami pass to the epaxial muscles, those of the ventral rami to the hypaxial muscles. The visceral ramus (also called ramus communicans) connects with a sympathetic ganglion. It carries visceral motor fibers from the spinal cord into the sympathetic system, these constituting the white ramus; and also carries visceral sensory fibers from the ganglion into the cord, these forming the gray ramus (Fig. 68). The white and gray rami together form the visceral or communicating ramus, which serves typically to connect each spinal ganglion with the adjacent ganglion of the sympathetic system.

The spinal nerves are paired and segmentally arranged. There are in general as many pairs of spinal nerves as body segments below the head. In connection with the paired appendages the ventral rami of several successive spinal nerves form a network or plexus from which the nerves to the appendage arise. This innervation shows: that the limb muscles arise from the hypaxial parts of the myotomes, since the ventral rami supply only hypaxial muscles; that the limb muscles are derived from several myotomes, since there is but one spinal nerve to each segment of the body; and that the muscles of the appendages have undergone much torsion and change of position, resulting in a crisscross arrangement of their nerves, since each nerve retains its innervation to the muscle which it originally supplied.

The cranial nerves are much less typical in arrangement than the spinal nerves. Most of them do not contain the four classes of fibers. They are attached to the brain by roots, but these are irregularly arranged. Those cranial nerves that contain somatic sensory fibers bear a ganglion corresponding to a spinal ganglion. The composition and functions of the cranial nerves will be described in the dissections. There are ten cranial nerves in anamniotes and twelve in amniotes, but the additional two are not new formations. The cranial nerves are not segmentally arranged, although probably so arranged in the beginning.

5. The segmentation of the head.-It was previously stated that the vertebrate head was originally segmented, but the segmentation is now much obscured. In attempting to work out the head segmentation, the brain, the cranial nerves, the visceral arches, and the true head muscles (not visceral muscles) have been studied. The brain is plainly segmented, particularly in its posterior part; these segments are called neuromeres. Probably the posterior neuromeres are true segmental divisions. The cranial nerves are now much altered from their original condition but they were formerly segmental nerves, and a certain amount of evidence has consequently been obtained from a study of them. The visceral arches are plainly segmentally arranged, but the relation of their segmentation to that of the head as a whole is not entirely clear. It is generally believed that the gill slits are intersegmental in position, i.e., occur at the myosepta. The best evidence has been obtained from the study of the myotomes of the head. In cyclostomes all of these form muscles of the adult, but in other vertebrates many of them disappear in embryonic stages. Those which persist form the muscles of the eyeball and the intrinsic musculature of the tongue. (The student should recollect that the apparent head muscles are visceral muscles, derived from the hypomeres, and not from the myotomes.) The various lines of evidence lead to the following conclusions: that there are four head segments in front of the ear of which the first (anterior head cavity) is evanescent, while the next three (named r, 2, and 3) give rise to the muscles of the eyeball; behind the ear the number of head segments appears to be variable, about $6-8$ in forms above cyclostomes, more in cyclostomes. These postotic segments develop no muscles above 
cyclostomes except that the intrinsic muscles of the tongue appear to be derived from them. These matters will be clearer after a study of the cranial nerves. (See also Fig. 69.)

6. The development of the sense organs. - There are three chief sense organs to be considered here-the nose, the eye, and the ear. The nose arises as a pair of invaginations of the ectoderm of the anterior end of the head. In fishes these persist as a pair of simple olfactory sacs not connected with the mouth. From Amphibia onward the olfactory sacs become connected with the oral cavity by the nasal passages which are then both olfactory and respiratory. The olfactory function is generally limited to the dorsal region of the nasal sac. In most vertebrates the walls of the nasal passages project into the passages as curiously rolled or folded structures, the turbinals or conchae, which serve to increase the olfactory surface and to warm, strain, and moisten the air passing in.

The eyes arise in part as evaginations of the diencephalon. The tip of the evagination then invaginates producing a double-walled cup, the optic cup. (See K, Fig. 228, p. 2I3.)

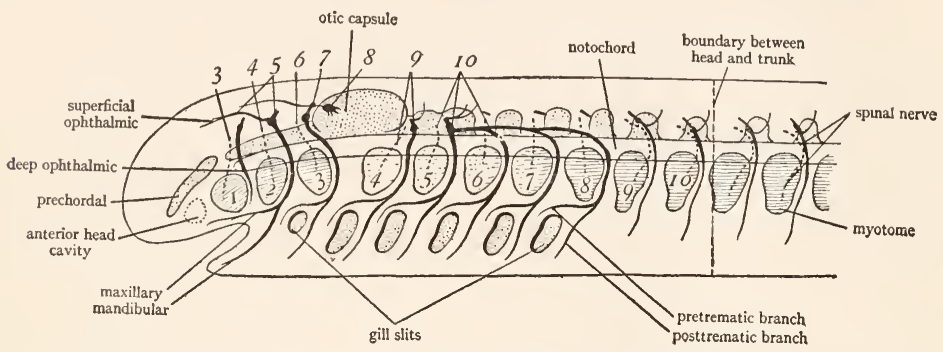

Fig. 69.-Diagram to illustrate the segmentation of the vertebrate head and the relation of the cranial nerves to the segmentation. The numbers above the figure designate the cranial nerves; the numbers in the figure are situated on the head myotomes; the sensory part of the nerves is represented by heavy continuous lines; the motor part by broken lines. The anterior head cavity is the first myotome and therefore the myotome which is numbered $I$ is really the second myotome and so on; but as the myotomes were numbered before the anterior head cavity was discovered, the old numbers are generally retained. The myotomes numbered $I, 2$, and 3 produce the eye muscles; those numbered 4,5 , and 6 degenerate in the majority of vertebrates; those from 7 on probably contribute to the tongue musculature but never form typical parietal muscles such as occur in the trunk. It is seen from the figure that the third cranial nerve and the deep ophthalmic branch of the fifth belong to the first (really second) head segment; the fourth and remainder of the fifth to the second (third) segment; the sixth and seventh to the third (fourth) segment; the ninth to the fourth (fifth) segment; and the tenth to the fifth to eighth (sixth to ninth) segments. The gill slits are intersegmental in location. The relation of the cranial nerves to the gill slits should also be noted. (After Goodrich in Part IX of Lankester's Treatise of Zoölogy, courtesy of the Macmillan Company.)

The internal wall of the optic cup develops into the retina or light-perceiving layer of the eye. The external wall of the cup becomes the pigment layer of the retina. The optic nerve arises in the retina and passes down the stalk of the cup into the brain. The retina is a part of the brain wall, as shown by its manner of formation. The lens of the eye arises from an invagination of the ectoderm over the optic cup. The surface ectoderm then remains as the conjunctiva of the eyeball. The other parts or coats of the eyeball (sclera, cornea, chorioid, and iris) are developed from the mesenchyme surrounding the optic cup.

The vertebrate ear in its complete form consists of three chambers, the internal ear, the middle ear, and the external ear. The internal ear alone is present in fishes. It arises as a saclike invagination of the ectoderm of the head at the level of the hindbrain. This 
sac sinks internally and by constrictions differentiates into various chambers and ducts which will be seen in the dissection. The middle ear appears in Amphibia. It is a chamber produced by an evagination from the first visceral pouch and remains connected with the pharyngeal cavity by the stalk of the outgrowth called the auditory (Eustachian) tube. The middle ear contains little bones for transmitting the sound; there are three of these in mammals, which were treated with the skull. The external ear begins in reptiles and birds and is complete in mammals. It consists of a passage invaginated from the region of the first gill slit; this passage is the external auditory meatus. The inner end of this passage comes in contact with the wall of the middle ear, the two walls then fusing to form a membrane of double origin, the tympanic membrane, commonly called the eardrum. The external orifice of the meatus is surrounded by an outgrowth, the pinna (generally called "ear"), for catching sound waves.

For more complete accounts of the development, comparative anatomy, and functions of the central nervous systems and sense organs, the appropriate chapters in $\mathrm{K}, \mathrm{W}$, or $\mathrm{Wd}$ should be consulted.

\section{B. THE NERVOUS SYSTEM AND SENSE ORGANS OF ELASMOBRANCHS}

I. The spinal nerves and fin plexi.-Remove all of the viscera including the kidneys and reproductive organs from the pleuroperitoneal cavity. Note against the dorsal coelomic wall dorsal to the pleuroperitoneum the white nerves passing out at segmental intervals. These are the ventral rami of the spinal nerves. They lie along the myocommata. In the spiny dogfish they are buried in the muscle and will be revealed by cutting along the myocommata. Farther laterally they.emerge to the internal surface. Trace the ventral rami into the hypaxial muscles.

In the regions of the paired fins the ventral rami supply the muscles of the fins and are more or less united with each other to form a plexus. The plexus for the posterior appendage is the lumbosacral plexus, for the anterior appendage, the cervicobrachial plexus. These plexi are as follows:

Dogfish: The lumbosacral plexus to the pelvic fin is found by cutting through the skin on the dorsal side of the base of the fin. On carefully separating the fin muscles from those of the trunk the nerves of the plexus are seen as white cords passing into the base of the fin. They are more or less imbedded in connective tissue which should be carefully cleaned away. There are ten nerves passing into the fin of which, however, only the last ones are united by cross-branches to form a plexus. The first of the ten is called the collector nerve. Trace it forward and note that it is formed by the union of branches from the ventral rami anterior to the fin.

The cervicobrachial plexus to the pectoral fin is located by cutting through the skin at the base of the fin on the ventral side. On separating the skin from the muscles of the trunk nerves will be seen passing in the connective tissue to the pectoral fin. Proceed carefully forward, carrying your cut into the coelom at the side of the esophagus. The plexus is then seen to consist of a number of nerves (nine in the smooth dogfish, eleven in the spiny species) passing from the 
spinal cord into the fin. Only the first four or five of these, situated on the dorsal side of the bag formed by the posterior cardinal sinus, are united by crossbranches to form a true plexus, the posterior ones passing directly into the fin.

Skate: A large number of ventral rami supply the pectoral fin, the anterior ones uniting to a plexus. Strip off the pleuroperitoneum at the level of the subclavian artery and note there the enormous nerve trunk of the brachial plexus. It is formed by the union (within the neural canal) of a large number of ventral rami. This will be seen later. Follow out the nerve trunk to the pectoral fin. It lies along the posterior side of the curved cartilage (propterygium) which is situated in the pectoral fin about halfway from the mid-dorsal line to the margin. Cut through skin and muscles on the dorsal side of the animal along the posterior and lateral side of this cartilage and expose the trunk. It supplies only the anterior part of the pectoral fin. The posterior part as already noted is supplied by direct ventral rami, not forming a plexus. The lumbosacral plexus for the pelvic fin is located as follows. Remove the skin from the base of the fin on the dorsal side. This exposes a fan-shaped layer of muscles. Cut through this and just ventral to it will be found a number of nerves which diverge into the fin muscles.

2. The sense organs.-For the rest of the section a large separate head will generally be provided. In that case the specimens used up to this point may be discarded. A very careful dissection of this head, on which the student will be graded, is required.

a) The ampullae of Lorenzini: It has already been noted that the skin of the head is perforated by pores, from which mucus exudes under pressure. Note the distribution of the pores. Remove a piece of skin from a region bearing pores (in the skate from the ventral side of the head) and note that each pore leads into a canal of varying length lying beneath the skin. Each canal, named the canal of Lorenzini, terminates in a little bulb, the ampulla of Lorenzini, which is supplied by a nerve, a delicate white fiber easily seen attached to the ampulla. The function of this sensory apparatus appears to be the perception of vibration and pressure in the surrounding water.

b) The lateral line system: In fishes and Amphibia (larval stages only of land Amphibia) there is present a system of sense organs, called the lateral line system, which is related to the aquatic mode of life. This system is completely lost, together with the nerves which supply it, in the land vertebrates. It consists of the lateral line canals and the lateral line nerves. The lateral line canals in fishes consist of tubes situated on the inner surface of the skin onto which they open by pores. In the canals are sensory cells which closely resemble the sensory cells of the ear, and, in fact, the lateral line system appears to be related to the internal ear both morphologically and functionally. The function of the lateral line system is believed to be the perception of water vibrations of low frequency 
Dogfish: Along the trunk the system consists of the lateral lines, which mark the position of a canal. Find the lateral line on the head. Remove the skin at this place, noting the underlying canal and the pores connecting the canal with the surface. Trace the lateral line forward, removing the skin as you proceed. At the level of the spiracles the canals of the two lateral lines are connected by the supratemporal canal. Anterior to this, each forks into a supraorbital canal passing forward above the eye and an infraorbital canal passing ventrally between the eye and the spiracle and then forward below the eye. Trace the supraorbital canal to the end of the rostrum; here it turns and proceeds posteriorly again parallel to its former course and becomes continuous with the infraorbital canal. The latter gives off a hyomandibular branch running posteriorly along the sides of the jaws, and turns to the ventral surface of the rostrum, passing first posterior to the nostril and then turning forward between the two nostrils. There is also a short mandibular canal under the skin just behind the lower jaw; it is not connected with the other canals.

Skate: The lateral line system is more complex than in the dogfish and more difficult to follow. The lateral line canal runs on the dorsal surface just lateral to the mid-dorsal spines. Remove the skin at this place and identify the canal. Trace it forward, removing the skin as you proceed. At the posterior end of the cartilage (propterygium) of the anterior part of the pectoral fin it gives off two canals which proceed posteriorly over the surface of the fin. It then proceeds above the eye as the supraorbital canal, apparently connecting with its fellow by a cross-union on the posterior part of the skull. The supraorbital canal passes in front of the eye and as the infraorbital canal below the eye. In the region of the eye it gives off branches over the rostrum and a long branch which proceeds posteriorly along the lateral margin of the fin. On the ventral side of the skate there is a prominent canal passing just lateral to the gill slits. Trace this forward, noting branches behind and in front of the nostril and on the ventral surface of the rostrum. On the surface of the pectoral fins after removal of the skin the numerous, very long canals of Lorenzini are noticeable.

Draw, showing distribution of the canals.

c) The olfactory organs: These consist of a pair of olfactory sacs on the ventral side of the rostrum, opening externally by the nostril or external naris, with which are associated various flaps of skin. Dissect the skin away from one olfactory sac and cut away the flaps so that you can look into the sac. Note the numerous plates or lamellae arranged in rows inside of the sac; these are covered with olfactory epithelium, the sense of smell being quite keen in fishes. Prove to yourself that the olfactory sac is closed internally, having no communication with the oral cavity.

Draw, showing the lamellae. 
d) The eye muscles: Remove the tissue from about the eye on the same side of the animal as under $c$ ) and completely expose the eyeball. In doing this, first cut away the upper eyelid (or in the skate the skin over the eye), noting that the inner lining of the eyelid is continuous with a thin layer (conjunctiva) which adheres closely to the external surface of the eyeball. Next cut away very carefully the cartilage between the eye and the brain, which is seen as a white lobed structure in the median region, and also the cartilage in front of the eye. Do not injure the brain and do not cut into the elevation dorsal to the spiracle. The stout, white bands seen in this dissection are cranial nerves. In the skate very little cutting is required. The large, somewhat spherical body exposed is the eyeball. In the dogfishes it is imbedded in a gelatinous material which should be carefully cleaned out.

The eyeball reposes in a cavity, the orbit, to the walls of which it is attached by muscular bands, the eye muscles. They are voluntary muscles derived from the myotomes of the second, third, and fourth segments of the head. There are six of these eye muscles which should be identified as follows. From the dorsal view four of them will be seen. The one which is attached to the anterior wall of the orbit is the superior oblique. The other three originate from the posterolateral angle of the orbit and are named recti muscles. The most anterior one is the internal or medial rectus; its insertion on the eyeball is covered dorsally by the superior oblique. The next rectus muscle is the superior rectus, more dorsally situated than the others. The third, the external (or lateral) rectus, is inserted on the posterior surface of the eyeball. Next, raise the eyeball dorsally and note that the conjunctiva or most superficial coat over the external surface of the eyeball is continuous with the lining of the lower lid. Cut through this and free the eyeball ventrally, cleaning out the gelatinous and fibrous tissue which will be found here. On lifting the eyeball the remaining two eye muscles will be seen. The inferior oblique originates from the anteromedial corner of the orbit, the inferior rectus from the posteromedial angle of the orbit; both are inserted in contact with each other on the middle of the ventral surface of the eyeball. The white cords seen among the eye muscles are nerves.

Draw the eyeball and its muscles from dorsal view, showing as many of the muscles as possible.

The eye muscles originate from the orbit and are inserted on the eyeball. Their action is to turn the eyeball in various directions. As already stated they are derived from three head segments and are in most vertebrates practically the only muscles developed from the head myotomes. These three muscleforming myotomes are designated the first, second, and third, although they are in reality the second, third, and fourth of the head myotomes, since the true first segment was not noticed until later. According to the recent investigations of Neal, the third myotome gives rise to part of the external rectus, the second to the rest of the external rectus and to the superior oblique, while the 
first myotome produces the other four muscles. This account differs slightly from that given in the textbooks.

e) The structure of the eyeball: Cut through the eye muscles at the insertions and remove the eyeball. As already noted the outermost coat covering the front of the eyeball is the conjunctiva which is deflected onto the inner surface of the eyelids. Note the free edge of the conjunctiva clinging to the eyeball where the eyelids were cut. The conjunctiva is the epidermis of the skin and not one of the true coats of the eye. The outermost coat of the eyeball is the sclera or sclerotic coat, a very tough membrane composed of connective tissue. The front part of the sclera is transparent and is named the cornea; the conjunctiva is inseparably fused to the outer surface of the cornea. Through the transparent cornea can be seen an opening, the pupil. Cut off the dorsal side of the eyeball so that you can look within the cavity. Place the larger piece under water. The large spherical body in the interior is the crystalline lens. Note that internal to the sclera is a black coat, the chorioid coat, and internal to this a soft, often collapsed, greenish layer, the retina. Follow the chorioid coat to the front of the eye and note that there it is separated from the cornea forming a black curtain, the iris, in the center of which is an opening, the pupil. The iris divides the cavity of the eyeball into an external cavity, the anterior chamber of the eye between the iris and the cornea, and an internal chamber, the cavity of the vitreous humor, between the lens and the retina. The anterior chamber contains a fluid, the aquaeous humor; the cavity of the vitreous humor contains a gelatinous material, the vitreous humor or vitreous body, collapsed in the preserved specimen. The lens in life is attached to the margins of the pupil and also to the margins of the retina; and the small space between these two points of attachment of the lens forms the posterior chamber of the eye.

Draw the section, showing the structures of the eye.

The retina is the nervous part of the eye containing the sensory cells (rods and cones) which are stimulated by light. The lens and the two humors focus the light upon the retina. The focus is changed in fishes by moving the lens back and forth. The pupil regulates the amount of light admitted. The coats of the eye serve for protection and to darken the interior.

In the orbit after removal of the eyeball note the origins of the six eye muscles, the optic pedicel, a cartilaginous stalk situated among the rectus muscles and helping support the eyeball, and the optic nerve, a stout white stalk located in front of the rectus muscles. The stout white band in the floor of the orbit is the infraorbital nerve.

f) The internal ear: The ear in fishes consists only of the internal ear or membranous labyrinth. This is imbedded in the otic region of the skull. In the dogfishes and skate it is situated between the spiracle and the mid-dorsal line. A pronounced elevation of the chondrocranium is present at this place. In the median line between the two elevations will be found a pair of small holes in 
the skin. Upon removing the skin bearing these holes the endolymphatic fossa of the chondrocranium will be found beneath it. In this fossa are two ducts, the endolymphatic ducts, which open on the skin by the two holes just mentioned. These ducts connect the cavity of the internal ear with the surface. Very carefully shave off with a scalpel the cartilage of the elevation containing the ear, working on the same side as before. There will soon be noticed a canal in the cartilage containing a delicate curved tube. This tube is the anterior vertical semicircular duct. Continue removing the cartilage, without injuring this duct. The muscles posterior to the ear may also be removed. Another tube will soon be uncovered posterior to the first one; this is the posterior vertical semicircular duct. There will next be revealed a thin-walled chamber, the utriculus, from which these two ducts spring. Continue picking away the cartilage in small pieces, leaving all parts in place. A third duct, the horizontal semicircular duct, lying below and lateral to the others, will next be exposed. When the cartilage has been removed as far as possible, the parts of the internal ear may be identified. The central chamber to which the ducts are attached consists of a smaller dorsoanterior portion, the utriculus, from whose dorsal tip the anterior vertical and horizontal ducts spring; and a larger ventro-posterior part, the sacculus, from which the posterior vertical duct takes origin. The sacculus fits into a rounded depression in the cartilage. The semicircular ducts are slender tubes, curved in a semicircle and each terminating in a rounded sac, the ampulla. The ampullae of the anterior vertical and horizontal ducts are in contact with and communicate with each other and also with the anterior extension of the ventral part of the utriculus. This extension is called the recessus utriculi. Both ends of the posterior vertical canal join the sacculus. In each ampulla will be seen a white sensory patch or crista, to which a branch of the auditory nerve is attached. Larger sensory patches, called maculae, also occur in the recessus utriculi and in the sacculus. Inside the sacculus, a white mass of sand grains or crystalline material, the otolith, is visible. The movements of these grains may be concerned in equilibration. The endolymphatic duct opens from the medial side of the sacculus but the connection is difficult to find.

Draw, showing parts of the internal ear. After the drawing has been made, the sacculus may be opened and the otolith examined.

The internal ear has two functions, that of hearing and that of equilibration. The whole structure is filled with a fluid, the endolymph, while the channels in the cartilage are filled with perilymph. Changes in pressure in the endolymph due either to the impinging of sound waves on the head or to changes in the position of the head appear to be the stimuli which excite the sensory cells of the cristae and maculae, producing in the first case the sensation of hearing and in the second sensations of the animal's position in the water, enabling it to keep in the desired position. According to the experiments of Maxwell, both (or either) the cristae and the maculae control equilibration. The capacity to perceive sounds is presumably limited to the cristae. 
3. The dorsal aspect of the brain.-The brain is now to be exposed by carefully picking away the cartilage in small pieces from its roof. The cranial nerves, white strands passing through the cartilage, must not be injured. One side of the head has thus far been left intact for the study of the cranial nerves. This side is now to be exposed along with the brain as far as necessary. Remove the upper eyelid as directed under the eye but leave all structures intact. In removing the cartilage between the brain and the eye the following nerves will be noted: the superficial ophthalmic nerve running in the wall of the orbit near the dorsal surface; the small trochlear nerve passing through the back wall of the orbit to the superior oblique eye muscle; in the skate the larger oculomotor nerve accompanying the trochlear. Dissect forward to the olfactory sacs, exposing them dorsally, leaving the ophthalmic nerve in place. Remove the skin behind the spiracle and note the hyomandibular nerve passing posterior to the spiracle; this nerve is also to be preserved. To expose the posterior part of the brain the internal ears of both sides may be cut through and the mass of muscles posterior to the ear removed as much as necessary. Nerves will be seen passing through the cartilage in the ventral part of the ear, but are not to be dissected out for the present. In short, the dorsal side of the brain is to be fully exposed, leaving all of the more superficial nerves intact. The dorsal aspect of the brain will then be studied first, and the cranial nerves afterward.

The brain is situated in a cavity in the chondrocranium, which it only partially fills. It is covered by a delicate membrane, the primitive meninx, in which the blood vessels of the brain are situated. The meninx is connected by strands with the membrane lining the cartilaginous walls of the cranial cavity. The space between brain and chondrocranium is filled in life by a fluid.

The most anterior part of the brain is the large olfactory bulb, a nervous mass situated in contact with the dorsal walls of the olfactory sacs. From the olfactory sac a number of very short fibers, which together constitute the olfactory nerve, pass into the olfactory bulb. The olfactory bulb is spherical in the dogfishes, elongated in the skate. The olfactory bulb is connected with the next part of the brain by a stalk, the olfactory tract, which is short in the smooth dogfish. The olfactory tracts pass to enlarged, rounded lobes, the olfactory lobes, at the anterior (or lateral in the skate) end of the main mass of the brain. Posterior to (dogfish) or medial to (skate) the olfactory lobes is situated another pair of lobes, the cerebral hemispheres. The cerebral hemispheres and the olfactory lobes are separated only by a faint groove. All of the parts thus far mentioned belong to the telencephalon. The olfactory bulbs, tracts, and lobes together constitute the rhinencephalon or smell portion of the brain. In fishes the cerebral hemispheres are also largely concerned with the sense of smell.

Posterior to the cerebral hemispheres is a depressed region, the diencephalon or thalamencephalon, very narrow in the smooth dogfish. The roof of this is thin and discolored, due to the fact that it consists entirely of a plexus of blood 
vessels, called a chorioid plexus. This particular plexus is named the chorioid plexus of the third ventricle. The diencephalon serves chiefly as a center of co-ordination of the principal sensations, such as sight, hearing, and skin sensations, its ventral portions being devoted to smell and taste. The diencephalon is in lower vertebrates the chief controlling region of the brain. The optic nerve passes from the orbit into the ventral surface of the diencephalon. It is quite conspicuous in the skate and will be seen in the dogfishes by gently pressing the diencephalon to one side. Posterior to the diencephalon is the midbrain or mesencephalon, consisting dorsally of two rounded lobes, the optic lobes or corpora bigemina. The optic lobes are centers for the visual, auditory, and general skin sensations, and these are connected with corresponding centers in the diencephalon. A nerve, the trochlear or fourth cranial nerve, arises from the posterior borders of the optic lobe on each side and passes forward to an eye muscle. By gently pressing the optic lobes to one side, a nerve, the oculomotor or third cranial nerve, will be seen emerging from the ventral surface of the midbrain and passing to the orbit.

Posterior to the optic lobes and somewhat overhanging them is the large cerebellum or metencephalon. In the spiny dogfish and skate this is slightly divided into four quadrants by faint longitudinal and transverse grooves; in the smooth dogfish it is marked by several transverse grooves. The cerebellum is a center for the co-ordination of motor impulses, including the maintenance of equilibrium. No nerves are attached to the cerebellum. Posterior to the cerebellum the elongated remaining section of the brain is the medulla oblongata or myelencephalon. It is continuous posteriorly with the spinal cord. The anterior part of the roof of the medulla is thin and discolored and consists of a chorioid plexus, that of the fourth ventricle. The anterior end of the medulla extends forward at the sides of and below the cerebellum as two earlike projections, sometimes called the auricles. Remove the chorioid plexus from the roof of the auricles and the medulla. The large cavity revealed is the cavity of the fourth ventricle. The auricles together with the portion of the dorsal part of the medulla just posterior to them constitute the acustico-lateral areas, or primary centers of the lateral line system and of hearing and equilibration. On lifting the posterior end of the cerebellum it will be seen that the auricles are continuous with the cerebellum and with each other, below the overhanging posterior portion of the cerebellum. By way of this connection with the cerebellum the impulses which come in from the lateral line system and internal ear are conveyed to the cerebellum after making a relay in the acustico-lateral area. The entire dorsal rim of the medulla, including the acustico-lateral areas, forms an elongated strip on each side which is known as the somatic sensory column. This column as its name implies contains centers and tracts associated with all of the body senses except smell, taste, and vision, which as we have seen are disposed of 
in the more anterior portions of the brain. Near the anterior end of the somatic sensory column, at the middle of the acustico-lateral area, will be seen, by pressing the latter toward the middle, the roots of a number of nerves. These are the roots of the fifth, seventh, and eighth cranial nerves, to be studied in more detail later. At the posterior end of the somatic sensory column, just anterior to the point where the walls of the medulla close together, will be noted the stout root of the tenth cranial nerve. In the lateral wall of the medulla, just ventral to the somatic sensory column, is another longitudinal area marked by a row of rounded elevations; this area is the visceral sensory column. As its name implies, it is associated with sensations from the viscera. In fishes the gills are important visceral structures so that a considerable portion of this column is connected with the gills; in fact, each of the little elevations is said to be a center for one visceral arch. Ventral to the visceral sensory column is the very slender visceral motor column, from which impulses go to the visceral muscles. We have already learned that the visceral muscles are the muscles of the gill region. In the floor of the fourth ventricle are the two conspicuous somatic motor columns, separated by a median groove. The somatic motor columns are the places of origin of impulses to the somatic or parietal muscles. As previously stated, these in the head consist chiefly of the six eye muscles.

The fourth ventricle narrows posteriorly and is finally roofed over by the fusion of the walls of the medulla. Shortly beyond this point the medulla is continuous with the spinal cord. The posterior end of the medulla marks the posterior end of the brain but is not sharply defined.

Draw the dorsal aspect of the brain. Place it in the middle of the page so that the cranial nerves can be added later.

4. The cranial nerves.-There are ten cranial nerves in fishes. They are to be dissected with great care and their distribution noted and drawn. This distribution is in general similar in all vertebrates except that certain nerve trunks present in fishes disappear in the land vertebrates. One of the most striking examples of homology is found in this distribution of the cranial nerves which still in man continue to supply the same parts as in the fish.

Add to your drawing of the dorsal side of the brain an outline of the head of the animal, putting in outline the olfactory sacs, eyes, ears, and gill slits. As you dissect the cranial nerves according to the directions to be given immediately, add each to this drawing, showing as accurately as possible the location and course of each of these nerves and the parts of the head which they supply. It is necessary to enter a nerve on one side only, and by using the two sides of the drawing for different nerves it will be possible to enter all of them.

a) The first or olfactory nerve: This nerve has already been noted. It arises from the olfactory cells in the lamellae of the olfactory sac and passes by very short branches into the olfactory bulb. These branches are practically invisible. 
From the olfactory bulb, after a relay, the olfactory impulses pass along the olfactory tracts to certain parts of the brain described above. The olfactory nerve is a pure sensory nerve.

b) The second or optic nerve: The optic nerve arises in the retina of the eye and passes through the coats of the eye, emerging ventral to the internal rectus muscle. Find it there on the intact eye by pressing this muscle against the eyeball. It is a stout, white trunk which pierces the cartilage of the orbit and passes to the ventral side of the diencephalon. It may be seen here by gently raising the diencephalon. The optic nerve is not really a nerve, for the retina in which it arises is a part of the brain wall, and in the retina there are several relays of nerve cells between the rods and cones and the cells of origin of the optic nerve. The optic nerve is really a tract of the brain. It carries sensory impulses only, visual impulses, and discharges them into certain parts of the diencephalon and optic lobes.

c) The fourth or trochlear nerve: The trochlear nerve arises in the midbrain and emerges in the groove between the optic lobes and the cerebellum. Trace it on the side where the eye is still intact. It passes forward in the cranial cavity to about the level of the cerebral hemispheres; it then turns abruptly laterally, pierces the wall of the orbit, and is distributed to the superior oblique muscle of the eyeball. It is the motor nerve of this muscle and carries only somatic motor impulses. Although it appears externally to emerge from the roof of the midbrain, the motor cells from which it originates are in fact in the floor of the midbrain in a forward extension of the somatic motor column.

d) The third or oculomotor nerve: The oculomotor nerve arises from the floor of the midbrain. It is readily noticed in the skate, ascending to the orbit near the preceding nerve. In the dogfishes it is deeply situated and is seen by pressing the cerebellum away from the wall of the orbit. Follow it into the orbit on both sides, getting its general relations first on the side where the eyeball was removed. It emerges into the orbit very near the insertion of the superior rectus muscle and is situated ventral to the superficial ophthalmic nerve, already noted. It should not be confused with the deep ophthalmic nerve which is in contact with it as it enters the orbit; the deep ophthalmic nerve runs through the orbit in contact with the medial surface of the eyeball. This nerve will be better seen on the intact side. Observe the branches given by the oculomotor nerve immediately after its entrance into the orbit to the internal and superior recti muscles. On the intact side now loosen the eyeball and cut the insertions of the superior oblique and superior rectus close to the eyeball. Identify the deep ophthalmic nerve passing in the spiny dogfish dorsal to the internal rectus lying against the eyeball and in the smooth dogfish and skate ventral to the internal rectus. Free and preserve this nerve. Cut through the insertion of the inferior rectus and the optic nerve and, pressing the eyeball outward, note the branch of the oculomotor nerve, which passes along the posterior side of the inferior rectus muscle, 
turns ventral to it, and then runs forward in the floor of the orbit to the inferior oblique. Note also that the branch which supplies the inferior rectus gives off a branch, one of the ciliary nerves, which passes into the eyeball in company with an artery. Along this nerve small brown masses can be noticed; they are the ciliary ganglia belonging to the sympathetic system. The deep ophthalmic nerve also supplies ciliary branches into the eyeball. The function of the ciliary nerves is to control the smooth muscles of the iris, regulating the size of the pupil; they are visceral motor nerves, making a relay in sympathetic ganglia.

It will be seen from the foregoing account that the oculomotor nerve supplies four eye muscles, namely, those which develop from the so-called first head myotome. It is a somatic motor nerve originating from the somatic motor column in the floor of the midbrain. It also, however, carries with it sympathetic fibers of visceral motor function.

e) The sixth or abducens nerve: The abducens originates from the somatic motor column on the ventral surface of the anterior end of the medulla. Its origin will be seen later. It penetrates the orbit at the point of origin of the external rectus muscle and passes along the ventral surface of this muscle to which its fibers are distributed. It will be seen as a white ridge on the ventral surface of the muscle.

It will now be seen that the third, fourth, and sixth cranial nerves are somatic motor nerves to the muscles of the eyeball. The reason for the allotment of three cranial nerves to six small muscles has already been indicated. To every segment of the vertebrate body there should be theoretically a pair of motor and sensory nerves. Since as already explained the six eye muscles come from three head myotomes, it is readily understood that a somatic motor nerve should be present to supply the derivatives of each of these myotomes. The sensory nerves of these segments will be indicated later.

f) The fifth or trigeminus nerve: The trigeminus is a very large nerve with four main branches in elasmobranchs (three in land vertebrates). The trigeminus is attached to the medulla near the anterior end of the somatic sensory column, just behind the auricles of the medulla. Its roots here are inextricably mingled with the roots of the seventh and eighth nerves, the three together forming a conspicuous mass at the place stated. The trigeminus passes through the adjacent wall of the orbit and should be followed into the orbit by carefully picking away the cartilage around it. As soon as it penetrates the orbit the trigeminus divides into four branches. The first of these, the superficial ophthalmic branch, is part of the superficial ophthalmic trunk which has already been mentioned several times. This large trunk passes forward in the dorsal part of the cartilage of the medial wall of the orbit. Trace it forward. It passes out of the orbit through the ophthalmic foramen in the chondrocranium, and above the olfactory bulb. Only a small part of this trunk is trigeminal; this is sensory to the skin dorsal to the orbit. The second branch of the trigeminus is the deep 
ophthalmic nerve. This passes through the orbit ventral to the preceding and leaves the orbit by the orbitonasal canal. Upon tracing it forward it will be seen to join the superficial ophthalmic and to be distributed in common with it. Both of these branches of the trigeminus are pure somatic sensory nerves, arising from sense organs of the skin.

The two remaining branches of the trigeminus lie in the floor of the orbit. To see them remove the eyeball or study the side where the eyeball was previously removed. A broad white band, the infraorbital trunk, is seen in the floor of the orbit, passing obliquely laterally. This trunk in the dogfishes is composed of the mixed fibers of the maxillary branch of the trigeminus and the buccal branch of the seventh nerve (see below). In the orbit the larger and more medial portion of the trunk is the maxillary branch, but farther out this becomes inextricably mingled with the buccal nerve. In the skate the infraorbital trunk is divisible into three trunks, of which the outer one is the maxillary branch of the trigeminus, the middle one the mandibular branch of the trigeminus, and the inner one the buccal branch of the seventh nerve. As before, however, it should be remembered that there is an admixture of fibers of the fifth and seventh nerves in these trunks. Trace the maxillary branch of the trigeminus and buccal branch of the seventh out from the orbit, along the ventral surface of the rostrum. The branches pass to the region below and in front of the eye, to the medial side of the nostril (in the skate to the lateral side of the nostril also) and to the angle of the jaws. In the smooth dogfish there is a conspicuous branch along the lower jaw which appears to be a part of the mandibular branch of the trigeminus. The maxillary branch is sensory to the skin of the rostrum, while the buccal nerve supplies the infraorbital lateral line canal and near-by ampullae of Lorenzini.

The fourth branch of the trigeminus is the mandibular branch. In the dogfishes it separates from the infraorbital trunk where the latter enters the orbit from the brain and passes along the posterior wall of the orbit. In the smooth dogfish part of it seems to accompany the infraorbital trunk forward and then curves to the lower jaw as described above. The mandibular nerve is seen to branch to various muscles in the floor of the orbit (these are gill-arch muscles) and on following it out of the orbit, will be seen to be distributed to muscles of the lower jaw and to send a sensory branch to the skin of the lower jaw, this branch being situated just behind the teeth. In the skate the position of the mandibular nerve was described above as between the maxillary and the buccal nerves. Follow it forward. It curves around the angle of the jaw and supplies muscles of the lower jaw and the adjacent skin.

It will be observed that all of the branches of the fifth nerve are somatic sensory nerves coming from various sensory organs of the skin, except the mandibular nerve which also contains some motor branches to muscles. As those muscles are visceral muscles, this part of the fifth nerve belongs to the visceral motor system. The deep ophthalmic nerve appears 
to have been the sensory part of the oculomotor nerve, but it has been absorbed by the trigeminus which has spread beyond its original distribution. The deep ophthalmic alone persists in higher forms as the ophthalmic branch of the trigeminus, the superficial ophthalmic branch disappearing. The remainder of the trigeminus is the sensory portion of the trochlear nerve (see Fig. 69, p. 300 ). It is important to note further that the trigeminus is the nerve of the upper and lower jaws. Since the jaws and associated parts constitute the first visceral arch, the trigeminus is said to be the nerve of the first visceral arch. It is the sensory nerve of this arch and also the motor nerve of its visceral muscles.

g) The seventh or facial nerve: This nerve is intimately related to the trigeminus. It arises in common with the latter from the anterior end of the medulla and divides into three main branches. Two of these branches pass through the orbit in common with the trigeminus. The superficial ophthalmic branch of the facial nerve accompanies the same branch of the trigeminus and forms the greater part of the superficial ophthalmic trunk, supplying the supraorbital lateral line canal and adjacent ampullae of Lorenzini. The buccal branch of the facial nerve, as already noted, forms in the orbit the outer half of the infraorbital trunk and supplies the infraorbital lateral line canal and near-by ampullae of Lorenzini. These two branches of the facial nerve are sensory branches which are lost when the lateral line system disappears. The third branch of the facial nerve is the large hyomandibular trunk which has already been located posterior to the spiracle. Trace it inward toward the brain, cutting tissues in its path. It turns ventrally and runs through the anterior part of the ear capsule deep down. Follow it by removing the cartilage of the ear capsule in small pieces. The nerve passes ventral to some of the branches of the nerve of the ear and joins the anterior end of the medulla in common with the trigeminus root. Near the brain it has an enlargement or ganglion (geniculate ganglion). From this ganglion is given off the palatine nerve. It will be found by dissecting carefully around and on the ventral surface of the ganglion. In the skate it is easily seen. It runs forward below the orbit along the roof of the mouth where it supplies the taste buds and the lining epithelium in general. Now trace the hyomandibular outward, past the spiracle. It turns ventrally and breaks up into branches on the side of the head. These branches supply the hyomandibular and mandibular lateral line canals, ampullae and similar sense organs, the muscles of the hyoid arch, and the lining of the floor of the mouth cavity and tongue.

The hyomandibular is seen to be a mixed nerve with lateral line, visceral motor, and visceral sensory components. The sensory part of the facial nerve is supposed to belong to the same segment of the head as that of which the abducens constitutes the motor nerve. The facial nerve is the nerve of the first gill slit (spiracle) and of the second visceral arch (hyoid arch) and of the muscles belonging to this arch. The lateral line parts of the facial nerve which in fishes compose the greater part of it are lost in land vertebrates, only the palatine and certain parts of the hyomandibular branch persisting as the facial nerve of land vertebrates. 
h) The eighth or auditory nerve: The auditory nerve is a pure somatic sensory nerve extending from the internal ear to the brain. It enters the anterior end of the medulla and is there mingled with the roots of the fifth and seventh nerves. Follow it into the internal ear on the side opposite that on which the hyomandibular was dissected. Note its branches to each ampulla and the fanlike arrangement of the branchlets to the crista of each ampulla. The auditory nerve also collects a number of branches from the walls of the sacculus and utriculus. It carries impulses for hearing and equilibration into the acustico-lateral area of the medulla to which it will be found to be attached. There is no motor nerve corresponding to the auditory nerve.

i) The ninth or glossopharyngeal nerve: This nerve passes through the floor of the middle of the ear capsule (where it is likely to be mistaken for a part of the auditory), parallel to the hyomandibular nerve. Pare away as much of the ear capsule as is necessary to reveal it. Find its attachment to the medulla posterior to the auditory nerve. Trace it out of the ear capsule. Just before it exits from the ear capsule it bears a swelling, the petrosal ganglion. Insert a knife blade into the second (first typical) gill slit (in the skate into the dorsal wall of the corresponding visceral pouch) and slit the gill cleft open dorsally. The petrosal ganglion will now be seen to be located near the upper limits of the cleft. Dissect the nerve from the ganglion toward the gill slit. It very soon divides into three branches, two smaller anterior ones, and a larger posterior one. The most anterior branch is the pretrematic branch and passes to the anterior wall of the visceral pouch to which it is a sensory nerve. The second branch is posterior to the pretrematic branch; it is named the pharyngeal branch and is a sensory nerve to the mouth cavity. (This branch appears to be lacking in the skate.) The third and largest is the post-trematic branch. It passes to the posterior wall of the visceral pouch and is both sensory and motor, its motor components supplying the muscles of the third visceral arch. The glossopharyngeal nerve is the nerve of the second visceral pouch and of the third visceral arch.

j) The tenth or vagus nerve: The vagus nerve is the very large trunk passing through the posterior border of the ear capsule. It is attached to the sides of the posterior part of the medulla. Dissect it out and follow its course. It passes to the anterior cardinal sinus, the wall of which is formed in the dogfishes of a tough membrane. Open up the anterior cardinal sinus by a deep cut through the muscles medial to the visceral pouches. Follow the vagus nerve into the anterior cardinal sinus. In the dogfishes it divides into two trunks at the point where it penetrates the tough wall of the sinus. The medially situated trunk is the lateral branch of the vagus and passes posteriorly internal to the lateral line whose canal it supplies. The lateral trunk is the visceral branch of the vagus, which continues along the anterior cardinal sinus. In the skate the vagus runs for a short distance in the sinus before dividing into a more dorsal lateral branch, which passes posteriorly internal to the lateral line canal to which 
it is distributed, and a more ventral visceral branch. In all three forms, open up the remaining visceral pouches as directed for the ninth nerve and determine the distribution of the visceral branch of the vagus. With the walls of the sinus well spread open note the four branches crossing the floor of the sinus to the visceral pouches. Dissect out each of these and observe that each bears a ganglion, beyond which it divides into three branches: an anterior pretrematic branch, a middle pharyngeal branch, and a posterior post-trematic branch. The pharyngeal branch seems to be missing in the skate. As in the case of the ninth nerve, the pre- and post-trematic branches embrace the visceral pouch, which lies between them; all three branches have the same functions as described for the ninth nerve. We thus see that the vagus nerve supplies the remaining visceral arches, beginning with the fourth, and the remaining visceral pouches, beginning with the third. After supplying the gill apparatus the visceral branch of the vagus passes on into the pericardial and pleuroperitoneal cavities, supplying the heart, digestive tract, and other viscera.

Our study of the cranial nerves shows that they bear a definite relation to the original segmentation of the head and to the gill apparatus. The fifth, seventh, ninth, and tenth cranial nerves are gill-arch nerves, each associated with one or more particular visceral arches. In the evolution of the vertebrates each nerve continues to supply its particular arch or arches and all derivatives thereof. Since the muscles of the visceral arches are visceral muscles, the motor components of the gill-arch nerves in all vertebrates are visceral motor in nature. The somatic motor components for the fifth and seventh nerves, as already stated, are separated into special nerves, the eye-muscle nerves. There are no somatic motor elements for the ninth and tenth cranial nerves, since the myotomes which should give rise to voluntary muscles for their segments are degenerate in all vertebrates above cyclostomes (Fig. 69, p. 300).

5. The occipital, hypobranchial, and first spinal nerves.-Very carefully expose the spinal cord posterior to the medulla by shaving away the cartilage of the neural arches in thin slices. On the dorsolateral surface of the cord note the little swellings, the dorsal or spinal ganglia, attached to the cord by the sensory or dorsal root. In the skate the spinal ganglia are elongated. Between the first spinal ganglion and the root of the vagus note two or three small roots springing from the side of the medulia. These are the occipital nerves. They innervate some muscles of this region and also help to form the hypobranchial nerve described below, and contribute to the cervicobrachial plexus. On pressing the spinal cord to one side the ventral or motor roots of the spinal nerves will be seen arising from the ventrolateral region of the cord, at the same level as the occipital nerves. The ventral roots are formed by the union of several small rootlets coming from the cord. The most anterior ventral roots are situated anterior to the dorsal roots which belong to the same segment.

In the dogfish the union of dorsal and ventral roots to form a spinal nerve is not easy to follow. It may usually be seen by carefully paring down the cartilage along the side of the spinal cord. In the skate the union is easily followed; 
the roots pass through the cartilage at the side of the cord and unite as they exit from the cartilage. A large number of the most anterior spinal nerves (really the ventral rami of the spinal nerves, the dorsal rami being very slender in the skate) are then seen to unite to form the very large nerve of the brachial plexus previously noted.

The hypobranchial nerve is a trunk formed by contributions from the occipital nerves and the first spinal nerves. In the dogfishes it may be located as follows. Insert one blade of the scissors in the angle of the jaw and cut back across the gill slits through to the side of the esophagus as was done in an early stage of the dissection (if the same specimen is still being used, this cut will already have been made). Open the flap thus formed and expose the roof of the mouth. Make a longitudinal cut through the mucous membrane of the roof in the median dorsal line. Strip the membrane laterally carrying with it the free dorsal ends of the gill cartilages which will be readily located just lateral to the mid-dorsal line. The visceral branch of the vagus nerve, which was already seen from the other side, is now exposed. It lies along the thin ventral wall of the anterior cardinal sinus. Emerging from the muscle now exposed in the roof of the mouth will be seen the ventral rami of the spinal nerves. In the spiny dogfish two of these (they appear as one but will be found to consist of two on dissecting them toward the median line) pass obliquely toward the visceral branch of the vagus and enter its sheath, thus appearing to join it. The trunk they form can, however, be readily separated from the vagus. This trunk is the hypobranchial nerve. In the smooth dogfish the several most anterior ventral rami unite to form a large trunk, the hypobranchial nerve. This nerve lies just anterior to the nerves of the cervicobrachial plexus to which it contributes branches. It passes to the dorsal side of the visceral branch of the vagus (which may be noted proceeding to the esophagus) posterior to the last visceral pouch, and turning ventrally courses along the floor of the mouth, supplying muscles.

In the skate the hypobranchial nerve leaves the spinal cord in common with the trunk of the brachial plexus. Locate this trunk again in the anterior wall of the pleuroperitoneal cavity, dorsal to the pleuroperitoneum and just behind the cartilage of the pectoral girdle. The entire trunk with the exception of one branch passes out dorsal to the cartilage to the pectoral fin. This one branch, the hypobranchial nerve, turns forward and is distributed to the muscles of the floor of the mouth.

The occipital and hypobranchial nerves are probably the homologues of the twelfth or hypoglossal nerve of amniotes, having been taken into the cranium in those forms. The muscles supplied by the hypobranchial nerve are believed to be derived from the most posterior of the head myotomes, and in higher forms become the intrinsic muscles of the tongue.

6. The ventral aspect of the brain.-Carefully free the brain from the chondrocranium. First cut through the olfactory tracts and gently lift the ante- 
rior end of the brain. You will next see the two optic nerves entering the ventral surface of the diencephalon. Cut through them and lift the brain farther. Next, pare away the wall of the orbit on one side. It will then be seen that certain structures attached to the ventral surface of the diencephalon extend ventrally into a deep pit (sella turcica) in the floor of the cranial cavity. Take especial care to lift these out intact. Then cut through the remainder of the cranial nerves and cut across the spinal cord and lift the brain out of the cranial cavity.

Examine the ventral surface of the brain. Note the forward continuation of the internal carotid artery on the midventral line of the brain. It forks around the ventral part of the diencephalon (farther posteriorly in the skate) and passes forward to the telencephalon, distributing many branches to all parts of the brain as well as to the orbit. The ventral surface of the brain presents nothing new except as regards the diencephalon, where several additional structures are visible. The two optic nerves are seen attached to the anterior end of the ventral surface of the diencephalon; as they enter the latter they cross, the crossed region being named the optic chiasma. From the chiasma a broad band, the optic tract, extends dorsad and caudad into the dorsal part of the diencephalon and into the optic lobes. It is readily seen, especially in the spiny dogfish, by scraping off the primitive meninx at this place. It is thus evident that the visual impulses pass into these two portions of the brain. Posterior to the optic chiasma the floor of the diencephalon bulges ventrally and posteriorly as the infundibulum, consisting in large part of two rounded lobes, the inferior lobes. These are devoted mainly to smell and taste. From between the two inferior lobes a stalk projects caudad and widens into a soft sac. This whole structure is the hypophysis or pituitary body. The dorsal part of the sac has thinner walls, generally discolored because of contained blood; it is called the vascular sac or saccus vasculosus. The hypophysis is generally more or less torn in removing the brain. It is known to be a gland of internal secretion in mammals and probably has the same function in other vertebrates. Dorsal to the vascular $\mathrm{sac}$ the roots of the oculomotor nerves will be found springing from the floor of the midbrain. The ventral surface of the remainder of the brain presents nothing new. The roots of the cranial nerves should be identified on the medulla. On the ventral surface of the medulla will be found the roots of the sixth nerves. If not identifiable on the brain, they will usually be found adhering to the floor of the cavity from which the brain was removed.

Draw a profile view of the brain.

7. The sagittal section and the ventricles of the brain.- The brain like the remainder of the central nervous system is hollow. Its cavities are known as ventricles and are continuous with each other by means of narrow passages. The fourth or last ventricle of the brain has already been identified as the cavity within the medulla oblongata. The ventral portion of this ventricle is named 
from its shape the fossa rhomboidea. Bisect the brain by a median sagittal cut. Examine the cut surface, best under water. From the fourth ventricle a narrow passage, the aqueduct of the brain, extends anteriorly. It communicates with the cavity of the cerebellum, the cerebellar ventricle, and the cavities of the optic lobes, the optic ventricles. The thick floor of the midbrain ventral to the aqueduct forms the cerebral peduncles. The aqueduct opens into the cavity of the diencephalon, the third ventricle. The roof of the diencephalon is very thin and consists of a chorioid (vascular) plexus which is folded into the cavity. The anterior part of this roof in the dogfishes extends dorsally into a sac, the paraphysis, resting against the telencephalon. It is regarded as part of the latter. Posterior to the paraphysis, a thin transverse partition, the velum transversum, is seen in the roof of the diencephalon, particularly in the spiny dogfish. This marks the dorsal boundary between diencephalon and telencephalon. The small thickened region of the diencephalon just in front of the anterior end of the optic lobe is the habenula, a smell center. From the habenula a slender process, the pineal body, may be seen in favorable specimens, extending dorsally, just back of the paraphysis. The entire roof of the diencephalon, including chorioid plexus, habenula, and pineal body, is named the epithalamus and is concerned chiefly with smell. The lateral walls of the diencephalon constitute the thalamus, an important correlation center for various body senses. The ventral part of the diencephalon is named the hypothalamus. It consists of the infundibulum, including the inferior lobes, the hypophysis, and the mammillary bodies. The former should be identified on the section. The mammillary bodies are the thickened part of the ventral wall above the vascular sac. The hypothalamus is concerned with smell and taste. Note that the cavity of the third ventricle extends into all parts of the hypothalamus. The third ventricle connects by a passage, the foramen of Monro, or interventricular foramen, with the cavity in each half of the telencephalon. (In the skate, the telencephalon is solid.) These two cavities are named the first and second or lateral ventricles. They extend out into the olfactory bulbs through the olfactory tracts. Cut into the telencephalon to see its ventricles.

Draw the sagittal section.

\section{THE NERVOUS SYSTEM AND SENSE ORGANS OF NECTURUS}

I. The spinal nerves. - The spinal nerves are best found as follows. Make a longitudinal cut along the side of the body below the lateral line. Cut through the external and internal oblique muscles and separate this mass of muscle from the thin layer of transverse muscles lying next to the coelom. The ventral rami of the spinal nerves will now be seen running between the oblique and transverse muscles, along the myosepta, and supplying the hypaxial muscles. Trace one of these toward the vertebral column, cutting away muscles from its course. It lies just behind the rib which may be cut away. The nerve may be traced up to the vertebra where it is imbedded in an orange-colored material. On 
carefully clearing this away the dorsal ganglion of the spinal nerve will be found imbedded in it. It is a rounded brownish body from which spring two nerves, the ventral ramus just followed and the smaller dorsal ramus which supplies the epaxial muscles.

2. The limb plexi.-The ventral rami of the spinal nerves form a plexus for each limb, the motor nerves for the limb muscles arising from this plexus. The brachial plexus is located as follows. Make a cut in the ventral body wall just medial to the base of the fore limb. Separate the pectoral and shoulder muscles from the sternohyoid muscle. The brachial plexus is then easily seen running posterior to the scapula. It consists of the ventral rami of three spinal nerves $(3,4$, and 5$)$ which have cross-connections with each other. Beyond the plexus, nerves proceed into the fore limb.

The lumbosacral plexus is located by cutting through the skin and both layers of oblique muscles longitudinally just dorsal to the base of the hind limb. On separating the oblique from the transverse muscle layers the plexus is exposed. It consists of the ventral rami of three (sometimes four) spinal nerves. The anterior one of the three gives off a slight branch to the nerve next posterior to it, sometimes receives a contribution from the ramus anterior to it, and as the crural nerve, passes into the limb. The middle of the three nerves is much the stoutest, and after receiving contributions from the nerve next posterior to it enters the limb as the ischiadic (sciatic) nerve.

3. The sense organs of the head.- - Owing to the small size of the eye and its similarity to that of other vertebrates it will not be investigated.

a) Lateral line system: Lateral line sense organs are present in Necturus but impractical to find in gross dissection. They are situated along lines similar to those of fishes.

b) Nose: Probe into the external naris and follow your probe with the cut, opening the entire nasal passage to the internal naris. Note the folds or olfactory lamellae in the interior of the passage. Unlike the condition in fishes, the olfactory sac opens into the oral cavity and has both olfactory and respiratory functions.

c) Ear: The ear as in fishes consists of the internal ear only, imbedded in the otic region of the skull. Expose the dorsal surface of the skull by cleaning away the muscles. Locate the otic capsules, one at either side of the posterior end of the skull. Cautiously shave away the cartilage here and locate the three semicircular ducts and vestibule as done in the dogfish. There are three of the former-anterior vertical, posterior vertical, and horizontal-each with an ampulla, all arranged exactly as in the dogfish. The vestibule is divided indistinctly into a dorsal utriculus, from which the ducts spring, and a ventral sacculus. The latter contains a crystalline mass, the otolith.

4. The dorsal aspect of the brain.-Remove the roof of the skull. This is best done by stripping it off in slivers with a knife. After the brain is revealed 
study its dorsal surface. The brain is covered by a membrane, the primitive meninx, which is more or less divisible into the pia mater, a delicate pigmented membrane adhering to the brain, and an outer dura mater, which is separated from the skull by the peridural space.

The most anterior portion of the brain consists of the two elongated lobes of the telencephalon. These are very indistinctly subdivided into anterior olfactory lobes and posterior cerebral hemispheres. The olfactory lobes include the bulbs and tracts of the dogfish. Between and behind the posterior ends of the telencephalic lobes is a thin roof containing a chorioid plexus. The anterior part of this bears a process, the paraphysis, projecting forward in the groove between the two cerebral hemispheres. It is regarded as a part of the telencephalon. The very small region posterior to this is the roof of the diencephalon. It bears a dorsally projecting process, the pinal body or epiphysis, which lies immediately posterior to the paraphysis. Posterior to the diencephalon are the two elongated optic lobes, representing the dorsal part of the mesencephalon. Behind this is another chorioid plexus, a dark pigmented membrane. This may be removed. There is then revealed the triangular cavity of the fourth ventricle. The anterior end of this cavity is overhung by a narrow shelf at the posterior margins of the optic lobes. This shelf is the cerebellum. The remainder of the brain consists of the medulla oblongata which incloses the cavity of the fourth ventricle. The medulla extends forward at the sides of the optic lobes as projections, the greater part of which consists of the acustico-lateral area. The walls of the medulla are divided into dorsal or sensory portions and ventral or motor portions, the latter forming a broad band on either side of the median ventral groove. The further subdivisions of these are not evident in Necturus.

Draw the dorsal view of the brain.

5. The cranial nerves.-

a) The first or olfactory nerve: This is a stout band passing from the olfactory sac to the anterior end of the telencephalon.

b) The second or optic nerve: This small nerve may be seen in the floor of the cranial cavity by pressing the telencephalon to one side. It passes obliquely caudad to the ventral surface of the diencephalon.

c) The eye muscle nerves: The third or oculomotor, fourth or trochlear, and sixth or abducens nerves are so small in Necturus as to be scarcely discernible in gross dissection. They originate from the same regions of the brain and supply the same eye muscles as in elasmobranchs.

d) The fifth or trigeminus nerve: The fifth nerve is the large trunk arising from the side of the anterior end of the medulla. Trace it out through the skull. It passes in front of the otic capsule and immediately enters a large ganglion, the semilunar or Gasserian ganglion of the trigeminus. From the ganglion three large nerves are given off, the ophthalmic, maxillary, and mandibular branches. The former, corresponding to the deep ophthalmic of fishes, passes forward through the quadrate cartilage and runs forward anteriorly along- 
side the frontal bones. The maxillary nerve runs along the margin of the upper jaw. The mandibular nerve passes laterally to the angle of the jaws and then turns forward along the lower jaw.

e) The seventh or facial nerve: This arises just behind the trigeminus and sends a branch forward to join the latter at the semilunar ganglion. This branch of the facial passes out with the ophthalmic nerve as the superficial ophthalmic branch of the facial and with the maxillary nerve as the buccal branch. Both of these go to lateral line organs. The greater part of the facial arises from the medulla in common with the auditory nerve ventral to the above-named branch. This common acustico-facial trunk of facial and auditory passes into the anterior part of the otic capsule. From here the main trunk of the facial or hyomandibular may be followed laterally. It branches to muscles, lateral line organs, etc.

f) The eighth or auditory nerve: This arises in common with the facial and is distributed to the internal ear. Its branches are readily noted in the otic capsule.

g) The ninth or glossopharyngeal and tenth or vagus nerves: These arise together from the medulla posterior to the acustico-facial trunk, by three roots. The common trunk passes along the posterior margin of the ear capsule and enters a large ganglion. From this several nerves arise which may be traced into the external gills (these nerves are not homologous with the pre- and posttrematic branches of elasmobranchs), and to the visceral arches. The most posterior branch of the vagus gives off a lateral branch which passes to the lateral line which it accompanies. The vagus also supplies the viscera.

Add the cranial nerves to your drawing of the brain, as far as seen.

6. Ventral aspect of the brain.-Remove the brain by cutting across the spinal cord, the olfactory, and other nerves. The ventral view reveals some additional parts of the diencephalon. At the anterior end of the ventral surface of the diencephalon is the small optic chiasma formed by the optic nerves. Posterior to this is the large infundibulum from the posterior end of which projects the hypophysis. The ventral surface of the midbrain consists of the two cerebral peduncles.

Draw the ventral view.

\section{THE NERVOUS SYSTEM AND SENSE ORGANS OF THE TURTLE}

I. The spinal nerves, the sympathetic system, and the spinal cord.-Remove all of the viscera from the pleuroperitoneal cavity, leaving the large neck muscles in the mid-dorsal region undisturbed. Identify the spinal nerves as the white cords passing along the sutural lines of the costal plates. The sympathetic chain should be identified as a white cord or cords located on the sides of the mass of neck muscles. ${ }^{\text {}}$

1 The fact that the sympathetic system was not described in elasmobranchs and Necturus should not be taken to indicate that the system is absent in those forms. It is present but impractical to dissect out. 
a) Spinal nerves and limb plexi: Carefully expose the spinal nerves of the trunk, avoiding injury to the sympathetic system. These nerves, called the dorsal spinal nerves, run along the sutures between the costal plates of the carapace. In most cases each consists of two branches, a smaller dorsal ramus and a larger ventral ramus. On tracing these toward the vertebral column they will be found to come from a large ganglion, the dorsal or spinal ganglion, situated in contact with the center of the centrum.

Expose the brachial plexus in the depression between the neck and the dorsal end of the scapula. It is generally formed by the cross-unions between the ventral rami of the last four cervical spinal nerves and the first dorsal spinal nerve. The last-named nerve may be identified as the one in front of the first typical rib (really the second rib). The four cervical nerves form a complex network on the surface of the shoulder muscles. From this network the large median nerve proceeds along the anterior surface of these muscles and the smaller ulnar and radial nerves along the posterior surface; the radial is the most dorsally situated one. The first dorsal nerve sends a branch near its ganglion to the brachial plexus and is then distributed to the carapace just posterior to the fore limb.

The next six dorsal nerves are similar to the first description given. The ventral rami of the eighth, ninth, and tenth dorsal nerves together with the two sacral nerves form the lumbosacral plexus for the hind limb. This lies on the medial surface of the muscles covering the ilium and is found by carefully separating the ilium with its muscles from the median region. The branches from the three dorsal nerves unite to a sort of knot from which several nerves proceed to the anterior part of the leg. The two sacral nerves, receiving also a contribution from the tenth dorsal nerve, unite to form a large trunk, the sciatic nerve, situated among the muscles on the posterior side of the leg.

There is a pair of caudal spinal nerves corresponding to each caudal vertebra, but these need not be looked for. There are nine pairs of cervical nerves which will be found by looking in the neck at the same level as the level of emergence of the nerves of the brachial plexus.

b) The sympathetic system: Locate the vagus nerve in the neck. It is the conspicuous white cord running along the side of the neck. Trace it posteriorly. The sympathetic trunk is bound with it but at about the level of the first nerve of the brachial plexus separates from the vagus and enters a swelling or ganglion, the middle cervical ganglion. The sympathetic cord proceeds dorsally from this ganglion and lies on the ventral surface of the brachial plexus where it presents two successive swellings, which together constitute the inferior cervical ganglion. Observe branches from these ganglia. The sympathetic cord passes to the ganglion of the first dorsal spinal nerve to which its own ganglion is fused. It then proceeds as a delicate white cord across the second rib and again forms a ganglion which is fused to the ganglion of the second dorsal nerve. The sympathetic cord then passes more ventrally, lying on the side of the long neck muscles. 
Follow it here and note the ganglia which it bears at intervals and the branches from these ganglia. Note particularly the branches between the sympathetic ganglia and the adjacent spinal ganglia. These branches constitute the ramus communicans and consist of the visceral motor and visceral sensory fibers passing between the sympathetic and central nervous systems. The ganglia and branches of the sympathetic are particularly noticeable in the urogenital region.

Draw spinal nerves, plexi, and sympathetic system as far as seen.

2. The sense organs of the head.-

a) The nasal cavities: The external nares lead into wide chambers, the nasal cavities. Cut off the external nares and the roof of the skull posterior to them, thus revealing the nasal cavities. They are separated by a median septum, partly bony. From the ventral region of the septum a conspicuous fold projects into the nasal cavity. On the posterior wall of the nasal cavity is a slight projection, a concha or turbinal. Posterior to this the nasal cavity connects by a passage with the roof of the mouth cavity, the nasal cavities thus serving as respiratory passages.

b) The eye: Although the eye is small it can be dissected with a little care. Its parts are very similar to those of the fish eye. Make an incision through the skin around one eye and with the bone scissors remove the skull dorsal to and between the eyes. The two eyes are seen to be close together, separated by a median membranous interorbital septum. Near this septum on each side runs an artery. On the anterior dorsal surface of the eyeball is a gland, the Harderian gland. Over the posterior and ventral surface of the eyeball extends the much larger lacrimal or tear gland. Remove these glands, thus exposing the surface of the eyeball and the eye muscles. Extending from the interorbital septum to the dorsal surface of the eyeball is the superior oblique muscle. Posterior to this and inserted in the eyeball near it is the superior rectus. Between and ventral to these two is the internal rectus. Passing above the internal rectus are two nerves, the trochlear to the superior oblique muscle and the ophthalmic branch of the trigeminus. Loosen the eyeball ventrally and raising it as far as possible examine the ventral surface. The anterior part of this surface is covered by a flat muscle, the pyramidalis, which originates on the eyeball and passes to the eyelids and nictitating membrane. Remove this and clean the ventral surface of the eyeball. The inferior oblique and inferior rectus muscles are then seen converging to their insertions on the ventral surface of the eyeball. The external rectus is posterior to them.

Remove the eyeball and open it by cutting off its dorsal side. Identify the coats of the eyeball, the lens, the cavities of the eye, and the two humors as in the elasmobranch eye, as the structure is practically identical with the latter. Note, however, the difference in the shape of the lens of the turtle eye.

c) The ear: The ear consists of two parts, a middle ear and an internal ear. The former is located posterior to the angle of the jaws internal to a circular area 
of skin. Remove this piece of skin and find beneath it a smaller cartilaginous circular plate, the tympanic membrane or eardrum. Make a cut around the margin of this and carefully raise it. Attached to its internal surface, posterior to the center, is a rod-shaped bone, the columella, whose inner end is fastened to the wall of a large cavity. This cavity is the tympanic cavity or cavity of the middle ear. It is an evagination from the first visceral pouch. Ventral to the inner end of the columella is a slit bounded by raised lips. This slit is the opening of the auditory tube connecting the pharyngeal cavity with the cavity of the middle ear and representing the stalk of the evagination by which the latter was formed. Considerably internal to the point of attachment of the inner end of the columella lies the internal ear. It will be more definitely located later. It is similar in structure to the internal ear of elasmobranchs.

3. Dorsal aspect of the brain.- - Remove the roof of the skull and expose the brain. The brain is covered by a tough membrane, the dura mater. On cutting carefully through this a more delicate membrane, the pia mater, will be found adhering to the brain. It is mo:e or less pigmented and contains numerous blood vessels. Strands cross between the two membranes. The two membranes are derived by the splitting of the original single membrane of the brain, the primitive meninx of fishes. The space between the dura mater and pia mater is the $s u b$ dural space; between the former and the skull, the peridural space. Remove the dura mater from the dorsal surface of the brain.

The brain possesses the same divisions as in the preceding forms, but the relative proportions of the parts are somewhat altered. The anterior end consists of the two olfactory lobes, which include the olfactory bulbs and tracts seen in the elasmobranch; the whole olfactory apparatus is evidently much reduced in the turtle as compared with the fish. Posterior to the olfactory lobes and separated from them by a slight groove are the two cerebral hemispheres, relatively much larger than in the elasmobranchs and Necturus. Between the posterior ends of the two cerebral hemispheres is the diencephalon, its roof consisting of a chorioid plexus, which projects dorsally as a sac that adheres to the dura mater and is generally torn off in removing the latter. This sac consists of the paraphysis and the epiphysis. When it is removed the diencephalon is seen as a depressed area posterior and ventral to the cerebral hemispheres. Posterior to the diencephalon are the two rounded optic lobes, belonging to the midbrain. Behind them is the cerebellum, smaller than in elasmobranchs but larger than in Amphibia. Posterior to the cerebellum and partly overhung by it is the medulla oblongata. Its thin dorsal roof contains as usual a chorioid plexus. On removing the roof of the medulla, the cavity of the fourth ventricle is revealed and the cerebellum is then seen to project like a roof over the anterior part of the medulla. The dorsal rims of the medulla consist as usual of the somatic sensory columns, and on raising the cerebellum the anterior ends of these columns which are auditory in nature are seen to be connected with the cerebel- 
lum. In the floor of the medulla the two somatic motor columns are conspicuous, one on either side of the midventral groove.

Draw the dorsal aspect of the brain.

4. The cranial nerves.-The dissection of the cranial nerves is a matter of some difficulty and the following description is consequently not complete.

a) The olfactory nerves: These are the two stout nerves extending from the dorsal portions of the olfactory sacs to the anterior end of the olfactory lobes of the brain.

b) The optic nerves: On cutting through the olfactory nerves and raising the anterior end of the brain, the optic nerves are seen as two stout trunks situated below the cerebral hemispheres and passing out of the orbit.

c) The trochlear nerve: This small nerve arises on each side of the brain in the dorsolateral angle between the optic lobe and the cerebellum. It passes ventrally and forward, and will be seen by pressing the cerebral hemisphere away from the skull. It lies behind the larger oculomotor nerve. To find the course of the trochlear nerve in the orbit expose the undissected eye as before, clearing away the glands. Cut through the superior oblique muscle at its point of insertion on the eyeball and find below it the trochlear nerve terminating in this muscle. Medial to the trochlear is the ophthalmic branch of the trigeminus.

d) The oculomotor nerve: This nerve originates from the floor of the midbrain immediately in front of the trochlear and is seen by pressing the cerebral hemisphere away from the skull. Loosen the ventral side of the eyeball and raise it. Among the loose tissues between the eyeball and the floor of the orbit, generally adhering to the eyeball, is a nerve, the maxillary branch of the trigeminus. Free this from the eyeball. Cut away the pyramidal muscle, and raising the eyeball and pressing it as far medially as possible, separate the inferior and external rectus muscles and find between and above them the stout white trunk of the optic nerve. The oculomotor is in contact with the ventral surface of the optic nerve and branches to the same four eye muscles as in the dogfish. These are not readily followed.

e) The trigeminus nerve: This is a stout trunk whose origin from the anterior end of the medulla will be seen by pressing the cerebellar region of the brain away from the skull. The trunk passes laterally and enters its ganglion, the semilunar ganglion, which lies in a depression in the medial wall of the skull. The trigeminus has three branches, the ophthalmic, the maxillary, and the mandibular, distributed to the orbit and nose, the upper, and the lower jaw, respectively. Remove the eyeball on the side where it is still present leaving the ophthalmic and maxillary nerves intact. Cut through the roots of the nerves anterior to the trigeminus so as to raise the brain and bend it away from the side being dissected. Follow the ophthalmic nerve forward and note its distribution to the nasal sacs. Follow it posteriorly toward the root of the trigeminus. It enters the skull, runs in company with the trochlear nerve between the dura mater anc 
the skull, and finally joins the semilunar ganglion. Follow the maxillary nerve posteriorly. Besides the branch below the eyeball already noted there is a branch in the floor of the orbit running obliquely forward. These two branches unite to form the main trunk of the maxillary nerve at the posterior end of the orbit. Trace this nerve posteriorly among the muscles to where it pierces the skull. At this point it is joined by the mandibular branch of the trigeminus. Trace this laterally. After branching into adjacent muscles, the mandibular nerve proceeds ventrally and enters the lower jaw. Mandibular and maxillary branches pass together through a foramen in the skull and connect with the semilunar ganglion.

f) The facial and auditory nerves: These arise together from the side of the medulla just behind the root of the trigeminus and immediately separate into an anterior facial nerve and a posterior auditory. The latter is distributed to the internal ear. This is situated in the skull opposite the acustico-facial root. This part of the skull may be broken open with the bone forceps. The semicircular ducts, ampullae, and vestibule of the internal ear will be noted. The auditory nerve will be seen branching among these structures. The facial nerve passes through the anterior part of the ear capsule and will be seen again later.

g) The glossopharyngeal nerve: This arises by a small root from the medulla immediately posterior to the acustico-facial root and passes out through the posterior part of the ear capsule.

h) The vagus, the spinal accessory, and the hypoglossal: The vagus and spinal accessory (eleventh) nerves arise together by a number of roots from the side of the medulla posterior to the preceding nerve, the more anterior roots belonging to the vagus and the posterior ones to the accessory. On cutting through these roots the more ventrally situated roots of the hypoglossal (twelfth) nerve will be seen. The three nerves pass out from the skull close together.

i) The abducens nerve: Cut through all of the nerve roots on one side of the brain and tilt the brain toward the opposite side. The abducens nerves will be seen springing from the ventral surface of the medulla at about the same level as the acustico-facial root.

The seventh, ninth, tenth, and twelfth nerves may be traced farther as follows. Turn the head ventral side up and remove the skin and superficial muscles from the hyoid apparatus. Locate the anterior and posterior horns of the hyoid. On the side of the neck, near the dorsal end of the anterior horn and posterior to it, the hypoglossal nerve will be seen emerging. It branches into the muscles over the anterior horn and sends a branch forward into the tongue muscles. Very near the point of emergence of the hypoglossal but situated more deeply and nearer to the cartilage of the anterior horn will be found the glossopharyngeal nerve. It runs between the two horns toward the median ventral line and supplies adjacent muscles and lining of the mouth cavity. Lateral 
to these nerves, a branch of the facial will be found crossing the anterior horn near its dorsal end and passing into the muscles lying along the posterior border of the mandible.

Make a median longitudinal incision through the whole floor of the mouth and pharyngeal cavities and open the two flaps so that the roof of these cavities is revealed. Locate the vagus (really vago-sympathetic) trunk in the neck and trace it anteriorly to its point of exit from the skull, removing the mucous membrane from the roof of the pharyngeal cavity. The vago-sympathetic trunk passes to the dorsal side of the hypoglossal nerve seen above and there enters a ganglion, the superior cervical ganglion of the sympathetic. From this ganglion numerous branches pass out. Internal to the hypoglossal locate the glossopharyngeal nerve, the carotid artery being situated between the two. Slightly anterior to these will be found the facial nerve, as it exits from the skull. Its branches pass to the muscles between the anterior horn of the hyoid and the lower jaw, one of them curving over the ventral surface of the horn as noted above. The vagus nerve proceeds posteriorly and supplies the heart and other viscera. It will be noted that the facial, glossopharyngeal, and vagus nerves are much reduced, owing to the loss of the lateral line system and the gill apparatus. Note, however, that these nerves continue to supply the remains of the visceral arches and their visceral muscles.

Add the cranial nerves to your drawing of the brain.

5. Ventral aspect of the brain.-Remove the brain from the skull and examine the ventral surface. On the ventral surface of the diencephalon note the optic chiasma, the infundibulum just behind this, with the hypophysis projecting ventrally from the latter. Note the roots of the abducens and hypogossal nerves arising from the ventral surface of the medulla.

6. Median sagittal section.-Make a median sagittal section and study the cut surface. Identify the fourth ventricle in the medulla, the aqueduct or passage below the cerebellum, the optic ventricle in the optic lobe, the third ventricle in the diencephalon. Note the increased size of the diencephalon as compared with the elasmobranch, and the backward extension of the cerebral hemisphere over the diencephalon. The diencephalon is divided into epithalamus, thalamus, and hypothalamus as in the dogfish, each including the parts previously enumerated. Note that the cerebral hemisphere presents a solid medial wall, called the septum. Cut into the roof or pallium of the hemisphere. Note its cavity, the lateral ventricle, and the large mass protruding from the floor into the ventricle; this mass is the corpus striatum.

The functions of the parts of the turtle brain are similar to those stated for the elasmobranch brain. The cerebral hemisphere is still largely olfactory, although its lateral surface is beginning to assume the functions characteristic of the mammalian hemisphere.

Draw the section. 


\section{E. THE NERVOUS SYSTEM AND SENSE ORGANS OF THE PIGEON}

I. The spinal nerves and the sympathetic system.-Carefully remove the remaining viscera from one-half of the trunk. Note the ventral rami of the spinal nerves passing laterally along the dorsal body wall between the ribs in the trunk region. Trace them toward the vertebral column and note, at the points where they emerge from the vertebrae, the ganglia of the sympathetic system lying on the spinal nerves and the delicate white cords of the sympathetic system extending between the ganglia along the sides of the vertebral column.

a) Spinal nerves and limb plexi: In the neck the cervical spinal nerves will be seen by separating the vertebral column from the skin. They pass out at segmental intervals. The vagus nerve is the white cord which passes ventral to the proximal portions of the cervical nerves.

The ventral rami of the last cervical nerves together with that of the first of the trunk form the brachial plexus to the wing. This is a network, formed by the union of branches of four stout nerves, which receives a small branch from the succeeding nerve.

The next five ventral rami pass out between the ribs. Following them is the lumbosacral plexus, divisible into three parts, the lumbar, the sacral, and the pudendal plexus. The lumbar plexus is formed by three nerves; from it nerves pass into the thigh. The sacral plexus arises from the union of five nerves, the first of which is the same as the third nerve contributing to the lumbar plexus. These five unite to produce a large trunk, the sciatic nerve, which passes along the dorsal side of the thigh between the muscles, and proceeds down the leg. It will be found by separating the muscles along the middle of the dorsal surface of the thigh. It courses alongside the femoral artery and vein.

The remaining spinal nerves posterior to the sacral plexus form the pudendal plexus and pass obliquely posteriorly to the tail and cloacal region.

b) The sympathetic system: This has already been identified on the sides of the vertebral column. It consists on each side of a chain of two cords and segmental ganglia. One of the cords passes ventral to the head of the rib, the other dorsal to it. A sympathetic ganglion lies fused to each spinal nerve in the trunk region as the latter emerges from the vertebral column. On scraping off one of these sympathetic ganglia, the spinal ganglion belonging to the spinal nerve will be found dorsal to it. At about the middle of the rib-bearing region a plexus of nerves and ganglia will be seen extending ventrally from the main sympathetic cords and surrounding the dorsal aorta and its main branches to the digestive tract. This is the coeliac plexus. Posterior to this region the sympathetic cords are reduced and consist of a single trunk on each side. A sympathetic cord accompanies the pudendal plexus and has a ganglion in the middle of this plexus. Anteriorly the sympathetic cords pass across the ventral side of the brachial plexus, having ganglionic enlargements on the latter, and then enter the vertebrarterial canals. 
2. The sense organs of the head.-

a) The nasal cavities: Open one nasal cavity by a longitudinal slit just above the margin of the upper jaw from the external naris to the head. Note the median septum between the two nasal cavities and the swellings, the turbinals or conchae, projecting from the septum into the nasal cavity. There are three turbinals in a row: the first two large and conspicuous, the third and most posterior one consisting only of a small rounded swelling on the roof of the cavity in close contact with the posterior end of the second concha. Only this third concha is provided with olfactory epithelium. Beyond the conchae the nasal passages connect with the pharyngeal cavity.

b) The eye: Cut through the skin around the eyeball and also remove the roof of the skull between the two eyes. Note the relatively large size of the eyeballs and the interorbital septum between them. Along the dorsal margin of the septum course the two olfactory nerves. Press the eyeball outwardly away from the skull. Two thin, flat muscles will be seen extending to the eyeball from the orbit; the anterior one is the superior oblique, the posterior one the superior rectus. Cut through the superior oblique at its insertion on the eyeball and press it against the orbit. The internal rectus will now be seen extending to the eyeball ventral to the superior oblique. The white nerve crossing the orbit against the internal surface of the superior oblique is the ophthalmic branch of the trigeminus. Dorsal to it the smaller trochlear nerve is seen terminating on the superior oblique. The thin sheet of muscles on the surface of the eyeball is the quadrate, a muscle of the eyelids. On the anterior surface of the eyeball ventral to the superior oblique is a white fatlike mass, the Harderian gland. Press the eyeball posteriorly and find anterior to this gland, against the anterior wall of the orbit, the inferior oblique muscle. On pulling the eyeball forward the external rectus is seen extending to the posterior surface of the eyeball. Free the ventral margin of the eyeball. In the posterior ventral region on raising the eyeball may be seen a small gland, the lacrimal gland. Two muscles will be seen on the ventral surface of the eyeball. The anterior one is the inferior rectus, the posterior one the external rectus. On cutting through the inferior rectus the pyramid, a muscle of the eyelids, will be revealed internal to it. Cut through all of the rectus muscles and the inferior oblique at their insertions on the eyeball and remove the eyeball, severing the optic nerve. The pyramid and quadrate muscles are now more readily seen extending on the surface of the eyeball to the optic nerve; the quadrate muscle is broad and dorsally situated, the pyramid narrow and ventral. They are concerned in operating the nictitating membrane. In the orbit note the extent of the Harderian gland.

Cut off the dorsal part of the eyeball and identify the structures of the eye. Note the sclerotic coat, continuing as the transparent cornea over the exposed part of the eye; the conjunctiva, passing over the external surface of the cornea and continuing onto the eyelids; the black chorioid coat internal to the sclerotic 
and forming the iris in front; the soft retina. Note the peculiar ridged structure, the pecten, projecting from the chorioid coat through the retina in the medial wall of the eyeball and extending to the lens. Its function is unknown. Loosen the lens and observe that it is encircled by a structure formed chiefly from the chorioid coat. This structure, which holds the lens in place, is called the ciliary body. It is marked by radiating ridges, the ciliary processes, and contains muscles, the ciliary muscles, which change the shape and position of the lens. Note the shape of the lens, nearly flat externally, more convex internally. The chambers of the eye and the two humors are as in the dogfish. Peel off the iris from the cornea and note the stiff, bony ring encircling the cornea; it is composed of a number of sclerotic bones.

Draw, showing structure of the eye.

c) The ear: The ear of birds consists of three parts-the external ear, the middle ear, and the internal ear. The external ear comprises the passage, the external auditory meatus, situated below and behind the eye. Cut into this on the same side of the head on which the eye was dissected and find at its internal end a circular transparent membrane, the tympanic membrane. Through the membrane the columella can be seen extending from its internal surface inwardly. Remove the tympanic membrane, noting the columella adhering to its internal surface. The cavity of the middle ear is now exposed; medially and ventrally it is connected to the pharnygeal cavity by the auditory tube; posterior and slightly dorsal to it is situated the internal ear. The inner end of the columella adjoins a tiny bone, the stapes, which fits into an opening, the fenestra ovalis or vestibuli, which leads into the internal ear. Look for these at the inner end of the columella. Next, carefully break away in small pieces the spongy bone behind the middle ear. Three bony semicircular canals are revealed. Each of them contains a membranous semicircular duct, as will be seen by breaking open one of them. The three ducts are situated in the same planes and have the same names as in elasmobranchs. The remaining structures of the internal ear, consisting of two small chambers-the utriculus and the sacculus - are too difficult to dissect.

3. Dorsal aspect of the brain.-Expose the brain, removing the roof of the skull and the side of the skull where the sense organs were dissected, including the medial wall of the orbit. Note: the dura mater inclosing the brain; on removing this, the very delicate pia mater next to the brain substance. The origin of these membranes is the same as given for the turtle, and the spaces bounding them have the same names. Unlike the condition in the preceding forms, the brain completely fills the cranial cavity.

The brain is short and broad and strongly curved, in correlation with the biped gait. The curvature results from flexures of the brain in three regions. The chief or primary flexure occurs in the region of the midbrain, with the result that the posterior part of the brain is bent nearly at right angles to the anterior 
part. The second or muchal flexure takes place in the medulla, bending the medulla at an angle to the spinal cord. The pontal flexure in the region ventral to the cerebellum bends the brain in the opposite direction from the other two flexures, with the result that this region of the brain is depressed.

The anterior end of the brain consists of the two very small olfactory lobes. Posterior to them are the large cerebral hemispheres separated by a deep sagittal fissure. These are so enlarged posteriorly as to completely conceal the diencephalon from dorsal view, with the exception of the delicate pineal body which is seen in the posterior end of the sagittal fissure. The large optic lobes of the midbrain are ventral to the posterior ends of the cerebral hemispheres. Posterior to the hemispheres is the curved cerebellum, marked by transverse grooves. Posterior and ventral to this is the medulla oblongata, its anterior end depressed beneath the cerebellum. The roof of the medulla is composed as usual of a chorioid plexus.

Draw the brain from the side.

4. The cranial nerves.-These are somewhat difficult to follow in detail. Work on the side left intact. There are twelve pairs of cranial nerves.

a) The olfactory nerves: These are two stout and elongated nerves passing from the nasal sacs along the dorsal margin of the interorbital septum to the olfactory lobes.

b) The optic nerves: On the side where the wall of the orbit was removed note the stout white optic tract in front of the optic lobe. Follow this toward the orbit and find the optic nerve connected with its anterior end.

c) The trochlear nerve: The cranial origin of this nerve is difficult to see at the present stage of the dissection. It arises in the deep groove between the optic lobe and the cerebellum, and passes ventrally between the optic lobe and medulla. It runs forward in the floor of the cranial cavity to the orbit. To find it in the orbit, expose the intact eye as before. Cut through the superior oblique at its insertion on the eyeball and lifting it note the trochlear nerve passing to it and spreading out on its ventral surface. The ophthalmic branch of the trigeminus runs close to the trochlear nerve.

d) The oculomotor nerve: The cranial origin of this nerve will be seen later. It branches to the inferior oblique, superior, inferior, and internal rectus muscles Remove the eye which is still in place, cutting the eye muscles as near the eyeball as possible and preserving the ophthalmic nerve intact. Look for the branches of the oculomotor among the eye muscles in question. The branch to the inferior oblique in the floor of the orbit is the most conspicuous of them.

e) The abducens nerve: This will be found on examining the posterior surface of the external rectus muscle. This nerve also supplies the pyramid and quadrate muscles.

f) The trigeminus nerve: This has three branches, the ophthalmic, the maxillary, and the mandibular. The ophthalmic has already been noted in the dorsal 
part of the orbit. Follow it forward, noting its distribution to the walls of the nasal cavities. In the floor of the orbit, near its outer margin, locate the maxillary nerve. Trace it forward, noting its branches to the orbit and upper jaw. Trace the maxillary nerve posteriorly, carefully cutting away tissues in its path. Caudad of the orbit it is joined by the mandibular nerve. Trace this, noting branches to muscles and main trunk passing into the lower jaw. Trace the common trunk of the maxillary and mandibular nerve toward the skull and note that they are joined in the skull by the ophthalmic nerve. At the point of union is the semilunar ganglion, lying in the skull. From the ganglion the trigeminus nerve may be traced to its origin from the side of the medulla below the optic lobe.

g) The facial nerve: This arises from the medulla just back of the root of the trigeminus and passes through the anterior part of the ear capsule, where it will be found by scraping away the latter.

h) The auditory nerve: This arises close to the facial and passes out with it into the ear capsule to the various parts of which it is distributed.

i) The glossopharyngeal and the vagus: These nerves arise close together just behind the ear capsule and will be found there by carefully dissecting in the muscles. The glossopharyngeal is the smaller of the two and anterior in position. It enters a ganglion, the petrosal ganglion, beyond which it is distributed to the palate, pharynx, and larynx. The vagus nerve is considerably larger than the glossopharyngeal. It passes laterally parallel and posterior to the glossopharyngeal and enters its ganglion, the jugular ganglion, which is united with the petrosal ganglion, the two forming a mass. Beyond this the vagus turns posteriorly and passes down the neck, supplying respiratory system, heart, and other viscera. Portions of the sympathetic system are intermingled with the ninth and tenth nerves.

j) The spinal accessory and the hypoglossal: The former passes out with the vagus and is distributed to certain muscles. The hypoglossal is found just posterior to the vagus. It is distributed to certain neck muscles and sends a branch forward to the tongue.

5. Ventral aspect of the brain.-Remove the brain from the cranial cavity, preserving the roots of the cranial nerves as far as possible. Those not kept attached to the brain will be found in the cranial cavity.

Note form of the olfactory lobes and cerebral hemispheres from the ventral aspect. Between the optic lobes is the diencephalon. In the center of this is the optic chiasma, marked by cross lines. From the optic chiasma the strong white optic tracts pass laterad and dorsad to the optic lobes and dorsal part of the diencephalon. Behind the chiasma is a depressed area, the infundibulum from which the hypophysis extends ventrally. The latter is usually left behind in removing the brain, and will be found in a deep pit, the sella turcica, in the floor of the cranial cavity. The infundibulum bears a central cleft where the 
hypophysis was torn from it. At the sides of the infundibulum are the roots of the oculomotor nerves. Posterior to the diencephalon is the depressed medulla. Between the medulla and the optic lobe is the slender root of the trochlear nerve. On the ventral surface of the medulla are the roots of the abducens nerves; they should also be sought in the floor of the cranial cavity. On the sides of the medulla look for the roots of the fifth, seventh, eighth, ninth, and tenth nerves, situated in a row. The twelfth nerve arises from the ventral surface of the medulla about on the same level as the ninth and tenth roots. The eleventh nerve arises from the spinal cord by several roots and ascends to a position immediately behind the tenth root.

Draw the ventral view of the brain.

6. Sagittal section.-Make a median sagittal section of the brain and study the cut surface. In the medulla note the fourth ventricle overhung by the cerebellum. Note the thick ventral wall of the medulla and the pontal flexure causing a ventrally directed bend in the medulla. In the cerebellum observe the small cerebellar ventricle and the arrangement of the gray and white matter resulting in section in a treelike appearance, called the arbor vitae. Each fold of the cerebellum consists of a central plate of white matter surrounded by a thick covering of gray matter. Anterior to the cerebellum is a region consisting dorsally of the mesencephalon and ventrally of the diencephalon. The optic lobes do not appear in the section, but the median part of the midbrain forms the dorsal part of the section. A narrow cavity, the third ventricle, is present in the diencephalon and extends into the infundibulum. In front of the latter appears the optic chiasma. Note how the cerebral hemisphere arches back over the diencephalon and midbrain, and note the strong connection of the diencephalon with the hemisphere. The cavity of the cerebral hemisphere is not visible in the median section. The medial wall of the hemisphere is called the septum, its dorsal wall the pallium. Cut into the latter, noting its thinness, and find inside the cavity or lateral ventricle of the hemisphere and the great mass, the corpus striatum, bulging from the floor. The function of the corpus striatum is not definitely known, but it seems to have a steadying effect on voluntary movements, and the delicacy and precision of movement necessary in flight may account for the relatively enormous size of the corpus striatum in birds.

\section{F. THE NERVOUS SYSTEM AND SENSE ORGANS OF THE MAMMAL}

For the complete dissection of the nervous system a new specimen is necessary, but the greater part of this system can be worked out on the same specimen as used for preceding systems. If a new animal is provided, open it by a longitudinal cut from the perineum through the anterior end of the sternum. If the old specimen is used it will not be possible to see the branches of the sympathetic system and the vagus to the viscera or the peripheral distribution of some of the cranial nerves. In working on the nerves all structures other than nerves 
may be removed in order to expose the latter. In following one nerve, adjacent nerves must not be destroyed.

I. The spinal nerves, the sympathetic system, and the vagus.-

a) Cervical portion of the sympathetic and the vagus: Locate the vagus nerve at a point near the larynx. It lies alongside the carotid artery. The nerve crossing the vagus near the larynx and giving off branches into the sternohyoid, sternothyroid, and related muscles is the descending branch of the twelfth or hypoglossal nerve.

Rabbit: The vagus nerve and the cervical part of the sympathetic trunk lie together on the dorsal surface of the carotid artery. The vagus is larger and more lateral. To the medial side of the sympathetic trunk posterior to the larynx may be separated a delicate nerve, the cardiac branch of the vagus (depressor nerve of the heart). Trace the sympathetic posteriorly. Just in front of the subclavian artery it enters a ganglion, the inferior cervical ganglion. From this ganglion cords pass to either side of the subclavian artery, forming the ansa subclavia, and unite again to another ganglion, the first thoracic ganglion, situated posterior and dorsal to the artery.

Cat: The sympathetic trunk is inseparably bound with the vagus, the two forming a large vago-sympathetic trunk coursing lateral to the carotid artery and bound with it by a common sheath. Trace it caudad. Just in front of the first rib branches of sympathetic origin arise from the trunk and proceed toward the esophagus. Shortly posterior to this point the sympathetic separates from the vagus and generally enters a ganglion, the middle cervical ganglion, which lies in contact with the vagus. From this ganglion cords pass on either side of the subclavian artery, forming the ansa subclavia, and proceeding directly dorsally unite to form a large ganglion, the inferior cervical ganglion, which lies against the neck muscles between the heads of the first and second ribs.

From the inferior cervical ganglion in both animals cardiac branches pass to the heart. The conspicuous nerve lying lateral to the vagus is the phrenic nerve or nerve of the diaphragm. The right vagus just after passing ventral to the subclavian artery gives off the recurrent or posterior laryngeal nerve which runs anteriorly along the side of the trachea to the larynx. The left recurrent nerve arises much farther posteriorly from the left vagus.

b) The anterior cervical spinal nerves: The spinal nerves emerge from the spinal cord in pairs between successive vertebrae, passing out through the intervertebral foramina. Those of the cervical region are called the cervical nerves; there are eight pairs of them. The ventral rami of the first four cervical nerves are loosely united with each other to form the cervical plexus; the last four together with the first thoracic form the brachial plexus. As the first two are small and more or less mingled with the posterior cranial nerves they will not be studied at this stage of the dissection.

To expose the cervical nerves, pull the muscles which are inserted on the anterior end of the sternum (sternomastoid, sternohyoid, sternothyroid) laterally 
or cut across them where necessary, thus exposing the musculature of the vertebral column. Look along the side of this, dorsal to the carotid artery, and note the ventral rami of the spinal nerves emerging at intervals. At about the level of the posterior end of the larynx lies the third cervical nerve in the rabbit, fourth in the cat. As already stated, the nerves thus exposed are the ventral rami only; the dorsal rami are exposed only by more radical dissection, which will not be attempted here. The dorsal rami supply the epaxial musculature. Note the branches of the exposed ventral rami to the muscles of the side of the neck.

From the ventral ramus of the fourth cervical nerve (rabbit) and fifth cervical nerve (cat) arises the phrenic nerve. It passes posteriorly parallel to the vagus, in the rabbit close to the vertebral musculature. It receives a branch from the fifth (rabbit) or sixth (cat) cervical nerve and then continues posteriorly into the thorax. As it passes the sympathetic ganglia it receives contributions from them. In the thorax it lies at the side of the pericardial sac, just ventral to the root of the lung. Trace it posteriorly and note how it spreads on the surface of the diaphragm. The phrenic nerves are the motor nerves of the diaphragm; their origin from the cervical nerves shows that the muscles of the diaphragm are derived from cervical myotomes.

c) The brachial plexus: The ventral rami of the fourth to eighth (rabbit) or fifth to eighth (cat) cervical nerves together with the ventral ramus of the first thoracic nerve are united by intercommunicating branches, called ansae, to form the brachial plexus, which innervates the muscles of the shoulder, breast, fore limb, and diaphragm. The fourth cervical (rabbit), or fifth (cat), take part in plexus only through their contribution to the phrenic nerve.

To expose the brachial plexus cut through the pectoral muscles near the midventral line and separate the pectoral muscles from the underlying serratus muscle. The plexus lies in the axilla along with the axillary artery and vein. Then cut through the pectoral muscles as near as possible to their insertion on the humerus and separate them from the muscles of the upper arm. In this way the course of the nerves into the fore limb is exposed.

The connections of the nerves of the plexus are so intricate that it is impossible to describe them. The following points may be noted, however. Rabbit: The fifth cervical immediately sends a branch to the sixth cervical and then proceeds laterally into the neck muscles. The sixth cervical is a broad nerve which, after communicating with the seventh nerve, passes to the shoulder muscles. The seventh is smaller and after contributing to the eighth likewise innervates the shoulder muscles. The eighth cervical and first thoracic unite to one trunk as they emerge from the vertebral column. From this trunk arise the nerves of the limb. Cat: The sixth cervical has a broad connection with the seventh and then proceeds to the shoulder. The seventh and eighth cervicals and the first thoracic are very stout trunks which are intricately connected with each other and from which proreed the nerves of the fore lim! 
The chief nerves from the brachial plexus are the following:

I. The phrenic nerve. This was described above.

2. The suprascapular nerve. This is the most anterior nerve arising from the sixth cervical. The main part of this nerve passes between the supraspinatus and subscapular muscles to supply the supraspinatus and infraspinatus. In the cat a branch of this nerve passes over the shoulder to more superficial parts.

3. The ventral thoracic nerves. These nerves supply the pectoral muscles and will be found entering the inner surface of these muscles between the two incisions made above. They are the most ventral of the nerves of the plexus. There are two of these nerves-one arising from the seventh cervical, the other from the eighth cervical and first thoracic. The former is small in the rabbit.

4. The subscapular nerves. There are three of these, dorsally situated and passing into the inner surface of the shoulder. The first arises from the sixth cervical and passes to the subscapular muscle; the second arises from the seventh cervical and supplies chiefly teres major; the third comes from the seventh and eighth cervicals and runs posteriorly along the internal surface of the latissimus dorsi muscle.

5. The axillary nerve. This nerve originates chiefly from the seventh cervical. It passes through the upper part of the upper arm, ventral to the triceps and emerging on the lateral surface of the upper arm supplies chiefly the deltoid muscles.

6. The dorsal or long thoracic nerve. This nerve is best located by examining the outer surface of the serratus ventralis muscle. On tracing it anteriorly the nerve will be found to pass internal to the scalenes and to spring from the seventh cervical nerve close to the vertebral column.

7. The musculocutaneous nerve (cat only). This arises from the ventral surface of the sixth and seventh cervicals. It passes to the biceps muscle, forking as it approaches the muscle. The posterior branch continues along the surface of the muscle and at the elbow passes to the lateral surface of the arm and supplies the skin of the forearm.

8. The radial nerve. This is the largest nerve springing from the plexus. Seventh and eighth cervicals and first thoracic nerves contribute to its formation. It passes to the upper arm and coursing between the humerus and the triceps turns distally. It supplies many muscles of the fore limb.

9. The median nerve. This nerve lies posterior to the radial. It arises in the cat by branches from the last three nerves of the plexus and in the rabbit chiefly from the first thoracic. It passes to the upper arm, and then turns distally running along with the brachial artery.

ro. The ulnar nerve. This lies just posterior and parallel to the median nerve, originating chiefly from the first thoracic nerve. The ulnar and median nerves supply the limb distal to the elbow, although in the rabbit, the median nerve innervates the biceps. 
Ix. The medial cutaneous. This is the small nerve which runs in contact with the ulnar nerve. It turns superficially just above the elbow and is distributed to the skin of the forearm.

Draw, showing the main parts of the brachial plexus.

d) The thoracic portions of the vagus and the sympathetic: Trace the two vagi toward the heart. They pass dorsal to the roots of the lungs. The left vagus just caudad of the aortic arch gives off the left recurrent laryngeal nerve which turns cephalad, passing on the dorsal side of the aorta, and ascends along the side of the trachea. At the roots of the lungs the vagi give rise to a plexusthe pulmonary plexus - to the lungs. This plexus also extends to the heart as the cardiac plexus. The cardiac branches of the sympathetic system, noted above, join the cardiac plexus. The cardiac plexus is situated at the bases of the aorta and pulmonary arteries. In the rabbit the cardiac branches of the vagus may be traced into this plexus.

Caudad of the pulmonary plexus the two vagi in the rabbit continue posteriorly along the sides of the esophagus, to which they furnish small branches and penetrate the diaphragm. In the cat each vagus divides just posterior to the root of the lungs into dorsal and ventral branches. The ventral branches of the two sides immediately unite into one trunk which proceeds posteriorly, lying on the left ventrolateral surface of the esophagus. The two dorsal branches continue posteriorly, lying along the right and left sides of the esophagus; near the diaphragm on the dorsal side of the esophagus they unite to one trunk. In this manner are formed the dorsal and ventral divisions of the vagi; they pass through the diaphragm. In their course along the esophagus they furnish branches to it.

Locate again the inferior cervical ganglion. Note the communicating branches from this ganglion to the brachial plexus. In the cat a particularly stout branch extends anteriorly ventral to the bases of the sixth to eighth cervical nerves, giving branches to them. Trace the sympathetic trunk posteriorly from the inferior cervical ganglion. The contents of the pleural cavities may now be cleaned out. The sympathetic trunk is a white cord lying to each side of the vertebral column, passing ventral to the heads of the ribs. At segmental intervals generally in the places between the ribs, it presents a ganglionic enlargement.

e) The thoracic spinal nerves: The first thoracic nerve contributes to the brachial plexus as already learned. The ventral rami of the remaining thoracic nerves pass laterally as the intercostal nerves, lying along the posterior side of each rib. These nerves are readily exposed by running the point of an instrument along the posterior side of each rib, slitting open the fascia of the intercostal muscles. As each nerve emerges from the intervertebral foramen it receives one or two communicating branches (rami communicantes) from the adjacent sympathetic ganglion. These branches are rather delicate and the student may not be able to see them. The dorsal rami of the thoracic spinal nerves supply the 
epaxial muscles. To see them, turn the animal dorsal side up and carefully cut down through the mass of epaxial muscles close to the vertebrae. The dorsal rami will then be seen emerging from the vertebral column and penetrating the epaxial mass, accompanied by blood vessels. There are twelve or thirteen pairs of thoracic nerves.

f) The abdominal portions of the vagus and the sympathetic: Trace the vagi into the peritoneal cavity, removing the liver if not already done. In the rabbit the left vagus crosses the ventral surface of the esophagus obliquely to the right and is distributed to the lesser curvature and ventral surface of the stomach. The right vagus crosses the dorsal surface of the esophagus obliquely to the left and is distributed to the dorsal surface of the stomach. In the cat the ventral division of the vagus passes to the lesser curvature, the dorsal division to the greater curvature. In both cases the vagi form plexi on the stomach called the ventral and dorsal gastric plexi, which also connect with the nearby sympathetic plexi, described in the next paragraph.

Locate again the posterior part of the thoracic portion of the sympathetic trunk. Expose it and note the nerve, the greater splanchnic nerve, which arises from the sympathetic trunk on each side and passes obliquely ventrally toward the diaphragm. In the cat this nerve is accompanied by additional smaller nerves, the lesser splanchnic nerves, arising from the sympathetic shortly posterior to the origin of the greater splanchnic nerve. The splanchnic nerves pass to either side of the crura of the diaphragm into the peritoneal cavity. (The crura of the diaphragm are the muscular cords which fasten the diaphragm to the lumbar vertebrae.) Turn the abdominal viscera to the right and look on the left surface of the superior mesenteric artery near its origin from the aorta. Two prominent sympathetic ganglia will be found lying on the superior mesenteric artery. These are the coeliac and superior mesenteric ganglia; the former lies in front of or on the left surface of the artery; the latter behind or on the ventral surface of the vessel. The two ganglia are bound together by a strong connection. The splanchnic nerves of both sides may be traced into the coeliac ganglion. From this ganglion a prominent coeliac plexus will be seen extending toward the stomach, where it connects with the gastric plexi of the vagi. This great sympathetic plexus formed around and dorsal to the stomach is often called the solar plexus. From the coeliac and superior mesenteric ganglia and adjacent plexi also arise plexi for the liver, spleen, adrenal glands, gonads, and the great blood vessels. Some of these will probably be seen. The inferior mesenteric ganglion of the sympathetic system lies in the mesocolon alongside the inferior mesenteric artery. It is situated in the inferior mesenteric plexus from which networks extend to adjacent structures.

The main sympathetic trunk of the abdominal region should now be traced caudad from the place of origin of the splanchnic nerves. The two trunks descend deep dorsally lying in the groove between two muscle masses. At segmental intervals they have ganglionic enlargements from which nerves pass 
to the ganglia and plexi already noted. At the posterior end of the peritoneal cavity the sympathetic trunks gradually diminish and disappear.

g) The lumbar and sacral spinal nerves and the lumbosacral plexus: There are seven pairs of lumbar nerves and four (rabbit) or three (cat) pairs of sacral nerves. The ventral rami of the last four lumbar nerves form a lumbar plexus, those of the sacral nerves a sacral plexus; but since the two plexi are united with each other, they may be considered together as the lumbosacral plexus.

Remove all viscera from the peritoneal cavity, including the postcaval vein and aorta. In the dorsal wall is a muscular mass extending from the vertebrae to the pelvic girdle. This consists of a lateral larger muscle, the iliopsoas, and a smaller medial one, the psoas minor. In the rabbit the psoas minor is a slender muscle which occupies only the posterior part of the mid-dorsal region; its stout shining tendon passes to the dorsal side of the inguinal ligament. In the cat the psoas minor extends nearly as far anteriorly as the iliopsoas; it narrows posteriorly to a tendon, which passes obliquely laterally on the ventral surface of the iliopsoas which is thus exposed both medially and laterally to the tendon of the psoas minor. The psoas minor covers a part of the iliopsoas in both animals, and the greater part of the lumbar plexus is situated between the two muscles. Note the abdominal parts of the sympathetic cords between the posterior portions of these muscles.

Locate the last thoracic spinal nerve. It lies about one-half inch posterior to the last rib. The first nerve posterior to this on the dorsal wall is the ventral ramus of the first lumbar nerve. Shortly posterior to this is the second lumbar nerve. These two nerves pass to the muscles and skin of the abdominal wall; in the cat each divides into two branches. The third lumbar nerve emerges dorsal to the iliopsoas muscle and divides into a larger lateral branch to the abdominal wall and a more slender medial branch, which passes obliquely caudad, reaching and following the course of the iliolumbar artery and vein. The fourth lumbar nerve is the first of the lumbar plexus. It has two main branches, the lateral cutaneous nerve and the genitofemoral nerve. The former is the stout trunk which emerges between the iliopsoas and psoas minor muscles and accompanies the course of the iliolumbar artery and vein, passing to the thigh. The genitofemoral nerve is a long slender nerve which runs along the medial border of the psoas minor muscle, lateral to the sympathetic cords. In the posterior part of its course it accompanies the external iliac artery. It supplies the thigh and abdominal wall of and adjacent to the inguinal region. After locating these two branches of the fourth lumbar trace them toward the vertebral column, removing the psoas minor as far as necessary. Find the point of emergence of the fourth lumbar from the vertebral column and note the connection, very stout in the cat, between the fourth lumbar and the fifth.

The fifth lumbar contributes by means of its connection with the fourth lumbar to the lateral cutaneous branch named above and also forms a strong union with the sixth lumbar. To expose these remove the rest of the psoas 
minor. The common trunk formed by the union of branches from the fifth and sixth lumbar nerves passes laterally as the large femoral nerve. Trace this to the thigh. It courses along the center of the medial surface of the thigh in company with the femoral artery and vein. It innervates adjacent muscles of the thigh and then continues down the shank and foot as the saphenous nerve.

The obturator nerve arises from the connecting band between the sixth and seventh lumbar nerves and passes obliquely caudad, dorsal to the pubis, through the obturator foramen and into the gracilis and other muscles.

The seventh lumbar together with the first sacral unite to form a very large trunk, the sciatic nerve. The sixth lumbar and second sacral also contribute small branches to this nerve. Follow the sciatic nerve. It turns dorsally, passing between the ilium and the vertebral column. Thrust an instrument through the place where it turns and dissect where the instrument emerges on the dorsal side of the animal. On separating the muscles at this place the sciatic nerve is exposed. Expose it as near to the vertebral column as possible. The gluteal nerves will be seen separating from the anterior side of the main trunk and passing into the gluteus muscles. (The nerve on the posterior side of the sciatic trunk is the posterior cutaneous, described below.) Follow the sciatic nerve down the leg. After giving off branches in to the thigh muscles it divides shortly above the knee into a lateral branch, the peroneal nerve, which passes between the insertion of the biceps femoris and the gastrocnemius, and a more medial branch, the tibial nerve, passing between the two heads of the gastrocnemius.

The sacral nerves are united by ansae to form the sacral plexus. The first sacral also, as seen above, takes part in the formation of the sciatic nerve. The chief nerves arising from the sacral plexus are the pudendal nerve and the inferior haemorrhoidal. The latter arises in the cat from the point of union of the three sacral nerves and passes to the bladder and rectum. The pudendal nerve arises from the large trunk formed by the union of the second and third sacral nerves and may also in the cat receive a branch from the sciatic. This trunk passes laterally parallel and posterior to the sciatic. From it arises the pudendal nerve which turns toward the rectum and urogenital organs, and the posterior cutaneous nerve which continues laterally into the biceps femoris muscle. It will be found by turning the animal dorsal side up and looking where the sciatic nerve was exposed. The nerve in question lies immediately posterior to the sciatic nerve and enters the biceps femoris. The fourth sacral nerve in the rabbit is of moderate size; it passes laterally and then turns to the sides of the rectum which it innervates in common with the pudendal nerve.

Draw, showing the lumbosacral plexus.

The foregoing nerves are all the ventral rami of the lumbar and sacral nerves. To see the small dorsal rami of the lumbar nerves proceed as directed for the dorsal rami of the thoracic nerves. The caudal spinal nerves will not be considered. 
2. The spinal cord and the roots of the spinal nerves.-With the bone scissors cut out a piece of the vertebral column two or three inches long from the posterior thoracic and anterior lumbar region. Remove the epaxial muscles from this piece so as to expose the vertebrae, and with the bone scissors cut off the neural arches of the vertebrae, thus exposing the neural canal. In this canal, but not completely filling it, lies the spinal cord. Note that the spinal cord is loosely inclosed in a tough membrane, the dura mater, from which strands pass to the walls of the neural canal. The space between the dura mater and the spinal cord is the subdural space. Slit open the dura mater. The spinal cord is closely invested by a membrane, the pia mater, which cannot be separated from its surface. Between these two is a delicate membrane, the arachnoid, which is almost impossible to identify in gross dissection. The arachnoid and pia mater of mammals together correspond to the pia mater of lower vertebrates. The spaces around and between these membranes are filled in life with the cerebrospinal fluid, which is a modified lymph.

From the sides of the spinal cord observe the roots of the spinal nerves arising in pairs at segmental intervals. They are insheathed in the dura mater which follows them to their exit from the intervertebral foramina and is continuous with their sheaths outside of the vertebral column. Examine one of the roots in detail. Although it appears at first glance to be single, a little gentle picking in the center of the root with the point of a probe will reveal that it is composed of two parts. One of these, the dorsal root, is attached to the dorsolateral region of the cord and near the intervertebral foramen bears a large oval swelling, the dorsal or spinal ganglion. The dorsal root carries sensory fibers only (except in fishes) and the nerve cells from which the sensory fibers originate are located in the spinal ganglion. The other root, the ventral root, arises from the ventrolateral region of the cord by several branches which unite to one trunk. The ventral root carries motor fibers only, arising from motor cells in the cord. The dorsal and ventral roots unite beyond the ganglion to form the spinal nerve, which then exits through the intervertebral foramen and divides into the dorsal ramus to the epaxial muscles and adjacent skin, the ventral ramus to the hypaxial muscles and adjacent skin, and the communicating rami to the sympathetic system. These rami were already seen.

Cut through the roots of the spinal nerves and remove a small section of the spinal cord for examination. Identify in the median dorsal line a groove, the dorsal median sulcus; in the median ventral line, another groove, the ventral median fissure. Lateral to the dorsal median sulcus is the dorsolateral sulcus, along which the dorsal roots enter the cord. The region between the dorsal median and dorsolateral sulci is called the dorsal funiculus. The lateral region of the cord between the dorsolateral sulcus and the line along which the ventral roots emerge is the lateral funiculus. Between this and the ventral median fissure is the ventral funiculus. 
Make a diagram through the cord showing its funiculi and the roots of the spinal nerves.

Make a clean cut across the cord and examine the cut surface. The section is divisible into a central darker material, the gray matter, shaped like a butterfly, in which the nerve cells of the cord are located; and a much thicker white material, the white matter, surrounding the gray matter and composed of nerve fibers. The white matter is subdivisible into the funiculi named above. Each funiculus consists of a number of tracts or bundles of fibers whose functions are known, but these tracts are not visibly differentiated from each other.

3. The peripheral distribution of the posterior cranial nerves. - In this section will be described the peripheral course of the fifth, seventh, and ninth to twelfth cranial nerves. For the complete dissection of these, it is necessary to have a specimen of which the head is intact, but most of them can be found, in part at least, on the same specimen on which the previous dissections were made.

a) The eleventh or spinal accessory nerve: This nerve supplies the sternomastoid, cleidomastoid, levator scapulae ventralis, and trapezius muscles. It is a pure motor nerve and is apparently derived from the vagus.

Rabbit: Separate the sternomastoid and cleidomastoid on the one hand from the basioclavicularis and levator scapulae ventralis on the other. Running near the dorsal border of the levator scapulae ventralis and parallel to it is the spinal accessory nerve. Branches of the second to fourth spinal nerves pass ventral to it and unite with it by branches. Trace it posteriorly and note its branches on the inner surface of the trapezius. Trace it anteriorly and note branches to the levator scapulae ventralis, sternomastoid, and cleidomastoid.

Cat: Cut through the clavotrapezius near its origin and deflect it ventrally, thus exposing the levator scapulae ventralis. On the inner surface of the clavotrapezius along the dorsal border of the levator scapulae ventralis runs the main part of the spinal accessory nerve. Trace it posteriorly, noting branches into the trapezius muscles and the levator scapulae ventralis. Trace it anteriorly. It passes dorsal to the second cervical nerve to which it is connected by a network, and near this region gives branches to the sternomastoid and cleidomastoid muscles. It then passes through the cleidomastoid muscle.

b) The vagus, the sympathetic, and the hypoglossal nerves: Follow the vagus and sympathetic anteriorly. Stretch the head forward by cutting across the lateral muscles of the neck. At about the level of the posterior end of the larynx the vagus and carotid artery are crossed ventrally by the descending branch of the hypoglossal or twelfth cranial nerve. This passes obliquely caudad toward the median line and supplies the sternohyoid, sternothyroid, and thyrohyoid muscles. Continue forward. At about the place where the common carotid artery divides into external and internal carotids a conspicuous nerve is seen crossing the ventral surface of the vagus and carotid artery and curving anteriorly. This is the main part of the hypoglossal nerve. Follow it forward. It 
passes to the dorsal side of the mylohyoid muscle, which may be cut, and innervates the muscles of the tongue.

About halfway between the descending branch and main part of the hypoglossal nerve, but deeper dorsally and passing to the dorsal side of the carotid artery, is situated the superior laryngeal branch of the vagus nerve. It runs obliquely caudad to the larynx which it penetrates, passing through the fibers of the thyrohyoid muscle.

Follow the vagus and sympathetic once more. At the place where the descending branch of the hypoglossal crosses them the two separate in the cat. Shortly anterior to this the vagus in both animals presents an elongated swelling, the nodosal ganglion. At about the same level, but more medial in position, the sympathetic trunk enters the superior cervical ganglion of the sympathetic, an elongated pinkish body. The two ganglia lie just posterior to the hypoglossal as it curves forward into the tongue.

The hypoglossal, the accessory, the vagus, and the sympathetic are all involved in a plexus in which the first cervical nerves also take part.

c) The ninth or glossopharyngeal nerve: This lies very close to the main part of the hypoglossal nerve but more deeply dorsal. Dissect directly internal to the hypoglossal where it curves anteriorly to the tongue. The glossopharyngeal is a smaller nerve lying dorsal to the hypoglossal along the sides of the pharynx anterior to the larynx. It is situated between the two horns of the hyoid. It divides into two branches: a smaller pharyngeal branch passing medially into the pharynx and a main lingual branch which enters the tongue. The former is a motor nerve to muscles of the pharynx, while the lingual branch is a nerve of taste.

Follow the nerves thus far described toward the point where they emerge from the skull. They are found to converge to a point to the medial side of the tympanic bulla. Here the ninth, tenth, and eleventh nerves emerge from the brain through the jugular foramen, located on the medial side of the bulla. The twelfth nerve emerges near the others through the hypoglossal foramen (consisting of several openings in the rabbit).

d) The seventh or facial nerve: The main part of this nerve is very superficial in position. It emerges at the posterior end of the masseter muscle at the base of the ear, in a sort of depression. On carefully searching in this region it will be found as a stout white band, in contact with the main part of the external carotid artery. At this place the facial gives off a branch to the posterior part of the digastric muscle, and the posterior auricular nerve to the pinna. (The large nerve to the pinna which may be noticed dorsal to this branch of the facial is the great auricular nerve originating in the cervical plexus.) The facial then proceeds forward, branching over the external surface of the masseter muscle, and passes to the lips and region of the eye. It supplies the various parts of the platysma muscle, which it may be recalled is a dermal muscle of the head 
and neck, serving to move the ears, lips, eyelids, whiskers, etc. The platysma muscle is a visceral muscle originally belonging to the hyoid arch, hence its innervation by the facial nerve.

e) The fifth or trigeminus nerve: This nerve has three main branches: the ophthalmic, the maxillary, and the mandibular. The former is best studied with the eye since it passes into the orbit.

To locate the mandibular branch of the trigeminus proceed as follows, freeing one half of the mandible. Cut through the attachment of the digastric to the mandible and deflect the digastric backward. Cut through the attachments of all of the muscles along the medial surface of the mandible, keeping the knife against the bone. Next, free the lateral or outer surface of the body of the mandible from muscle attachments, chiefly the masseter. Cut through the symphysis of the mandible (place of junction of the two halves of the mandible at their anterior tips). Carefully bend the half of the mandible thus freed outward, so as to expose the side of the muscular mass which forms the floor of the mouth and pharyngeal cavities. The main part of the mandibular branch of the trigeminus, the inferior alveolar nerve, will now be seen passing into a foramen, the mandibular foramen, situated on the medial surface of the mandible. In the rabbit the mylohyoid nerve, another branch of the mandibular, will be noted to the medial side of the inferior alveolar and proceeding ventrally to muscles of the floor of the mouth cavity. The inferior alveolar nerve runs in the interior of the mandible supplying the teeth and then emerges through the mental foramen on the lateral surface of the mandible at the level of the diastema. There the nerve, now named the mental nerve, may be found and followed into the lower lip.

Trace the inferior alveolar nerve posteriorly. It converges toward another branch of the mandibular nerve, the lingual nerve, which should then be followed forward. It passes into the tongue, lying close to the hypoglossal. The lingual branch of the trigeminus innervates the mucous membrane of the tongue, but is not a nerve of taste.

Follow both lingual and inferior alveolar nerves centrally again. In front of the tympanic bulla behind the point where the body of the mandible bends dorsally into the ramus of the mandible will be seen the auriculotemporal branch of the mandibular nerve joining the other two. On tracing it peripherally it is found to pass to the skin of the cranial side of the pinna and in the cat also sends branches along the side of the face in company with the branches of the facial.

The tympanic bulla may now be exposed. Emerging from the bulla will be found a slender nerve which very soon joins the lingual branch of the mandibular. This is the chorda tympani (so called because it runs in the tympanic membrane), a branch of the facial nerve. Its fibers pass out with the lingual nerve and supply the taste buds on the anterior part of the tongue; it also innervates the sublingual and submaxillary salivary glands. 
Besides the branches of the mandibular nerve here named there are branches to the muscles of mastication, namely, the temporal, the masseter, the anterior part of the digastric, and the pterygoids.

Remove the half of the mandible. This will reveal additional branches of the mandibular nerve. One of these, the buccinator, will probably be noticed extending to the angle of the mouth, where it supplies the masseter muscle and the lips. The main trunk of the maxillary nerve, the second branch of the trigeminus, may now be sought. It is a very stout trunk lying at the sides of the palate in front of and more deeply situated than the main trunk of the mandibular nerve. It is somewhat concealed by an artery (internal maxillary) which runs along its ventral surface and should be removed. The maxillary nerve is then revealed as a large trunk which passes forward along the side of the hard palate and disappears dorsal to the teeth. Cut away the zygomatic arch on the same side on which the half of the mandible was removed; in the rabbit cut away also the ridge which holds the molar and premolar teeth. By this operation the contents of the orbit are revealed. Note in the cat the small reddish infraorbital salivary gland lying close to the maxillary nerve. In the rabbit the very large reddish mass of the Harderian gland with the smaller yellowish mass of the infraorbital salivary gland anterior to it are readily noticed. The maxillary nerve should now be investigated. It is seen to divide into a large main trunk, the infraorbital nerves, and a small medial branch, the sphenopalatine nerve, which passes into the hard palate. The infraorbital nerves pass forward above the teeth, which they supply, and emerge through the infraorbital foramen, situated internal to the root of the zygomatic arch. On separating the upper lip from the teeth the foramen is readily found and the nerve is seen emerging from it to supply the upper lip and side of the nose. Follow the sphenopalatine nerve toward the palate, cutting away the bone. It connects with a ganglion, the sphenopalatine ganglion of the sympathetic system. This ganglion lies near the sphenopalatine foramen. The chief branch of the sphenopalatine nerve is the palatine branch which passes into the hard palate by a foramen. In the cat this nerve arises before the ganglion is reached, in the rabbit beyond the ganglion. Other branches of the sphenopalatine nerve pass from the ganglion into the nasal cavity.

4. The sense organs of the head.-

a) The eye, the eye muscles, and the nerves of the orbit: Dissect on the other side from that on which the cranial nerves were worked out. Identify upper and lower eyelids and the nictitating membrane, a fold projecting from the anterior corner of the eye. Make a slit through the junction of upper and lower lids at the posterior corner of the eye so that the eyelids can be pulled away from the eyeball. Note that the skin passes onto the inner surface of the eyelids and continues over the exposed surface of the eyeball, thus forming the outermost covering membrane, the conjunctiva, for this part of the eyeball. Make an 
incision through the skin above the eye and deflect the skin downward toward the eye on which you are working, stretching the skin away from the head. On the skin of the inner surface of the upper eyelid note a thin sheet of muscle fibers, proceeding in a somewhat circular direction. This is orbicularis oculi, a part of the platysma, and has the function of closing the eyelids.

Rabbit: Stretch the upper eyelid away from the head and clean away the connective tissue between it and the eyeball. A thin sheet of muscle will be found extending from beneath the supraorbital arch to the upper eyelid. This is the levator palpebrae superioris, which raises the eyelid. Repeat the foregoing directions on the lower eyelid, stretching the skin away from the eyeball. On the inner surface of the lower eyelid note the rest of the orbicularis oculi. The depressor palpebrae inferioris may be noted extending from the zygomatic arch to the lower eyelid; it lowers the eyelid. Remove the surrounding skin and eyelids, cutting them away from the eyeball. With the bone clippers cut away the supraorbital arch and clean away tissue between the dorsal surface of the eyeball and the orbit. A slender but strong muscle will now be seen extending from about the middle of the wall of the orbit to the dorsal surface of the eyeball; this is the superior oblique muscle. It separates the thin sheet of the levator palpebrae superioris into two parts which pass on either side of it. Trace the superior oblique to the wall of the orbit. Here there will be found a tendinous cord, the trochlea, over which the muscle passes. Next, remove the levator palpebrae superioris and find underneath its posterior portion the thin flat superior rectus muscle. The insertion of the superior oblique on the eyeball is concealed under the margin of the superior rectus.

Remove the half of the mandible and the zygomatic arch on the side on which you are working. This fully exposes the ventral side of the eyeball. Along the ventral surface of the outer part of the eyeball extends the yellowish infraorbital salivary gland. Medial to this extending beneath the eyeball is the larger Harderian gland, which pours its secretion onto the nictitating membrane. Remove these glands; note the white part of the Harderian gland extending far medially. The inferior oblique muscle is now seen extending to the eyeball from the anteroventral region of the orbit. Posterior to it is the inferior rectus muscle originating from the posteroventral region of the orbit. Note the branch of the oculomotor nerve running along the anterior border of the inferior rectus and supplying both muscles. The nerve which runs along the posterior border of the inferior rectus, innervating the lower eyelid, is the zygomatic branch of the maxillary nerve. Immediately behind the inferior rectus is the external or lateral rectus. The nerve passing along the posterior margin of the external rectus is the lacrimal branch of the maxillary. It passes to the lacrimal gland and to the skin between the eye and base of the pinna. The lacrimal gland is a small reddish body which will be found by pressing the eyeball forward and searching against the posterodorsal wall of the orbit. Two nerves pass the point 
of origin of the external rectus from the orbit. The larger is the oculomotor, the smaller the abducens. Cut through the insertions of the inferior oblique and inferior and external recti at the eyeball and deflect them ventrally. Above the inferior rectus the internal or medial rectus will be seen inserted on the eyeball. Look on the inner surface of the external rectus and find the abducens nerve, curving around the posterior border of the origin of this muscle and passing onto its surface. Return to the dorsal surface of the eyeball, cut through the insertion of the superior oblique at the eyeball, and press the eyeball ventrally. Two nerves will be seen on the medial wall of the orbit. The lower one is the trochlear nerve. Trace it to the medial surface of the superior oblique. The upper nerve is the frontal nerve, one of the main branches of the ophthalmic branch of the trigeminus. It passes to the dorsal part of the orbit and exits through the anterior supraorbital foramen to be distributed to the upper eyelid and skin in front of the orbit. It may have been cut in removing the supraorbital arch. The white part of the Harderian gland will be noted in the anterior part of the orbit. Cut through all of the insertions of the eye muscles at the eyeball and through the optic nerve, removing the eyeball. The optic nerve is the stout white trunk near the superior rectus. The muscles around the optic nerve, exclusive of those already identified, belong to the retractor bulbi. Find the main trunk of the oculomotor nerve and trace its branches to the retractor bulbi and superior and internal recti. The main nerve curves below the optic nerve. The nasociliary branch of the ophthalmic nerve may be noted passing between the superior oblique and the retractor bulbi. Its main portion, the ethmoidal nerve, leaves the orbit by a small foramen in front of the superior oblique muscle. On tracing this nerve posteriorly fine branches to the orbit may be seen.

Trace the nerves of the orbit to their exits from the skull. The third, fourth, and sixth nerves and the ophthalmic and maxillary branches of the trigeminus pass through the orbital fissure. The mandibular branch of the trigeminus passes through the foramen lacerum.

Cat: Remove the eyelids and the surrounding skin, cutting them away from the eyeball. Remove the half of the mandible and the zygomatic arch from the side on which you are working. Press the eyeball ventrally away from the supraorbital arch. In the anterodorsal angle of the orbit a strong fibrous connection will be found between the wall of the orbit and the eyeball. On investigating this it is found to consist of two fibrous bands which form a pulley; this is known as the trochlea. The tendon of the superior oblique muscle passes over the trochlea and is inserted on the eyeball. Its insertion is much expanded and extends caudad from the trochlea. Posterior to the insertion of the superior oblique is a thin flat muscle, the levator palpebrae superioris, or elevator of the upper eyelid. This passes to the dorsoposterior surface of the eyeball. Cut through this at its insertion. Posterior to this muscle in the dorsoposterior 
angle of the orbit is the flattened lacrimal gland. Cut out the nictitating membrane and examine its internal surface. It is found to be roughened, owing to the presence of the Harderian gland in its wall.

Turn to the ventral surface of the eyeball exposed by the removal of the mandible, the zygomatic arch, and part of the hard palate. Identify again the small reddish infraorbital salivary gland, situated back of the last tooth. On clearing away connective tissue and fat, the inferior oblique eye muscle will be seen extending from the anterior part of the orbit to the ventral surface of the eyeball. Ventral and at right angles to the inferior oblique is the inferior rectus. The branch of the oculomotor which innervates the inferior oblique runs along the posterior border of the inferior rectus. The zygomatic branch of the maxillary nerve passes along the posterior border of the inferior rectus to the lower eyelid, but may have been destroyed. Posterior to the inferior rectus is the external rectus; between and internal to them appears one of the four parts of the retractor bulbi muscle. Along the posterior border of the external rectus runs the lacrimal branch of the maxillary nerve, supplying the lacrimal gland and adjacent skin. On detaching the eyeball from the posterior wall of the orbit another part of the retractor bulbi will be seen next posterior to the external rectus; dorsal to this is the superior rectus. Cut through both obliques at their insertions and press the eyeball posteriorly. Note the internal rectus on the anterior surface of the eye and above it the remainder of the retractor bulbi.

Cut through all of the eye muscles and the optic nerve at their insertion on the eyeball and remove the latter. Note the four parts of the retractor bulbi around the optic nerve. Deflect the external rectus ventrally and note the abducens nerve ascending on its inner surface. Running along the ventral surface of the optic nerve note a slender nerve, the long ciliary branch of the ophthalmic branch of the trigeminus; it accompanies the optic nerve into the eyeball. Look on the inner surface of the inferior rectus for the branch of the oculomotor to this muscle. Note the ciliary ganglion of the sympathetic near this branch and observe branches between this ganglion and the oculomotor and long ciliary nerves and the short ciliary nerves passing from the ganglion along the optic nerve. Find the main trunk of the oculomotor ventral to the optic nerve at the place of passage of both through the wall of the orbit, and note branches of the oculomotor to the retractor bulbi and superior rectus. Bend all eye muscles except the superior oblique ventrally, leaving the superior oblique against the medial wall of the orbit. Crossing the inner surface of the superior oblique obliquely forward are two nerves. They are parts of the ophthalmic branch of the trigeminus. The lower one is the ethmoidal nerve; it passes through a foramen into the nasal cavity. The upper one is the infratrochlear nerve. It goes to the anterior part of the upper eyelid. Posterior to and parallel to the posterior margin of the superior oblique is the frontal branch of the ophthalmic. It innervates the upper eyelid and integument 
anterior to the eyelid. The trochlear nerve lies slightly dorsal and medial to the proximal portions of the ethmoidal and infratrochlear nerves. It runs obliquely dorsad and anteriorly, and enters the superior oblique muscle at about the middle of its posterior margin.

The structure of the eyeball may now be investigated. It is very similar to that of all vertebrates. The outer tough sclerotic coat or sclera is continuous with the transparent cornea covering the exposed surface of the eye. As found above, the cornea is covered externally by the conjunctiva. Cut off the top or dorsal side of the eyeball and look within. The large lens will be observed. Internal to the sclera is the black chorioid coat of the eye and internal to that the greenish-gray retina. Between the lens and the retina is a large chamber, the cavity of the vitreous humor, containing a gelatinous mass, the vitreous humor or vitreous body. Remove the lens. The chorioid coat terminates behind the cornea as a black curtain, the iris, bearing in its center a round hole, the pupil. The space between the cornea and the iris is called the anterior chamber of the eye and is filled in life with a fluid, the aqueous humor. The boundary between the iris and the rest of the chorioid coat constitutes a ring known as the ciliary body. It consists of two parts: a ring of thickened processes, the ciliary processes, next to the iris, and a ring of radially arranged ridges, the orbiculus ciliaris, extending to the main part of the chorioid coat. Both parts of the ciliary body contain the ciliary muscle; this is a smooth muscle having both meridional and circular fibers. It originates on the sclera, is inserted on the walls of the ciliary body, and has the function of changing the shape of the lens. By making a new cut parallel to the first around the equator of the eyeball the relations of cornea, iris, and ciliary body will be more clearly observable. Note the marked thickening due to the ciliary body. Examine the lens. Note its biconvex form as compared with the spherical form of the lens of the fish eye. Around the equator of the lens will be found the torn attachment of a membrane. This membrane holds the vitreous body. Where it is attached to the lens it exhibits parallel ridges, the zonular fibers, which in life fit into the hollows between the ciliary processes. The zonular fibers constitute the suspensory ligament of the lens which passes from the lens to the ciliary processes. By means of this ligament traction can be exerted on the lens and its shape altered to some extent. The small space between the suspensory ligament and the iris is the posterior chamber of the eye. Peel the lens and note that it is composed of concentric coats or lamellae like the coats of an onion, each lamella being composed of lens fibers.

Draw the section of the eye.

b) The nasal cavties: Detach the head of the animal at the joint between the occipital condyles and the altas, and discard the body. Cut off the pinnae. Clear the dorsal surface of the skull down to the bone. With a saw, saw completely through the head slightly to one side of the median sagittal plane. Use 
the saw only for the bony parts. After having sawed through the roof of the skull, cut down through the brain with a single sliding stroke of a blunt knife like a table knife. The brain and skull should thus be cut in two, one part being slightly larger than the other. Wash the cut surfaces gently under the tap, and study the nasal cavities.

The nasal cavities are very long in the rabbit, shorter in the cat. They are divided into right and left cavities or fossae by a perpendicular plate, the septum of the nose, which is present on the larger section of the head. The septum consists of cartilage anteriorly and of thin bone posteriorly, the latter being the perpendicular plate of the ethmoid bone. On the smaller section the lateral and posterior walls of the nasal fossa are seen to be occupied by delicate scrolled and folded bones, the turbinated bones or conchae. In the rabbit these are readily divisable into an anterior concha, the inferior concha or maxilloturbinal, much folded and located on a separate small bone of the skull; a middle concha or nasoturbinal, a long single fold dorsal to the preceding and dependent from the nasal bone; and the superior concha or ethmoturbinal, part of the ethmoid bone. In the cat the turbinals are closely crowded together, but by prying them apart gently there can be distinguished a small anterior maxilloturbinal on the maxilla; above this a single fold, the nasoturbinal, dependent from the nasal bone; and a great mass of folds, the ethmoturbinal, filling the greater part of the nasal fossa. The ethmoturbinals are also called the ethmoid labyrinths, and the spaces inclosed by the bony folds are called the ethmoid cells. Definite passages known as the meatuses of the nose run between the conchae and conduct the air to the nasopharynx. They connect with the nasopharynx below the ethmoturbinals.

The posterior dorsal part of the nasal fossa is closed by the cribriform plate of the ethmoid which unites with the perpendicular plate of the ethmoid bone medially and with the parts of the ethmoid which bear the layrinths laterally. The anterior end of the brain (olfactory bulbs) is readily seen to abut against the cribriform plate, and through this plate the fibers of the olfactory nerve pass from the olfactory membrane covering the ethmoid labyrinths to the olfactory bulbs.

c) The structure of the ear: Carefully remove the brain from the two halves of the skull, preserving the latter. In doing this the roof of the skull may be cut away. Loosen the brain on all sides by passing a blunt instrument between the brain and the skull. The tough membrane, the dura mater, which covers the brain should be retained with the brain. Carefully cut the cranial nerves where they pass through the foramina of the skull, leaving their roots attached to the brain. Note the small round reddish body, the pituitary body, attached to the ventral surface of the brain, set into a depression in the floor of the skull; keep this body attached to the brain if possible. Preserve the two halves of the brain in a vessel of water or, if they are to be kept for some time, in weak formaldehyde. 
After removal of the brain examine the cavities of the skull on the larger piece. Anteriorly behind the cribriform plate is the small anterior or olfactory fossa in which the olfactory bulbs are situated. Posterior to this is the large middle or cerebral fossa lodging the cerebrum. Behind this is the smaller posterior or cerebellar fossa for the cerebellum. The cerebral and cerebellar fossae are partly separated by a bony ledge, the tentorium, which is continued in life by the dura mater. In the floor of the cerebral fossa in the basisphenoid bone is the sella turcica lodging the pituitary body. Note also the optic foramen in front of this and behind this, near the ventral end of the tentorium, the foramina for the passage of the third to sixth cranial nerves. In the wall of the cerebellar fossa observe an area of hard, white bone; this is the petrous portion of the temporal. In the center of this is a foramen for the passage of the auditory nerve into the internal ear. Above this in the rabbit is a depression, the floccular fossa, which lodges a part of the cerebellum called the flocculus. In removing the rabbit brain the flocculus is left behind in the fossa. In front of the ventral part of the petrous bone, just behind the tentorium, is the internal opening of the facial canal for the passage of the facial nerve. Behind the middle of the petrous bone is the jugular foramen for the passage of the ninth, tenth, and eleventh nerves. Behind this the twelfth nerve passes through one or more foramina.

The ear of mammals consists of three parts, the external, the middle, and the internal ear. The external ear includes the pinna or auricle and the external auditory meatus leading into the interior of the bulla; these have already been noted. The middle ear is situated in the tympanic bulla, the internal ear in the petrous portion of the temporal bone. Both are consequently in the wall of the cerebellar fossa. With the bone clippers remove this wall in one piece and discard the remainder of the skull. Clean away the muscles from its external surface, exposing the tympanic bulla.

Rabbit: With the bone clippers cut away the ventral wall of the tympanic bulla. A large cavity, the cavity of the middle ear or tympanic cavity, is revealed. In the lateral wall of this cavity is a ringlike elevation of bone across which is stretched a delicate membrane, the tympanic membrane or eardrum. By probing into the external auditory meatus determine that the meatus terminates at the eardrum, which closes its internal opening. The tympanic membrane has a nearly vertical position. Extending toward the tympanic membrane from the medial wall is a short calcareous process which supports the chorda tympani branch of the facial nerve as it crosses from the facial to the tympanic membrane. Anterior to the tympanic membrane is a depression in which are lodged the three little ear bones. These bones are so small and so deeply lodged in the depression that they cannot be distinctly seen, but on picking in the depression with a forceps it is usually possible to extract one or more of them. Compare them with $\mathrm{K}$, page 202 , Figure 2 Io. 
Cat: Remove the fleshy part of the external auditory meatus down to the tympanic bulla. The meatus will be found to terminate at an oval opening with a slightly elevated rim. Across the rim is stretched the delicate tympanic membrane or eardrum. The handle of the malleus or hammer is visible through the eardrum attached to its internal surface. Next, remove with the bone clippers the ventral wall of the bulla. The interior is the tympanic cavity of the middle ear. Note that it is divided by a bony plate into a larger posteroventral chamber and a smaller anterodorsal chamber. The latter is the one covered above by the tympanic membrane. Break open the plate of bone, exposing this cavity, which is called the tympanum proper and which contains the ear bones. Note the membrane which lines it and the eardrum forming its anterodorsal wall. From the posterodorsal region of the cavity a calcareous process projects toward the eardrum and carries the chorda tympani nerve, a branch of the facial, to the eardrum. From the internal surface of the eardrum the three little ear bones are plainly seen extending into a depression in the internal wall of the tympanum. These may be extracted and examined. Compare with K, page 202, Figure 2 I0.

There now remains between the bulla and the cerebellar fossa the hard white mass of the petrous bone already noted. This contains the internal ear. Owing to the complexity of the internal ear and its small size, a dissection of it is impractical, but its main parts can be seen by breaking away the petrous bone in small fragments. The tiny spirally coiled chamber in the bone is the cochlea, it contains a spiral tube, the cochlear duct, in which the organ of sound perception (organ of Corti) is located. In the thicker harder part of the petrous bone are semicircular channels, the semicircular canals, inclosing the semicircular ducts.

The internal ear is thus seen to be inclosed in channels in the petrous bone, consisting of the cochlea, the semicircular canals, and the vestibule or connecting chamber; together, these constitute the bony labyrinth. The internal ear proper, or membranous labyrinth, is contained in the bony labyrinth. Its parts are: the sacculus and utriculus inclosed in the vestibule; the semicircular ducts arising from the utriculus and situated inside of the semicircular canals, and the cochlear duct arising from the sacculus and inclosed in the cochlea. The cochlear duct is a new structure characteristic of mammals, although it begins to appear in birds. It is an outgrowth of the sacculus and is the real organ of hearing, the semicircular ducts serving equilibratory functions only.

\section{The structure of the brain.-}

a) The membranes or meninges of the brain: With the two halves of the brain previously removed before you, study the membranes of the brain. The brain is covered by a tough membrane, the dura mater. This consists of the dura mater of lower forms fused to the internal lining (periosteum) of the skull. A considerable space, the subdural space, is present between the dura mater and the other membranes of the brain. The dura mater dips down between the larger divisions of the brain. The surface of the brain is covered by the delicate pia mater, in 
which the blood vessels run. The pia mater follows closely all of the folds oi the brain surface. Between the pia mater and the dura mater is another membrane, the arachnoid, very delicate and difficult to see. It is best found covering the depressions on the surface of the brain, for the pia mater dips down into these depressions while the arachnoid passes over them. Between the arachnoid and the pia mater is the subarachnoid space crossed by a delicate web of tissue. All of the spaces between the meninges of the brain are filled in life with the cerebrospinal fluid.

b) The dorsal aspect of the brain: Remove the dura mater. Fit the two halves of the brain and study the dorsal surface. At the anterior end of the brain are the two olfactory bulbs, relatively small, rounded masses into whose anterior surfaces the fibers of the olfactory nerve enter. Posterior to them are the enlarged pear-shaped cerebral hemispheres. Their surfaces are much convoluted in the cat, consisting of folds, the gyri, with grooves, the sulci, between the gyri. The two hemispheres are separated from each other by a deep median sagittal fissure, the longitudinal cerebral fissure (which is on the larger piece of the brain). Gently spread open the fissure and note at its bottom a thick, white mass connecting the two hemispheres. This is the corpus callosum, a structure characteristic of the mammalian brain. It is composed of nerve fibers passing between the hemispheres. At the posterior end of the longitudinal fissure is a small reddish mass of folded tissue, which is part of the chorioid plexus of the roof of the diencephalon. The diencephalon or region of the brain posterior to the cerebral hemispheres is in mammals completely concealed from dorsal view by the posterior extension of the hemispheres above it. The posterior ends of the cerebral hemispheres are in contact with the cerebellum, a large mass with a much convoluted surface. Between the cerebellum and the cerebral hemispheres is the midbrain, also concealed from dorsal view by the hemispheres. It is readily revealed by bending the hemispheres and the cerebellum apart. It consists of four rounded lobes or hillocks known as the corpora quadrugemina or colliculi. The two anterior ones are named the superior colliculi, the two posterior ones the inferior colliculi. The cerebellum consists of a median lobe, the vermis or worm, and a pair of lateral lobes, the hemispheres. The vermis corresponds to the entire cerebellum of lower vertebrates. From each hemisphere in the rabbit arises by a narrow stalk another lobe, the flocculus, which as already seen is left behind in the floccular fossa of the petrous bone when the brain is removed from the skull. Identify on the hemispheres the cut surfaces where the flocculi were attached. Posterior to the cerebellum and partly overlapped by the vermis is the medulla oblongata. Lift the vermis and note the cavity of the fourth ventricle in the medulla. It is normally roofed over by a membrane, the medullary velum. which contains a chorioid plexus; this has probably been removed in sectioning the brain. In the cat the chorioid plexus projects on each side between cerebellum and medulla as a little tuft of vascular tissue. At each side of the 
posterior pointed end of the fourth ventricle is a tract terminating in a club-shaped area, the clava. Lateral to each clava is another elongated area, the tuberculum cuneatum. These two belong to the somatic sensory column. Anterior to them a white bundle passes toward the cerebellum, disappearing ventral to an elevation which lies just ventral to the hemisphere of the cerebellum. The bundle is the restiform body or posterior peduncle of the cerebellum which conveys impulses from the medulla and spinal cord to the cerebellum. The elevation over the restiform body is the area acustica or primary auditory center. The general resemblance of these structures to those found in the dogfish should be evident.

Draw the dorsal view of the brain.

c) The ventral aspect of the brain: Note the basilar artery (continuation of the two vertebral arteries) running in the midventral line and forming a circle around some structures in the center of the ventral surface. This circle, the circle of Willis, is joined on each side by the internal carotid artery. Note the arteries arising from the basilar and circle of Willis and distributed over the brain, coursing in the pia mater. The arteries should be removed.

At the anterior end of the ventral surface are the two olfactory bulbs. From each one a definite white tract, the olfactory tract, extends obliquely caudad and terminates posteriorly in a lobe, the pyriform lobe, which forms the posteroventral part of the cerebral hemispheres. The fissure or sulcus which separates the pyriform lobe from the rest of the cerebral hemisphere is called the rhinal fissure. Inclosed between the two pyriform lobes is the ventral side of the diencephalon, or thalamencephalon. At the anterior end of this is the optzc chiasma from which the optic nerves project. The region between the optic chiasma and the olfactory tracts is called the anterior perforated substance. Behind the optic chiasma is a slight rounded elevation, the tuber cinereum, from which the pituitary body or hypophysis depends by a stalk. In case the pituitary body was torn off in removing the brain, a slitlike aperture will be noticed in the center of the tuber cinereum marking the place of attachment of the pituitary body. Immediately posterior to the attachment of the pituitary body is an area, the mammillary body, not distinctly marked off from the tuber cinereum. Posterior to this is a depressed area, the posterior perforated substance, from which arise the two third, or oculomotor, nerves. From beneath (dorsal to) the pyriform lobes a thick white bundle will be seen passing obliquely backward on each side of the posterior perforated substance. These bundles are the cerebral peduncles, belonging to the nidbrain. The fourth or trochlear nerves arise on the side of the brain between the cerebellum and the inferior colliculi, and pass ventrally over the outer surface of the peduncles.

The remainder of the ventral surface of the brain belongs to the hindbrain, and consists of the pons and the medulla oblongata. The pons is the heavy band of fibers which crosses the ventral surface of the hindbrain immediately behind the posterior perforated substance. By following it around to the sides of the 
brain it will be seen to narrow to a white cord, the brachium pontis or middle peduncle of the cerebellum, which passes into the substance of the cerebellum. The pons is, in fact, a bridge between the two hemispheres of the cerebellum. It is the ventral part of the metencephalon of which the cerebellum is the dorsal portion. Immediately posterior to the brachium pontis and partly concealing it is the thick root of the trigeminus nerve. On close examination this will be seen to consist of a large dorsal portion, the sensory root (portio major) which consists of the somatic sensory fibers of the trigeminus, and a very small ventral portion, the motor root (portio minor) which contains the visceral motor fibers for the muscles of mastication (masseter, temporal, digastric, etc.). Posterior to the pons and of about half its width is another bundle of transverse fibers the trapezoid body. Close inspection will show that the trapezoid body originates from the area acustica or auditory center; it passes toward the median line but before reaching it, turns forward and disappears dorsal to the pons. The trapezoid body is the main tract which carries the auditory impulses to the more anterior portions of the brain. Attached to the side of the area acustica is the root of the eighth or auditory nerve. Just ventral to this and behind the root of the trigeminus is the root of the facial nerve emerging through the trapezoid body. In the median ventral line of the medulla is a groove, the median ventral fissure. Along each side of this runs a narrow bundle of fibers; each emerges dorsal to the posterior margin of the pons and proceeds straight posteriorly. These two tracts are the pyramids or somatic motor tracts; they convey impulses irom the cerebral hemispheres to the voluntary muscles. At the place where the pyramids emerge from above the pons are the roots of the sixth or abducens nerves. The small root of the ninth or glossopharyngeal nerve will be found at the posterior boundary of the acustic area, at the point where the restiform body passes dorsal to it and about on a line with the root of the eighth nerve. The equally small root of the tenth or vagus nerve lies immediately posterior to and on a line with the root of the ninth nerve. Posterior to the vagus are the numerous roots of the eleventh or spinal accessory nerve, arising in a line. The main root of the accessory ascends from the spinal cord, but is probably missing in the specimen. The roots of the twelfth or hypoglossal nerve emerge along the lateral border of the pyramid, posterior to the preceding roots.

Draw the ventral view of the brain, including the roots of the cranial nerves as far as you have seen them.

d) The median sagittal section: Now cut the larger half of the brain along the longitudinal cerebral fissure so as to obtain an exact median sagittal section. In making such a cut use a dull knife and pass it through the brain with one sliding stroke. Examine the cut surface. The cerebral hemisphere forms a thick roof which arches posteriorly above the diencephalon and midbrain. In the cerebral hemisphere identify the section of the corpus callosum. This is an obliquely placed longitudinal band of white material. Both anterior and posterior 
ends are enlarged, the former being named the genu, the latter the splenium. From about the middle of the corpus callosum a band of fibers, the fornix, curves ventrally. Between the fornix and the anterior half of the corpus callosum stretches a thin membrane, the septum pellucidum, consisting of two leaves. If the brain is sectioned exactly in the median sagittal plane, the section will pass between the two leaves of the septum pellucidum; but often the whole septum is left on one half; in this case a slitlike opening into a cavity, the lateral ventricle, will appear on the other half between the fornix and the corpus callosum. The fornix passes downward and soon turns (as the column of the fornix) into the interior of the brain where it is lost to view. Immediately in front of the point where it disappears is the section of a small round bundle, the anterior commissure. From the anterior commissure a delicate membrane, the lamina terminalis, extends ventrally to the optic chiasma. The fornix, the anterior commissure, and the lamina terminalis form the anter or boundary of a deep but narrow chamber, the third ventricle, which lies in the middle of the diencephalon. The cavity of the third ventricle extends ventrally into the tuber cinereum and the pituitary body.

The diencephalon is the massive region extending between the fornix and lamina terminalis and midbrain. It consists of three parts: a dorsal region, the epithalamus; a central and lateral region, the thalamus; and a ventral region, the hypothalamus. The hypothalamus includes the optic chiasma, the tuber cinereum, the mammillary body, and the hypophysis or pituitary body, all of which should be identified in the section. The epithalamus includes the structures in the roof of the diencephalon. These are: the chorioid plexus, a thin folded vascular membrane between the cerebral hemisphere and the diencephalon; the pineal body, a stalked body lying in the chorioid plexus; the habenula, a small mass just in front of the attachment of the pineal body to the diencephalon; and the posterior commissure, a small circular area just posterior to the habenula. The thalamus constitutes the greater part of the diencephalon. On the cut surface it presents a large, round mass, the intermediate mass or middle commissure; this is not really a commissure but merely the cut median mass of the thalamus. The greater part of the thalamus is concealed by the overhanging cerebral hemisphere. On the smaller piece of the brain remove the cerebral hemisphere and then examine the dorsal and lateral regions of the thalamus. Three low elevations are present. The most dorsal and medial one is the pulvinar. Lateral to this and whiter in color is the lateral geniculate body. A white band, the optic tract, is plainly seen ascending from the optic chiasma and terminating on the lateral geniculate body. Posterior and ventral to the lateral geniculate body is a smaller swelling, the medial geniculate body. Behind the geniculate bodies will be recognized the corpora quadrugemina as two low hillocks. Ventral to them runs the stout cerebral peduncle, the anterior part of which is crossed externally by the optic tract. 
Returning to the medial sagittal section, identify in the roof of the midbrain the two hillocks formed by the superior and inferior colliculi or corpora quadrugemina. Below them is a narrow passage, the aqueduct of the brain, which connects the third ventricle in the diencephalon with the fourth ventricle in the medulla. Below the aqueduct is the thick floor of the midbrain, the tegmentum. At the sides of this are the cerebral peduncles, not exposed in the section. In the section of the cerebellum note the curious branching treelike arrangement of the white matter, forming the arbor vitae or tree of life. This appearance is brought about by the fact that each fold of the cerebellar surface consists of gray matter or nerve cells, with a central plate of white matter or nerve fibers. The cerebellum fits into the fourth ventricle from which, however, it is separated in the normal condition by a membrane, the medullary velum. Part of this velum will probably be found below the anterior part of the cerebellum. Identify in the section the mass formed by the pons. The section of the medulla has nothing of additional interest.

Draw the sagittal section.

e) Further structure of the cerebral hemispheres: On the intact half of the brain begin to cut away the roof of the cerebral hemisphere in thin slices. Note that the superficial substance of the roof is gray, the interior white, a reversal of the condition found in lower vertebrates. This gray outer coat of the mammalian brain is called the cortex; it is gray because it consists of nerve cells which have migrated to the surface from their original, more central position. The white matter consists of fibers which carry impulses to and from the cortex. In the cat the cortex is much convoluted. Note that each convolution has a central core of white matter and a peripheral thick coat of gray matter. Continue to shave the brain ventrally until the corpus callosum is exposed. This is a narrow band of fibers conveying impulses between the two hemispheres. Remove the corpus callosum and the cortex lateral to it. This exposes the cavity of the cerebral hemisphere, called the lateral ventricle. It is filled by two conspicuous elevations. The anterior and smaller one, of a darker color, is the corpus striatum, a mass of gray matter. The posterior, larger one is the hippocampus. Remove the side of the hemisphere so as to expose the hippocampus. It is a curved body with an anterior free margin, the fimbria. Cut through the medial attachment of the hippocampus, raise the anterior border, and roll the hippocampus back. Observe that the part of the hippocampus still attached is continuous with the pyriform lobe. The hippocampus is a part of the original external surface of the brain, which has been invaginated into the interior. The turning back of the hippocampus reveals the thalamus Note the thick stalk extending from the thalamus into the cerebrum, immediately in front of the pulvinar and lateral geniculate body. Scrape the surface of this and note that it consists of a great mass of fibers radiating from the thalamus into the cerebral hemisphere. This is 
more evident in the rabbit than in the cat, since in the cat the fibers turn dorsally. This radiating mass is called the corona radiata.

f) Functions of the parts of the brain: As an aid in the understanding of the anatomy of the brain, a few statements may be made concerning the functions of the parts identified in the preceding sections. The olfactory bulbs, the olfactory tracts, the pyriform lobe, the tuber cinereum, the fornix, the habenulae, the mammillary body, and the hippocampus belong to the olfactory apparatus of the mammal. The olfactory impulses come along the olfactory nerves into the olfactory bulbs, which are the primary olfactory centers; they are then relayed along the olfactory tracts to the pyriform lobe, which is the secondary olfactory center; from the pyriform lobe they pass to the hippocampus, the tertiary olfactory center, or conscious center of smell. The hippocampus has extensive connections with other parts of the brain for reflex purposes. These connections occur by way of the fimbria, a mass of nerve fibers. The fimbria connects with the fornix and this in turn with the mammillary body. The habenula is also connected with the hippocampus. In the dogfish practically the whole of the telencephalon is concerned with smell, while here the olfactory functions occupy but a part of the telencephalon, the remainder having developed new connections and functions. The diencephalon is the great center of correlations in the mammalian brain. Its relation to the olfactory sense has already been noted. The lateral geniculate body is the primary optic center, in which, as we saw, the optic tracts terminate in part. From the lateral geniculate body the optic impulses pass to the cerebral hemisphere by way of the corona radiata. The pulvinar and the superior colliculus of the midbrain are also concerned in optic impulses, the latter being a reflex center for these impulses. The primary auditory center is located in the area acustica; from here the auditory impulses are carried, in part by the trapezoid body, to the inferior colliculus and the medial geniculate body, which constitute secondary and tertiary auditory centers. From the medial geniculate body the auditory impulses are carried in the corona radiata to the cerebral cortex. In a similar way other sensations such as pain, touch, temperature, pressure, consciousness of muscle and joint movements, and position of the body in space are carried by definite paths (which are for the most part invisible externally on the brain) to the thalamus from which they are relayed to the cerebral cortex. (The clava and tuberculum cuneatum are concerned with joint and muscle sense and steadiness of body movement and position.) It will thus be seen that practically all sensations make a relay in the diencephalon from which they ascend by a new path, the corona radiata, to the cerebral cortex. The corona radiata is thus the great pathway between the diencephalon and the cerebral cortex by means of which the sensations are projected, as it were, upon the cortex. It is further well known on which area of the cerebral cortex each sensation is projected. After all of the sensations have thus been localized upon the cortex, there still remains a considerable portion of the cortex to which no definite tracts from below can be traced. It is presumed that these areas are concerned with co-ordination, reason, emotion, etc.

The impulses toward voluntary movements originate in a definite part of the cortex, pass downward in the corona radiata into the cerebral peduncles, and appear on the ventral surface of the medulla as the pyramidal tracts or pyramids, which descend the whole length of the spinal cord and make connections with the motor cells of the ventral columns of the cord. The cerebral peduncles, besides carrying the pyramidal tracts, also carry large tracts from the cortex to the pons, where they pass into the cerebellum.

The cerebellum is the great center for equilibration and motor co-ordination. Its functions are involuntary and unperceived by the conscious mind. It is connected with the rest of the brain by means of its peduncles: the posterior peduncles or restiform bodies, 
which connect it with the medulla and spinal cord; the brachium pontis or middle peduncle, which joins the two sides of the cerebellum and also conveys tracts between the cerebellum and cerebral cortex; and the anterior peduncles (which were not seen in the dissection) which extend between the cerebellum and midbrain and thalamus. The cerebellum has extensive connections with the area acustica, since the impulses from the ampullae of the semicircular ducts, which are concerned with equilibration, terminate in the area acustica.

\section{G. SUMMARY OF THE NERVOUS SYSTEM AND THE SENSE ORGANS}

I. The nervous system and the nervous parts of the sense organs are derived from the ectoderm.

2. The nervous system is subdivided into central, peripheral, and sympathetic nervous systems. The first includes the brain and spinal cord, the second the cranial and spinal nerves, and the third the sympathetic cords, ganglia, and nerves.

3. The spinal cord consists of a central gray region and a peripheral white region subdivided into tracts. The spinal nerves arise in pairs from the cord at segmental intervals.

4. Each spinal nerve arises from the cord by two roots, a dorsal and a ventral. The dorsal root is sensory and bears a ganglion composed of sensory cells; the ventral root is motor. The two roots unite to form a spinal nerve.

5. The spinal nerve very soon divides into a dorsal ramus which passes to the epaxial muscles and adjacent skin, a ventral ramus which passes to the hypaxial muscles and adjacent skin, and a communicating ramus which connects with the sympathetic system.

6. The ventral rami of the spinal nerves are intricately united by cross-connections in the region of the appendages to form plexi from which the motor nerves to the muscles of the appendages arise. The chief plexi are the brachial plexus to the anterior appendages and the lumbosacral plexus to the posterior appendages

7. The sympathetic system consists chiefly of a paired ganglionated cord in the middorsal region of the body cavity. These are connected with the spinal and cranial nerves by communicating branches and with the viscera, glands, blood vessels, etc., by means of branches, networks or plexi, and ganglia.

8. The vertebrate brain consists at first of three vesicles, the forebrain or prosencephalon, the midbrain or mesencephalon, and the hindbrain or rhombencephalon. The first and third soon divide into two vesicles, making five in all.

9. The five vesicles of the adult vertebrate brain are arranged in a longitudinal series and are named, beginning anteriorly: the telencephalon, the diencephalon, the mesencephalon, the metencephalon, and the myelencephalon.

ro. The telencephalon differentiates into the olfactory bulbs, the olfactory tracts, olfactory lobes, and cerebral hemispheres. The hemispheres are lateral expansions of the telencephalon. The olfactory part of the telencephalon is of paramount importance in the lower vertebrates, but later becomes subordinated to the cerebral hemispheres. These latter increase in size in the vertebrate scale until in mammals they cover most of the remaining parts of the brain. In mammals the two hemispheres are connected by the corpus callosum.

II. The diencephalon differentiates into the hypothalamus, the thalamus, and the epithalamus. The hypothalamus includes the optic chiasma, optic nerves, and nervous parts of the eye (these are often regarded, however, as belonging to the telencephalon), the infundibulum, and the mammillary body. The infundibulum is an evagination from the floor of the diencephalon; its most ventral portion unites with an outgrowth from the roof of the mouth, the two together forming the pituitary body. The thalamus includes the 
greater central mass of the diencephalon and in mammals is subdivided into several parts. The epithalamus consists of the pineal body and other evaginations from the roof of the diencephalon, and adjacent parts.

12. The mesencephalon or midbrain is composed dorsally of the optic lobes, of which there are two in most vertebrates, four in mammals, named the corpora quadrugemina. Ventrally the midbrain consists of the tegmentum and the cerebral peduncles.

I3. The metencephalon includes the cerebellum dorsally and the pons ventrally. The pons is distinctly developed only in mammals.

14. The myelencephalon becomes the medulla oblongata.

I 5. The brain is hollow, its cavities being known as ventricles. The first two ventricles are in the cerebral hemispheres, the third ventricle in the diencephalon, the fourth in the medulla. In lower vertebrates ventricles are also present in the cerebellum and midbrain. The ventricles are connected with each other.

I6. The brain of fishes and Amphibia is provided with ten cranial nerves, that of amniotes with twelve. The last two in amniotes are probably not new formations.

17. The first or olfactory nerve extends from the olfactory epithelium in the nose to the olfactory bulbs.

18. The second or optic nerve extends from the retina to the diencephalon. It is not a true nerve but a tract of the brain.

I9. The third or oculomotor nerve is a motor nerve to the inferior oblique, superior, inferior, and internal recti and some accessory muscles of the eyeball. It originates in the midbrain.

20. The fourth or trochlear nerve is a motor nerve to the superior oblique muscle of the eyeball. It arises from the midbrain.

2I. The fifth or trigeminus nerve is the chief somatic sensory nerve of the head and the nerve of the first visceral or mandibular arch. It has in all vertebrates three branches: the ophthalmic (deep ophthalmic of fishes) branch to the orbit and nasal region; the maxillary to the upper jaws and roof of the mouth and pharyngeal cavities; the mandibular to the lower jaw and floor of these cavities. The first two are pure somatic sensory nerves; the mandibular also contains visceral motor fibers to the visceral muscles belonging to the lower jaw. In fishes there is an additional branch of the trigeminus, the superficial ophthalmic, which disappears later. The trigeminus arises from the anterior end of the medulla.

22. The sixth or abducens nerve is the motor nerve of the external rectus muscle of the eyeball. It takes origin in the floor of the medulla.

23. The seventh or facial nerve is the nerve of the second or hyoid visceral arch. It is large in fishes because it includes branches for the lateral line system. These disappear in land vertebrates, leaving only that part of the facial which is named the hyomandibular nerve in fishes. This nerve is a sensory nerve (taste) to the pharyngeal cavity and a visceral motor nerve to the muscles of the hyoid arch. The facial springs from the medulla.

24. The eighth or auditory nerve is a sensory nerve extending from the internal ear to the acustic area of the medulla.

25. The ninth or glossopharyngeal nerve is the nerve of the third visceral arch. In the land vertebrates it is reduced but continues to supply sensory fibers to the floor of the pharyngeal cavity and visceral motor fibers to pharyngeal muscles. It is attached to the medulla.

26. The tenth or vagus nerve is the nerve of the remaining visceral arches. In fishes it also includes a large branch for the lateral line. With the loss of the lateral line system and the branchial mode of breathing the vagus is much reduced but continues to supply the corresponding region of the pharynx, being a sensory nerve to the lining of this region and a 
visceral motor nerve to the corresponding muscles. In addition, the vagus nerve is distributed extensively to the heart, lungs, stomach, and other viscera. The vagus arises from the medulla.

27. The eleventh or spinal accessory nerve of amniotes is probably a part of the vagus isolated as a separate nerve. It springs from the medulla and upper spinal cord and is a motor nerve to certain visceral muscles.

28. The twelfth or hypoglossal nerve of amniotes is probably a spinal nerve which has become included in the cranial cavity. It arises in the medulla and innervates the muscles of the tongue.

29. The chief sense organs of the head are the eyes, ears, and nose.

30. The nose at first consists of a pair of olfactory sacs not communicating with the mouth cavity. In land vertebrates they establish connections with the mouth cavity for respiratory purposes. The nasal cavities thereafter have both respiratory and olfactory functions, the latter being limited to the posterior regions of the cavities.

3r. In higher vertebrates, particularly mammals, the walls of the nasal cavities develop complex outgrowths, the turbinals or conchae, for the purpose of increasing both respiratory and olfactory surfaces.

32 The eyes are compound structures. The nervous part of the eye is formed by an evagination from the brain. The lens of the eye is an invagination from the adjacent ectoderm. The coats of the eye, sclera, and chorioid, are formed in the surrounding mesenchyme. The eye is moved by muscles which are very constant in arrangement in the different vertebrate classes, except that in mammals the superior oblique operates by means of a pulley.

33. The ear consists of internal, middle, and external portions. The internal ear is an invagination from the ectoderm. It differentiates into the three semicircular ducts, the sacculus, the utriculus, and the endolymphatic duct. Fishes and many urodeles possess only the internal ear.

34. The middle ear is added to the internal ear beginning with Amphibia. It consists of a chamber developed by outgrowth from the first visceral pouch. The outer wall of this chamber comes in contact with the skin, producing a double-walled membrane, the tympanic membrane. Within the middle ear is a chain of ear bones, two in number in most vertebrates, three in mammals.

35. Beginning with reptiles the tympanic membrane sinks into the skull, leaving a passage, the external auditory meatus, extending from the tympanic membrane to the exterior. This passage is deepened in birds and mammals, and in the latter a fold of skin, the pinna, develops around the external rim of the meatus. Pinna and meatus constitute the external ear.

36. The internal ear of mammals is more complicated than that of other vertebrates, owing to the development of a spiral outgrowth, the cochlear duct, from the sacculus. 


\section{APPENDIX A}

\section{PRONUNCIATION AND DERIVATION OF TECHNICAL WORDS}

Some compound words and names combined of two or more words are given under their components. Some common prefixes are also given. (L, Latin; G, Greek; F, French.)

A (G prefix, without)

$\mathrm{Ab}$ ( $\mathrm{L}$ prefix, away from)

Abdomen, abdominal-ab dough' men, ab dom' i nal ( $\mathrm{L}$, of uncertain origin)

Abducens-ab due' senz (L, $a b$, from; duco, lead)

Acanthias - a kan' the as ( $\mathrm{G}$, acantha, thorn)

Acentrous - a sen' trous (G, a, without; kentron, center)

Acetabulum-ass i tab' yu lum (L, name of a kind of cup)

Acipenser-ass i pen' ser (L, a sturgeon)

Acromion-a krow' me on (G, akros, top; omos, shoulder)

Actinopterygii-ak' ti nop' ter yg'ee eye (G, $a k$ tinos, ray; pterygion, wing, fin)

Acustico-a kous' ti koh' (G, akoustikos, related to hearing)

Ad-(L prefix, toward, upon)

Adductor-a duck' tore (L, ad, to; duco, lead)

Adrenal-add ree' nal (L, ad, upon; renes, kidneys)

Afferent-aff'err ent ( $\mathrm{L}, a d$, to; fero, bear)

Ali-al' ee (L prefix, ala, wing)

Allantois-a lan' toe iss (G, allas, sausage; eidos, (orm)

Alveolar, alveolus, alveoli-al vee' oh lar, -lus, -lie (L, a little cavity)

A mia - aim' ee ah ( $G$, a kind of fish)

Amnion, amniota, amniote- $\mathrm{am}^{\prime}$ nee on, $\mathrm{am}^{\prime}$ nee $\mathrm{oh}^{\prime}$ tah, am' nee oat ( $\mathrm{G}, \mathrm{a}$ membrane of the embryo)

Amphibia - am fib' ee ah (G, amphi, double; bios, life)

Amphicoelous - am' fee see' lous (G, amphi, double; koilos, hollow)

Amphioxus - $\mathrm{am}^{\prime}$ fee ox' us (G, amphi, both; oxys, sharp)

Amphiplatyan-am' fee pla' tee an (G, amphi, both; platys, flat)

Ampulla, ampullae-am pull' ah, am pull' ee (L, flask)

Anamnia-an am' nee ah (G, an, without; amnion, embryonic membrane)

Anastomosis - a nass' toh mow' sis ( $G$, an opening)

Anconeus-an' ko nee' us ( $\mathrm{L}$, ancon, the bend of the arm)

Ankylosis-an' kee lo' sis (G, ankylos, bent)
Ansa-ann' sah (L, a handle)

Anura-a new' rah (G, an, without; oura, tail)

Anus-ay' nus ( $L$, ring)

Aorta, aortic-ay or' tah, -tik (G, aorte, to lift)

Aponeurosis-ap oh' new row' sis (G, apo, from; neuron, tendon)

Apophysis - a poff' y sis ( $G$, an outgrowth)

Aqueduct- $\mathrm{k}^{\prime}$ we duct (L, aqua, water; duco, lead)

Aqueous - $a y^{\prime}$ kwee us (L, aqua, water)

Arachnoid-a rack' noid (G, arachne, spider)

Archenteron-ar ken' ter on (G, archos, chief, first; entcron, intestine)

Arcualia-ar' kiu ale' ee ah (L, arcus, bow)

Arteriosus-ar teer' ee oh' sus (L, arteria, artery)

Arytenoid-ar' ee tee' noid ( $\mathrm{G}$, arytaina, pitcher, funnel)

Astragalus - ass trag' a lus ( $\mathrm{G}$, an ankle bone)

Atlas - at' lass (G, tlao, to bear)

Atrium-ay' tree um (L, a court)

Auditory-aw' di toe ry (L, auditorius, pertaining to hearing)

Auricle, auricular-aw' ree kal, aw rik' yu lar (L, auricula, a little ear)

Aves-ay' veez (L, birds)

Axilla, axillary-axe ill' a h, axe' i lay ree (L, axilla, a little axis)

Azygos-az' ee gos (G, a, without; zygon, yoke)

Balanoglossus-bahl' an oh gloss' us ( $\mathrm{G}$, balanos, acorn; glossa, tongue)

Basalia-bah sail' ee ah (L, basis, the base)

Basi-base' ee (L, prefix, at the base of)

Basilar-bass' ee lar (L, basis, the base)

Biceps-buy' seps (L, bi, two; caput, head)

Blastocoel-blas' toe seal (G, blastos, germ; koillos, hollow)

Blastoderm-blas' toe derm (G, blastos, germ; derma, skin)

Blastula-blas' tiu lah (L, a little germ)

Brachial, brachialis, brachium-bray' kee al, -kee $\mathrm{ay}^{\prime}$ lis, -kee um (L, brachium, arm)

Brachiocephalic-bray' kee oh se phal' ik (L, brachium, arm; cephalicus, pertaining to the head)

Branchia, branchiae, branchial-bran' kee ah, -kee ee, -kee al (G, branchia, gills) 
Branchiostegal-bran' kee oss' te gal (G, branchia, gills; stego, to cover)

Bronchus, bronchi, bronchial-bron' kus, bron' kai, bron' kee al (G, bronchos, windpipe)

Buccal-buck' al (L, bucca, cheek or mouth)

Buccinator-buck' si nay' tor (L, pertaining to blowing a trumpet)

Bulla-bull' ah (L, a round seal or locket)

Bursa-burr' sah (L, a purse)

Caecum, caeca-see' kum, see' kah (L, caecus, blind)

Calcaneus, kal kay' nee us (L, calx, heel)

Canine-kay nine' (L, canis, dog)

Capillary-kap' ee lay' ry (L, capillus, hair)

Capitular, capitulum-ka pit' yu lar, -yu lum, (L, a small head)

Carapace-kair' a pace (F, probably from L, capa, hood)

Cardia, cardiac-kar' dee ah, -dee ak (G, kardia, heart)

Carina-ka rye' nah (L, keel)

Carnivora, carnivore-kar niv' oh rah, kar' ni vore (L, caro, flesh; voro, devour)

Carotid-ka rot' id (G, karos, stupor)

Carpales, carpus-kar pay' les, kar' pus (G, karpos, wrist)

Caudal-kaw' dal (L, cauda, tail)

Cava, caval-kave' ah, -al (L, cavus, hollow)

Cavernosa, cavernous-kav' er no' sah, -nous (L, caverna, hollow)

Centrale, centralia-sen tray' lee, -tray' lee ah (L, centralis, central)

Centrum-sen' trum (L, center)

Cephalic-se phal' ik (G, kephale, head)

Cephalization-seph' al i $\mathrm{za}^{\prime}$ tion (G, kephale, head)

Cephalochordata- $\mathrm{seph}^{\prime}$ a low chor day' tah (G, kephale, head; chorde, string)

Cerato-ser' a toe (G, prefix, keras, horn)

Cere-sear (L, cera, wax)

Cerebellum-ser' e bell' um (L, a little prain)

Cerebral, cerebrum-ser' e bral, ser' e brum (L, brain)

Cervical-ser' vi kal (L, cervix, neck)

Chelonia-kee low' nee ah ( $\mathrm{G}$, chelone, turtle)

Chiasma-kai as' ma ( $\mathrm{G}$, cross-mark)

Choana, choanae-koh' a nah, -a nee (G, funnel)

Chondrocranium-kon' dro cray' nee um (G, chondros, cartilage; kranion, skull)

Chondrostei-kon dross' tee eye (G, chondros, cartilage; osteon, bone)

Chordata, chordate-kor day' tah, kor' date (L, chorda, cord or string)

Chordocentrous-kor' dough sen' trous (L, chorda, cord; centrum, center)

Chorioid (or choroid)-koh' ree oid, koh' roid (G, chorion, membrane)
Chorion-koh' ree on ( $\mathrm{G}$, membrane)

Ciliary-sill' ee a ry (L, cilium, eyelid)

Cinereum-si nee' ree um (L, ashy)

Ciona-sigh' oh na ( $\mathrm{G}$, kion, pillar)

Circulatory-sir' kiu la tow' ree ( $\mathrm{L}$, circulo, to form a circle)

Cirrus, cirri-sir' rus, -ree (L, cirrus, tuft, lock of hair)

Clava-clay' va (L, branch, club)

Clavicle-klav' i kel (L, claricula, little key)

Clavo-clay' voh (L combining form, clavis, key, referring to the clavicle)

Cleido-kly' dough ( $\mathrm{G}$ combining form, kleis, key, referring to the clavicle)

Cleithrum-klyth' rum ( $\mathrm{G}$, kleithron, bar, gate)

Clitoris-kly' to riss ( $\mathrm{G}$, kleio, to close)

Cloaca-klo ay' kah (L, sewer)

Coccyx, coccygeal-kock' six, -sij' ee al (G word)

Cochlea-kock' lee ah (L, snail)

Coeliac-see' lee ak (G, koilia, stomach)

Coelom-see' loam (G, koilos, hollow)

Colon-koh' lon (G, kolon, member)

Columella-kol' yu mell' ah (L, little column)

Concha-kon' ka (L, shell)

Condyle-kon' dill ( $\mathrm{L}$, condylus, knuckle)

Conjunctiva-kon' junk tie' va ( $\mathrm{L}$, conjunctus, join together)

Copula-kop' yu lah (L, cum, together; apo, bind)

Coracoid-kor' a koid ( $\mathrm{G}$, korakoeides, like a crow's beak)

Corium-koh' ree um (L, leather)

Cornea, corneum-kor' nee ah, -um (L, corneus, horny)

Cornu, cornua-kor' niu, kor' niu ah (L, horn)

Corona, coronary - koh row' nah, kor' oh nay ry (L, crown or wreath)

Coronoid-kor' oh noid (G, korone, crow)

Corpora, corpus - kor' po rah, kor' pus (L, body)

Costal-kos' tal (L, costa, rib)

Cranial, craniate, cranium-kray' nee al, -nee ate, -nee um (G, kranion, skull)

Cribriform-krib' ree form (L, cribrum, sieve)

Cricoid-kry' coid (G, kriksos, ring)

Crista-kris' tah (L, crest)

Crocodilia-krok' oh dill' ee ah (L, crocodile)

Crossopterygii-cross sop' ter yg' ee eye (G, krossoi, fringe; pteron, wing)

Crura, crural, crus-kru' rah, kru' ral, kruss (L, crus, leg)

Ctenoid-ten' oid (G, ktein, comb)

Cuneiform-kiu' nee i form (L, cuneus, wedge)

Cutaneous-kiu tay' nee us (L, cutis, skin)

Cuvier-kiu vyay (name of French anatomist)

Cycloid-sigh' kloid (G, kyklos, circle)

Cyclostomata, cyclostome-sigh' klo stow' ma tah, -stome (G, kyklos, circle; stoma, mouth)

Cystic - sis' tik (G, kystis, bladder) 
Deferens-deff' er enz ( $\mathrm{L}$, defero, carry away)

Deltoid-dell' toid ( $G$, delta, fourth letter of the Greek alphabet, triangular in form)

Dentary-den' ta ree (L, dens, tooth)

Dentine-den' tin (or teen) (L, dens, tooth)

Dermal, dermis-derr' mal, derr' miss (G, derma, skin)

Dermatome-derr' ma tome (G, derma, skin; tomos, cutting)

Diaphragm-dye' a framm (G, dia, between; phragnymi, to inclose)

Diastema-dye' a stee' mah (G, interval)

Digastric-dye gas' trik (G, di, two; gaster, belly)

Digitigrade- -dij' i ti grade (L, digitus, finger, toe; gradus, step, walk)

Diphycercal-diff' ee sir' kel (G, diphyes, twofold; kerkos, tail)

Diplospondyly-dip' low spon' dy lee (G, diploos, double; spondylos, vertebra)

Duodenum-diu' oh dee' num (L, duodeni, twelve) Dura-diu' rah (L, hard)

Ect, ecto-ekt, ek' toh ( $\mathrm{G}$ prefix, the outer, outside)

Edentata-ee' den $\operatorname{tay}^{\prime}$ tah (L, $e$, without; dens, tooth)

Efferent-eff' er ent (L, effero, to bear away from)

Efferentia-eff' er en' shi a (L, effero, to bear away from)

Elasmobranch, elasmobranchii-ee las' mow brank, -mow bran' kee eye (G, elasmos, plate; branchia, gills)

Encephalon-en sef' a lon (G, enkephalos, brain)

End, endo-end, en' dough (G prefix, within, inside)

Endostyle-en' dough style (G, endo, within; stylos, column)

Entoderm-en' toe derm (G, endo, within; derma, skin)

Ep, epi-epp, epp' ee (G, prefix, upon)

Epaxial-epp axe' i al (G, epi, upon; L, axis)

Epidermis-epp' ee derr' miss (G, epi, upon; derma, skin)

Epididymis-epp' ee did' $^{\prime}$ y miss (G, epi, upon; didymos, testis)

Epimere-epp' ee mere (G, epi, upon; meros, part)

Epiphysis, epiphyseal-e piff' ee sis, epp' ee fis' e al (G, an outgrowth)

Epiploic, epiploicum-epp' ee plo' ik, -plo' ee kum (G, epiploon, omentum)

Epistropheus-epp' e stro' fee us (G, epi, upon; strepho, turn)

Esophagus, esophageal-ee soph' a gus, ee' so faj' ee al ( $\mathrm{G}$, oisophagos, gullet)

Ethmoid-eth' moid (G, ethmoe, sieve)

Eustachian-you stay' kee an (after Eustachius, an anatomist)

Ex-(L prefix, out, outside)

Excretory-eks' kree toh ry (L, ex, out; cerno, separate)
Facet-fas' et (L, facies, face)

Facial-fay' shal (L, facies, face)

Falciform-fal' see form (L, fal $x$, sickle)

Fallopian-fa loh' pee an (after Fallopio, an anatomist)

Fascia - fash' ee ah (L, a band or bandage)

Fasciculus, fasciculi-fa sik' yu lus, -yu lye (L, a little bundle)

Fauces- $\mathrm{fa}^{\prime}$ sees (L, throat)

Femoral, femoris-fem' oh ral, -oh riss (L, femur, thigh)

Femur -fee' mur (L, thigh)

Fenestra-fee nes' trah (L, window)

Fibula, fibulare-fib' yu lah, -yu lay' ree (L, fibula, clasp, buckle)

Filoplume-fill' (or file) oh plume (L, filum, thread; pluma, feather)

Fimbria-fim' bree ah (L, border)

Flocculus-flock' yu lus (L, a little piece of wool)

Follicle-foll' i kel (L, folliculus, a little bag)

Fontanelle-fon' ta nell' (F, a little fountain)

Foramen, foramina - fo ray' men, -ram' ee nah (L, an opening)

Fornix - for' niks (L, an arch or vault)

Fossa-foss' ah (L, a pit or cavity)

Frenulum-fren' yu lum (L, a little bridle or bit)

Frontal-frun' tal (L, frons, brow)

Fundus-fun' dus (L, the bottom)

Fungiform-fun' ji form (L, fungus, a mushroom)

Funiculus-fiu nik' yu lus (L, a small rope or cord)

Furcula-fur' kiu lah (L, a little fork)

Ganglion-gan' glee on ( $G$, a tumor)

Ganoid-gan' oid ( $\mathrm{G}$, ganos, bright)

Gasserian-ga see' ri an (from a physician Gasser)

Gastric, gastro-gas' trik, gas' troh (L, gaster, stomach)

Gastrocentrous-gas' troh sen' trous (L, gaster, stomach; centrum, center)

Gastrocnemius-gas' trok nee' me us ( $G$, gaster, stomach; kneme, shank)

Gastrocoel-gas' troh seal (G, gaster, stomach; koilos, hollow)

Gastrula-gas' trew lah (L, a little stomach)

Geniculate-ji nik' yu late (L, genu, knee)

Geniohyoid-ji nye' oh high' oid (G, geneion, chin; upsilon, a $\mathrm{Y}$-shaped letter of the Greek alphabet)

Genital-jen'i tal (L, gigno, to reproduce)

Genu-jee' new (L, knee)

Germinativum-jerr' mi nay tiv um (L, germino, to sprout)

Glans-glanz (L, an acorn)

Glenoid-glee' noid ( $\mathrm{G}$, glene, a socket)

Glomerulus-ylow mer' yu lus (L, glomus, a ball of yarn)

Glossopharyngeal-gloss' oh fa rin' jee al (or fare' in jee' al) (G, glossa, tongue; pharynx, pharynx) 
Glottis-glott' iss (G, glotta, tongue)

Gluteus-glu tee' us (G, gloutos, rump)

Gnathostomata, gnathostome-nath' oh stow' ma tha, nath' oh stome (G, gnathos, jaw; stoma, mouth)

Gonad-gonn' ad (G, gonos, seed)

Graafian-grahf' ee an (after de Graaf, a Dutch physician)

Gracilis-grass'i lis (L, slender)

Gubernaculum-giu' ber nak' yu lum (L, a rudder)

Gyrus, gyri-jye' rus, -rye (G, gyros, round)

Habenula - ha ben' yu la (L, a little band)

Haemal-hem' al (G, haima, blood)

Hamulus-ham' yu lus ( $\mathrm{L}$, a little hook)

Hemichordata-hem' i core day' tah (L, hemi, half; chorda, cord)

Hepatic, hepato-he pat' ik, hep' a toe (G, hepar, liver)

Hermaphroditic-her maff' row dit ik (G, Hermes, Mercury; A phrodite, Venus)

Heterocercal-het' er oh ser' kal (G, heteros, different; kerkos, tail)

Heterocoelous-het' er oh see' lous ( $G$, heteros, different; koilos, hollow)

Heterodont-het' er oh dahnt ( $\mathrm{G}$, heteros, different; odon, tooth)

Heteronomous-het' er on' oh mous (G, heteros, different; nomos, law)

Hilum-high' lum (L, eye of the bean)

Hippocampus-hip' pol kam' pus (G, hippos, horse; kampos, sea-monster)

Holoblastic-holl' oh blas' tik (G, holos, whole; blastos, germ)

Holostei-ho loss' tee eye (G, holos, whole; osteon, bone)

Homocercal-home' oh $\operatorname{ser}^{\prime} \mathrm{kal}$ (G, homos, the same; kerkos, tail)

Homonomous - hoh mon' oh mous ( $\mathrm{G}$, homos, the same; nomos, law)

Humerus-hiu' mer us ( $\mathrm{L}$, the bone of the upper arm)

Hy, hyo-high, high' oh (prefix referring to the hyoid)

Hyoid, hyal-high' oid, high' all (G, upsilon, a Y shaped letter of the Greek alphabet)

Hyp, hypo-hipp, high' poh (G or L prefix, below, less than)

Hypaxial-hip axe' ee al (G, hypo, below; uxis, axis)

Hypogossal-high' poh gloss' al (G, hypo, below; glossa, tongue)

Hypomere-high' poh mere (G, hypo, below; meros, part)

Hypophysis-he poff' ee sis (G, hypo, below; phyo, to cause to grow)

Hypural-hip your' al (G, hypo, below, oura, tail)
Ichthyopsida-ik' thy op' si dah (G, ichthys, fish; opsis, appearance)

Ileum-ill' e um (G, eilo, twist)

Iliac-ill' i ak (L, ilium, flank)

Ilium-ill' ee um (L, flank)

Incisor, incisiva, incisive-inn sigh' sor, -sigh'si vah, -sigh' sive (L, incido, to cut into)

Incus-inn' kuss (L, anvil)

Infra-inn' frah ( $\mathrm{L}$ prefix, below)

Infundibulum - inn' fun dib' yu lum ( $\mathrm{L}$, funnel)

Inguinal-inn' gwi nal (L, inguen, groin)

Innominate-inn nomm' i nate (L, innominatus, without a name)

Integument-inn teg' u ment ( $\mathrm{L}$, intego, to cover)

Inter-inn' ter ( $\mathrm{L}$ prefix, between)

Intercalary-inn terr' $\mathrm{ka}$ lay ree $(\mathrm{L}$, intercalo, to put between)

Intestine, intestinal-inn tess' tin, inn tess' ti nal, (L, intestinus, inside)

Intra-inn' trah (L prefix, within)

Invagination-inn $\mathrm{vaj}^{\prime} \mathrm{i}$ nay' shun ( $\mathrm{L}$, in, in; vagina, sheath)

Iris-eye' riss (G, rainbow)

Ischial, ischium-iss' kee al, iss' kee um (G, ischion . hip)

Isolecithal-eye' so less' i thal (G, isos, equal; lekithos, yolk)

Iter-eye' ter (L, passage)

Jejunum-jee jew' num (L, hungry)

Jugal一jew' gal (L, jugum, yoke)

Jugular-jew' giu lar (L, jugulum, the collar bone)

Labia, labial-lay' bee ah, -bee al ( $\mathbf{L}$, labium, lip)

Lacertilia-lass' err till' ee ah (L, lacerta, lizard)

Lacrimal (also spelled lacrymal)-lack' ree mal (L lacrima, tear)

Lagena-la jee' nah (G, lagynos, flask)

Lamella, la mellae-la mell' ah,-mell' ee (L, lamina, a thin sheet)

Lamina-lamm' ee nah ( $\mathrm{L}$, a thin plate)

Laryngeal, larynx-la rin' jee al (or lar' in jee al) lar' inks (G, larynx, gullet)

Latissimus-la tiss' i muss (L, the broadest)

Lepidosteus-lepp' i doss' tee us ( $\mathrm{G}$, lepis, scale; osteon, bone)

Levator-le vay' tor ( $\mathrm{L}$, a lifter)

Lienal, lieno-lie ay' nal, lie ay' no ( $\mathrm{L}$, lien, spleen)

Linea-linn' ee ah (L, line)

Lingual-linn' gwal (L, lingua, tongue)

Lissamphibia-liss' am fib' ee ah ( $\mathrm{G}$, lissos, smooth; amphibios, double life)

Lobule-lobb' yule (G, lobos, lobe)

Longissimus-lon jiss' ee mus ( $L$, the longest)

Lorenzini-loh ren zee' nee (Italian anatomist)

Lumbar-lumm' bar (L, lumbus, loin)

Lutea, lutein-liu' tee ah, -tee in ( $\mathrm{L}$, luteus, yellow) 
Lymphatic-limm fat' ik (L, lympha, clear water) Lymphocytes-limph' oh sites (L, lympha, clear water; G, kytos, hollow place)

Major-may' jor (L, the greater)

Malar-may' lar (L, mala, cheek)

Malleolus, malleoli-ma lee' oh lus, -oh lye (L, little hammer)

Malleus-mahl' ee us ( $L$, hammer)

Malpighian, malpighii-mahl pig' ee an, -ee eye (after Malpighi, an Italian biologist)

Mamillary (or mammillary)-mam' ill lay ree (L, mamma, breast)

Mammal-mam' mal (L, mamma, breast)

Mammalia-ma may' lee ah (L, mamma, breast)

Mammary-mam' a ree (L, mamma, breast)

Mandible, mandibular-man' di bl, man dib' yu $\operatorname{lar}(\mathrm{L}$, mandibula, jaw)

Manubrium-ma niu' bree um ( $\mathrm{L}$, handle)

Manus-may' nuss ( $L$, hand)

Marsupial, marsupialia-marr siu' pee al, mar siu' pee $a y^{\prime}$ lee ah (L, marsupium, pouch)

Masseter-ma see' ter ( $\mathrm{G}$, maseter, a chew)

Mastoid-mass' toid (G, mastos, breast)

Mater-may' ter ( $L$, mother)

Maxilla, maxillary-maks ill' ah, maks' i lay ree (L, maxilla, jawbone)

Maximus-maks' i mus (L, the largest)

Meatus-mee ate' us (L, passage)

Mediastinal, mediastinum-mee' dee ass tie' nal, -num ( $\mathrm{L}$, mediastinus, being in the middle)

Medius-mee' di us ( $\mathrm{L}$, middle)

Medulla, medullary-me dull' lah, medd' u lay ree (L, medulla, marrow, pith)

Meninx, meninges-mee' ninks, mee nin' jees ( $G$, membrane)

Mental-men'tal (L, mentum, chin)

Meroblastic-merr' oh blas' tik (G, meros, part; blastos, germ)

Mes, meso-mess, mess' oh (G prefix, the middle)

Mesenchyme-mess' en kime ( $G$, mesos, middle; enchyma, in a fluid)

Mesenteric, mesentery-mess' en tare' ik, -y (G, mesos, middle; enteron, gut)

Mesoderm-mess' oh derm (G, mesos, middle; derma, skin)

Mesogaster-mess' oh gas' ter (G, mesos, middle; gaster, stomach)

Mesomere-mess' oh mere (G, mesos, middle; meros, part)

Mesopterygium-mess' op terr $\mathrm{yg}^{\prime}$ ee um (G, mesos, middle; pteron, wing)

Mesorchium-mess or' kee um (G, mesos, middle; orchis, testis)

Mesotubarium-mess' oh tiu bay' ri um (G, mesos, middle; L, tubus, tube)

Mesovarium-mess oh vay' ree um (G, mesos, middle; L, ovum, egg)
Met, meta-met, met' ah (L or G prefix, between. after, reversely)

Metacarpal-met' a kar' pal (G, meta, after; karpos, wrist)

Metacromion-met' a krow' mee on (G, meta, after; akromion, point of the shoulder)

Metamere, metamerism-met' a mere, met' $^{\prime}$ a mer izm (G, meta, after; meros, part)

Metapleural-met' a plu' ral (G, meta, after; pleura, side)

Metapterygium-met ap' ter $\mathrm{yg}^{\prime}$ ee um (G, meta, after; pteron, wing)

Metatarsal-met' a tar' sal (G, meta, after; tarsos, a flat surface)

Minimus-min'i mus ( $L$, the least)

Minor-my' nor ( $\mathrm{L}$, the lesser)

Mitral-my' tral (F, mitre, a peaked cap)

Molar-mow' lar (L, mola, millstone)

Monotremata, monotreme-mon' oh tremm' a tah, -treem (G, monos, one; trema, hole or opening)

Müllerian-me lerr' i an (after Müller, a German physiologist)

Multifidus-mull tiff' i dus (L, many cleft)

Myelon-my' e lon (G, marrow)

Mylohyoid-my' low high' oid (G, myle, mill; upsilon, the letter $y$ )

Myocomma, myocommata-my' o komm' ah, -komm' a tah ( $\mathrm{G}$, mys, muscle; komma, that which is cut off)

Myotome-my' oh tome (G, mys, muscle; tome, cutting)

Naris, Nares-nay' riss, nay' rees (L, nostril)

Necturus-nek too' rus (G, nektos, swimming; oura, tail)

Nephros-neff' ross (G, kidney)

Nephrostome-neff' row stome (G, nephros, kidney; stoma, mouth)

Nephrotome-neff' row tome (G, nephros, kidney; tome, cutting)

Neural-niu'ral (G, neuron, nerve)

Neurocoel-niu' row seal (G, neuron, nerve; koilos, hollow)

Nictitating - nik' ti tay' ting (L, nicto, wink)

Nodosal-no dose' al (L, nodosus, knotty)

Notocentrous-no' toe sen' trous (G, notos, back; kentron, center)

Notochord-no' toe kord (G, notos, back; chorde, string)

Nuchal-new' kal (L, nucha, nape of the neck)

Oblongata-ob lon gay' tah (L, oblongus, oblong)

Obturator-ob tiu ray' ter (L, obturo, to close, shut)

Occipital-ok sip' i tal (L, occiput, back of the head)

Oculomotor-ok' yu loh mow' tor ( $\mathrm{L}$, oculus, eye motor, mover) 
Odontoid-o don' toid ( $\mathrm{G}$, odous, tooth)

Oleeranon-oh' lee kray' non ( $\mathrm{G}$, olene, ulna; kranion, skull)

Olfactory - ol fak' toh ree (L, olfacere, to smell)

Omentum-oh men' tum (L, fat skin)

Omo, om-oh' mow, ohm (G prefix, omos, shoulder)

Opercular, operculum-o per' kiu lar, -lum (L, operculum, lid)

Ophidia-o fid' ee ah (G, ophis, serpent)

Ophthalmic - off thal' mik (G, ophthalmos, eye)

Opisto, opist-o pis' tho, o pist' (G prefix, opisthen, behind)

Oral-oh' ral (L, os, mouth)

Orbicularis-or bik' yu lay' riss (L, orbiculus, a little circle)

Orbital, orbito--or' bi tal, or' bi toe (L, orbita, orbit)

Ostium-oss' tee um (L, mouth or entrance)

Otic-oh' tik (G, otikos, pertaining to the ear)

Otolith-oh' toe lith (G, ous, ear; lithos, stone)

Ovarian, ovary-ova' ree an, oh' va ree (L, ovum, egg)

Oviduct-oh' vi duct (L, ovum, egg; ductus, duct)

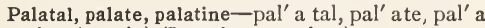
tiyn (or -tin) (L, palatum, palate)

Pallium-pahl' ee um (L, cloak)

Palpebra-pahl' pe brah (L, eyelid)

Pancreas, pancreatic-pan' kree ass, pan' kree at' ik (G, pas, all; kreas, flesh)

Papilla, papillae-pa pill' ah, -ee (L, nipple)

Para-par' ah (G prefix, beside, near)

Paraphysis-pa raff' ee sis (G, para, beside; phyo, produce)

Parietal-pa rye' ee tal (L, paries, wall)

Parotid-pa rot' id (G, para, beside; ous, ear)

Patella-pa tell' ah (L, a small pan or dish)

Pecten-pek' ten (L, comb)

Pectineal-pek'ti nee' al (L, pecten, comb)

Pectoral, pectoralis- pek' $^{\prime}$ toe ral, -ray' liss (L, pectoralis, referring to the chest)

Pellucidum-pe liu' see dum (L, translucent)

Pelvic, pelvis-pell' vik, -viss (L, pelvis, basin, the pelvis)

Penis-pee' nis (L, male external sex organ)

Peri-perr' ee ( $\mathrm{L}$ or $\mathrm{G}$ prefix, around)

Pericardial, pericardium-per' ee kar' dee al, -um (G, peri, around; kardia, heart)

Perineum-perr' i nee' um (L, perineon, origin uncertain)

Peripheral-pe riff' er al (G, peri, around; phero, bear)

Peritoneal, peritoneum-perr' i toe nee'al, -um (G, peri, around; teino, stretch)

Peroneal, peroneus-perr oh nee' al,-us (G, perone, pin of a brooch, referring to the fibula)
Petrosal, petrous-pe trow' sal, pee' trous ( $\mathrm{L}$ petrosus, rocky)

Phalanges, phalanx-fay lan' gees, fay' lanks (G. phalanx, battle-line)

Pharyngeal, pharynx - fa rin' jee al (or far' in jee' all) far' ynks (G, pharynx, throat)

Phrenic-fren'ik (G, phren, diaphragm)

Phylum-fye' lum (G, phylon, race, tribe)

Pia_pie' ah (L, pious, kind)

Pineal-pin' ee al (L, pinea, pine cone)

Pinna-pin' ah (L, feather)

Pisces-piss' ees (L, fish)

Pituitary-pi tiu' i tay ree (L, pituita, mucus)

Placenta, placentalia-pla sen' tah, plass' en tay' lee ah (L, placenta, cake)

Placoid-plak' oid (G, plax, plate)

Plantaris-plan tay'riss (L, planta, sole of the foot)

Plantigrade-plan' ti grade (L, planta, sole; gradus, walk)

Plastron-plass' tron (F, a breastplate)

Platysma-pla tiz' mah (G, flat plate)

Pleura, pleural-plew' rah,-ral (G, pleura, rib, side)

Plexus-pleks' us (L, in terweaving)

Plumulae-plew' miu lee ( $L$, little feather)

Pneumatic-niu mat' ik (G, pneuma, breath, spirit)

Polyodon-polee' oh don (G, polys, many; odous, teeth)

Polypterus-polip' ter us (G, polys, many; pteron, wing)

Pons-ponz (L, bridge)

Portal-pour' tal (L, porta, gate)

Post-(L prefix, behind, after)

Pre-pree (L prefix, before)

Prepuce-pree' pyuse (F, probably from L, pre, before; and $\mathrm{G}$, posthion, penis)

Primate-pry' mate (L, primus, first)

Pro-proh (L or G prefix, before)

Procoelous-prob'see lous (G, pro, before; koilos, hollow)

Proctodaeum-prok' toe dee' um (G, proktos, anus; daio, divide)

Prostate-pross' tayte (G. prostates, in the front rank)

Proventriculus-proh' ven trick' yu lus (L, pro, before; venter, belly)

Pseudo-sue' do (G prefix, pseudes, false)

Pterygium-te ryg' ee um (G, pterygion, a little wing or fin or projection, from pteron, wing)

Pterygo, pterygoid-terr' i go, -goid (G, pteron, wing)

Pterylae-terr' y lee (G, pteron, feather, wing; hyle, wood)

Pubic, pubis-piu' bik, -bis (L, pubes, hair, by inference, maturity)

Pulmonary-pull' mow nay' ree (L, pulmon, lung)

Pulvinar-pul vine' ar (L, pulvinus, cushion) 
Pygal-pye' gal (G, pyge, rump)

Pygostyle-pye' go style (G, pyge, rump; stylos, column)

Pylorus-pye loh' rus (G, pyloros, gatekeeper)

Quadrate-kwad' rate (L, quadratus, square)

Quadriceps-kwad' ree seps (L, quattuor, four; caput, head)

Rachis-ray' kis (G, spine)

Radial, radiale, radius-ray' dee al, ray' dee ay' lee, -dee us (L, radius, ray)

Ramus, rami-ray' mus, ray' mee (L, a branch)

Raphe-ray'fee (G, seam)

Rectrices-rek try'sees (L, rectus, straight)

Rectum, rectus-rek' tum,-tus (L, rectus, straight)

Remiges-rem'i jeez (L, remus, oar)

Renal-ree' nal (L, renes, kidneys)

Roptilia, reptile-rep till' ee ah, rep' till (L, reptilus, reptile, from repo, creep)

Respiratory-re spire' a toh' ree (or res' pee rah toh' ree) (L, respiro, breathe back)

Rete-ree' tee (L, net)

Retina-ret' i nah (L, rete, net)

Retro-ret' roh (L prefix, back, backward)

Rhinal-rye' nal (G, rhis, nose)

Rhomb, rhombo-romb, romm' boh (L or G prefix, referring to a geometric figure, a kind of parallelogram)

Rhomboideus-rom boy' dee us (L, rhombus, or G, rhombos, rhomboid in form)

Rhyncocephalia-rin' koh see fay' lee ah (G, rhynchos, snout; kephale, head)

Rodent, rodentia-row' dent, row den' she ah (L, rodens, gnawing)

Rostral, rostrum-ross' tral, -trum (L, rostrum, beak)

Sacculus-sak' yu lus (L, little sac)

Sacral, sacrum-say' cral, -krum (L, sacer, sacred)

Sagittal-saj' i tal (L, sagitta, arrow)

Salivary-sall' i vay' ree (L, saliva, spit)

Sartorius-sar toe' ree us (L, sartor, tailor)

Sauropsida-sah ropp' si dah (G, sauros, lizard; opsis, appearance)

Scalene, scalenes-skay' lean, skay' leans (G, skalenos, uneven)

Scapula-skap' yu lah (L, shoulder blade)

Sciatic-sigh at' ik (L, sciaticus, originally ischiadicus, from $\mathrm{G}$, ischion, hip)

Sclera-sklay' rah (G, skleros, hard)

Sclerotic-skle rot' ik (G, skleros, hard)

Sclerotome-skle' roh tome (G, skleros, hard; tome, cutting)

Scrotal, scrotum-skroh' tal, -tum (L, uncertain origin)

Scute-skiut (L, scutum, shield)

Sella-sell' ah (L, a seat or saddle)
Semilunar-semm' i liu' nar (L, semi, half; luna, moon)

Seminal-semm'i nal (L, semen, seed)

Seminiferous-semm' i niff' er ous (L, semen, seed; fero, to bear)

Septum-sepp' tum (L, fence, wall)

Serosa-see row'sah (L, serum, serum)

Serratus - se rate' us (L, serra, saw)

Sesamoid-sess' a moid (G, sesamon, a plant. referring to the shape of the seeds)

Sinus-sigh' nus (L, sinus, curve)

Skeletogenous-skell' e toj' ee nous (G, skeleton, from skello, make dry)

Solar-soh' lar (L, sol, the sun)

Soleus-soh' lee us (L, solea, sole of the foot)

Somatic-soh mat' ik (G, soma, body)

Somatopleure-soh' ma toe plure (G, soma, body; pleura, side)

Somite-soh' might (G, soma, body)

Spermatic-sperr mat'ik (L, sperma, sperm)

Sphenodon-sfee' noh don (G, sphen, wedge; odon, tooth)

Sphenoid-sfee' noid (G, sphen, wedge)

Spiracle-spear' ah kel (or spire' ah kel) (L, spiraculum, air hole)

Splanchnic-splank' nik (G, splanchnon, one of the viscera)

Splanchnopleure-splank' noh plure (G, splanchnon, a viscus; pleura, side)

Splenial, splenic-splee' nee al, splenn' ik (G, splen, spleen)

Splenius-splee' nee us (G, splenion, bandage)

Squamata-skwa may' tah (L, squama, scale)

Squamosal-sk wa moh'sal (L, squama, scale)

Stapes-stay' peez (L, stirrup)

Stegocephala-steg' oh seff' ah lah (G, stego, cover; kephale, head)

Sternebra, sternebrae-stir' ne brah, -bree (G, sternon, breastbone; L, vertebra, joint)

Sterno-stir' noh (G combining word, sternon, breastbone)

Sternum-stir' numm (G, sternon, breastbone)

Stomodaeum-stow' moh dee um (G, stoma, mouth; daio, divide)

Stratum-stray' tum (L, a spread or cover)

Striatum-stry ay' tum (L, stria, furrow)

Stylo-sty' loh (G combining word, stylos, column)

Sub-subb (L prefix, under)

Subclavian-subb clay' vee an (L, sub, under; clavis, key, referring to the clavicle)

Sulcus, sulci-sull' kuss, sull' sigh (L, a furrow or groove)

Supine-sue' pine (L, supino, to put on the back)

Supra-siu' prah (L prefix, above)

Suture-siu' chur (L, suo, to sew)

Symphysis-simm' fee sis (G, union) 
Syn- $\sin$ (L or G prefix, together, with)

Synotic-sy not' ik (G, syn, together; ous, ear)

Syrinx $-\operatorname{sir}^{\prime}$ inks (G, pipe)

Tarsal, tarsalia, tarsus- $\operatorname{tar}^{\prime}$ sal, tar say' lee ah, tar'sus (G, tarsos, a flat surface)

Tectum-tek' tum ( $L$, tego, to cover)

Tegmentum-teg men' tum (L, tego, to cover)

Tel, tele-tell, tell' ee (G, tele, far)

Teleost, teleostei-tell' ee ost, tell' ee os' tee eye ( $\mathrm{G}$, teleos, whole, perfect; osteon, bone)

Teleostome, teleostomi-tell' ee oh stome, tell' ee oss' toe my (G, teleos, perfect; stoma, mouth)

Telolecithal-tell' oh less' i thal ( $\mathrm{G}$, telos, end; lekithos, yolk)

Temporal-tem' poh ral ( $\mathrm{L}$, tempus, temple)

Tendon-ten' don ( $\mathrm{L}$, tendo, to stretch)

Tentorium-ten toh' ree um ( $\mathrm{L}$, tendo, to stretch)

Teres-tee' reez ( $L$, round)

Testes, testis- tess' teez, tess' tiss (L, testis)

Thalamus - thal' a mus (L, chamber)

Thecodont-thee' koh dont (G, theke, sheath; odon, tooth)

Thoracic, thoraco-thoh ras' ik, thoh' ra koh (L, thorax, thorax)

Thorax-thoh' raks (L, thorax)

Thymus-thy' mus (G, thymon, thyme)

Thyroid (or thyreoid) - thy' roid, thy' ree oid (G, thyreos, shield)

Tibia, tibiale, tibialis-tibb' ee ah, tibb' ee ay'lee, $-a y^{\prime}$ lis (L, tibia, the shin bone)

Tonsil-ton' sill (L, tonsilla, tonsil)

Trabecula, trabeculae- tra bek $^{\prime}$ yu lah, -lee (L, little beam)

Trachea-tray' kee ah (G, trachys, rough)

Trapezius-tra pee' zee us (G, trapeza, table)

Trapezoid-tra' pe zoid (G, trapeza, table)

Trematic-tree mat' ik ( $\mathrm{G}$, trema, hole, opening)

Triceps-try'seps (L, tres, three; caput, head)

Tricuspid-try kuss' pid (L, tres, three; cuspis, point)

Trigeminus - try jem' $\mathrm{i}$ nus ( $\mathrm{L}$, three at a birth, triple)

Trochanter-troh kan' ter (G, trochos, wheel)

Trochlea, trochlear-trock' lee ah, -lee ar (L, trochlea, pulley)

Tuber-tiu' ber ( $\mathrm{L}$, a swelling)

Tubercular, tuberculum-tiu burr' kiu lar, -lum (L, tuberculum, a little swelling)

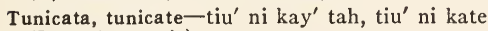
( $\mathrm{L}$, tunica, tunic)

Turbinal-turr' bi nal ( $\mathrm{L}$, turbo, anything that whirls)

Turcica-turr' see kah (L, turkish)

Tympanic, tympanum-tim pan' $i k$, tim $^{\prime}$ pa num (L, tympanum, drum)

Ulna, ulnar, ulnare-ull' nah, ull' nar, ull nay' re (L. ulna, elbow)
Umbilical, umbilicus—um bill' i kel, um bi lye' kus ( $\mathrm{L}$, umbilicus, navel)

Uncinate-unn' see nate ( $\mathrm{L}$, uncus, hook)

Unguligrade- unn giu' li grade (L, ungula, hoof; gradus, walk)

Ureter-yu ree' ter (G, ouron, urine)

Urethra-yu ree' thrah ( $\mathrm{G}$, ouron, urine)

Urinary-yu ri nay' ree ( $\mathrm{L}$, urina, urine)

Urodela, urodele-yu' row dee' lah, yu' row deal ( $\mathrm{G}$, oura, tail; delos, evident)

Urogenital-yu' row jen' i tal (G, ouron, urine; $\mathrm{L}$, genitalis, genital)

Uropygiurs, uropygial-yu' row pij' i um, -i al (G, oura, tail; pyge, rump)

Urostyle-yu' row style (G, oura, tail; stylos, column)

Uterus, uterine-yu' ter us, $\mathrm{yu}^{\prime}$ ter inn (or -ein) (L, uterus, womb)

Utriculus-yu trik' yu lus (L, a little bag)

Vagina-va jye' nah (L, a sheath)

Vagus-vay' guss ( $\mathrm{L}$, wandering)

Vallate-vall' ate (L, vallo, to surround with a wall)

Vas, vasa-vass, vay' sah ( $L$, a duct)

Vastus-vass' tus (L, vast)

Velum-vee' lum (L, veil)

Venosus-vee noh'sus (L, vena, vein)

Venous-vee' nous (L, vena, vein)

Ventricle-ven' tree kel (L, venter, belly)

Vermis-verr' mis (L, worm)

Vertebra, vertebrae-verr' te brah, -bree (L, a joint)

Vertebrata, vertebrate-verr' te bray' tah, -brate (L, verlebra, joint)

Vesical-vess'i kal (L, vesica, bladder)

Vibrissa, vibrissae-vie briss' ah, -ee (L, hairs in the nostrils)

Villi, villus-vill' eye, -us (L, shaggy hair)

Viscera, visceral-viss' err ah, -al (L, viscus, internal organ)

Viscus-viss' kus (L, internal organ)

Vitelline-vi (or vie) tell' inn (L, vitellus, yolk)

Vitreous-vit' ree ous (L, glassy)

Vomer-voh' mer (L, ploughshare)

Vulva-vull'vah (L, covering)

Wolffian-wolf' ee an (after K. F. Wolff, German anatomist)

Xiph, xiphi-ziff, ziff' ee (G, xiphos, sword, used in combining words)

Zyg, zygo-zigg, zye' go (or zigg' oh) (G combining word, zygon, yoke)

Zygapophysis-zye' (or zigg) ga poff' ee sis (G, zygon, yoke; apophysis, process)

Zygomatic-zye' go (or zigg' oh) mat' ik (G, zygoma, yoke or bar) 


\title{
Appendix B
}

\section{PREPARATION OF MATERIALS}

I. Killing the specimens.-Necturus is best killed by placing in hot water; turtles by injection of ether or chloroform into the cloaca or better the trachea; birds and mammals by inclosing them in a tightly closed vessel with a wad of cotton soaked in ether or chloroform. In handling turtles pull the head forward by inserting a stout hook behind the jaw, and pry open the mouth.

2. Preparation of skeletons.--Skeletons are best prepared from fresh materials or, in the case of marine forms, those that have been preserved in brine. It is difficult to prepare skeletons from specimens that have been preserved in formalin. To prepare a skeleton remove the skin, all of the viscera, and as much of the muscles as possible and soak the specimen in water. The remaining flesh will decay and may be removed with a stiff brush or forceps. This process of maceration in cold water takes some time. 'The process may be much shortened, and tough specimens are more easily prepared by immersing the specimen for a few hours in hot or boiling water to which gold dust or the following soap solution has been added. Kingsley gives the following formula for the soap solution:

\author{
75 gms. of hard soap \\ 12 gms. of potassium nitrate (saltpeter) \\ I 50 c.c. of strong ammonia \\ 2,000 c.c. of distilled or soft water.
}

Mix thoroughly. In using, take one part of the soap solution to three or four parts of water. The length of time required before the flesh will separate easily from the bones varies with different animals and is shorter for small or young specimens than for large and old ones. Davison gives the time for the cat as two to four hours in boiling water or better three to six hours in water kept at 75 to $90^{\circ} \mathrm{C}$. He also states that if the bones are heated for only one to two hours at a temperature not above $85^{\circ} \mathrm{C}$., the ligaments will be preserved, and when the skeleton is dried the ligaments will harden and hold the bones together. Skeletons containing a considerable amount of cartilage should not, of course, be boiled but treated only with moderately hot water. For fishes a few minutes' treatment with hot water is generally sufficient. Cartilaginous skeletons should be preserved in weak formalin or in 70 per cent alcohol.

3. Injection of the circulatory system.-To render the blood vessels conspicuous and more easily followed it is advisable and in fact practically necessary that the arteries at least be injected with a colored solution. Injection syringes for this purpose may be obtained from dealers in laboratory supplies, or an ordinary rubber atomizer bulb may be used. A glass cannula is inserted in the vessel to be injected. A cannula is simply a piece of glass tubing drawn out in the flame at one end to a size suitable for the blood vessel into which it is to be inserted. It is also desirable that the end to be inserted in the vessel be slightly enlarged as it will then hold more securely in the blood vessel. Loosen the vessel to be injected from the surrounding tissues, pass a cord under it, and tie the cord in a loose single knot above the vessel. With a fine scissors make a V-shaped cut into the blood vessel, having the cut extend not more than halfway through the vessel, and immediately insert the cannula in to the vessel. Tighten the knot around the cannula and blood vessel by pulling on the two ends of the cord, but make only a single knot. The cannula is connerted with the injection syringe or rubber bulb by a 
piece of rubber tubing. The whole system should be filled with the injection fluid in advance, in order to avoid forcing air into the blood vessels. After everything is ready inject the solution into the blood vessel by a steady but not too forceful pressure on the syringe or bulb. The success of the injection should be determined by examining the small vessels of the skin or in the intestinal walls. When these are deeply colored the injection is complete and the cannula is then withdrawn, the cord being immediately tightened around the blood vessel and tied with a double knot to prevent leakage. In case only the arterial system is injected, as is usually the case, the blood should not be let out of the animal; the injection will force the blood around into the veins and distend them. If the systemic veins are to be injected, the blood should be drained from the animal by opening the vein, at the place where it is to be injected.

The following formula for the injection fluid is given by Davison:

$$
\begin{aligned}
& \text { 100 c.c. of water } \\
& 20 \text { c.c. of glycerin } \\
& 20 \text { c.c. of concentrated formalin } \\
& 85 \text { gms. of corn starch } \\
& \text { sufficient coloring matter to give a deep color. }
\end{aligned}
$$

Stir thoroughly and strain through fine cheesecloth. All lumps must be removed. As the starch settles to the bottom on standing, the mixture must be stirred before using. Coloring matter used in the injection mass is: for red color, vermilion (mercuric sulphide); for yellow color, chrome yellow (lead chromate); for blue color, Prussian blue. The injection fluid will keep indefinitely.

Injection is usually made into the following vessels: in elasmobranchs, the arteries are injected by way of the caudal artery, exposed by cutting across the tail; the hepatic portal system is injected by way of the hepatic portal vein near the liver backward or in both directions or into the posterior mesenteric vein forward. Injection of the systemic veins is more difficult and is usually not done; a method is given by Rand in the American Naturalist. Vol. XXXIX (1905). In Necturus the arteries are most easily injected by way of the bulbus arteriosus. The hepatic portal system should be injected in both directions into the hepatic portal vein just before it enters the liver. The postcaval can be injected, if desired, through the large hepatic vein seen on the ventral surface of the liver just behind the transverse septum. The arterial system of the turtle is injected either backward by way of one of the carotids or into the left aorta away from the heart. It is practically impossible to work out the renal portal and hepatic portal systems on preserved turtles unless they are injected. Injection is very easily made of both of these systems by way of the abdominal vein. The plastron is, of course, to be removed by sawing across the bridges. Insert the cannula into one of the abdominal veins about halfway between the heart and pelvis and inject forward. Injection of the arterial system of the pigeon can be done by way of the pectoral artery toward the heart. According to Parker the systemic veins and the hepatic portal system may be injected by way of the coccygeo-mesenteric vein, in both directions. The arterial system of mammals is injected either by way of the carotid or the femoral artery, the cannula being directed toward the heart. The veins are usually not injected, but it is stated that they may be injected by way of the external jugular toward the heart. The hepatic portal system may be injected by way of the hepatic portal vein near its entrance into the liver. In injecting arteries and veins in the same specimen, differently colored injection fluids should, of course, be used.

4. Preservation of specimens.-Specimens are usually preserved in 5 per cent formalin (5 c.c. of commercial formalin plus 95 c.c. of water). A slit should be made in the body cavity or the formalin should be injected into the body cavity through a small opening to insure preservation of the viscera. A portion of the roof of the skull should be removed in the smaller 
specimens in order that the brain may harden. In the case of mammals additional measures are necessary in order to insure preservation. Mammals should be embalmed. This is done by injecting an embalming fluid into the blood vessels before the injection fluid is sent in. The embalming fluid may consist of 5 per cent formalin or better 5 per cent formalin plus one-sixth its volume of glycerin. A still better but more expensive embalming fluid is one used for human bodies, with the following formula:

\begin{tabular}{|c|c|c|c|c|}
\hline & & & & Parts by Vol \\
\hline Formalin & . & . & & . . $\quad 1.5$ \\
\hline Carbolic acid (melted crystals) & & . & . & 2.5 \\
\hline 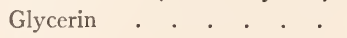 & . & . & . & . $\quad 10.0$ \\
\hline er & & . & . & . . 86.0 \\
\hline
\end{tabular}

The embalming fluid is injected preferably into the femoral artery through a cannula in the same way as already described for the injection fluid. Bensley recommends that the injection of the embalming fluid should be done, not with a syringe, but by attaching the cannula to a receptacle containing the embalming fluid elevated about three feet and allowing the fluid to run into the vessel under this pressure for about two hours. The animal should be arranged in a position suitable for dissection, with the limbs spread wall apart and the head tilted backward. After the embalming the injection fluid is run into the same cannula by attaching the syringe to it; Bensley recommends that twenty-four hours elapse between the embalming and the injection with the colored fluid.

If the animal has been thoroughly embalmed, it will keep without being immersed in a preserving fluid. It should be prevented from becoming dry by being placed in air-tight receptacles or wrapped in cloths saturated with the formalin-glycerin solution. It is best to sponge the hair with a mixture of alcohol and water containing 2 per cent formalin. The animals may, however, if preferred, be immersed in I per cent formalin solution. During the dissection the animals should be kept wrapped in cloths moistened with formalin glycerin.

Further details on these matters will be found in: Bensley, Practical Anatomy of the Rabbit (University of Toronto Press); Reighard and Jennings, Anatomy of the Cat (Henry Holt and Company); and Davison, Mammalian Anatomy (P. Blakiston's Son and Company).

5. Dealers.-Preserved and injected material necessary in the course can be obtained from:

The General Biological Supply House, I 177 East 55th Street, Chicago.

Supply Department, Marine Biological Laboratory, Woods Hole, Massachusetts.

The Angler's Company, 1534 West Lake Street, Chicago.

Live Necturus, turtles, pigeons, etc., are also cbtainable from the first and third dealers named above. The General Biological Supply House can also furnish prepared skeletons of any forms. For the names of other dealers consult Science, Transactions of the American Microscopical Society, and similar journals. 


\section{INDEX}

Abdominal pores 166

Abdominal vein: development of 203 , 229-30, 24I; dogfish 209; mammals 271; Necturus 22.3, 224-25; pigeon 248 ; skate 211 ; turtle $230^{-}$ $32,233,24 \mathrm{I}$

Abducens nerve: definition of 360 ; elasmobrancb 3II; mammal 355; Necturus 320; pigeon 33I; turtle 326

Abduction, definition of $\mathrm{I}_{3 \mathrm{I}}$

Accessory mesonephric ducts 281, 282, 283

Accessory urinary bladder 285,286

Acetabulum 81, 82, 83

Acipenser: classification of 6; external anatomy 21: scales 49; skull ror; vertebral column $63-6_{4}$

Acrania, definition of 8

Acromion process 93

Action of muscle, definition of 136

Acustic area 354,358

Acustico-facial nerve: Necturus 321 ; turtle 326

Acustico-lateral area: elasmobrancb 308; Necturus 320

Adduction, definition of $\mathrm{I} 3 \mathrm{I}$

Afferent brancblal arteries: dogfisb 215; Necturus 227; skate 215

A gnathostomata, definition of 8

Air-sacs, pigeon $178-8 \mathrm{I}$

Allantois $163,276,292-94$; blood vessels of 203

Alligator: atlas 70-71; axis 71 ; jaw II3-14, II5; pectoral girdle 9I; ribs 71-72; skull Io6, I I $2-16$; sternum or; vertebral column $70-72$

Alveolus, jaw 116, I 24

Alveolus, lung 176,188

A mblystoma, classilication of 7

A mia: classification of 6; external anatomy 22; scales 49; skull 102; vertebral column 64

Amnion 202, 203, 204

Amniota: definition of 8 ; embryonic membranes of $292^{-}-94$

Amphibia: blastula 32 ; cleavage 32 ; development of 32, 34, 37-39; exoskeleton 50; fore limb 90 ; gastrula 34; hind limb 81 ; pectoral girdle $80-00 ;$ pelvic girdle $80-8 \mathrm{r}$ ribs 69; sternum 86, 90 ; vertebra column 68-69. See further under Necturus

Amphicoelous vertebra $65,67,69,76$

Amphioxus 0-I I: blastula 32; classification of 6 ; cleavage 32 ; development of $32,34,36-37$; external anatomy 9-10; gastrula 34; internal anatomy ro-I I

Amphiplatyan vertebra 76

Ampulla, ear: elasmobranchs 306: Necturus 319

Ampullae of Lorenzini 302

Analogy 3-4

Anamniota, definition of 8

Ankle. See Tarsus

Ankylosis, definition of 94

Ansa, definition of 335

Ansa subclavia 334

Anterior cardinal vein: development of 203; dogfisb 200; mammal 269; Necturus 225, 227; pigeon 247; skate $2 \mathrm{II}$; turtle $24^{\circ}$

Anterior cbamber, eye 305,349

Anterior vena cava See Precaval

Antibrachium 88. See further under Fore limb
Anura: classification of 7 ; coelom 159, I96; pectoral girdle 90; sternum oo; vertebral column 69

Aorta: development of $202-3$; mammal 248, 257, 260-64; pigeon 244 245; turtle 235. See further under Dorsal aorta and Ventral aorta

Aortic arches: development of 202-3 elasmobranchs 214-18; mammal 267-60; Necturus 226-27, 229; pigeon 247; turtle $235-37,240$

Aponeurosis, definition of 136

Apophyses, definition of 62

Aqueduct, brain: elasmobranchs 318 ; mammal 357; turtle 327

Aqueous humor 305,349

Arachnoid $34 \mathrm{I}, 353$

Arbor vitae: mammal 357; pigeon

333
Arcade: infratemporal 113 ; supratemporal I 12

Arch of aorta: mammal 257; pigeon 245

Arcualia 59-61, 69; alligator $7 \mathrm{I}$; Stegoce phala 64 ; sturgeon $63-64$

Area acustica 35

Armadillo: classification of 7 ; exoskeleton 55

Arterial ligament: mammal 260, 260; turtle 236,240

Arterial system: development of 202-3; elasmobranchs $214^{-21}$ mammal 257-64; Necturus 226-28 pigeon 244-46; turtle 235-38 Artery, definition of 200

Arytenoid cartilages: mammal 187 ; Necturus 17I; pigeon 178; turtle I 75,176

Ascending vena cava. See Postcaval

Astragalus 84

Atlas 6o, 70, 71, 73-74

Auditory nerve, 360 : elasmobranchs 314; mammal 355; Necturus 321 ; pigeon 332 ; turtle 326

Auditory tube 162,301 : mammal 186 ; pigeon 177; turtle 175, 324

Auricle: elasmobrancb 207,221 ; mammal 248, 265-66; Vecturis, 222, 220; pigeon 24I, 2.46: turtle 230,238

Auricular appendage 248

Axes of body I

Axilla 135

Axillary artery: cat 259; pigeon 244; rabbit 258; turtle 235-36

Axillary fossa 135

Axillary vein: cat $254-55$; rabbit 252; turtle 234

Axis, vertebral column $70,71,74$

Azygos vein: cat 253; development of 269 ; rabbit 251

Balanoglossus: classification of 6 ; external anatomy I4

Basal plate, of skull 97: elasmobranchs 98 ; Necturus iI I

Basalia: pectoral fins 89 ; pelvic fins 80

Basals. See Basalia

Basidorsal, definition of 59,60

Basiventral, definition of 59,60

Bícuspid valve 266

Bilateral symmetry

Bile duct 163: elasmobrancb 164 : mammal 191, 192; Nerturus 170; pigeon 182 ; turtle 174

Birds: exoskeleton 25, 52-54; external anatomy 25-27; fore limb 02 ; hind limb 83 ; pectoral girdle 9r; pelvic girdle $82-83$; sternum 01-02; vertebral column $72 \rightarrow 73$. See further under Pigeon

Blastocoel 32

Blastoderm 34, 36

Blastopore 34,36

Blastula 32: amniotes 34; A mphihia 32; A mphioxus 32

Blood: definition of 200 ; origin of 201

Blood-vascular system 200

Body cavity. See Coelom

Bone 58

Bony labyrinth 352

Bowfin. See Amia

Bowman's capsule 275

Brachial artery: cat 250; Necturus 228; pigeon 244; rabbit 258

Brachial plexus: definition of 299; dogfisb 30I-2; mammal 335-37; Necturus 319; pigeon 328; skate 302 ; turtle 323

Brachial vein: cat 255; Necturus 225; pigeon 243; rabbit 252; skate $21 \mathrm{I}$; turtle 234

Bracbiocepbalic artery: cat 258-60; pigeon 244-45; rabbit 257-58 . turtle 235-36

Brachiocephalic vein: cat 254-55

Brachium 87. See under Fore limb

Brain: definition of 206 ; development of 296-07; elasmobranchs 307-9. functions of 358-59; mammal 352 59; Necturus $319-21$; parts of 206 97, 359-6o; pigeon 330-31, 332-3.3; turtle 324-25, 327

Branchia. See Gill

Branchiostegal membrane 20, 21,22

Branchiostegal rays 20

Breastbone. See Sternum

Bronchus: mammal 189, 266; pigeon I 83 ; turtle 176

Buccal nerve. elasmobrancbs 312 . 313; Necturus 321

Bulbourethral gland 291

Bulbus arteriosus: definition of 226 : Necturus 222,226

Bursa of Fabricius 287

Caecum: definition of $16 \mathrm{r}$; mammal I03; pigeon 182; turtle 174

Calcaneus 84

Canals of lateral line system: elasmo branchs 302-3; Necturus 310

Canals of Lorenzini 302

Canine teeth I 25

Capillary, definition of 200

Capitulum: of humerus 93; of rib 60 . 71,75

Capsules of sense organs $97,104-5$

Carapace 25, 51

Cardia 100

Cardiac nerve 334,337

Cardiac plexus 337

Carina 91,92

Carotid artery: elasmobranchs 21718; mammal 250-60; Necturus 227; pigeon $244^{-45}$; turtle $235-36$

Carpales 88

Carpus 88: bird 92; mammal 94; turtle 9I

Cartilage bone $57-58$

Cartilage bones of skull $\mathrm{rO}_{3}-5$

Cat: arterial system 257, 258-60, 262-64; brain 352-50; circulatory system $248-66$; classification of 7 . coelom 187-90, 196-98; cranial nerves 334, 337, $33^{8}, 344^{2-48}$ digestive tract $\mathrm{I}_{3}-86, \mathrm{I}^{8} 0-94$; $\mathrm{ex}$ ternal anatomy 27-29; fore limb 
93-94; heart $188,248,265-66$; hind limb $84-85$; hyoid apparatus I 26 I86; jaw I 1 8-19, I24; muscles I 3657; nervous system 333-59; pectoral girdle 92-93; pelvic girdle $83-$ 84 ; respiratory system $186-80$; ribs 75 ; sense organs $345-52$; skull i i626; spinal nerves $334-42$; sternum 93; sympathetic system 334-45, 337-39; teeth I24-25; urogenital system $288-02$; venous system $250-$ $51,253-57,265$; vertebral column $73-76$

Caval fold 188

Cavernous bodies. See Corpora cavernosa

Central nervous system: definition of 206; development of $206-97$; elasmobranchs 307-0; functional parts of 297-98; mammal 34I, 352-59; Necturus 319-21; pigeon $330-31,332-33$; turtle $324-25,327$

Centrales 79, 88. See under Limbs

Centrum: formation of 61 ; types of 76

Cephalization 3

Cere 25

Cerebellar fossa I 2I, 35 I

Cerebellum: elasmobranchs 308; mammal 353,357 ; Necturus 320; pigeon 331 , 333 ; turtle 324

Cerebral fossa I 2I, $35 \mathrm{I}$

Cerebral hemisphere: elasmobranchs 307,318 ; mammal $353,355-56$, 357-58; Nechurus 320; pigeon 331 , 332,333 ; turtle 324,327

Cerebral peduncle: elasmobranch 318 ; mammal 354; Necturus 32 I

Cerebrospinal fluid $34 \mathrm{I}, 353$

Cervical plexus, mammal 334

Cervicobrachial plexus: dogfish $301-2$; skate 302

Chevron bones: alligator 70 ; mammal 76

Choana: definition of I18; mammal I 86

Chondrocranium: bones derived from ro3-5; development of 96-97; dogfish 97-roo; Necturus rog, III

Chordae tendinae: mammal 266 ; pigeon 247

Chordate: characters of 5 ; classification of $6-7$; embryonic development of $3 \mathrm{I}-44$

Chordocentrous vertebra 65

Chorioid coat, eye: development 300 ; elasmobranch 305; mammal 349; pigeon $329-30$

Chorioid plexus, brain: elasmobranchs 308; mammal 353, 356; Necturus 320; turtle 324

Chorion 202-94

Ciliary body: mammal 349; pigeon

Ciliary ganglion: cat 348 ; elasmobranch 31 I

Ciliary nerves: cat 348 ; elasmobranch 3 II

Ciona $12-13$

Circulation through heart: elasmobranch 221-22; mammal 266-67; Necturus 229; pigeon 247; turtle $239^{-40}$

Circulatory system 200-272: development 20I-7; elasmobranchs 20722; mammal 248-72; Necturus 222-30; parts of 200-20r; pigeon $241-48$; summary $270-72$; turtle $230-4 \mathrm{I}$

Cladoselache 79

Classification of chordates $6-8$

Clava 354,358

Clavicle 87,88 ; bird 91; frog 9o; mammal 02

Cleavage $32-34$

Cleithrum 87.88

Clitoris: mammal 289, 290; turtle 285

Cloaca I6I, 270-80: dogfish I65, 28I: mammals 279-80; Necturus 160 284 ; pigeon 182,287 ; skate 165 , 282 ; turtle $175,285-86$

Coccyx: frog $60 ; \operatorname{man} 76$
Cochlea 352

Cochlear duct 352

Coeliac artery: cat 262; dogfish 219 , 220; pigeon 245; rabbit 260-6I; skate 220 ; turtle 236

Coeliac ganglion 338

Coeliac plexus: mammal 338; pigeon 328

Coelom 158-59, 195-98: definition of I58; development of $37-38,39-40$ 44; elasmobranchs $163-64, \mathrm{I} 66-67$; mammal r87-00, I07; Necturus 168-69, 170-71; pigeon $180-83$, 197; summary 195-99; turtle 172 -

Coelomoduct 273

Collector nerve 301

Colliculus 353

Colon: definition of 161 ; mammal 193-94; turtle 174

Columella: pigeon 330 ; turtle 324

Columnae carnae 247

Common cardinal vein: development of 203; dogfish 207, 200; mammal 260; Neclurus 222, 225; pigeon 247; skate 210 : turtle 240

Common carotid artery: mammal $257,259-60$; pigeon 244-45

Common iliac artery: rabbit 263 ; turtle $2.37-38$

Common iliac vein: cat 265

Comparisons, circulatory system: mammal 266-70; Necturus 229-30; pigeon 247-48; turtle $240^{\circ}-4$ I

Concha 105, 300; mammal I 21-22, 350; pigeon 329; turtle 323

Condyle: of limbs 83,84 ; of skull $99, I_{09}, I_{4}, I_{17}$

Condyloid process, jaw 124

Conjunctiva: elasmobranch 304; mammal 345, 349; pigeon 329

Contour feather 25, 52-54

Conus arteriosus: elasmobranch 207 221; Necturus 222, 229; turtle 230, 239

Convergence 4

Coprodaeum 287

Coracoid 86 . See Pectoral girdle

Coracoid process 86,92

Corium 45,46

Cornea: development of 300 ; elasmobranch 305; mammal 349; pigeon 329

Coronary artery, of heart: elasmobranch 215, 216-17; mammal 257; turtle 235

Coronary ligament 160,166 , 195-06; elasmobranch 166; mammal ro2; Necturus 170; pigeon 18r; turtle 173

Coronary sinus 253,265

Coronary vein: cat 253 ; rabbit 251

Coronoid process: of jaw 124 ; of ulna 93

Corpora bigemina 308. See Optic lobes

Corpora cavernosa 205; turtle 285; mammal $291-92$

Corpora lutea 289

Corpora quadrugemina 353,360

Corpus callosum $353,355^{-56}$

Corpus striatum: function, 3.33; mammal 358 ; pigeon 333 ; turtle 327

Corpuscles 200

Cortex, brain 357

Cotylosauria: classification of 7 ; jaw 108; pectoral girdle 87,88 ; skull I06, I 12

Cowper's gland $29 \mathrm{I}$

Cranial nerves 296, 299. 360-6I: elasmobranch $309-15 ;$ mammal 334 , 337. $338,342-45,346-47,348$; Necturus 320-2I; pigeon $33 \mathrm{I}-32$; turtle $325-27$

Cranium. See Skull

Cribriform plate $\mathbf{1 2 1}, 350$

Cricoid cartilage: mammal 187; pigeon 178 ; turtle 176

Crista 306

Crop I6r. 178
Crossopterygii: classification of 6 ; fins 79,89 ; ribs 67 ; skull 102

Crura: of diaphragm 338; of penis 202

Cryptobranchus: classification of 7 ; fore limb go; hind limb 8I; pectoral girdle 80-00; pelvic girdle 8o-8I; vertebral column $68-60$

Ctenoid scale 49,50

Cycloid scale 49,50

Cyclostomata: anatomy 14-16; classification of 6

Cystic duct $191-92$

Delamination 36

Dental formula 125

Dentine: scales 47 ; teeth 49

Depressor, definition of 133,141

Dermal bone 58. See Membrane bone

Dermal exoskeleton, definition of 47

Dermal papilla: for feather 52-53; for hair 55; for placoid scale 47

Dermatome 43,45

Dermis $45,46:$ in formation of feathers $52-54$; in formation of fish scales $47,49,50$; in formation of hair 55; in formation of reptile plates 50

Descending vena cava. See Precaval

Descent of testes, $200,29 \mathrm{I}$

Development of chordates $3 \mathrm{I}-44$

Diaphragm 159, 188, 189, 197

Diastema 125

Diencephalon 297, 359-60; elasmobranch 307-8, 317, 318; mammal $353,354,356,357,358$; Necturus, 320 , 32I; pigeon 33I, 333; turtle 324,327

Digestive system 150-09: elasmobranch I64-66, I67-68; general I59-63; mammal 183-86, 189-94; Necturus 169-70; parts of $161-63$; pigeon 176-77, I80, I81-82; summary ro8-90: turtle 173-75

Digitigrade walk 28

Diphycercal tail 23

Diplospondyly $6 \mathrm{r}, 64$

Divergence 4

Dog: skull to6

Dogfish: arterial system 214-2I; brain 307-0, 317-18; chondrocranium $97-00$; classification of 6 ; coelom 163-64, 166-67; cranial nerves 309-15; digestive system I64-66, I67-68; exoskeleton 48; external anatomy $16-17$; fins 17 , 80, 89; gills 168; heart 207, 221-22; mesenteries 165-66; muscles I 2830; myotomes 64, I 29: nervous system 3or-I8; pectoral fin 80 ; pectoral girdle 88-89; pelvic fin 80; pelvic girdle 79-80; respiratory system I67-68; ribs 66; spinal nerves 3or-2; urogenital system 280-83; venous system 207-10; 2I 2-I 4 ; vertebral column 64-66; visceral skeleton $09^{-100}$

Dorsal aorta 202: elasmobranchs 218-21; mammal 260-64; Necturus 227-28; pigeon 244-46; turtle 237-38

Dorsal column, spinal cord 297

Dorsal ramus, spinal nerve 299

Down feather $52-53$

Duct of Cuvier 203. See Common cardinal vein

Duodenum: elasmobranch 164 ; mammal 192-93; Necturus 169; pigeon I 8 I-82; turtle 174

Dura mater: mammal 341, 352, pigeon 330 , turtle 324

Ear 30, 300-301, 36r: development of 300-301; elasmobranch 305-6; functions of 306; lizard 23; mammal 27, 350-52; Necturus 22, 3 I9; parts of $30,300-301,36 \mathrm{r}$; pigeon $25-26,330$; teleost 20 ; turtle 24 . $323-24.326$ 
Ear bones 127, 301: mammal I 20, 351, 352; Necturus I10; pigeon 33 ; turtle 324

Ear drum 162 . See Tympanic membrane

Ectoderm: definition of 34 ; derivatives of $4 \mathrm{I}$

Efferent branchial arteries: elasmobranchs 216-I8; Necturus 227

Egg: cleavage of 32 ; kinds of 31

Elasmobranchii: chondrocranium $97^{-}$ IoO; circulatory system 207-22; classification of 6 ; coelom I63-64, I66-67; digestive system I64-66, I67-68; exoskeleton 47-48; external anatomy 16-19; mesenteries 165-66; nervous system 30I-18 pectoral fin 80 ; pectoral girdle 88-89; pelvic fin 80 ; pelvic girdle 79-80; respiratory system $167^{-68}$; urogenital system $280-83$; vertebral column 64-66; visceral skeleton $99-$ roo

Elbow ${ }^{*}$ birds 92 ; mammals 93

Elevator, definition of 133,140

Embryo: cat 294; dogfish 294

Embryology $31-43$ : amniotes 34,36 , 37-39; Amphibia 32, 34, 35, 37-39; A mphioxus 32, 34, 36

Embryonic membranes 292-94

Enamel: scale 47 , teeth 49

Endolymphatic ducts $17,98,306$

Endolymphatic fossa 08

Endoskeleton 57-127: chondrocranium $96-99$; definition of 45,57 ; gill arches 99-100; limbs 78-94; parts of 57; pectoral girdle $85-94$; pelvic girdle $78-85$; ribs $57-77$; skull 06-1 27; sternum 85-94; vertebral column $57-66$; visceral skeleton 90-roo

Endostyle I I, I3, I6I

Entoderm: definition of 34 ; derivatives of $4 \mathrm{I}-42$

Entoglossal cartilage 177

Epicondyles 84, 93

Epicoracoid 87

Epidermis 45: in formation of feathers $52-53,54$; of hair 55 ; of placoid scales 47 ; of reptilian scales

Epididymis 276; elasmobranch 283 ; mammal 202; tuitle 286

Epiglott is 186

Epimere 39, 41. 43

Epiphysis 297, 360: elasmobranch 318; mammal 356; Necturus 320; pigeon $33 \mathrm{I}$; turtle 324

Episternum 87. See Pectoral girdle

Epistropheus. See Axis

Epithalamus 359, 360: elasmobranch 3. 8; mammal 356; turtle 327

Epithelial bodies 162

Esophagus 16r: elasmobranch r67; mammal 187, r89; Necturus 17I; pigeon I77; turtle 175

Ethmoid plate 97, I04, I09, I I I

Ethmoid region of skull ro4: alligator II5; mammal I20-22, 350; Necturus I I

Eustachian tube. See Auditory tube

Exoskeleton 45-56; Amphibia 50 ; birds $52-54$; definition of 45 ; development of 47 ; fishes $47-50$; mammals $54-55$; reptiles $50-52$; summary 55-56; turtle $5 \mathrm{I}-52$

Extension, definition of $I_{3}$

External anatomy 9-30: $A m i a$ 22; Amphioxus 9-10; Balanoglossus 14 cyclostomes $14-\mathbf{r} 5$; dogfish $16-18$; elasmobranchs I6-I0; ganoids 21 gar pike $2 \mathrm{r}$; lamprey 4 $^{-1} 5$; lizard 23-24; mammal 27-29; Necturus 22-23; pigeon 25-27; skate $18-19$; spoonbill 2I; sturgeon 2I; summary 29-30; teleost I9-2I; tunicate II-I 2; turtle $24-25$

External auditory meatusi $6_{2}, 301$ : alligator I I 2; mammal 27, I I 7, 35 I pigeon $25,33 \circ$
External carotid artery: elasmobranch 21 7; mammal 259-60; Necturus 227; pigeon 245

External ear 30, 30r, 36r: lizard 23; mammal 27, 351; pigeon $25-26,330$

External genitalia 29, 289, 290

External iliac artery: mammal 26364 ; turtle 238

External iliac vein: mammal $264^{-65}$

External jugular vein: cat 254; Necturus 225; rabbit 252; turtle 234

Eye: development of 300,361 ; elasmobranch 304-5; mammal 345-49; pigeon 329-30; turtle 323

Eyeball: elasmobranch $30_{4}-5$; mammal 349; pigeon 329-30; turtle 323

Eyelids: dogfish 17 ; lizard 23; mammals 27; muscles of, in mammals 346,347 ; pigeon 25; turtle 24

Eye muscles 299, 3II: cat $347-48$; elasmobranch $304^{-5}$; pigeon 329 ; rabbit $346-47$; turtle 323

Facet 7 1, 74, 75

Facial nerve 313,360 : elasmobranch 313; mammal 343-44, 355; $\mathrm{Nec}$ turus 321 ; pigeon 332 ; turtle 326 , 327

Falciform ligament ro6: elasmobranch r66; mammal 102; $\mathrm{Nec}$ turus I70; pigeon I80; turtle 173

Fallopian tube. See Uterine tube

Fascia 1,30, 134, 136

Fasciculus $135^{-3} 6$

Feat hers $25-26,52-54$

Femoral artery: mammal 264; Necturus 228; pigeon 246

Femoral vein: mammal 265: pigeon 243; turtle 231

Femur 79. See Hind limb

Fenestra, of skull 97

Fibula 70. See Hind limb

Fibulare 70. See Hind limb

Filiform papilla 185

Filoplume $25,52,54$

Fimbria 357,358

Fin-fold theory 78-79

Fin rays: elasmobranch 18, 80; teleost 20

Fins: Amphioxus 9; cyclostomes r4; dogfish 17-18, 80, 89; ganoid 89; Necturus 23; skate $18-19$; teleost 20

Fishes: classification of 6; exoskeleton 47-50; external ana tomy $16-2 I$ fins $17-18,20$; ribs $66,67-68$; scales 47-50; vertebral column 64-68. See further under Elasmobranchii, Ganoid, and Teleost

Flexion, definition of $I_{3} I$

Flexures of brain, pigeon $33^{\circ}-31$

Floccular fossa $35 \mathrm{I}$

Flocculus $35 \mathrm{I}$

Foliate papilla 185

Follicle: feather 25, 52; hair 54

Fontanelle 07,08

Foramen epiploicum 102

Foramen magnum 98, rog. See under Skull

Foramina of skull: cat 122-23; rabbit I 23-24

Fore limb 87-88: bird 92; mammal 93-94; turtle 9r; urodeles 90

Forebrain. See Prosencephalon

Form, principles of $\mathbf{I}-3$

Fornix 356,358

Fossa rhomboidea 3r8. See under Medulla oblongata

Frenulum I 8

Frog: pectoral girdle 9o; sternum 9०; vertebral column 60

Fungiform papilla 185

Funiculi, of spinal cord $341-42$

Furcula or

Gall bladder I63: elasmobranch r64; mammal ro1-02: Necturus 170; turtle 174
Ganglion, definition of 206

Ganoid fishes: classification of 6 ; exoskeleton 49; external anatomy 21-22; limbs from fins 79; pectoral fin 89 ; skull roI-3; vertebral column $63-64$

Ganoid scales 21, 49

Ganoin 2 I, 49

Gar pike. See Lepidosteus

Gasserian ganglion. See Semilunar ganglion

Gastric plexus 3.38

Gastrocentrous vertebra 71

Gastrocoel 34: amniotes 35; $A m$. phibia $35 ;$ Amphioxus 34

Gastrula 34: amniotes $36 ;$ Amphibia 34; A mphioxus 34

Geniculate bodies 356,358

Geniculate ganglion $3 \mathrm{I}_{3}$

Germinal disk 3 I

Germ layers 34,36 ; development of $3^{6-44}$

I6I: elasmobranch 168; $\mathrm{Nec}$ iurus 22; teleost 20

Gill arch 99-100, 105, 107, I 26+27: alligator II3, II5-I6; cartilage bones from ro5; dogfish 99-100: mammal 126; membrane bones to 107; Necturus I I I-I 2; nerves 315: pigeon I77-78; turtle II 5-I6, I75

Gill slits 5, I6I: Amphoxus ro-1x: Balanoglossus 14; dogfish I7, r68; lamprey 15; Necturus 22, 171; skate I0, I68; teleost 20

Gizzard $180, \mathbf{r} 8 \mathbf{r}, \mathbf{r} 82$

Glans, of penis: mammal 29I; turtle 286

Glenoid fossa oo. See Pectoral girdle

Glomerulus 273, 274, 275

Glossopharyngeal nerve 360 : elasmobranch 314; mammal 343, 355; Necturus 321; pigeon 332 ; turtle 326,327

Glottis: mammal r86; Necturus I 7 I: pigeon I77; turtle 175

Gnathwstomata, definition of 8

Gonad $276-78$

Graafian follicles 280

Gray matter 296, 297, 342

Gubernaculum 202

Gyrus 353

Habenula $318,356,35^{8}$

Haemal arch 60, 6I See Vertebral column

Haemal canal 6o, 65. See Vertebral column

Haemal spine 6o, 6r. See Vertebral column

Hair $27,54-55$

Harderian gland: mammal 345,346 , 348 ; pigeon 329 ; turtle 323

Haustra I 93

Head 3, 29, 299-300; segmentation of 299-300

Heart: development of $20 \mathrm{I}-2$; elasmobranch $166-67,207,22 \mathrm{I}-22$; mammal I87, I88, 248-49, 265-67; Necturus I70-71, 222, 228-20: pigeon $180,24 I_{-42}, 246-47$; turtle I $72,230,238-30$

Hepatic portal system 204, 205, 270: development of 204-5; dogfish 212-I3; mammal 249-5I; Necturus 223; pigeon 242; skate 213; turtle 232-33

Hepatic portal vein 204: cat $250-5 \mathrm{I}$; dogfish 212-13; Necturus 223; pigeon 242 , rabbit $249^{-50}$; skate 213; turtle 232-33

Hepatic veins 204: dogfish 208; mammal 255-56; Necturus 222, 226; pigeon 24.3 ; skate 2 Io; turtle 234

Heterocercal tail I7

Heterocoelous vertebra 72

Heterodont I 24

Heteronomy 2

Hilus 288

Hind limb 70: birds 83; mammals $84-85$; turtle $8 \mathrm{I}-82$; urodele $8 \mathrm{I}$ 
Hindbrain. See Rhombencephalon

Hippocampus, $357,35^{8}$

Holoblastic cleavage 32

Homocercal tail 20,68

Homodont 116

Homology 3

Homonomy 2

Humerus 88 . See Fore limb

Hyoid apparatus: alligator II5: mammal I26, 186-87; pigeon 177-78; turtle $115-16,175$

Hyoid arch 100, 105: bones derived from ro5; elasmobranch 100: Necturus $111-12$

Hyoidean artery 21 7-1 8

Hyomandibular 100, 105; as stapes 120

Hyomandibular nerve 307,313

Hypobranchial nerve 316

Hypogastric vein. See Internal iliac vein

Hypoglossal nerve 36r: mammal 334 $342-43,355$; pigeon 332; turtle 326,327

Hypomere $39,4 \mathrm{I}$; derivatives of 44

Hypophysis $16 \mathrm{r}, 350$; elasmobranch 317; mammal 351, 354; Necturus $32 \mathrm{r}$; turtle 327

Hypothalamus 350 : elasmobranch 318; mammal 356 ; turtle 327

Hypural bone 68

Ichthyopsida, definition of 8

Ileocolic valve 194

Ileum 161,193

Iliac artery: Necturus 228; turtle $237-38$

Iliac vein: dogfish 209; pigeon 243; skate 211 ; turtle 23I-32

llium 79. See Pelvic girdle

Incisor 125

Inferior cervical ganglion: mammal 334 ; turtle 322

Inferior lobes, of brain 317

Inferior mesenteric artery: dogfish 219; mammal 263; pigeon 245, 246 ; skate 220 ; turtle 237

Inferior mesenteric ganglion 338

Inferior mesenteric vein: cat 250: pigeon 242, 243: rabbit 240

Infra-orbital gland $184,345,346,348$

Infra-orbital nerve 305,312

Infraspinous fossa 93

Infratemporal fossa 112

Infundibulum, of brain 359: elasmobranch 317 ; mammal 354; Necturus 321; pigeon 332; turtle 327

Inguinal canal 290

Inguinal ligament 135

Inguinal region 135

Innominate artery. See Brachiocephalic artery

lnnominate bone: birds 82 ; mammals 83

Insertion, of niuscle $131, \mathrm{I}_{3} 6$

Interauricular septum: mammal 265 ; Necturus 220; pigeon 246; turtle 238

Intercalary arch 6r, 65

Interclavicle 87, 88. See Pectoral girclle

Interdorsal 59,60

Inter haemal arch 6 r

Intermedium 70, 88. See under Limbs

Internal carotid artery: mammal 259, 354; Necturus 227; pigeon 245

Internal ear 300-30r,36r: development of 300-301; elasmobranch 305-6; function 306; mammal 352 ; Necturus 319; pigeon 330; turtle 324,326

Internal iliac artery: mammal 263-64; pigeon 246; turtle 238

Internal iliac vein: mammal $264-65$; pigeon 243; turtle 232

Internal jugular vein 27I; cat 255 ; Necturus 225, 227; rabbit 252.253: turtle 234

Interorbital septum: pigeon 329 : turtle 323
Interventral 59,60

Interventricular septum: pigeon $\mathbf{2 4} 6$; turtle 230; mammal 266

Intervertebral foramen 76

Intestine 161,163 : elasmobranch $164-$ 65; mammal 192-04; Necturus I69-70; pigeon I8I-82; turtle

$174-75$
Intratarsal joint: birds 83 ; reptiles 82 Invagination, definition of 34,36

Involution, definition of 36

Iris 300: development of 300 ; elasmobranch 305; mammal 349; pigeon 330

Ischium 70. See Pelvic girdle

Isolecithal, definition of $3 \mathbf{I}$

Isthmus, of fauces 186

Jaw 105-7: alligator 113, II5; dog. fish 09-100; mammal I I 8-10, I 24; membrane bones of 107, 108: Necturus 1 I0, 111

Jugular ganglion 332

Jugular vein: Necturus 225; pigeon 242-43

Kidneys 273-76, 294; development of 273; dogfish 208, 280-81; mammal 190, 288; Necturus 169, 223, 284; pigeon 243, 287; skate 2II, 282; turtle 232, 28

Kneecap. See Patella

Lacrimal gland: cat 348; pigeon 329 ; rabbit 346; turtle 323

Lamprey: classification of 6; external anatomy 14-15; internal anatomy I5-16

Larynx 199: cartilages of 105; mam. mal 1 86-87; Necturus 171; pigeon I 78; turtle $175-76$

Lateral column, spinal cord 297

Lateral line canals: dogfish 302-3; skate 303; Necturus 319

Lateral line nerve: elasmobranch 3 I $_{4}$ : Necturus $32 \mathrm{I}$

Lateral line system: dogfish 302-3; Necturus 310; skate 303

Lens: development of 300 ; elasmobranch 305; mammal 349; pigeon 330; turtle 323

Lepidosteus: classification of 6 ; external anatomy $2 \mathrm{I}$; scales 40 ; skull 102-3

Ligament of Botallus. See Arterial ligament

Ligaments: of digestive tract. See Mesenteries; of testis 289; of uterus 280

Limbs $27-28,79,86,87-88$ : bird 26 , 83,02 ; endoskeleton of 70,86 , 87-88; lizard 24; mammal 27-28, $84-85,93-94$; Necturus 23, 81, 90; origin of 78-79; position of 23,24 , $26,27.28$; torsion of $23,24,26$, 27 28; turtle 25, $81-82$, 9r; urodeles $8 \mathrm{r}, 00$

Linea alba 120

Liver $163,195-96,204$ : development of $163,195-96$, elasmobranch 164 ; mammal 190, I9I-92; Necturus I60, I70; pigeon I8I; turtle 173

Lizard: classification of $7 ;$ external anatomy 23-24; jaw 108; pectoral girdle 88 ; scales $23,50-5 \mathrm{I}$

Lorenzini: ampullae 302 ; canals 302

Lumbosacral plexus: dogfish 30I-2; mammal 339-40; Necturus 319; pigeon 328 ; skate 302 ; turtle 322

Lung $162-63,196-97$, 199: mammal 188, 189; Necturus 169. 170, 171; pigeon 183 ; turtle 176

Lymph 201

Lymphatic system 200-20I

Lymph glands 200; of intestine 194

Lymph hearts 200

Lymph nodules 194

Lymphocytes 200
Malleolus 83, 84

Malpighian body 275

Mammalia: circulatory system 248 70; coelom 159, 187-90, 190-98 digestive system 183-86, 18994; exoskeleton 54-55; external anatomy 27-20; fore limb 03-04; hind limb $84-85$; muscular system 133-57; nervous system 333-50 pectoral girdle $88,92-93$; pelvic girdle $83-84$; respiratory system 186-88; ribs 75; skull I1 6-26; sternum 93; urogenital system 28892; vertebral column 73-76. See also under Cat and Rabbit

Mammary glands $\mathbf{1 3 5}$

Mammillary body: elasmobranch 318; mammal $354,35^{8}$

Mandible. See Jaw

Mandibular a rch 99-100, 105, 107

Mandibular foramen 124

Mandibular fossa 118

Mandibula nerve: elasmobranch 312; mammal 344-45; Neclurus 320-21; pigeon 33I-32; turtle 325,326

Marsupialia: classification of 7 : oviducts $278-79$

Maxillary nerve: elasmobranch 312 mammal $345,346,348 ;$ Necturus 320-2I; pigeon 33I-32; turtle 325,326

Meatus, of nose $35^{\circ}$

Meckel's cartilage 90, 105, 107; alli gator II5; elasmobranch 00-100: Necturus III

Mediastinal septum r 88, 197

Mediastinum I $88, \mathbf{1} 89$

Medulla oblongata 297, 360: elasmobranch 308-9, 317-18; mammal 353-54; Necturus 320 ; pigeon 331 ; turtle $324-25$

Medullary velum 353,357

Membrane bones: definition of $57-58$; of ganoids ror- 3 ; of pectoral girdle 37-88; of skull 100-103, 105-7. See also under Skull and Pectoral girdle

Membranes of brain. See Meninges

Membranous labyrinth. See Internal ear

Meninges: elasmobranch 307; mam mal 34I, 352-53; Necturus 320: pigeon 330; turtle 324

Mental foramen 124

Meroblastic development $3 \mathrm{I}$ : cleav. age 34 ; egg 31 ; gastrula 36 ; later development $30-41$

Mesencephalon 207, 360; elasmobranch 308; mammal 353; Necturu. 320 ; pigeon 331; turtle 324

Mesenchyme: definition of 42: prod ucts of 44

Mesenteries 158 166; definition of 1 58, 166; development of $39,40,43$ elasmobranchs 165-66; mammal I00-94; Necturus 160-70; pigeon r80-82; turtle $173-76$

Mesoblastic somite 39, $4 \mathrm{I}$

Mesocardia 158,202

Mesoderm: development of 36,37 $38,39,4 \mathbf{1}-44$

Mesomere: derivatives of $43-44$; development of $39,4 \mathrm{I}, 43^{-44}$; in formation of kidneys $273-76$

Mesonephric duct 275, 276, 295 dogfish 281, 283; mammal 292; Necturus 284; pigeon 287; skate 282, 283; turtle 286

Mesonephros 274-75, 295: dogfish 280; mammal 292; Necturus 284 skate 282; turtle 286

Mesopterygium 80

Metacarpals 88 . See Fore linb

Metacromion process 93

Metamere 2

Metamerism 2

Metanephric duct. See Ureter

Metanephros 276, 294: mammal 288; pigeon 287 ; turtle 285.286 
Metapterygium 80,80

Metatarsals 70. See Hind limb

Metencephalon 297. See Cerebellum

Midbrain. See Mesencephalon

Middle cervical ganglion: mammal 334; turtle 322

Middle ear 30 , 301, $36 \mathrm{r}$ : development of 301 ; lizard 23 ; mammal $351-52$; pigeon 25, 330; turtle 24, 323-24. See also Tympanic cavity

Mitral valve: mammal 266; pigeon 246

Molar gland 184

Molar teeth 125

Molgula 12,13

Monodelphia 7, 279

Monotremata: classification of 7 ; oviducts 278 ; pectoral girdle 88

Motor, definition of $297^{-98}$

Mouth cavity. See Oral cavity

Müllerian duct. See Oviduct

Muscles: development of 43, I 28; epaxial I 28; hypaxial I28; insertion $13 \mathrm{I}$; involuntary 128 ; origin I3I; parietal 128; parts of 135; somatic I 28; visceral I 28, I 29; voluntary I 28

Muscles, of eye. See Eye muscles

Muscles, of mammals: acromiotrapezius 147; adductor femoris I54; adductor longus 153, I54; 2dductor magnus I53; anconeus I 50; basioclavicularis 140; biceps brachii 150; biceps temoris 15I, 153; brachialis 150; caudofemoralis I53; cephalobrachial I42; clavobrachialis I42; clavodeltoid I40, 148 ; clavotrapezius 142,147 ; cleidomastoid I4O, I I I; cutaneous maximus I 34; deltoid I $40, I_{45}, I_{4} 8$; depressor conchae posterior 140 digastric I4I, I42; extensor antibrachii 150; extensor digitorum longus 155, 156; extensor hallucis longus 155; external oblique 135 36 , I37; flexor digitorum longus 155, I57; gastrocnemius $155,1_{5}$; geniohyoid I42; gluteus maximus I51, 153; gluteus medius 152 , 153 ; gracilis 152,154 ; iliocostalis 146 I49; iliopsoas 339; infraspinatus I 45, I48; intercostals I46, I49; internal oblique 137; latissimus dorsi $1_{3} 6,1_{44}, 1_{47}$; levator scapulae 149 ; levator scapulae ventralis I 44, I 47 ; longissimus I46, I49: masseter $140,1_{42}$; multifidus 137 ; mylohyoid I4I, I42; panniculus carnosus 134; pectoantibrachialis 130; pectoralis major 139; pectoralis minor 139 ; pectoralis primus I39; peroneus 155, 156; plantaris 155, I56; platysma $134-35,140$; psoas minor 339; quadriceps femoris 152,154 ; rectus abdominis I37; rectus femoris 152, I54; rhomboideus 144, I47; rhomboideus capitis 145, 147; sacrospinalis 137 sartorius 152 , 154 ; scalenes 145 , I49; semimembranosus I53, I54; semispinalis dorsi $\mathbf{I}_{4} 6, \mathbf{1 4 9}$; semitendinosus 153, I55; serratus dorsalis 146 , 149 ; serratus ventralis I45, I48; soleus 155 , I 56 ; splenius I44, I47; spinotrapezius I47; sternohyoid I 40 , I4I; sternomastoid $1_{4} 0, I_{4}$ I; sternothyroid I 4 I, I42; subscapularis I $45, I_{4} 8$; supraspinatus I44, I48; temporal I42; tensor fasciae latae I5I, I53: tenuissinus 155; teres major I45, I48; teres minor I45, I48; thyrohyoid I4I, I43; tibialis anterior I55, 156; tibialis posterior 157; transverse 137 ; trapezius $144,1_{47}$; triceps brachii I5O; vastus intermedius 152, 154; vastus lateralis 152,154 ; vastus medialis 152 . I54; xiphibumeralis $I_{39}$
Muscular system I 28: dogfish I 28-30; mammal I 33-57; Necturus I 30-33; pigeon $170^{-80}$

Myeloncephalon 297. See Medulla oblongata

Myocomma, definition of 43,129

Myoseptum 43, I 29

Myotome 43, 128: Amphioxus 9; dogfish I20; head 209-300; Nec turus $\mathrm{I} 30^{\circ}$

Nares: alligator II 2, II3; elasmobranch 303 ; lizard 23; mammal 26 , 117-18; Necturus 22, 171; pigeon 25, 177; turtle 24, 175

Nasal cavities 300, 36I: elasmobranch 303; mammal 349-50; Necturus 310; pigeon 320; turtle 323

Nasopalatine duct 185

Nasopharynx I 86

Neclurus: arterial system 226-28; chondrocranium III; circulatory system 222-30; classification of 7; coelom I69; digestive system I68-7I; external anatomy 22-23; fore limb oO; gill arches III, II heart 222, 228-29; hind limb 8r; jaws I10, III; mesenteries 169-70; muscular system 130-33; nervous system 3I8-2I; pectoral girdle 89-0o; pelvic girdle $80-8 \mathrm{r}$; respira. tory system I7I; ribs 69; skull IO7-12; urogenital system $283-84$; venous system 222-26; vertebral column $68-69$

Nephrostome 273,275

Nephrotome $39,4 \mathrm{I}$

Nervous system $296-361$ : development of 296-97, 359-6r; elasmobranchs 301-18; functional components 297-08; mammal 333-50; Necturus 318-21; parts of 296; pigeon 328-33; summary 359-6I; turtle $32 \mathrm{I}-28$

Neural arch 6o, 6I See Vertebral column

Neural canal 60, 65. See Vertebral column

Neural crest 206

Neural folds 36,38

Neural spine 60, 6r. See Vertebral column

Neural tube, development of 36,38 ; 296-97

Neuromere 299-300

Nodosal ganglion 343

Nose. See Nasal cavities

Notocentrous vertebra 60

Notochord 5: development of 37,39; in skull $96-08$; in vertebral column $59^{-6} \mathrm{I}, 63,65$

Jblique septum r 59, I8 r, 196, I99

Obturator foramen: bird 82 ; mammal 84 ; turtle $8 \mathrm{t}$

Occipital condyle 08, I00, II $7, I_{3}$

Occipital nerves 315

Occipital region of skull $\mathrm{IO}_{4}$ : alligator II 4 ; mammal irg: Necturus roo

Oculomotor nerve 360 : cat, 348,354 ; elasmobranch $308,310-11$; Necturus 320; pigeon 331; rabbit 34647,354 ; turtle 325

Odontoid process: alligator $7 \mathrm{I}$; mammal 74 ; turtle 72

Olecranon: bird 92; mammal 93

Olfactory bulb: elasmobranch 307 ; mammal $353,354,35^{8}$

Olfactory capsule $97, \mathrm{IO4}_{-5}$ : alligator I15; mammal I21-22; Necturus, III; in development of skull 97, 104-5. See also Nasal cavities

Olfactory fossa of skull $35 \mathrm{I}$

Olfactory lobe: elasmobranch 307 ; Necturus 320; pigeon 331-32; turtle 324
Olfactory nerve 360: elasmobranch 307, 309-10; mammal 353: Necturus 320; pigeon 329, 33r; turtle 325

Olfactory sacs: development of 300 361 ; elasmobranch 303 . See also Nasal cavities

Olfactory tract: elasmobranch 307 ; mammal 354

Omentum, greater: 100, 191, 198

Omentum, lesser: 166 , I9I

Operculum 20

Ophthalmic nerve 360 : cat $348-49$ elasmobranch 307,3 II-I 2,313 Necturus 320-21; pigeon 329 331-32; rabbit 347; turtle 323 . $325-56$

Opisthocoelous 76

Optic capsule 97, ro5

Optic chiasma: elasmobranch 317; mammal 354; Necturus 321; pigeon 332; turtle 327

Optic lobes: elasmobranch 308,318 ; mammal 353; Necturus 320; pigeon $33 \mathrm{I}$; turtle 324

Optic nerve 360 : cat 348 ; elasmobranch 305, 3 10; Necturus 320 ; pigeon 3.31; rabbit 347 ; turtle 325

Optic pedicel 08,305

Optic tract: elasmobranch 317 ; mammal 356, 358; pigeon 332

Oral cavity: elasmobranch $167-68$; mammal 183-85; Necturus I 7 , pigeon $176-77$; turtle 175

Oral glands 161 : mammal $183-84$

Orbit, definition of 98 . See under Skull

Orbital fossa II 7

Origin, of muscle $131, I_{3} 6$

Ossification $58, \mathrm{IO}_{3}$

Ostium, of oviduct 278 : dogfish $28 \mathrm{I}$ mammal 289; Necturus 284; pigeon 287 ; skate $28 \mathrm{I}$; turtle 284

Ostrich, sternum 02

Otic capsule 97, I04: alligator II4: mammal 120; Necturus 109-10

Otolith 306,310

Ovary 278: dogfish 280; mammal 280; Necturus 283; pigeon 286; skate $28 \mathrm{I}$; turtle 284

Oviducal gland 282

Oviduct 278-70: dogfish 280-8I; mammal 278-79, 289; Necturus 283-84; pigeon 286, 287; skate $28 \mathrm{I}-82$; turtle 284

Palate II8, II9: mammal I84-85 pigeon $176-77$

Palatoquadrate cartilage. See Ptery. goquadrate cartilage

Pallium: pigeon 333 ; turtle 327

Pancreas 163 , I99: elasmobranch I64; mammal r92; Necturus I60, I70; pigeon 182 ; turtle 174

Papillae, of tongue 185

Papillary muscles 266

Parachordal 97: bones derived from IO4

Paraphysis 297, 318: elasmobranch 318; Necturus 320; turtle 324

Parathyroids $\mathrm{I}_{2}$

Parietal peritoneum I 58, I66, 190, I9:

Parotid gland $14 \mathrm{I}, \mathrm{I} 84$

Patella: bird 83 ; mammal 84

Pecten, of eye 330

Pectoral artery 244

Pectoral fin: elasmobranch 89 : Polypterus 89

Pectoral girdle 78-79, 86-87: birds 9r; elasmobranch 88-89; frog 90; mammals 92-93; membrane bones of 87 ; origin of 79 ; parts of $86-87$; reptiles $90-01$; urodeles $89-00$

Pectoral vein 242

Peduncles of cerebellum: elasmobranch 308; mammal 354355 . 358-59

Pelvic fin: elasmobranchs 18, I9, 80

Pelvic girdle $78-79$ : birds 82,83 ; elasmobranchs 79-80; mammals 
$83-84$; origin of $79 ;$ parts of 79 ; turtle $8 \mathrm{I}$; urodeles $80-8 \mathrm{I}$

Penis 295: mammal 29, 29I-92: turtle 285,286

Pericardial cavity I50, I60, I05-07, I09: elasmobranch 166-67; mammal 187, 188; Necturus 170-71; pigeon I80; turtle 172

Pericardial sac r6o, 196-07; mammal I88; pigeon 180; turtle $172-73$

Pericardio-peritoneal canal 167 : dogfish 222; skate 210,222

Pericardium: elasmobranch I 66-67; mammal r 88; Necturus I70; pigeon I80; turtle I $72^{2}$

Periderm 52, 53, 54

Perimysium $\mathrm{I}_{3} 6$

Perineum 20

Peripheral nervous system 200,296 298-99,359, 360-6r: elasmobranchs 30I-2, 300-I6; mammal 334-45, 346-49; Necturus 318-19, 320-21; pigeon $328,33 \mathrm{I}-32$; turtle $32 \mathrm{I}-23$, $325^{-27}$

Peritoneal cavity $150-60$, I96-97, I09: mammal I80-00; pigeon $180-$ 82

Petrosal ganglion: elasmobranch $3{ }_{4}$; pigeon 332

Peyer's patches I94

Phalanges $79,88$. See under Limb

Pharynx I61-62, I98-99: elasmobranch I 68; mammal I 85-86; Necturus I7I; pigeon I77; turtle I75

Phrenic nerve $334^{-35}$

Pia mater: mammal 34I, 352-5.3; Necturus 320; pigeon 330; turtle 324

Pigeon: air sacs $178-81$; arterial system 244-46; circulatory system $24 \mathrm{I}-48$; classification of 7,25 ; coelom I $80-81, \quad 182-83$; digestive system 1 76-77, 180, I81-82; exoskeleton 52-54; external anatomy 25-26; feathers $25,52-54$; fore limb 02; heart 24I-42, 246-47; hind limb $83 ;$ muscles $179-80$ nervous system 328-33; pectora girdle 91 ; pelvic girdle $82-83$; respiratory system $177-81$; ribs 73 sternum 91 ; syrinx 183 ; urogenita system 286-87; venous system 242-44; vertebral column 72-73

Pineal body. See Epiphysis

Pinna 27, 30, 301, 351, 361

Pisces 6. See Fishes

Pituitary body. See Hypophysis

Placenta 204

Placental mamnal

Placoid scale $47^{-4} 8$

Planes of body I

Plantigrade walk 28

Plasma 200

Plastron 25, 52

Pleura I88; mammal I 88, r 80 , pigeon 183

Pleural cavity I59, I96-97, 199: mammal 187-89; pigeon $181,182-$ 83

Pleuroperitoneal cavity 159, 195-99: elasmobranch I6 $_{3}-64 ;$ Necturus I 68-69; turtle $\mathrm{I}_{72}-73$

Plexus of spinal nerves 299, 350: dogfish 30I-2; mammal $335-37$, 339-40; Necturus 319; pigeon 328 ; skate 302 ; turtle 322

Pneumatic foramen 02,170

Polyodon: classification of 6; external anatomy $2 \mathrm{I}$

Polypterus: classification of 6 ; pectoral fin and girdle 89 ; ribs 67 ; skull ror

Pons $354^{-55}$

Portal system 200, 204, 204-6. See further under Hepatic portal system and Renal portal system

Postaxial border of limb 2

Postcaval vein: development of 220-30, 24I, 247, 268-70, 27I; mammal $255-56,264-65,268-70$;
Necturus 225-26; pigeon 242, 24344, 247; turtle 233, 234-35, 237, 24I

Posterior cardinal vein $203,270,27 \mathrm{I}$ : dogfish 208; mammal 260; Necturus 226, 220; pigeon 247; skate 2IO-II; turtle 240

Posterior chamber of eye 305,349

Posterior vena cava. See Postcaval

Preaxial border of limb 23

Precaval vein 27I; cat 253-55; pigeon 242-43, 247; rabbit 25I-53; turtle $233-34,240$

Prechordal cartilage 97 ; bones derived from 104

Premolar teeth I 25

Prepuce 29, 291-02

Procoelous vertebra $69,70,76$

Procoracoid 86. See under Pectoral girdle

Procricoid cartilage 178

Proctodaeum 160 ; pigeon 287

Prone, definition of 28

Pronephric duct 273, 275, 294, 295

Pronephros $273-74,275,294$

Propterygium 80, 89

Prosencephalon 207

Prostate gland $29 \mathrm{I}$

Proventriculus 181,182

Pseudocentrous vertebrae 60

Pterygoid fossa II 3, II 8

Pterygoquadrate cartilage 99. I05, I07, I IO, II 4

Pubis 79. See Pelvic girdle

Pudendal plexus, pigeon 328

Pulmonary artery 267, 272: mammal 248, 257; Necturus 227; pigeon 244, 245; turtle 235, 236

Pulmonary plexus 337

Pulmonary veins 200, 272: mammal 256-57; Necturus 226; pigeon 242, 244; turtle 235

Pulp: feather 52 ; scale 47

Pulvinar 356,358

Pupil: elasmobranch 305 ; mammal 349

Pygostyle 73

Pylorus: elasmobranch 164 ; mammal I 9o, 194; Necturus I69

Pyramid, of brain $355,35^{8}$

Pyriform lobe $354,35^{8}$

Rabbit: arterial system 257-58, 26064; brain 352-59; circulatory system 240-65; classification of 7 ; coelom I 87-89, I96-98; cranial nerves $334,337,338,342-45,346-$ $47,354^{-55}$; digestive system $18_{3}-$ 86, 180-9.4; external anatomy 2729; fore limb 93-94; heart 188 , 248, 265-66; hind limb 84-85; hyoid apparatus I 26, I86; jaw II8-19, I24; muscular system 134-56; nervous system 333-59; pectoral girdle 92-93; pelvic girdle $83-84$; respiratory system 186-89; ribs 75; sense organs 345-52; skull II6-26; spinal nerves $334-42$; sternum 93 ; sympathetic system $334-35,337-39$, $343-44$; teeth I25; urogenital system 288-92; venous system 249-50, $250-53,255-57,264-65$; vertebral column $73 \rightarrow 76$

Radiale 88. See Fore limb

Radialia: pectoral fin 89; pelvic fin 80

Radials. See Radialia

Radius 88. See Fore limb

Ramus communicans 299:mammal 337 turtle 323

Raphe 133,136

Rectal gland 165

Rectrices 26

Remiges 26

Renal corpuscle 275

Renal portal system 204-6, 214, 224 , 229, $240-4 \mathrm{I}, 247,269-70,27 \mathrm{I}$ : dogfish 214 ; mammal 260-70; Necturus 223-25, 220; pigeon 247; skate $2 \mathrm{I}_{4}$; turtle $23 \mathrm{O}^{-} 32,24 \mathrm{O}^{-} 4 \mathrm{I}$
Renal portal vein 206: elasmobranch 214; Necturus 223-24, 220; pigeon $243-44,247$; turtle $23 \mathrm{I}-32,24 \mathrm{O}^{-4} \mathrm{I}$ Reptilia: classification of 7; exoskeleton 50-52; fore limb or; hind limb $8 \mathrm{I}-82$; pectoral girdle 90-9I; pelvic girdle $8 \mathrm{I}$; skull II 2-I6; sternum 9I; vertebral column 70-72. See further under Alligator, Lizard, and Turtle

Respiratory system I 6I-63, I $98-99$ : elasmobranchs I67-68; mammal I86-88; Necturus I7I; pigeons 17781; summary I08 -09 ; turtle I 75-76

Restiform body: elasmobranch 308 ; mammal 354,358

Rete testis 276

Retina 30r, 36r: elasmobranch 305; mammal 349; pigeon 330

Retroperitoneal, definition of 176

Rhinencephalon: elasmobranchs 307

Rhombencephalon 207

Ribs 62, 77: alligator 71-72; $A m$ phibia 69; birds 73 ; dogfish 66; kinds of 62,77 ; mammals 75 ; origin of 62 ; teleost $67-68$; turtle 72

Roots of spinal nerves 298, 359: elasmobranchs 315, 3I6; mammal $34 \mathrm{I}$

Rostrum 17,97

Sacculus, of ear: elasmobranch 306 ; mammal 352; Necturus 319; pigeon 330

Sacculus rotundus 103

Sacral plexus, pigeon 328

Sacrum 69 See under Vertebral column

Salivary glands I6r: mammal r $83-84$

Sauropsida, definition of 8

Scales: ctenoid 49-50; cycloid 49; fishes $21,22,47-50$; ganoid 2I, 49; mammals 55 ; reptiles $23,24,25$, 50-52

Scapula 86. See Pectoral girdle

Sciatic nerve: mammal $340 ; \mathrm{Nec}$ turus 310; pigeon 328; turtle 322

Sciatic vein: pigeon 243; turtle 23I

Sclera 300: elasmobranch 305 ; mam. mal 349; pigeon 320

Sclerotic coat. See Sclera

Sclerotome 43 ; in development of vertebrae $58-6$ I

Scrotum 20, 200

Scute: armadillo 55; reptiles 50-52

Segment 2

Segmentation 2: of head 299-300, 315; of circulatory system 204, 206

Segmentation cavity 32

Sella turcica I 2I, 35 I

Semicircular canals: mammal 352 ; pigeon $33^{\circ}$

Semicircular ducts: elasmobranch 306; mammal 352; Necturus 319; pigeon 330; turtle 326

Semilunar ganglion: Necturus 320 ; pigeon 332: turtle 325,326

Semilunar valves: elasmobranch $22 \mathrm{I}$; mammal 266; Necturus 229; pigeon 246,247 ; turtle 239

Seminal vesicle: elasmobranch 283 ; rabbit 291

Seminiferous tubules 276

Sense organs: development of 300 301 , 36I; elasmobranchs 302-6; mammal $345^{-52}$; Necturus 319; pigeon 329-30; turtle $323^{-24}$

Sensory, definition of $297-08$

Septum, of brain: mammal 356; pigeon 333 ; turtle 327

Septum, of nose: mammal II 7, I II 350 ; turtle 323

Septum pellucidum 356

Serosa, definition of 158

Sesamoid bone 83

Seymouria: pectoral girdle 88 ; skull I06

Shell gland 28

Sinus venosus: elasmobranch 207 22I; mammal 248; Necturus 222 228-20: turtle 230,238 
Skate: arterial system 215-10; brain $307-9,316-18$; circulatory system $210-11,213,214,215-19$; classification of 6 ; coelom 163-67; cranial nerves 309-16; digestive system I64-66; exoskeleton 47 ; external anatomy $18-19$; gills 168; heart 207, 221-22; nervous system $301-18$; respiratory system I68; scales 47 ; sense organs $302-6$; spinal nerves $301-2$; urogenital system $28 \mathrm{r}-83$; venous system $210-11,213,214$

Skeletogenous regions 57

Skeletogenous septa 57,58

Skeleton: definition of 45, 57; exoskeleton 45-56; gill arches $99^{-}$ I00, III-I 2; girdles and appendages $78-94$; ribs $57-77$; skull 96-1 27; sternum 85-86, 90-93, 95; summary 76-77, 94-95; 1 26-27, vertebral column 57-77; visceral skeleton $99-100$

Skin $45^{-55}$ : microscopic structure of $45-46$

Skull 96-97, 99, 100-101, 103-7: alligator I I 2-I6; bones of 103-7; cartilage bones of $103-5$; definition of 96; elasmobranchs 97-100; ganoids ror-3; mammal II6-26; membrane bones of $100-103,105-7$;

Necturus ro7-1 2; origin of $96-97$

Solar plexus 338

Somatopleure, definition of $4 \mathrm{I}$

Somatic mesoderm 36,4 I

Somatic motor column 309, 325

Somatic muscles 128

Somatic sensory column: elasmobranch 308-9; mammal 354; turtle 324

Somite 2

Spermatic cord 290

Sperm sac 283

Sphenodon: classification of 7 ; vertebral column 7

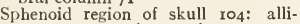
gator $\mathrm{II}_{4}{ }^{-15}$; bones of $\mathrm{r}_{4}$; mammal 120

Sphenopalatine ganglion 345

Spinal accessory nerve $36 \mathrm{r}$ : mammal 342,355 ; pigeon 332 ; turtle 326

Spinal cord 206-08: definition of 206 . development of 296-97; functional components 298; mammal $34 \mathrm{r}$

Spinal ganglion 208: elasmobranchs 315; mammal 341; Necturus 319; pigeon 328 ; turtle 322

Spinal nerves 296, 298-99, 359; components 208; definition of 206 ; elasmobranchs 3 OI-2, 315-16; mammal $334-42 ;$ Necturus $318-19$; parts of 298-99; pigeon 328; turtle $32 \mathrm{I}-23$

Spiracle 17, 168

Spiral valve $164^{-6}$

Splanchnic mesoderm $36,4 \mathrm{I}$

Splanchnic nerves 338

Splanchnocranium 90-100

Splanchnopleure $4 \mathrm{I}$

Spleen 200: elasmobranch I64; mammal r9o; Necturus I69; pigeon I82; turtle I74

Spoonbill. See Polyodon

Stegocephala: classification of 7 ; exoskeleton 50; jaw I08; pectoral girdle 87,88 ; skull ror, ro6; vertebral column 64

Sternebra 93

Sternum $78,85-86,95$ : alligator or; bird $9 \mathrm{I}$ : definition of 78 ; frog 90 ; mammal 93; origin of $85-86$; urodeles 90

Stomach I6r: elasmobranch I64; mammal r9o-9r; Necturus 169; pigeon 181,182 ; turtle 174

Stratum corneum 45,46 See Exoskeleton

Stratum germinativum 45,46 . See Exoskeleton

Sturgeon. See Acipenser
Subcardinal vein $204-6,224,270-71$ : elasmobranchs 214; in formation of postcaval vein 229, 24I, 268, $270-71$

Subclavian artery: cat 258-59; elasmobranch 218; Necturus 227; pigeon 244 ; rabbit $257-58$; turtle

Subclavian vein: cat 254; dogfish 209; Necturus 225; pigeon 242-43; rabbit $25 \mathrm{I}$; turtle 234

Subintestinal vein $203,205,206,270$

Sublingual gland 184

Submaxillary gland 140,184

Subscapular fossa 93

Sulcus 353

Summary: appendages $94-06$; circulatory system 270-72; coelom $195^{-}$ 99; digestive system 198-99; exoskeleton 55-56; external anatomy 20-30; girdles 94-06; muscular system 157; nervous system 359-6r; respiratory system 198-99; ribs 77 ; sense organs $36 \mathrm{I}$; skull I 26-27; sternum 05; urogenital system 294-95: vertebral column 76-77

Superior cervical ganglion: mammal 343; turtle 327

Superior mesenteric artery: cat 262 ; pigeon 245 ; rabbit 261 ; turtle 237

Superior mesenteric ganglion 338

Superior mesenteric vein: cat 250; pigeon 242 ; rabbit 249

Supine, definition of 28

Supracondyloid foramen 93

Suprascapula 86-87. See Pectoral girdle

Supraspinous fossa 93

Supratemporal arcade 106, II 2

Supratemporal fossa II 2

Suspensory ligament: of lens 349 of liver. See Falciform ligament

Suture, definition of $\mathbf{1 0 8 ,}$ I 17

Swim bladder 163

Symmetry $1-2$

Sympathetic system 206, 299, 359: mammal $334^{-35}, 337-39,343^{-44}$ pigeon 328,332 ; turtle $321,322-23$, 327

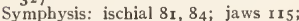
pubic 81,84

Synotic tectum 97, 104, I II

Synsacrum 73

Syrinx 183

Systemic veins 200, 203-4: definition of 200 ; development $2 \mathrm{O}_{3}-4$; dogfish 207-10; mammal 25I-56, 264 65: Necturus 225-26; pigeon 24244; skate 2IO-II; turtle 233-35. 237

Tarsales 79. See Hind limb

Tarsus 79: bird 83 ; mammal $84-85$; turtle 82

Teeth 48-49; alligator I 16; homology of 48-49; mammal $\mathrm{r}_{24}-25 ; \mathrm{Nec}$ turus 109, 171; structure of $4^{8-49}$

Tegmentum 357

Telencephalon 297. 359: elasmobranch 307, 318; Necturus 320. See Cerebral hemisphere

Teleostei: classification of 6 ; external anatomy I9-2I; pectoral girdle 87,89 ; ribs $67-68$; scales $49^{-} 50^{\circ}$ vertebral column $67-68$

Teleostomi: classification of 6 . See further under Ganoids

Telolecithal, definition of $3 \mathrm{I}$

Temiporal fossa 117

Tendon, definition of 136

Tendon of Achilles 155

Tentorium I 21, 35 I

Testis 276,295 : descent of 290 ; elasmobranch 282; mammal 290 292; pigeon 287; turtle 286

Thalamencephalon. See Diencephalon

Thalamus 359-60; elasmobranch 318 ; mammal 356,358 ; turtle 327
Thecodont II6, 124

Thoracic duct 260

Thorax 27, $187-89$ : muscles of 139 . I $44-50$

Thymus 162: mammal 189; turtle 235

Thy roid cartilage 142,187

Thyroid gland 161, 162: elasmobranch 215; mammal I87; turtle 235

Tibia 79, 8o. See Hind limb

Tibiale 79, 8o. See Hind limb

Tongue: elasmobranch I68; mammal 185; Necturus 171; pigeon 177; turtle 175

Tonsils 162,186

Trabecula 97. See Prechordal

Trabeculae carnae 266

Trachea $162-63$, 199: mammal 187 ; Necturus 1 71 ; pigeon $177,178,183$; turtle $\mathbf{I 7 5}$

Transverse septum $159,195-98,203-$ 4: elasmobranch 166, I67; mam mal I89, 197; Necturus 170, I71; pigeon I80-81, r97; turtle 173,106

Trapezoid body $355,35^{8}$

Tricuspid valve 266

Trigeminus nerve 360 : elasmobranch 3II-I3; mammal 344-45, 346, 347, 348 , 355; Necturus 320-21; pigeon $331-32$; turtle $325-26$

Trochanter 83,84

Trochlea: of eye 346,347 ; of humerus 93

Trochlear nerve 360 : elasmobranch $307,308,3$ ro: mammal 347,349 , 354; Necturus 320; pigeon 329,331 ; tuitle 323,325

Tuber cinereum 35

Tuberculum of rib $60,71,75$

Tuberculum cuneatum 354,358

Tunicata: classification of 6 ; external anatomy $1 \mathrm{I}^{-12}$; internal anatomy I 2-13: pharynx I3 $_{3}$

Turbinals 300 . See Conchae

Turbinated bones I2I-22. See Conchae

Turtle: arterial system $235-38$; brain $324^{-25}, 327$; carapace $5 \mathrm{I}-52$; circulatory system $230-4 \mathrm{r}$; classification of 7 ; coelom 172-73; cranial nerves 325-27; digestive system 173-75; exoskeleton 5I-52; external anatomy 24-25; fore limb $9 r$; heart 230 238-39; hind limb 8I-82; hyoid apparatus $115-16$; nervous system 321-28; pectoral girdle $90-91$; pelvic girdle 81 ; plastron 52; respiratory system $175-76$; sense organs $323-25$; spinal nerves 321-23; sympathetic system 32223; urogenital system 284-86; venous system $230-35,237$; vertebral column 72

Tympanic bulla $117, \mathrm{I} 19, \mathrm{r} 20,35 \mathrm{I}^{-52}$ Tympanic cavity 162, 300-301, 361: mammal 35I-52; pigeon 330; turtle 324

Tympanic membrane 162, 301, 36r: cat 352 ; pigeon 330 ; rabbit $35 \mathrm{I}$; turtle 324

Ulna 88. See Fore limb

Ulnare 88. See Fore limb

Umbilical vein 203,294

Uncinate process 73

Unguligrade walk 28

Ureter 276, 294: mammal 288; pigeon 287 ; turtle 285,286

Urethra $279,288,290,29 \mathrm{I}$

Urethral groove 286

Urinary bladder $163,276,292,295$ : mammal roo, 194, 288; Necturus I69, I 70, 284: skate 282; turtle I $74,285,286$

Urodaeum 287

Urodela: classification of 7 ; external anatomy 22-23; fore limb 90 ; hind limb $8 \mathrm{r}$; pectoral girdle $89-90$; pelvic girdle 8o-81; vertebral 

